for

\title{
Copper(I)-Catalyzed Asymmetric Alkylation of Unsymmetrical Secondary Phosphines
}

Shuai Zhang, Jun-Zhao Xiao, Yan-Bo Li, Chang-Yun Shi, and Liang Yin*

CAS Key Laboratory of Synthetic Chemistry of Natural Substances, Centre for Excellence in Molecular Synthesis, Shanghai Institute of Organic Chemistry, University of Chinese Academy of Sciences, Chinese Academy of Sciences, 345 Lingling Road, Shanghai 200032, China.

liangyin@sioc.ac.cn

Table of Contents

1. General Information 2

2. Substrate Synthesis 2

3. Optimization of the Reaction Conditions 5

4. Catalytic Asymmetric Alkylation of Unsymmetrical Secondary Phosphines 7

5. 0.5 mmol-Scale Reaction 104

6. Preparation of Copper(I)-Complexes 16, 17, and $18 \quad 105$

7. Determination of the Absolute Configurations of the Products 115

$\begin{array}{ll}\text { 8. Ligand-Accelerating Effect } & 119\end{array}$

9. References 120

10. NMR Spectra Data 121 


\section{General Information}

All reagents were obtained commercially unless otherwise noted. Nuclear Magnetic Resonance (NMR) spectra were acquired on an Agilent 400, Bruker 400 or Bruker 500 spectrometer. For ${ }^{1} \mathrm{H}$ NMR, chemical shifts were reported in $\delta \mathrm{ppm}$ referenced to an internal $\mathrm{SiMe}_{4}$ standard. For ${ }^{19} \mathrm{~F} \mathrm{NMR}, \mathrm{CFCl}_{3}$ was used as the reference with chemical shift at $0 \mathrm{ppm}$. For ${ }^{13} \mathrm{C}$ NMR, chemical shifts were reported in the scale relative to NMR solvent $\left(\mathrm{CDCl}_{3}: \delta 77.0 \mathrm{ppm}\right)$ as an internal reference. ${ }^{31} \mathrm{P}$ NMR spectra were referenced externally to phosphoric acid. Multiplicities are reported using the following abbreviations: $\mathrm{br}=$ broad, $\mathrm{s}=$ singlet, $\mathrm{d}=$ doublet $\mathrm{t}$ $=$ triplet, $\mathrm{q}=$ quartet, $\mathrm{p}=$ pentet, $\mathrm{m}=$ multiplet. High-resolution mass spectra (ESI) were measured on Thermo Scientific LTQ FT Ultra FT-MS. High-resolution mass spectra (MALDI) were measured on Voyager-DE STR. Infrared (IR) spectra were recorded on Thermo Scientific Nicolet iS5 FT-IR. Optical rotation was measured using a $1 \mathrm{~mL}$ cell with $1.0 \mathrm{dm}$ path length on a JASCO P-1030 polarimeter. HPLC analysis was conducted on a Shimadzu HPLC system equipped with Daicel chiral-stationary-phase columns $(\phi 4.6 \mathrm{~mm} \times 250 \mathrm{~mm})$.

\section{Substrate Synthesis}

\subsection{Preparation of Diarylphosphines 1.}

$\mathbf{1 a}, \mathbf{1 e}, \mathbf{1 h}, \mathbf{1 i}, \mathbf{1} \mathbf{j}$ and $\mathbf{1 k}$ were known compounds ${ }^{[1]}$.

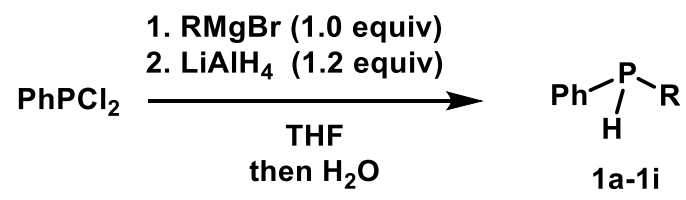

General preparation for HPPhR (1a-1i): A dried $250 \mathrm{~mL}$ round-bottom flask equipped with a magnetic stirring bar was charged with $\mathrm{PhPCl}_{2}(3.4 \mathrm{~mL}, 25 \mathrm{mmol}, 1.0$ equiv) and THF (40 mL) under $\mathrm{N}_{2}$ atmosphere. After the mixture was cooled to $-78^{\circ} \mathrm{C}$, Grignard reagent $\mathrm{RMgBr}(25 \mathrm{mmol}$, 1.0 equiv, in $40 \mathrm{~mL}$ THF) was added dropwise. Then the reaction mixture was stirred at $-78^{\circ} \mathrm{C}$ for 2 hours. After the mixture was allowed to warm up to $0{ }^{\circ} \mathrm{C}, \mathrm{LiAlH}_{4}(1.14 \mathrm{~g}, 30 \mathrm{mmol}, 1.2$ equiv, in $20 \mathrm{~mL}$ THF) was added dropwise. Then the resulting reaction mixture was stirred at room temperature for 2 hours and degassed $\mathrm{H}_{2} \mathrm{O}(8 \mathrm{~mL})$ was added slowly. Then the mixture was filtered to give a clear solution and the solids were washed quickly with anhydrous $\mathrm{Et}_{2} \mathrm{O}(50 \mathrm{~mL})$ for three times. The organic layers were combined and the resulting solution was evaporated quickly under reduced pressure to give crude product, which was purified by distillation under reduced pressure to give the pure secondary phosphine. These phosphines were stocked in a glove box for further use. Note: These phosphines are very irritant and should be handled with extra care. 


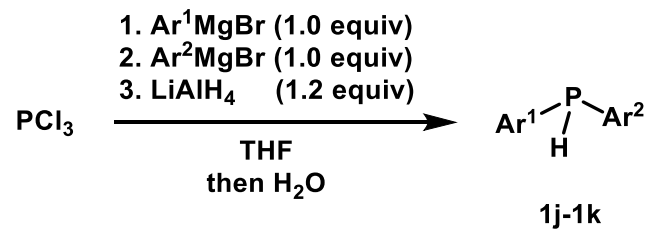

General preparation for $\operatorname{HPAr}^{1} \mathrm{Ar}^{2}(\mathbf{1 j} \mathbf{j}-\mathbf{1 k})$ : A dried $250 \mathrm{~mL}$ round-bottom flask equipped with a magnetic stirring bar was charged with $\mathrm{PCl}_{3}(1.74 \mathrm{~mL}, 20 \mathrm{mmol}, 1.0$ equiv) and THF (40 mL) under $\mathrm{N}_{2}$ atmosphere. After the mixture was cooled to $-78{ }^{\circ} \mathrm{C}$, Grignard reagent $\operatorname{Ar}^{1} \mathrm{MgBr}(20$ mmol, 1.0 equiv, in $20 \mathrm{~mL}$ THF) was added dropwise. Then the reaction mixture was stirred at -78 ${ }^{\circ} \mathrm{C}$ for 2 hours. Then Grignard reagent $\mathrm{Ar}^{2} \mathrm{MgBr}$ (20 mmol, 1.0 equiv, in $20 \mathrm{~mL}$ THF) was added dropwise and the resulting reaction mixture was stirred at $-78{ }^{\circ} \mathrm{C}$ for 2 hours and at room temperate for additional 2 hours. After the mixture was cooled to $0{ }^{\circ} \mathrm{C}, \mathrm{LiAlH}_{4}(0.9 \mathrm{~g}, 24 \mathrm{mmol}$, 1.2 equiv, in $10 \mathrm{~mL}$ THF) was added dropwise. Then the resulting reaction mixture was stirred at room temperature for 2 hours and degassed $\mathrm{H}_{2} \mathrm{O}(6 \mathrm{~mL})$ was added slowly at $0{ }^{\circ} \mathrm{C}$. After the mixture was stirred at room temperature for half of an hour, it was filtered to give a clear solution and the solids were washed quickly with anhydrous $\mathrm{Et}_{2} \mathrm{O}(50 \mathrm{~mL})$ for three times. The organic layers were combined and the resulting solution was evaporated quickly under reduced pressure to give crude product, which was purified by distillation under reduced pressure to give the pure secondary phosphine. These phosphines were stocked in a glove box for further use. Note: These phosphines are very irritant and should be handled with extra care.

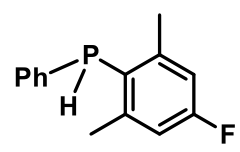

$1 b$

${ }^{1}$ H NMR (500 MHz, $\left.\mathbf{C D C l}_{3}\right) \delta 7.23(\mathrm{~s}, 5 \mathrm{H}), 6.83(\mathrm{dd}, J=9.7,1.9 \mathrm{~Hz}, 2 \mathrm{H}), 5.10(\mathrm{~s}, 1 \mathrm{H}), 2.43(\mathrm{~s}$, $6 \mathrm{H})$.

${ }^{13} \mathbf{C}$ NMR(126 MHz, $\left.\mathbf{C D C l}_{3}\right) \delta 162.99(\mathrm{~d}, J=247.9 \mathrm{~Hz}), 145.66(\mathrm{dd}, J=13.6,8.0 \mathrm{~Hz}), 133.92(\mathrm{~d}$, $J=12.8 \mathrm{~Hz}), 131.88(\mathrm{~d}, J=16.5 \mathrm{~Hz}), 128.47(\mathrm{~d}, J=5.9 \mathrm{~Hz}), 127.78,127.24(\mathrm{dd}, J=11.7,3.2 \mathrm{~Hz})$, $114.98(\mathrm{dd}, J=20.2,3.6 \mathrm{~Hz}), 23.35(\mathrm{dd}, J=11.9,1.8 \mathrm{~Hz})$.

${ }^{31}$ P NMR (162 MHz, $\left.\mathbf{C D C l}_{3}\right) \delta-78.21$.

${ }^{19}$ F NMR (376 MHz, $\left.\mathrm{CDCl}_{3}\right) \delta-109.27$.

HRMS (ESI) $\mathbf{m} / \mathbf{z}$ [M+H] $]^{+}$: calcd. 233.0890, found. 233.0891.

IR (film): $v_{\max }\left(\mathrm{cm}^{-1}\right)$ 3069, 2971, 2327, 1597, 1466, 1296, 1139, 1023, 859, 725, 694.

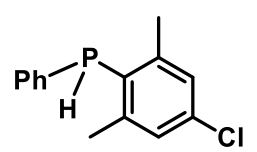

$1 \mathrm{c}$

${ }^{1}$ H NMR (500 MHz, $\left.\mathbf{C D C l}_{3}\right) \delta 7.23(\mathrm{~d}, J=2.9 \mathrm{~Hz}, 5 \mathrm{H}), 7.10(\mathrm{~d}, J=2.1 \mathrm{~Hz}, 2 \mathrm{H}), 5.09(\mathrm{~s}, 1 \mathrm{H})$, $2.40(\mathrm{~s}, 6 \mathrm{H})$.

${ }^{13} \mathbf{C}$ NMR(126 MHz, $\left.\mathbf{C D C l}_{3}\right) \delta 144.59(\mathrm{~d}, J=12.9 \mathrm{~Hz}), 134.64,133.31(\mathrm{~d}, J=12.5 \mathrm{~Hz}), 132.19$ (d, $J=16.8 \mathrm{~Hz}), 130.66$ (d, $J=13.0 \mathrm{~Hz}), 128.50$ (d, $J=6.0 \mathrm{~Hz}), 127.99,127.97,23.09$ (d, $J=$ $11.4 \mathrm{~Hz})$.

${ }^{31}$ P NMR (162 MHz, CDCl $) \delta-77.97$.

HRMS (ESI) m/z [M+H] $]^{+}$: calcd. 249.0594, found. 249.0593. 
IR (film): $v_{\max }\left(\mathrm{cm}^{-1}\right)$ 3069, 2973, 2328, 1573, 1459, 1435, 1252, 1134, 1025, 869, 725, 693.

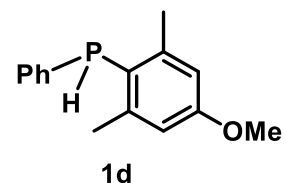

${ }^{1}$ H NMR $\left(\mathbf{5 0 0} \mathbf{~ M H z}, \mathbf{C D C l}_{\mathbf{3}}\right) \delta 7.21(\mathrm{~d}, J=4.0 \mathrm{~Hz}, 5 \mathrm{H}), 6.68(\mathrm{~d}, J=2.0 \mathrm{~Hz}, 2 \mathrm{H}), 5.28(\mathrm{~s}, 1 \mathrm{H})$, $3.78(\mathrm{~s}, 3 \mathrm{H}), 2.42(\mathrm{~s}, 6 \mathrm{H})$.

${ }^{13} \mathbf{C}$ NMR(126 MHz, $\left.\mathbf{C D C l}_{3}\right) \delta 160.05,145.01(\mathrm{~d}, J=13.7 \mathrm{~Hz}), 135.03(\mathrm{~d}, J=13.3 \mathrm{~Hz}), 131.57$ $(\mathrm{d}, J=16.1 \mathrm{~Hz}), 128.33(\mathrm{~d}, J=5.5 \mathrm{~Hz}), 127.40,122.52(\mathrm{~d}, J=9.6 \mathrm{~Hz}), 113.73(\mathrm{~d}, J=3.9 \mathrm{~Hz})$, $54.92,23.47(\mathrm{~d}, J=12.0 \mathrm{~Hz})$.

${ }^{31}$ P NMR (162 MHz, $\left.\mathbf{C D C l}_{3}\right) \delta-82.21$.

HRMS (ESI) $\mathbf{m} / \mathbf{z}$ [M+H] ${ }^{+}$: calcd. 245.1090, found. 245.1088.

IR (film): $v_{\max }\left(\mathrm{cm}^{-1}\right)$ 3055, 2939, 2361, 1595, 1468, 1311, 1193, 1115, 959, 749, 694.

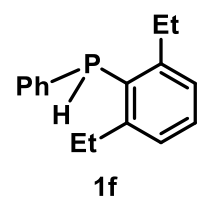

${ }^{1} \mathbf{H}$ NMR $\left(\mathbf{5 0 0} \mathbf{M H z}, \mathbf{C D C l}_{\mathbf{3}}\right) \delta 7.30(\mathrm{t}, J=7.6 \mathrm{~Hz}, 1 \mathrm{H}), 7.24-7.19(\mathrm{~m}, 5 \mathrm{H}), 7.14(\mathrm{dd}, J=7.6,2.5$ $\mathrm{Hz}, 2 \mathrm{H}), 5.18(\mathrm{~s}, 1 \mathrm{H}), 2.81$ (q, $J=7.5 \mathrm{~Hz}, 4 \mathrm{H}), 1.14(\mathrm{t}, J=7.5 \mathrm{~Hz}, 6 \mathrm{H})$.

${ }^{13} \mathbf{C}$ NMR(126 MHz, CDCl $) \delta 149.29(\mathrm{~d}, J=12.0 \mathrm{~Hz}), 135.19(\mathrm{~d}, J=12.8 \mathrm{~Hz}), 131.92(\mathrm{~d}, J=$ $16.6 \mathrm{~Hz}), 130.05(\mathrm{~d}, J=12.8 \mathrm{~Hz}), 129.56,128.32(\mathrm{~d}, J=5.9 \mathrm{~Hz}), 127.57,126.57$ (d, $J=3.3 \mathrm{~Hz})$, $29.33(\mathrm{~d}, J=11.6 \mathrm{~Hz}), 16.01$.

${ }^{31}$ P NMR (162 MHz, $\left.\mathbf{C D C l}_{3}\right) \delta-81.54$.

HRMS (ESI) $\mathbf{m} / \mathbf{z}$ [M+H] $]^{+}$: calcd. 243.1297, found. 243.1296.

IR (film): $v_{\max }\left(\mathrm{cm}^{-1}\right)$ 3053, 2964, 2871, 2330, 1585, 1460, 1434, 896, 799, 722, 694.

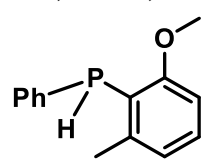

$1 \mathrm{~g}$

${ }^{1}$ H NMR (500 MHz, $\left.\mathbf{C D C l}_{3}\right) \delta$ 7.42-7.34 (m, 2H), 7.25-7.18 (m, 4H), $6.85(\mathrm{dd}, J=7.6,2.6 \mathrm{~Hz}$, $1 \mathrm{H}), 6.78-6.71(\mathrm{~m}, 1 \mathrm{H}), 5.53(\mathrm{~d}, J=213.4 \mathrm{~Hz}, 1 \mathrm{H}), 3.75(\mathrm{~s}, 3 \mathrm{H}), 2.43(\mathrm{~s}, 3 \mathrm{H})$.

${ }^{13} \mathbf{C}$ NMR(126 MHz, $\left.\mathbf{C D C l}_{3}\right) \delta 160.71(\mathrm{~d}, J=5.7 \mathrm{~Hz}), 143.49(\mathrm{~d}, J=13.0 \mathrm{~Hz}), 134.41(\mathrm{~d}, J=$ $11.9 \mathrm{~Hz}), 133.16(\mathrm{~d}, J=17.4 \mathrm{~Hz}), 129.75,128.10(\mathrm{~d}, J=6.4 \mathrm{~Hz}), 127.74,122.97$ (d, $J=3.0 \mathrm{~Hz})$, 121.96 (d, $J=13.8 \mathrm{~Hz}), 108.18,55.80,22.52$ (d, $J=12.3 \mathrm{~Hz})$.

${ }^{31}$ P NMR (162 MHz, $\left.\mathbf{C D C l}_{3}\right) \delta-86.18$

HRMS (ESI) $\mathbf{m} / \mathbf{z}$ [M+H] $]^{+}$: calcd. 231.0933, found. 231.0932.

IR (film): $v_{\max }\left(\mathrm{cm}^{-1}\right)$ 3054, 2937, 2330, 1568, 1463, 1256, 1084, 924, 774, 720, 694.

2.2. Preparation of alkyl iodides

$\mathbf{4 g}, \mathbf{4 h}, \mathbf{4 i}, \mathbf{4 j}, \mathbf{4 m}$ and $6 \mathbf{f}$ were prepared according to known methods ${ }^{[2,3,4,5,6]}$. 


\section{Optimization of the Reaction Conditions}

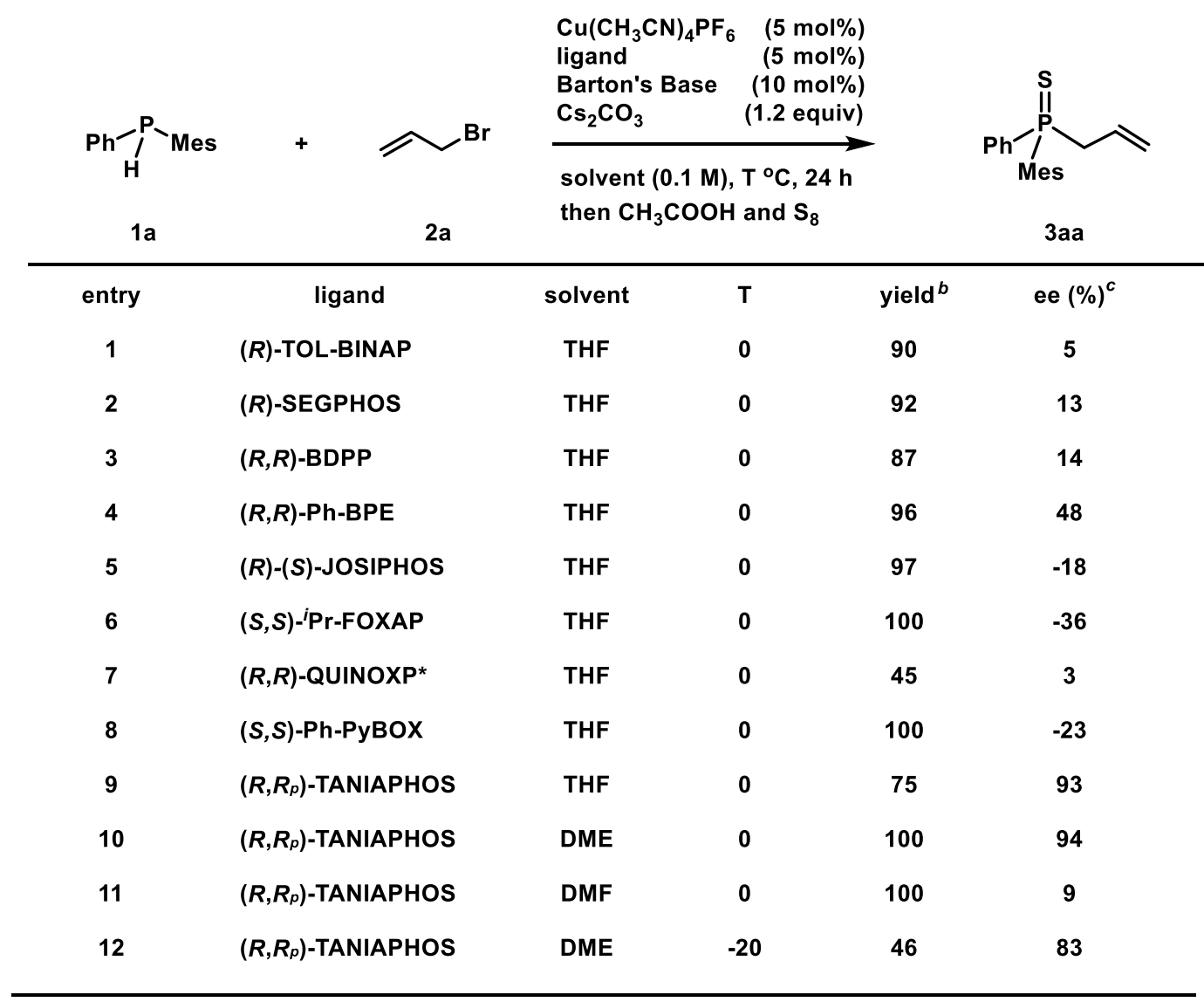

a 1a: $0.1 \mathrm{mmol}, 2 \mathrm{a}: 0.15 \mathrm{mmol} .{ }^{b}$ Determined by ${ }^{1} \mathrm{H}$ NMR analysis of reaction crude mixture using $\mathrm{CH}_{2} \mathrm{Br}_{2}$ as internal standard. ${ }^{c}$ Determined by chiral-stationary-phase HPLC analysis. 


\begin{tabular}{|c|c|c|c|c|c|c|}
\hline \multirow{2}{*}{$\begin{array}{r}\mathrm{Ph}_{\mathrm{H}}^{-\mathrm{P}} \\
\mathrm{1a} \\
\text { entry }\end{array}$} & \multirow{2}{*}{$\begin{array}{c}+ \\
\text { solvent }\end{array}$} & \multirow{2}{*}{$\frac{2 a}{\text { base }}$} & \multicolumn{3}{|c|}{ 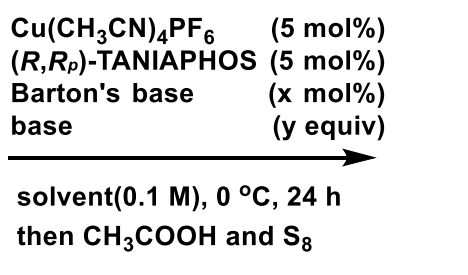 } & 3aa \\
\hline & & & $\mathbf{x}$ & y & yield $^{b}$ & ee $(\%)^{c}$ \\
\hline 1 & THF & $\mathrm{Cs}_{2} \mathrm{CO}_{3}$ & 0 & 1.2 & 60 & 93 \\
\hline 2 & THF & $\mathrm{Cs}_{2} \mathrm{CO}_{3}$ & 0 & 1.5 & 90 & 93 \\
\hline 3 & THF & $\mathrm{KO}^{t} \mathrm{Bu}$ & 0 & 1.5 & 93 & $<5$ \\
\hline 4 & THF & $\mathrm{K}_{2} \mathrm{CO}_{3}$ & 0 & 1.5 & 20 & 75 \\
\hline 5 & THF & $\mathrm{Cs}_{2} \mathrm{CO}_{3}$ & 0 & 2.0 & 96 & 92 \\
\hline 6 & THF & $\mathrm{Cs}_{2} \mathrm{CO}_{3}$ & 10 & 1.2 & 75 & 93 \\
\hline 7 & THF & $\mathrm{Cs}_{2} \mathrm{CO}_{3}$ & 20 & 1.2 & 96 & 91 \\
\hline 8 & DME & $\mathrm{Cs}_{2} \mathrm{CO}_{3}$ & 10 & 1.2 & 100 & 94 \\
\hline
\end{tabular}

a 1a: $0.1 \mathrm{mmol}, 2 \mathrm{2a}: 0.15 \mathrm{mmol}$. ${ }^{b}$ Determined by ${ }^{1} \mathrm{H}$ NMR analysis of reaction crude mixture using $\mathrm{CH}_{2} \mathrm{Br}_{2}$ as internal standard. ${ }^{c}$ Determined by chiral-stationary-phase HPLC analysis.

\begin{tabular}{|c|c|c|c|c|c|c|}
\hline \multirow{2}{*}{$\begin{array}{l}\mathrm{Ph}_{\mathrm{H}}-\mathrm{P} \\
1 \mathrm{H}\end{array}$} & \multirow[t]{2}{*}{. } & \multirow{2}{*}{$\begin{array}{c}{ }^{n} \text { Bul } \\
4 c \text { (x equiv) }\end{array}$} & \multicolumn{3}{|c|}{$\begin{array}{ll}\mathrm{Cu}\left(\mathrm{CH}_{3} \mathrm{CN}\right)_{4} \mathrm{PF}_{6} & (5 \mathrm{~mol} \%) \\
\left(R, R_{p}\right)-\mathrm{TANIAPHOS} & (5 \mathrm{~mol} \%) \\
\text { Barton's base } & \text { (y mol\%) } \\
\mathrm{Cs}_{2} \mathrm{CO}_{3} & \text { (z equiv) } \\
\end{array}$} & \multirow{2}{*}{ 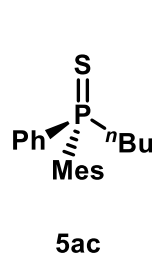 } \\
\hline & & & \multicolumn{3}{|c|}{$\begin{array}{l}\mathrm{DME}(0.1 \mathrm{M}), 0^{\circ} \mathrm{C}, \mathrm{t} \mathrm{h} \\
\text { then } \mathrm{CH}_{3} \mathrm{COOH} \text { and } \mathrm{S}_{8}\end{array}$} & \\
\hline entry & $\mathbf{x}$ & $y$ & z & $t(h)$ & yield $^{b}$ & ee $(\%)^{c}$ \\
\hline 1 & 1.5 & 10 & 1.2 & 24 & 40 & 91 \\
\hline 2 & 3.0 & 10 & 1.2 & 40 & 50 & 91 \\
\hline 3 & 3.0 & 20 & 1.2 & 40 & 60 & 91 \\
\hline 4 & 3.0 & 10 & 2.0 & 40 & 80 & 91 \\
\hline $5^{d}$ & 3.0 & 10 & 3.0 & 40 & 95 & 91 \\
\hline
\end{tabular}

a 1a: 0.1 mmol. ${ }^{b}$ Determined by ${ }^{1} \mathrm{H}$ NMR analysis of reaction crude mixture using $\mathrm{CH}_{2} \mathrm{Br}_{2}$ as internal standard. ${ }^{c}$ Determined by chiral-stationary-phase HPLC analysis. ${ }^{d}$ Isolated yield. 


\section{Catalytic Asymmetric Alkylation of Unsymmetrical Secondary}

\section{Phosphines}

General procedures for the catalytic asymmetric alkylation of unsymmetrical secondary phosphines.

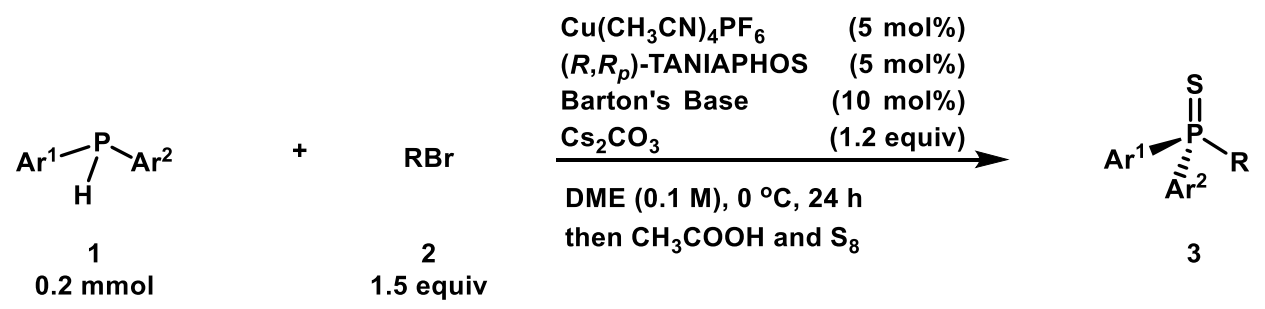

Procedure A:

A dried $25 \mathrm{~mL}$ Schlenk tube equipped with a magnetic stirring bar was charged with $\left[\mathrm{Cu}\left(\mathrm{CH}_{3} \mathrm{CN}\right)_{4}\right] \mathrm{PF}_{6}\left(3.7 \mathrm{mg}, 0.01 \mathrm{mmol}, 0.05\right.$ equiv) and $\left(R, R_{\mathrm{P}}\right)$-TANIAPHOS $(6.9 \mathrm{mg}, 0.01 \mathrm{mmol}$, 0.05 equiv) in a glove box under Ar atmosphere. Anhydrous DME $(2.0 \mathrm{~mL})$ was added via a syringe. The mixture was stirred at room temperature for 15 minutes to give a yellow catalyst solution. Then 1 ( $0.2 \mathrm{mmol}, 1.0$ equiv) and $\mathrm{Cs}_{2} \mathrm{CO}_{3}(78.0 \mathrm{mg}, 0.24 \mathrm{mmol}, 1.2$ equiv) were added sequentially and the Schlenk tube was taken out of the glove box. After the mixture was cooled to $0{ }^{\circ} \mathrm{C}, 2$ ( $0.3 \mathrm{mmol}, 1.5$ equiv) and Barton's Base ( $40 \mu \mathrm{L}$ ( $0.5 \mathrm{M}$ in DME), $0.02 \mathrm{mmol}, 0.1$ equiv) was added sequentially. The resulting reaction mixture was stirred at $0{ }^{\circ} \mathrm{C}$ for 24 hours. Then the reaction mixture was quenched by acetic acid $(40 \mu \mathrm{L})$ and $\mathrm{S}_{8}$ and the reaction mixture was stirred for additional 30 minutes at room temperature. After solvent was removed under reduced pressure, the residue was purified by silica gel column chromatography (petroleum ether/ethyl acetate $=$ 50/1) to give the desired product.

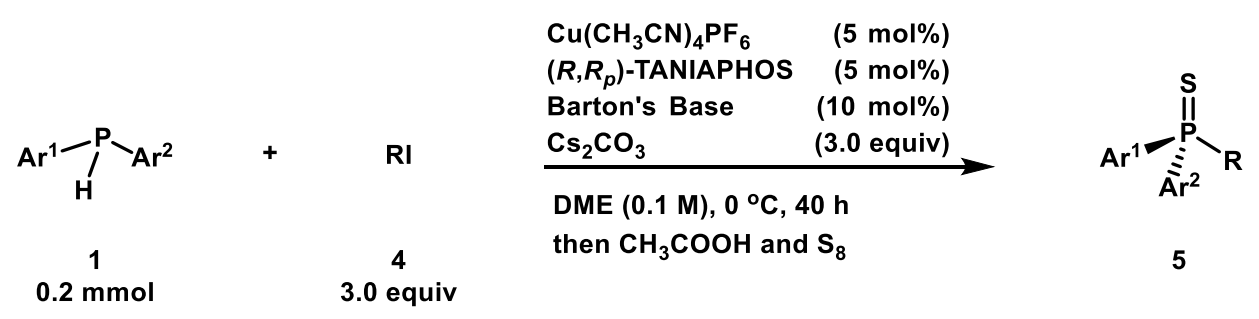

Procedure B:

A dried $25 \mathrm{~mL}$ Schlenk tube equipped with a magnetic stirring bar was charged with $\left[\mathrm{Cu}\left(\mathrm{CH}_{3} \mathrm{CN}\right)_{4}\right] \mathrm{PF}_{6}\left(3.7 \mathrm{mg}, 0.01 \mathrm{mmol}, 0.05\right.$ equiv) and $\left(R, R_{\mathrm{P}}\right)$-TANIAPHOS $(6.9 \mathrm{mg}, 0.01 \mathrm{mmol}$, 0.05 equiv) in a glove box under Ar atmosphere. Anhydrous DME $(2.0 \mathrm{~mL})$ was added via a syringe. The mixture was stirred at room temperature for 15 minutes to give a yellow catalyst solution. Then 1 ( $0.2 \mathrm{mmol}, 1.0$ equiv) and $\mathrm{Cs}_{2} \mathrm{CO}_{3}(195.0 \mathrm{mg}, 0.6 \mathrm{mmol}, 3.0$ equiv) were added sequentially and the Schlenk tube was taken out of the glove box. After the mixture was cooled to $0{ }^{\circ} \mathrm{C}, 4$ (0.6 mmol, 3.0 equiv) and Barton's Base ( $40 \mu \mathrm{L}$ (0.5 M in DME), $0.02 \mathrm{mmol}, 0.1$ equiv) was added sequentially. The resulting reaction mixture was stirred at $0{ }^{\circ} \mathrm{C}$ for 40 hours. Then the reaction mixture was quenched by acetic acid $(80 \mu \mathrm{L})$ and $\mathrm{S}_{8}$ and the reaction mixture was stirred for additional 30 minutes at room temperature. After solvent was removed under reduced pressure, the residue was purified by silica gel column chromatography (petroleum ether/ethyl acetate $=$ 50/1-15/1) to give the desired product. 


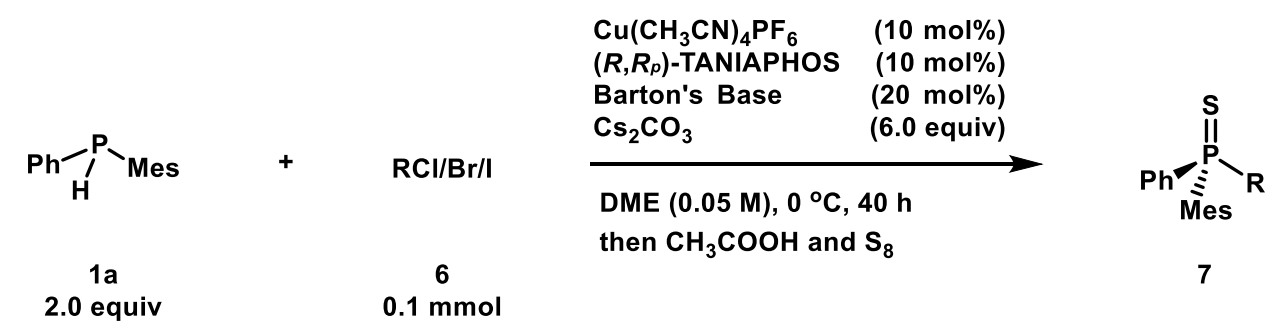

Procedure C:

A dried $25 \mathrm{~mL}$ Schlenk tube equipped with a magnetic stirring bar was charged with $\left[\mathrm{Cu}\left(\mathrm{CH}_{3} \mathrm{CN}\right)_{4}\right] \mathrm{PF}_{6}\left(3.7 \mathrm{mg}, 0.01 \mathrm{mmol}, 0.1\right.$ equiv) and $\left(R, R_{\mathrm{P}}\right)$-TANIAPHOS (6.9 mg, $0.01 \mathrm{mmol}$, 0.1 equiv) in a glove box under Ar atmosphere. Anhydrous DME (2.0 mL) was added via a syringe. The mixture was stirred at room temperature for 15 minutes to give a yellow catalyst solution. Then $1 \mathrm{a}$ (46 $\mu \mathrm{L}, 0.2 \mathrm{mmol}, 2.0$ equiv) and $\mathrm{Cs}_{2} \mathrm{CO}_{3}$ (195.0 mg, $0.6 \mathrm{mmol}, 6.0$ equiv) were added sequentially and the Schlenk tube was taken out of the glove box. After the mixture was cooled to $0{ }^{\circ} \mathrm{C}, 6$ ( $0.1 \mathrm{mmol}, 1.0$ equiv) and Barton's Base (40 $\mu \mathrm{L}$ (0.5 M in DME), $0.02 \mathrm{mmol}, 0.2$ equiv) was added sequentially. The resulting reaction mixture was stirred at $0{ }^{\circ} \mathrm{C}$ for 40 hours. Then the reaction mixture was quenched by acetic acid $(80 \mu \mathrm{L})$ and $\mathrm{S}_{8}$ and the reaction mixture was stirred for additional 30 minutes at room temperature. After solvent was removed under reduced pressure, the residue was purified by silica gel column chromatography (petroleum ether/ethyl acetate $=30 / 1-8 / 1$ ) to give the desired product.

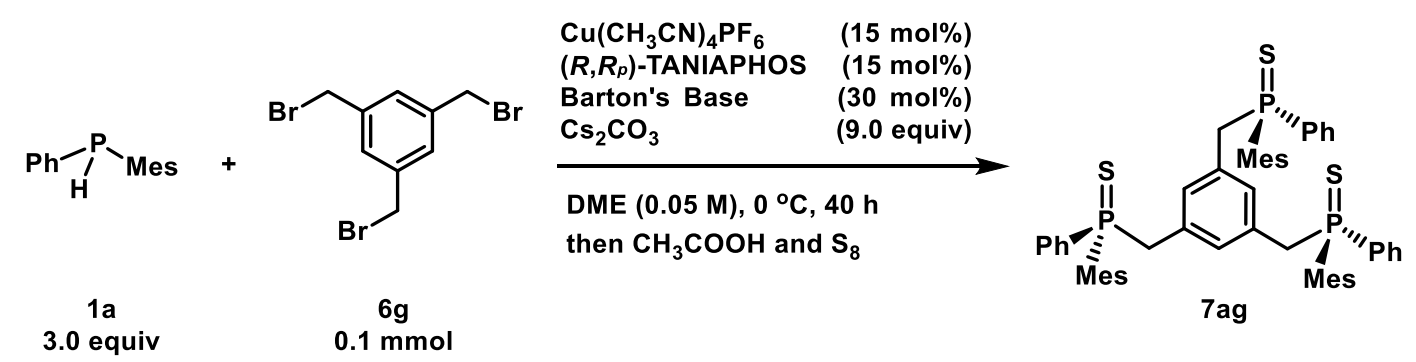

Procedure D:

A dried $25 \mathrm{~mL}$ Schlenk tube equipped with a magnetic stirring bar was charged with $\left[\mathrm{Cu}\left(\mathrm{CH}_{3} \mathrm{CN}\right)_{4}\right] \mathrm{PF}_{6}\left(5.6 \mathrm{mg}, 0.015 \mathrm{mmol}, 0.15\right.$ equiv) and $\left(R, R_{\mathrm{P}}\right)$-TANIAPHOS (10.3 mg, 0.015 mmol, 0.15 equiv) in a glove box under Ar atmosphere. Anhydrous DME $(2.0 \mathrm{~mL})$ was added via a syringe. The mixture was stirred at room temperature for 15 minutes to give a yellow catalyst solution. Then 1a (69 $\mu \mathrm{L}, 0.3 \mathrm{mmol}, 3.0$ equiv) and $\mathrm{Cs}_{2} \mathrm{CO}_{3}(293.0 \mathrm{mg}, 0.9 \mathrm{mmol}, 9.0$ equiv) were added sequentially and the Schlenk tube was taken out of the glove box. After the mixture was cooled to $0{ }^{\circ} \mathrm{C}, \mathbf{6 g}(0.1 \mathrm{mmol}, 1.0$ equiv) and Barton's Base (60 $\mu \mathrm{L}$ (0.5 M in DME), $0.03 \mathrm{mmol}$, 0.3 equiv) was added sequentially. The resulting reaction mixture was stirred at $0{ }^{\circ} \mathrm{C}$ for 40 hours. Then the reaction mixture was quenched by acetic acid $(80 \mu \mathrm{L})$ and $\mathrm{S}_{8}$ and the reaction mixture was stirred for additional 30 minutes at room temperature. After solvent was removed under reduced pressure, the residue was purified by silica gel column chromatography (petroleum ether/ethyl acetate $=6 / 1$ ) to give the desired product $\mathbf{7 a g}$.

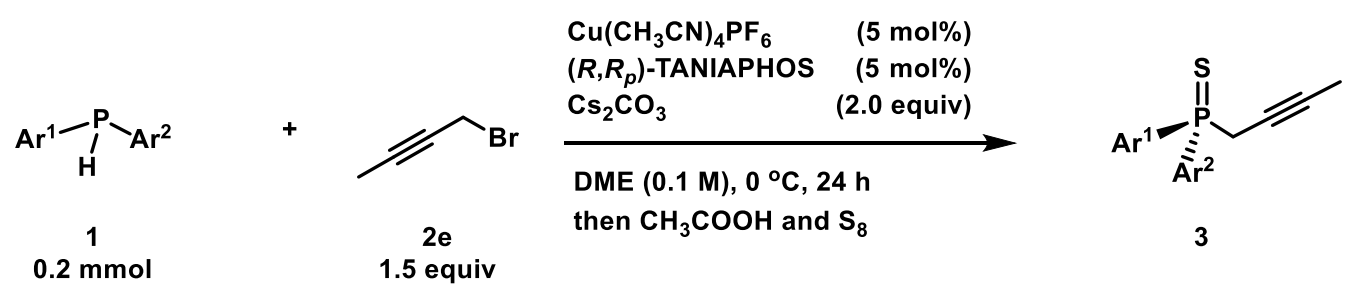


Procedure E:

A dried $25 \mathrm{~mL}$ Schlenk tube equipped with a magnetic stirring bar was charged with $\left[\mathrm{Cu}\left(\mathrm{CH}_{3} \mathrm{CN}\right)_{4}\right] \mathrm{PF}_{6}\left(3.7 \mathrm{mg}, 0.01 \mathrm{mmol}, 0.05\right.$ equiv) and $\left(R, R_{\mathrm{P}}\right)$-TANIAPHOS $(6.9 \mathrm{mg}, 0.01 \mathrm{mmol}$, 0.05 equiv) in a glove box under Ar atmosphere. Anhydrous DME $(2.0 \mathrm{~mL})$ was added via a syringe. The mixture was stirred at room temperature for 15 minutes to give a yellow catalyst solution. Then 1 ( $0.2 \mathrm{mmol}, 1.0$ equiv) and $\mathrm{Cs}_{2} \mathrm{CO}_{3}(130.0 \mathrm{mg}, 0.4 \mathrm{mmol}, 2.0$ equiv) were added sequentially and the Schlenk tube was taken out of the glove box. After the mixture was cooled to $0{ }^{\circ} \mathrm{C}, 2 \mathrm{e}(26 \mu \mathrm{L}, 0.3 \mathrm{mmol}, 1.5$ equiv) was added sequentially. The resulting reaction mixture was stirred at $0{ }^{\circ} \mathrm{C}$ for 24 hours. Then the reaction mixture was quenched by acetic acid $(40 \mu \mathrm{L})$ and $\mathrm{S}_{8}$ and the reaction mixture was stirred for additional 30 minutes at room temperature. After solvent was removed under reduced pressure, the residue was purified by silica gel column chromatography (petroleum ether/ethyl acetate $=50 / 1$ ) to give the desired product. 


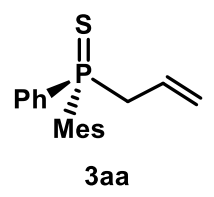

3aa: Procedure A, $57.7 \mathrm{mg}$, 95\% yield, light yellow solid.

${ }^{1}$ H NMR (400 MHz, CDCl $) \delta$ 7.79-7.68 (m, 2H), 7.51-7.36 (m, 3H), $6.85(\mathrm{~d}, J=4.0 \mathrm{~Hz}, 2 \mathrm{H})$, 5.51-5.31 (m, 1H), 5.03-4.93 (m, 1H), 4.90-4.78 (m, 1H), 3.46-3.25 (m, 2H), $2.30(\mathrm{~s}, 6 \mathrm{H}), 2.27$ (s, 3H).

${ }^{13} \mathbf{C}$ NMR(101 MHz, $\left.\mathbf{C D C l}_{3}\right) \delta 141.41(\mathrm{~d}, J=10.2 \mathrm{~Hz}), 140.85(\mathrm{~d}, J=2.8 \mathrm{~Hz}), 134.13(\mathrm{~d}, J=$ $79.4 \mathrm{~Hz}), 131.21(\mathrm{~d}, J=11.1 \mathrm{~Hz}), 130.77(\mathrm{~d}, J=9.9 \mathrm{~Hz}), 130.74(\mathrm{~d}, J=2.9 \mathrm{~Hz}), 128.63(\mathrm{~d}, J=$ $81.0 \mathrm{~Hz}), 128.10(\mathrm{~d}, J=12.3 \mathrm{~Hz}), 127.73(\mathrm{~d}, J=8.5 \mathrm{~Hz}), 120.89(\mathrm{~d}, J=13.5 \mathrm{~Hz}), 44.72(\mathrm{~d}, J=$ $51.1 \mathrm{~Hz}), 23.96(\mathrm{~d}, J=5.1 \mathrm{~Hz}), 20.78$.

${ }^{31}$ P NMR (162 MHz, $\left.\mathbf{C D C l}_{\mathbf{3}}\right) \delta 41.05$.

HRMS (ESI) $\mathbf{m} / \mathbf{z}[\mathbf{M}+\mathbf{H}]^{+}$: calcd. 301.1174, found. 301.1175 .

IR (film): $v_{\max }\left(\mathrm{cm}^{-1}\right) 3051,2961,2923,2861,1603,1437,1417,1100,1060,918,843,750,716$, 649, 579.

Optical rotation: $[\alpha]_{\mathrm{D}}^{25}=-108.43\left(c=1.000, \mathrm{CHCl}_{3}, 94 \%\right.$ ee $)$.

HPLC: DAICEL CHIRALPAK ID, hexane $/ i-\mathrm{PrOH}=9 / 1$, flow rate: $1.0 \mathrm{~mL} / \mathrm{min}, \lambda=254 \mathrm{~nm}$, $\mathrm{t}_{\mathrm{R}}($ major $)=8.9 \mathrm{~min}, \mathrm{t}_{\mathrm{R}}($ minor $)=9.9 \mathrm{~min}, 94 \%$ ee .
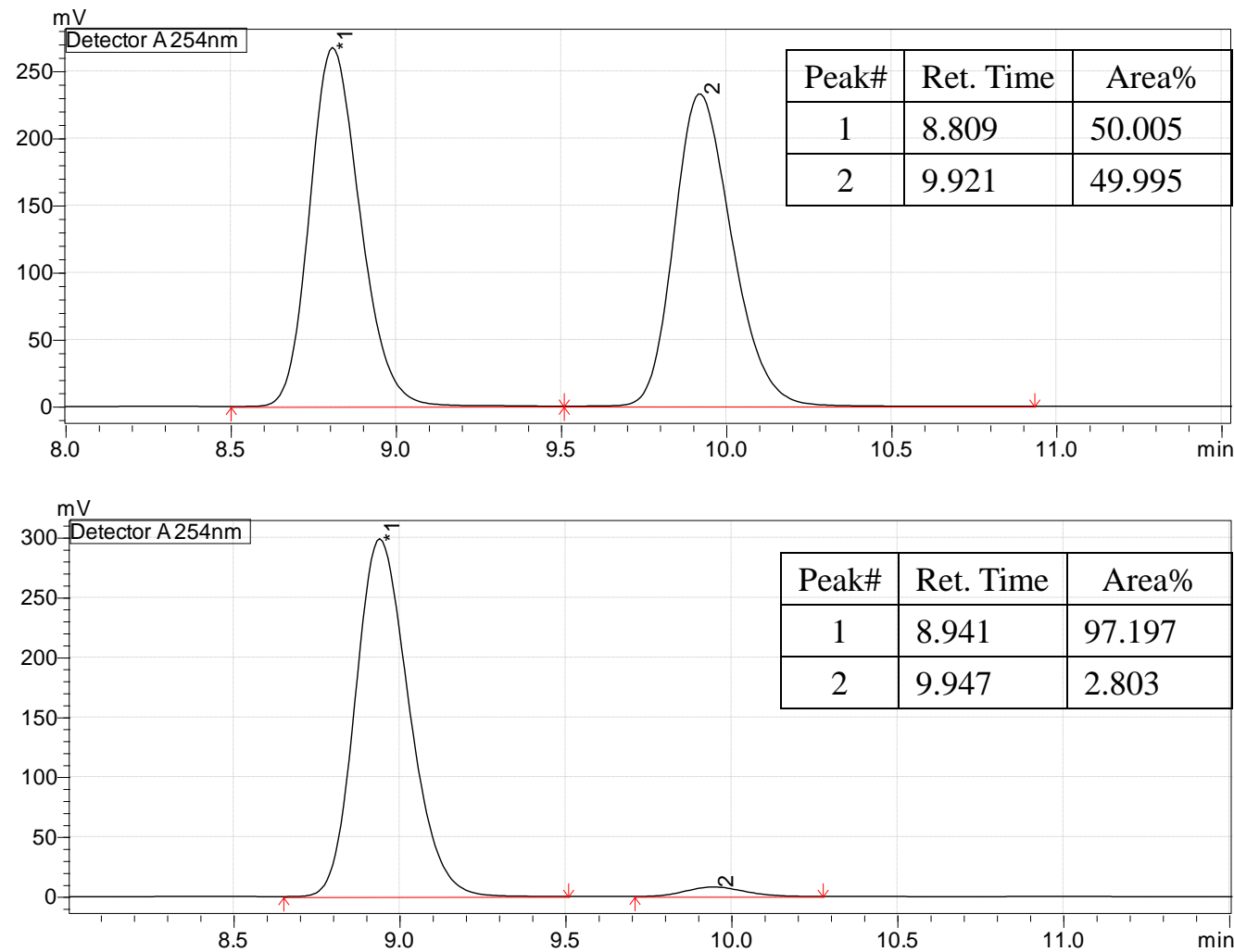


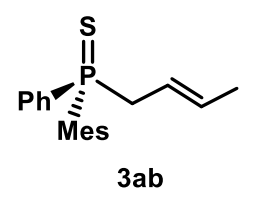

3ab: Procedure A, $53.4 \mathrm{mg}, 85 \%$ yield, light yellow liquid.

${ }^{1} \mathbf{H}$ NMR (400 MHz, CDCl $\left.{ }_{3}\right) \delta 7.73-7.65(\mathrm{~m}, 2 \mathrm{H}), 7.46-7.38(\mathrm{~m}, 3 \mathrm{H}), 6.84(\mathrm{~d}, J=4.0 \mathrm{~Hz}, 2 \mathrm{H})$, 5.19-4.99 (m, 2H), 3.30-3.21 (m, 2H), $2.30(\mathrm{~s}, 6 \mathrm{H}), 2.27(\mathrm{~s}, 3 \mathrm{H}), 1.49-1.42(\mathrm{~m}, 3 \mathrm{H})$.

${ }^{13}$ C NMR(101 MHz, CDCl $) \delta 141.42(\mathrm{~d}, J=10.2 \mathrm{~Hz}), 140.70(\mathrm{~d}, J=2.7 \mathrm{~Hz}), 134.32(\mathrm{~d}, J=$ $79.5 \mathrm{~Hz}), 132.01$ (d, $J=13.8 \mathrm{~Hz}), 131.13(\mathrm{~d}, J=11.0 \mathrm{~Hz}), 130.70(\mathrm{~d}, J=9.7 \mathrm{~Hz}), 130.56(\mathrm{~d}, J=$ $3.0 \mathrm{~Hz}), 128.67(\mathrm{~d}, J=79.6 \mathrm{~Hz}), 127.88(\mathrm{~d}, J=12.2 \mathrm{~Hz}), 119.80(\mathrm{~d}, J=8.9 \mathrm{~Hz}), 43.87(\mathrm{~d}, J=$ $50.8 \mathrm{~Hz}), 23.91(\mathrm{~d}, J=5.0 \mathrm{~Hz}), 20.74(\mathrm{~d}, J=1.7 \mathrm{~Hz}), 17.92(\mathrm{~d}, J=3.0 \mathrm{~Hz})$.

${ }^{31}$ P NMR (162 MHz, $\left.\mathbf{C D C l}_{3}\right) \delta 41.97$.

HRMS (ESI) $\mathbf{~ m / z ~ [ M + H ] ~}]^{+}$: calcd. 315.1331, found. 315.1331.

IR (film): $v_{\max }\left(\mathrm{cm}^{-1}\right)$ 3026, 2958, 2924, 2852, 1603, 1454, 1436, 1377, 1100, 1027, 963, 841, 749, $718,649,592$

Optical rotation: $[\alpha]_{\mathrm{D}}^{25}=-88.03\left(c=1.046, \mathrm{CHCl}_{3}, 85 \%\right.$ ee $)$.

HPLC: DAICEL CHIRALPAK IF-3, hexane $/ i$-PrOH $=49 / 1$, flow rate: $1.0 \mathrm{~mL} / \mathrm{min}, \lambda=254 \mathrm{~nm}$, $\mathrm{t}_{\mathrm{R}}($ major $)=19.2 \mathrm{~min}, \mathrm{t}_{\mathrm{R}}($ minor $)=20.1 \mathrm{~min}, 85 \%$ ee.
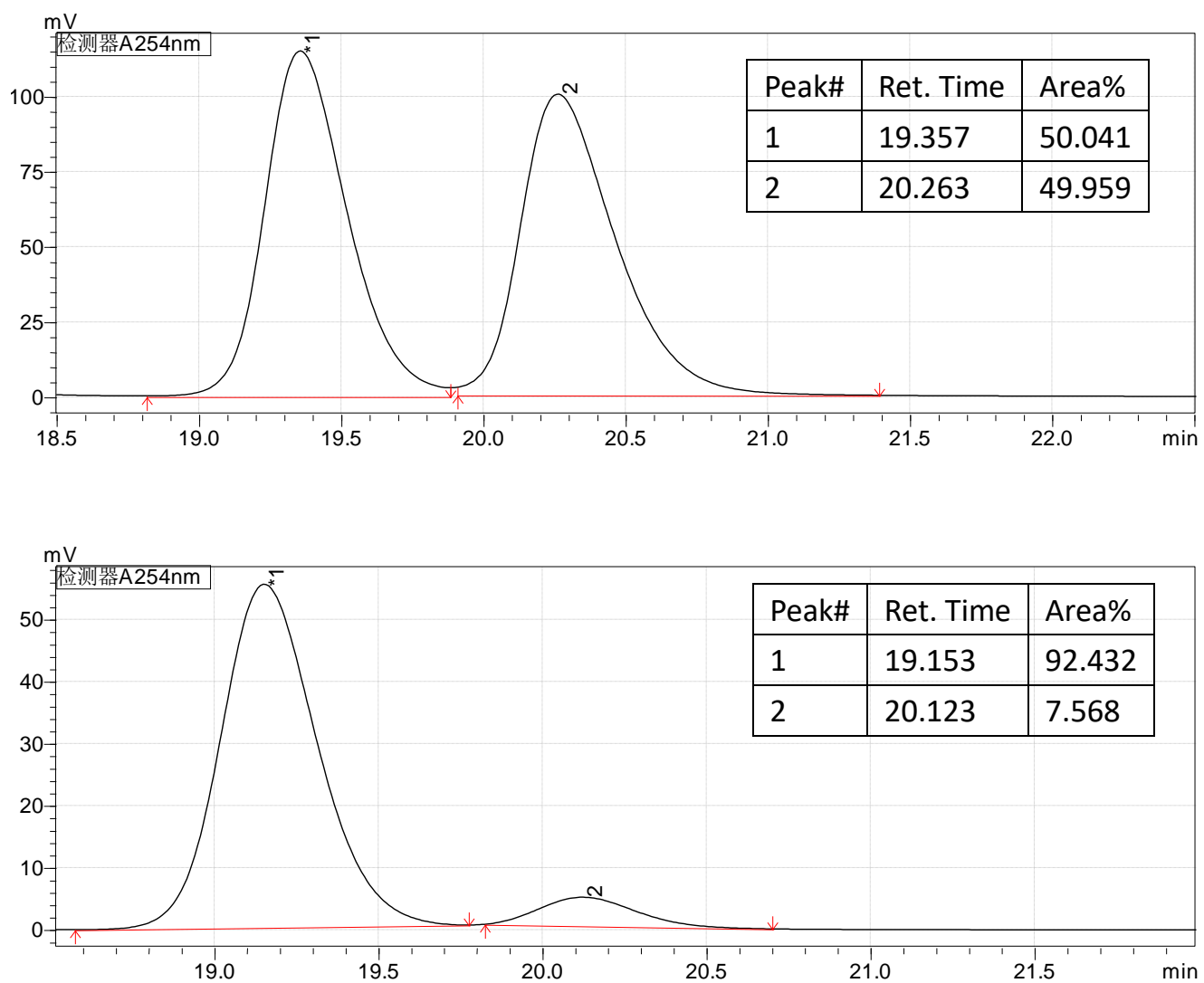


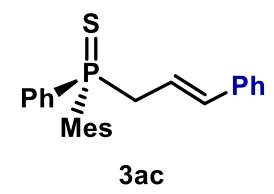

3ac: Procedure A, $45.1 \mathrm{mg}, 60 \%$ yield, light yellow liquid.

${ }^{1}$ H NMR (400 MHz, $\left.\mathbf{C D C l}_{3}\right) \delta$ 7.79-7.65 (m, 2H), 7.48-7.34 (m, 3H), 7.23-7.13 (m, 3H), 7.10$7.03(\mathrm{~m}, 2 \mathrm{H}), 6.86(\mathrm{~d}, J=4.0 \mathrm{~Hz}, 2 \mathrm{H}), 6.03(\mathrm{dd}, J=15.8,5.7 \mathrm{~Hz}, 1 \mathrm{H}), 5.83-5.68(\mathrm{~m}, 1 \mathrm{H}), 3.59-$ $3.36(\mathrm{~m}, 2 \mathrm{H}), 2.34(\mathrm{~s}, 6 \mathrm{H}), 2.28(\mathrm{~s}, 3 \mathrm{H})$.

${ }^{13} \mathbf{C}$ NMR(101 MHz, CDCl $) \delta 141.49(\mathrm{~d}, J=10.0 \mathrm{~Hz}), 140.94(\mathrm{~d}, J=2.9 \mathrm{~Hz}), 136.81(\mathrm{~d}, J=3.9$ Hz), 135.64 (d, $J=13.8 \mathrm{~Hz}), 134.40(\mathrm{~d}, J=79.8 \mathrm{~Hz}), 131.26(\mathrm{~d}, J=11.1 \mathrm{~Hz}), 130.79$ (d, $J=2.8$ Hz), 130.69 (d, $J=9.8 \mathrm{~Hz}), 128.67(\mathrm{~d}, J=79.8 \mathrm{~Hz}), 128.31(\mathrm{~d}, J=1.1 \mathrm{~Hz}), 128.14(\mathrm{~d}, J=12.2$ $\mathrm{Hz}), 127.36(\mathrm{~d}, J=1.5 \mathrm{~Hz}), 126.10(\mathrm{~d}, J=2.3 \mathrm{~Hz}), 119.27$ (d, $J=9.8 \mathrm{~Hz}), 44.42(\mathrm{~d}, J=50.0 \mathrm{~Hz})$, $24.02(\mathrm{~d}, J=5.0 \mathrm{~Hz}), 20.83(\mathrm{~d}, J=1.5 \mathrm{~Hz})$.

${ }^{31}$ P NMR (162 MHz, $\left.\mathbf{C D C l}_{3}\right) \delta 41.81$.

HRMS (ESI) $\mathbf{m} / \mathbf{z}[\mathbf{M}+\mathbf{H}]^{+}$: calcd. 377.1487, found. 377.1487.

IR (film): $v_{\max }\left(\mathrm{cm}^{-1}\right)$ 3025, 2956, 2924, 2853, 1603, 1455, 1436, 1377, 1099, 1026, 962, 874, 750, 693, 651, 591.

Optical rotation: $[\alpha]_{\mathrm{D}}{ }^{25}=-76.88\left(c=1.153, \mathrm{CHCl}_{3}, 78 \%\right.$ ee $)$.

HPLC: DAICEL CHIRALPAK IG-3, hexane $/ i-\mathrm{PrOH}=2 / 1$, flow rate: $0.6 \mathrm{~mL} / \mathrm{min}, \lambda=254 \mathrm{~nm}$, $\mathrm{t}_{\mathrm{R}}($ major $)=13.9 \mathrm{~min}, \mathrm{t}_{\mathrm{R}}($ minor $)=16.4 \mathrm{~min}, 78 \%$ ee .

\begin{tabular}{|l|l|l|}
\hline Peak\# & Ret. Time & Area\% \\
\hline 1 & 13.862 & 49.958 \\
\hline 2 & 16.342 & 50.042 \\
\hline
\end{tabular}
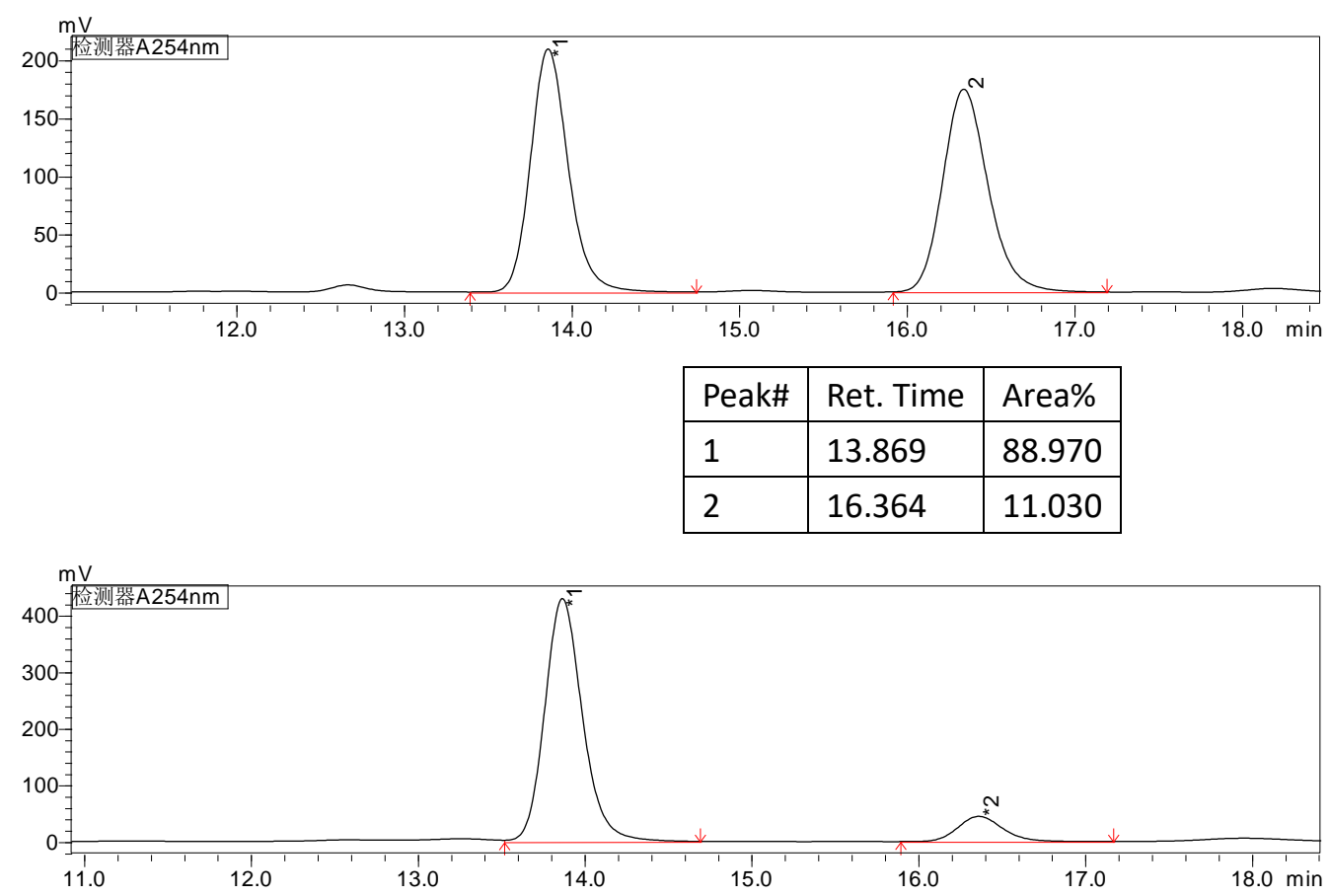


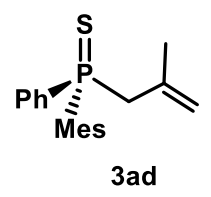

3ad: Procedure A, $55.2 \mathrm{mg}, 88 \%$ yield, light yellow liquid.

${ }^{1}$ H NMR (400 MHz, CDCl $\left.{ }_{3}\right) \delta 7.82-7.69(\mathrm{~m}, 2 \mathrm{H}), 7.49-7.34(\mathrm{~m}, 3 \mathrm{H}), 6.84(\mathrm{~d}, J=4.0 \mathrm{~Hz}, 2 \mathrm{H})$, $4.65(\mathrm{dt}, J=5.4,1.8 \mathrm{~Hz}, 1 \mathrm{H}), 4.38(\mathrm{~d}, J=5.7 \mathrm{~Hz}, 1 \mathrm{H}), 3.51(\mathrm{dd}, J=17.5,13.1 \mathrm{~Hz}, 1 \mathrm{H}), 3.25$ (dd, $J=13.1,11.4 \mathrm{~Hz}, 1 \mathrm{H}), 2.30(\mathrm{~s}, 6 \mathrm{H}), 2.26(\mathrm{~s}, 3 \mathrm{H}), 1.53(\mathrm{~d}, J=3.2 \mathrm{~Hz}, 3 \mathrm{H})$.

${ }^{13}$ C NMR(101 MHz, CDCl $) \delta 141.22(\mathrm{~d}, J=10.1 \mathrm{~Hz}), 140.55(\mathrm{~d}, J=2.9 \mathrm{~Hz}), 136.35(\mathrm{~d}, J=9.5$ $\mathrm{Hz}), 134.08$ (d, $J=79.3 \mathrm{~Hz}), 131.24(\mathrm{~d}, J=11.1 \mathrm{~Hz}), 131.12(\mathrm{~d}, J=9.9 \mathrm{~Hz}), 130.70(\mathrm{~d}, J=3.0$ $\mathrm{Hz}), 129.73$ (d, $J=80.5 \mathrm{~Hz}), 127.99$ (d, $J=12.2 \mathrm{~Hz}), 117.09$ (d, $J=11.0 \mathrm{~Hz}), 46.83$ (d, $J=48.6$ $\mathrm{Hz}), 24.53$ (d, $J=2.1 \mathrm{~Hz}), 24.14(\mathrm{~d}, J=4.6 \mathrm{~Hz}), 20.73$ (d, $J=1.5 \mathrm{~Hz})$.

${ }^{31}$ P NMR (162 MHz, $\left.\mathbf{C D C l}_{3}\right) \delta 40.92$.

HRMS (ESI) $\mathbf{~ m / z ~ [ M + H ~}]^{+}$: calcd. 315.1331, found. 315.1331.

IR (film): $v_{\max }\left(\mathrm{cm}^{-1}\right)$ 3051, 2958, 2924, 2853, 1604, 1455, 1437, 1377, 1100, 1027, 893, 850, 749, $693,641,595$.

Optical rotation: $[\alpha]_{\mathrm{D}}^{25}=-75.37\left(c=1.086, \mathrm{CHCl}_{3}, 86 \%\right.$ ee $)$.

HPLC: DAICEL CHIRALPAK ID, hexane $/ i-\mathrm{PrOH}=19 / 1$, flow rate: $1.0 \mathrm{~mL} / \mathrm{min}, \lambda=254 \mathrm{~nm}$, $\mathrm{t}_{\mathrm{R}}($ major $)=9.3 \mathrm{~min}, \mathrm{t}_{\mathrm{R}}($ minor $)=10.8 \mathrm{~min}, 86 \%$ ee.
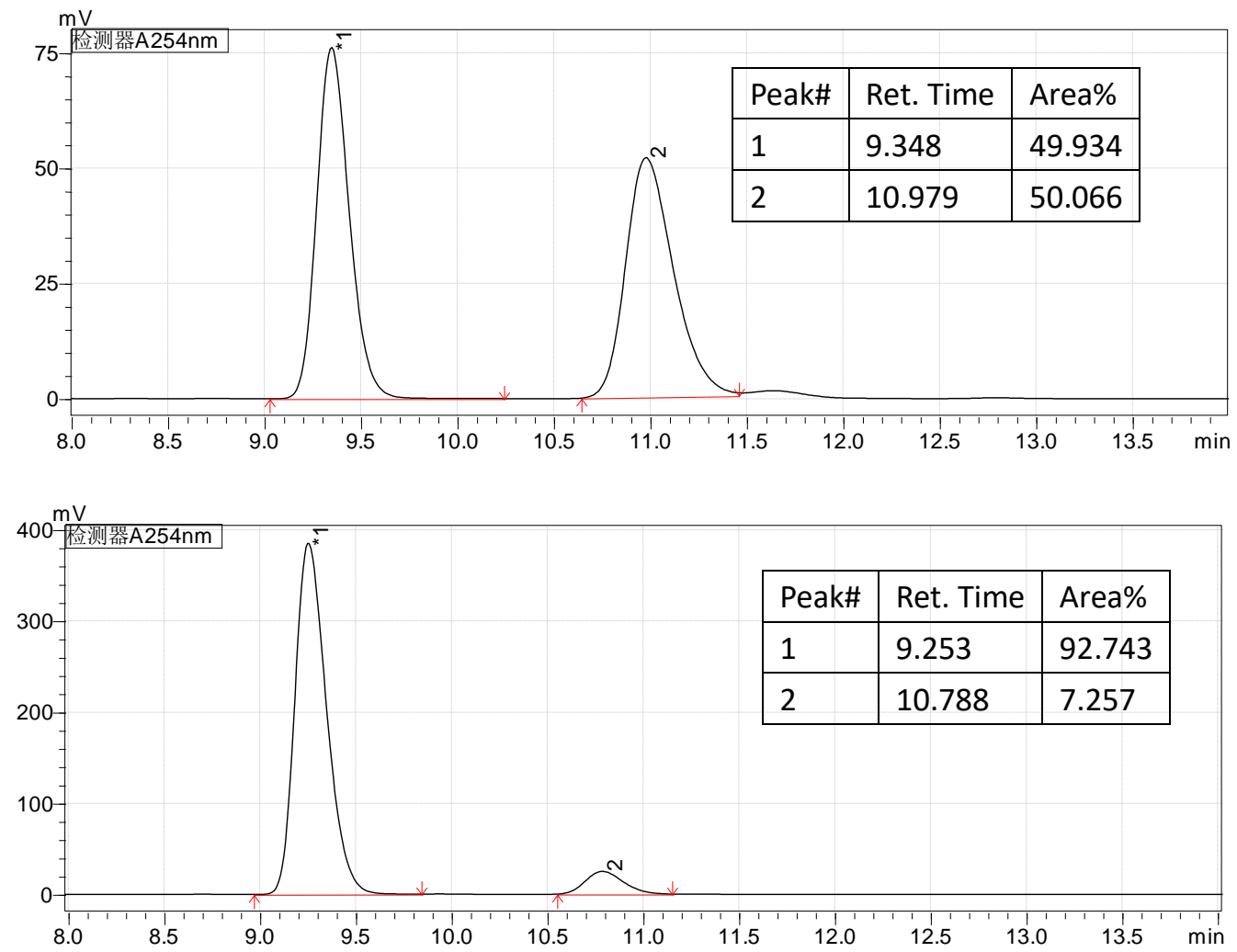


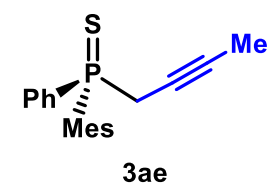

3ae: Procedure E, $54.9 \mathrm{mg}, 88 \%$ yield, light yellow solid.

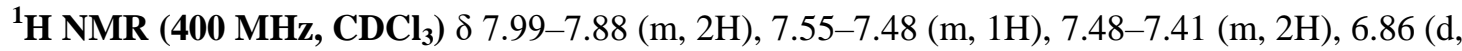
$J=4.1 \mathrm{~Hz}, 2 \mathrm{H}), 3.56(\mathrm{~m}, 1 \mathrm{H}), 3.03(\mathrm{~m}, 1 \mathrm{H}), 2.34(\mathrm{~s}, 6 \mathrm{H}), 2.28(\mathrm{~s}, 3 \mathrm{H}), 1.68-1.62(\mathrm{~m}, 3 \mathrm{H})$.

${ }^{13} \mathbf{C}$ NMR(101 MHz, $\left.\mathbf{C D C l}_{3}\right) \delta 141.78(\mathrm{~d}, J=10.1 \mathrm{~Hz}), 140.99(\mathrm{~d}, J=2.9 \mathrm{~Hz}), 134.54(\mathrm{~d}, J=$ $80.4 \mathrm{~Hz}), 131.14(\mathrm{~d}, J=3.0 \mathrm{~Hz}), 131.04(\mathrm{~d}, J=11.3 \mathrm{~Hz}), 130.56(\mathrm{~d}, J=9.8 \mathrm{~Hz}), 127.97$ (d, $J=$ $12.3 \mathrm{~Hz}), 126.69$ (d, $J=84.0 \mathrm{~Hz}), 80.62(\mathrm{~d}, J=8.7 \mathrm{~Hz}), 71.01(\mathrm{~d}, J=13.0 \mathrm{~Hz}), 34.53(\mathrm{~d}, J=53.6$ $\mathrm{Hz}), 23.15(\mathrm{~d}, J=5.5 \mathrm{~Hz}), 20.85(\mathrm{~d}, J=1.4 \mathrm{~Hz}), 3.46(\mathrm{~d}, J=3.1 \mathrm{~Hz})$.

${ }^{31}$ P NMR (162 MHz, CDCl $)$ ) 39.48 .

HRMS (ESI) $\mathbf{m} / \mathbf{z}[\mathbf{M}+\mathbf{H}]^{+}$: calcd. 313.1174, found. 313.1175.

IR (film): $v_{\max }\left(\mathrm{cm}^{-1}\right)$ 3052, 2960, 2918, 1603, 1436, 1393, 1099, 1028, 826, 723, 693, 666, 598.

Optical rotation: $[\alpha]_{\mathrm{D}}{ }^{25}=4.29\left(c=1.000, \mathrm{CHCl}_{3}, 94 \%\right.$ ee $)$.

HPLC: DAICEL CHIRALPAK ID, hexane $/ i-\mathrm{PrOH}=9 / 1$, flow rate: $1.0 \mathrm{~mL} / \mathrm{min}, \lambda=254 \mathrm{~nm}$, $\mathrm{t}_{\mathrm{R}}($ major $)=17.6 \mathrm{~min}, \mathrm{t}_{\mathrm{R}}($ minor $)=19.2 \mathrm{~min}, 94 \%$ ee .
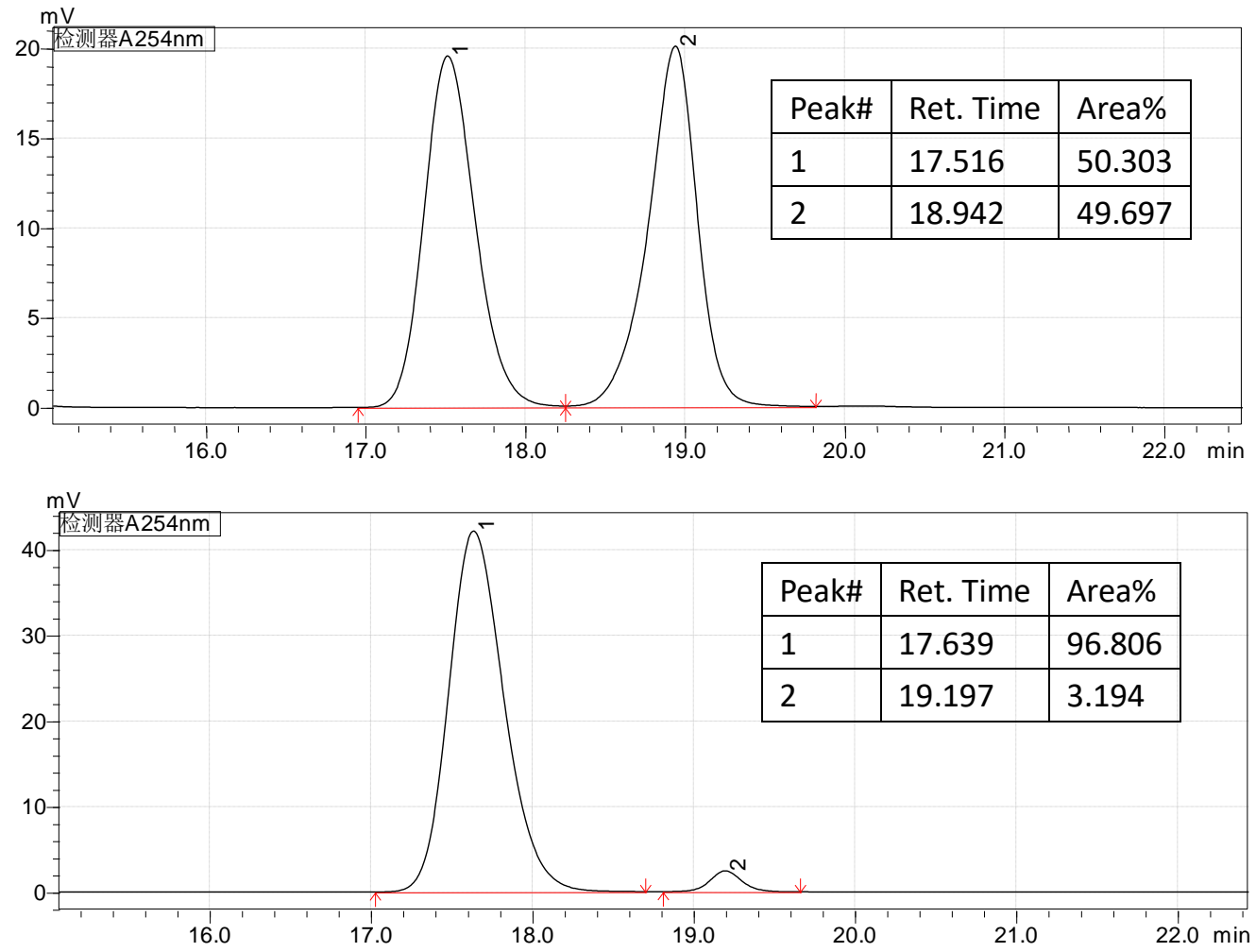


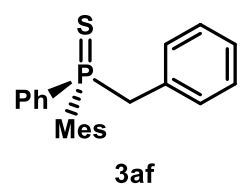

3af: Procedure A, $66.5 \mathrm{mg}, 95 \%$ yield, light yellow solid

${ }^{1}$ H NMR (400 MHz, CDCl $)_{3}$ ) 7.47-7.38 (m, 2H), 7.37-7.30 (m, 1H), 7.25-7.18 (m, 2H), 7.09$7.03(\mathrm{~m}, 1 \mathrm{H}), 7.02-6.94(\mathrm{~m}, 2 \mathrm{H}), 6.88(\mathrm{~d}, J=3.8 \mathrm{~Hz}, 2 \mathrm{H}), 6.77(\mathrm{dd}, J=7.4,2.1 \mathrm{~Hz}, 2 \mathrm{H}), 4.03(\mathrm{dd}$, $J=15.9,13.0 \mathrm{~Hz}, 1 \mathrm{H}), 3.71(\mathrm{dd}, J=12.9,10.7 \mathrm{~Hz}, 1 \mathrm{H}), 2.35(\mathrm{~s}, 6 \mathrm{H}), 2.29(\mathrm{~s}, 3 \mathrm{H})$.

${ }^{13} \mathbf{C}$ NMR(101 MHz, $\left.\mathbf{C D C l}_{3}\right) \delta 141.47(\mathrm{~d}, J=10.0 \mathrm{~Hz}), 140.77(\mathrm{~d}, J=2.9 \mathrm{~Hz}), 133.77(\mathrm{~d}, J=$ $80.1 \mathrm{~Hz}), 131.30(\mathrm{~d}, J=11.1 \mathrm{~Hz}), 131.05(\mathrm{~d}, J=7.4 \mathrm{~Hz}), 130.62(\mathrm{~d}, J=9.9 \mathrm{~Hz}), 130.60$ (d, $J=$ $5.3 \mathrm{~Hz}), 130.55(\mathrm{~d}, J=4.5 \mathrm{~Hz}), 129.17(\mathrm{~d}, J=80.4 \mathrm{~Hz}), 127.86(\mathrm{~d}, J=12.3 \mathrm{~Hz}), 127.25(\mathrm{~d}, J=$ $3.4 \mathrm{~Hz}), 126.50(\mathrm{~d}, J=4.0 \mathrm{~Hz}), 45.55(\mathrm{~d}, J=47.1 \mathrm{~Hz}), 24.31(\mathrm{~d}, J=4.7 \mathrm{~Hz}), 20.82(\mathrm{~d}, J=1.4$ $\mathrm{Hz})$.

${ }^{31}$ P NMR (162 MHz, CDCl 3 ) $\delta 43.26$.

HRMS (ESI) $\mathbf{m} / \mathbf{z}$ [M+H] $]^{+}$: calcd. 351.1331, found. 351.1331 .

IR (film): $v_{\max }\left(\mathrm{cm}^{-1}\right) 3028,2922,2857,1602,1452,1437,1101,846,772,695,645$.

Optical rotation: $[\alpha]_{\mathrm{D}}^{25}=-189.57\left(c=1.000, \mathrm{CHCl}_{3}, 87 \%\right.$ ee $)$.

HPLC: DAICEL CHIRALPAK ID, hexane $/ i-\mathrm{PrOH}=9 / 1$, flow rate: $1.0 \mathrm{~mL} / \mathrm{min}, \lambda=254 \mathrm{~nm}$, $\mathrm{t}_{\mathrm{R}}($ major $)=10.6 \mathrm{~min}, \mathrm{t}_{\mathrm{R}}($ minor $)=12.3 \mathrm{~min}, 87 \%$ ee .
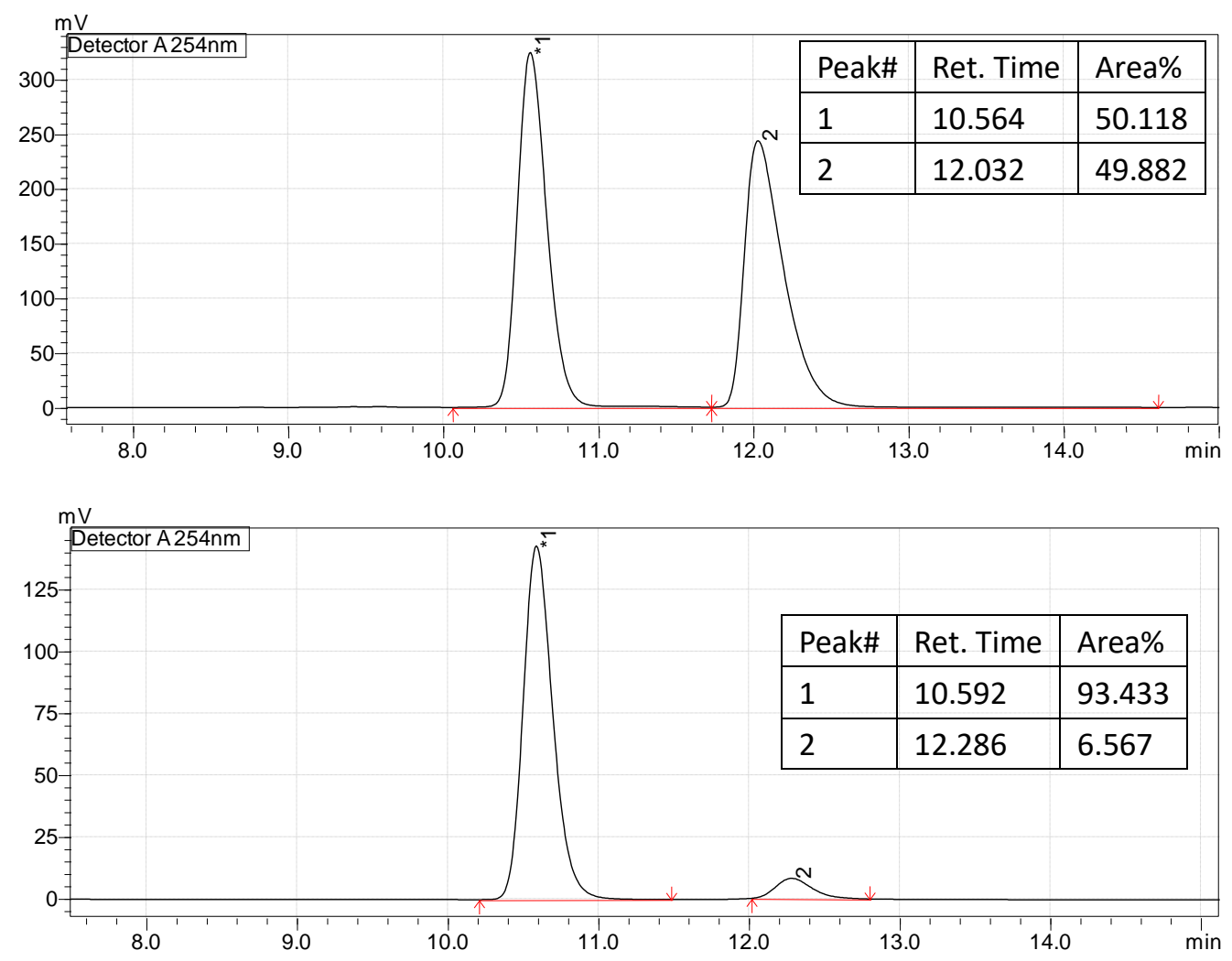


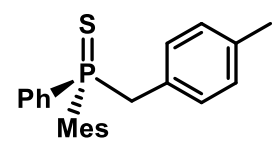

3 ag

3ag: Procedure A, $66.9 \mathrm{mg}$, 92\% yield, light brown solid.

${ }^{1}$ H NMR (400 MHz, $\mathbf{C D C l}_{3}$ ) $\delta$ 7.48-7.40 (m, 2H), 7.39-7.33 (m, 1H), 7.26-7.21 (m, 2H), 6.87 (d, $J=3.7 \mathrm{~Hz}, 2 \mathrm{H}), 6.80(\mathrm{~d}, J=7.8 \mathrm{~Hz}, 2 \mathrm{H}), 6.63(\mathrm{dd}, J=8.0,2.5 \mathrm{~Hz}, 2 \mathrm{H}), 3.99(\mathrm{dd}, J=15.5,13.2$ $\mathrm{Hz}, 1 \mathrm{H}), 3.69$ (dd, $J=12.9,10.5 \mathrm{~Hz}, 1 \mathrm{H}), 2.34$ (s, 6H), 2.28 (s, 3H), 2.20 (d, J=2.2 Hz, 3H).

${ }^{13}$ C NMR(101 MHz, CDCl $) \delta 141.50(\mathrm{~d}, J=10.0 \mathrm{~Hz}), 140.74(\mathrm{~d}, J=2.8 \mathrm{~Hz}), 136.14(\mathrm{~d}, J=4.3$ $\mathrm{Hz}), 133.91(\mathrm{~d}, J=80.0 \mathrm{~Hz}), 131.30(\mathrm{~d}, J=11.1 \mathrm{~Hz}), 130.75(\mathrm{~d}, J=9.8 \mathrm{~Hz}), 130.52(\mathrm{~d}, J=2.2$ $\mathrm{Hz}), 130.51$ (d, $J=5.5 \mathrm{~Hz}), 129.28$ (d, $J=79.7 \mathrm{~Hz}), 128.03(\mathrm{~d}, J=3.5 \mathrm{~Hz}), 127.86(\mathrm{~d}, J=12.4$ $\mathrm{Hz}), 127.76$ (d, $J=8.5 \mathrm{~Hz}), 45.07$ (d, $J=47.2 \mathrm{~Hz}), 24.35(\mathrm{~d}, J=4.7 \mathrm{~Hz}), 20.97$ (d, $J=1.4 \mathrm{~Hz})$, $20.83(\mathrm{~d}, J=1.4 \mathrm{~Hz})$.

${ }^{31}$ P NMR (162 MHz, $\left.\mathbf{C D C l}_{3}\right) \delta 43.23$.

HRMS (ESI) $\mathbf{~ m / z ~ [ M + H ] ~}]^{+}$: calcd. 365.1487, found. 365.1487.

IR (film): $v_{\max }\left(\mathrm{cm}^{-1}\right) 3047,2967,2926,1604,1436,1101,1031,844,822,694,640$.

Optical rotation: $[\alpha]_{\mathrm{D}}^{25}=-193.92\left(c=1.000, \mathrm{CHCl}_{3}, 90 \%\right.$ ee $)$.

HPLC: DAICEL CHIRALPAK ID, hexane $/ i-\mathrm{PrOH}=9 / 1$, flow rate: $1.0 \mathrm{~mL} / \mathrm{min}, \lambda=254 \mathrm{~nm}$, $\mathrm{t}_{\mathrm{R}}($ major $)=10.1 \mathrm{~min}, \mathrm{t}_{\mathrm{R}}($ minor $)=12.1 \mathrm{~min}, 90 \%$ ee.

\begin{tabular}{|l|l|l|}
\hline Peak\# & Ret. Time & Area\% \\
\hline 1 & 10.160 & 50.273 \\
\hline 2 & 12.136 & 49.727 \\
\hline
\end{tabular}
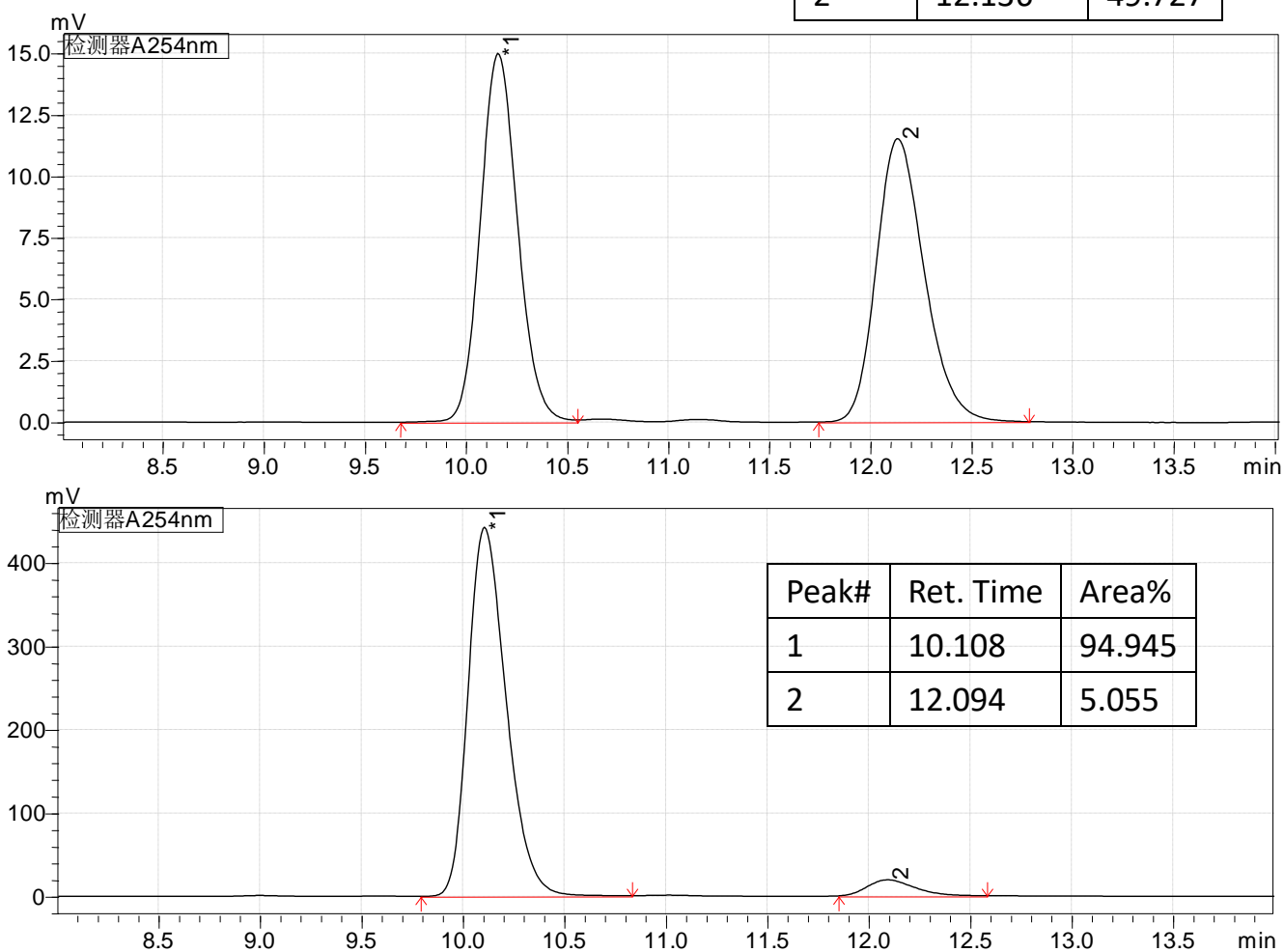


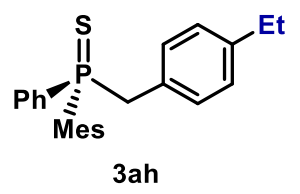

3ah: Procedure A, $71.8 \mathrm{mg}, 95 \%$ yield, light yellow solid.

${ }^{1}$ H NMR (400 MHz, CDCl $_{3}$ ) $\delta$ 7.46-7.38 (m, 2H), 7.38-7.32 (m, 1H), 7.24-7.18 (m, 2H), 6.87 (d, $J=4.0 \mathrm{~Hz}, 2 \mathrm{H}), 6.82(\mathrm{~d}, J=7.8 \mathrm{~Hz}, 2 \mathrm{H}), 6.66(\mathrm{dd}, J=8.1,2.8 \mathrm{~Hz}, 2 \mathrm{H}), 3.99(\mathrm{dd}, J=15.7,13.0$ $\mathrm{Hz}, 1 \mathrm{H}), 3.69(\mathrm{dd}, J=13.0,10.4 \mathrm{~Hz}, 1 \mathrm{H}), 2.49(\mathrm{qd}, J=7.6,2.0 \mathrm{~Hz}, 2 \mathrm{H}), 2.38-2.32(\mathrm{~m}, 6 \mathrm{H}), 2.28$ $(\mathrm{s}, 3 \mathrm{H}), 1.12(\mathrm{t}, J=7.6 \mathrm{~Hz}, 3 \mathrm{H})$.

${ }^{13} \mathbf{C}$ NMR(101 MHz, CDCl $) \delta 142.54(\mathrm{~d}, J=4.2 \mathrm{~Hz}), 141.48(\mathrm{~d}, J=10.0 \mathrm{~Hz}), 140.71(\mathrm{~d}, J=2.9$ $\mathrm{Hz}), 133.87(\mathrm{~d}, J=80.1 \mathrm{~Hz}), 131.27(\mathrm{~d}, J=11.1 \mathrm{~Hz}), 130.65(\mathrm{~d}, J=9.7 \mathrm{~Hz}), 130.50(\mathrm{~d}, J=5.5$ $\mathrm{Hz}), 130.47(\mathrm{~d}, J=2.6 \mathrm{~Hz}), 129.22(\mathrm{~d}, J=79.6 \mathrm{~Hz}), 128.03(\mathrm{~d}, J=7.6 \mathrm{~Hz}), 127.80(\mathrm{~d}, J=12.2$ $\mathrm{Hz}), 126.79(\mathrm{~d}, J=3.6 \mathrm{~Hz}), 45.17(\mathrm{~d}, J=47.2 \mathrm{~Hz}), 28.27(\mathrm{~d}, J=1.4 \mathrm{~Hz}), 24.30(\mathrm{~d}, J=4.6 \mathrm{~Hz})$, $20.79(\mathrm{~d}, J=1.5 \mathrm{~Hz}), 15.47(\mathrm{~d}, J=1.8 \mathrm{~Hz})$.

${ }^{31}$ P NMR (162 MHz, CDCl $) \delta 43.34$.

HRMS (ESI) $\mathbf{m} / \mathbf{z}$ [M+H] ${ }^{+}$: calcd. 379.1644, found. 379.1644 .

IR (film): $v_{\max }\left(\mathrm{cm}^{-1}\right)$ 3023, 2963, 2926, 2871, 1603, 1512, 1437, 1101, 845, 748, 693, 646, 596.

Optical rotation: $[\alpha]_{\mathrm{D}}{ }^{25}=-195.62\left(c=1.000, \mathrm{CHCl}_{3}, 87 \%\right.$ ee $)$.

HPLC: DAICEL CHIRALPAK ID, hexane $/ i-\mathrm{PrOH}=9 / 1$, flow rate: $1.0 \mathrm{~mL} / \mathrm{min}, \lambda=254 \mathrm{~nm}$, $\mathrm{t}_{\mathrm{R}}($ major $)=9.5 \mathrm{~min}, \mathrm{t}_{\mathrm{R}}($ minor $)=11.3 \mathrm{~min}, 87 \%$ ee.
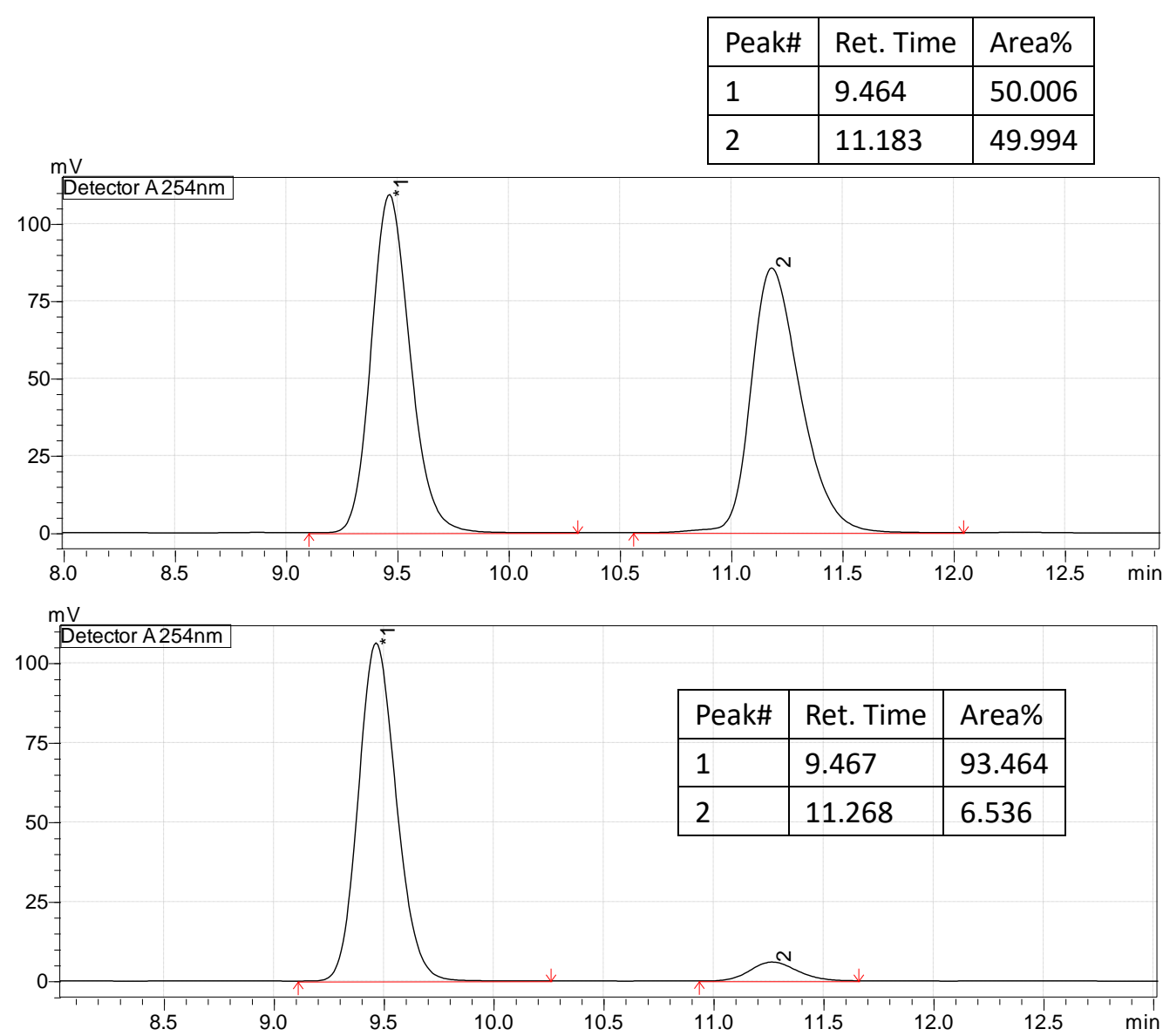


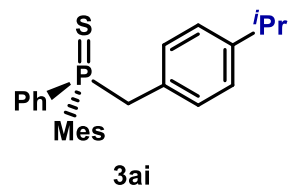

3ai: Procedure A, $74.4 \mathrm{mg}, 95 \%$ yield, yellow solid.

${ }^{1}$ H NMR (400 MHz, CDCl $\left.{ }_{3}\right) \delta$ 7.44-7.30 (m, 3H), 7.23-7.16 (m, 2H), $6.87(\mathrm{~d}, J=4.0 \mathrm{~Hz}, 2 \mathrm{H})$, $6.86-6.81(\mathrm{~m}, 2 \mathrm{H}), 6.68(\mathrm{dd}, J=8.2,2.8 \mathrm{~Hz}, 2 \mathrm{H}), 3.98(\mathrm{dd}, J=15.7,13.0 \mathrm{~Hz}, 1 \mathrm{H}), 3.69(\mathrm{dd}, J=$ 13.0, $10.4 \mathrm{~Hz}, 1 \mathrm{H}), 2.81-2.68(\mathrm{~m}, 1 \mathrm{H}), 2.35(\mathrm{~s}, 6 \mathrm{H}), 2.28(\mathrm{~s}, 3 \mathrm{H}), 1.14(\mathrm{dd}, J=6.9,2.3 \mathrm{~Hz}, 6 \mathrm{H})$.

${ }^{13} \mathbf{C}$ NMR(101 MHz, CDCl $) \delta 147.27(\mathrm{~d}, J=4.2 \mathrm{~Hz}), 141.60(\mathrm{~d}, J=9.9 \mathrm{~Hz}), 140.82(\mathrm{~d}, J=2.8$ $\mathrm{Hz}), 133.97$ (d, $J=80.1 \mathrm{~Hz}), 131.37(\mathrm{~d}, J=11.0 \mathrm{~Hz}), 130.69$ (d, $J=9.8 \mathrm{~Hz}), 130.53$ (d, $J=3.1$ $\mathrm{Hz}), 130.52$ (d, $J=5.4 \mathrm{~Hz}) 129.29$ (d, $J=79.7 \mathrm{~Hz}), 128.33$ (d, $J=7.6 \mathrm{~Hz}), 127.85$ (d, $J=12.3 \mathrm{~Hz}$ ), $125.44(\mathrm{~d}, J=3.5 \mathrm{~Hz}), 45.39$ (d, $J=47.0 \mathrm{~Hz}), 33.59,24.40(\mathrm{~d}, J=4.7 \mathrm{~Hz}), 23.94(\mathrm{dd}, J=8.0,1.6$ $\mathrm{Hz}), 20.90(\mathrm{~d}, J=1.5 \mathrm{~Hz})$.

${ }^{31}$ P NMR (162 MHz, $\left.\mathbf{C D C l}_{3}\right) \delta 43.44$.

HRMS (ESI) $\mathbf{m} / \mathbf{z}$ [M+H] $]^{+}$: calcd. 393.1800, found. 393.1800 .

IR (film): $v_{\max }\left(\mathrm{cm}^{-1}\right) 3051,3024,2959,2923,1603,1511,1455,1437,1287,1100,1053,846$, 749, 692, 646, 597, 556 .

Optical rotation: $[\alpha]_{\mathrm{D}}^{25}=-179.06\left(c=1.000, \mathrm{CHCl}_{3}, 87 \%\right.$ ee $)$.

HPLC: DAICEL CHIRALPAK OD-H, hexane $/ i-\mathrm{PrOH}=19 / 1$, flow rate: $1.0 \mathrm{~mL} / \mathrm{min}, \lambda=254 \mathrm{~nm}$, $\mathrm{t}_{\mathrm{R}}($ major $)=6.3 \mathrm{~min}, \mathrm{t}_{\mathrm{R}}($ minor $)=8.8 \mathrm{~min}, 87 \%$ ee.
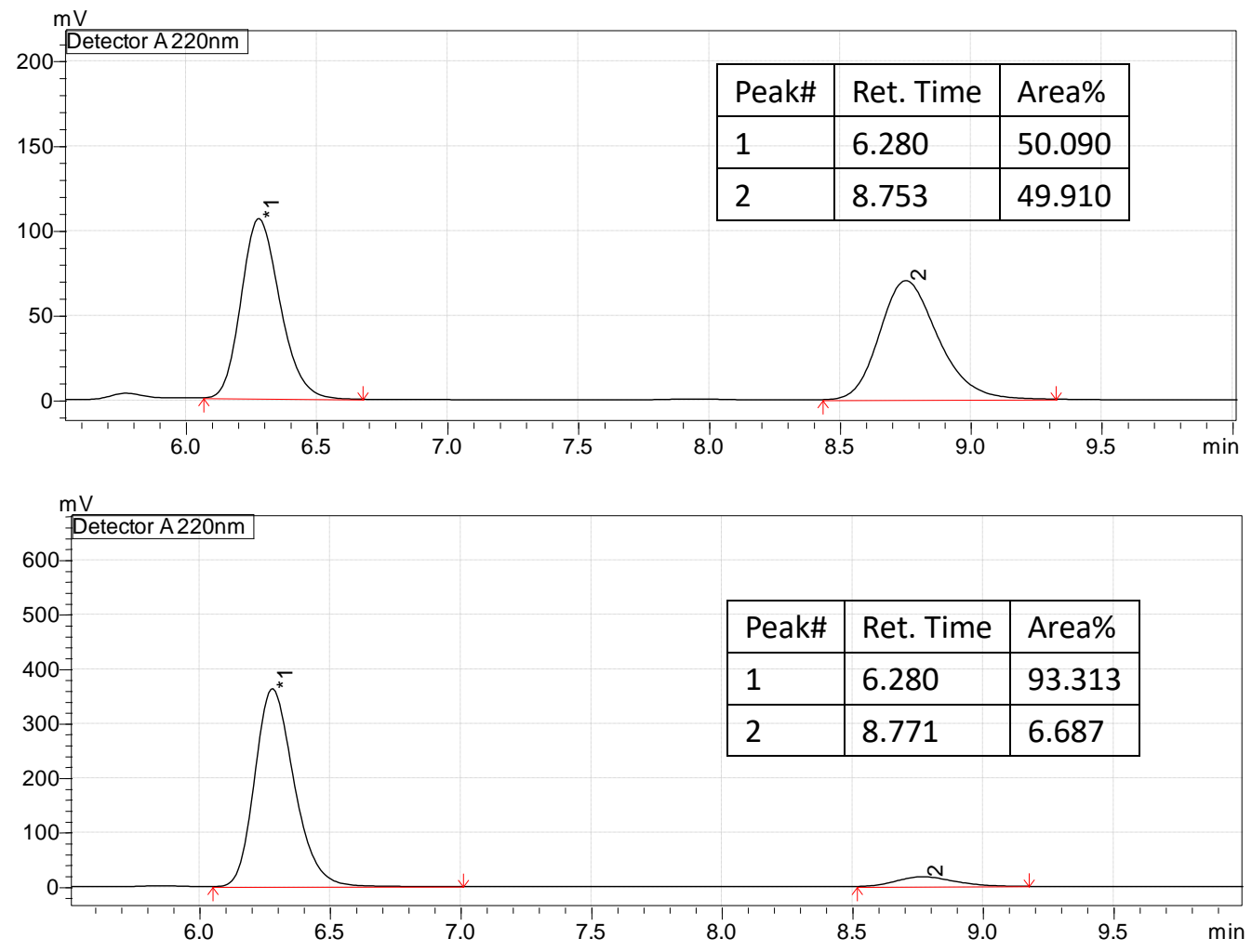


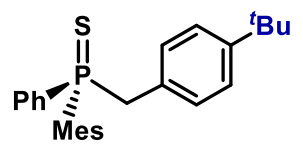

3aj

3aj: Procedure A, $77.1 \mathrm{mg}, 95 \%$ yield, orange liquid.

${ }^{1}$ H NMR (400 MHz, CDCl $_{3}$ ) $\delta$ 7.42-7.30 (m, 3H), 7.23-7.15 (m, 2H), 7.03-6.96 (m, 2H), $6.88(\mathrm{~d}$, $J=4.0 \mathrm{~Hz}, 2 \mathrm{H}), 6.68(\mathrm{dd}, J=8.4,2.8 \mathrm{~Hz}, 2 \mathrm{H}), 3.98(\mathrm{dd}, J=15.7,13.0 \mathrm{~Hz}, 1 \mathrm{H}), 3.70(\mathrm{dd}, J=13.0$, $10.5 \mathrm{~Hz}, 1 \mathrm{H}), 2.35(\mathrm{~s}, 6 \mathrm{H}), 2.29(\mathrm{~s}, 3 \mathrm{H}), 1.21(\mathrm{~s}, 9 \mathrm{H})$.

${ }^{13} \mathbf{C}$ NMR(101 MHz, CDCl $) \delta 149.42(\mathrm{~d}, J=4.2 \mathrm{~Hz}), 141.51(\mathrm{~d}, J=10.0 \mathrm{~Hz}), 140.74(\mathrm{~d}, J=2.9$ $\mathrm{Hz}), 133.83(\mathrm{~d}, J=80.4 \mathrm{~Hz}), 131.27(\mathrm{~d}, J=11.1 \mathrm{~Hz}), 130.56(\mathrm{~d}, J=9.6 \mathrm{~Hz}), 130.43(\mathrm{~d}, J=2.9$ $\mathrm{Hz}), 130.12(\mathrm{~d}, J=5.5 \mathrm{~Hz}), 129.11(\mathrm{~d}, J=79.7 \mathrm{~Hz}), 127.89(\mathrm{~d}, J=7.4 \mathrm{~Hz}), 127.73(\mathrm{~d}, J=12.4$ $\mathrm{Hz}), 124.21(\mathrm{~d}, J=3.5 \mathrm{~Hz}), 45.23(\mathrm{~d}, J=47.0 \mathrm{~Hz}), 34.16(\mathrm{~d}, J=1.2 \mathrm{~Hz}), 31.16,24.31(\mathrm{~d}, J=4.6$ $\mathrm{Hz}), 20.81(\mathrm{~d}, J=1.5 \mathrm{~Hz})$.

${ }^{31}$ P NMR (162 MHz, CDCl 3 ) $\delta 43.45$.

HRMS (ESI) $\mathbf{m} / \mathbf{z}[\mathbf{M}+\mathbf{H}]^{+}$: calcd. 407.1957 , found. 407.1957.

IR (film): $v_{\max }\left(\mathrm{cm}^{-1}\right)$ 3056, 2962, 2870, 1603, 1454, 1437, 1102, 1022, 835, 705, 692, 645, 596.

Optical rotation: $[\alpha]_{\mathrm{D}}^{25}=-144.61\left(c=1.560, \mathrm{CHCl}_{3}, 85 \%\right.$ ee $)$.

HPLC: DAICEL CHIRALPAK ID, hexane $/ i-\mathrm{PrOH}=9 / 1$, flow rate: $1.0 \mathrm{~mL} / \mathrm{min}, \lambda=254 \mathrm{~nm}$, $\mathrm{t}_{\mathrm{R}}($ major $)=7.9 \mathrm{~min}, \mathrm{t}_{\mathrm{R}}($ minor $)=9.2 \mathrm{~min}, 85 \%$ ee.
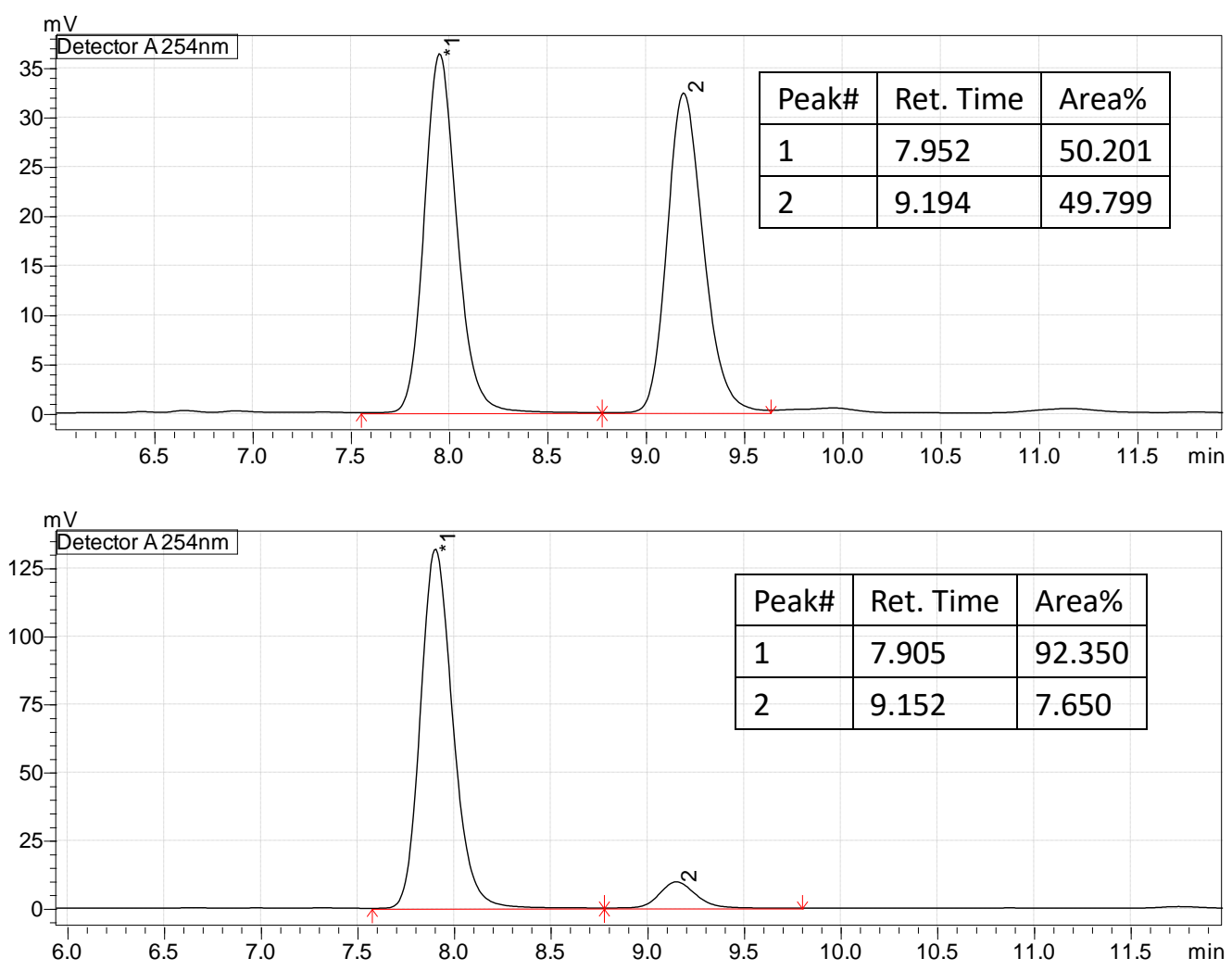


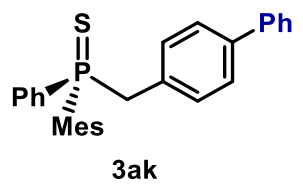

3ak: Procedure A, $80.9 \mathrm{mg}, 95 \%$ yield, brown yellow solid.

${ }^{1}$ H NMR (400 MHz, CDCl $_{3}$ ) $\delta$ 7.53-7.41 (m, 4H), 7.40-7.26 (m, 4H), 7.25-7.19 (m, 4H), 6.88 (d, $J=4.0 \mathrm{~Hz}, 2 \mathrm{H}), 6.82(\mathrm{dd}, J=8.3,2.7 \mathrm{~Hz}, 2 \mathrm{H}), 4.06(\mathrm{dd}, J=15.9,12.9 \mathrm{~Hz}, 1 \mathrm{H}), 3.75(\mathrm{dd}, J=12.9$, $10.6 \mathrm{~Hz}, 1 \mathrm{H}), 2.36$ (s, 6H), 2.29 (s, 3H).

${ }^{13} \mathbf{C}$ NMR(101 MHz, CDCl $) \delta 141.50(\mathrm{~d}, J=10.0 \mathrm{~Hz}), 140.83(\mathrm{~d}, J=2.9 \mathrm{~Hz}), 140.59(\mathrm{~d}, J=1.8$ $\mathrm{Hz}), 139.19(\mathrm{~d}, J=4.2 \mathrm{~Hz}), 133.80(\mathrm{~d}, J=80.1 \mathrm{~Hz}), 131.32(\mathrm{~d}, J=11.1 \mathrm{~Hz}), 130.97(\mathrm{~d}, J=5.5$ $\mathrm{Hz}), 130.68$ (d, $J=9.9 \mathrm{~Hz}), 130.61(\mathrm{~d}, J=4.0 \mathrm{~Hz}), 130.13$ (d, $J=7.6 \mathrm{~Hz}), 129.13$ (d, $J=80.2 \mathrm{~Hz})$, 128.58, $127.91(\mathrm{~d}, J=12.3 \mathrm{~Hz}), 127.05,126.80,125.90(\mathrm{~d}, J=3.5 \mathrm{~Hz}), 45.31(\mathrm{~d}, J=46.9 \mathrm{~Hz})$, $24.32(\mathrm{~d}, J=4.8 \mathrm{~Hz}), 20.82(\mathrm{~d}, J=1.5 \mathrm{~Hz})$.

${ }^{31}$ P NMR (162 MHz, $\left.\mathbf{C D C l}_{3}\right) \delta 43.20$.

HRMS (ESI) $\mathbf{m} / \mathbf{z}$ [M+H] $]^{+}$: calcd. 427.1644, found. 427.1640.

IR (film): $v_{\max }\left(\mathrm{cm}^{-1}\right)$ 3052, 3028, 2968, 2920, 1603, 1486, 1436, 1101, 1027, 844, 768, 694, 601. Optical rotation: $[\alpha]_{\mathrm{D}}^{25}=-249.11\left(c=1.000, \mathrm{CHCl}_{3}, 84 \%\right.$ ee $)$.

HPLC: DAICEL CHIRALPAK ID, hexane $/ i-\mathrm{PrOH}=9 / 1$, flow rate: $1.0 \mathrm{~mL} / \mathrm{min}, \lambda=254 \mathrm{~nm}$, $\mathrm{t}_{\mathrm{R}}($ major $)=13.9 \mathrm{~min}, \mathrm{t}_{\mathrm{R}}($ minor $)=15.8 \mathrm{~min}, 84 \%$ ee .
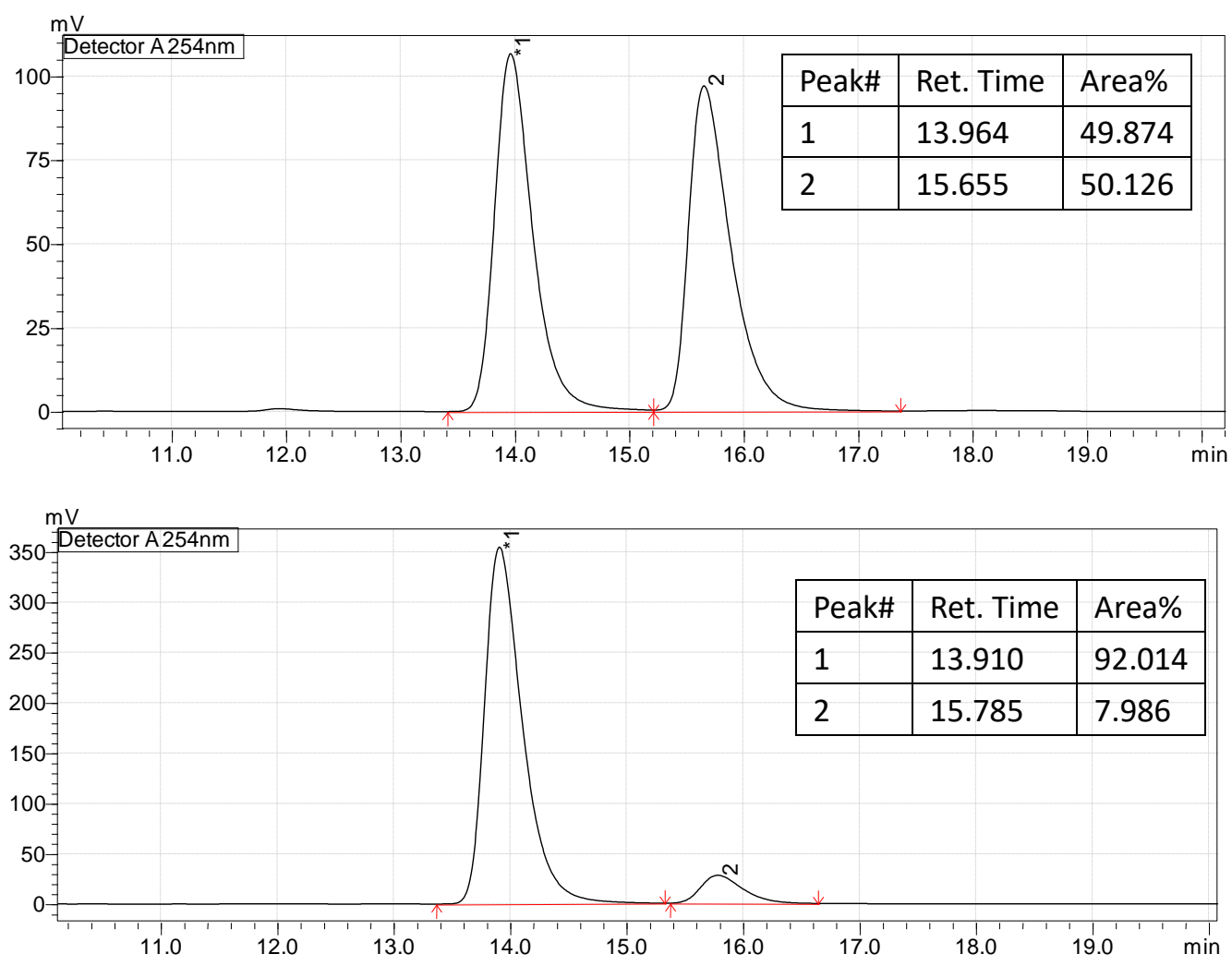


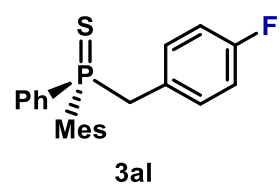

3al: Procedure A, $66.9 \mathrm{mg}, 91 \%$ yield, white solid.

${ }^{1}$ H NMR (400 MHz, CDCl $)_{3} \delta$ 7.45-7.34 (m, 3H), 7.28-7.22 (m, 2H), $6.88(\mathrm{~d}, J=3.8 \mathrm{~Hz}, 2 \mathrm{H})$, 6.76-6.64 (m, 4H), 3.99 (dd, $J=15.3,13.3 \mathrm{~Hz}, 1 \mathrm{H}), 3.68(\mathrm{dd}, J=13.0,10.4 \mathrm{~Hz}, 1 \mathrm{H}), 2.34(\mathrm{~s}, 6 \mathrm{H})$, $2.29(\mathrm{~s}, 3 \mathrm{H})$.

${ }^{13}$ C NMR(101 MHz, CDCl $) \delta 161.81(\mathrm{dd}, J=245.5,4.3 \mathrm{~Hz}), 141.49(\mathrm{~d}, J=10.1 \mathrm{~Hz}), 140.93(\mathrm{~d}$, $J=2.8 \mathrm{~Hz}), 133.67(\mathrm{~d}, J=80.1 \mathrm{~Hz}), 132.02(\mathrm{dd}, J=8.0,5.3 \mathrm{~Hz}), 131.35(\mathrm{~d}, J=11.1 \mathrm{~Hz}), 130.70$ $(\mathrm{d}, J=3.0 \mathrm{~Hz}), 130.59$ (d, $J=9.8 \mathrm{~Hz}), 128.95(\mathrm{~d}, J=80.4 \mathrm{~Hz}), 128.01(\mathrm{~d}, J=12.3 \mathrm{~Hz}), 126.75$ (dd, $J=7.4,3.2 \mathrm{~Hz}), 114.18(\mathrm{dd}, J=21.4,3.4 \mathrm{~Hz}), 44.73(\mathrm{~d}, J=47.3 \mathrm{~Hz}), 30.76(\mathrm{~d}, J=138.6 \mathrm{~Hz})$, $24.32(\mathrm{~d}, J=4.7 \mathrm{~Hz}), 20.84(\mathrm{~d}, J=1.4 \mathrm{~Hz})$.

${ }^{31}$ P NMR (162 MHz, CDCl $) \delta 43.23(\mathrm{~d}, J=6.5 \mathrm{~Hz})$.

${ }^{19}$ F NMR (376 MHz, $\left.\mathbf{C D C l}_{3}\right) \delta-115.94--116.09(\mathrm{~m})$.

HRMS (ESI) $\mathbf{m} / \mathbf{z}$ [M+H] $]^{+}$: calcd. 369.1237, found. 369.1237.

IR (film): $v_{\max }\left(\mathrm{cm}^{-1}\right)$ 3056, 2964, 2923, 1603, 1507, 1437, 1221, 1101, 835, 747, 594.

Optical rotation: $[\alpha]_{\mathrm{D}}{ }^{25}=-183.62\left(c=1.000, \mathrm{CHCl}_{3}, 88 \%\right.$ ee $)$.

HPLC: DAICEL CHIRALPAK ID, hexane $/ i-\mathrm{PrOH}=9 / 1$, flow rate: $1.0 \mathrm{~mL} / \mathrm{min}, \lambda=254 \mathrm{~nm}$, $\mathrm{t}_{\mathrm{R}}($ major $)=8.9 \mathrm{~min}, \mathrm{t}_{\mathrm{R}}(\operatorname{minor})=10.6 \mathrm{~min}, 88 \%$ ee.
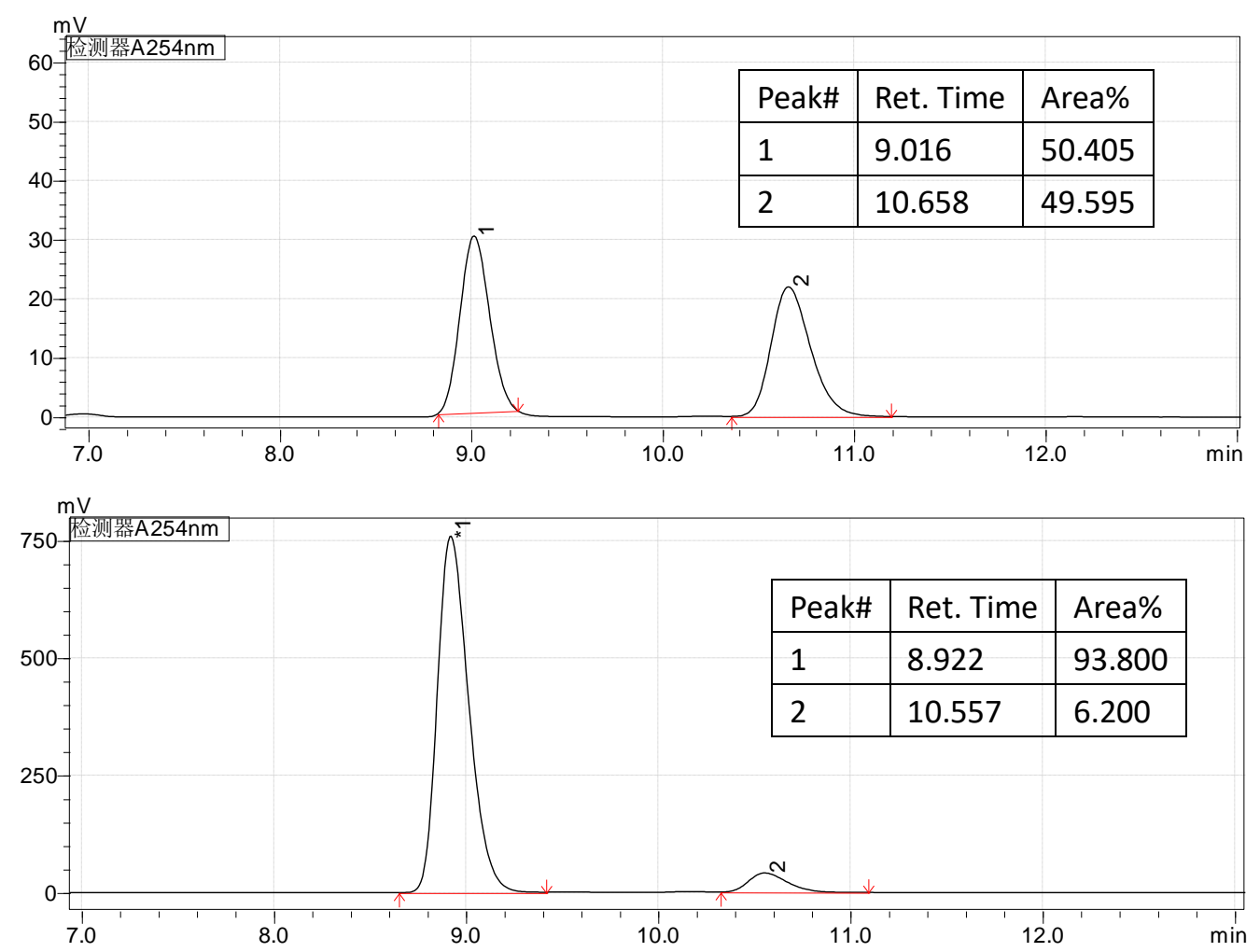


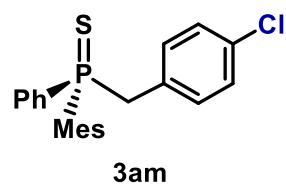

3am: Procedure A, $72.9 \mathrm{mg}, 95 \%$ yield, yellow solid.

${ }^{1}$ H NMR (400 MHz, CDCl ${ }_{3}$ ) $\delta$ 7.48-7.35 (m, 3H), 7.31-7.21 (m, 2H), 6.99-6.92 (m, 2H), 6.88 (d, $J=4.1 \mathrm{~Hz}, 2 \mathrm{H}), 6.68(\mathrm{dd}, J=8.5,2.8 \mathrm{~Hz}, 2 \mathrm{H}), 3.99(\mathrm{dd}, J=15.8,13.0 \mathrm{~Hz}, 1 \mathrm{H}), 3.67(\mathrm{dd}, J=13.0$, $10.5 \mathrm{~Hz}, 1 \mathrm{H}), 2.34$ (s, 6H), 2.29 (s, 3H).

${ }^{13}$ C NMR(101 MHz, CDCl $) \delta 141.48(\mathrm{~d}, J=10.1 \mathrm{~Hz}), 140.98(\mathrm{~d}, J=2.8 \mathrm{~Hz}), 133.57(\mathrm{~d}, J=$ $80.1 \mathrm{~Hz}), 132.72$ (d, $J=4.7 \mathrm{~Hz}), 131.82(\mathrm{~d}, J=5.3 \mathrm{~Hz}), 131.37$ (d, $J=11.1 \mathrm{~Hz}), 130.79$ (d, $J=$ $3.0 \mathrm{~Hz}), 130.62$ (d, $J=9.8 \mathrm{~Hz}), 129.59(\mathrm{~d}, J=7.5 \mathrm{~Hz}), 128.88(\mathrm{~d}, J=80.9 \mathrm{~Hz}), 128.05(\mathrm{~d}, J=$ $12.3 \mathrm{~Hz}), 127.41$ (d, $J=3.5 \mathrm{~Hz}), 44.91(\mathrm{~d}, J=47.0 \mathrm{~Hz}), 24.30$ (d, $J=4.8 \mathrm{~Hz}), 20.84(\mathrm{~d}, J=1.5$ $\mathrm{Hz})$.

${ }^{31}$ P NMR (162 MHz, $\left.\mathbf{C D C l}_{3}\right) \delta 42.93$.

HRMS (ESI) $\mathbf{~ m / z ~ [ M + H ] ~}{ }^{+}$: calcd. 385.0941, found. 385.0941.

IR (film): $v_{\max }\left(\mathrm{cm}^{-1}\right)$ 3050, 2963, 2923, 1603, 1490, 1437, 1263, 1096, 1027, 846, 702, 665, 593.

Optical rotation: $[\alpha]_{\mathrm{D}}{ }^{25}=-220.24\left(c=1.014, \mathrm{CHCl}_{3}, 87 \%\right.$ ee $)$.

HPLC: DAICEL CHIRALPAK OD-H, hexane $/ i-\mathrm{PrOH}=49 / 1$, flow rate: $1.0 \mathrm{~mL} / \mathrm{min}, \lambda=254 \mathrm{~nm}$, $\mathrm{t}_{\mathrm{R}}($ major $)=9.3 \mathrm{~min}, \mathrm{t}_{\mathrm{R}}($ minor $)=16.1 \mathrm{~min}, 87 \%$ ee .
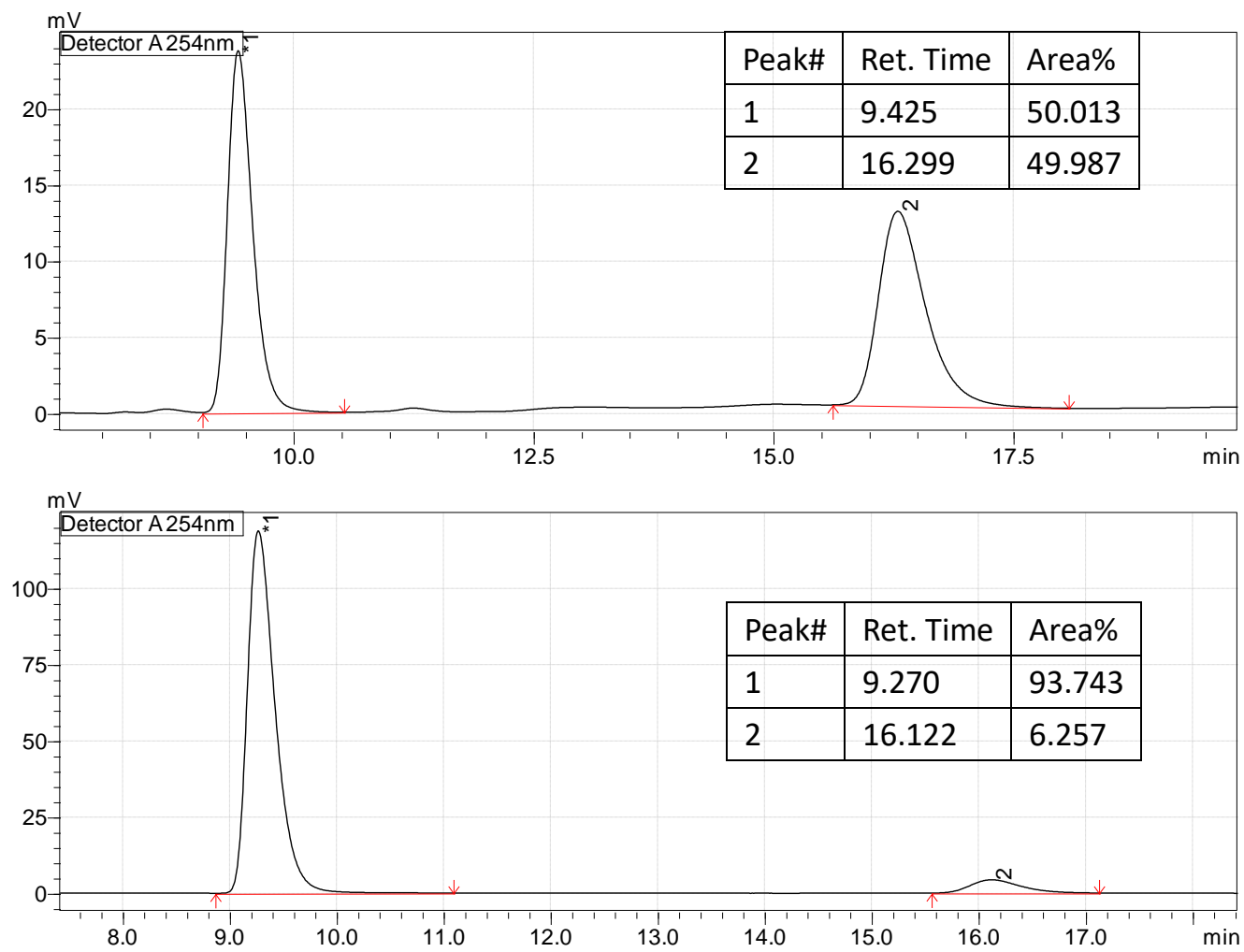


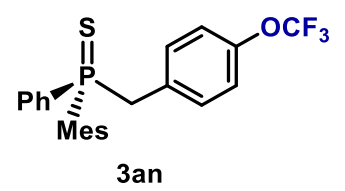

3an: Procedure A, $82.4 \mathrm{mg}, 95 \%$ yield, white solid.

${ }^{1}$ H NMR (400 MHz, CDCl $)_{3} \delta$ 7.44-7.32 (m, 3H), 7.26-7.18 (m, 2H), $6.89(\mathrm{~d}, J=4.1 \mathrm{~Hz}, 2 \mathrm{H})$, 6.86-6.76 (m, 4H), $4.01(\mathrm{dd}, J=15.8,12.9 \mathrm{~Hz}, 1 \mathrm{H}), 3.70(\mathrm{dd}, J=13.0,10.6 \mathrm{~Hz}, 1 \mathrm{H}), 2.35(\mathrm{~s}, 6 \mathrm{H})$, $2.29(\mathrm{~s}, 3 \mathrm{H})$.

${ }^{13} \mathbf{C}$ NMR(101 MHz, CDCl $) \delta 148.02(\mathrm{dt}, J=3.9,1.9 \mathrm{~Hz}), 141.48(\mathrm{~d}, J=10.1 \mathrm{~Hz}), 141.03(\mathrm{~d}, J$ $=3.0 \mathrm{~Hz}), 133.52(\mathrm{~d}, J=80.3 \mathrm{~Hz}), 131.71(\mathrm{~d}, J=5.3 \mathrm{~Hz}), 131.36(\mathrm{~d}, J=11.2 \mathrm{~Hz}), 130.75(\mathrm{~d}, J=$ $3.0 \mathrm{~Hz}), 130.46(\mathrm{~d}, J=9.8 \mathrm{~Hz}), 130.00(\mathrm{~d}, J=7.3 \mathrm{~Hz}), 128.75(\mathrm{~d}, J=81.2 \mathrm{~Hz}), 128.00(\mathrm{~d}, J=$ $12.3 \mathrm{~Hz}), 120.28(\mathrm{dd}, J=258.1,1.3 \mathrm{~Hz}), 119.73(\mathrm{~d}, J=3.4 \mathrm{~Hz}), 45.00(\mathrm{~d}, J=47.0 \mathrm{~Hz}), 24.27(\mathrm{~d}, J$ $=4.9 \mathrm{~Hz}), 20.81(\mathrm{~d}, J=1.5 \mathrm{~Hz})$.

${ }^{31}$ P NMR (162 MHz, CDCl 3 ) $\delta 43.12$.

${ }^{19}$ F NMR (376 MHz, $\left.\mathrm{CDCl}_{3}\right) \delta-57.94$.

HRMS (ESI) $\mathbf{m} / \mathbf{z}[\mathbf{M}+\mathbf{H}]^{+}$: calcd. 435.1154 , found. 435.1154.

IR (film): $v_{\max }\left(\mathrm{cm}^{-1}\right)$ 2970, 2929, 1604, 1507, 1455, 1261, 1222, 1164, 1101, 847, 693, 648, 592.

Optical rotation: $[\alpha]_{\mathrm{D}}{ }^{25}=-160.21\left(c=1.029, \mathrm{CHCl}_{3}, 88 \%\right.$ ee $)$.

HPLC: DAICEL CHIRALPAK ID, hexane $/ i-\mathrm{PrOH}=9 / 1$, flow rate: $1.0 \mathrm{~mL} / \mathrm{min}, \lambda=254 \mathrm{~nm}$, $\mathrm{t}_{\mathrm{R}}($ major $)=6.3 \mathrm{~min}, \mathrm{t}_{\mathrm{R}}($ minor $)=7.1 \mathrm{~min}, 88 \%$ ee .
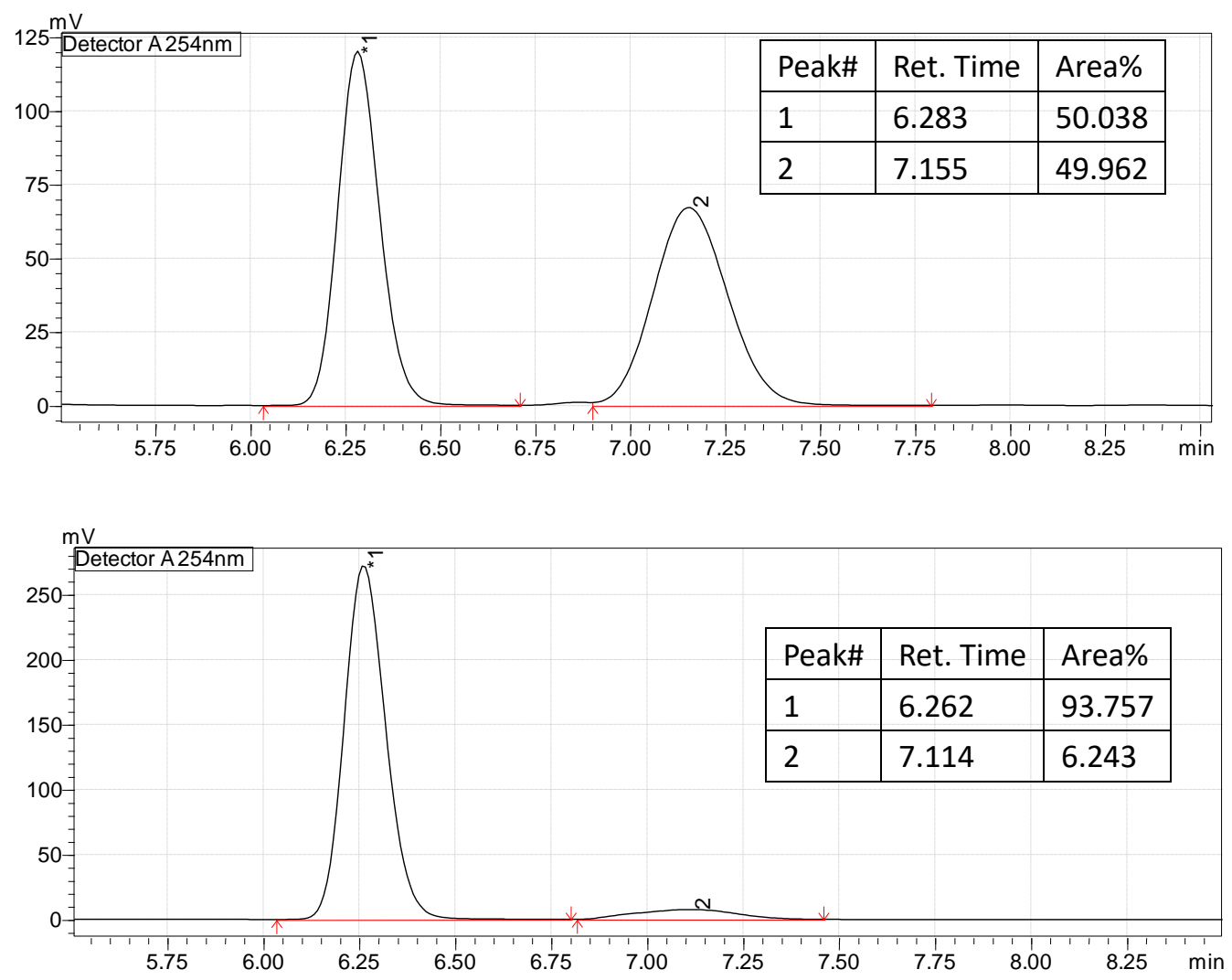


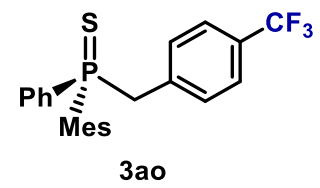

3ao: Procedure A, $71.1 \mathrm{mg}, 85 \%$ yield, light yellow solid

${ }^{1} \mathbf{H}$ NMR (400 MHz, $\left.\mathbf{C D C l}_{3}\right) \delta$ 7.45-7.34 (m, 3H), 7.26-7.20 (m, 4H), 6.93-6.84 (m, 4H), 4.07 (dd, $J=16.2,12.8 \mathrm{~Hz}, 1 \mathrm{H}), 3.75$ (dd, $J=12.8,10.9 \mathrm{~Hz}, 1 \mathrm{H}), 2.35$ (s, 6H), 2.30 (s, 3H).

${ }^{13} \mathbf{C}$ NMR(101 MHz, CDCl $\left.{ }_{3}\right) \delta 141.47(\mathrm{~d}, J=10.2 \mathrm{~Hz}), 141.10(\mathrm{~d}, J=2.8 \mathrm{~Hz}), 135.70-135.11$ (m), $133.42(\mathrm{~d}, J=80.1 \mathrm{~Hz}), 131.42(\mathrm{~d}, J=11.2 \mathrm{~Hz}), 130.89(\mathrm{~d}, J=2.9 \mathrm{~Hz}), 130.80(\mathrm{~d}, J=5.3$ Hz), 130.53 (d, $J=9.8 \mathrm{~Hz}), 128.69$ (dd, $J=32.3,3.9 \mathrm{~Hz}), 128.69$ (d, $J=81.8 \mathrm{~Hz}), 128.06$ (d, $J=$ $12.5 \mathrm{~Hz}$ ), 124.10 (dd, $J=272.0,1.5 \mathrm{~Hz}), 124.03$ (p, $J=3.7 \mathrm{~Hz}), 45.45$ (d, $J=46.7 \mathrm{~Hz}), 24.27$ (d, $J$ $=4.9 \mathrm{~Hz}), 20.82(\mathrm{~d}, J=1.5 \mathrm{~Hz})$.

${ }^{31}$ P NMR (162 MHz, CDCl 3 ) $\delta 42.84(\mathrm{q}, J=2.8 \mathrm{~Hz})$.

${ }^{19}$ F NMR (376 MHz, $\left.\mathbf{C D C l}_{3}\right) \delta-62.49(\mathrm{~d}, J=2.8 \mathrm{~Hz})$.

HRMS (ESI) $\mathbf{m} / \mathbf{z}[\mathbf{M}+\mathbf{H}]^{+}$: calcd. 419.1205 , found. 419.1205 .

IR (film): $v_{\max }\left(\mathrm{cm}^{-1}\right)$ 3056, 2964, 2924, 1615, 1604, 1437, 1324, 1123, 1067, 845, 693, 651, 597. Optical rotation: $[\alpha]_{\mathrm{D}}{ }^{25}=-159.31\left(c=1.132, \mathrm{CHCl}_{3}, 85 \%\right.$ ee $)$.

HPLC: DAICEL CHIRALPAK ID, hexane $/ i-\mathrm{PrOH}=9 / 1$, flow rate: $1.0 \mathrm{~mL} / \mathrm{min}, \lambda=254 \mathrm{~nm}$, $\mathrm{t}_{\mathrm{R}}($ major $)=6.9 \mathrm{~min}, \mathrm{t}_{\mathrm{R}}($ minor $)=7.9 \mathrm{~min}, 85 \%$ ee .
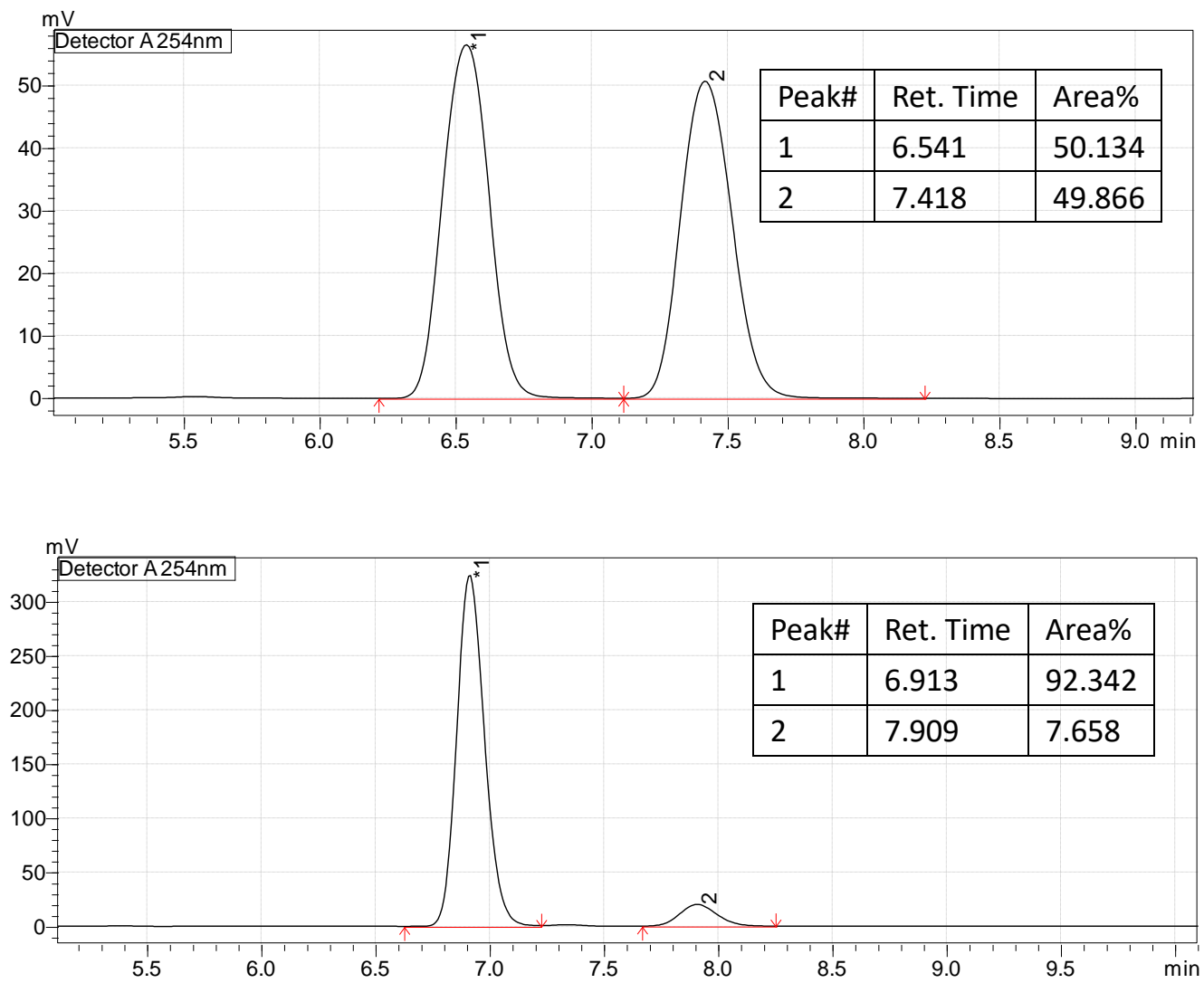


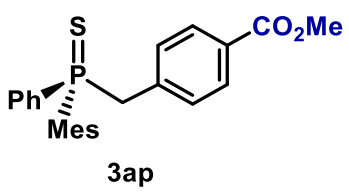

3ap: Procedure A, $73.4 \mathrm{mg}, 90 \%$ yield, light yellow solid.

${ }^{1}$ H NMR (400 MHz, CDCl $)$ ) $8.69-7.63(\mathrm{~m}, 2 \mathrm{H}), 7.47-7.33$ (m, 3H), 7.26-7.20 (m, 2H), $6.89(\mathrm{~d}$ $J=4.1 \mathrm{~Hz}, 2 \mathrm{H}), 6.84(\mathrm{dd}, J=8.3,2.6 \mathrm{~Hz}, 2 \mathrm{H}), 4.08(\mathrm{dd}, J=16.4,12.7 \mathrm{~Hz}, 1 \mathrm{H}), 3.85(\mathrm{~s}, 3 \mathrm{H}), 3.76$ (dd, $J=12.8,10.9 \mathrm{~Hz}, 1 \mathrm{H}), 2.35(\mathrm{~s}, 6 \mathrm{H}), 2.29(\mathrm{~s}, 3 \mathrm{H})$.

${ }^{13} \mathbf{C}$ NMR(101 MHz, CDCl $) \delta 166.93(\mathrm{~d}, J=1.8 \mathrm{~Hz}), 141.45(\mathrm{~d}, J=10.2 \mathrm{~Hz}), 141.00(\mathrm{~d}, J=3.0$ Hz), 136.63 (d, $J=7.6 \mathrm{~Hz}), 133.48$ (d, $J=80.3 \mathrm{~Hz}), 131.38(\mathrm{~d}, J=11.1 \mathrm{~Hz}), 130.84(\mathrm{~d}, J=3.0$ Hz), 130.56 (d), 130.53 (d, $J=4.8 \mathrm{~Hz}), 128.87$ (d, $J=81.9 \mathrm{~Hz}), 128.43$ (d, $J=3.4 \mathrm{~Hz}), 128.24$ (d, $J=3.9 \mathrm{~Hz}), 128.05(\mathrm{~d}, J=12.3 \mathrm{~Hz}), 51.91,45.67(\mathrm{~d}, J=46.5 \mathrm{~Hz}), 24.26(\mathrm{~d}, J=4.9 \mathrm{~Hz}), 20.82(\mathrm{~d}$, $J=1.7 \mathrm{~Hz})$.

${ }^{31}$ P NMR (162 MHz, $\left.\mathbf{C D C l}_{3}\right) \delta 42.70$.

HRMS (ESI) $\mathbf{m} / \mathbf{z}[\mathbf{M}+\mathbf{H}]^{+}$: calcd. 409.1386 , found. 409.1387.

IR (film): $v_{\max }\left(\mathrm{cm}^{-1}\right)$ 3060, 2950, 2923, 1720, 1606, 1436, 1278, 1103, 848, 777, 696, 655, 594.

Optical rotation: $[\alpha]_{\mathrm{D}}^{25}=-185.69\left(c=1.090, \mathrm{CHCl}_{3}, 82 \%\right.$ ee $)$.

HPLC: DAICEL CHIRALPAK IF-3, hexane $/ i-\mathrm{PrOH}=23 / 2$, flow rate: $0.5 \mathrm{~mL} / \mathrm{min}, \lambda=254 \mathrm{~nm}$, $\mathrm{t}_{\mathrm{R}}($ minor $)=47.8 \mathrm{~min}, \mathrm{t}_{\mathrm{R}}($ major $)=52.3 \mathrm{~min}, 82 \%$ ee
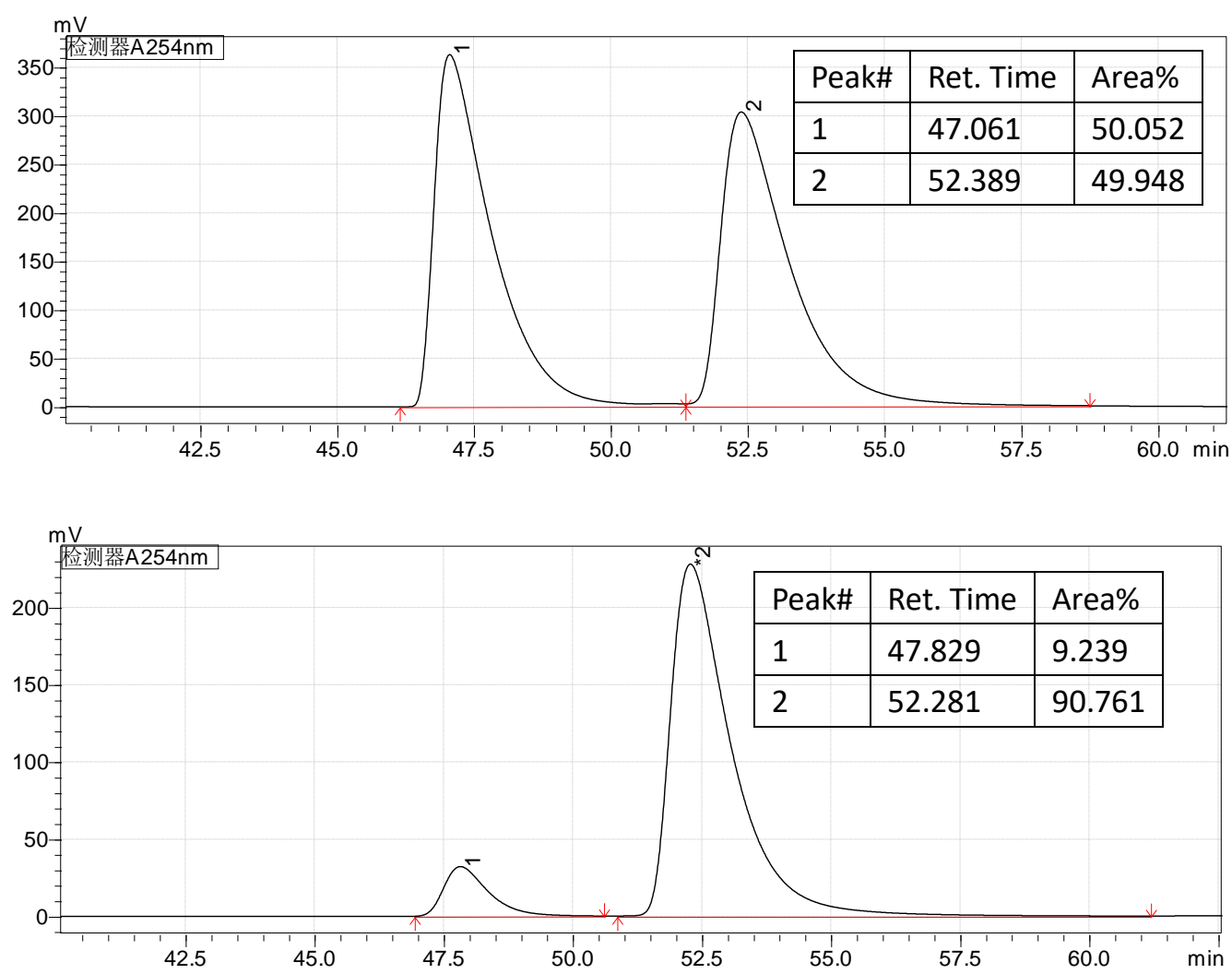


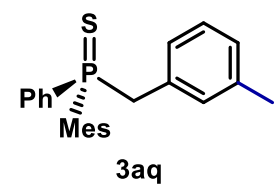

3aq: Procedure A, $69.9 \mathrm{mg}, 96 \%$ yield, light yellow solid.

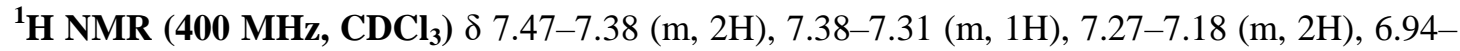
$6.84(\mathrm{~m}, 4 \mathrm{H}), 6.67-6.61(\mathrm{~m}, 1 \mathrm{H}), 6.42(\mathrm{~d}, J=2.4 \mathrm{~Hz}, 1 \mathrm{H}), 3.97(\mathrm{dd}, J=15.9,12.9 \mathrm{~Hz}, 1 \mathrm{H}), 3.67$ (dd, $J=12.9,10.6 \mathrm{~Hz}, 1 \mathrm{H}), 2.35$ (s, 6H), 2.29 (s, 3H), 2.07 (s, 3H).

${ }^{13} \mathbf{C}$ NMR(101 MHz, CDCl $) \delta 141.45(\mathrm{~d}, J=10.2 \mathrm{~Hz}), 140.72(\mathrm{~d}, J=2.8 \mathrm{~Hz}), 136.67(\mathrm{~d}, J=3.5$ $\mathrm{Hz}), 133.90(\mathrm{~d}, J=80.1 \mathrm{~Hz}), 131.49(\mathrm{~d}, J=5.5 \mathrm{~Hz}), 131.27(\mathrm{~d}, J=11.1 \mathrm{~Hz}), 130.77(\mathrm{~d}, J=7.2$ Hz), 130.68 (d, $J=9.6 \mathrm{~Hz}), 130.46(\mathrm{~d}, J=2.9 \mathrm{~Hz}), 129.19$ (d, $J=80.1 \mathrm{~Hz}), 127.75$ (d, $J=12.5$ $\mathrm{Hz}), 127.71(\mathrm{~d}, J=5.1 \mathrm{~Hz}) 127.19(\mathrm{~d}, J=4.1 \mathrm{~Hz}), 127.07(\mathrm{~d}, J=3.6 \mathrm{~Hz}), 45.53(\mathrm{~d}, J=47.0 \mathrm{~Hz})$, $24.30(\mathrm{~d}, J=4.7 \mathrm{~Hz}), 21.00,20.79(\mathrm{~d}, J=1.3 \mathrm{~Hz})$.

${ }^{31}$ P NMR (162 MHz, $\mathbf{C D C l}_{3}$ ) $\delta 43.28$.

HRMS (ESI) $\mathbf{m} / \mathbf{z}$ [M+H] $]^{+}$: calcd. 365.1487, found. 365.1487 .

IR (film): $v_{\max }\left(\mathrm{cm}^{-1}\right)$ 3024, 2957, 2923, 2854, 1603, 1454, 1437, 1100, 1027, 842, 694, 646, 607. Optical rotation: $[\alpha]_{D}{ }^{25}=-173.76\left(c=1.220, \mathrm{CHCl}_{3}, 87 \%\right.$ ee $)$.

HPLC: DAICEL CHIRALPAK OD-H, hexane $/ i-\mathrm{PrOH}=97 / 3$, flow rate: $1.0 \mathrm{~mL} / \mathrm{min}, \lambda=254 \mathrm{~nm}$, $\mathrm{t}_{\mathrm{R}}($ major $)=9.8 \mathrm{~min}, \mathrm{t}_{\mathrm{R}}($ minor $)=16.1 \mathrm{~min}, 87 \%$ ee .
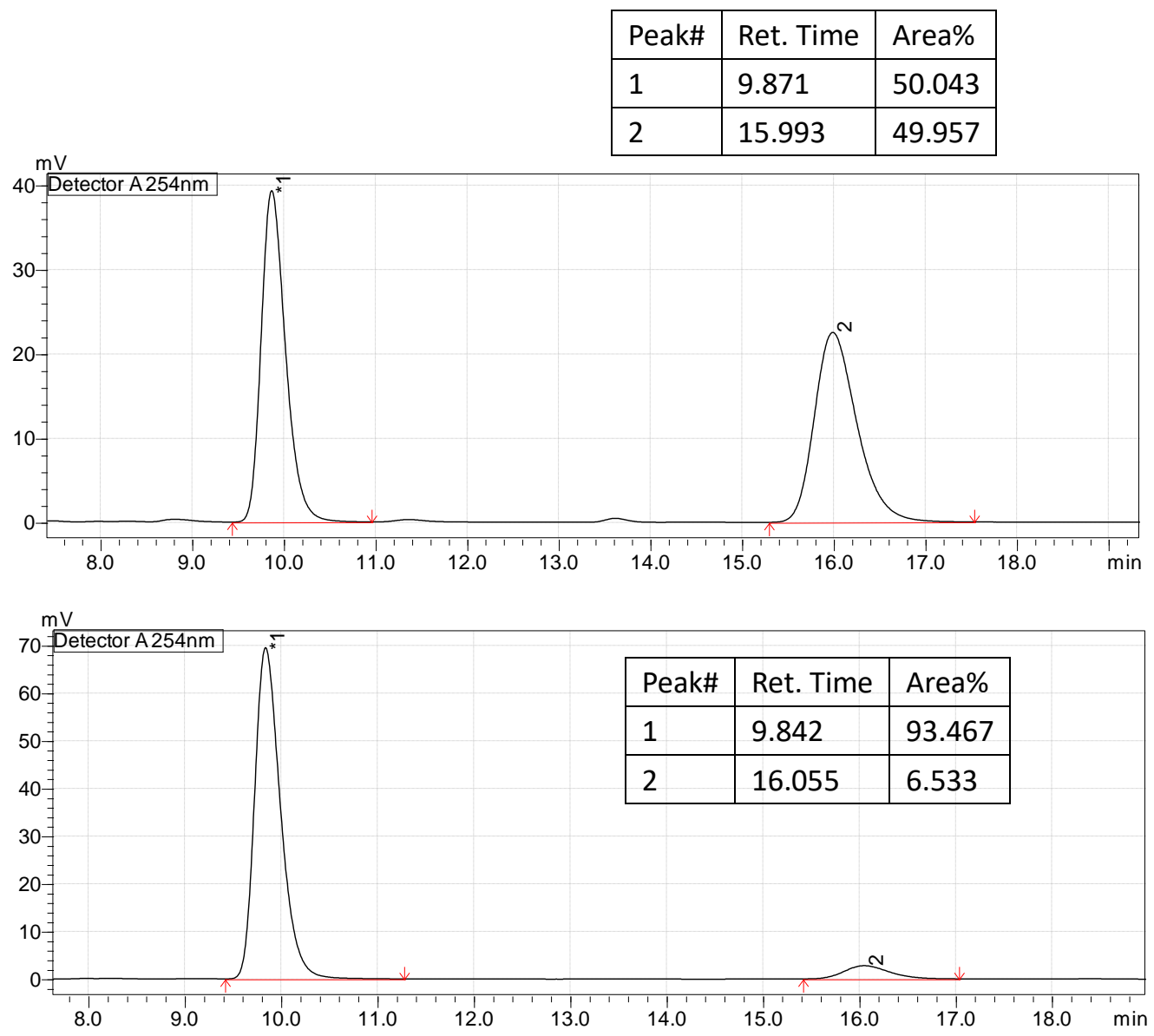


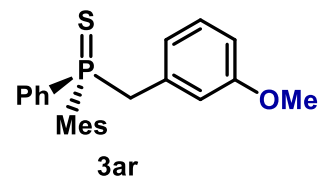

3ar: Procedure A, $72.9 \mathrm{mg}$, 96\% yield, light yellow solid.

${ }^{1}$ H NMR (400 MHz, CDCl $_{3}$ ) $\delta$ 7.49-7.41 (m, 2H), 7.39-7.32 (m, 1H), 7.26-7.20 (m, 2H), 6.92$6.85(\mathrm{~m}, 3 \mathrm{H}), 6.65-6.59(\mathrm{~m}, 1 \mathrm{H}), 6.42-6.36(\mathrm{~m}, 1 \mathrm{H}), 6.33(\mathrm{q}, J=2.3 \mathrm{~Hz}, 1 \mathrm{H}), 4.01$ (dd, $J=16.0$, $12.9 \mathrm{~Hz}, 1 \mathrm{H}), 3.69$ (dd, $J=13.0,10.6 \mathrm{~Hz}, 1 \mathrm{H}), 3.57$ (s, 3H), 2.35 (s, 6H), 2.29 (s, 3H).

${ }^{13} \mathbf{C}$ NMR(101 MHz, CDCl $) \delta 158.52(\mathrm{~d}, J=3.5 \mathrm{~Hz}), 141.46(\mathrm{~d}, J=10.2 \mathrm{~Hz}), 140.79(\mathrm{~d}, J=3.0$ Hz), 133.86 (d, $J=79.9 \mathrm{~Hz}), 132.47(\mathrm{~d}, J=7.5 \mathrm{~Hz}), 131.31(\mathrm{~d}, J=11.1 \mathrm{~Hz}), 130.67$ (d, $J=9.8$ Hz), 130.54 (d, $J=3.0 \mathrm{~Hz}), 129.21(\mathrm{~d}, J=80.5 \mathrm{~Hz}), 128.14(\mathrm{~d}, J=3.4 \mathrm{~Hz}), 127.89$ (d, $J=12.2$ $\mathrm{Hz}), 123.20(\mathrm{~d}, J=5.7 \mathrm{~Hz}), 115.35(\mathrm{~d}, J=5.3 \mathrm{~Hz}), 113.11(\mathrm{~d}, J=4.1 \mathrm{~Hz}), 54.93,45.60(\mathrm{~d}, J=$ $47.0 \mathrm{~Hz}), 24.28(\mathrm{~d}, J=4.7 \mathrm{~Hz}), 20.80(\mathrm{~d}, J=1.5 \mathrm{~Hz})$.

${ }^{31}$ P NMR (162 MHz, CDCl $) \delta 43.10$.

HRMS (ESI) $\mathbf{m} / \mathbf{z}$ [M+H] $]^{+}$: calcd. 381.1436, found. 381.1436.

IR (film): $v_{\max }\left(\mathrm{cm}^{-1}\right) 3051,2959,2921,2833,1601,1486,1436,1263,1166,1101,1042,843$, 737, 692, 645, 585.

Optical rotation: $[\alpha]_{\mathrm{D}}^{25}=-149.69\left(c=1.000, \mathrm{CHCl}_{3}, 85 \%\right.$ ee $)$.

HPLC: DAICEL CHIRALPAK OD-H, hexane $/ i-\mathrm{PrOH}=97 / 3$, flow rate: $1.0 \mathrm{~mL} / \mathrm{min}, \lambda=254 \mathrm{~nm}$, $\mathrm{t}_{\mathrm{R}}($ major $)=11.0 \mathrm{~min}, \mathrm{t}_{\mathrm{R}}($ minor $)=22.9 \mathrm{~min}, 85 \%$ ee
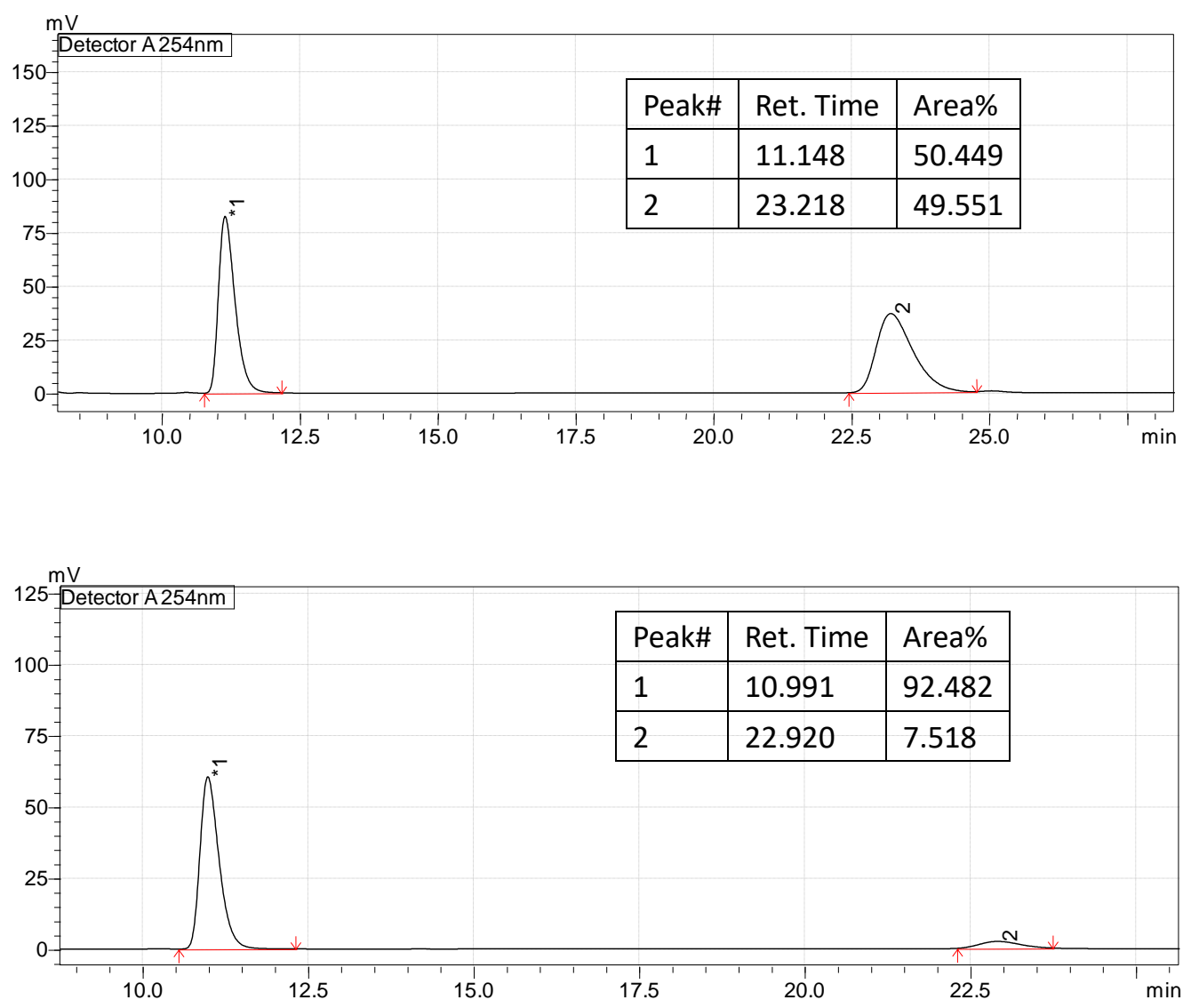


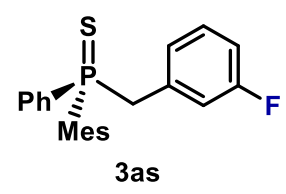

3as: Procedure A, $70.6 \mathrm{mg}, 96 \%$ yield, light yellow liquid.

${ }^{1} \mathbf{H}$ NMR (400 MHz, $\left.\mathbf{C D C l}_{3}\right) \delta$ 7.49-7.39 (m, 2H), 7.42-7.32 (m, 1H), 7.28-7.22 (m, 2H), 7.00$6.90(\mathrm{~m}, 1 \mathrm{H}), 6.88(\mathrm{~d}, J=4.0 \mathrm{~Hz}, 2 \mathrm{H}), 6.80-6.70(\mathrm{~m}, 1 \mathrm{H}), 6.63-6.56(\mathrm{~m}, 1 \mathrm{H}), 6.50-6.41(\mathrm{~m}, 1 \mathrm{H})$, $4.01(\mathrm{dd}, J=16.1,12.9 \mathrm{~Hz}, 1 \mathrm{H}), 3.69$ (dd, $J=13.0,10.7 \mathrm{~Hz}, 1 \mathrm{H}), 2.34$ (d, $J=1.2 \mathrm{~Hz}, 6 \mathrm{H}), 2.29$ (s, $3 \mathrm{H})$.

${ }^{13} \mathbf{C}$ NMR(101 MHz, CDCl $) \delta 161.76(\mathrm{dd}, J=244.9,3.8 \mathrm{~Hz}), 141.44(\mathrm{~d}, J=10.1 \mathrm{~Hz}), 140.95(\mathrm{~d}$, $J=3.0 \mathrm{~Hz}), 133.57(\mathrm{~d}, J=80.2 \mathrm{~Hz}), 133.57(\mathrm{t}, J=7.8 \mathrm{~Hz}), 131.36(\mathrm{~d}, J=11.2 \mathrm{~Hz}), 130.78(\mathrm{~d}, J=$ $3.0 \mathrm{~Hz}), 130.54(\mathrm{~d}, J=9.7 \mathrm{~Hz}), 128.93(\mathrm{~d}, J=81.0 \mathrm{~Hz}), 128.50(\mathrm{dd}, J=8.1,3.6 \mathrm{~Hz}), 128.00(\mathrm{~d}, J$ $=12.3 \mathrm{~Hz}), 126.39(\mathrm{dd}, J=5.5,2.8 \mathrm{~Hz}), 117.38(\mathrm{dd}, J=22.2,5.3 \mathrm{~Hz}), 113.44(\mathrm{dd}, J=21.0,3.9$ $\mathrm{Hz}), 45.34$ (dd, $J=47.0,1.9 \mathrm{~Hz}), 24.26(\mathrm{~d}, J=4.9 \mathrm{~Hz}), 20.81(\mathrm{~d}, J=1.4 \mathrm{~Hz})$.

${ }^{31}$ P NMR (162 MHz, CDCl $) \delta 42.83(\mathrm{~d}, J=3.1 \mathrm{~Hz})$.

${ }^{19}$ F NMR (376 MHz, $\left.\mathbf{C D C l}_{3}\right) \delta-114.25--114.37(\mathrm{~m})$.

HRMS (ESI) $\mathbf{m} / \mathbf{z}[\mathbf{M}+\mathbf{H}]^{+}$: calcd. 369.1237, found. 369.1237.

IR (film): $v_{\max }\left(\mathrm{cm}^{-1}\right)$ 3058, 2963, 2924, 2858, 1604, 1588, 1484, 1445, 1254, 1101, 942, 843, 786, 686, 613.

Optical rotation: $[\alpha]_{\mathrm{D}}{ }^{25}=-160.09\left(c=1.000, \mathrm{CHCl}_{3}, 86 \%\right.$ ee $)$.

HPLC: DAICEL CHIRALPAK OD-H, hexane $/ i-\mathrm{PrOH}=97 / 3$, flow rate: $1.0 \mathrm{~mL} / \mathrm{min}, \lambda=254 \mathrm{~nm}$, $\mathrm{t}_{\mathrm{R}}($ major $)=8.6 \mathrm{~min}, \mathrm{t}_{\mathrm{R}}($ minor $)=15.5 \mathrm{~min}, 86 \%$ ee
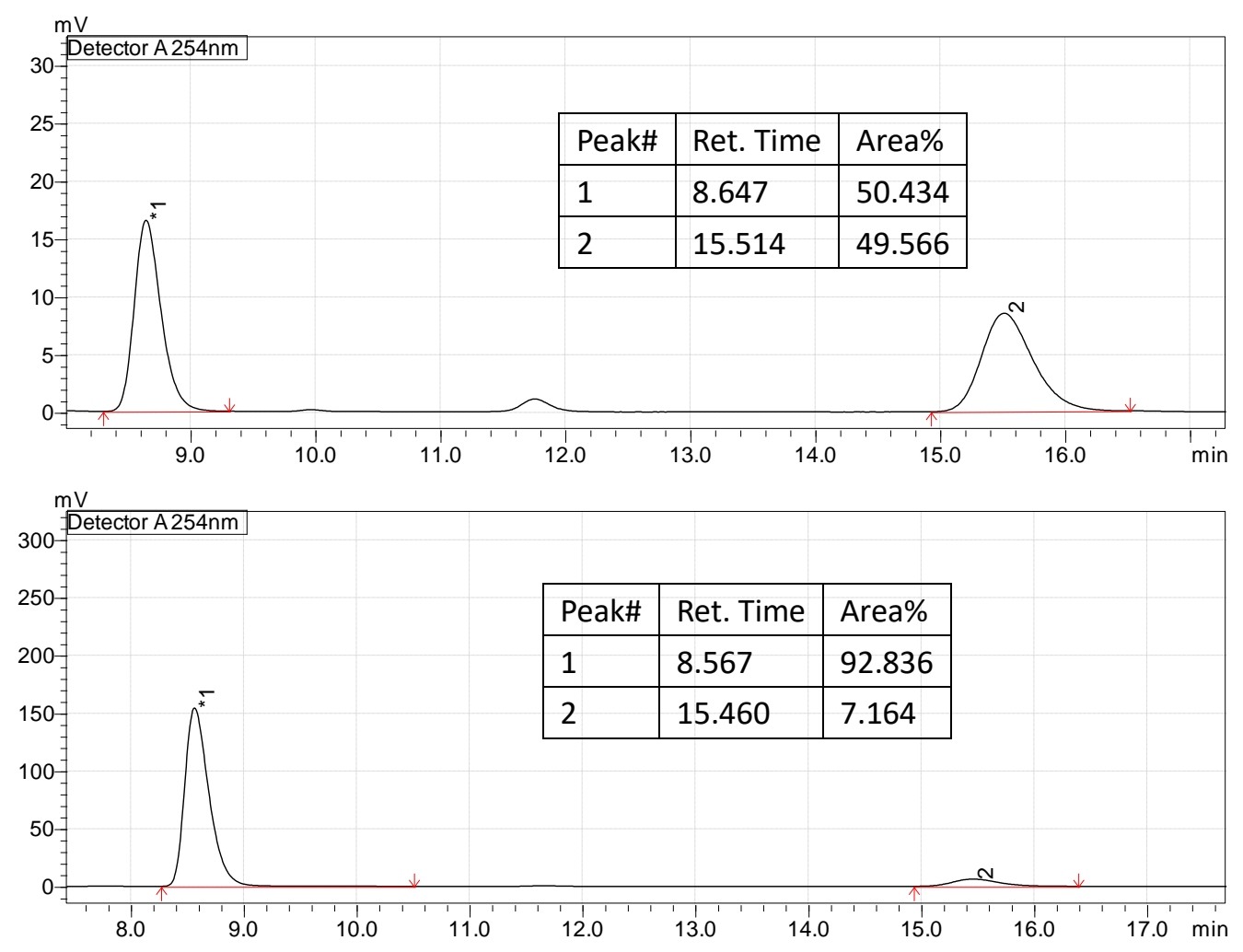


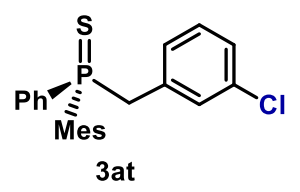

3at: Procedure A, $72.9 \mathrm{mg}, 95 \%$ yield, light yellow liquid.

${ }^{1}$ H NMR (400 MHz, CDCl $)$ ) $8.47-7.35$ (m, 3H), 7.31-7.22 (m, 2H), 7.08-7.00 (m, 1H), 6.95 (t, $\mathrm{J}=7.8 \mathrm{~Hz}, 1 \mathrm{H}), 6.89(\mathrm{~d}, \mathrm{~J}=4.1 \mathrm{~Hz}, 2 \mathrm{H}), 6.82-6.74(\mathrm{~m}, 1 \mathrm{H}), 6.57(\mathrm{q}, \mathrm{J}=2.2 \mathrm{~Hz}, 1 \mathrm{H}), 3.98(\mathrm{dd}, \mathrm{J}$ $=15.9,12.9 \mathrm{~Hz}, 1 \mathrm{H}), 3.65(\mathrm{dd}, \mathrm{J}=12.9,10.6 \mathrm{~Hz}, 1 \mathrm{H}), 2.35(\mathrm{~s}, 6 \mathrm{H}), 2.29(\mathrm{~s}, 3 \mathrm{H})$.

${ }^{13} \mathbf{C}$ NMR(101 MHz, $\left.\mathbf{C D C l}_{3}\right) \delta 141.47(\mathrm{~d}, J=10.2 \mathrm{~Hz}), 140.99(\mathrm{~d}, J=2.9 \mathrm{~Hz}), 133.53(\mathrm{~d}, J=$ $79.4 \mathrm{~Hz}), 133.10(\mathrm{~d}, J=7.2 \mathrm{~Hz}), 132.95(\mathrm{~d}, J=4.1 \mathrm{~Hz}), 131.37$ (d, $J=11.3 \mathrm{~Hz}), 130.87$ (d, $J=$ $3.0 \mathrm{~Hz}), 130.55(\mathrm{~d}, J=9.8 \mathrm{~Hz}), 130.51(\mathrm{~d}, J=5.5 \mathrm{~Hz}), 128.87$ (d, $J=5.3 \mathrm{~Hz}), 128.83(\mathrm{~d}, J=81.3$ $\mathrm{Hz}), 128.36(\mathrm{~d}, J=3.4 \mathrm{~Hz}), 128.03(\mathrm{~d}, J=12.3 \mathrm{~Hz}), 126.68(\mathrm{~d}, J=4.1 \mathrm{~Hz}), 45.35(\mathrm{~d}, J=46.9 \mathrm{~Hz})$, $24.29(\mathrm{~d}, J=4.9 \mathrm{~Hz}), 20.83(\mathrm{~d}, J=1.5 \mathrm{~Hz})$.

${ }^{31}$ P NMR (162 MHz, $\left.\mathbf{C D C l}_{3}\right) \delta 42.92$.

HRMS (ESI) $\mathbf{m} / \mathbf{z}$ [M+H] $]^{+}$: calcd. 385.0941, found. 385.0941.

IR (film): $v_{\max }\left(\mathrm{cm}^{-1}\right)$ 3060, 2958, 2924, 2853, 1603, 1596, 1455, 1437, 1100, 873, 787, 696, 646, 607.

Optical rotation: $[\alpha]_{\mathrm{D}}^{25}=-146.04\left(c=1.000, \mathrm{CHCl}_{3}, 84 \%\right.$ ee $)$.

HPLC: DAICEL CHIRALPAK ID, hexane $/ i-\mathrm{PrOH}=9 / 1$, flow rate: $1.0 \mathrm{~mL} / \mathrm{min}, \lambda=254 \mathrm{~nm}$, $\mathrm{t}_{\mathrm{R}}($ major $)=9.7 \mathrm{~min}, \mathrm{t}_{\mathrm{R}}($ minor $)=10.9 \mathrm{~min}, 84 \%$ ee.
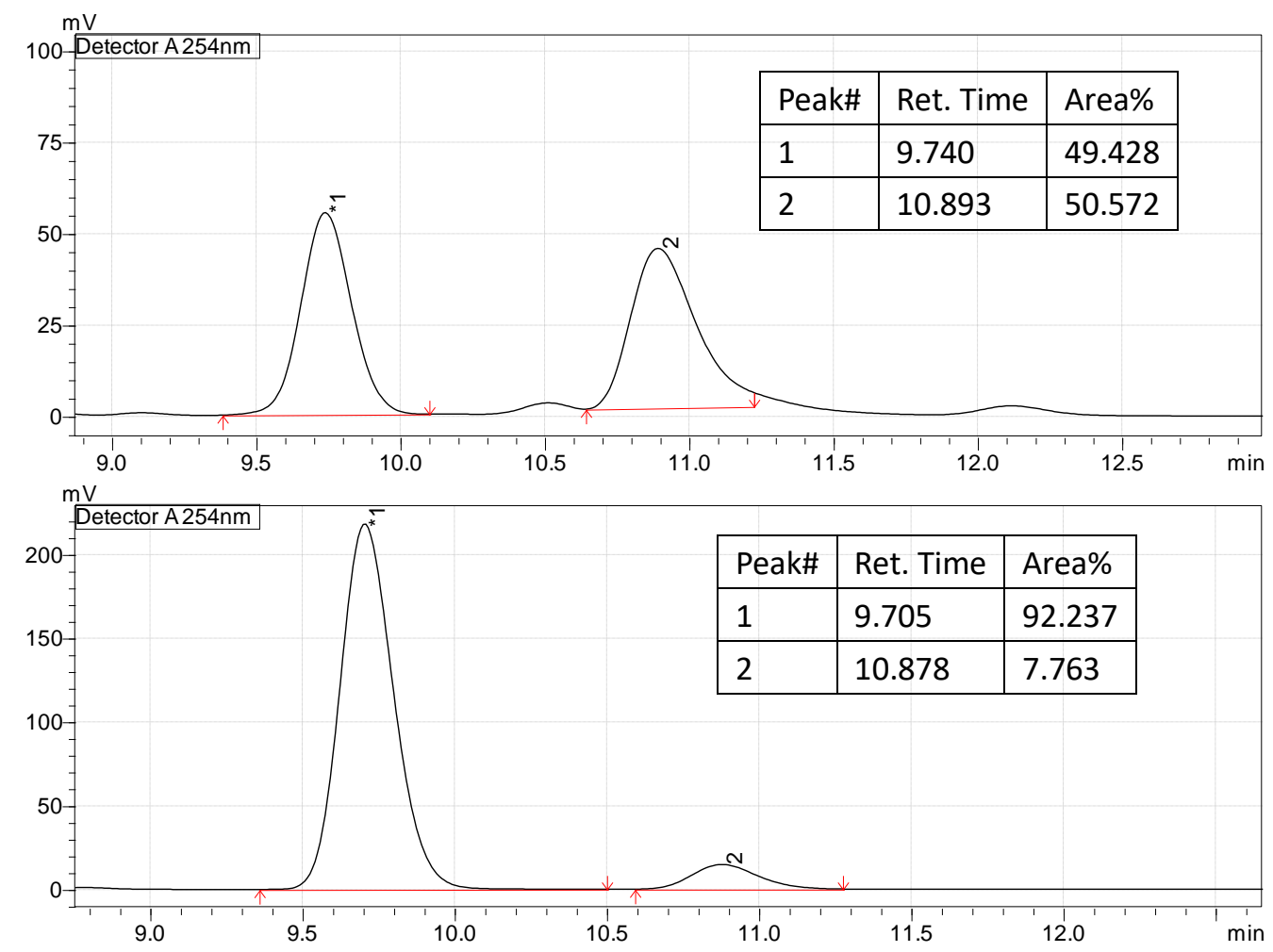


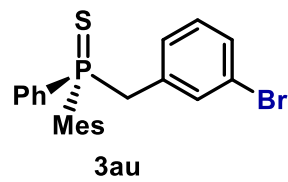

3au: Procedure A, $77.2 \mathrm{mg}, 90 \%$ yield, white solid.

${ }^{1}$ H NMR (400 MHz, CDCl $\left.{ }_{3}\right) \delta$ 7.47-7.36 (m, 3H), 7.31-7.24 (m, 2H), 7.22-7.15 (m, 1H), 6.95$6.82(\mathrm{~m}, 4 \mathrm{H}), 6.67(\mathrm{q}, J=2.0 \mathrm{~Hz}, 1 \mathrm{H}), 3.97(\mathrm{dd}, J=15.8,12.8 \mathrm{~Hz}, 1 \mathrm{H}), 3.64(\mathrm{dd}, J=12.9,10.6$ $\mathrm{Hz}, 1 \mathrm{H}), 2.35$ (s, 6H), 2.29 (s, 3H).

${ }^{13} \mathbf{C}$ NMR(101 MHz, $\left.\mathbf{C D C l}_{3}\right) \delta 141.44(\mathrm{~d}, J=10.2 \mathrm{~Hz}), 140.98(\mathrm{~d}, J=3.0 \mathrm{~Hz}), 133.46(\mathrm{~d}, J=$ $80.4 \mathrm{~Hz}), 133.36$ (d, $J=5.4 \mathrm{~Hz}), 133.32(\mathrm{~d}, J=7.3 \mathrm{~Hz}), 131.35$ (d, $J=11.1 \mathrm{~Hz}), 130.92(\mathrm{~d}, J=$ $3.0 \mathrm{~Hz}), 130.51(\mathrm{~d}, J=9.7 \mathrm{~Hz}), 129.55(\mathrm{~d}, J=4.0 \mathrm{~Hz}), 129.32(\mathrm{~d}, J=5.3 \mathrm{~Hz}), 128.75(\mathrm{~d}, J=81.2$ $\mathrm{Hz}), 128.64(\mathrm{~d}, J=3.6 \mathrm{~Hz}), 128.02(\mathrm{~d}, J=12.5 \mathrm{~Hz}), 121.11(\mathrm{~d}, J=4.2 \mathrm{~Hz}), 45.34(\mathrm{~d}, J=46.7 \mathrm{~Hz})$, $24.29(\mathrm{~d}, J=4.9 \mathrm{~Hz}), 20.83(\mathrm{~d}, J=1.5 \mathrm{~Hz})$.

\section{${ }^{31}$ P NMR (162 MHz, $\left.\mathbf{C D C l}_{3}\right) \delta 42.99$.}

HRMS (ESI) $\mathbf{m} / \mathbf{z}[\mathbf{M}+\mathbf{H}]^{+}$: calcd. 429.0436, found. 429.0433.

IR (film): $v_{\max }\left(\mathrm{cm}^{-1}\right)$ 3053, 2960, 2923, 2853, 1603, 1567, 1437, 1263, 1100, 1072, 856, 786, 691, 645,605 .

Optical rotation: $[\alpha]_{\mathrm{D}}^{25}=-119.50\left(c=1.080, \mathrm{CHCl}_{3}, 85 \%\right.$ ee $)$.

HPLC: DAICEL CHIRALPAK ID, hexane $/ i-\mathrm{PrOH}=9 / 1$, flow rate: $1.0 \mathrm{~mL} / \mathrm{min}, \lambda=254 \mathrm{~nm}$, $\mathrm{t}_{\mathrm{R}}($ major $)=10.9 \mathrm{~min}, \mathrm{t}_{\mathrm{R}}($ minor $)=12.5 \mathrm{~min}, 85 \%$ ee .
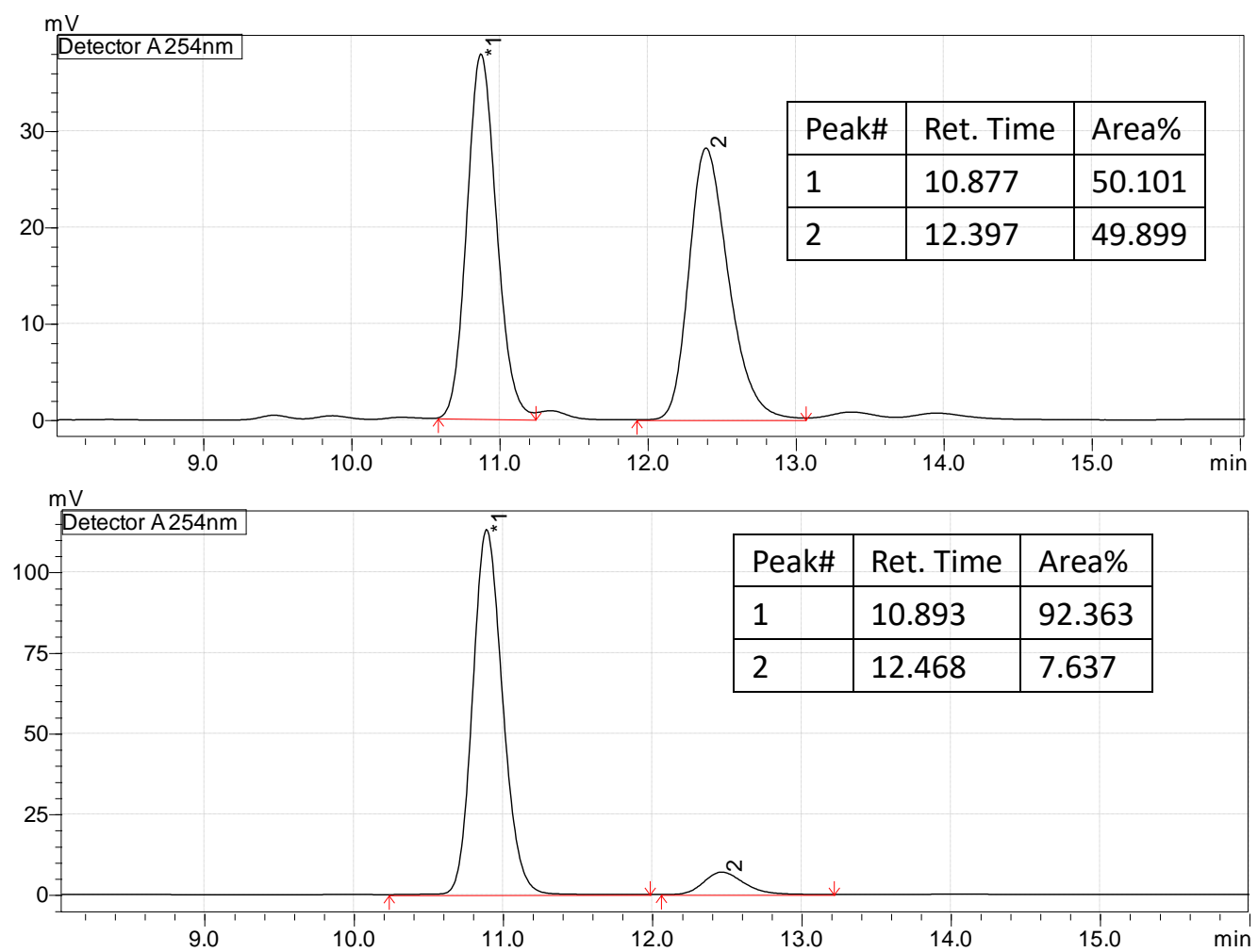


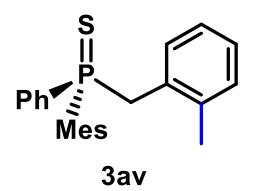

3av: Procedure A, $71.3 \mathrm{mg}, 98 \%$ yield, white solid.

${ }^{1}$ H NMR (400 MHz, $\left.\mathbf{C D C l}_{3}\right) \delta$ 7.55-7.45 (m, 2H), 7.41-7.35 (m, 1H), 7.28-7.21 (m, 2H), 7.02$6.96(\mathrm{~m}, 1 \mathrm{H}), 6.96-6.92(\mathrm{~m}, 1 \mathrm{H}), 6.89(\mathrm{~d}, J=4.0 \mathrm{~Hz}, 2 \mathrm{H}), 6.85-6.79(\mathrm{~m}, 1 \mathrm{H}), 6.72-6.65(\mathrm{~m}, 1 \mathrm{H})$, $4.17(\mathrm{dd}, J=15.9,13.5 \mathrm{~Hz}, 1 \mathrm{H}), 3.78(\mathrm{dd}, J=13.5,11.2 \mathrm{~Hz}, 1 \mathrm{H}), 2.37$ (s, 6H), 2.29 (s, 3H), 2.09 (s, 3H).

${ }^{13} \mathbf{C}$ NMR(101 MHz, CDCl $) \delta 141.54(\mathrm{~d}, J=10.1 \mathrm{~Hz}), 140.69(\mathrm{~d}, J=2.9 \mathrm{~Hz}), 137.84(\mathrm{~d}, J=6.1$ Hz), 134.19 (d, $J=79.1 \mathrm{~Hz}), 131.43(\mathrm{~d}, J=11.1 \mathrm{~Hz}), 130.96(\mathrm{~d}, J=9.9 \mathrm{~Hz}), 130.90(\mathrm{~d}, J=4.7$ $\mathrm{Hz}), 130.72(\mathrm{~d}, J=3.0 \mathrm{~Hz}), 130.02(\mathrm{~d}, J=3.2 \mathrm{~Hz}), 129.96(\mathrm{~d}, J=7.7 \mathrm{~Hz}), 129.18(\mathrm{~d}, J=79.8 \mathrm{~Hz})$, $127.87(\mathrm{~d}, J=12.2 \mathrm{~Hz}), 126.81(\mathrm{~d}, J=3.8 \mathrm{~Hz}), 124.81(\mathrm{~d}, J=3.5 \mathrm{~Hz}), 41.26(\mathrm{~d}, J=47.9 \mathrm{~Hz})$, 24.46 (d, $J=4.6 \mathrm{~Hz}), 20.80(\mathrm{~d}, J=1.6 \mathrm{~Hz}), 20.46(\mathrm{~d}, J=1.8 \mathrm{~Hz})$.

${ }^{31}$ P NMR (162 MHz, CDCl $) \delta 43.12$.

HRMS (ESI) $\mathbf{m} / \mathbf{z}[\mathbf{M}+\mathbf{H}]^{+}$: calcd. 365.1487, found. 365.1487 .

IR (film): $v_{\max }\left(\mathrm{cm}^{-1}\right)$ 3020, 2956, 2924, 2854, 1603, 1455, 1436, 1377, 1264, 1100, 847, 772, 744, 696, 644, 591.

Optical rotation: $[\alpha]_{\mathrm{D}}^{25}=-128.46\left(c=1.125, \mathrm{CHCl}_{3}, 77 \%\right.$ ee $)$.

HPLC: DAICEL CHIRALPAK OD-H, hexane $/ i-\mathrm{PrOH}=19 / 1$, flow rate: $1.0 \mathrm{~mL} / \mathrm{min}, \lambda=254 \mathrm{~nm}$, $\mathrm{t}_{\mathrm{R}}($ major $)=6.4 \mathrm{~min}, \mathrm{t}_{\mathrm{R}}($ minor $)=12.6 \mathrm{~min}, 77 \%$ ee.
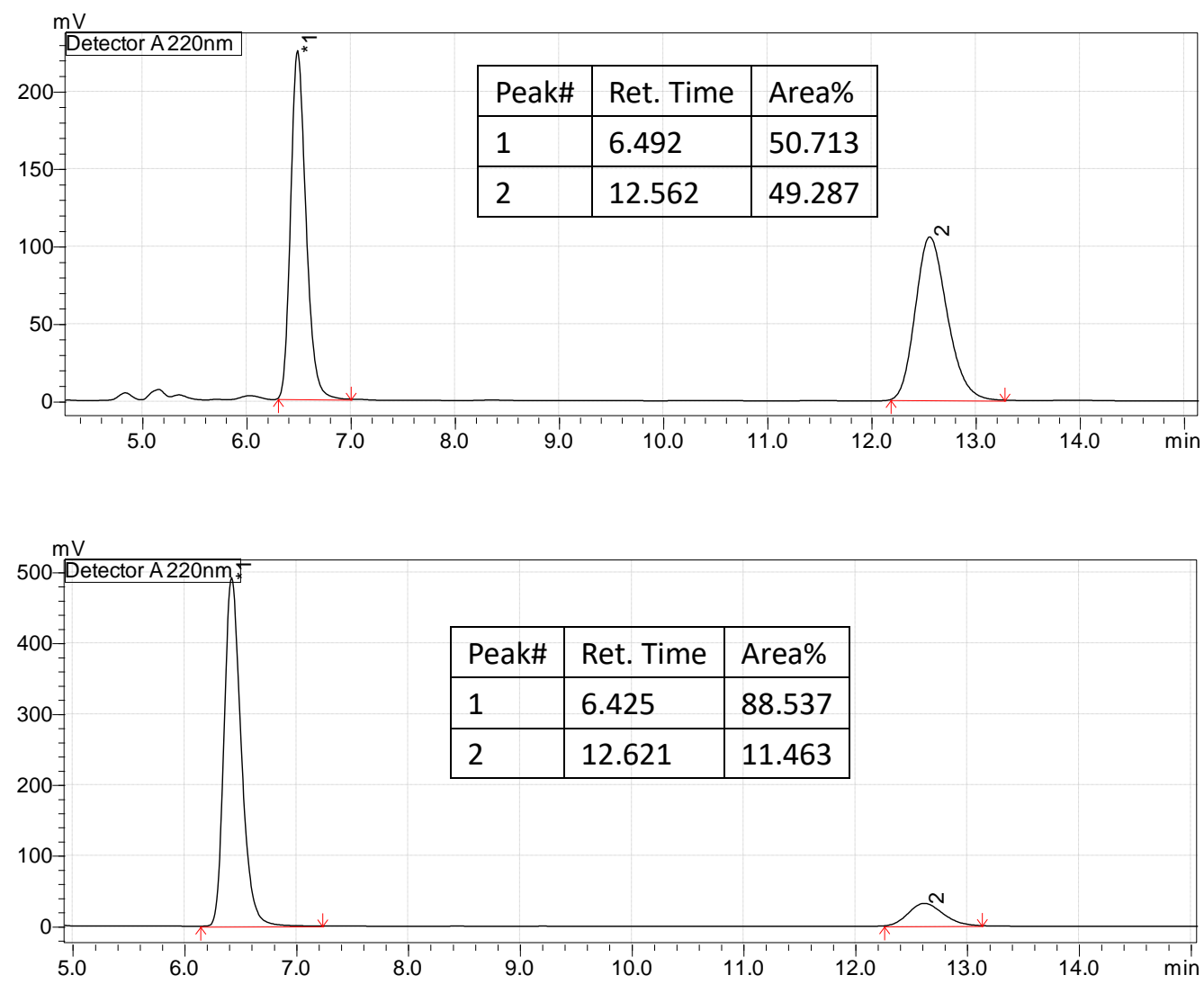


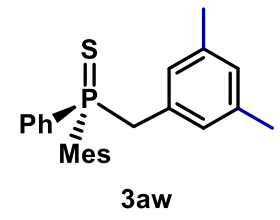

3aw: Procedure A, $71.8 \mathrm{mg}, 95 \%$ yield, light yellow solid.

${ }^{1}$ H NMR (400 MHz, CDCl $_{3}$ ) $\delta$ 7.48-7.38 (m, 2H), 7.40-7.31 (m, 1H), 7.29-7.20 (m, 2H), $6.88(\mathrm{~d}$, $J=4.0 \mathrm{~Hz}, 2 \mathrm{H}), 6.69(\mathrm{~d}, J=2.6 \mathrm{~Hz}, 1 \mathrm{H}), 6.30(\mathrm{t}, J=2.1 \mathrm{~Hz}, 2 \mathrm{H}), 3.92(\mathrm{dd}, J=15.8,12.8 \mathrm{~Hz}, 1 \mathrm{H})$, $3.63(\mathrm{dd}, J=12.9,10.6 \mathrm{~Hz}, 1 \mathrm{H}), 2.35$ (s, 6H), 2.29 (s, 3H), 2.06 (s, 6H).

${ }^{13} \mathbf{C}$ NMR(101 MHz, CDCl $) \delta 141.48(\mathrm{~d}, J=9.9 \mathrm{~Hz}), 140.70(\mathrm{~d}, J=2.7 \mathrm{~Hz}), 136.51(\mathrm{~d}, J=3.5$ Hz), 134.09 (d, $J=80.2 \mathrm{~Hz}), 131.28(\mathrm{~d}, J=11.1 \mathrm{~Hz}), 130.80(\mathrm{~d}, J=9.7 \mathrm{~Hz}), 130.56(\mathrm{~d}, J=7.3$ $\mathrm{Hz}), 130.41(\mathrm{~d}, J=3.0 \mathrm{~Hz}), 129.28(\mathrm{~d}, J=79.7 \mathrm{~Hz}), 128.65(\mathrm{~d}, J=5.4 \mathrm{~Hz}), 128.08(\mathrm{~d}, J=4.1 \mathrm{~Hz})$, 127.69 (d, $J=12.3 \mathrm{~Hz}), 45.53$ (d, $J=46.9 \mathrm{~Hz}), 24.35$ (d, $J=4.9 \mathrm{~Hz}), 20.93,20.80$ (d, $J=1.5 \mathrm{~Hz})$.

${ }^{31} \mathbf{P}$ NMR (162 MHz, $\left.\mathbf{C D C l}_{3}\right) \delta 43.24$.

HRMS (ESI) $\mathbf{m} / \mathbf{z}$ [M+H] $]^{+}$: calcd. 379.1644, found. 379.1644 .

IR (film): $v_{\max }\left(\mathrm{cm}^{-1}\right)$ 3019, 2966, 2918, 1604, 1437, 1404, 1100, 1028, 849, 735, 697, 653, 594.

Optical rotation: $[\alpha]_{\mathrm{D}}^{25}=-160.12\left(c=1.000, \mathrm{CHCl}_{3}, 82 \%\right.$ ee $)$.

HPLC: DAICEL CHIRALPAK ID, hexane $/ i-\mathrm{PrOH}=9 / 1$, flow rate: $1.0 \mathrm{~mL} / \mathrm{min}, \lambda=254 \mathrm{~nm}$, $\mathrm{t}_{\mathrm{R}}($ major $)=9.0 \mathrm{~min}, \mathrm{t}_{\mathrm{R}}($ minor $)=10.2 \mathrm{~min}, 82 \%$ ee
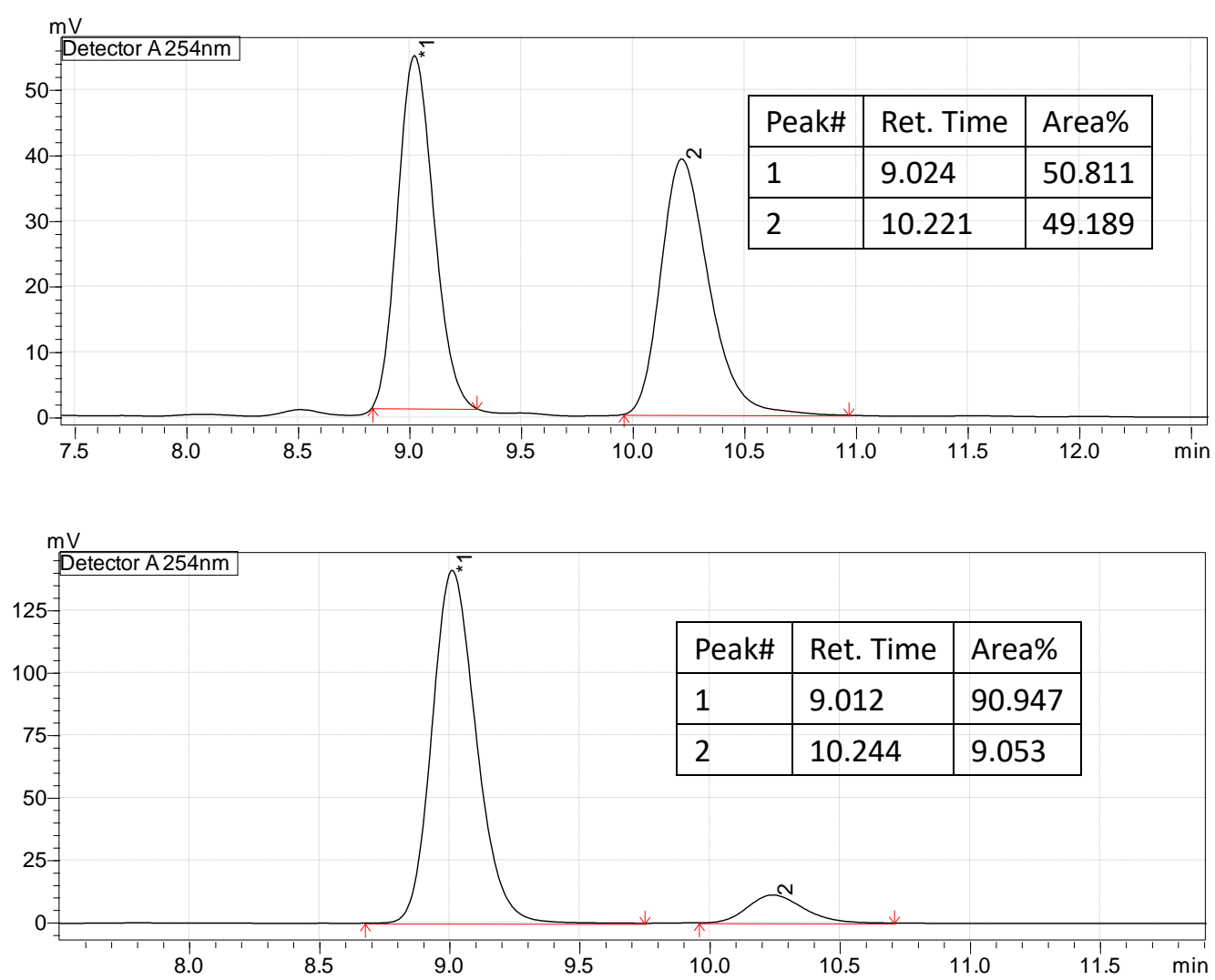


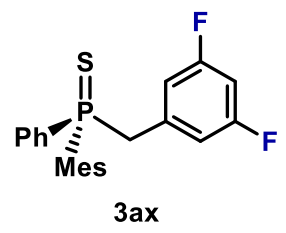

3ax: Procedure A, $73.3 \mathrm{mg}$, 95\% yield, brown liquid.

${ }^{1}$ H NMR (400 MHz, CDCl $)_{3} \delta$ 7.51-7.34 (m, 3H), 7.32-7.23 (m, 2H), $6.89(\mathrm{~d}, J=4.1 \mathrm{~Hz}, 2 \mathrm{H})$, 6.56-6.45 (m, 1H), 6.38-6.27 (m, 2H), $3.98(\mathrm{dd}, J=16.2,12.9 \mathrm{~Hz}, 1 \mathrm{H}), 3.65(\mathrm{dd}, J=12.9,10.8$ $\mathrm{Hz}, 1 \mathrm{H}), 2.34$ (s, 6H), 2.29 (s, 3H).

${ }^{13} \mathbf{C}$ NMR(101 MHz, $\left.\mathbf{C D C l}_{3}\right) \delta 161.74(\mathrm{ddd}, J=247.3,12.9,3.8 \mathrm{~Hz}), 141.39(\mathrm{~d}, J=10.3 \mathrm{~Hz})$, $141.10(\mathrm{~d}, J=3.0 \mathrm{~Hz}), 135.32-134.78(\mathrm{~m}), 133.34(\mathrm{~d}, J=80.3 \mathrm{~Hz}), 131.39(\mathrm{~d}, J=11.3 \mathrm{~Hz})$, $131.00(\mathrm{~d}, J=3.0 \mathrm{~Hz}), 130.45(\mathrm{~d}, J=9.9 \mathrm{~Hz}), 128.65(\mathrm{~d}, J=82.3 \mathrm{~Hz}), 128.14(\mathrm{~d}, J=12.5 \mathrm{~Hz})$, $113.68-113.19$ (m), 102.02 (td, $J=25.2,3.8 \mathrm{~Hz}), 45.37$ (dt, $J=47.6,2.3 \mathrm{~Hz}), 24.22$ (d, $J=5.0$ $\mathrm{Hz}), 20.81(\mathrm{~d}, J=1.8 \mathrm{~Hz})$.

${ }^{31}$ P NMR (162 MHz, CDCl 3 ) $\delta 42.41(\mathrm{~d}, J=3.3 \mathrm{~Hz})$.

${ }^{19}$ F NMR (376 MHz, $\left.\mathbf{C D C l}_{3}\right) \delta-111.29(\mathrm{td}, J=8.5,2.9 \mathrm{~Hz})$.

HRMS (ESI) $\mathbf{m} / \mathbf{z}[\mathbf{M}+\mathbf{H}]^{+}$: calcd. 387.1142 , found. 387.1142 .

IR (film): $v_{\max }\left(\mathrm{cm}^{-1}\right)$ 3054, 2956, 2952, 1620, 1597, 1455, 1318, 1117, 986, 856, 750, 693, 595.

Optical rotation: $[\alpha]_{\mathrm{D}}{ }^{25}=-140.24\left(c=1.114, \mathrm{CHCl}_{3}, 82 \%\right.$ ee $)$.

HPLC: DAICEL CHIRALPAK OD-H, hexane $/ i-\mathrm{PrOH}=49 / 1$, flow rate: $1.0 \mathrm{~mL} / \mathrm{min}, \lambda=254 \mathrm{~nm}$, $\mathrm{t}_{\mathrm{R}}($ major $)=9.3 \mathrm{~min}, \mathrm{t}_{\mathrm{R}}($ minor $)=16.1 \mathrm{~min}, 82 \%$ ee .
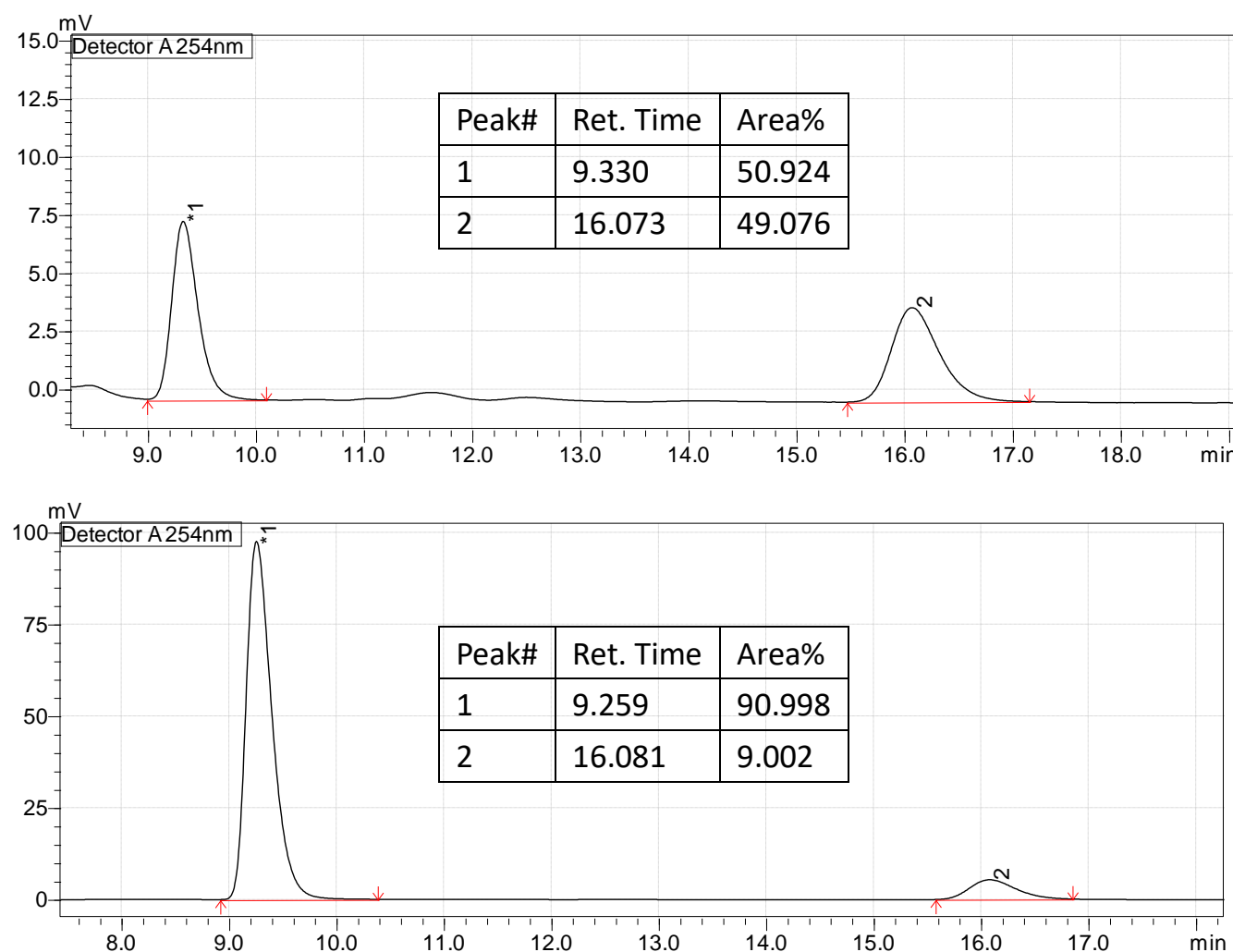


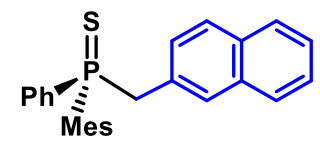

3ay

3ay: Procedure A, $78.4 \mathrm{mg}, 98 \%$ yield, white solid.

${ }^{1}$ H NMR (400 MHz, CDCl $)$ ) 7.70-7.63 (m, 1H), 7.53-7.48 (m, 1H), 7.47-7.28 (m, 6H), 7.19$7.10(\mathrm{~m}, 3 \mathrm{H}), 6.93-6.86(\mathrm{~m}, 3 \mathrm{H}), 4.18(\mathrm{dd}, J=15.9,12.9 \mathrm{~Hz}, 1 \mathrm{H}), 3.85(\mathrm{dd}, J=12.9,10.6 \mathrm{~Hz}$, $1 \mathrm{H}), 2.37$ (s, 6H), 2.29 (s, 3H).

${ }^{13} \mathbf{C}$ NMR(101 MHz, $\left.\mathbf{C D C l}_{3}\right) \delta 141.61(\mathrm{~d}, J=10.1 \mathrm{~Hz}), 140.94(\mathrm{~d}, J=2.9 \mathrm{~Hz}), 133.88(\mathrm{~d}, J=$ $80.1 \mathrm{~Hz}), 132.74(\mathrm{~d}, J=3.6 \mathrm{~Hz}), 132.07(\mathrm{~d}, J=3.0 \mathrm{~Hz}), 131.45(\mathrm{~d}, J=11.1 \mathrm{~Hz}), 130.81(\mathrm{~d}, J=$ $9.9 \mathrm{~Hz}), 130.70(\mathrm{~d}, J=3.0 \mathrm{~Hz}), 129.88(\mathrm{~d}, J=7.2 \mathrm{~Hz}), 129.30(\mathrm{~d}, J=80.0 \mathrm{~Hz}), 128.81(\mathrm{~d}, J=4.2$ $\mathrm{Hz}), 128.69(\mathrm{~d}, J=7.9 \mathrm{~Hz}), 128.00(\mathrm{~d}, J=12.4 \mathrm{~Hz}), 127.69(\mathrm{~d}, J=1.6 \mathrm{~Hz}), 127.41(\mathrm{~d}, J=1.9 \mathrm{~Hz})$, $126.62(\mathrm{~d}, J=2.7 \mathrm{~Hz}), 125.62(\mathrm{~d}, J=1.5 \mathrm{~Hz}), 125.56(\mathrm{~d}, J=2.0 \mathrm{~Hz}), 45.87(\mathrm{~d}, J=46.9 \mathrm{~Hz})$, $24.45(\mathrm{~d}, J=4.7 \mathrm{~Hz}), 20.93(\mathrm{~d}, J=1.5 \mathrm{~Hz})$.

${ }^{31}$ P NMR (162 MHz, CDCl $) \delta 43.27$.

HRMS (ESI) $\mathbf{m} / \mathbf{z}[\mathbf{M}+\mathbf{H}]^{+}$: calcd. 401.1487, found. 401.1488 .

IR (film): $v_{\max }\left(\mathrm{cm}^{-1}\right)$ 3053, 2967, 2921, 1602, 1507, 1454, 1437, 1404, 1265, 1100, 856, 817, 735, 692, 647, 591.

Optical rotation: $[\alpha]_{\mathrm{D}}^{25}=-198.34\left(c=1.000, \mathrm{CHCl}_{3}\right.$, ee $\left.=83 \%\right)$.

HPLC: DAICEL CHIRALPAK OD-H, hexane $/ i-\mathrm{PrOH}=19 / 1$, flow rate: $1.0 \mathrm{~mL} / \mathrm{min}, \lambda=254 \mathrm{~nm}$, $\mathrm{t}_{\mathrm{R}}($ major $)=12.4 \mathrm{~min}, \mathrm{t}_{\mathrm{R}}($ minor $)=17.4 \mathrm{~min}, \mathrm{ee}=83 \%$.
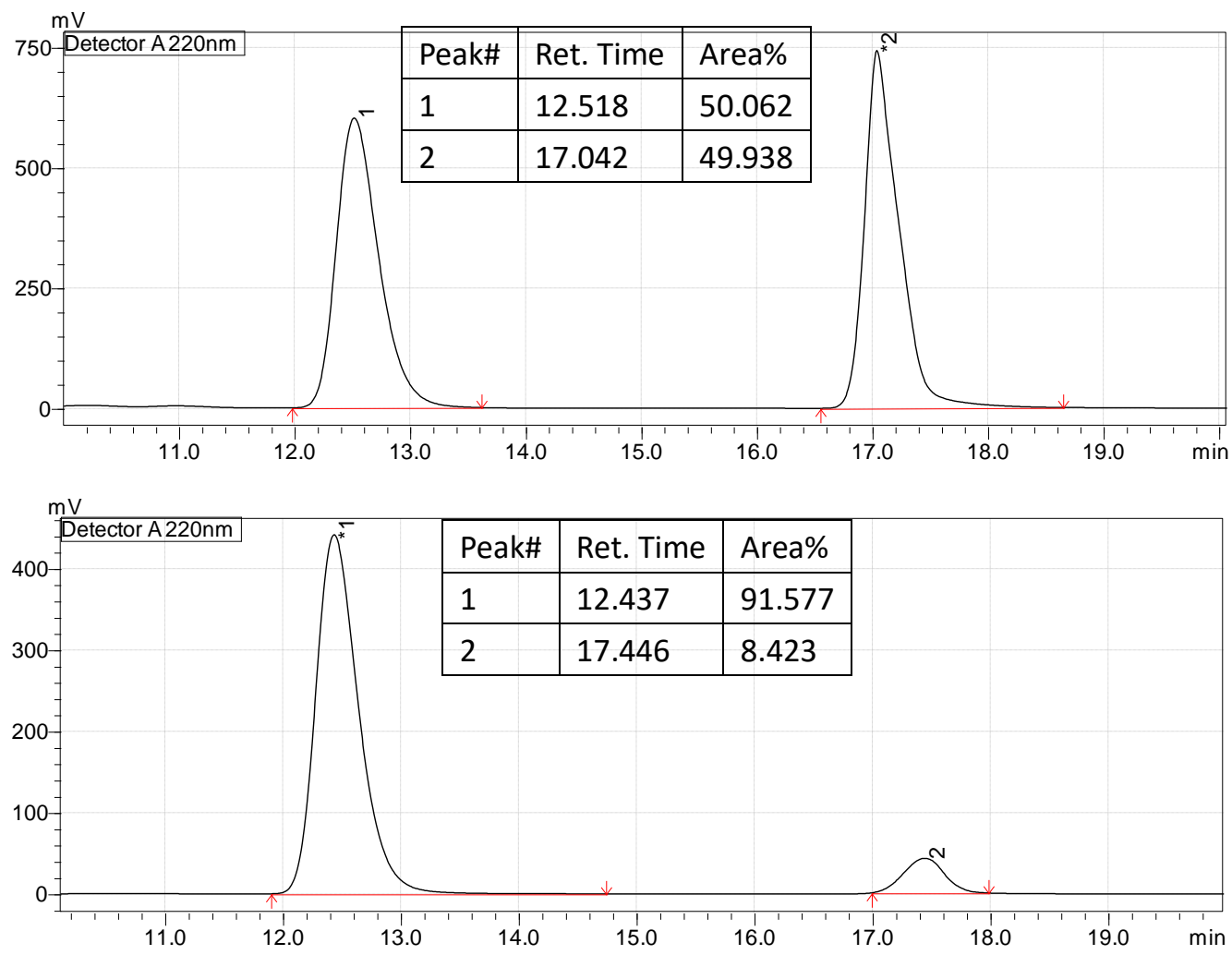


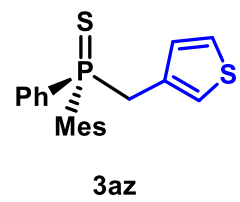

3az: Procedure A, $60.5 \mathrm{mg}, 85 \%$ yield, brown solid.

${ }^{1}$ H NMR (400 MHz, $\left.\mathbf{C D C l}_{3}\right) \delta$ 7.51-7.41 (m, 2H), 7.41-7.32 (m, 1H), 7.30-7.21 (m, 3H), 6.93 $(\mathrm{dd}, J=5.0,3.0 \mathrm{~Hz}, 1 \mathrm{H}), 6.87(\mathrm{~d}, J=4.0 \mathrm{~Hz}, 2 \mathrm{H}), 6.63(\mathrm{~d}, J=3.7 \mathrm{~Hz}, 1 \mathrm{H}), 6.52(\mathrm{dt}, J=5.1,1.4$ $\mathrm{Hz}, 1 \mathrm{H}), 4.01$ (dd, $J=15.3,13.5 \mathrm{~Hz}, 1 \mathrm{H}), 3.84(\mathrm{dd}, J=13.5,10.4 \mathrm{~Hz}, 1 \mathrm{H}), 2.33(\mathrm{~s}, 6 \mathrm{H}), 2.29(\mathrm{~s}$, $3 \mathrm{H})$.

${ }^{13} \mathbf{C}$ NMR(101 MHz, CDCl $) \delta 141.44(\mathrm{~d}, J=10.2 \mathrm{~Hz}), 140.84(\mathrm{~d}, J=2.8 \mathrm{~Hz}), 134.11(\mathrm{~d}, J=$ $80.7 \mathrm{~Hz}), 131.26(\mathrm{~d}, J=11.1 \mathrm{~Hz}), 130.60(\mathrm{~d}, J=6.5 \mathrm{~Hz}), 130.59(\mathrm{~d}, J=3.1 \mathrm{~Hz}), 130.40(\mathrm{~d}, J=$ $9.8 \mathrm{~Hz}), 129.34(\mathrm{~d}, J=3.4 \mathrm{~Hz}), 128.98(\mathrm{~d}, J=79.8 \mathrm{~Hz}), 127.92(\mathrm{~d}, J=12.2 \mathrm{~Hz}), 123.91(\mathrm{~d}, J=$ $8.1 \mathrm{~Hz}), 123.78(\mathrm{~d}, J=2.0 \mathrm{~Hz}), 40.94(\mathrm{~d}, J=48.9 \mathrm{~Hz}), 24.22(\mathrm{~d}, J=4.9 \mathrm{~Hz}), 20.81(\mathrm{~d}, J=1.5$ $\mathrm{Hz})$.

${ }^{31}$ P NMR (162 MHz, CDCl 3 ) $\delta 42,28$.

HRMS (ESI) $\mathbf{m} / \mathbf{z}$ [M+H] ${ }^{+}$: calcd. 357.0895 , found. 357.0895 .

IR (film): $v_{\max }\left(\mathrm{cm}^{-1}\right)$ 3050, 2965, 2921, 2862, 1603, 1454, 1436, 1101, 939, 873, 787, 694, 644.

Optical rotation: $[\alpha]_{\mathrm{D}}{ }^{25}=-169.25\left(c=1.120, \mathrm{CHCl}_{3}, 90 \%\right.$ ee $)$.

HPLC: DAICEL CHIRALPAK OD-H, hexane $/ i-\mathrm{PrOH}=97 / 3$, flow rate: $1.0 \mathrm{~mL} / \mathrm{min}, \lambda=254 \mathrm{~nm}$, $\mathrm{t}_{\mathrm{R}}($ major $)=10.8 \mathrm{~min}, \mathrm{t}_{\mathrm{R}}($ minor $)=13.5 \mathrm{~min}, 90 \%$ ee .
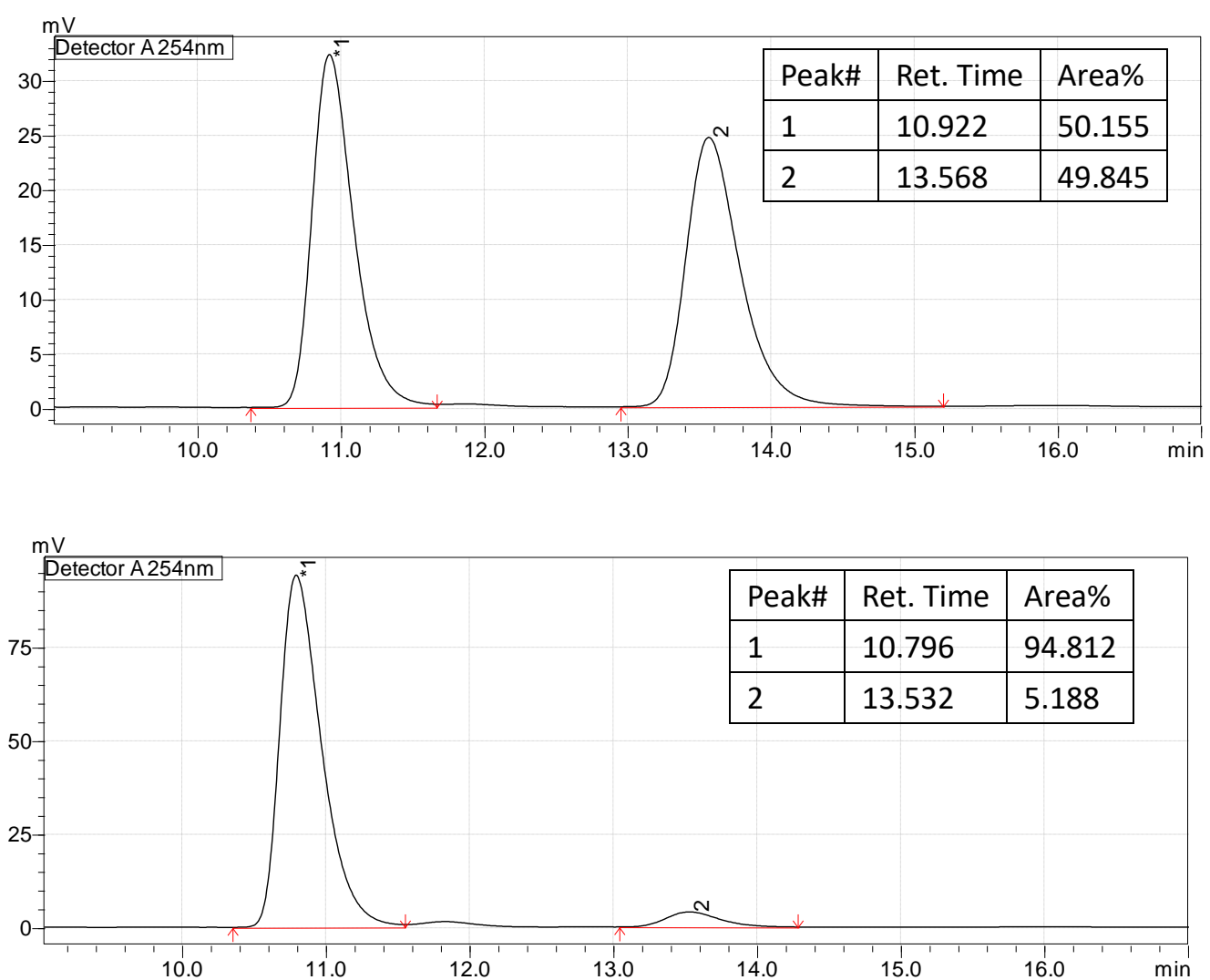


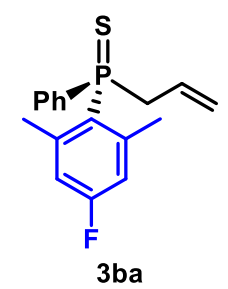

3ba: Procedure A, $56.0 \mathrm{mg}, 92 \%$ yield, light yellow solid.

${ }^{1} \mathbf{H}$ NMR (500 MHz, $\left.\mathbf{C D C l}_{3}\right) \delta$ 7.76-7.66 (m, 2H), 7.52-7.46 (m, 1H), 7.46-7.40 (m, 2H), 6.76 (dd, $J=9.3,3.1 \mathrm{~Hz}, 2 \mathrm{H}), 5.46-5.32(\mathrm{~m}, 1 \mathrm{H}), 4.99(\mathrm{dd}, J=10.2,4.3 \mathrm{~Hz}, 1 \mathrm{H}), 4.91-4.78(\mathrm{~m}, 1 \mathrm{H})$, $3.47-3.24(\mathrm{~m}, 2 \mathrm{H}), 2.34(\mathrm{~s}, 6 \mathrm{H})$.

${ }^{13} \mathbf{C}$ NMR(101 MHz, $\left.\mathbf{C D C l}_{3}\right) \delta 163.42(\mathrm{dd}, J=252.1,3.4 \mathrm{~Hz}), 144.85(\mathrm{dd}, J=11.4,8.5 \mathrm{~Hz})$, $133.81(\mathrm{~d}, J=80.1 \mathrm{~Hz}), 131.07(\mathrm{~d}, J=3.0 \mathrm{~Hz}), 130.80(\mathrm{~d}, J=9.9 \mathrm{~Hz}), 128.34(\mathrm{~d}, J=12.3 \mathrm{~Hz})$, $128.20(\mathrm{dd}, J=82.1,3.4 \mathrm{~Hz}) 127.45(\mathrm{~d}, J=8.4 \mathrm{~Hz}), 121.32(\mathrm{~d}, J=13.7 \mathrm{~Hz}), 117.16(\mathrm{dd}, J=20.5$, $11.8 \mathrm{~Hz}), 44.75(\mathrm{~d}, J=51.4 \mathrm{~Hz}), 24.32(\mathrm{dd}, J=4.9,1.6 \mathrm{~Hz})$.

${ }^{31}$ P NMR (162 MHz, $\left.\mathbf{C D C l}_{3}\right) \delta 40.80$.

${ }^{19}$ F NMR (376 MHz, $\left.\mathbf{C D C l}_{3}\right) \delta-110.91$.

HRMS (ESI) $\mathbf{m} / \mathbf{z}$ [M+H] $]^{+}$: calcd. 305.0924, found. 305.0924.

IR (film): $v_{\max }\left(\mathrm{cm}^{-1}\right)$ 3053, 3014, 2977, 2931, 1599, 1455, 1437, 1418, 1292, 1145, 1100, 843, 670, 575, 496.

Optical rotation: $[\alpha]_{\mathrm{D}}{ }^{25}=-93.06\left(c=1.066, \mathrm{CHCl}_{3}, 93 \%\right.$ ee $)$.

HPLC: DAICEL CHIRALPAK ID, hexane $/ i-\mathrm{PrOH}=19 / 1$, flow rate: $1.0 \mathrm{~mL} / \mathrm{min}, \lambda=254 \mathrm{~nm}$, $\mathrm{t}_{\mathrm{R}}($ major $)=8.5 \mathrm{~min}, \mathrm{t}_{\mathrm{R}}($ minor $)=9.3 \mathrm{~min}, 93 \%$ ee.

\begin{tabular}{|l|l|l|}
\hline Peak\# & Ret. Time & Area\% \\
\hline 1 & 8.354 & 50.338 \\
\hline 2 & 9.054 & 49.662 \\
\hline
\end{tabular}

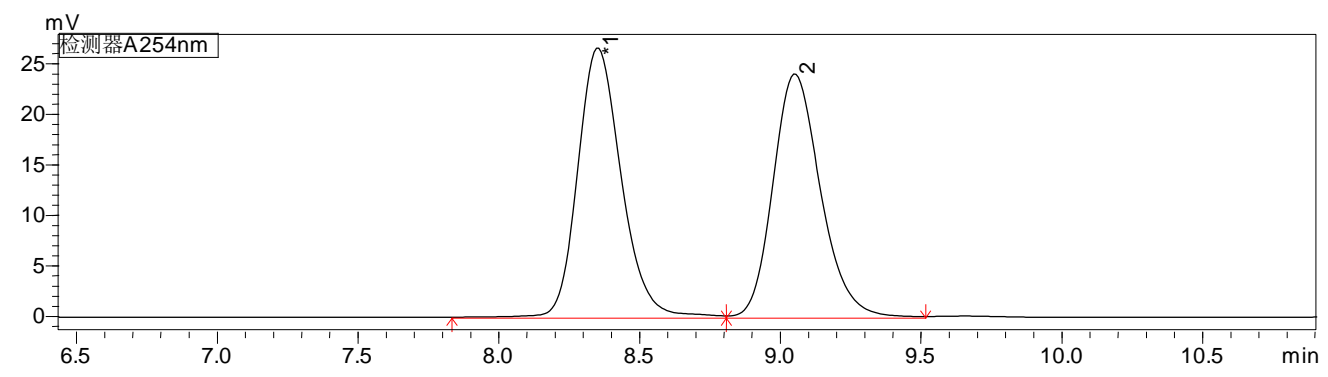

\begin{tabular}{|l|l|l|}
\hline Peak\# & Ret. Time & Area\% \\
\hline 1 & 8.499 & 96.288 \\
\hline 2 & 9.296 & 3.712 \\
\hline
\end{tabular}

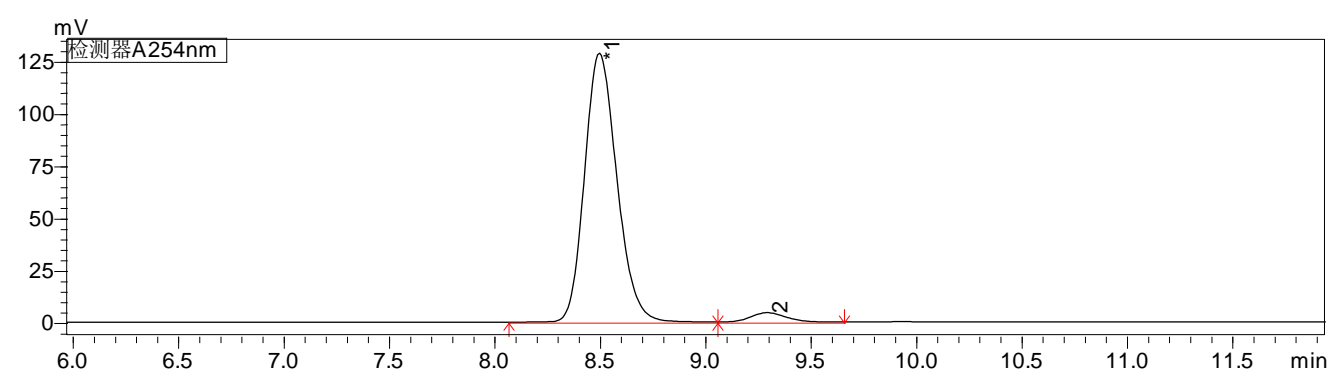




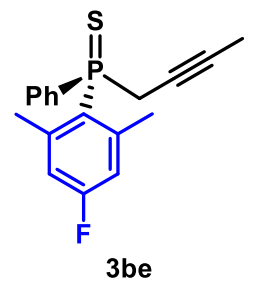

3be: Procedure E, $53.1 \mathrm{mg}$, 91\% yield, light yellow solid.

${ }^{1} \mathbf{H}$ NMR (500 MHz, $\left.\mathbf{C D C l}_{\mathbf{3}}\right) \delta$ 7.99-7.86 (m, 2H), 7.59-7.51 (m, 1H), 7.51-7.42 (m, 2H), 6.77 (dd, $J=9.3,3.2 \mathrm{~Hz}, 2 \mathrm{H}), 3.62-3.50(\mathrm{~m}, 1 \mathrm{H}), 3.08-2.94(\mathrm{~m}, 1 \mathrm{H}), 2.38(\mathrm{~s}, 6 \mathrm{H}), 1.71-1.63(\mathrm{~m}, 3 \mathrm{H})$.

${ }^{13} \mathbf{C}$ NMR(101 MHz, $\left.\mathbf{C D C l}_{3}\right) \delta 163.51(\mathrm{dd}, J=252.2,3.5 \mathrm{~Hz}), 145.24(\mathrm{dd}, J=11.5,8.7 \mathrm{~Hz})$, $134.24(\mathrm{~d}, J=80.9 \mathrm{~Hz}), 131.43(\mathrm{~d}, J=3.0 \mathrm{~Hz}), 130.51(\mathrm{~d}, J=9.9 \mathrm{~Hz}), 128.16(\mathrm{~d}, J=12.4 \mathrm{~Hz})$, $126.10(\mathrm{dd}, J=85.7,3.4 \mathrm{~Hz}), 116.93(\mathrm{dd}, J=20.4,12.0 \mathrm{~Hz}), 81.00(\mathrm{~d}, J=8.7 \mathrm{~Hz}), 70.80(\mathrm{~d}, J=$ $13.3 \mathrm{~Hz}), 34.56(\mathrm{~d}, J=54.2 \mathrm{~Hz}), 23.42(\mathrm{dd}, J=5.3,1.6 \mathrm{~Hz}), 3.45(\mathrm{~d}, J=3.1 \mathrm{~Hz})$.

${ }^{31} \mathbf{P}$ NMR (162 MHz, CDCl $) \delta 38.91$.

${ }^{19}$ F NMR (376 MHz, $\left.\mathbf{C D C l}_{3}\right) \delta-110.69$.

HRMS (ESI) m/z [M+H] ${ }^{+}$: calcd. 317.0924, found. 317.0924.

IR (film): $v_{\max }\left(\mathrm{cm}^{-1}\right)$ 3054, 2974, 1588, 1455, 1293, 1146, 1099, 860, 694, 599.

Optical rotation: $[\alpha]_{\mathrm{D}}{ }^{25}=20.80\left(c=1.062, \mathrm{CHCl}_{3}, 93 \%\right.$ ee $)$.

HPLC: DAICEL CHIRALPAK IA-3, hexane $/ i-\mathrm{PrOH}=139 / 1$, flow rate: $0.7 \mathrm{~mL} / \mathrm{min}, \lambda=254 \mathrm{~nm}$, $\mathrm{t}_{\mathrm{R}}($ major $)=19.4 \mathrm{~min}, \mathrm{t}_{\mathrm{R}}($ minor $)=17.0 \mathrm{~min}, 93 \%$ ee .

\begin{tabular}{|l|l|l|}
\hline Peak\# & Ret. Time & Area\% \\
\hline 1 & 16.766 & 50.135 \\
\hline 2 & 19.036 & 49.865 \\
\hline
\end{tabular}

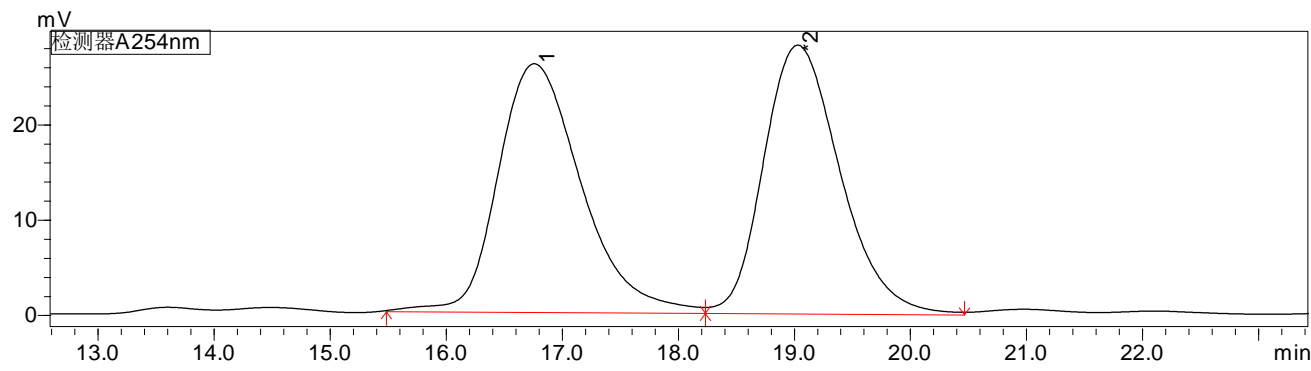

\begin{tabular}{|l|l|l|}
\hline Peak\# & Ret. Time & Area\% \\
\hline 1 & 16.995 & 3.359 \\
\hline 2 & 19.353 & 96.641 \\
\hline
\end{tabular}

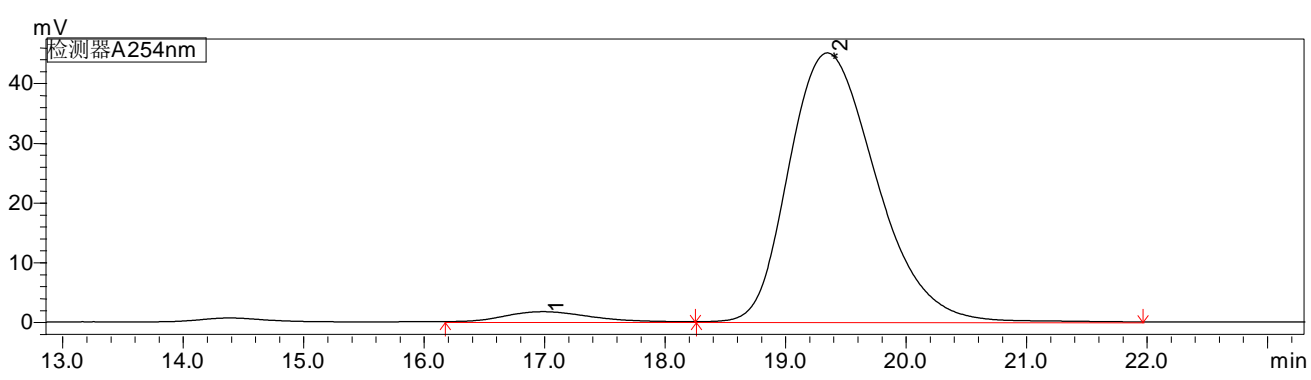




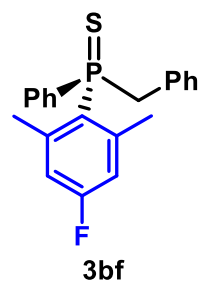

3bf: Procedure A, $67.8 \mathrm{mg}, 92 \%$ yield, light yellow solid.

${ }^{1} \mathbf{H}$ NMR (500 MHz, $\mathbf{C D C l}_{3}$ ) $\delta$ 7.46-7.33 (m, 3H), 7.28-7.21 (m, 2H), 7.11-7.04 (m, 1H), 7.03$6.94(\mathrm{~m}, 2 \mathrm{H}), 6.83-6.71(\mathrm{~m}, 4 \mathrm{H}), 4.03(\mathrm{dd}, J=15.9,12.9 \mathrm{~Hz}, 1 \mathrm{H}), 3.71(\mathrm{dd}, J=13.0,10.7 \mathrm{~Hz}$, $1 \mathrm{H}), 2.38(\mathrm{~s}, 6 \mathrm{H})$.

${ }^{13} \mathbf{C}$ NMR(101 MHz, $\left.\mathbf{C D C l}_{3}\right) \delta 163.32(\mathrm{dd}, J=251.9,3.4 \mathrm{~Hz}), 144.82(\mathrm{dd}, J=11.4,8.4 \mathrm{~Hz})$, $133.37(\mathrm{~d}, J=80.6 \mathrm{~Hz}), 130.78(\mathrm{~d}, J=3.0 \mathrm{~Hz}), 130.72-130.46(\mathrm{~m}), 128.59$ (dd, $J=81.4,3.3 \mathrm{~Hz})$, $128.01(\mathrm{~d}, J=12.3 \mathrm{~Hz}), 127.33(\mathrm{~d}, J=3.5 \mathrm{~Hz}), 126.66(\mathrm{~d}, J=4.1 \mathrm{~Hz}), 117.15(\mathrm{dd}, J=20.3,11.8$ $\mathrm{Hz}), 45.47(\mathrm{~d}, J=47.5 \mathrm{~Hz}), 24.58(\mathrm{dd}, J=4.6,1.6 \mathrm{~Hz})$.

${ }^{31}$ P NMR (162 MHz, $\left.\mathbf{C D C l}_{3}\right) \delta 42.96$.

${ }^{19}$ F NMR (376 MHz, $\left.\mathrm{CDCl}_{3}\right) \delta-111.01$.

HRMS (ESI) $\mathbf{m} / \mathbf{z}[\mathbf{M}+\mathbf{H}]^{+}$: calcd. 355.1080, found. 355.1080.

IR (film): $v_{\max }\left(\mathrm{cm}^{-1}\right) 3058,2975,1599,1452,1293,1102,848,773,696,599$.

Optical rotation: $[\alpha]_{\mathrm{D}}{ }^{25}=-142.93\left(c=1.356, \mathrm{CHCl}_{3}, 84 \%\right.$ ee $)$.

HPLC: DAICEL CHIRALPAK AD-H, hexane $/ i-\mathrm{PrOH}=49 / 1$, flow rate: $1.0 \mathrm{~mL} / \mathrm{min}, \lambda=254 \mathrm{~nm}$, $\mathrm{t}_{\mathrm{R}}($ major $)=16.2 \mathrm{~min}, \mathrm{t}_{\mathrm{R}}($ minor $)=13.3 \mathrm{~min}, 84 \%$ ee .

\begin{tabular}{|l|l|l|}
\hline Peak\# & Ret. Time & Area\% \\
\hline 1 & 13.337 & 50.024 \\
\hline 2 & 16.227 & 49.976 \\
\hline
\end{tabular}
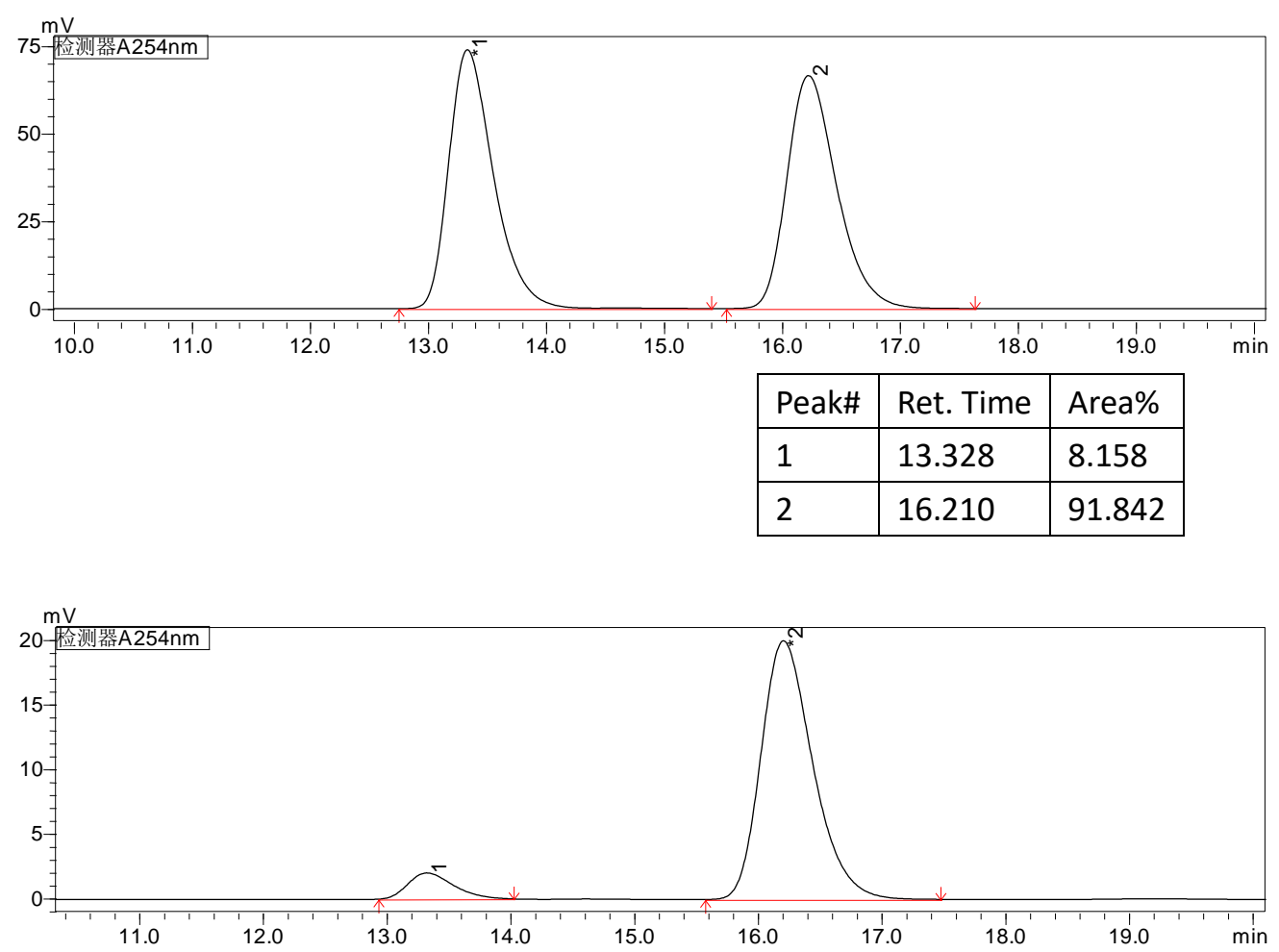


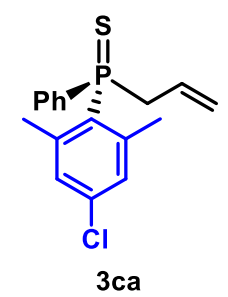

3ca: Procedure A, $62.9 \mathrm{mg}$, 94\% yield, light yellow solid.

${ }^{1}$ H NMR (500 MHz, CDCl $)$ ) 7.75-7.65 (m, 2H), 7.52-7.46 (m, 1H), 7.46-7.39 (m, 2H), 7.04 (d, $J=3.3 \mathrm{~Hz}, 2 \mathrm{H}), 5.47-5.31(\mathrm{~m}, 1 \mathrm{H}), 5.05-4.92(\mathrm{~m}, 1 \mathrm{H}), 4.90-4.80(\mathrm{~m}, 1 \mathrm{H}), 3.47-3.22(\mathrm{~m}, 2 \mathrm{H})$, $2.32(\mathrm{~s}, 6 \mathrm{H})$.

${ }^{13} \mathbf{C ~ N M R}\left(101 \mathrm{MHz}, \mathbf{C D C l}_{3}\right) \delta 143.46(\mathrm{~d}, J=11.0 \mathrm{~Hz}), 136.63(\mathrm{~d}, J=3.5 \mathrm{~Hz}), 133.57(\mathrm{~d}, J=$ $79.9 \mathrm{~Hz}), 131.15(\mathrm{~d}, J=2.9 \mathrm{~Hz}), 130.88(\mathrm{~d}, J=80.0 \mathrm{~Hz}), 130.79(\mathrm{~d}, J=9.9 \mathrm{~Hz}), 130.14$ (d, $J=$ $11.2 \mathrm{~Hz}), 128.38(\mathrm{~d}, J=12.4 \mathrm{~Hz}), 127.33(\mathrm{~d}, J=8.5 \mathrm{~Hz}), 121.42(\mathrm{~d}, J=13.8 \mathrm{~Hz}), 44.69$ (d, $J=$ $51.3 \mathrm{~Hz}), 24.02(\mathrm{~d}, J=4.9 \mathrm{~Hz})$.

${ }^{31}$ P NMR (162 MHz, $\left.\mathbf{C D C l}_{\mathbf{3}}\right) \delta 41.01$.

HRMS (ESI) $\mathbf{m} / \mathbf{z}$ [M+H] $]^{+}$: calcd. 321.0628, found. 321.0628 .

IR (film): $v_{\max }\left(\mathrm{cm}^{-1}\right)$ 3014, 2977, 1573, 1437, 1248, 1100, 874, 667, 615.

Optical rotation: $[\alpha]_{\mathrm{D}}{ }^{25}=-91.21\left(c=1.258, \mathrm{CHCl}_{3}, 87 \%\right.$ ee $)$.

HPLC: DAICEL CHIRALPAK ID, hexane $/ i-\mathrm{PrOH}=19 / 1$, flow rate: $1.0 \mathrm{~mL} / \mathrm{min}, \lambda=254 \mathrm{~nm}$, $\mathrm{t}_{\mathrm{R}}($ major $)=8.7 \mathrm{~min}, \mathrm{t}_{\mathrm{R}}($ minor $)=9.6 \mathrm{~min}, 87 \%$ ee.

\begin{tabular}{|l|l|l|}
\hline Peak\# & Ret. Time & Area\% \\
\hline 1 & 8.644 & 49.746 \\
\hline 2 & 9.554 & 50.254 \\
\hline
\end{tabular}

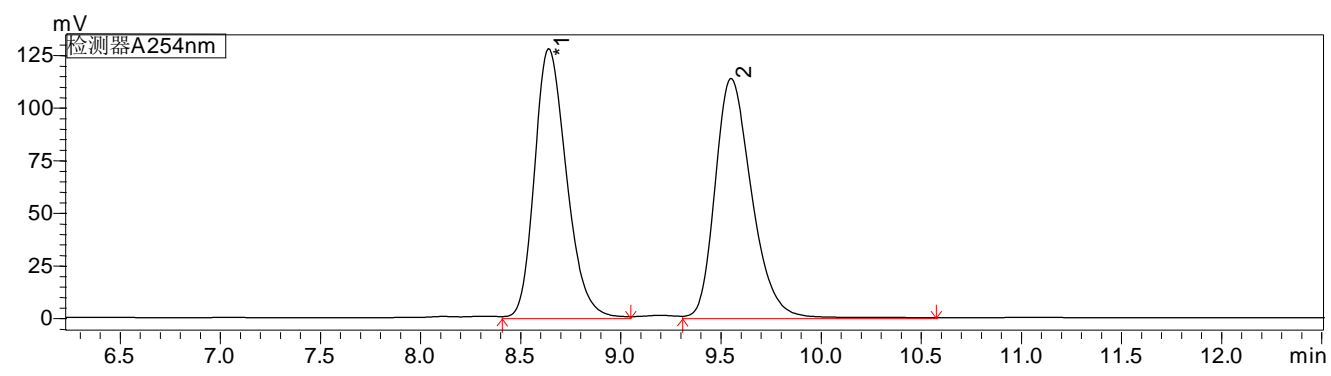

\begin{tabular}{|l|l|l|}
\hline Peak\# & Ret. Time & Area\% \\
\hline 1 & 8.662 & 93.297 \\
\hline 2 & 9.610 & 6.703 \\
\hline
\end{tabular}

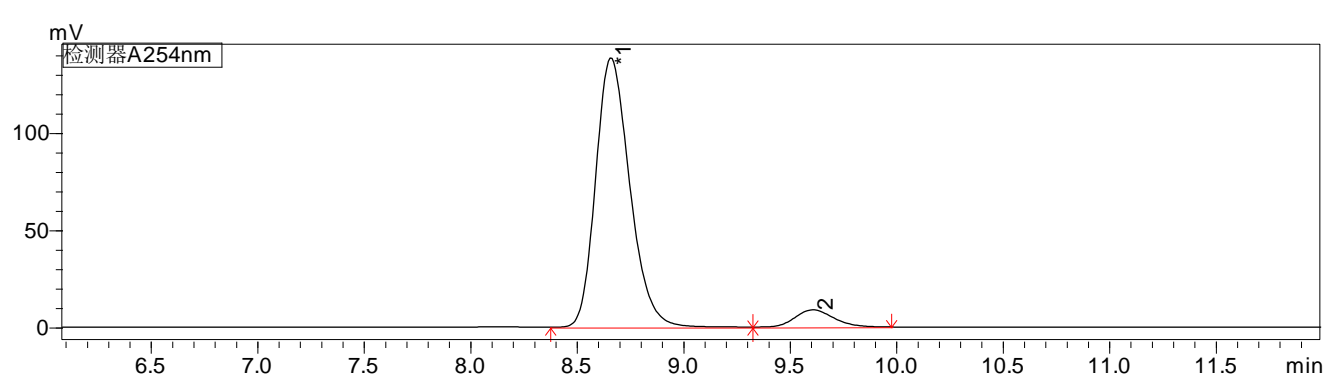




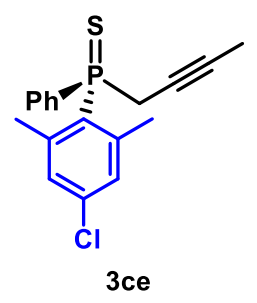

3ce: Procedure E, $61.0 \mathrm{mg}$, 92\% yield, light yellow solid.

${ }^{1} \mathbf{H}$ NMR (500 MHz, $\left.\mathbf{C D C l}_{3}\right) \delta$ 7.98-7.87 (m, 2H), 7.58-7.50 (m, 1H), 7.50-7.42 (m, 2H), $7.06(\mathrm{~d}$, $J=3.5 \mathrm{~Hz}, 2 \mathrm{H}), 3.61-3.50(\mathrm{~m}, 1 \mathrm{H}), 3.09-2.93(\mathrm{~m}, 1 \mathrm{H}), 2.36(\mathrm{~s}, 6 \mathrm{H}), 1.70-1.63(\mathrm{~m}, 3 \mathrm{H})$.

${ }^{13} \mathbf{C}$ NMR(101 MHz, $\left.\mathbf{C D C l}_{3}\right) \delta 143.82(\mathrm{~d}, J=10.7 \mathrm{~Hz}), 136.70(\mathrm{~d}, J=3.8 \mathrm{~Hz}), 134.01(\mathrm{~d}, J=$ $80.8 \mathrm{~Hz}), 131.49$ (d, $J=3.0 \mathrm{~Hz}), 130.51(\mathrm{~d}, J=9.7 \mathrm{~Hz}), 129.90(\mathrm{~d}, J=11.5 \mathrm{~Hz}), 128.84(\mathrm{~d}, J=$ $83.9 \mathrm{~Hz}), 128.18(\mathrm{~d}, J=12.5 \mathrm{~Hz}), 81.06(\mathrm{~d}, J=8.8 \mathrm{~Hz}), 70.69(\mathrm{~d}, J=13.4 \mathrm{~Hz}), 34.52(\mathrm{~d}, J=54.1$ $\mathrm{Hz}), 23.11(\mathrm{~d}, J=5.3 \mathrm{~Hz}), 3.48(\mathrm{~d}, J=3.2 \mathrm{~Hz})$.

${ }^{31}$ P NMR (162 MHz, $\left.\mathbf{C D C l}_{3}\right) \delta 39.09$.

HRMS (ESI) $\mathbf{m} / \mathbf{z}$ [M+H] $]^{+}$: calcd. 333.0628, found. 333.0629.

IR (film): $v_{\max }\left(\mathrm{cm}^{-1}\right)$ 3054, 2973, 1573, 1454, 1396, 1248, 1098, 874, 693, 664, 615.

Optical rotation: $[\alpha]_{\mathrm{D}}{ }^{25}=-1.65\left(c=1.220, \mathrm{CHCl}_{3}, 88 \%\right.$ ee $)$.

HPLC: DAICEL CHIRALPAK OD-H, hexane $/ i-\mathrm{PrOH}=199 / 1$, flow rate: $1.0 \mathrm{~mL} / \mathrm{min}, \lambda=254$ $\mathrm{nm}, \mathrm{t}_{\mathrm{R}}($ major $)=15.8 \mathrm{~min}, \mathrm{t}_{\mathrm{R}}($ minor $)=13.4 \mathrm{~min}, 88 \%$ ee .

\begin{tabular}{|l|l|l|}
\hline Peak\# & Ret. Time & Area\% \\
\hline 1 & 13.472 & 50.209 \\
\hline 2 & 15.926 & 49.791 \\
\hline
\end{tabular}

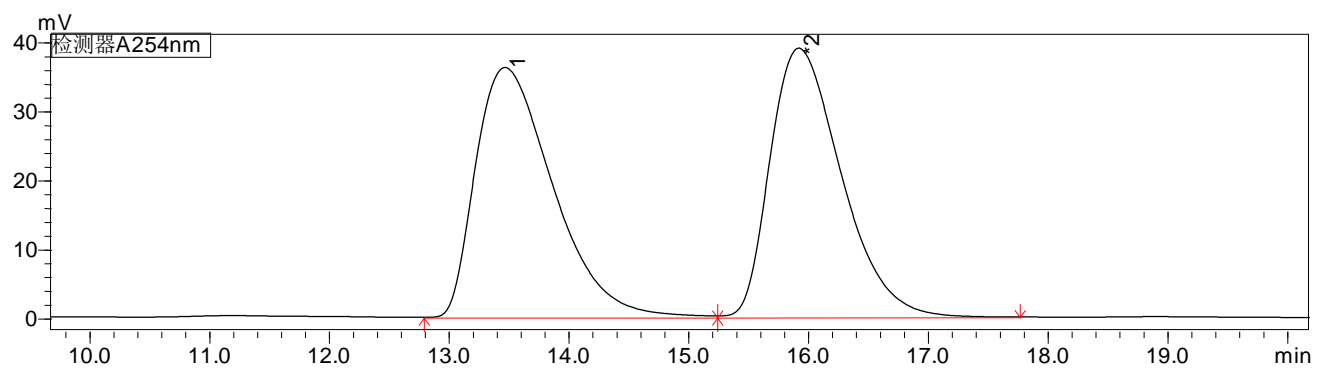

\begin{tabular}{|l|l|l|}
\hline Peak\# & Ret. Time & Area\% \\
\hline 1 & 13.410 & 6.052 \\
\hline 2 & 15.753 & 93.948 \\
\hline
\end{tabular}

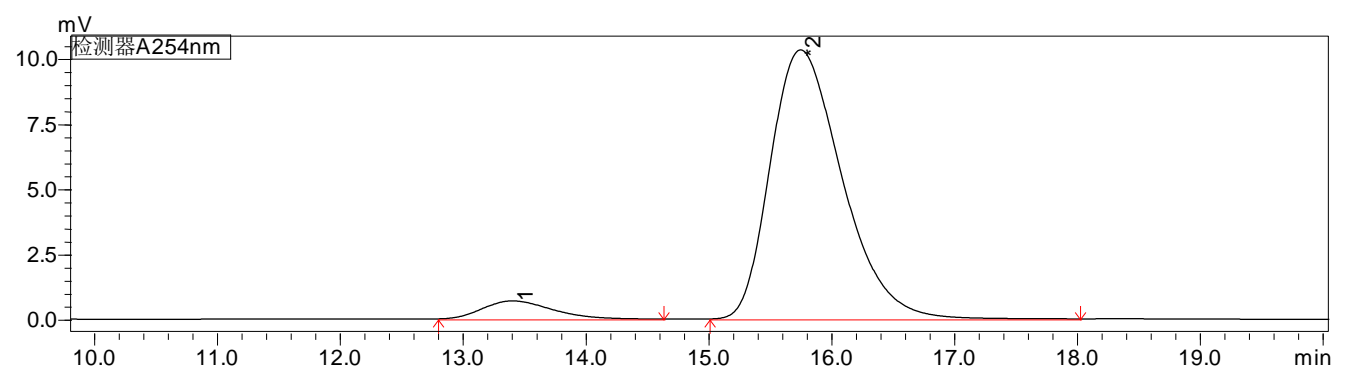




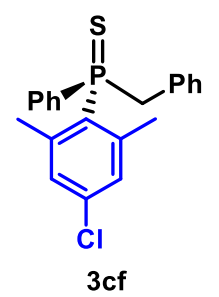

3cf: Procedure A, $64.6 \mathrm{mg}, 87 \%$ yield, light yellow solid.

${ }^{1}$ H NMR (500 MHz, CDCl $)$ ) $8.44-7.34(\mathrm{~m}, 3 \mathrm{H}), 7.26-7.21(\mathrm{~m}, 2 \mathrm{H}), 7.10-7.04(\mathrm{~m}, 3 \mathrm{H}), 6.99$ (t, $J=7.5 \mathrm{~Hz}, 2 \mathrm{H}), 6.76(\mathrm{dd}, J=7.8,2.7 \mathrm{~Hz}, 2 \mathrm{H}), 4.02(\mathrm{dd}, J=15.9,12.9 \mathrm{~Hz}, 1 \mathrm{H}), 3.71(\mathrm{dd}, J=12.9$, $10.6 \mathrm{~Hz}, 1 \mathrm{H}), 2.36(\mathrm{~s}, 6 \mathrm{H})$.

${ }^{13} \mathbf{C ~ N M R}\left(101 \mathrm{MHz}, \mathbf{C D C l}_{3}\right) \delta 143.54(\mathrm{~d}, J=10.7 \mathrm{~Hz}), 136.58(\mathrm{~d}, J=3.6 \mathrm{~Hz}), 133.23(\mathrm{~d}, J=$ $80.8 \mathrm{~Hz}), 131.40(\mathrm{~d}, J=79.6 \mathrm{~Hz}), 130.96(\mathrm{~d}, J=3.0 \mathrm{~Hz}), 130.71(\mathrm{~d}, J=5.3 \mathrm{~Hz}), 130.67(\mathrm{~d}, J=$ $7.3 \mathrm{~Hz}), 130.59,130.23$ (d, $J=11.2 \mathrm{~Hz}), 128.14$ (d, $J=12.3 \mathrm{~Hz}), 127.45$ (d, $J=3.6 \mathrm{~Hz}), 126.79$ (d, $J=4.1 \mathrm{~Hz}), 45.55(\mathrm{~d}, J=47.3 \mathrm{~Hz}), 24.37$ (d, $J=4.5 \mathrm{~Hz})$.

${ }^{31} \mathbf{P}$ NMR (162 MHz, CDCl $) \delta 43.17$.

HRMS (ESI) $\mathbf{m} / \mathbf{z}[\mathbf{M}+\mathbf{H}]^{+}$: calcd. 371.0783, found. 371.0785 .

IR (film): $v_{\max }\left(\mathrm{cm}^{-1}\right)$ 3057, 2975, 1573, 1467, 1247, 1099, 874, 695, 594.

Optical rotation: $[\alpha]_{\mathrm{D}}{ }^{25}=-142.04\left(c=1.292, \mathrm{CHCl}_{3}, 75 \%\right.$ ee $)$.

HPLC: DAICEL CHIRALPAK ID, hexane $/ i-\mathrm{PrOH}=19 / 1$, flow rate: $1.0 \mathrm{~mL} / \mathrm{min}, \lambda=254 \mathrm{~nm}$, $\mathrm{t}_{\mathrm{R}}($ major $)=10.8 \mathrm{~min}, \mathrm{t}_{\mathrm{R}}($ minor $)=12.3 \mathrm{~min}, 75 \%$ ee .

\begin{tabular}{|l|l|l|}
\hline Peak\# & Ret. Time & Area\% \\
\hline 1 & 10.839 & 50.091 \\
\hline 2 & 12.141 & 49.909 \\
\hline
\end{tabular}

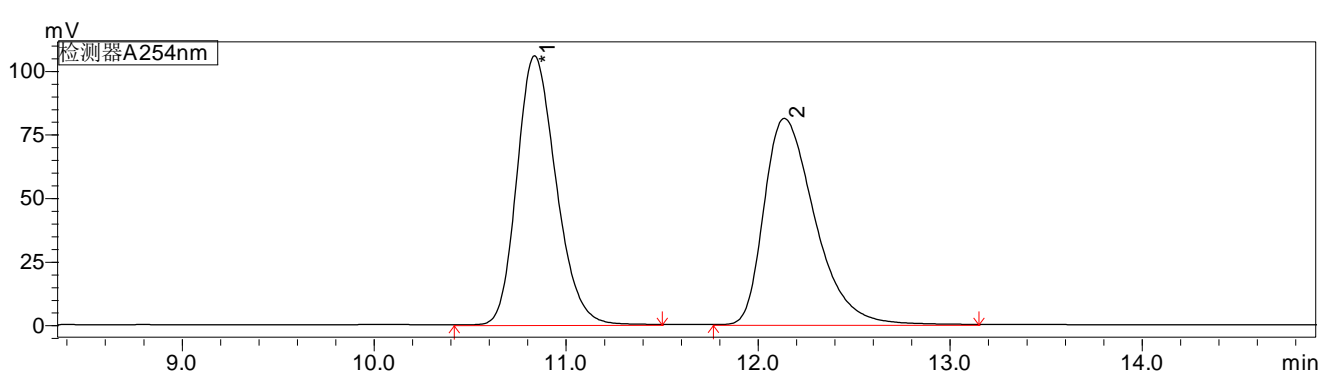

\begin{tabular}{|l|l|l|}
\hline Peak\# & Ret. Time & Area\% \\
\hline 1 & 10.795 & 87.521 \\
\hline 2 & 12.257 & 12.479 \\
\hline
\end{tabular}

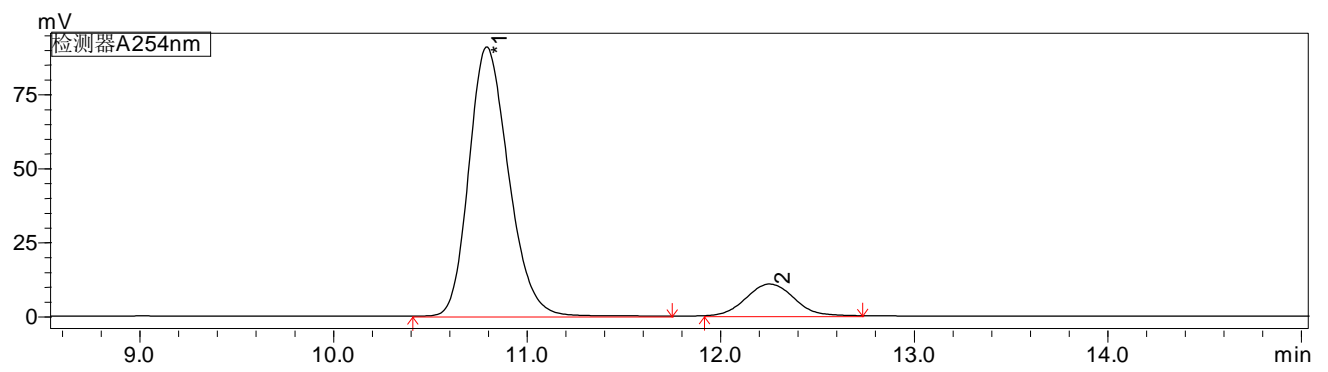




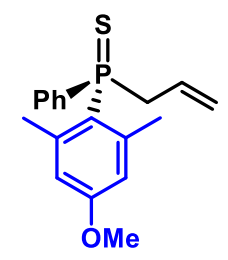

3da

3da: Procedure A, $52.2 \mathrm{mg}, 93 \%$ yield, light yellow solid.

${ }^{1} \mathbf{H}$ NMR (500 MHz, $\left.\mathbf{C D C l}_{\mathbf{3}}\right) \delta$ 7.77-7.70 (m, 2H), 7.49-7.38 (m, 3H), $6.57(\mathrm{~d}, J=3.4 \mathrm{~Hz}, 2 \mathrm{H})$, 5.48-5.35 (m, 1H), 5.01-4.94 (m, 1H), 4.87-4.78 (m, 1H), $3.79(\mathrm{~s}, 3 \mathrm{H}), 3.43-3.24(\mathrm{~m}, 2 \mathrm{H}), 2.32$ $(\mathrm{s}, 6 \mathrm{H})$.

${ }^{13} \mathbf{C}$ NMR(101 MHz, $\left.\mathbf{C D C l}_{3}\right) \delta 160.78(\mathrm{~d}, J=3.1 \mathrm{~Hz}), 143.74(\mathrm{~d}, J=11.2 \mathrm{~Hz}), 134.31(\mathrm{~d}, J=$ $80.0 \mathrm{~Hz}), 130.78(\mathrm{~d}, J=9.9 \mathrm{~Hz}), 130.74(\mathrm{~d}, J=3.1 \mathrm{~Hz}), 128.12(\mathrm{~d}, J=12.3 \mathrm{~Hz}), 127.80(\mathrm{~d}, J=$ $8.5 \mathrm{~Hz}), 123.43(\mathrm{~d}, J=84.8 \mathrm{~Hz}), 120.89$ (d, $J=13.7 \mathrm{~Hz}), 115.66(\mathrm{~d}, J=11.8 \mathrm{~Hz}), 55.02,44.80$ (d, $J=51.5 \mathrm{~Hz}), 24.38(\mathrm{~d}, J=4.7 \mathrm{~Hz})$.

${ }^{31}$ P NMR (162 MHz, $\left.\mathrm{CDCl}_{3}\right) \delta 40.71$.

HRMS (ESI) $\mathbf{m} / \mathbf{z}[\mathbf{M}+\mathbf{H}]^{+}$: calcd. 317.1123, found. 317.1123 .

IR (film): $v_{\max }\left(\mathrm{cm}^{-1}\right)$ 2964, 2934, 1593, 1437, 1306, 1168, 1090, 841, 751, 654.

Optical rotation: $[\alpha]_{\mathrm{D}}{ }^{25}=-105.20\left(c=1.044, \mathrm{CHCl}_{3}, 93 \%\right.$ ee $)$.

HPLC: DAICEL CHIRALPAK ID, hexane $/ i-\mathrm{PrOH}=19 / 1$, flow rate: $1.0 \mathrm{~mL} / \mathrm{min}, \lambda=254 \mathrm{~nm}$, $\mathrm{t}_{\mathrm{R}}($ major $)=19.7 \min , \mathrm{t}_{\mathrm{R}}($ minor $)=23.9 \mathrm{~min}, 93 \%$ ee .

\begin{tabular}{|l|l|l|}
\hline Peak\# & Ret. Time & Area\% \\
\hline 1 & 19.627 & 50.003 \\
\hline 2 & 23.740 & 49.997 \\
\hline
\end{tabular}
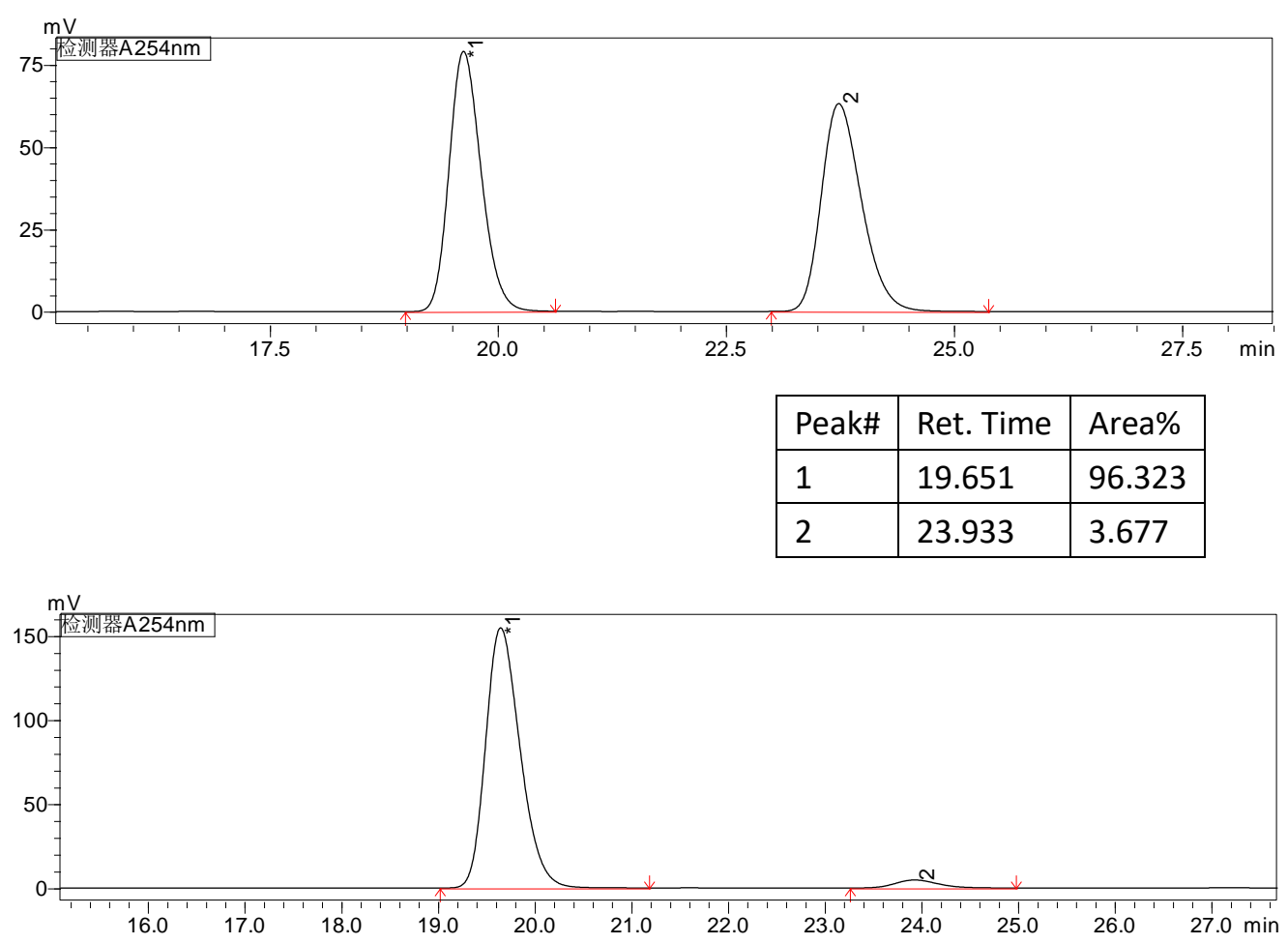


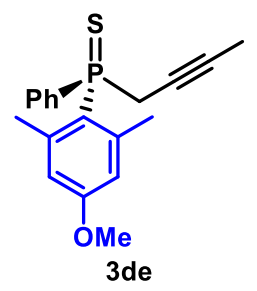

3de: Procedure E, $41.0 \mathrm{mg}$, 58\% yield, light yellow solid.

${ }^{1} \mathbf{H}$ NMR (500 MHz, CDCl $\left.{ }_{3}\right) \delta$ 7.99-7.88 (m, 2H), 7.56-7.48 (m, 1H), 7.48-7.40 (m, 2H), $6.58(\mathrm{~d}$, $J=3.5 \mathrm{~Hz}, 2 \mathrm{H}), 3.80(\mathrm{~s}, 3 \mathrm{H}), 3.61-3.49(\mathrm{~m}, 1 \mathrm{H}), 3.07-2.95(\mathrm{~m}, 1 \mathrm{H}), 2.36(\mathrm{~s}, 6 \mathrm{H}), 1.70-1.62(\mathrm{~m}$, $3 \mathrm{H})$.

${ }^{13} \mathbf{C}$ NMR(101 MHz, $\left.\mathbf{C D C l}_{3}\right) \delta 160.95(\mathrm{~d}, J=3.0 \mathrm{~Hz}), 144.18(\mathrm{~d}, J=11.3 \mathrm{~Hz}), 134.79(\mathrm{~d}, J=$ $80.9 \mathrm{~Hz}), 131.17(\mathrm{~d}, J=3.0 \mathrm{~Hz}), 130.62(\mathrm{~d}, J=9.9 \mathrm{~Hz}), 128.01(\mathrm{~d}, J=12.4 \mathrm{~Hz}), 121.50$ (d, $J=$ $88.2 \mathrm{~Hz}), 115.53(\mathrm{~d}, J=11.9 \mathrm{~Hz}), 80.66(\mathrm{~d}, J=8.7 \mathrm{~Hz}), 71.10(\mathrm{~d}, J=13.0 \mathrm{~Hz}), 55.02,34.64(\mathrm{~d}, J$ $=54.1 \mathrm{~Hz}), 23.58(\mathrm{~d}, J=5.4 \mathrm{~Hz}), 3.50(\mathrm{~d}, J=3.1 \mathrm{~Hz})$.

${ }^{31}$ P NMR (162 MHz, $\left.\mathbf{C D C l}_{\mathbf{3}}\right) \delta 39.11$.

HRMS (ESI) $\mathbf{m} / \mathbf{z}$ [M+H] ${ }^{+}$: calcd. 329.1123, found. 329.1124 .

IR (film): $v_{\max }\left(\mathrm{cm}^{-1}\right) 2964,2916,1593,1455,1307,1168,1092,856,722,666$.

Optical rotation: $[\alpha]_{\mathrm{D}}^{25}=-4.43\left(c=0.820, \mathrm{CHCl}_{3}, 90 \%\right.$ ee $)$.

HPLC: DAICEL CHIRALPAK ID, hexane $/ i-\mathrm{PrOH}=9 / 1$, flow rate: $1.0 \mathrm{~mL} / \mathrm{min}, \lambda=254 \mathrm{~nm}$, $\mathrm{t}_{\mathrm{R}}($ major $)=17.5 \mathrm{~min}, \mathrm{t}_{\mathrm{R}}($ minor $)=20.2 \mathrm{~min}, 90 \%$ ee.

\begin{tabular}{|l|l|l|}
\hline Peak\# & Ret. Time & Area\% \\
\hline 1 & 17.481 & 49.913 \\
\hline 2 & 20.195 & 50.087 \\
\hline
\end{tabular}

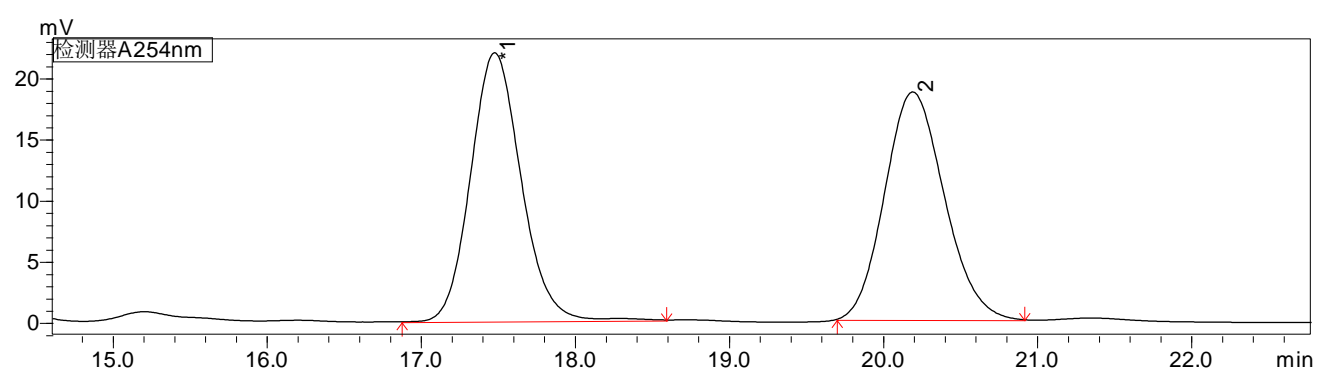

\begin{tabular}{|l|l|l|}
\hline Peak\# & Ret. Time & Area\% \\
\hline 1 & 17.465 & 94.800 \\
\hline 2 & 20.219 & 5.200 \\
\hline
\end{tabular}

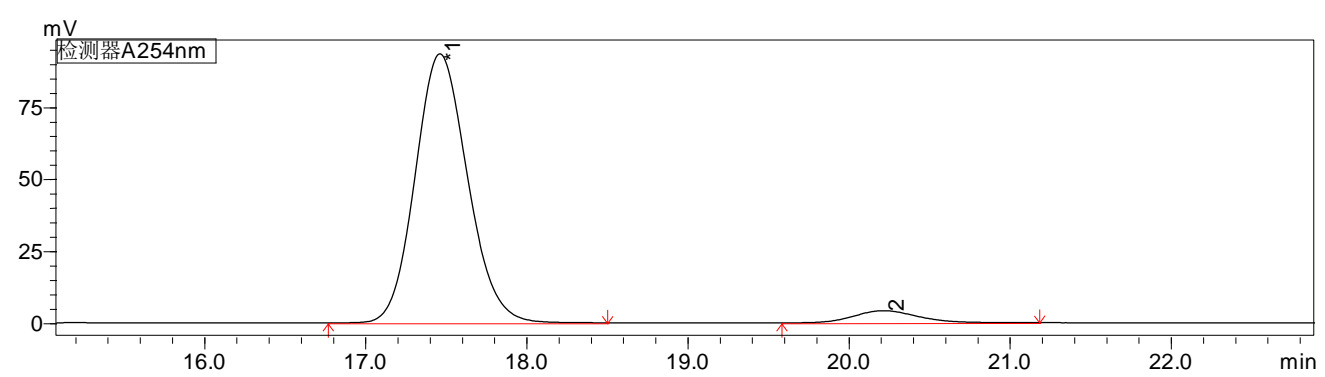




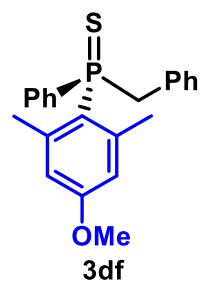

3df: Procedure A, $50.6 \mathrm{mg}, 69 \%$ yield, light yellow solid.

${ }^{1} \mathbf{H}$ NMR (500 MHz, $\mathbf{C D C l}_{3}$ ) $\delta$ 7.46-7.39 (m, 2H), 7.38-7.32 (m, 1H), 7.25-7.20 (m, 2H), 7.10$7.03(\mathrm{~m}, 1 \mathrm{H}), 6.99(\mathrm{t}, J=7.5 \mathrm{~Hz}, 2 \mathrm{H}), 6.81-6.70(\mathrm{~m}, 2 \mathrm{H}), 6.60(\mathrm{~d}, J=3.3 \mathrm{~Hz}, 2 \mathrm{H}), 4.02(\mathrm{dd}, J=$ $15.9,12.9 \mathrm{~Hz}, 1 \mathrm{H}), 3.80(\mathrm{~s}, 3 \mathrm{H}), 3.70(\mathrm{dd}, J=13.0,10.6 \mathrm{~Hz}, 1 \mathrm{H}), 2.37(\mathrm{~s}, 6 \mathrm{H})$.

${ }^{13} \mathbf{C}$ NMR(101 MHz, $\left.\mathbf{C D C l}_{3}\right) \delta 160.85(\mathrm{~d}, J=3.0 \mathrm{~Hz}), 143.88(\mathrm{~d}, J=11.1 \mathrm{~Hz}), 134.08(\mathrm{~d}, J=$ $80.8 \mathrm{~Hz}), 131.18(\mathrm{~d}, J=7.3 \mathrm{~Hz}), 130.74(\mathrm{~d}, J=3.9 \mathrm{~Hz}), 130.67,130.62(\mathrm{~d}, J=3.0 \mathrm{~Hz}), 127.96(\mathrm{~d}$, $J=12.4 \mathrm{~Hz}), 127.35(\mathrm{~d}, J=3.4 \mathrm{~Hz}), 126.60(\mathrm{~d}, J=4.0 \mathrm{~Hz}), 124.04(\mathrm{~d}, J=84.3 \mathrm{~Hz}), 115.84(\mathrm{~d}, J$ $=11.6 \mathrm{~Hz}), 55.12,45.68(\mathrm{~d}, J=47.6 \mathrm{~Hz}), 24.80(\mathrm{~d}, J=4.6 \mathrm{~Hz})$.

${ }^{31} \mathbf{P}$ NMR (162 MHz, $\left.\mathbf{C D C l}_{\mathbf{3}}\right) \delta 42.91$.

HRMS (ESI) $\mathbf{m} / \mathbf{z}[\mathbf{M}+\mathbf{H}]^{+}$: calcd. 367.1280, found. 367.1280.

IR (film): $v_{\max }\left(\mathrm{cm}^{-1}\right)$ 3057, 2964, 1593, 1494, 1305, 1168, 1090, 846, 696, 650, 596.

Optical rotation: $[\alpha]_{\mathrm{D}}{ }^{25}=-134.07\left(c=1.032, \mathrm{CHCl}_{3}, 80 \%\right.$ ee $)$.

HPLC: DAICEL CHIRALPAK ID, hexane $/ i-\mathrm{PrOH}=9 / 1$, flow rate: $1.0 \mathrm{~mL} / \mathrm{min}, \lambda=254 \mathrm{~nm}$, $\mathrm{t}_{\mathrm{R}}($ major $)=18.1 \mathrm{~min}, \mathrm{t}_{\mathrm{R}}($ minor $)=22.6 \mathrm{~min}, 80 \%$ ee .

\begin{tabular}{|l|l|l|}
\hline Peak\# & Ret. Time & Area\% \\
\hline 1 & 18.089 & 49.997 \\
\hline 2 & 22.477 & 50.003 \\
\hline
\end{tabular}

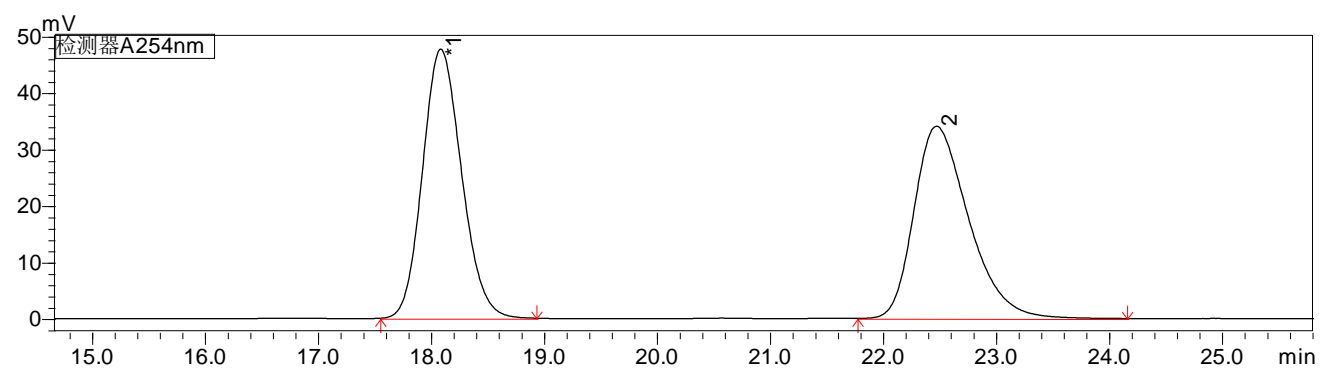

\begin{tabular}{|l|l|l|}
\hline Peak\# & Ret. Time & Area\% \\
\hline 1 & 18.063 & 90.068 \\
\hline 2 & 22.582 & 9.932 \\
\hline
\end{tabular}

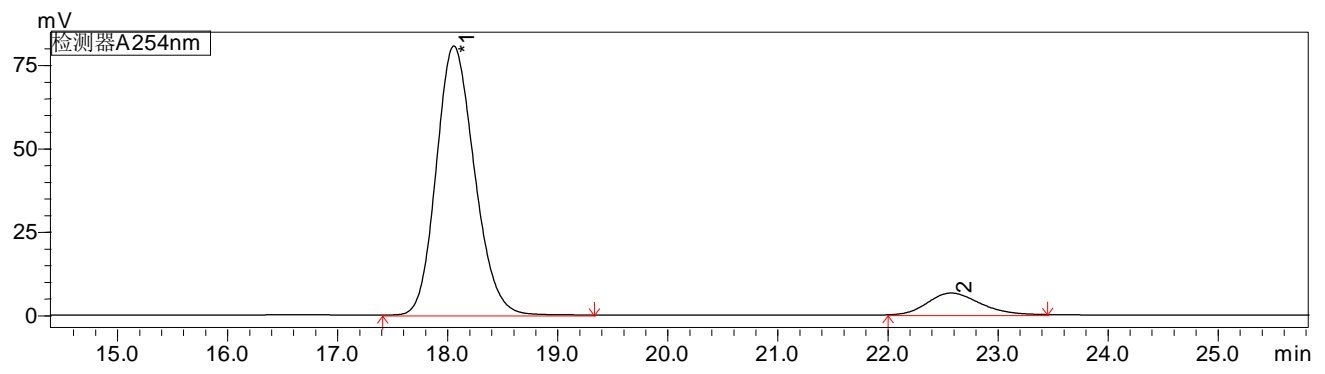




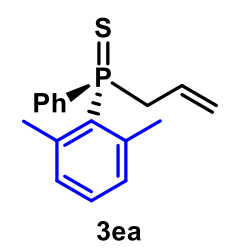

3ea: Procedure A, $52.9 \mathrm{mg}$, 92\% yield, light yellow solid.

${ }^{1}$ H NMR (400 MHz, $\mathbf{C D C l}_{3}$ ) $\delta$ 7.80-7.66 (m, 2H), 7.53-7.35 (m, 3H), 7.28-7.19 (m, 1H), 7.07$6.97(\mathrm{~m}, 2 \mathrm{H}), 5.50-5.31(\mathrm{~m}, 1 \mathrm{H}), 4.98(\mathrm{dd}, J=10.2,4.2 \mathrm{~Hz}, 1 \mathrm{H}), 4.85(\mathrm{dd}, J=17.0,5.2 \mathrm{~Hz}, 1 \mathrm{H})$, 3.49-3.22 (m, 2H), $2.34(\mathrm{~s}, 6 \mathrm{H})$.

${ }^{13} \mathbf{C}$ NMR(101 MHz, $\left.\mathbf{C D C l}_{3}\right) \delta 141.50(\mathrm{~d}, J=9.9 \mathrm{~Hz}), 133.91(\mathrm{~d}, J=79.5 \mathrm{~Hz}), 131.94(\mathrm{~d}, J=$ $79.0 \mathrm{~Hz}), 130.86(\mathrm{~d}, J=3.0 \mathrm{~Hz}), 130.78(\mathrm{~d}, J=9.9 \mathrm{~Hz}), 130.70(\mathrm{~d}, J=2.8 \mathrm{~Hz}), 130.38(\mathrm{~d}, J=$ $10.7 \mathrm{~Hz}), 128.18(\mathrm{~d}, J=12.4 \mathrm{~Hz}), 127.60(\mathrm{~d}, J=8.5 \mathrm{~Hz}), 121.08(\mathrm{~d}, J=13.6 \mathrm{~Hz}), 44.70(\mathrm{~d}, J=$ $51.0 \mathrm{~Hz}), 24.10(\mathrm{~d}, J=5.0 \mathrm{~Hz})$.

${ }^{31}$ P NMR (162 MHz, $\left.\mathbf{C D C l}_{3}\right) \delta 41.19$.

HRMS (ESI) $\mathbf{m} / \mathbf{z}$ [M+H] $]^{+}$: calcd. 287.1018, found. 287.1018 .

IR (film): $v_{\max }\left(\mathrm{cm}^{-1}\right)$ 2955, 2924, 2853, 1633, 1588, 1453, 1436, 1377, 1100, 784, 693, 638, 606.

Optical rotation: $[\alpha]_{\mathrm{D}}^{25}=-105.24\left(c=1.040, \mathrm{CHCl}_{3}, 92 \%\right.$ ee $)$.

HPLC: DAICEL CHIRALPAK IC-3, hexane $/ i-\mathrm{PrOH}=19 / 1$, flow rate: $0.5 \mathrm{~mL} / \mathrm{min}, \lambda=254 \mathrm{~nm}$, $\mathrm{t}_{\mathrm{R}}($ major $)=25.0 \mathrm{~min}, \mathrm{t}_{\mathrm{R}}($ minor $)=26.8 \mathrm{~min}, 92 \%$ ee .
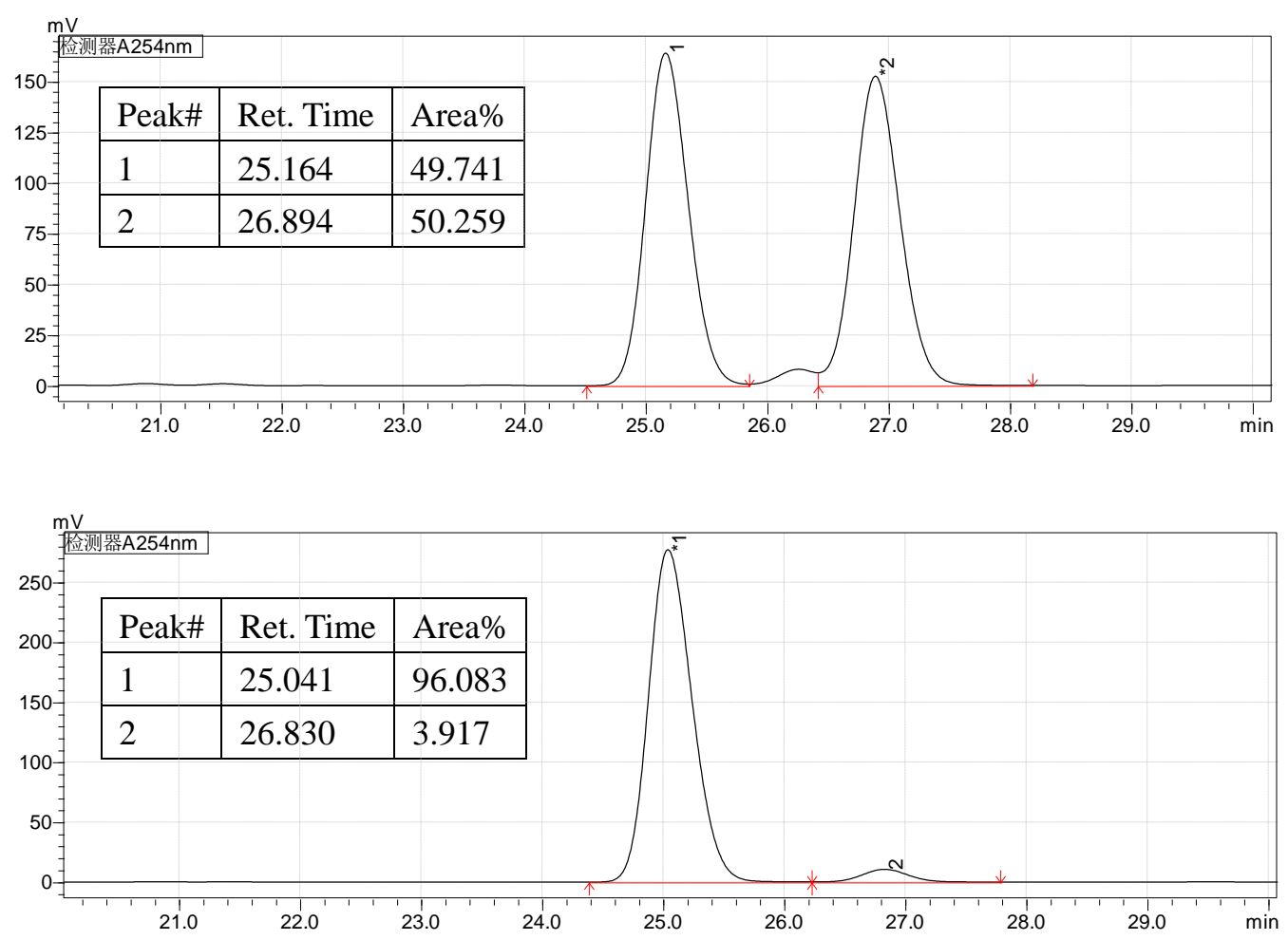


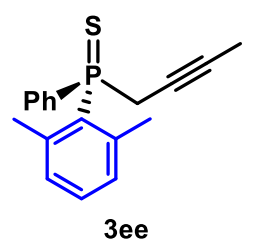

3ee: Procedure E, $59.0 \mathrm{mg}$, 99\% yield, light yellow solid.

${ }^{1}$ H NMR (500 MHz, CDCl $) \delta 7.93(\mathrm{dd}, J=13.1,7.6 \mathrm{~Hz}, 2 \mathrm{H}), 7.57-7.49(\mathrm{~m}, 1 \mathrm{H}), 7.49-7.40(\mathrm{~m}$, $2 \mathrm{H}), 7.26(\mathrm{~d}, J=5.9 \mathrm{~Hz}, 1 \mathrm{H}), 7.04(\mathrm{dd}, J=7.7,4.4 \mathrm{~Hz}, 2 \mathrm{H}), 3.65-3.51(\mathrm{~m}, 1 \mathrm{H}), 3.11-2.96(\mathrm{~m}$, $1 \mathrm{H}), 2.38(\mathrm{~s}, 6 \mathrm{H}), 1.69-1.61(\mathrm{~m}, 3 \mathrm{H})$.

${ }^{13} \mathbf{C}$ NMR(101 MHz, CDCl $) \delta 141.95(\mathrm{~d}, J=9.9 \mathrm{~Hz}), 134.44(\mathrm{~d}, J=80.3 \mathrm{~Hz}), 131.29(\mathrm{~d}, J=3.0$ $\mathrm{Hz}), 130.85(\mathrm{~d}, J=2.8 \mathrm{~Hz}), 130.60(\mathrm{~d}, J=9.8 \mathrm{~Hz}), 130.20(\mathrm{~d}, J=11.0 \mathrm{~Hz}), 130.02(\mathrm{~d}, J=82.0$ Hz), 128.09 (d, $J=12.4 \mathrm{~Hz}), 80.80(\mathrm{~d}, J=8.7 \mathrm{~Hz}), 70.95$ (d, $J=13.0 \mathrm{~Hz}), 34.59$ (d, $J=53.5 \mathrm{~Hz})$, $23.31(\mathrm{~d}, J=5.6 \mathrm{~Hz}), 3.49(\mathrm{~d}, J=3.1 \mathrm{~Hz})$.

${ }^{31}$ P NMR (162 MHz, $\left.\mathbf{C D C l}_{3}\right) \delta 39.49$.

HRMS (ESI) $\mathbf{m} / \mathbf{z}$ [M+H] $]^{+}$: calcd. 299.1018, found. 299.1018 .

IR (film): $v_{\max }\left(\mathrm{cm}^{-1}\right)$ 3054, 2963, 1453, 1100, 827, 708, 659, 628 .

Optical rotation: $[\alpha]_{\mathrm{D}}{ }^{25}=17.89\left(c=0.993, \mathrm{CHCl}_{3}, 92 \%\right.$ ee $)$.

HPLC: DAICEL CHIRALPAK ID, hexane $/ i-\mathrm{PrOH}=9 / 1$, flow rate: $1.0 \mathrm{~mL} / \mathrm{min}, \lambda=254 \mathrm{~nm}$, $\mathrm{t}_{\mathrm{R}}($ major $)=9.5 \mathrm{~min}, \mathrm{t}_{\mathrm{R}}($ minor $)=10.4 \mathrm{~min}, 92 \%$ ee

\begin{tabular}{|l|l|l|}
\hline Peak\# & Ret. Time & Area\% \\
\hline 1 & 9.510 & 50.148 \\
\hline 2 & 10.338 & 49.852 \\
\hline
\end{tabular}

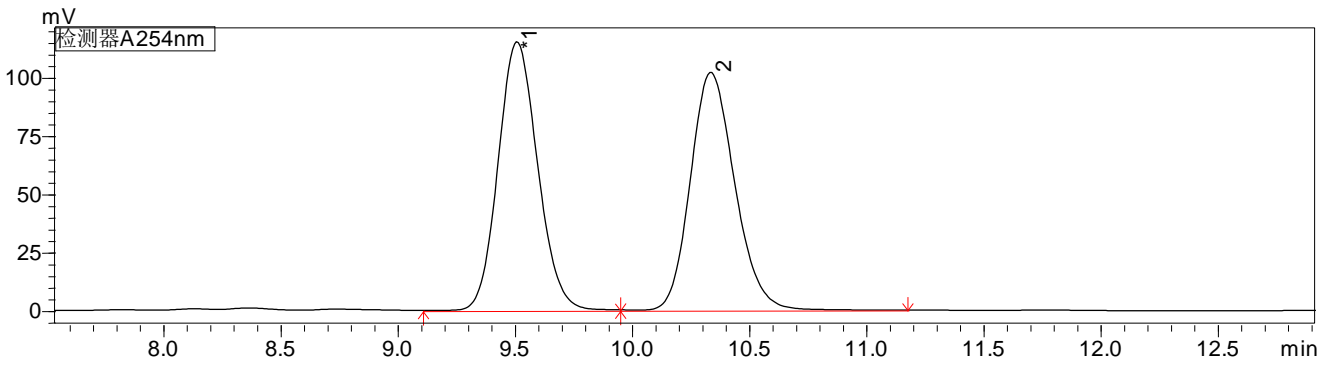

\begin{tabular}{|l|l|l|}
\hline Peak\# & Ret. Time & Area\% \\
\hline 1 & 9.523 & 96.071 \\
\hline 2 & 10.376 & 3.929 \\
\hline
\end{tabular}

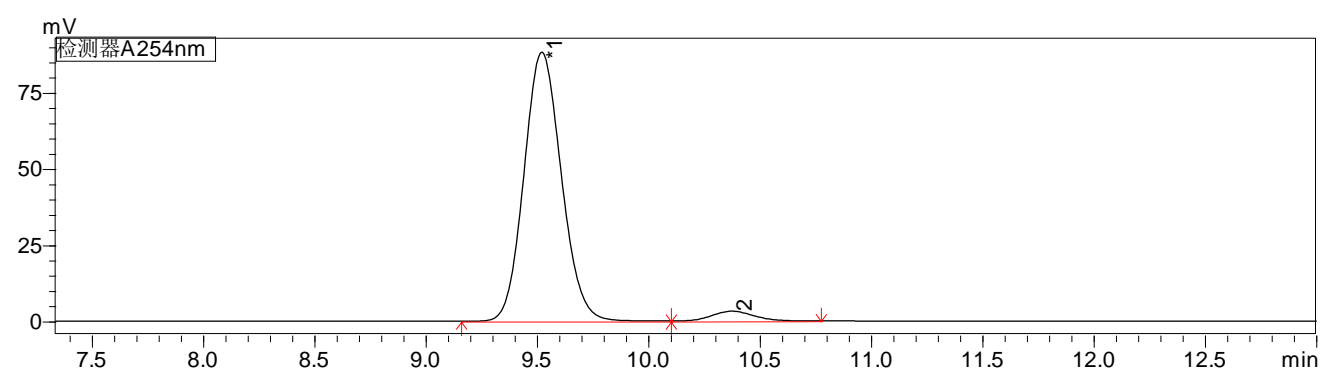




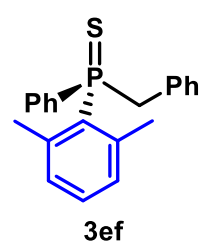

3ef: Procedure A, $66.6 \mathrm{mg}$, 99\% yield, light yellow solid.

${ }^{1}$ H NMR (500 MHz, $\left.\mathbf{C D C l}_{\mathbf{3}}\right) \delta 7.41(\mathrm{dd}, J=13.1,7.6 \mathrm{~Hz}, 2 \mathrm{H}), 7.38-7.33(\mathrm{~m}, 1 \mathrm{H}), 7.27-7.19(\mathrm{~m}$, $3 \mathrm{H}), 7.10-7.02(\mathrm{~m}, 3 \mathrm{H}), 6.99(\mathrm{t}, J=7.5 \mathrm{~Hz}, 2 \mathrm{H}), 6.77(\mathrm{dd}, J=7.9,2.7 \mathrm{~Hz}, 2 \mathrm{H}), 4.05(\mathrm{dd}, J=15.9$, $13.0 \mathrm{~Hz}, 1 \mathrm{H}), 3.73$ (dd, $J=12.9,10.6 \mathrm{~Hz}, 1 \mathrm{H}), 2.39$ (s, 6H).

${ }^{13} \mathbf{C}$ NMR(101 MHz, $\left.\mathbf{C D C l}_{3}\right) \delta 141.54(\mathrm{~d}, J=9.6 \mathrm{~Hz}), 133.54(\mathrm{~d}, J=79.9 \mathrm{~Hz}), 132.46(\mathrm{~d}, J=$ $78.3 \mathrm{~Hz}), 130.94(\mathrm{~d}, J=7.5 \mathrm{~Hz}), 130.68-130.61(\mathrm{~m}), 130.56$ (d, $J=2.7 \mathrm{~Hz}), 130.46$ (d, $J=10.6$ $\mathrm{Hz}), 127.92(\mathrm{~d}, J=12.3 \mathrm{~Hz}), 127.30(\mathrm{~d}, J=3.4 \mathrm{~Hz}), 126.56(\mathrm{~d}, J=4.1 \mathrm{~Hz}), 45.52(\mathrm{~d}, J=46.9 \mathrm{~Hz})$, $24.42(\mathrm{~d}, J=4.8 \mathrm{~Hz})$.

${ }^{31}$ P NMR (162 MHz, $\left.\mathrm{CDCl}_{3}\right) \delta 43.38$.

HRMS (ESI) $\mathbf{m} / \mathbf{z}[\mathbf{M}+\mathbf{H}]^{+}$: calcd. 337.1174 , found. 337.1174 .

IR (film): $v_{\max }\left(\mathrm{cm}^{-1}\right)$ 3056, 2972, 1494, 1452, 1102, 911, 846, 772, 696, 630, 592.

Optical rotation: $[\alpha]_{\mathrm{D}}{ }^{25}=-146.41\left(c=1.332, \mathrm{CHCl}_{3}, 84 \%\right.$ ee $)$.

HPLC: DAICEL CHIRALPAK ID, hexane $/ i-\mathrm{PrOH}=9 / 1$, flow rate: $1.0 \mathrm{~mL} / \mathrm{min}, \lambda=254 \mathrm{~nm}$, $\mathrm{t}_{\mathrm{R}}($ major $)=9.6 \mathrm{~min}, \mathrm{t}_{\mathrm{R}}($ minor $)=10.3 \mathrm{~min}, 84 \%$ ee.

\begin{tabular}{|l|l|l|}
\hline Peak\# & Ret. Time & Area\% \\
\hline 1 & 9.614 & 50.005 \\
\hline 2 & 10.242 & 49.995 \\
\hline
\end{tabular}

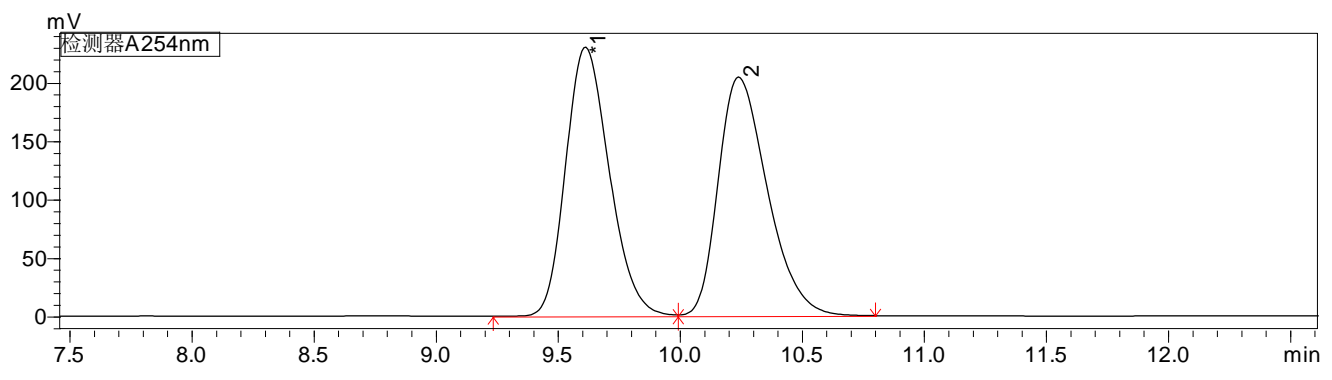

\begin{tabular}{|l|l|l|}
\hline Peak\# & Ret. Time & Area\% \\
\hline 1 & 9.624 & 92.048 \\
\hline 2 & 10.324 & 7.952 \\
\hline
\end{tabular}

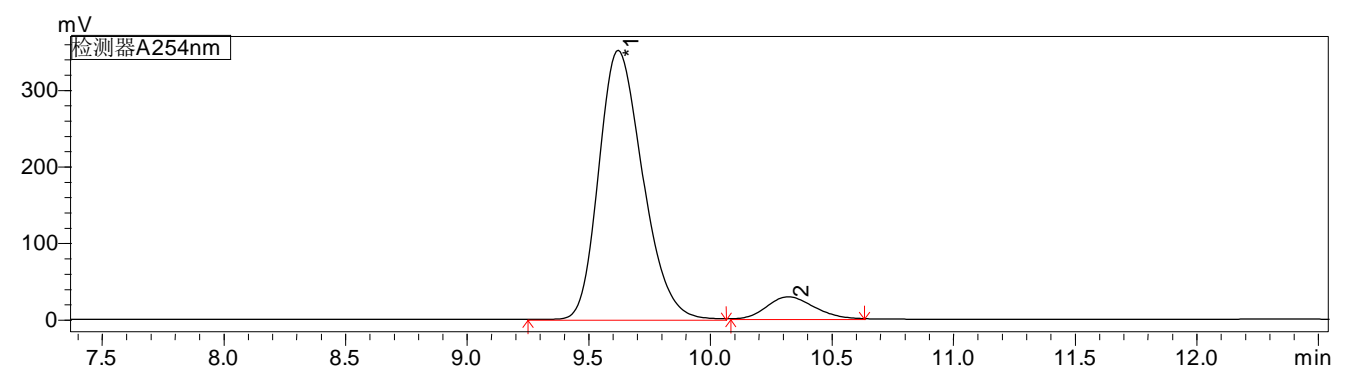




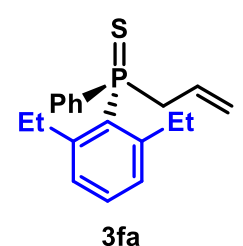

3fa: Procedure A, $60.4 \mathrm{mg}, 96 \%$ yield, light yellow solid.

${ }^{1}$ H NMR (500 MHz, $\left.\mathbf{C D C l}_{3}\right) \delta$ 7.71-7.63 (m, 2H), 7.47-7.32 (m, 4H), $7.13(\mathrm{dd}, J=7.7,4.3 \mathrm{~Hz}$, $2 \mathrm{H}), 5.49-5.36(\mathrm{~m}, 1 \mathrm{H}), 5.02-4.92(\mathrm{~m}, 1 \mathrm{H}), 4.86-4.75(\mathrm{~m}, 1 \mathrm{H}), 3.48-3.36(\mathrm{~m}, 1 \mathrm{H}), 3.36-3.26(\mathrm{~m}$, $1 \mathrm{H}), 2.87-2.71(\mathrm{~m}, 4 \mathrm{H}), 1.05(\mathrm{t}, J=7.4 \mathrm{~Hz}, 6 \mathrm{H})$.

${ }^{13}$ C NMR(101 MHz, CDCl $) \delta 147.84(\mathrm{~d}, J=10.2 \mathrm{~Hz}), 135.04(\mathrm{~d}, J=79.6 \mathrm{~Hz}), 131.42(\mathrm{~d}, J=$ $79.3 \mathrm{~Hz}), 131.05(\mathrm{~d}, J=2.7 \mathrm{~Hz}), 130.72(\mathrm{~d}, J=9.5 \mathrm{~Hz}), 130.66(\mathrm{~d}, J=2.6 \mathrm{~Hz}), 128.04(\mathrm{~d}, J=8.0$ $\mathrm{Hz}), 127.93(\mathrm{~d}, J=6.6 \mathrm{~Hz}), 127.80(\mathrm{~d}, J=8.4 \mathrm{~Hz}), 120.90(\mathrm{~d}, J=13.7 \mathrm{~Hz}), 44.71(\mathrm{~d}, J=50.8 \mathrm{~Hz})$, $28.72(\mathrm{~d}, J=5.6 \mathrm{~Hz}), 15.37$.

${ }^{31}$ P NMR (162 MHz, CDCl 3 ) $\delta 40.31$.

HRMS (ESI) $\mathbf{m} / \mathbf{z}[\mathbf{M}+\mathbf{H}]^{+}$: calcd. 315.1331, found. 315.1331.

IR (film): $v_{\max }\left(\mathrm{cm}^{-1}\right)$ 2966, 2930, 1456, 1437, 1099, 917, 802, 706, 642, 615.

Optical rotation: $[\alpha]_{\mathrm{D}}{ }^{25}=-43.17\left(c=1.612, \mathrm{CHCl}_{3}, 83 \%\right.$ ee $)$.

HPLC: DAICEL CHIRALPAK AD-H, hexane $/ i-\mathrm{PrOH}=19 / 1$, flow rate: $1.0 \mathrm{~mL} / \mathrm{min}, \lambda=220 \mathrm{~nm}$, $\mathrm{t}_{\mathrm{R}}($ major $)=4.8 \mathrm{~min}, \mathrm{t}_{\mathrm{R}}($ minor $)=5.0 \mathrm{~min}, 83 \%$ ee.

\begin{tabular}{|l|l|l|}
\hline Peak\# & Ret. Time & Area\% \\
\hline 1 & 4.779 & 49.405 \\
\hline 2 & 4.985 & 50.595 \\
\hline
\end{tabular}

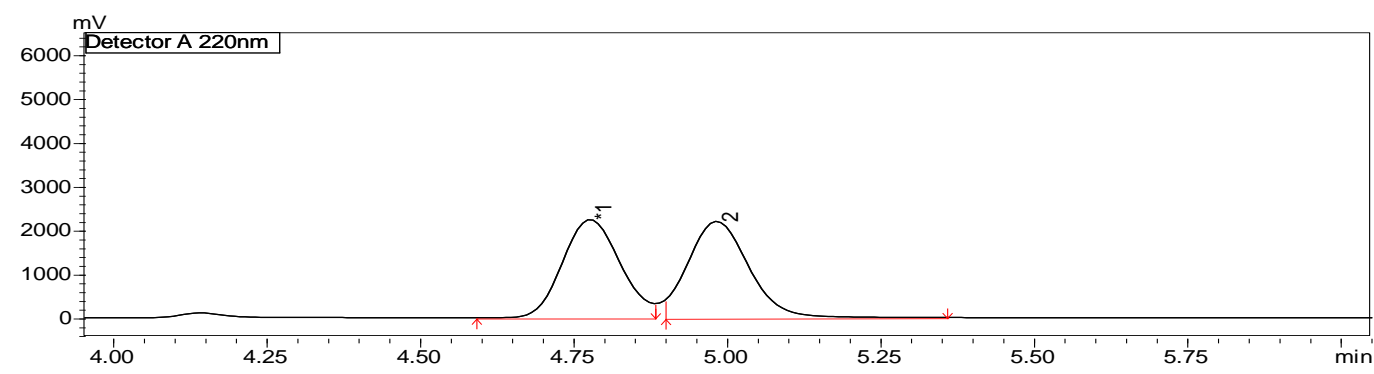

\begin{tabular}{|l|l|l|}
\hline Peak\# & Ret. Time & Area\% \\
\hline 1 & 4.771 & 91.525 \\
\hline 2 & 4.973 & 8.475 \\
\hline
\end{tabular}

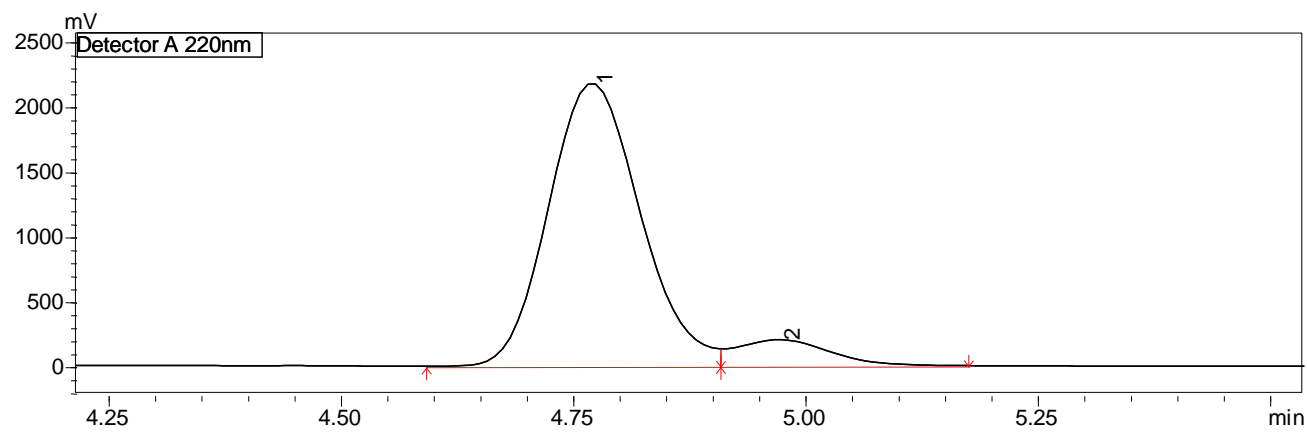




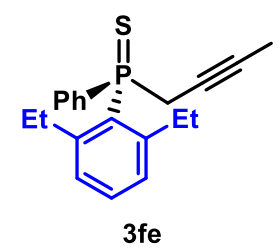

3fe: Procedure E, $50.3 \mathrm{mg}, 77 \%$ yield, light yellow solid.

${ }^{1}$ H NMR (500 MHz, $\mathbf{C D C l}_{3}$ ) $\delta$ 7.89-7.75 (m, 2H), 7.52-7.46 (m, 1H), 7.46-7.39 (m, 2H), 7.39$7.34(\mathrm{~m}, 1 \mathrm{H}), 7.15(\mathrm{dd}, J=7.7,4.5 \mathrm{~Hz}, 2 \mathrm{H}), 3.60-3.47(\mathrm{~m}, 1 \mathrm{H}), 3.15-3.01(\mathrm{~m}, 1 \mathrm{H}), 2.90-2.72(\mathrm{~m}$, $4 \mathrm{H}), 1.62(\mathrm{dt}, J=6.0,2.6 \mathrm{~Hz}, 3 \mathrm{H}), 1.09(\mathrm{t}, J=7.5 \mathrm{~Hz}, 6 \mathrm{H})$.

${ }^{13} \mathbf{C}$ NMR(101 MHz, CDCl $) \delta 148.17(\mathrm{~d}, J=9.9 \mathrm{~Hz}), 135.40(\mathrm{~d}, J=80.8 \mathrm{~Hz}), 131.22(\mathrm{~d}, J=3.0$ $\mathrm{Hz}), 131.03(\mathrm{~d}, J=3.0 \mathrm{~Hz}), 130.45(\mathrm{~d}, J=9.6 \mathrm{~Hz}), 128.97,127.84(\mathrm{~d}, J=12.4 \mathrm{~Hz}), 127.62(\mathrm{~d}, J=$ $11.0 \mathrm{~Hz}), 81.17(\mathrm{~d}, J=8.8 \mathrm{~Hz}), 71.05(\mathrm{~d}, J=12.6 \mathrm{~Hz}), 34.79(\mathrm{~d}, J=53.1 \mathrm{~Hz}), 28.33(\mathrm{~d}, J=6.0$ $\mathrm{Hz}), 15.29,3.44(\mathrm{~d}, J=3.1 \mathrm{~Hz})$.

${ }^{31}$ P NMR (162 MHz, $\left.\mathbf{C D C l}_{3}\right) \delta 38.95$.

HRMS (ESI) $\mathbf{m} / \mathbf{z}$ [M+H] $]^{+}$: calcd. 327.1331, found. 327.1330 .

IR (film): $v_{\max }\left(\mathrm{cm}^{-1}\right)$ 2965, 2931, 1456, 1098, 825, 750, 710, 658 .

Optical rotation: $[\alpha]_{\mathrm{D}}{ }^{25}=-1.22\left(c=1.224, \mathrm{CHCl}_{3}, 84 \%\right.$ ee $)$.

HPLC: DAICEL CHIRALPAK IA, hexane $/ i-\mathrm{PrOH}=9 / 1$, flow rate: $1.0 \mathrm{~mL} / \mathrm{min}, \lambda=220 \mathrm{~nm}$, $\mathrm{t}_{\mathrm{R}}($ major $)=4.8 \mathrm{~min}, \mathrm{t}_{\mathrm{R}}($ minor $)=4.4 \mathrm{~min}, 84 \%$ ee.

\begin{tabular}{|l|l|l|}
\hline Peak\# & Ret. Time & Area\% \\
\hline 1 & 4.418 & 50.191 \\
\hline 2 & 4.786 & 49.809 \\
\hline
\end{tabular}

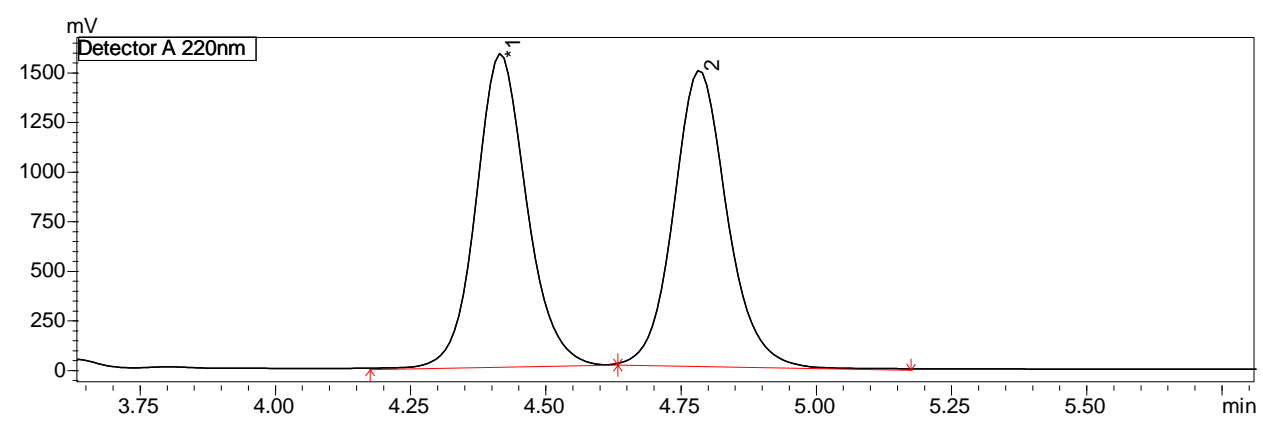

\begin{tabular}{|l|l|l|}
\hline Peak\# & Ret. Time & Area\% \\
\hline 1 & 4.416 & 8.245 \\
\hline 2 & 4.782 & 91.755 \\
\hline
\end{tabular}

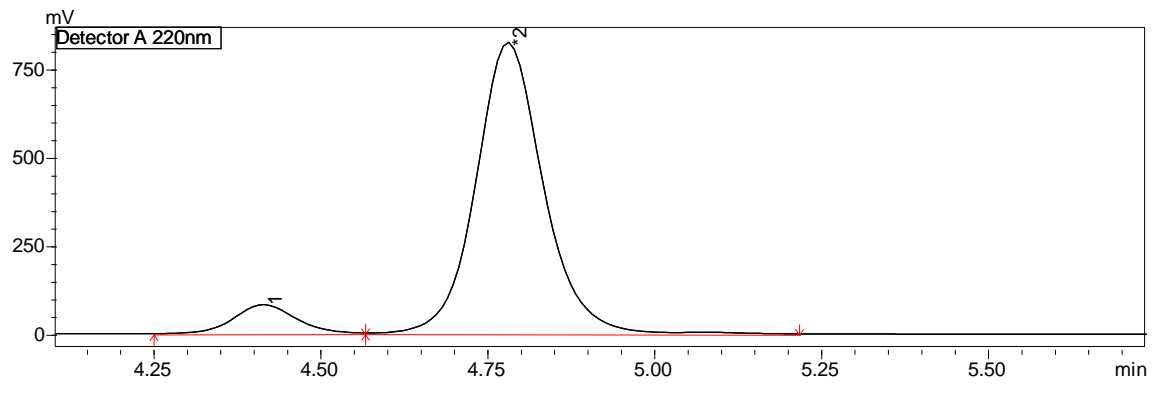




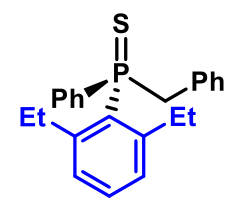

3ff

3ff: Procedure A, $71.4 \mathrm{mg}, 98 \%$ yield, light yellow solid.

${ }^{1}$ H NMR (500 MHz, $\mathbf{C D C l}_{3}$ ) $\delta$ 7.42-7.30 (m, 4H), 7.24-7.19 (m, 2H), 7.19-7.13 (m, 2H), 7.10$7.04(\mathrm{~m}, 1 \mathrm{H}), 6.99(\mathrm{t}, J=7.7 \mathrm{~Hz}, 2 \mathrm{H}), 6.75(\mathrm{dd}, J=7.6,2.7 \mathrm{~Hz}, 2 \mathrm{H}), 4.04(\mathrm{dd}, J=15.4,12.8 \mathrm{~Hz}$, $1 \mathrm{H}), 3.74(\mathrm{dd}, J=12.8,10.5 \mathrm{~Hz}, 1 \mathrm{H}), 2.93-2.72(\mathrm{~m}, 4 \mathrm{H}), 1.08(\mathrm{t}, J=7.4 \mathrm{~Hz}, 6 \mathrm{H})$.

${ }^{13} \mathbf{C}$ NMR(101 MHz, CDCl $) \delta 147.90(\mathrm{~d}, J=10.0 \mathrm{~Hz}), 134.63(\mathrm{~d}, J=80.1 \mathrm{~Hz}), 131.95(\mathrm{~d}, J=$ $78.9 \mathrm{~Hz}), 130.98(\mathrm{~d}, J=3.0 \mathrm{~Hz}), 130.96(\mathrm{~d}, J=7.3 \mathrm{~Hz}), 130.73(\mathrm{~d}, J=5.4 \mathrm{~Hz}), 130.62(\mathrm{~d}, J=9.7$ $\mathrm{Hz}), 130.48(\mathrm{~d}, J=3.0 \mathrm{~Hz}), 127.99(\mathrm{~d}, J=10.7 \mathrm{~Hz}), 127.77(\mathrm{~d}, J=12.2 \mathrm{~Hz}), 127.28(\mathrm{~d}, J=3.4$ $\mathrm{Hz}), 126.58(\mathrm{~d}, J=4.0 \mathrm{~Hz}), 45.39(\mathrm{~d}, J=46.8 \mathrm{~Hz}), 28.96(\mathrm{~d}, J=5.4 \mathrm{~Hz}), 15.39$.

${ }^{31}$ P NMR (162 MHz, $\left.\mathbf{C D C l}_{3}\right) \delta 42.22$.

HRMS (ESI) $\mathbf{m} / \mathbf{z}$ [M+H] $]^{+}$: calcd. 365.1487, found. 365.1484 .

IR (film): $v_{\max }\left(\mathrm{cm}^{-1}\right)$ 2965, 2929, 1454, 1437, 1100, 849, 770, 695, 632, 516.

Optical rotation: $[\alpha]_{\mathrm{D}}^{25}=-131.25\left(c=1.016, \mathrm{CHCl}_{3}, 86 \%\right.$ ee $)$.

HPLC: DAICEL CHIRALPAK AD-H, hexane $/ i-\mathrm{PrOH}=19 / 1$, flow rate: $1.0 \mathrm{~mL} / \mathrm{min}, \lambda=220 \mathrm{~nm}$, $\mathrm{t}_{\mathrm{R}}($ major $)=6.2 \mathrm{~min}, \mathrm{t}_{\mathrm{R}}($ minor $)=7.2 \mathrm{~min}, 86 \%$ ee.

\begin{tabular}{|l|l|l|}
\hline Peak\# & Ret. Time & Area\% \\
\hline 1 & 6.234 & 50.848 \\
\hline 2 & 7.209 & 49.152 \\
\hline
\end{tabular}

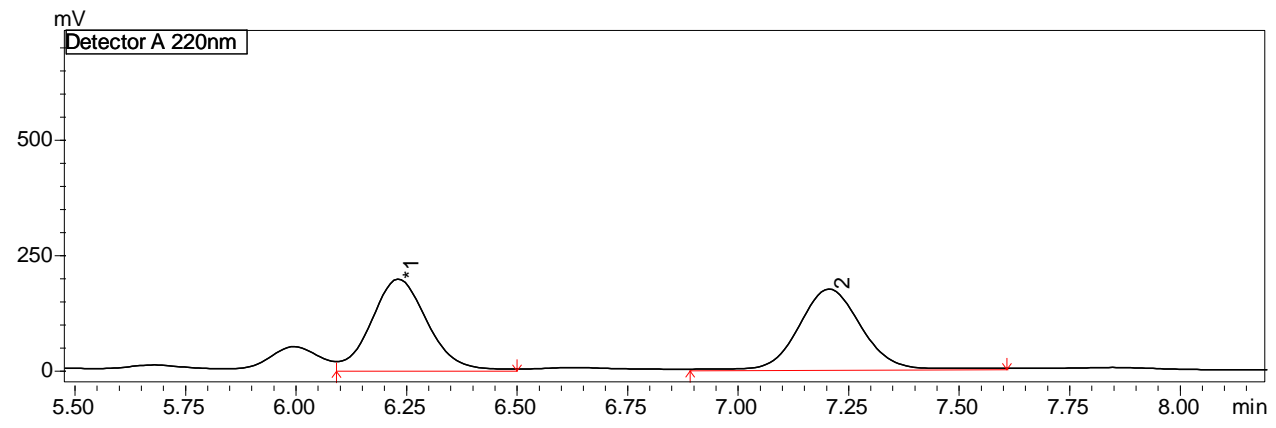

\begin{tabular}{|l|l|l|}
\hline Peak\# & Ret. Time & Area\% \\
\hline 1 & 6.232 & 93.019 \\
\hline 2 & 7.207 & 6.981 \\
\hline
\end{tabular}

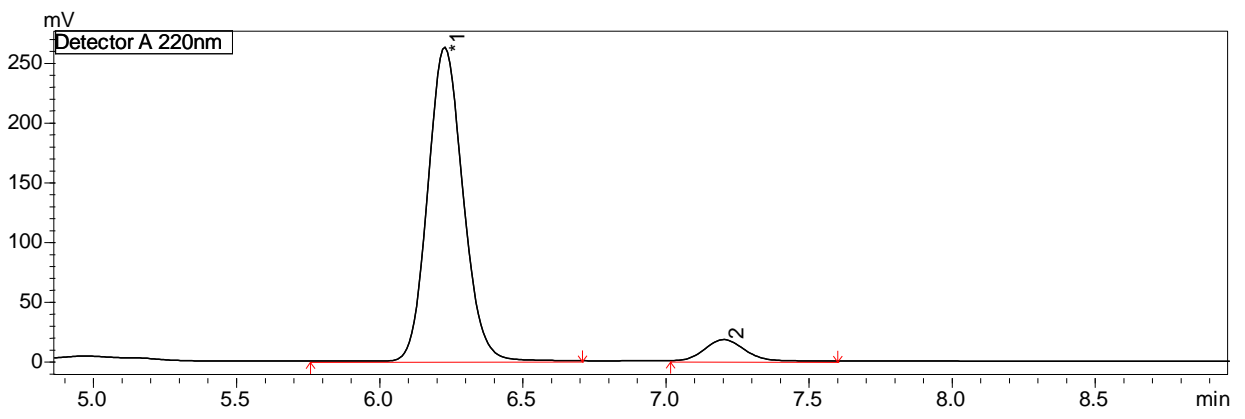




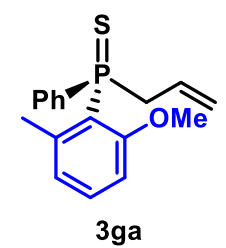

3ga: Procedure A, $61.3 \mathrm{mg}, 88 \%$ yield, light yellow solid.

${ }^{1} \mathbf{H}$ NMR (500 MHz, $\left.\mathbf{C D C l}_{\mathbf{3}}\right) \delta$ 7.80-7.70 (m, 2H), 7.49-7.37 (m, 3H), 7.35-7.29 (m, 1H), 6.83 (dd, $J=8.5,5.0 \mathrm{~Hz}, 1 \mathrm{H}), 6.75(\mathrm{dd}, J=7.7,3.8 \mathrm{~Hz}, 1 \mathrm{H}), 5.64-5.51(\mathrm{~m}, 1 \mathrm{H}), 5.00$ (dd, $J=10.1,4.4$ $\mathrm{Hz}, 1 \mathrm{H}), 4.87-4.78(\mathrm{~m}, 1 \mathrm{H}), 3.87$ (s, 3H), 3.56-3.35 (m, 2H), 2.05 (s, 3H).

${ }^{13} \mathbf{C ~ N M R}\left(101 \mathrm{MHz}, \mathbf{C D C l}_{3}\right) \delta 160.87(\mathrm{~d}, J=3.4 \mathrm{~Hz}), 144.20(\mathrm{~d}, J=6.6 \mathrm{~Hz}), 133.97(\mathrm{~d}, J=80.0$ $\mathrm{Hz}), 132.36$ (d, $J=2.2 \mathrm{~Hz}), 130.80(\mathrm{~d}, J=10.1 \mathrm{~Hz}), 130.71(\mathrm{~d}, J=3.0 \mathrm{~Hz}), 128.66(\mathrm{~d}, J=9.0 \mathrm{~Hz})$, $128.01(\mathrm{~d}, J=12.3 \mathrm{~Hz}), 125.41(\mathrm{~d}, J=9.9 \mathrm{~Hz}), 120.51(\mathrm{~d}, J=14.2 \mathrm{~Hz}), 119.98(\mathrm{~d}, J=79.3 \mathrm{~Hz})$, $108.87(\mathrm{~d}, J=6.5 \mathrm{~Hz}), 55.33,45.02(\mathrm{~d}, J=53.2 \mathrm{~Hz}), 22.56(\mathrm{~d}, J=6.5 \mathrm{~Hz})$.

${ }^{31}$ P NMR (162 MHz, $\left.\mathbf{C D C l}_{3}\right) \delta 39.44$.

HRMS (ESI) $\mathbf{m} / \mathbf{z}$ [M+H] $]^{+}$: calcd. 303.0967, found. 303.0967.

IR (film): $v_{\max }\left(\mathrm{cm}^{-1}\right) 3054,2965,2837,1587,1569,1464,1436,1264,1101,1076,841,776,666$, 587.

Optical rotation: $[\alpha]_{\mathrm{D}}{ }^{25}=-117.45\left(c=1.226, \mathrm{CHCl}_{3}, 82 \%\right.$ ee $)$.

HPLC: DAICEL CHIRALPAK ID, hexane $/ i-\mathrm{PrOH}=4 / 1$, flow rate: $1.0 \mathrm{~mL} / \mathrm{min}, \lambda=254 \mathrm{~nm}$, $\mathrm{t}_{\mathrm{R}}($ major $)=8.0 \mathrm{~min}, \mathrm{t}_{\mathrm{R}}($ minor $)=9.4 \mathrm{~min}, 82 \%$ ee .

\begin{tabular}{|l|l|l|}
\hline Peak\# & Ret. Time & Area\% \\
\hline 1 & 8.051 & 49.853 \\
\hline 2 & 9.385 & 50.147 \\
\hline
\end{tabular}
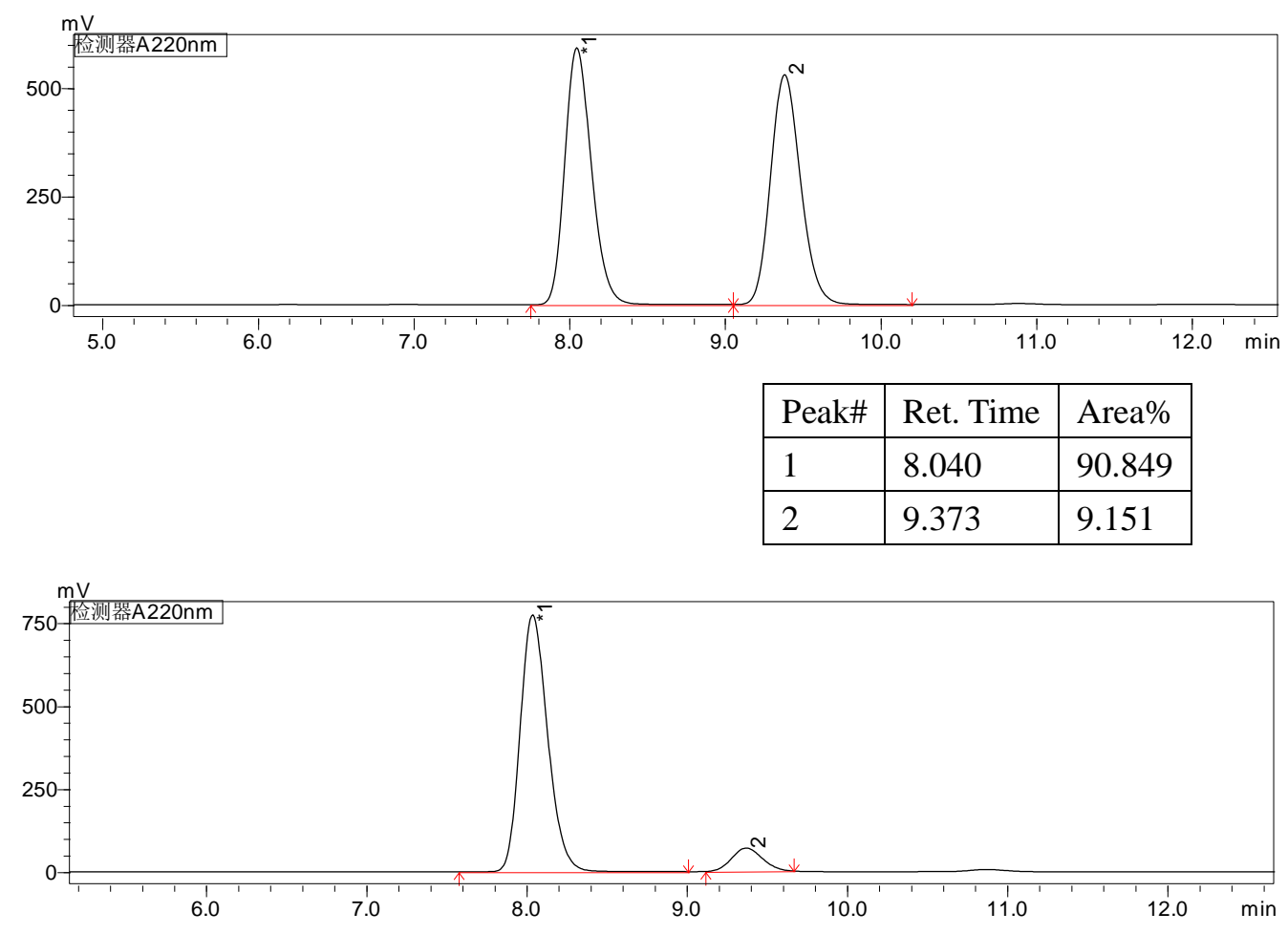


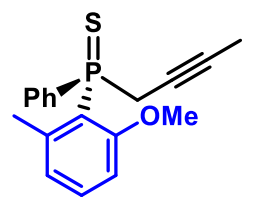

3ge

3ge: Procedure E, $48.8 \mathrm{mg}, 88 \%$ yield, light yellow solid.

${ }^{1}$ H NMR (500 MHz, $\left.\mathbf{C D C l}_{\mathbf{3}}\right) \delta 7.91(\mathrm{dd}, J=13.3,7.6 \mathrm{~Hz}, 2 \mathrm{H}), 7.55-7.47(\mathrm{~m}, 1 \mathrm{H}), 7.47-7.39(\mathrm{~m}$, $2 \mathrm{H}), 7.33(\mathrm{t}, J=8.0 \mathrm{~Hz}, 1 \mathrm{H}), 6.84(\mathrm{dd}, J=8.5,5.2 \mathrm{~Hz}, 1 \mathrm{H}), 6.76(\mathrm{dd}, J=7.7,4.0 \mathrm{~Hz}, 1 \mathrm{H}), 3.87(\mathrm{~s}$, $3 \mathrm{H}), 3.72-3.58(\mathrm{~m}, 1 \mathrm{H}), 3.20-3.07(\mathrm{~m}, 1 \mathrm{H}), 2.00(\mathrm{~s}, 3 \mathrm{H}), 1.70-1.60(\mathrm{~m}, 3 \mathrm{H})$.

${ }^{13} \mathbf{C}$ NMR(101 MHz, CDCl $) \delta 161.28(\mathrm{~d}, J=3.0 \mathrm{~Hz}), 143.97(\mathrm{~d}, J=7.0 \mathrm{~Hz}), 134.13(\mathrm{~d}, J=81.2$ $\mathrm{Hz}), 132.59(\mathrm{~d}, J=2.1 \mathrm{~Hz}), 131.17(\mathrm{~d}, J=3.0 \mathrm{~Hz}), 130.84(\mathrm{~d}, J=9.9 \mathrm{~Hz}), 128.02(\mathrm{~d}, J=12.3 \mathrm{~Hz})$ $125.27(\mathrm{~d}, J=10.5 \mathrm{~Hz}), 118.75(\mathrm{~d}, J=83.0 \mathrm{~Hz}), 109.05(\mathrm{~d}, J=6.5 \mathrm{~Hz}), 79.14(\mathrm{~d}, J=9.4 \mathrm{~Hz})$, $71.72(\mathrm{~d}, J=13.3 \mathrm{~Hz}), 55.69,33.78(\mathrm{~d}, J=57.1 \mathrm{~Hz}), 22.44$ (d, $J=6.5 \mathrm{~Hz}), 3.53(\mathrm{~d}, J=3.2 \mathrm{~Hz})$.

${ }^{31}$ P NMR (162 MHz, CDCl $) \delta 37.98$.

HRMS (ESI) $\mathbf{m} / \mathbf{z}$ [M+H] $]^{+}$: calcd. 315.0967, found. 315.0967.

IR (film): $v_{\max }\left(\mathrm{cm}^{-1}\right)$ 3055, 2916, 1569, 1465, 1267, 1100, 1077, 911, 829, 693, 665.

Optical rotation: $[\alpha]_{\mathrm{D}}{ }^{25}=-41.26\left(c=0.976, \mathrm{CHCl}_{3}, 50 \%\right.$ ee $)$.

HPLC: DAICEL CHIRALPAK AD-H, hexane $/ i-\mathrm{PrOH}=19 / 1$, flow rate: $1.0 \mathrm{~mL} / \mathrm{min}, \lambda=254 \mathrm{~nm}$, $\mathrm{t}_{\mathrm{R}}($ major $)=22.9 \mathrm{~min}, \mathrm{t}_{\mathrm{R}}($ minor $)=26.2 \mathrm{~min}, 50 \%$ ee .

\begin{tabular}{|l|l|l|}
\hline Peak\# & Ret. Time & Area\% \\
\hline 1 & 23.097 & 50.045 \\
\hline 2 & 26.743 & 49.955 \\
\hline
\end{tabular}

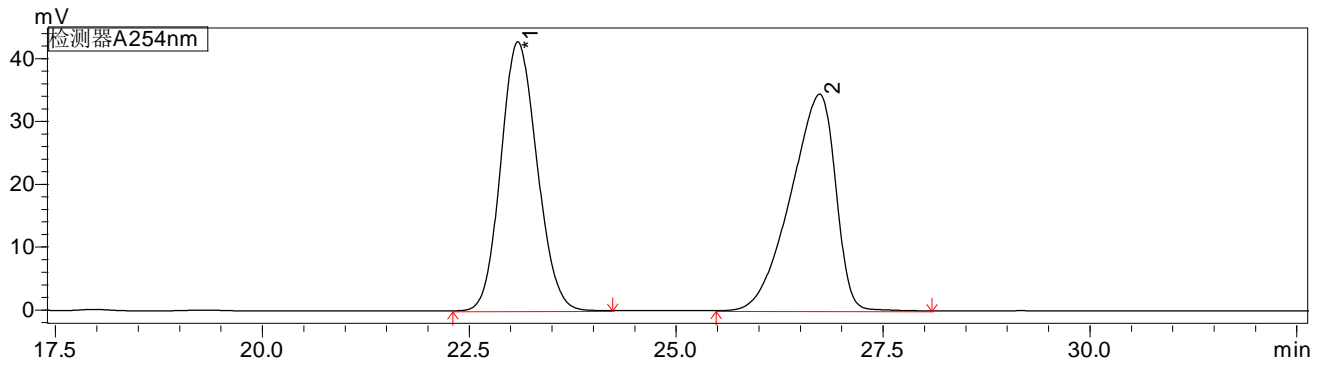

\begin{tabular}{|l|l|l|}
\hline Peak\# & Ret. Time & Area\% \\
\hline 1 & 22.862 & 74.852 \\
\hline 2 & 26.180 & 25.148 \\
\hline
\end{tabular}

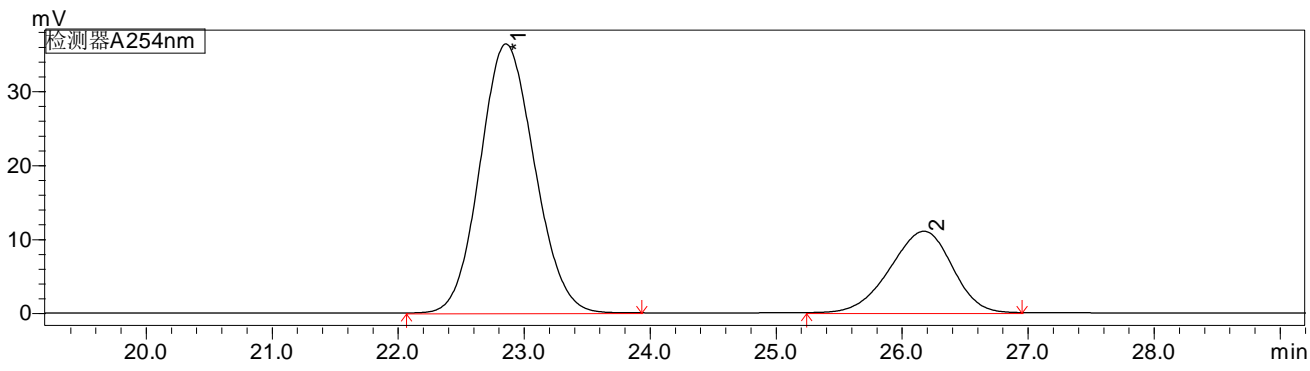




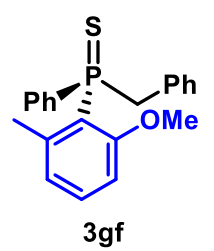

3gf: Procedure A, $67.5 \mathrm{mg}, 86 \%$ yield, light yellow solid.

${ }^{1}$ H NMR (500 MHz, $\left.\mathbf{C D C l}_{3}\right) \delta$ 7.51-7.41 (m, 2H), 7.39-7.30 (m, 2H), 7.26-7.22 (m, 2H), 7.11$7.04(\mathrm{~m}, 1 \mathrm{H}), 7.04-6.95(\mathrm{~m}, 2 \mathrm{H}), 6.87(\mathrm{dd}, J=8.5,5.0 \mathrm{~Hz}, 1 \mathrm{H}), 6.80(\mathrm{dd}, J=7.7,2.7 \mathrm{~Hz}, 2 \mathrm{H})$, 6.78-6.74 (m, 1H), 4.15 (dd, $J=18.5,13.8 \mathrm{~Hz}, 1 \mathrm{H}), 3.94-3.85(\mathrm{~m}, 4 \mathrm{H}), 2.03(\mathrm{~s}, 3 \mathrm{H})$.

${ }^{13} \mathbf{C}$ NMR(101 MHz, CDCl $) \delta 160.92(\mathrm{~d}, J=3.6 \mathrm{~Hz}), 144.39(\mathrm{~d}, J=6.5 \mathrm{~Hz}), 133.74(\mathrm{~d}, J=80.5$ $\mathrm{Hz}), 132.32$ (d, $J=2.2 \mathrm{~Hz}), 131.53(\mathrm{~d}, J=7.6 \mathrm{~Hz}), 130.80(\mathrm{~d}, J=5.7 \mathrm{~Hz}), 130.68$ (d, $J=10.0 \mathrm{~Hz})$, $130.55(\mathrm{~d}, J=3.0 \mathrm{~Hz}), 127.83(\mathrm{~d}, J=12.3 \mathrm{~Hz}), 127.21(\mathrm{~d}, J=3.5 \mathrm{~Hz}), 126.42(\mathrm{~d}, J=4.2 \mathrm{~Hz})$, $125.53(\mathrm{~d}, J=9.9 \mathrm{~Hz}), 120.45$ (d, $J=78.5 \mathrm{~Hz}), 109.00(\mathrm{~d}, J=6.6 \mathrm{~Hz}), 55.39,45.36$ (d, $J=49.1$ $\mathrm{Hz}), 22.68(\mathrm{~d}, J=6.1 \mathrm{~Hz})$.

${ }^{31}$ P NMR (162 MHz, CDCl $) \delta 41.79$.

HRMS (ESI) $\mathbf{m} / \mathbf{z}[\mathbf{M}+\mathbf{H}]^{+}$: calcd. 353.1123 , found. 353.1123 .

IR (film): $v_{\max }\left(\mathrm{cm}^{-1}\right)$ 3057, 2965, 1587, 14951464, 1265, 1102, 1074, 837, 696, 576.

Optical rotation: $[\alpha]_{\mathrm{D}}^{25}=-151.04\left(c=1.350, \mathrm{CHCl}_{3}, 73 \%\right.$ ee $)$.

HPLC: DAICEL CHIRALPAK AD-H, hexane $/ i-\mathrm{PrOH}=9 / 1$, flow rate: $1.0 \mathrm{~mL} / \mathrm{min}, \lambda=254 \mathrm{~nm}$, $t_{R}($ major $)=15.4 \min , t_{R}($ minor $)=19.7 \min , 73 \%$ ee .

\begin{tabular}{|l|l|l|}
\hline Peak\# & Ret. Time & Area\% \\
\hline 1 & 15.607 & 49.993 \\
\hline 2 & 20.122 & 50.007 \\
\hline
\end{tabular}

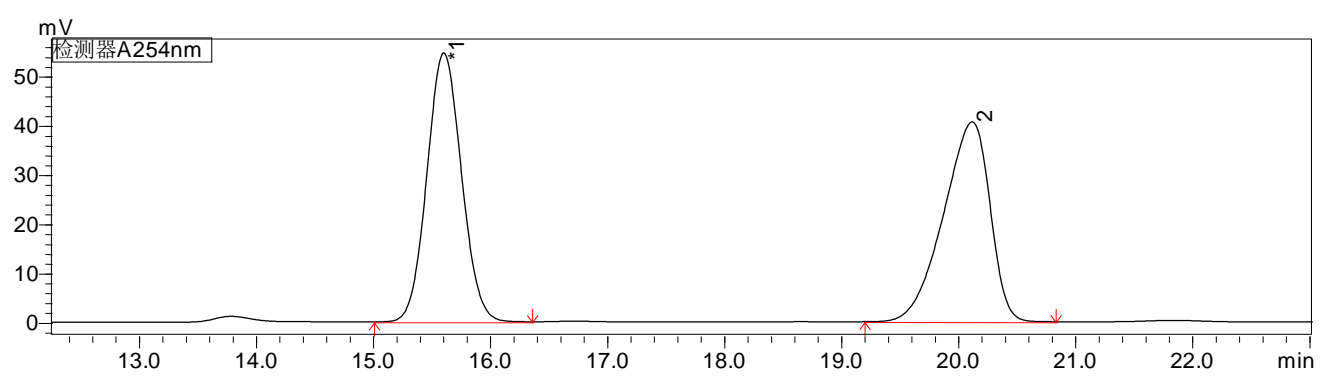

\begin{tabular}{|l|l|l|}
\hline Peak\# & Ret. Time & Area\% \\
\hline 1 & 15.378 & 86.414 \\
\hline 2 & 19.680 & 13.586 \\
\hline
\end{tabular}

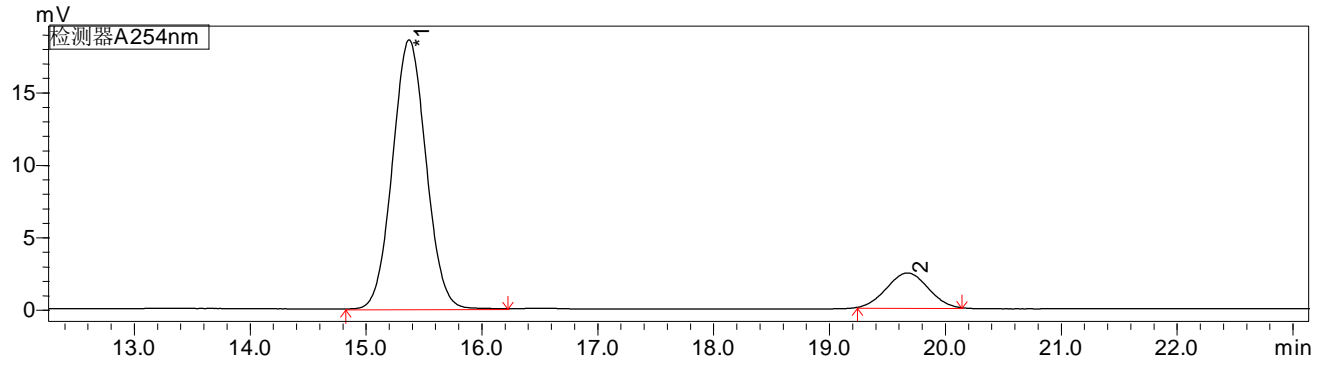




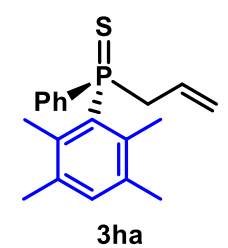

3ha: Procedure A, $59.0 \mathrm{mg}, 94 \%$ yield, yellow solid.

${ }^{1}$ H NMR (400 MHz, $\mathbf{C D C l}_{3}$ ) $\delta$ 7.74-7.61 (m, 2H), 7.49-7.34 (m, 3H), 7.06 (s, 1H), 5.39-5.22 (m, $1 \mathrm{H}), 4.93(\mathrm{dd}, J=10.1,4.0 \mathrm{~Hz}, 1 \mathrm{H}), 4.82(\mathrm{dd}, J=17.0,5.4 \mathrm{~Hz}, 1 \mathrm{H}), 3.44-3.20(\mathrm{~m}, 2 \mathrm{H}), 2.19$ (s, $6 \mathrm{H}), 2.16(\mathrm{~s}, 6 \mathrm{H})$.

${ }^{13} \mathbf{C}$ NMR(101 MHz, $\left.\mathbf{C D C l}_{3}\right) \delta 137.78(\mathrm{~d}, J=9.9 \mathrm{~Hz}), 135.47(\mathrm{~d}, J=11.8 \mathrm{~Hz}), 134.66(\mathrm{~d}, J=$ $79.7 \mathrm{~Hz}), 134.30(\mathrm{~d}, J=3.0 \mathrm{~Hz}), 132.33(\mathrm{~d}, J=82.4 \mathrm{~Hz}), 130.67(\mathrm{~d}, J=9.5 \mathrm{~Hz}), 130.52(\mathrm{~d}, J=$ $3.0 \mathrm{~Hz}), 127.98(\mathrm{~d}, J=12.2 \mathrm{~Hz}), 127.94(\mathrm{~d}, J=8.4 \mathrm{~Hz}), 120.67(\mathrm{~d}, J=13.4 \mathrm{~Hz}), 44.72(\mathrm{~d}, J=$ $51.9 \mathrm{~Hz}), 20.57(\mathrm{~d}, J=6.7 \mathrm{~Hz}), 20.01(\mathrm{~d}, J=1.8 \mathrm{~Hz})$.

${ }^{31}$ P NMR (162 MHz, $\left.\mathbf{C D C l}_{3}\right) \delta 40.88$.

HRMS (ESI) $\mathbf{m} / \mathbf{z}$ [M+H] $]^{+}$: calcd. 315.1331 , found. 315.1331.

IR (film): $v_{\max }\left(\mathrm{cm}^{-1}\right)$ 2954, 2924, 2854, 1633, 1459, 1437, 1381, 1099, 1017, 915, 840, 750, 694, 637, 589.

Optical rotation: $[\alpha]_{\mathrm{D}}^{25}=-69.52\left(c=1.152, \mathrm{CHCl}_{3}, 91 \%\right.$ ee $)$.

HPLC: DAICEL CHIRALPAK IC-3, hexane $/ i-\mathrm{PrOH}=19 / 1$, flow rate: $0.5 \mathrm{~mL} / \mathrm{min}, \lambda=254 \mathrm{~nm}$, $\mathrm{t}_{\mathrm{R}}($ major $)=30.7 \mathrm{~min}, \mathrm{t}_{\mathrm{R}}($ minor $)=32.2 \mathrm{~min}, 91 \%$ ee .
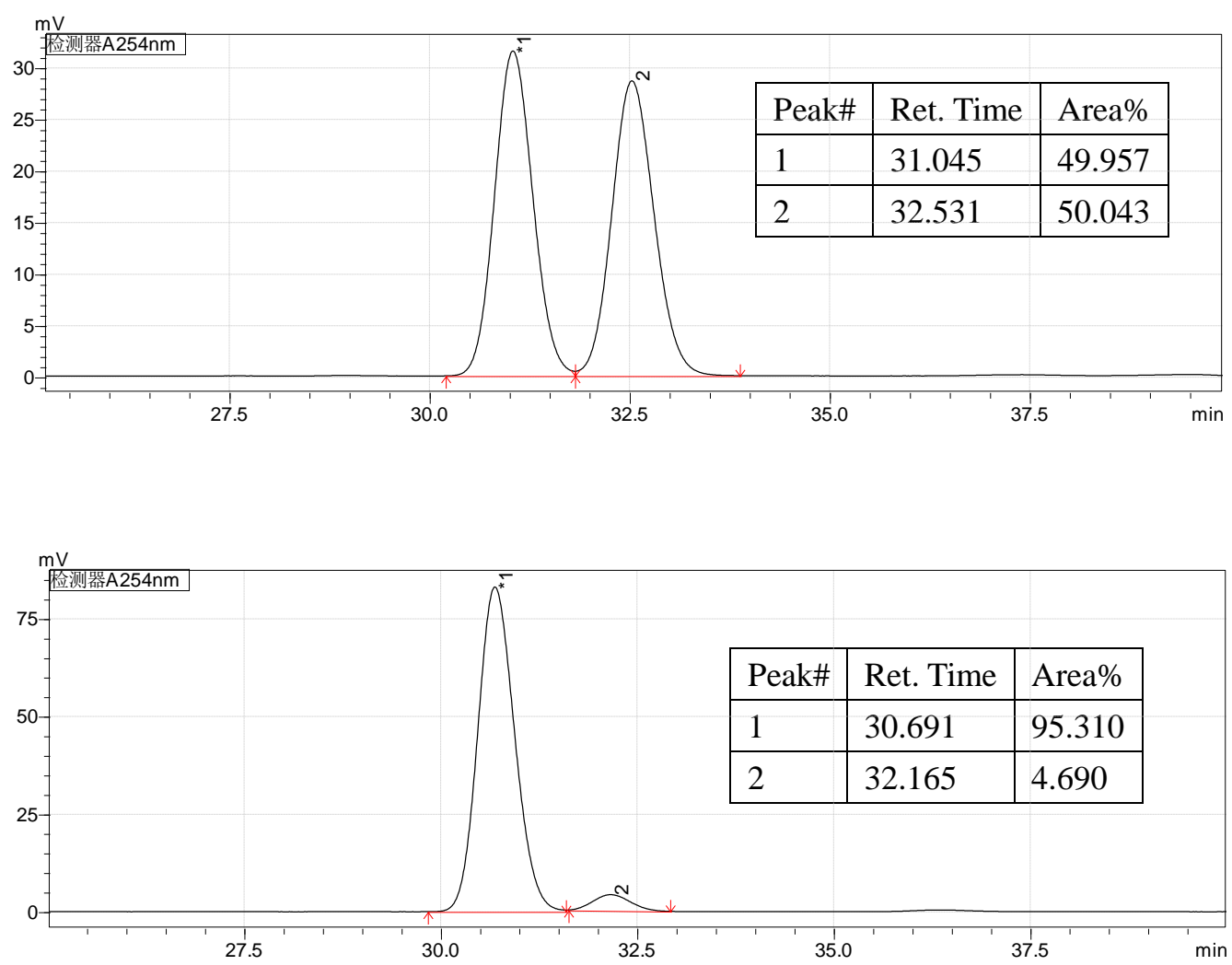


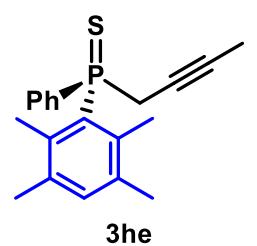

3he: Procedure E, $56.6 \mathrm{mg}$, 94\% yield, light yellow solid.

${ }^{1}$ H NMR (500 MHz, $\left.\mathbf{C D C l}_{\mathbf{3}}\right) \delta 7.86(\mathrm{dd}, J=12.9,7.6 \mathrm{~Hz}, 2 \mathrm{H}), 7.54-7.46(\mathrm{~m}, 1 \mathrm{H}), 7.45-7.39(\mathrm{~m}$, $2 \mathrm{H}), 7.07(\mathrm{~s}, 1 \mathrm{H}), 3.59-3.46(\mathrm{~m}, 1 \mathrm{H}), 3.06-2.95(\mathrm{~m}, 1 \mathrm{H}), 2.21(\mathrm{~s}, 6 \mathrm{H}), 2.18(\mathrm{~s}, 6 \mathrm{H}), 1.63-1.56(\mathrm{~m}$, $3 \mathrm{H})$.

${ }^{13} \mathbf{C}$ NMR(101 MHz, $\left.\mathbf{C D C l}_{3}\right) \delta 138.18(\mathrm{~d}, J=9.9 \mathrm{~Hz}), 135.55(\mathrm{~d}, J=80.7 \mathrm{~Hz}), 135.23(\mathrm{~d}, J=$ $11.9 \mathrm{~Hz}), 134.52(\mathrm{~d}, J=3.0 \mathrm{~Hz}), 130.89(\mathrm{~d}, J=3.0 \mathrm{~Hz}), 130.29$ (d, $J=85.3 \mathrm{~Hz}), 130.29$ (d, $J=$ $9.6 \mathrm{~Hz}), 127.96(\mathrm{~d}, J=12.2 \mathrm{~Hz}), 80.56(\mathrm{~d}, J=8.8 \mathrm{~Hz}), 70.98(\mathrm{~d}, J=13.0 \mathrm{~Hz}), 34.52(\mathrm{~d}, J=54.8$ $\mathrm{Hz}), 20.07$ (d, $J=1.8 \mathrm{~Hz}), 19.86(\mathrm{~d}, J=6.9 \mathrm{~Hz}), 3.42(\mathrm{~d}, J=3.4 \mathrm{~Hz})$.

${ }^{31}$ P NMR (162 MHz, CDCl $\left.\mathbf{3}\right) \delta 39.80$.

HRMS (ESI) $\mathbf{m} / \mathbf{z}$ [M+H] $]^{+}$: calcd. 327.1331, found. 327.1331.

IR (film): $v_{\max }\left(\mathrm{cm}^{-1}\right)$ 2916, 1458, 1437, 1098, 1018, 842, 749, 676, 651.

Optical rotation: $[\alpha]_{\mathrm{D}}{ }^{25}=13.33\left(c=0.943, \mathrm{CHCl}_{3}, 92 \%\right.$ ee $)$.

HPLC: DAICEL CHIRALPAK ID, hexane $/ i-\mathrm{PrOH}=9 / 1$, flow rate: $1.0 \mathrm{~mL} / \mathrm{min}, \lambda=254 \mathrm{~nm}$, $\mathrm{t}_{\mathrm{R}}($ major $)=10.4 \mathrm{~min}, \mathrm{t}_{\mathrm{R}}($ minor $)=8.9 \mathrm{~min}, 92 \%$ ee

\begin{tabular}{|l|l|l|}
\hline Peak\# & Ret. Time & Area\% \\
\hline 1 & 8.855 & 49.960 \\
\hline 2 & 10.389 & 50.040 \\
\hline
\end{tabular}

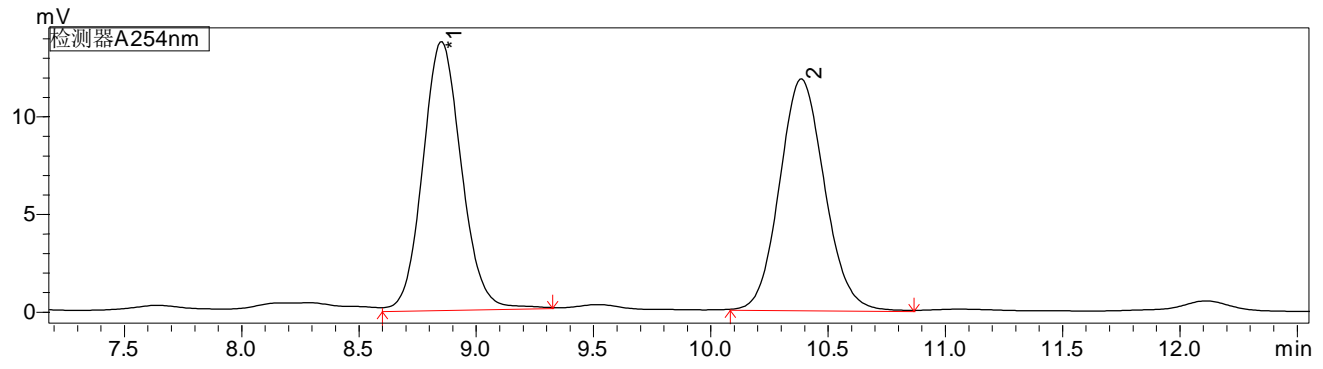

\begin{tabular}{|l|l|l|}
\hline Peak\# & Ret. Time & Area\% \\
\hline 1 & 8.877 & 4.198 \\
\hline 2 & 10.402 & 95.802 \\
\hline
\end{tabular}

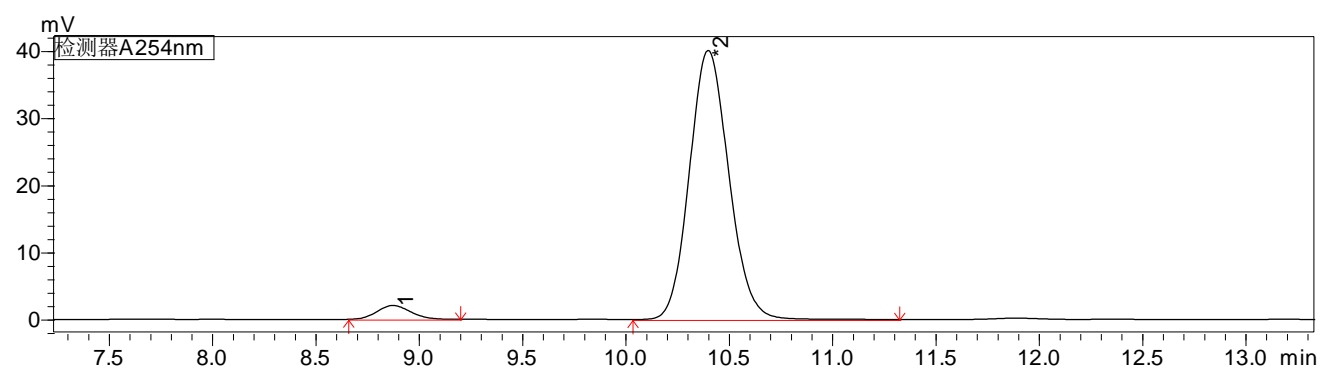




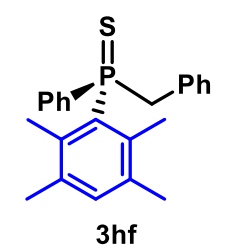

3hf: Procedure A, $64.4 \mathrm{mg}, 96 \%$ yield, light yellow solid.

${ }^{1} \mathbf{H}$ NMR (500 MHz, $\left.\mathbf{C D C l}_{\mathbf{3}}\right) \delta 7.40-7.34(\mathrm{~m}, 2 \mathrm{H}), 7.34-7.29(\mathrm{~m}, 1 \mathrm{H}), 7.19(\mathrm{td}, J=7.7,2.8 \mathrm{~Hz}$, 2H), 7.09 (s, 1H), 7.07-7.00 (m, 1H), 6.97 (t, $J=7.5 \mathrm{~Hz}, 2 \mathrm{H}), 6.75$ (dd, $J=7.7,2.6 \mathrm{~Hz}, 2 \mathrm{H}), 4.12$ (dd, $J=15.7,13.3 \mathrm{~Hz}, 1 \mathrm{H}), 3.60$ (dd, $J=13.3,10.5 \mathrm{~Hz}, 1 \mathrm{H}$ ), 2.25 (s, 6H), 2.19 (s, 6H).

${ }^{13} \mathbf{C}$ NMR(101 MHz, CDCl $) \delta 137.87(\mathrm{~d}, J=9.6 \mathrm{~Hz}), 135.61(\mathrm{~d}, J=11.8 \mathrm{~Hz}), 134.29(\mathrm{~d}, J=3.0$ $\mathrm{Hz}), 134.14(\mathrm{~d}, J=80.5 \mathrm{~Hz}), 133.13(\mathrm{~d}, J=81.7 \mathrm{~Hz}), 131.61(\mathrm{~d}, J=6.9 \mathrm{~Hz}), 130.61(\mathrm{~d}, J=9.3$ $\mathrm{Hz}), 130.39$ (d, $J=5.5 \mathrm{~Hz}), 130.30(\mathrm{~d}, J=3.0 \mathrm{~Hz}), 127.75(\mathrm{~d}, J=12.2 \mathrm{~Hz}), 127.37$ (d, $J=3.4 \mathrm{~Hz})$, $126.44(\mathrm{~d}, J=3.9 \mathrm{~Hz}), 45.27(\mathrm{~d}, J=48.3 \mathrm{~Hz}), 21.05(\mathrm{~d}, J=6.2 \mathrm{~Hz}), 20.11(\mathrm{~d}, J=1.6 \mathrm{~Hz})$.

${ }^{31}$ P NMR (162 MHz, $\left.\mathbf{C D C l}_{3}\right) \delta 43.13$.

HRMS (ESI) $\mathbf{m} / \mathbf{z}[\mathbf{M}+\mathbf{H}]^{+}$: calcd. 365.1487, found. 365.1487 .

IR (film): $v_{\max }\left(\mathrm{cm}^{-1}\right) 3057,2921,1454,1437,1101,1068,911,834,748,696,611$.

Optical rotation: $[\alpha]_{\mathrm{D}}{ }^{25}=-104.55\left(c=1.073, \mathrm{CHCl}_{3}, 82 \%\right.$ ee $)$.

HPLC: DAICEL CHIRALPAK IC-3, hexane $/ i-\mathrm{PrOH}=19 / 1$, flow rate: $0.5 \mathrm{~mL} / \mathrm{min}, \lambda=254 \mathrm{~nm}$, $\mathrm{t}_{\mathrm{R}}($ major $)=32.0 \mathrm{~min}, \mathrm{t}_{\mathrm{R}}($ minor $)=34.7 \mathrm{~min}, 82 \%$ ee.

\begin{tabular}{|l|l|l|}
\hline Peak\# & Ret. Time & Area\% \\
\hline 1 & 31.746 & 50.039 \\
\hline 2 & 34.350 & 49.961 \\
\hline
\end{tabular}

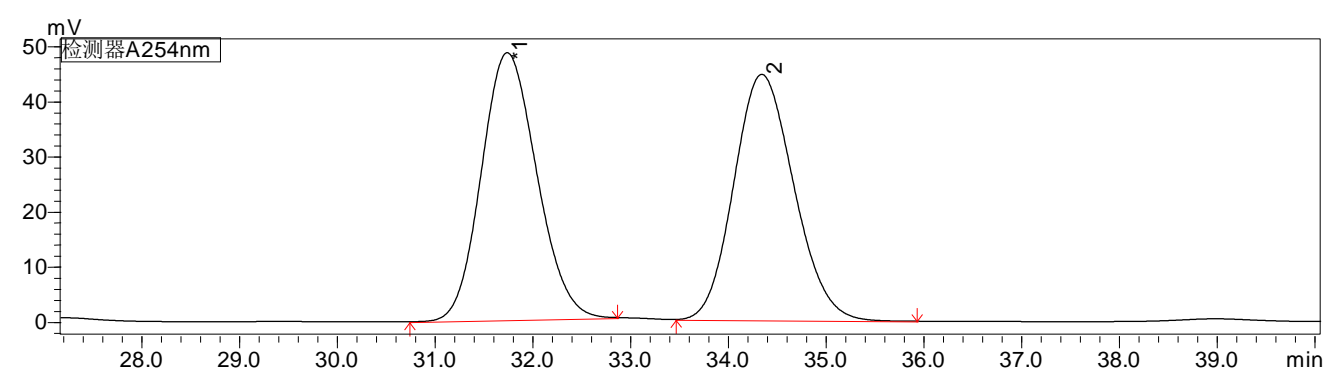

\begin{tabular}{|l|l|l|}
\hline Peak\# & Ret. Time & Area\% \\
\hline 1 & 31.955 & 91.089 \\
\hline 2 & 34.700 & 8.911 \\
\hline
\end{tabular}

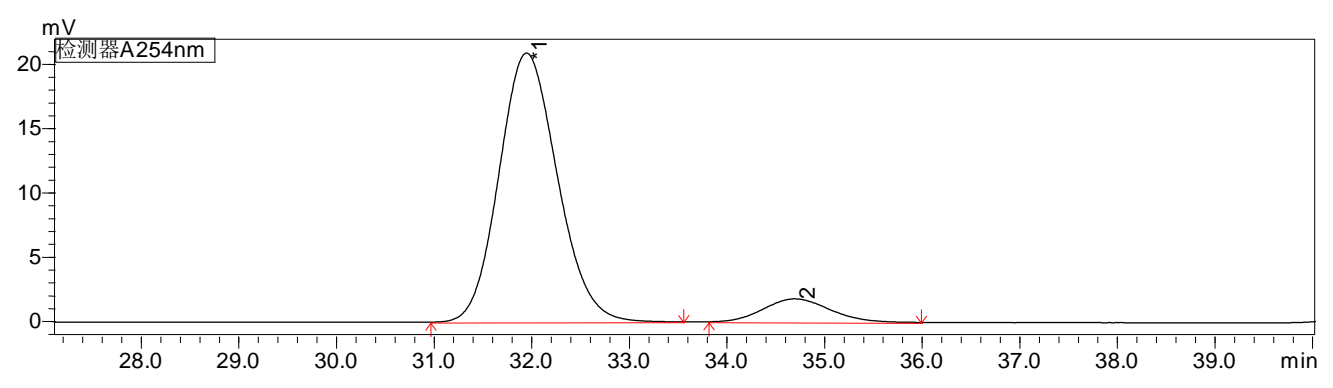




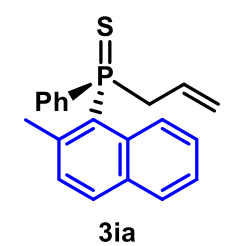

3ia: Procedure A, $56.8 \mathrm{mg}, 88 \%$ yield, light yellow solid.

${ }^{1}$ H NMR (400 MHz, $\left.\mathbf{C D C l}_{3}\right) \delta 8.41(\mathrm{~d}, J=8.6 \mathrm{~Hz}, 1 \mathrm{H}), 7.85-7.75(\mathrm{~m}, 2 \mathrm{H}), 7.75-7.67(\mathrm{~m}, 2 \mathrm{H})$, $7.49-7.28(\mathrm{~m}, 5 \mathrm{H}), 7.23(\mathrm{dd}, J=8.4,4.2 \mathrm{~Hz}, 1 \mathrm{H}), 5.49-5.33(\mathrm{~m}, 1 \mathrm{H}), 4.97$ (dd, $J=10.3,4.2 \mathrm{~Hz}$, $1 \mathrm{H}), 4.85(\mathrm{dd}, J=17.2,5.3 \mathrm{~Hz}, 1 \mathrm{H}), 3.65-3.50(\mathrm{~m}, 1 \mathrm{H}), 3.43-3.28(\mathrm{~m}, 1 \mathrm{H}), 2.42(\mathrm{~s}, 3 \mathrm{H})$.

${ }^{13} \mathbf{C}$ NMR(101 MHz, CDCl $) \delta 141.73(\mathrm{~d}, J=8.0 \mathrm{~Hz}), 134.08(\mathrm{~d}, J=79.8 \mathrm{~Hz}), 133.07(\mathrm{~d}, J=9.6$ Hz), 132.40 (d, $J=9.1 \mathrm{~Hz}), 131.92$ (d, $J=3.3 \mathrm{~Hz}), 130.97$ (d, $J=9.8 \mathrm{~Hz}), 130.90$ (d, $J=3.6 \mathrm{~Hz})$, $130.09(\mathrm{~d}, J=12.5 \mathrm{~Hz}), 128.54(\mathrm{~d}, J=1.2 \mathrm{~Hz}), 128.19(\mathrm{~d}, J=12.3 \mathrm{~Hz}), 127.68(\mathrm{~d}, J=8.4 \mathrm{~Hz})$, $127.26(\mathrm{~d}, J=80.0 \mathrm{~Hz}), 126.67(\mathrm{~d}, J=7.3 \mathrm{~Hz}), 125.63(\mathrm{~d}, J=66.6 \mathrm{~Hz}), 121.04(\mathrm{~d}, J=13.5 \mathrm{~Hz})$, $44.79(\mathrm{~d}, J=52.2 \mathrm{~Hz}), 24.58(\mathrm{~d}, J=6.0 \mathrm{~Hz})$.

${ }^{31}$ P NMR (162 MHz, CDCl $) \delta 38.69$.

HRMS (ESI) $\mathbf{m} / \mathbf{z}$ [M+H] ${ }^{+}$: calcd. 323.1018, found. 323.1018.

IR (film): $v_{\max }\left(\mathrm{cm}^{-1}\right)$ 3050, 2955, 2924, 2853, 1631, 1506, 1461, 1436, 1377, 1098, 812, 743, 630.

Optical rotation: $[\alpha]_{\mathrm{D}}{ }^{25}=-89.77\left(c=1.108, \mathrm{CHCl}_{3}, 86 \%\right.$ ee $)$.

HPLC: DAICEL CHIRALPAK ID, hexane $/ i-\mathrm{PrOH}=9 / 1$, flow rate: $1.0 \mathrm{~mL} / \mathrm{min}, \lambda=254 \mathrm{~nm}$, $\mathrm{t}_{\mathrm{R}}($ major $)=9.0 \mathrm{~min}, \mathrm{t}_{\mathrm{R}}($ minor $)=9.8 \mathrm{~min}, 86 \%$ ee.
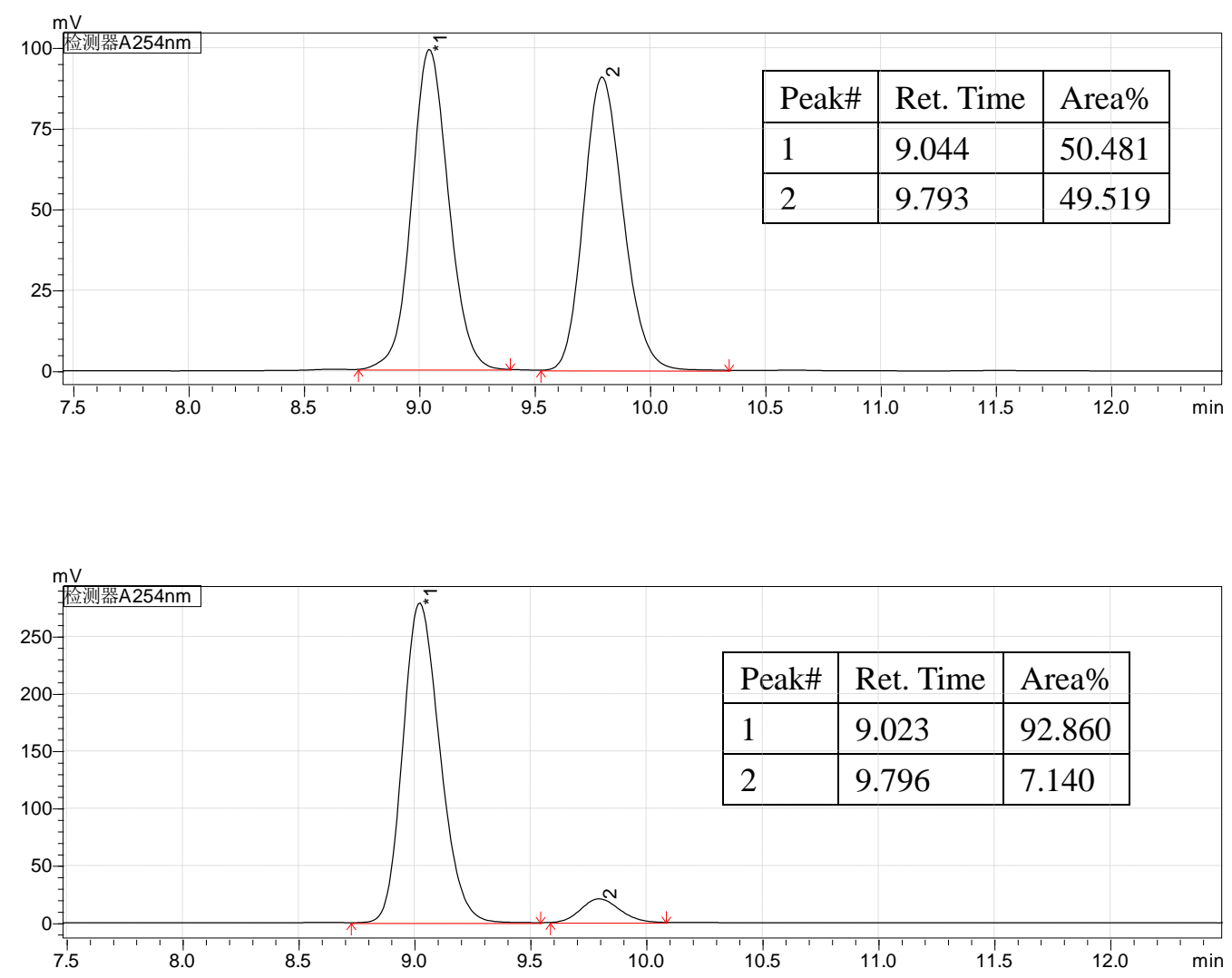


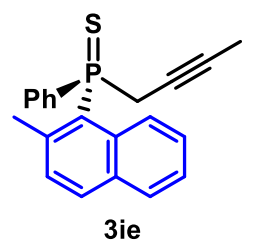

3ie: Procedure E, $54.1 \mathrm{mg}$, 95\% yield, light yellow solid.

${ }^{1}$ H NMR $\left(\mathbf{5 0 0} \mathbf{~ M H z}, \mathbf{C D C l}_{3}\right) \delta 8.14(\mathrm{~d}, J=8.7 \mathrm{~Hz}, 1 \mathrm{H}), 7.89-7.80(\mathrm{~m}, 3 \mathrm{H}), 7.77(\mathrm{~d}, J=8.1 \mathrm{~Hz}$, 1H), 7.52-7.44 (m, 1H), 7.43-7.32 (m, 3H), 7.32-7.27 (m, 1H), 7.21 (t, J = 7.8 Hz, 1H), 3.71$3.57(\mathrm{~m}, 1 \mathrm{H}), 3.29-3.17(\mathrm{~m}, 1 \mathrm{H}), 2.67(\mathrm{~s}, 3 \mathrm{H}), 1.54(\mathrm{dt}, J=5.5,2.6 \mathrm{~Hz}, 3 \mathrm{H})$.

${ }^{13} \mathbf{C}$ NMR(101 MHz, CDCl $) \delta 142.19(\mathrm{~d}, J=8.0 \mathrm{~Hz}), 134.81(\mathrm{~d}, J=80.9 \mathrm{~Hz}), 133.08(\mathrm{~d}, J=9.7$ $\mathrm{Hz}), 132.41(\mathrm{~d}, J=9.4 \mathrm{~Hz}), 132.19(\mathrm{~d}, J=3.3 \mathrm{~Hz}), 131.20(\mathrm{~d}, J=3.0 \mathrm{~Hz}), 130.77$ (d, $J=9.8 \mathrm{~Hz})$, $130.02(\mathrm{~d}, J=12.9 \mathrm{~Hz}), 128.43(\mathrm{~d}, J=1.4 \mathrm{~Hz}), 128.07(\mathrm{~d}, J=12.3 \mathrm{~Hz}), 126.81(\mathrm{~d}, J=8.3 \mathrm{~Hz})$, $125.56(\mathrm{~d}, J=67.1 \mathrm{~Hz}), 125.55(\mathrm{~d}, J=82.7 \mathrm{~Hz}), 81.17(\mathrm{~d}, J=8.9 \mathrm{~Hz}), 70.69(\mathrm{~d}, J=13.2 \mathrm{~Hz})$, $34.47(\mathrm{~d}, J=54.5 \mathrm{~Hz}), 23.71(\mathrm{~d}, J=6.1 \mathrm{~Hz}), 3.38(\mathrm{~d}, J=3.4 \mathrm{~Hz})$.

${ }^{31}$ P NMR (162 MHz, $\left.\mathbf{C D C l}_{3}\right) \delta 38.28$.

HRMS (ESI) $\mathbf{m} / \mathbf{z}$ [M+H] $]^{+}$: calcd. 335.1018, found. 335.1018.

IR (film): $v_{\max }\left(\mathrm{cm}^{-1}\right)$ 3051, 2915, 1478, 1437, 1098, 911, 814, 743, 668, 609.

Optical rotation: $[\alpha]_{\mathrm{D}}{ }^{25}=-15.01\left(c=0.900, \mathrm{CHCl}_{3}, 90 \%\right.$ ee $)$.

HPLC: DAICEL CHIRALPAK ID, hexane $/ i-\mathrm{PrOH}=9 / 1$, flow rate: $1.0 \mathrm{~mL} / \mathrm{min}, \lambda=254 \mathrm{~nm}$, $\mathrm{t}_{\mathrm{R}}($ major $)=12.3 \mathrm{~min}, \mathrm{t}_{\mathrm{R}}($ minor $)=13.2 \mathrm{~min}, 90 \%$ ee .

\begin{tabular}{|l|l|l|}
\hline Peak\# & Ret. Time & Area\% \\
\hline 1 & 12.311 & 49.692 \\
\hline 2 & 13.140 & 50.308 \\
\hline
\end{tabular}

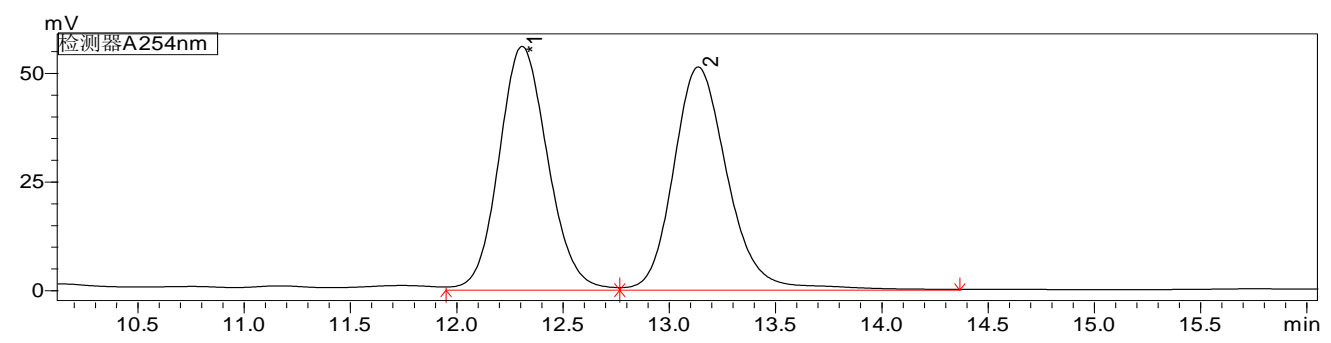

\begin{tabular}{|l|l|l|}
\hline Peak\# & Ret. Time & Area\% \\
\hline 1 & 12.321 & 94.863 \\
\hline 2 & 13.167 & 5.137 \\
\hline
\end{tabular}

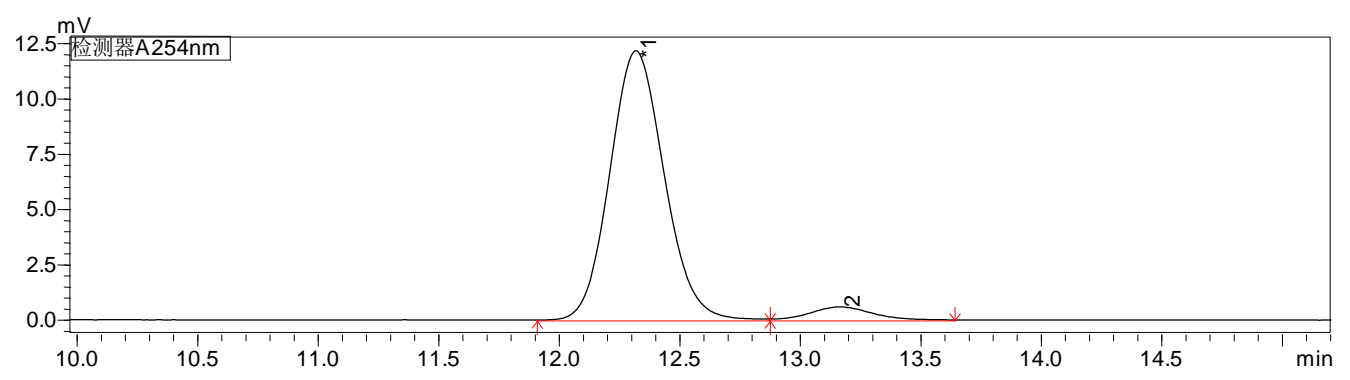




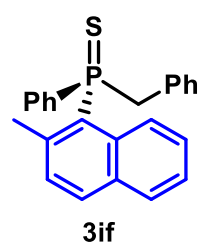

3if: Procedure A, $66.3 \mathrm{mg}, 89 \%$ yield, light yellow solid.

${ }^{1}$ H NMR $\left(\mathbf{5 0 0 ~ M H z}, \mathbf{C D C l}_{\mathbf{3}}\right) \delta 8.43(\mathrm{~d}, J=8.6 \mathrm{~Hz}, 1 \mathrm{H}), 7.84(\mathrm{~d}, J=8.4 \mathrm{~Hz}, 1 \mathrm{H}), 7.79(\mathrm{~d}, J=8.1$ $\mathrm{Hz}, 1 \mathrm{H}), 7.45-7.35(\mathrm{~m}, 3 \mathrm{H}), 7.34-7.25(\mathrm{~m}, 3 \mathrm{H}), 7.22-7.16(\mathrm{~m}, 2 \mathrm{H}), 7.10-7.03(\mathrm{~m}, 1 \mathrm{H}), 6.99$ (t, $J$ $=7.5 \mathrm{~Hz}, 2 \mathrm{H}), 6.77(\mathrm{dd}, J=7.6,2.6 \mathrm{~Hz}, 2 \mathrm{H}), 4.27(\mathrm{t}, J=14.3 \mathrm{~Hz}, 1 \mathrm{H}), 3.75(\mathrm{dd}, J=13.3,10.9 \mathrm{~Hz}$, $1 \mathrm{H}), 2.51(\mathrm{~s}, 3 \mathrm{H})$.

${ }^{13} \mathbf{C}$ NMR(101 MHz, $\left.\mathbf{C D C l}_{3}\right) \delta 141.79(\mathrm{~d}, J=8.0 \mathrm{~Hz}), 133.58(\mathrm{~d}, J=80.5 \mathrm{~Hz}), 133.13(\mathrm{~d}, J=9.8$ $\mathrm{Hz}), 132.51$ (d, $J=8.9 \mathrm{~Hz}), 131.89$ (d, $J=3.1 \mathrm{~Hz}), 131.19$ (d, $J=7.2 \mathrm{~Hz}), 130.93$ (d, $J=9.8 \mathrm{~Hz})$, $130.67(\mathrm{~d}, J=3.0 \mathrm{~Hz}), 130.50(\mathrm{~d}, J=5.6 \mathrm{~Hz}), 130.16(\mathrm{~d}, J=12.3 \mathrm{~Hz}), 128.56(\mathrm{~d}, J=1.2 \mathrm{~Hz})$, $128.07(\mathrm{~d}, J=20.3 \mathrm{~Hz}), 127.91(\mathrm{~d}, J=12.4 \mathrm{~Hz}), 127.43(\mathrm{~d}, J=3.4 \mathrm{~Hz}), 127.40(\mathrm{~d}, J=3.0 \mathrm{~Hz})$, $127.05(\mathrm{~d}, J=7.3 \mathrm{~Hz}), 126.64(\mathrm{~d}, J=4.1 \mathrm{~Hz}), 125.59(\mathrm{~d}, J=59.6 \mathrm{~Hz}), 45.48(\mathrm{~d}, J=48.4 \mathrm{~Hz})$, $25.00(\mathrm{~d}, J=5.6 \mathrm{~Hz})$.

${ }^{31}$ P NMR (162 MHz, $\left.\mathbf{C D C l}_{3}\right) \delta 40.78$.

HRMS (ESI) $\mathbf{m} / \mathbf{z}[\mathbf{M}+\mathbf{H}]^{+}$: calcd. 373.1174, found. 373.1175 .

IR (film): $v_{\max }\left(\mathrm{cm}^{-1}\right)$ 3054, 2923, 1599, 1437, 1100, 814, 696, 607, 501.

Optical rotation: $[\alpha]_{\mathrm{D}}{ }^{25}=-107.76\left(c=1.312, \mathrm{CHCl}_{3}, 81 \%\right.$ ee $)$.

HPLC: DAICEL CHIRALPAK ID, hexane $/ i-\mathrm{PrOH}=9 / 1$, flow rate: $1.0 \mathrm{~mL} / \mathrm{min}, \lambda=254 \mathrm{~nm}$, $\mathrm{t}_{\mathrm{R}}($ major $)=12.3 \mathrm{~min}, \mathrm{t}_{\mathrm{R}}($ minor $)=13.6 \mathrm{~min}, 81 \%$ ee .
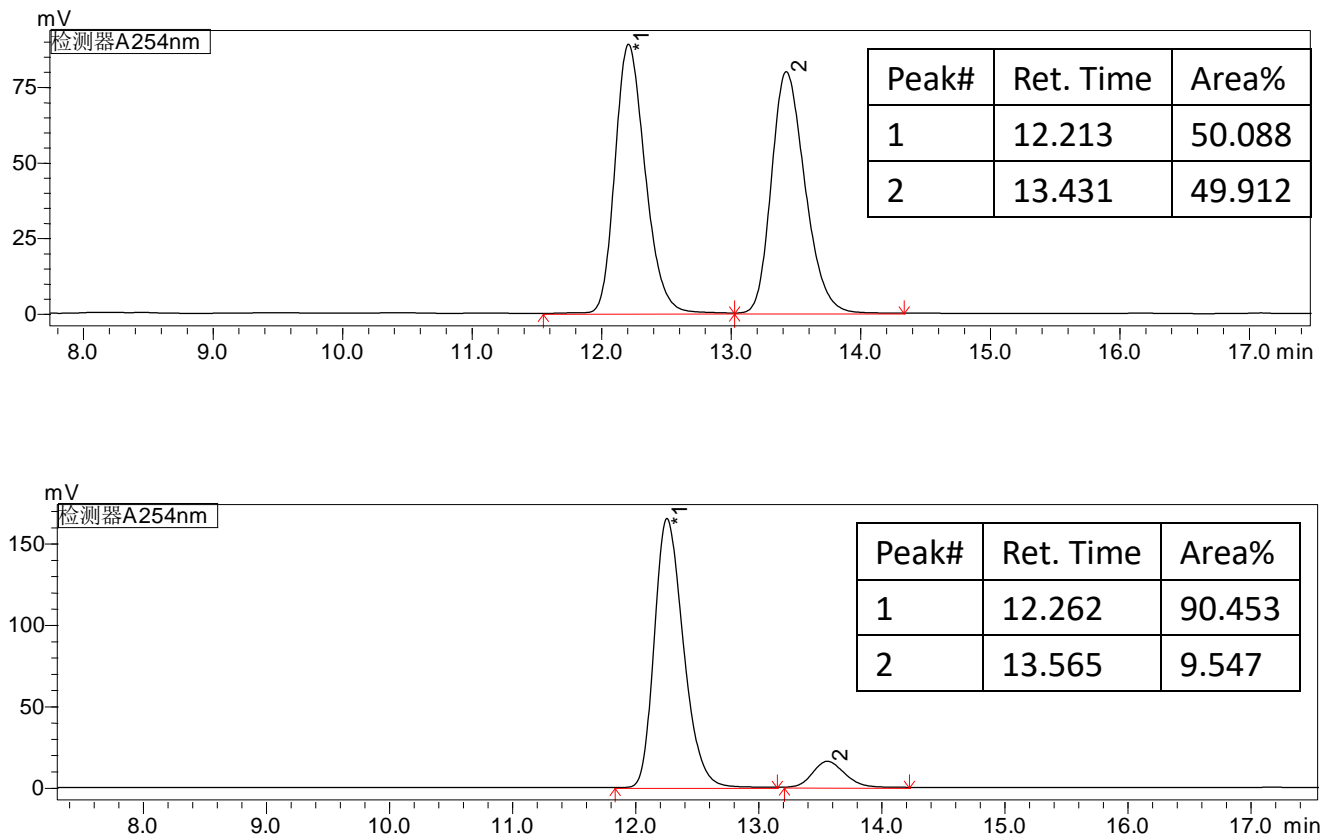


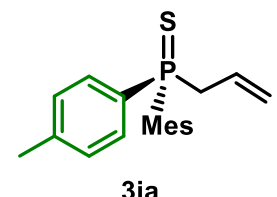

3ja: Procedure A, $51.6 \mathrm{mg}, 82 \%$ yield, light yellow solid.

${ }^{1} \mathbf{H}$ NMR (400 MHz, $\left.\mathbf{C D C l}_{3}\right) \delta$ 7.67-7.53 (m, 2H), 7.25-7.17 (m, 2H), $6.84(\mathrm{~d}, J=4.0 \mathrm{~Hz}, 2 \mathrm{H})$, 5.49-5.31 (m, 1H), 5.05-4.94 (m, 1H), 4.94-4.81 (m, 1H), 3.44-3.24 (m, 2H), $2.39(\mathrm{~s}, 3 \mathrm{H}), 2.30$ (s, 6H), $2.26(\mathrm{~s}, 3 \mathrm{H})$.

${ }^{13} \mathbf{C}$ NMR(101 MHz, CDCl $) \delta 141.44(\mathrm{~d}, J=10.0 \mathrm{~Hz}), 141.13(\mathrm{~d}, J=2.8 \mathrm{~Hz}), 140.76(\mathrm{~d}, J=3.0$ $\mathrm{Hz}), 131.20(\mathrm{~d}, J=11.2 \mathrm{~Hz}), 130.85(\mathrm{~d}, J=10.3 \mathrm{~Hz}), 130.74(\mathrm{~d}, J=82.2 \mathrm{~Hz}), 128.91(\mathrm{~d}, J=12.7$ $\mathrm{Hz}), 128.85(\mathrm{~d}, J=80.9 \mathrm{~Hz}), 127.87(\mathrm{~d}, J=8.5 \mathrm{~Hz}), 120.86(\mathrm{~d}, J=13.7 \mathrm{~Hz}), 44.76(\mathrm{~d}, J=51.2$ $\mathrm{Hz}), 23.98(\mathrm{~d}, J=5.2 \mathrm{~Hz}), 21.39(\mathrm{~d}, J=1.5 \mathrm{~Hz}), 20.78(\mathrm{~d}, J=1.3 \mathrm{~Hz})$.

${ }^{31}$ P NMR (162 MHz, $\left.\mathbf{C D C l}_{3}\right) \delta 40.71$.

HRMS (ESI) $\mathbf{m} / \mathbf{z}$ [M+H] ${ }^{+}$: calcd. 315.1331, found. 315.1330.

IR (film): $v_{\max }\left(\mathrm{cm}^{-1}\right)$ 2966, 2920, 1602, 1455, 1397, 1100, 917, 845, 809, 659, 613.

Optical rotation: $[\alpha]_{D}^{25}=-115.70\left(c=1.022, \mathrm{CHCl}_{3}, 93 \%\right.$ ee $)$.

HPLC: DAICEL CHIRALPAK AD-H, hexane $/ i-\mathrm{PrOH}=19 / 1$, flow rate: $1.0 \mathrm{~mL} / \mathrm{min}, \lambda=254 \mathrm{~nm}$, $\mathrm{t}_{\mathrm{R}}($ major $)=6.7 \mathrm{~min}, \mathrm{t}_{\mathrm{R}}($ minor $)=8.6 \mathrm{~min}, 93 \%$ ee.

\begin{tabular}{|l|l|l|}
\hline Peak\# & Ret. Time & Area\% \\
\hline 1 & 6.707 & 50.042 \\
\hline 2 & 8.663 & 49.958 \\
\hline
\end{tabular}

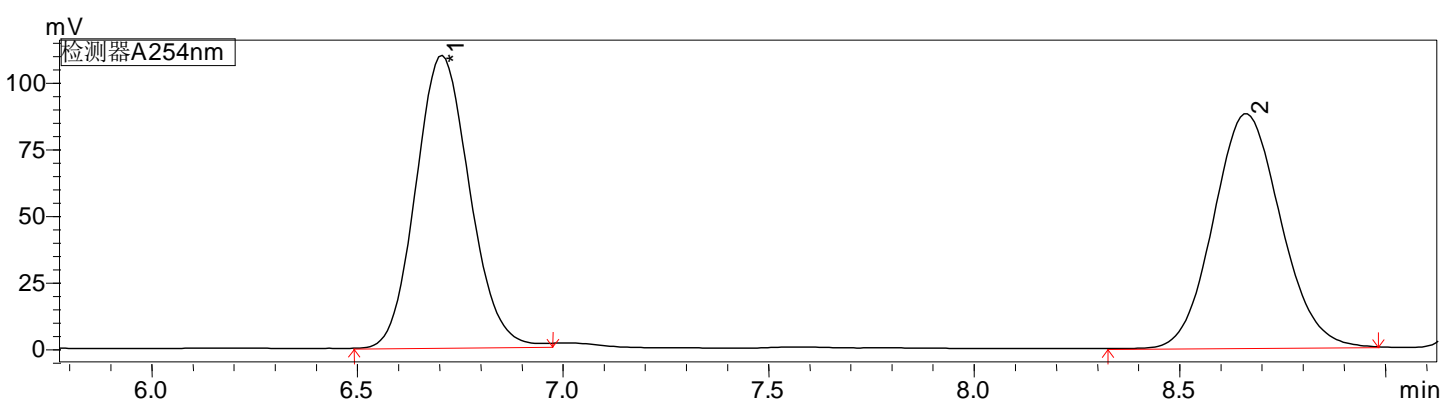

\begin{tabular}{|l|l|l|}
\hline Peak\# & Ret. Time & Area\% \\
\hline 1 & 6.695 & 96.511 \\
\hline 2 & 8.646 & 3.489 \\
\hline
\end{tabular}

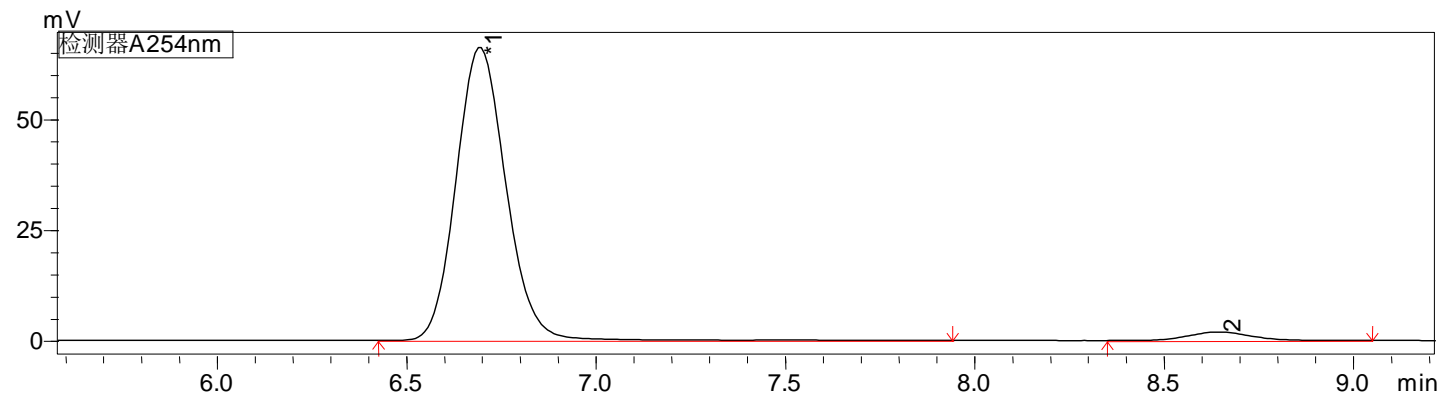




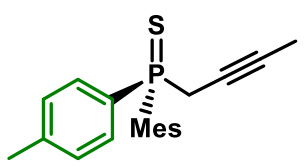

3je

3je: Procedure E, $47.7 \mathrm{mg}, 73 \%$ yield, light yellow solid.

${ }^{1}$ H NMR (400 MHz, CDCl $\left.{ }_{3}\right) \delta 7.91-7.70(\mathrm{~m}, 2 \mathrm{H}), 7.29-7.20(\mathrm{~m}, 2 \mathrm{H}), 6.85(\mathrm{~d}, J=4.2 \mathrm{~Hz}, 2 \mathrm{H})$, 3.64-3.46 (m, 1H), 3.09-2.91 (m, 1H), 2.41 (s, 3H), 2.34 (d, $J=1.2 \mathrm{~Hz}, 6 \mathrm{H}), 2.28(\mathrm{~s}, 3 \mathrm{H}), 1.66$ (dt, $J=5.6,2.6 \mathrm{~Hz}, 3 \mathrm{H})$.

${ }^{13} \mathbf{C}$ NMR(101 MHz, CDCl $) \delta 141.83(\mathrm{~d}, J=10.0 \mathrm{~Hz}), 141.58(\mathrm{~d}, J=2.9 \mathrm{~Hz}), 140.92(\mathrm{~d}, J=3.0$ Hz), $131.25(\mathrm{~d}, J=82.5 \mathrm{~Hz}) 131.05(\mathrm{~d}, J=11.4 \mathrm{~Hz}), 130.63(\mathrm{~d}, J=10.3 \mathrm{~Hz}), 128.79$ (d, $J=12.7$ $\mathrm{Hz}), 126.86(\mathrm{~d}, J=84.0 \mathrm{~Hz}), 80.50(\mathrm{~d}, J=8.6 \mathrm{~Hz}), 71.16(\mathrm{~d}, J=13.0 \mathrm{~Hz}), 34.69(\mathrm{~d}, J=53.8 \mathrm{~Hz})$, $23.17(\mathrm{~d}, J=5.5 \mathrm{~Hz}), 21.45(\mathrm{~d}, J=1.6 \mathrm{~Hz}), 20.87(\mathrm{~d}, J=1.5 \mathrm{~Hz}), 3.51(\mathrm{~d}, J=3.1 \mathrm{~Hz})$.

${ }^{31}$ P NMR (162 MHz, $\left.\mathbf{C D C l}_{3}\right) \delta 39.15$.

HRMS (ESI) $\mathbf{m} / \mathbf{z}$ [M+H] $]^{+}$: calcd. 327.1331, found. 327.1329.

IR (film): $v_{\max }\left(\mathrm{cm}^{-1}\right) 2963,2918,1603,1455,1397,1377,1099,829,808,676,628$.

Optical rotation: $[\alpha]_{\mathrm{D}}{ }^{25}=-6.08\left(c=0.946, \mathrm{CHCl}_{3}, 89 \%\right.$ ee $)$.

HPLC: DAICEL CHIRALPAK AD-H, hexane $/ i-\mathrm{PrOH}=19 / 1$, flow rate: $1.0 \mathrm{~mL} / \mathrm{min}, \lambda=254 \mathrm{~nm}$, $\mathrm{t}_{\mathrm{R}}($ major $)=8.2 \mathrm{~min}, \mathrm{t}_{\mathrm{R}}($ minor $)=13.7 \mathrm{~min}, 89 \%$ ee .

\begin{tabular}{|l|l|l|}
\hline Peak\# & Ret. Time & Area\% \\
\hline 1 & 8.214 & 50.002 \\
\hline 2 & 13.678 & 49.998 \\
\hline
\end{tabular}

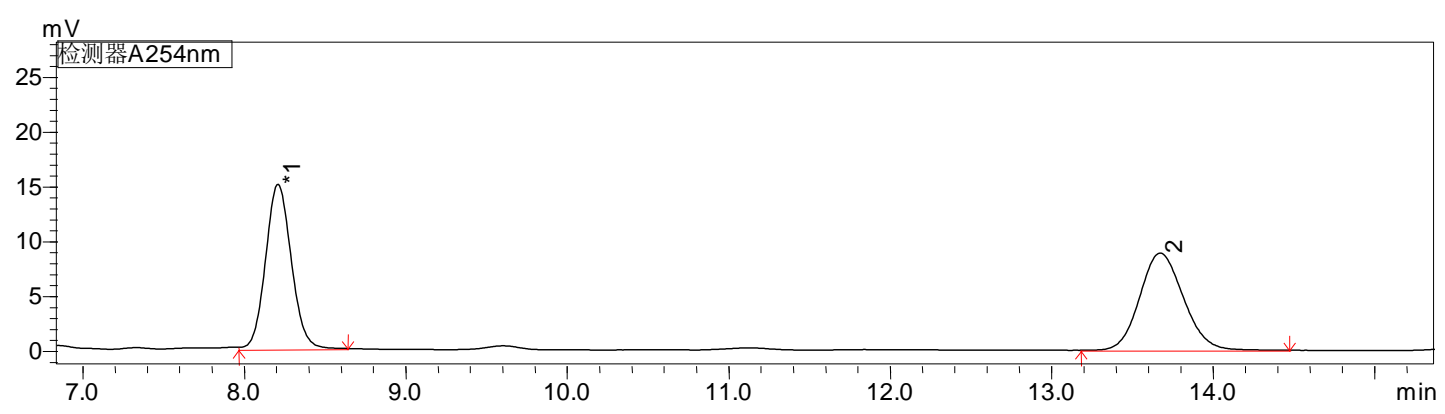

\begin{tabular}{|l|l|l|}
\hline Peak\# & Ret. Time & Area\% \\
\hline 1 & 8.220 & 94.408 \\
\hline 2 & 13.699 & 5.592 \\
\hline
\end{tabular}

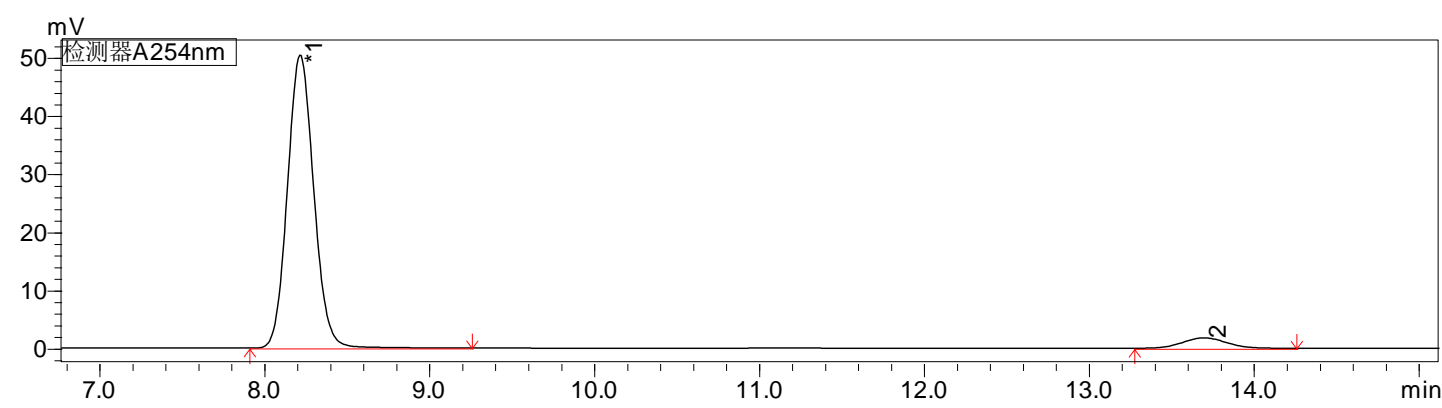




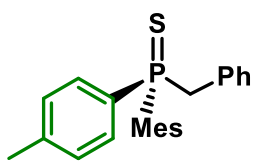

$3 \mathbf{j f}$

3jf: Procedure A, $56.8 \mathrm{mg}, 78 \%$ yield, light yellow solid.

${ }^{1}$ H NMR (400 MHz, CDCl $) \delta$ 7.35-7.26 (m, 2H), 7.11-6.94 (m, 5H), $6.87(\mathrm{~d}, J=4.0 \mathrm{~Hz}, 2 \mathrm{H})$, 6.82-6.74 (m, 2H), $4.01(\mathrm{dd}, J=15.9,12.9 \mathrm{~Hz}, 1 \mathrm{H}), 3.70(\mathrm{dd}, J=13.0,10.7 \mathrm{~Hz}, 1 \mathrm{H}), 2.35(\mathrm{~s}, 6 \mathrm{H})$, $2.33(\mathrm{~s}, 3 \mathrm{H}), 2.28$ (s, 3H).

${ }^{13} \mathbf{C}$ NMR(101 MHz, CDCl $) \delta 141.47(\mathrm{~d}, J=10.1 \mathrm{~Hz}), 140.90(\mathrm{~d}, J=3.0 \mathrm{~Hz}), 140.66(\mathrm{~d}, J=3.0$ $\mathrm{Hz}), 131.28(\mathrm{~d}, J=11.1 \mathrm{~Hz}), 131.17(\mathrm{~d}, J=7.5 \mathrm{~Hz}), 130.69(\mathrm{~d}, J=10.4 \mathrm{~Hz}), 130.66$ (d, $J=5.1$ $\mathrm{Hz}), 130.35(\mathrm{~d}, J=82.5 \mathrm{~Hz}), 129.35(\mathrm{~d}, J=80.4 \mathrm{~Hz}), 128.61(\mathrm{~d}, J=12.7 \mathrm{~Hz}), 127.22(\mathrm{~d}, J=3.4$ $\mathrm{Hz}), 126.47(\mathrm{~d}, J=4.1 \mathrm{~Hz}), 45.49(\mathrm{~d}, J=47.3 \mathrm{~Hz}), 24.31(\mathrm{~d}, J=4.7 \mathrm{~Hz}), 21.33(\mathrm{~d}, J=1.6 \mathrm{~Hz})$, $20.79(\mathrm{~d}, J=1.5 \mathrm{~Hz})$.

${ }^{31}$ P NMR (162 MHz, CDCl 3 ) $\delta 42.97$.

HRMS (ESI) $\mathbf{m} / \mathbf{z}$ [M+H] $]^{+}$: calcd. 365.1487, found. 365.1487 .

IR (film): $v_{\max }\left(\mathrm{cm}^{-1}\right) 3029,2921,1602,1495,1452,1398,1101,848,772,697,584$.

Optical rotation: $[\alpha]_{\mathrm{D}}^{25}=-178.25\left(c=1.176, \mathrm{CHCl}_{3}, 84 \%\right.$ ee $)$.

HPLC: DAICEL CHIRALPAK ID, hexane $/ i-\mathrm{PrOH}=9 / 1$, flow rate: $1.0 \mathrm{~mL} / \mathrm{min}, \lambda=254 \mathrm{~nm}$, $\mathrm{t}_{\mathrm{R}}($ major $)=11.8 \mathrm{~min}, \mathrm{t}_{\mathrm{R}}($ minor $)=14.0 \mathrm{~min}, 84 \%$ ee .

\begin{tabular}{|l|l|l|}
\hline Peak\# & Ret. Time & Area\% \\
\hline 1 & 11.850 & 49.968 \\
\hline 2 & 14.099 & 50.032 \\
\hline
\end{tabular}

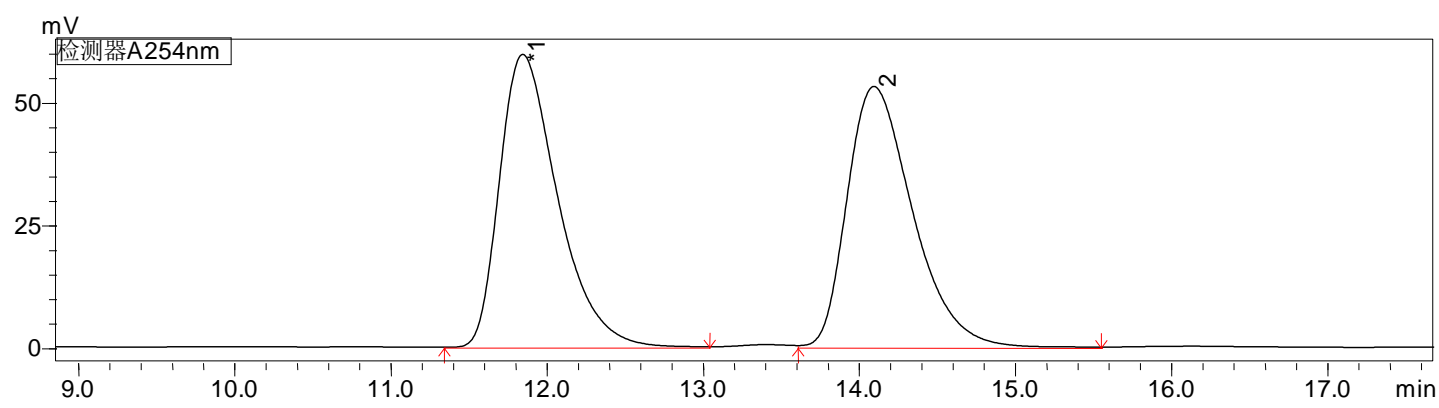

\begin{tabular}{|l|l|l|}
\hline Peak\# & Ret. Time & Area\% \\
\hline 1 & 11.775 & 91.868 \\
\hline 2 & 14.047 & 8.132 \\
\hline
\end{tabular}

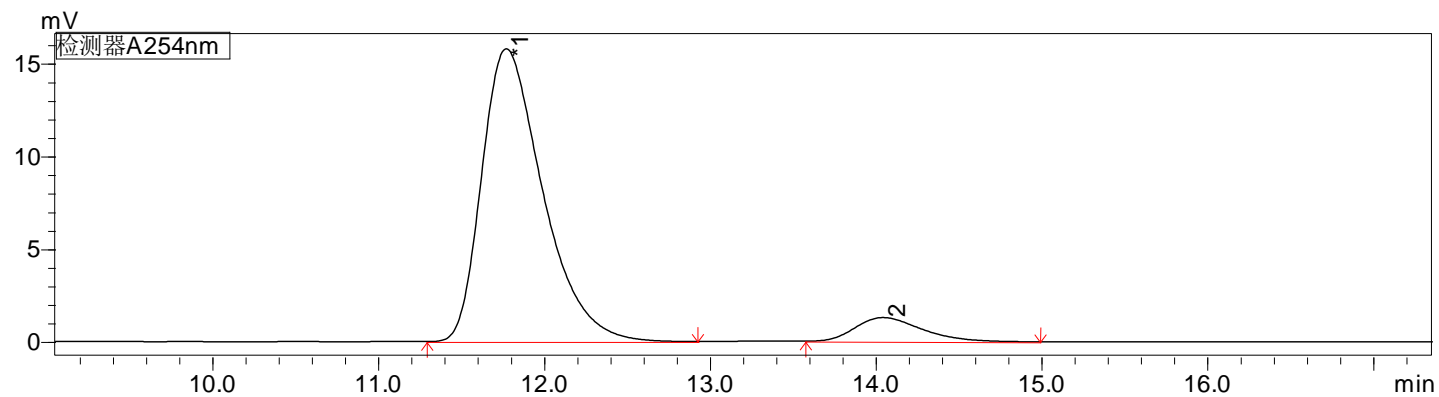




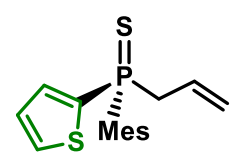

$3 \mathbf{k a}$

3ka: Procedure A, $64.0 \mathrm{mg}, 94 \%$ yield, light yellow solid.

${ }^{1}$ H NMR (500 MHz, $\left.\mathbf{C D C l}_{\mathbf{3}}\right) \delta$ 7.68-7.60 (m, 1H), 7.51-7.42 (m, 1H), 7.16-7.08 (m, 1H), $6.86(\mathrm{~d}$, $J=4.2 \mathrm{~Hz}, 2 \mathrm{H}), 5.65-5.51(\mathrm{~m}, 1 \mathrm{H}), 5.12-5.04(\mathrm{~m}, 1 \mathrm{H}), 5.03-4.94(\mathrm{~m}, 1 \mathrm{H}), 3.48-3.28(\mathrm{~m}, 2 \mathrm{H})$, $2.38(\mathrm{~s}, 6 \mathrm{H}), 2.27$ (s, 3H).

${ }^{13} \mathbf{C}$ NMR(101 MHz, $\left.\mathbf{C D C l}_{3}\right) \delta 141.36(\mathrm{~d}, J=10.7 \mathrm{~Hz}), 141.04(\mathrm{~d}, J=3.0 \mathrm{~Hz}), 137.54(\mathrm{~d}, J=$ $86.9 \mathrm{~Hz}), 135.53(\mathrm{~d}, J=9.1 \mathrm{~Hz}), 132.94(\mathrm{~d}, J=4.4 \mathrm{~Hz}), 131.24(\mathrm{~d}, J=11.5 \mathrm{~Hz}), 129.00$ (d, $J=$ $83.7 \mathrm{~Hz}), 127.89(\mathrm{~d}, J=13.8 \mathrm{~Hz}), 127.56(\mathrm{~d}, J=9.2 \mathrm{~Hz}), 121.03(\mathrm{~d}, J=14.1 \mathrm{~Hz}), 45.93(\mathrm{~d}, J=$ $54.5 \mathrm{~Hz}), 23.51(\mathrm{~d}, J=5.1 \mathrm{~Hz}), 20.75(\mathrm{~d}, J=1.5 \mathrm{~Hz})$.

${ }^{31}$ P NMR (162 MHz, $\left.\mathbf{C D C l}_{3}\right) \delta 32.49$.

HRMS (ESI) $\mathbf{m} / \mathbf{z}$ [M+H] $]^{+}$: calcd. 307.0739, found. 307.0739.

IR (film): $v_{\max }\left(\mathrm{cm}^{-1}\right)$ 2964, 2922, 1603, 1455, 1404, 1215, 996, 835, 716, 653, 622.

Optical rotation: $[\alpha]_{\mathrm{D}}{ }^{25}=-37.13\left(c=1.424, \mathrm{CHCl}_{3}, 90 \%\right.$ ee $)$.

HPLC: DAICEL CHIRALPAK ID, hexane $/ i-\mathrm{PrOH}=9 / 1$, flow rate: $1.0 \mathrm{~mL} / \mathrm{min}, \lambda=254 \mathrm{~nm}$, $\mathrm{t}_{\mathrm{R}}($ major $)=8.8 \mathrm{~min}, \mathrm{t}_{\mathrm{R}}($ minor $)=9.4 \mathrm{~min}, 90 \%$ ee .

\begin{tabular}{|l|l|l|}
\hline Peak\# & Ret. Time & Area\% \\
\hline 1 & 8.833 & 50.374 \\
\hline 2 & 9.435 & 49.626 \\
\hline
\end{tabular}

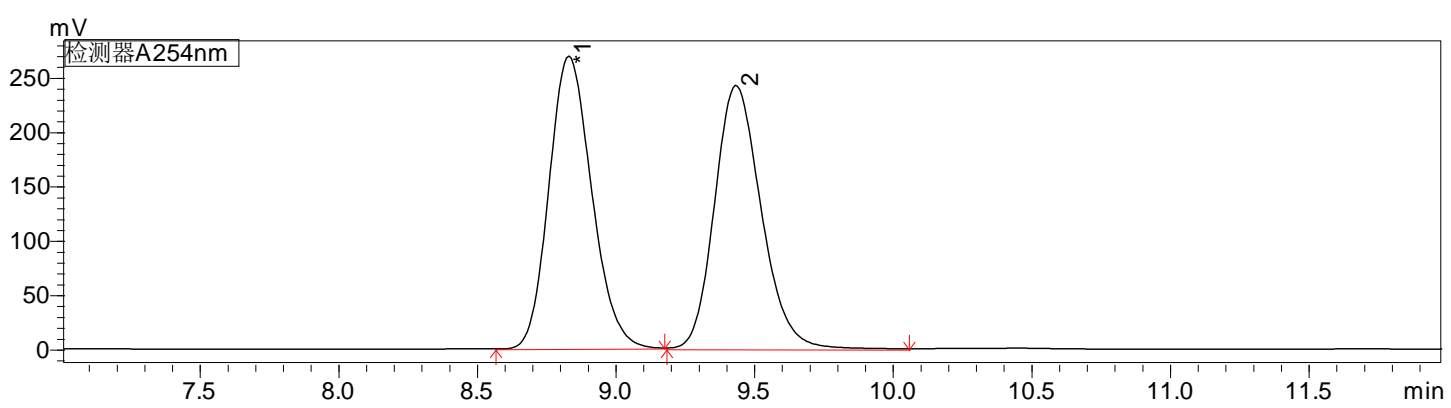

\begin{tabular}{|l|l|l|}
\hline Peak\# & Ret. Time & Area\% \\
\hline 1 & 8.837 & 95.174 \\
\hline 2 & 9.465 & 4.826 \\
\hline
\end{tabular}

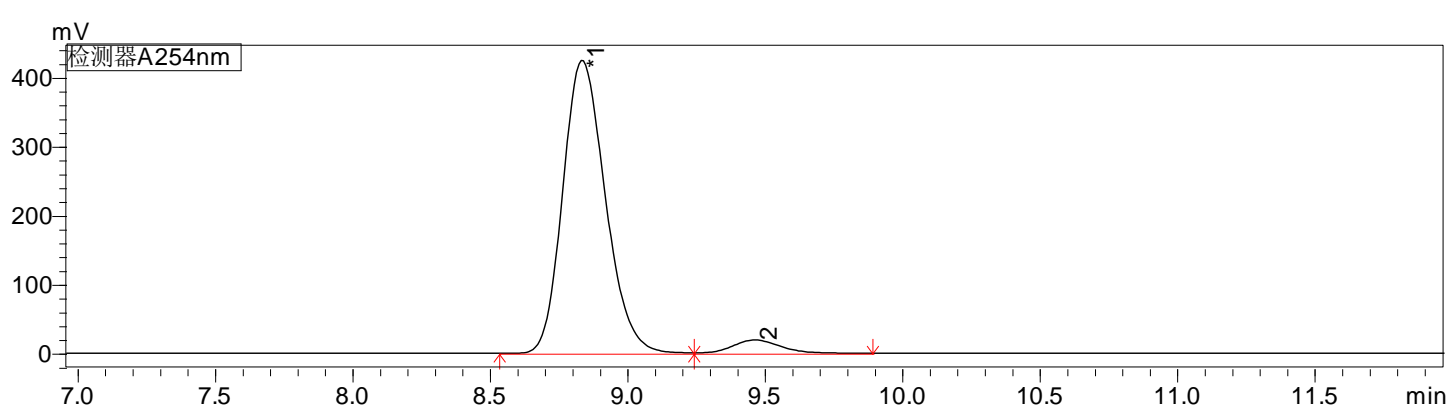




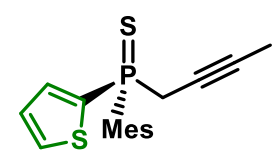

3ke

3ke: Procedure E, $64.9 \mathrm{mg}$, 92\% yield, light yellow solid.

${ }^{1}$ H NMR (500 MHz, CDCl $) \delta$ 7.78-7.61 (m, 2H), 7.21-7.10 (m, 1H), $6.85(\mathrm{~d}, J=4.4 \mathrm{~Hz}, 2 \mathrm{H})$, $3.72-3.55(\mathrm{~m}, 1 \mathrm{H}), 3.23-3.05(\mathrm{~m}, 1 \mathrm{H}), 2.39(\mathrm{~s}, 6 \mathrm{H}), 2.27(\mathrm{~s}, 3 \mathrm{H}), 1.69(\mathrm{dt}, J=6.4,2.6 \mathrm{~Hz}, 3 \mathrm{H})$.

${ }^{13} \mathbf{C}$ NMR(101 MHz, CDCl $) \delta 141.72(\mathrm{~d}, J=10.7 \mathrm{~Hz}), 141.12(\mathrm{~d}, J=3.0 \mathrm{~Hz}), 137.57(\mathrm{~d}, J=88.8$ $\mathrm{Hz}), 135.43(\mathrm{~d}, J=9.1 \mathrm{~Hz}), 133.34(\mathrm{~d}, J=4.5 \mathrm{~Hz}), 131.04(\mathrm{~d}, J=11.8 \mathrm{~Hz}), 127.85(\mathrm{~d}, J=14.0 \mathrm{~Hz})$, $127.26(\mathrm{~d}, J=87.2 \mathrm{~Hz}), 81.23(\mathrm{~d}, J=9.1 \mathrm{~Hz}), 70.71(\mathrm{~d}, J=14.0 \mathrm{~Hz}), 35.26(\mathrm{~d}, J=57.5 \mathrm{~Hz}), 22.64(\mathrm{~d}$, $J=5.4 \mathrm{~Hz}), 20.84(\mathrm{~d}, J=1.5 \mathrm{~Hz}), 3.53(\mathrm{~d}, J=3.3 \mathrm{~Hz})$.

${ }^{31}$ P NMR (162 MHz, $\left.\mathrm{CDCl}_{3}\right) \delta 32.04$.

HRMS (ESI) $\mathbf{m} / \mathbf{z}$ [M+H] $]^{+}$: calcd. 319.0739, found. 319.0739.

IR (film): $v_{\max }\left(\mathrm{cm}^{-1}\right)$ 2966, 2916, 1604, 1454. 1404, 1214, 1000, 853, 822, 718, 675, 599.

Optical rotation: $[\alpha]_{\mathrm{D}}^{25}=55.92\left(c=1.278, \mathrm{CHCl}_{3}, 90 \%\right.$ ee $)$.

HPLC: DAICEL CHIRALPAK AD-H, hexane $/ i-\mathrm{PrOH}=19 / 1$, flow rate: $1.0 \mathrm{~mL} / \mathrm{min}, \lambda=254 \mathrm{~nm}$, $\mathrm{t}_{\mathrm{R}}($ major $)=8.9 \mathrm{~min}, \mathrm{t}_{\mathrm{R}}($ minor $)=10.3 \mathrm{~min}, 90 \%$ ee.

\begin{tabular}{|l|l|l|}
\hline Peak\# & Ret. Time & Area\% \\
\hline 1 & 8.902 & 50.050 \\
\hline 2 & 10.346 & 49.950 \\
\hline
\end{tabular}

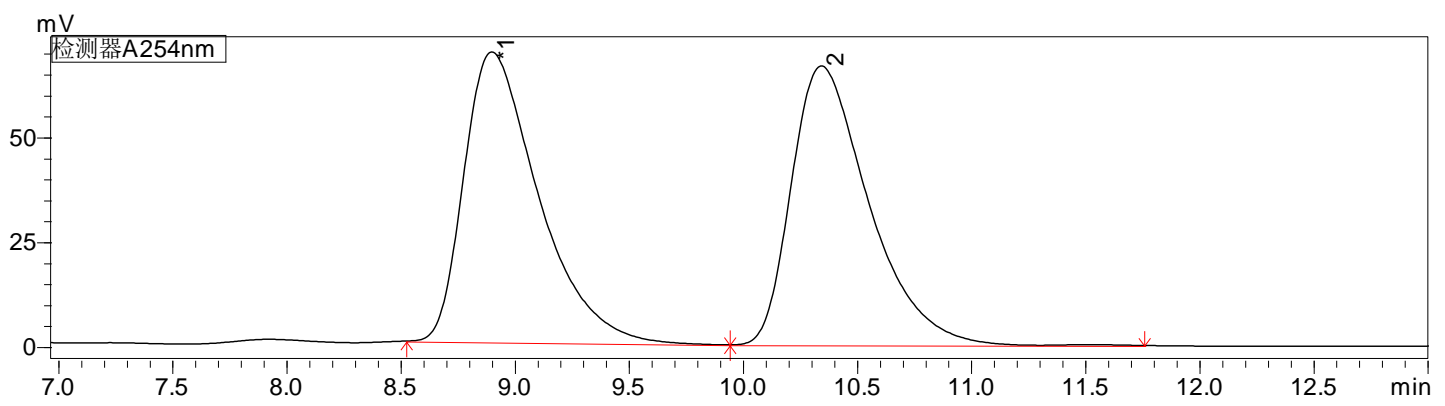

\begin{tabular}{|l|l|l|}
\hline Peak\# & Ret. Time & Area\% \\
\hline 1 & 8.899 & 94.839 \\
\hline 2 & 10.333 & 5.161 \\
\hline
\end{tabular}

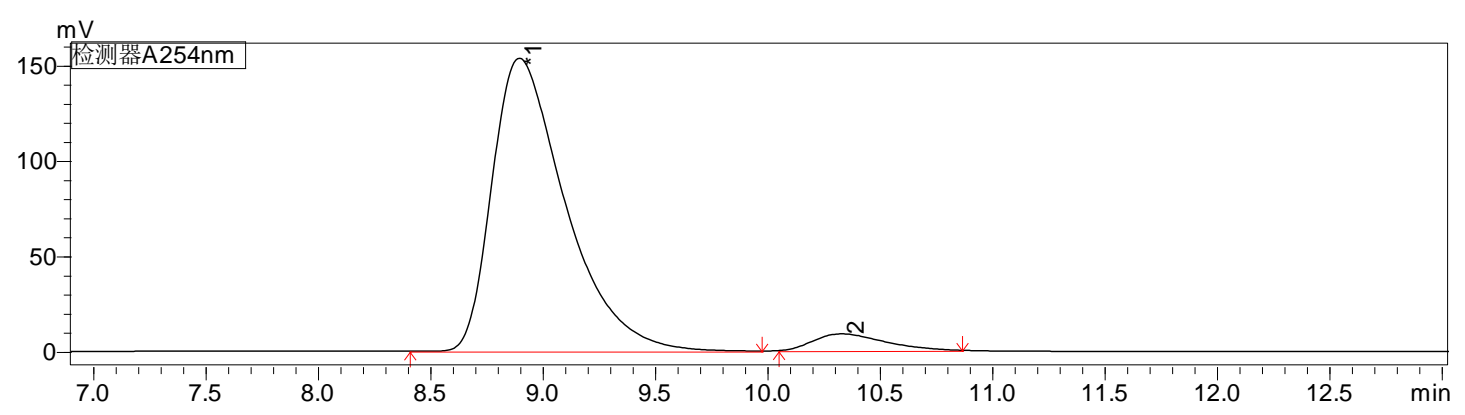




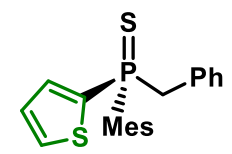

$3 \mathbf{k f}$

3kf: Procedure A, $78.0 \mathrm{mg}, 99 \%$ yield, light yellow solid.

${ }^{1}$ H NMR (500 MHz, CDCl $\left.)_{3}\right) \delta$ 7.53-7.47 (m, 1H), 7.16-7.03 (m, 4H), $6.94(\mathrm{t}, J=4.1 \mathrm{~Hz}, 3 \mathrm{H})$, $6.89(\mathrm{~d}, J=4.2 \mathrm{~Hz}, 2 \mathrm{H}), 4.04(\mathrm{dd}, J=15.2,13.1 \mathrm{~Hz}, 1 \mathrm{H}), 3.77(\mathrm{dd}, J=13.1,11.2 \mathrm{~Hz}, 1 \mathrm{H}), 2.43(\mathrm{~s}$, $6 \mathrm{H}), 2.29(\mathrm{~s}, 3 \mathrm{H})$.

${ }^{13} \mathbf{C}$ NMR(101 MHz, $\left.\mathbf{C D C l}_{3}\right) \delta 141.46(\mathrm{~d}, J=10.9 \mathrm{~Hz}), 141.01(\mathrm{~d}, J=3.0 \mathrm{~Hz}), 137.05(\mathrm{~d}, J=$ $87.8 \mathrm{~Hz}), 135.37(\mathrm{~d}, J=9.0 \mathrm{~Hz}), 132.74(\mathrm{~d}, J=4.4 \mathrm{~Hz}), 131.34(\mathrm{~d}, J=11.4 \mathrm{~Hz}), 131.20$ (d, $J=$ $8.3 \mathrm{~Hz}), 130.34(\mathrm{~d}, J=5.7 \mathrm{~Hz}), 129.71(\mathrm{~d}, J=83.0 \mathrm{~Hz}), 127.70(\mathrm{~d}, J=13.9 \mathrm{~Hz}), 127.43(\mathrm{~d}, J=$ $3.6 \mathrm{~Hz}), 126.72(\mathrm{~d}, J=4.1 \mathrm{~Hz}), 46.92(\mathrm{~d}, J=50.5 \mathrm{~Hz}), 23.96(\mathrm{~d}, J=4.7 \mathrm{~Hz}), 20.80(\mathrm{~d}, J=1.5$ $\mathrm{Hz})$.

${ }^{31}$ P NMR (162 MHz, CDCl 3 ) $\delta 34.66$.

HRMS (ESI) $\mathbf{m} / \mathbf{z}$ [M+H] $]^{+}$: calcd. 357.0895, found. 357.0893 .

IR (film): $v_{\max }\left(\mathrm{cm}^{-1}\right)$ 2970, 2920, 1602, 1495, 1452, 1404, 1215, 998, 832, 772, 697, 599.

Optical rotation: $[\alpha]_{\mathrm{D}}{ }^{25}=-76.37\left(c=1.546, \mathrm{CHCl}_{3}, 80 \%\right.$ ee $)$.

HPLC: DAICEL CHIRALPAK ID, hexane $/ i-\mathrm{PrOH}=9 / 1$, flow rate: $1.0 \mathrm{~mL} / \mathrm{min}, \lambda=254 \mathrm{~nm}$, $\mathrm{t}_{\mathrm{R}}($ major $)=10.5 \mathrm{~min}, \mathrm{t}_{\mathrm{R}}($ minor $)=11.1 \mathrm{~min}, 80 \%$ ee .

\begin{tabular}{|l|l|l|}
\hline Peak\# & Ret. Time & Area\% \\
\hline 1 & 10.456 & 50.036 \\
\hline 2 & 11.064 & 49.964 \\
\hline
\end{tabular}

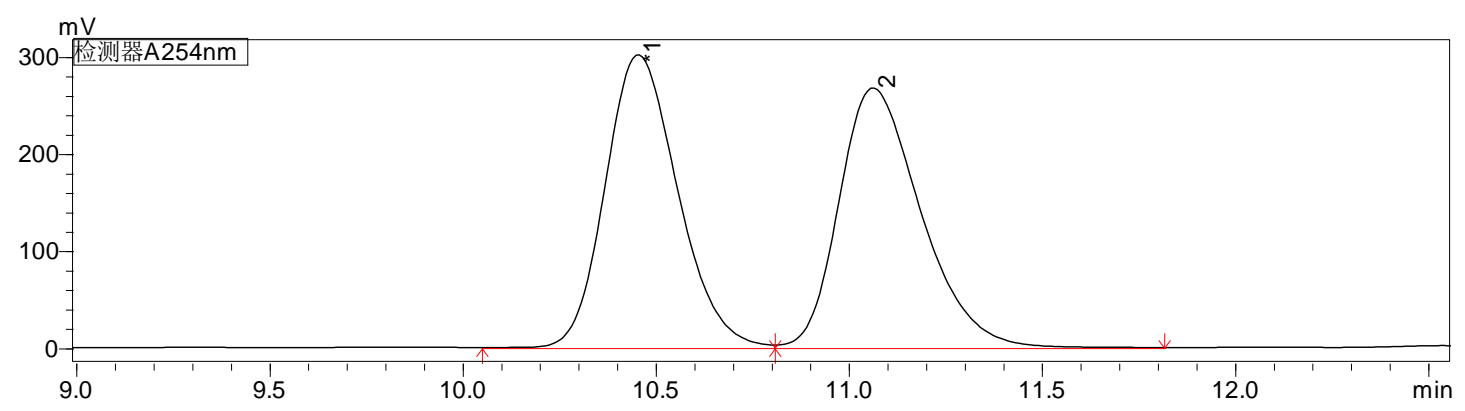

\begin{tabular}{|l|l|l|}
\hline Peak\# & Ret. Time & Area\% \\
\hline 1 & 10.476 & 89.863 \\
\hline 2 & 11.138 & 10.137 \\
\hline
\end{tabular}

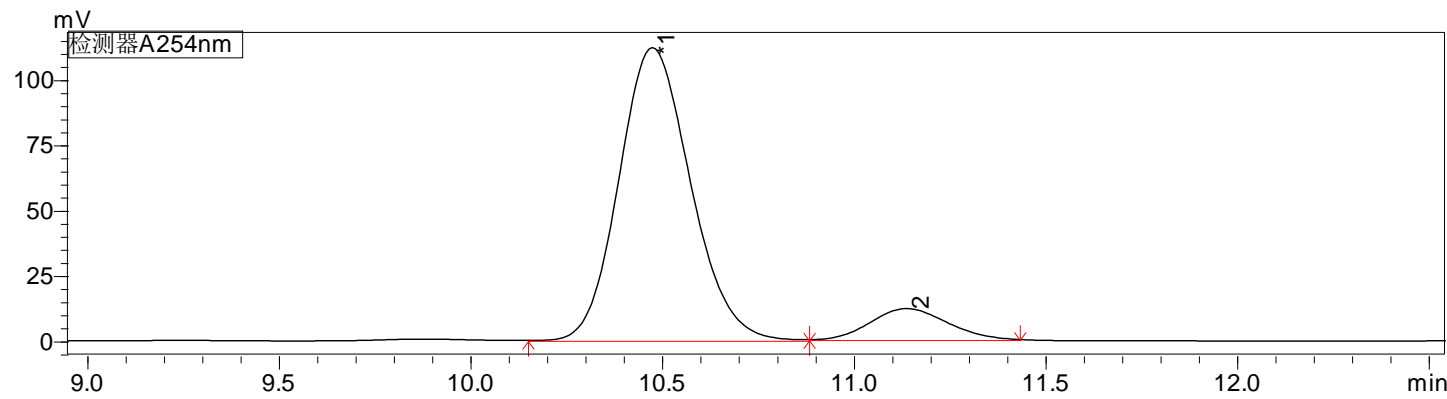




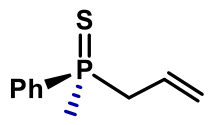

$31 a$

3la: Procedure A, $37.1 \mathrm{mg}, 95 \%$ yield, $0 \%$ ee, colorless liquid.

3la is a known compound ${ }^{[7]}$.

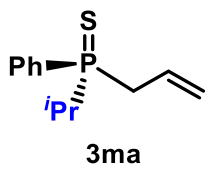

3ma: Procedure A, $30.1 \mathrm{mg}, 67 \%$ yield, colorless liquid.

${ }^{1}$ H NMR (400 MHz, $\left.\mathbf{C D C l}_{3}\right) \delta$ 7.91-7.80 (m, 2H), 7.56-7.45 (m, 3H), 5.79-5.63 (m, 1H), 5.19$5.01(\mathrm{~m}, 2 \mathrm{H}), 3.09-2.91(\mathrm{~m}, 2 \mathrm{H}), 2.49-2.34(\mathrm{~m}, 1 \mathrm{H}), 1.30$ (dd, $J=18.1,6.9 \mathrm{~Hz}, 3 \mathrm{H}), 0.98$ (dd, $J=$ $18.4,6.9 \mathrm{~Hz}, 3 \mathrm{H})$.

${ }^{13} \mathbf{C}$ NMR(101 MHz, CDCl $) \delta 131.49(\mathrm{~d}, J=9.1 \mathrm{~Hz}), 131.45(\mathrm{~d}, J=3.0 \mathrm{~Hz}), 129.48(\mathrm{~d}, J=72.8$ $\mathrm{Hz}), 128.35(\mathrm{~d}, J=11.4 \mathrm{~Hz}), 127.75(\mathrm{~d}, J=8.8 \mathrm{~Hz}), 120.45(\mathrm{~d}, J=12.1 \mathrm{~Hz}), 37.29(\mathrm{~d}, J=49.6$ $\mathrm{Hz}), 29.14(\mathrm{~d}, J=53.0 \mathrm{~Hz}), 15.68(\mathrm{dd}, J=14.1,1.6 \mathrm{~Hz})$.

${ }^{31}$ P NMR (162 MHz, CDCl $) \delta 55.16$.

HRMS (ESI) $\mathbf{m} / \mathbf{z}[\mathbf{M}+\mathbf{H}]^{+}$: calcd. 225.0861, found. 225.0862.

IR (film): $v_{\max }\left(\mathrm{cm}^{-1}\right)$ 2956, 2924, 2869, 1640, 1462, 1435, 1102. 1025, 916, 829,744, 693, 614.

Optical rotation: $[\alpha]_{\mathrm{D}}^{25}=0.26\left(c=1.140, \mathrm{CHCl}_{3}, 3 \%\right.$ ee $)$

HPLC: DAICEL CHIRALPAK ID, hexane $/ i-\mathrm{PrOH}=9 / 1$, flow rate: $1.0 \mathrm{~mL} / \mathrm{min}, \lambda=220 \mathrm{~nm}$, $t_{R}(\operatorname{minor})=6.3 \mathrm{~min}, t_{R}($ major $)=6.7 \mathrm{~min}, 3 \%$ ee.
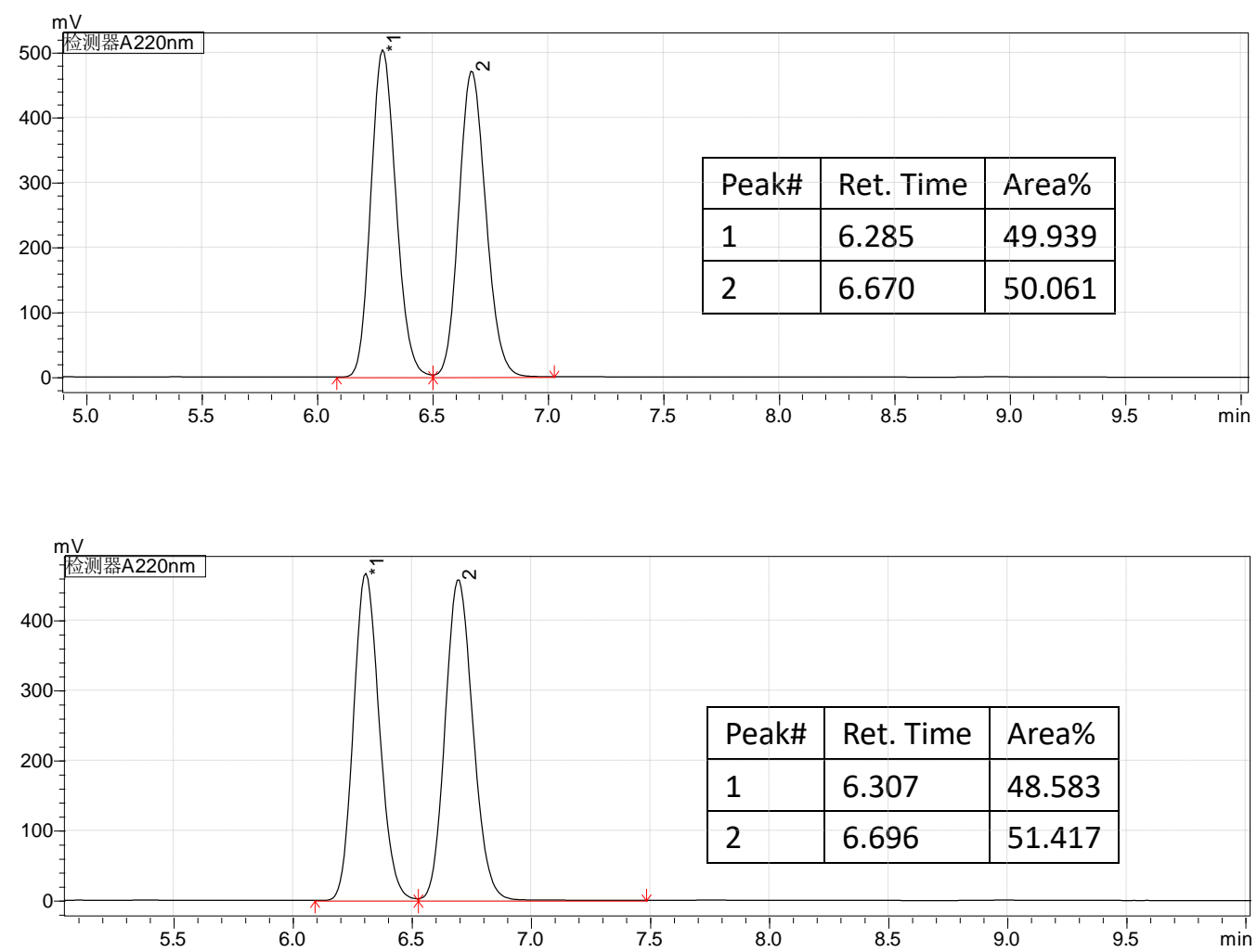


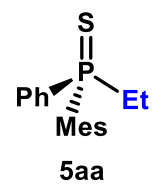

5aa: Procedure B, $54.6 \mathrm{mg}, 95 \%$ yield, light yellow solid.

${ }^{1}$ H NMR (400 MHz, CDCl $) \delta$ 7.81-7.71 (m, 2H), 7.53-7.39 (m, 3H), $6.84(\mathrm{~d}, J=3.9 \mathrm{~Hz}, 2 \mathrm{H})$, $2.68-2.53(\mathrm{~m}, 1 \mathrm{H}), 2.52-2.39(\mathrm{~m}, 1 \mathrm{H}), 2.30(\mathrm{~s}, 6 \mathrm{H}), 2.26(\mathrm{~s}, 3 \mathrm{H}), 0.89(\mathrm{dt}, J=20.7,7.3 \mathrm{~Hz}, 3 \mathrm{H})$.

${ }^{13}$ C NMR(101 MHz, CDCl $) \delta 141.57(\mathrm{~d}, J=10.1 \mathrm{~Hz}), 140.80(\mathrm{~d}, J=3.0 \mathrm{~Hz}), 134.18(\mathrm{~d}, J=$ $77.2 \mathrm{~Hz}), 131.30$ (d, $J=11.2 \mathrm{~Hz}), 130.78(\mathrm{~d}, J=9.9 \mathrm{~Hz}), 130.75(\mathrm{~d}, J=3.3 \mathrm{~Hz}), 128.82(\mathrm{~d}, J=$ $82.4 \mathrm{~Hz}), 128.33(\mathrm{~d}, J=11.9 \mathrm{~Hz}), 31.81(\mathrm{~d}, J=54.2 \mathrm{~Hz}), 24.02(\mathrm{~d}, J=5.0 \mathrm{~Hz}), 20.84(\mathrm{~d}, J=1.5$ $\mathrm{Hz}), 7.08(\mathrm{~d}, J=3.9 \mathrm{~Hz})$.

${ }^{31}$ P NMR (162 MHz, $\left.\mathbf{C D C l}_{3}\right) \delta 46.27$.

HRMS (ESI) $\mathbf{m} / \mathbf{z}[\mathbf{M}+\mathbf{H}]^{+}$: calcd. 289.1174, found. 289.1175.

IR (film): $v_{\max }\left(\mathrm{cm}^{-1}\right)$ 3054, 2968, 2929, 2875, 1604, 1454, 1436, 1101, 1028, 850, 776, 702, 645, 586.

Optical rotation: $[\alpha]_{\mathrm{D}}{ }^{25}=-94.79\left(c=0.867, \mathrm{CHCl}_{3}, 92 \%\right.$ ee $)$.

HPLC: DAICEL CHIRALPAK OD-H, hexane $/ i-\mathrm{PrOH}=97 / 3$, flow rate: $1.0 \mathrm{~mL} / \mathrm{min}, \lambda=254 \mathrm{~nm}$, $\mathrm{t}_{\mathrm{R}}($ major $)=6.9 \mathrm{~min}, \mathrm{t}_{\mathrm{R}}($ minor $)=9.1 \mathrm{~min}, 92 \%$ ee.

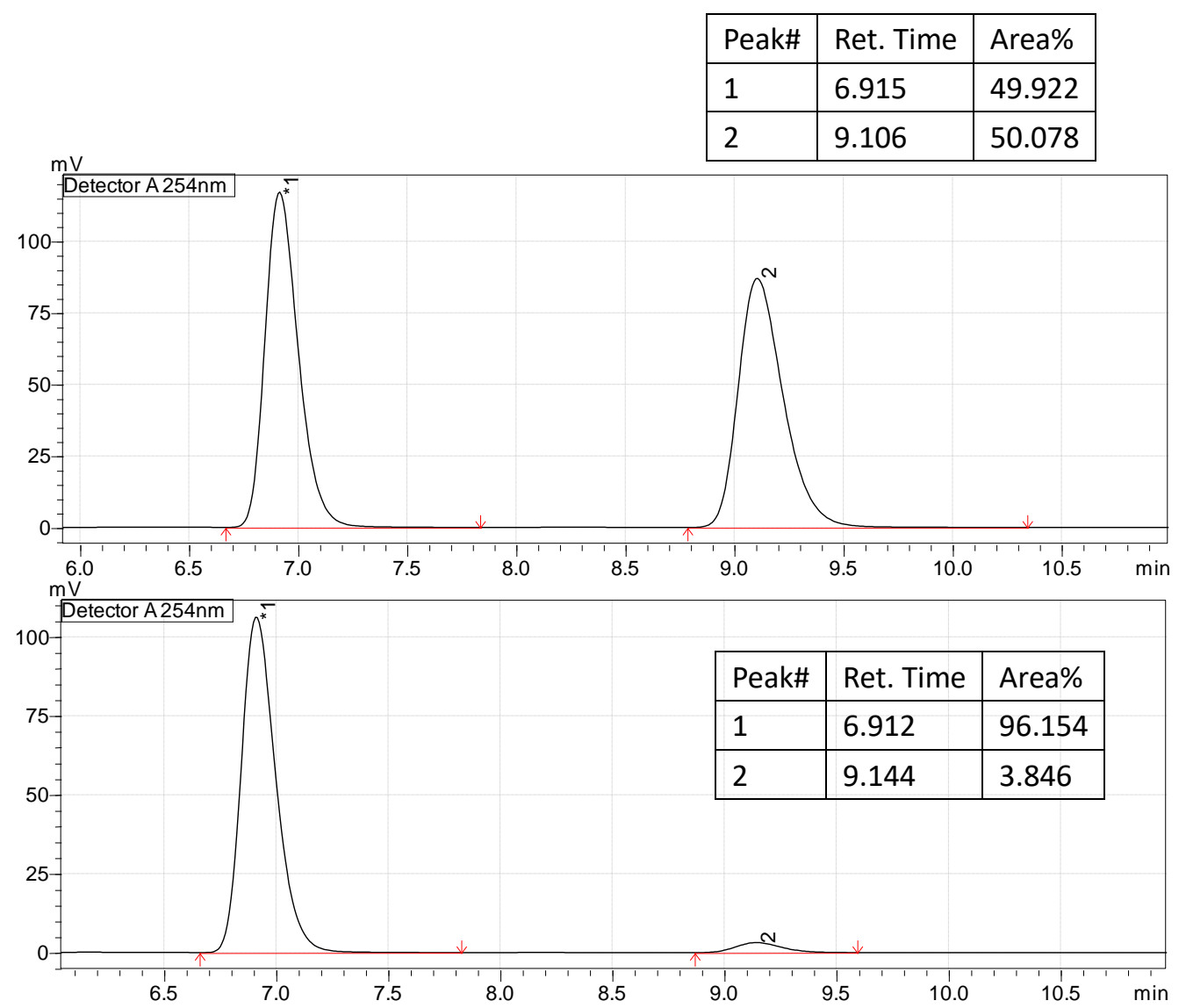




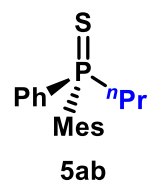

5ab: Procedure B, $54.3 \mathrm{mg}, 90 \%$ yield, white solid.

${ }^{1}$ H NMR (400 MHz, CDCl $) \delta$ 7.83-7.70 (m, 2H), 7.52-7.37 (m, 3H), $6.83(\mathrm{~d}, J=3.9 \mathrm{~Hz}, 2 \mathrm{H})$, $2.61-2.38(\mathrm{~m}, 2 \mathrm{H}), 2.30(\mathrm{~s}, 6 \mathrm{H}), 2.26(\mathrm{~s}, 3 \mathrm{H}), 1.80-1.61(\mathrm{~m}, 1 \mathrm{H}), 0.91(\mathrm{td}, J=7.2,1.7 \mathrm{~Hz}, 3 \mathrm{H})$, $0.87-0.71(\mathrm{~m}, 1 \mathrm{H})$.

${ }^{13} \mathbf{C}$ NMR(101 MHz, $\left.\mathbf{C D C l}_{3}\right) \delta 141.45(\mathrm{~d}, J=10.0 \mathrm{~Hz}), 140.68(\mathrm{~d}, J=2.9 \mathrm{~Hz}), 134.65(\mathrm{~d}, J=$ $77.1 \mathrm{~Hz}), 131.21(\mathrm{~d}, J=11.1 \mathrm{~Hz}), 130.64(\mathrm{~d}, J=3.3 \mathrm{~Hz}), 130.57(\mathrm{~d}, J=10.3 \mathrm{~Hz}), 128.82(\mathrm{~d}, J=$ $81.8 \mathrm{~Hz}), 128.26(\mathrm{~d}, J=12.0 \mathrm{~Hz}), 40.80(\mathrm{~d}, J=53.0 \mathrm{~Hz}), 23.90(\mathrm{~d}, J=5.0 \mathrm{~Hz}), 20.75(\mathrm{~d}, J=1.5$ $\mathrm{Hz}), 16.57(\mathrm{~d}, J=3.0 \mathrm{~Hz}), 15.37(\mathrm{~d}, J=19.0 \mathrm{~Hz})$.

${ }^{31}$ P NMR (162 MHz, CDCl $\left.\mathbf{3}\right) \delta 43.29$.

HRMS (ESI) $\mathbf{m} / \mathbf{z}[\mathbf{M}+\mathbf{H}]^{+}$: calcd. 303.1331, found. 303.1332 .

IR (film): $v_{\max }\left(\mathrm{cm}^{-1}\right)$ 3054, 2961, 2929, 2871, 1604, 1454, 1436, 1099, 1032, 843, 750, 694, 593.

Optical rotation: $[\alpha]_{\mathrm{D}}{ }^{25}=-75.41\left(c=1.080, \mathrm{CHCl}_{3}, 90 \%\right.$ ee $)$.

HPLC: DAICEL CHIRALPAK OD-H, hexane $/ i-\mathrm{PrOH}=97 / 3$, flow rate: $1.0 \mathrm{~mL} / \mathrm{min}, \lambda=254 \mathrm{~nm}$, $\mathrm{t}_{\mathrm{R}}($ major $)=6.0 \mathrm{~min}, \mathrm{t}_{\mathrm{R}}($ minor $)=7.7 \mathrm{~min}, 90 \%$ ee.

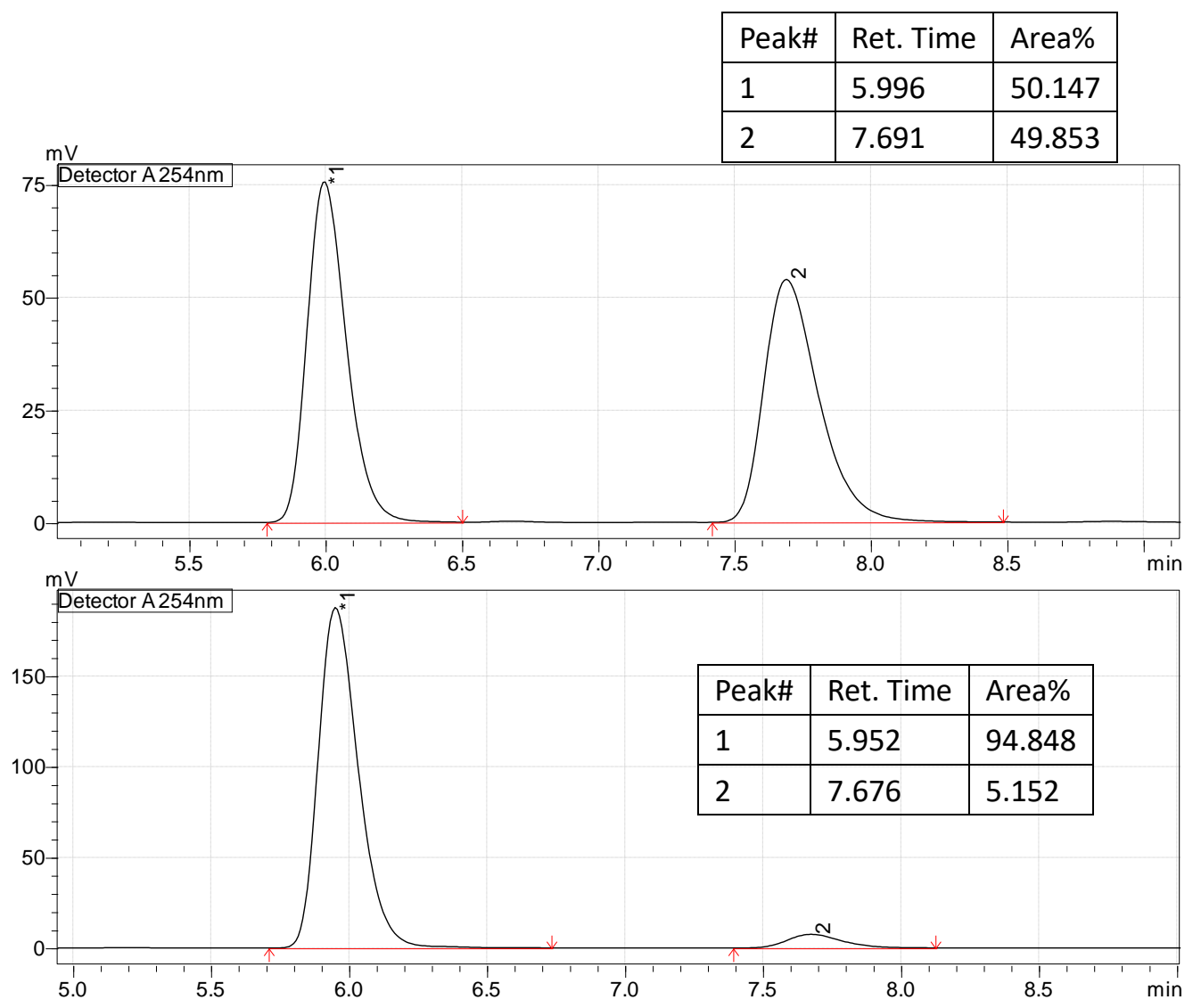




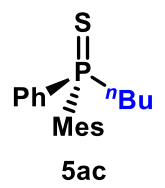

5ac: Procedure B, $60.0 \mathrm{mg}, 95 \%$ yield, light yellow liquid.

${ }^{1}$ H NMR (400 MHz, CDCl $) \delta$ 7.81-7.72 (m, 2H), 7.51-7.39 (m, 3H), $6.83(\mathrm{~d}, J=3.9 \mathrm{~Hz}, 2 \mathrm{H})$, $2.64-2.37(\mathrm{~m}, 2 \mathrm{H}), 2.29(\mathrm{~s}, 6 \mathrm{H}), 2.26(\mathrm{~s}, 3 \mathrm{H}), 1.75-1.57(\mathrm{~m}, 1 \mathrm{H}), 1.39-1.21(\mathrm{~m}, 2 \mathrm{H}), 0.79(\mathrm{t}, J=$ $7.3 \mathrm{~Hz}, 3 \mathrm{H}), 0.76-0.68(\mathrm{~m}, 1 \mathrm{H})$.

${ }^{13}$ C NMR(101 MHz, CDCl $) \delta 141.50(\mathrm{~d}, J=10.2 \mathrm{~Hz}), 140.71(\mathrm{~d}, J=2.9 \mathrm{~Hz}), 134.69(\mathrm{~d}, J=$ $77.2 \mathrm{~Hz}), 131.26(\mathrm{~d}, J=11.1 \mathrm{~Hz}), 130.68(\mathrm{~d}, J=2.6 \mathrm{~Hz}), 130.64(\mathrm{~d}, J=10.1 \mathrm{~Hz}), 128.93(\mathrm{~d}, J=$ $81.9 \mathrm{~Hz}), 128.30(\mathrm{~d}, J=12.1 \mathrm{~Hz}), 38.49(\mathrm{~d}, J=53.2 \mathrm{~Hz}), 24.87(\mathrm{~d}, J=3.3 \mathrm{~Hz}), 23.96(\mathrm{~d}, J=5.1$ $\mathrm{Hz}), 23.87$ (d, $J=17.6 \mathrm{~Hz}), 20.79$ (d, $J=1.4 \mathrm{~Hz}), 13.57$.

${ }^{31}$ P NMR (162 MHz, CDCl$) \delta 43.70$.

HRMS (ESI) $\mathbf{~ m / z ~ [ M + H ] ~}]^{+}$: calcd. 317.1487, found. 317.1488.

IR (film): $v_{\max }\left(\mathrm{cm}^{-1}\right)$ 3058, 2957, 2927, 2870, 1604, 1455, 1436, 1100, 850, 749, 694, 647, 593.

Optical rotation: $[\alpha]_{\mathrm{D}}{ }^{25}=-69.85\left(c=1.033, \mathrm{CHCl}_{3}, 91 \%\right.$ ee $)$.

HPLC: DAICEL CHIRALPAK OD-H, hexane $/ i-\mathrm{PrOH}=97 / 3$, flow rate: $1.0 \mathrm{~mL} / \mathrm{min}, \lambda=254 \mathrm{~nm}$, $\mathrm{t}_{\mathrm{R}}($ major $)=5.4 \mathrm{~min}, \mathrm{t}_{\mathrm{R}}($ minor $)=6.9 \mathrm{~min}, 91 \%$ ee.
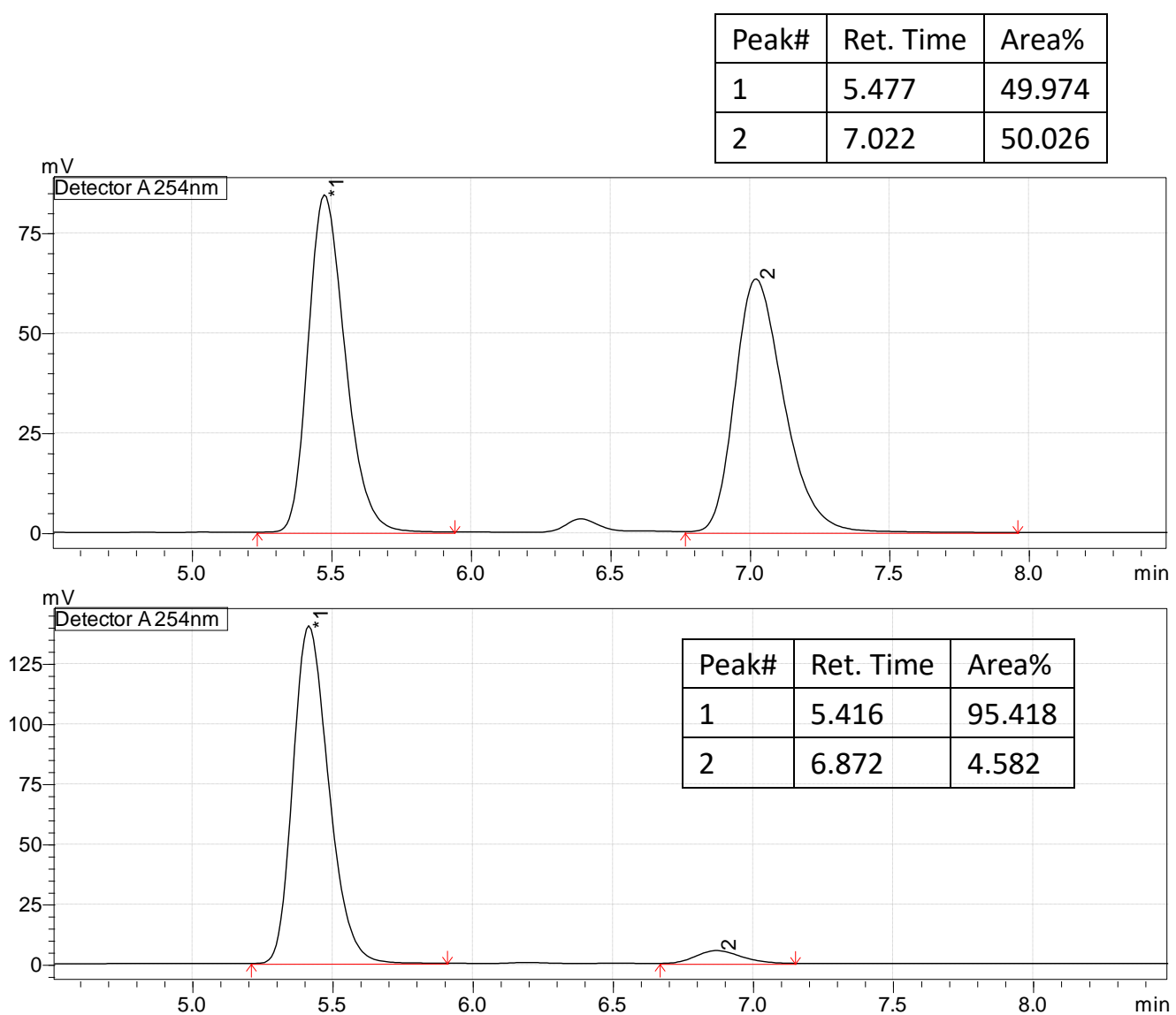


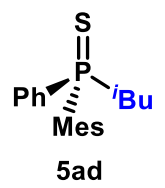

5ad: Procedure B, $49.3 \mathrm{mg}, 78 \%$ yield, white solid.

${ }^{1}$ H NMR (400 MHz, CDCl $)_{3} \delta$ 7.87-7.78 (m, 2H), 7.51-7.40 (m, 3H), $6.81(\mathrm{~d}, J=4.0 \mathrm{~Hz}, 2 \mathrm{H})$, $2.67-2.54(\mathrm{~m}, 1 \mathrm{H}), 2.41-2.32(\mathrm{~m}, 1 \mathrm{H}), 2.28(\mathrm{~s}, 6 \mathrm{H}), 2.25(\mathrm{~s}, 3 \mathrm{H}), 1.75-1.61(\mathrm{~m}, 1 \mathrm{H}), 1.09(\mathrm{~d}, J=$ $6.7 \mathrm{~Hz}, 3 \mathrm{H}), 0.57$ (dd, $J=6.7,1.2 \mathrm{~Hz}, 3 \mathrm{H})$.

${ }^{13} \mathbf{C}$ NMR(101 MHz, $\left.\mathbf{C D C l}_{3}\right) \delta 141.23(\mathrm{~d}, J=10.2 \mathrm{~Hz}), 140.46(\mathrm{~d}, J=2.9 \mathrm{~Hz}), 134.98(\mathrm{~d}, J=$ $77.0 \mathrm{~Hz}), 131.25$ (d, $J=11.1 \mathrm{~Hz}), 130.96(\mathrm{~d}, J=10.0 \mathrm{~Hz}), 130.73(\mathrm{~d}, J=3.0 \mathrm{~Hz}), 130.05$ (d, $J=$ $81.7 \mathrm{~Hz}), 128.33(\mathrm{~d}, J=12.0 \mathrm{~Hz}), 45.63(\mathrm{~d}, J=51.9 \mathrm{~Hz}), 24.93(\mathrm{~d}, J=11.0 \mathrm{~Hz}), 24.85(\mathrm{~d}, J=6.5$ $\mathrm{Hz}), 24.57(\mathrm{~d}, J=2.6 \mathrm{~Hz}), 24.15(\mathrm{~d}, J=4.9 \mathrm{~Hz}), 20.75(\mathrm{~d}, J=1.5 \mathrm{~Hz})$.

${ }^{31}$ P NMR (162 MHz, $\left.\mathbf{C D C l}_{\mathbf{3}}\right) \delta 42.29$.

HRMS (ESI) $\mathbf{m} / \mathbf{z}[\mathbf{M}+\mathbf{H}]^{+}$: calcd. 317.1487, found. 317.1487.

IR (film): $v_{\max }\left(\mathrm{cm}^{-1}\right) 3058,2955,2925,2867,1603,1455,1437,1404,1170,1099,1059,844$, 750, 695, 643, 596.

Optical rotation: $[\alpha]_{\mathrm{D}}{ }^{25}=-81.09\left(c=1.000, \mathrm{CHCl}_{3}, 91 \%\right.$ ee $)$.

HPLC: DAICEL CHIRALPAK OD-H, hexane $/ i-\mathrm{PrOH}=97 / 3$, flow rate: $1.0 \mathrm{~mL} / \mathrm{min}, \lambda=254 \mathrm{~nm}$, $\mathrm{t}_{\mathrm{R}}($ major $)=5.0 \mathrm{~min}, \mathrm{t}_{\mathrm{R}}($ minor $)=6.7 \mathrm{~min}, 91 \%$ ee .

\begin{tabular}{|l|l|l|}
\hline Peak\# & Ret. Time & Area\% \\
\hline 1 & 4.982 & 49.844 \\
\hline 2 & 6.679 & 50.156 \\
\hline
\end{tabular}
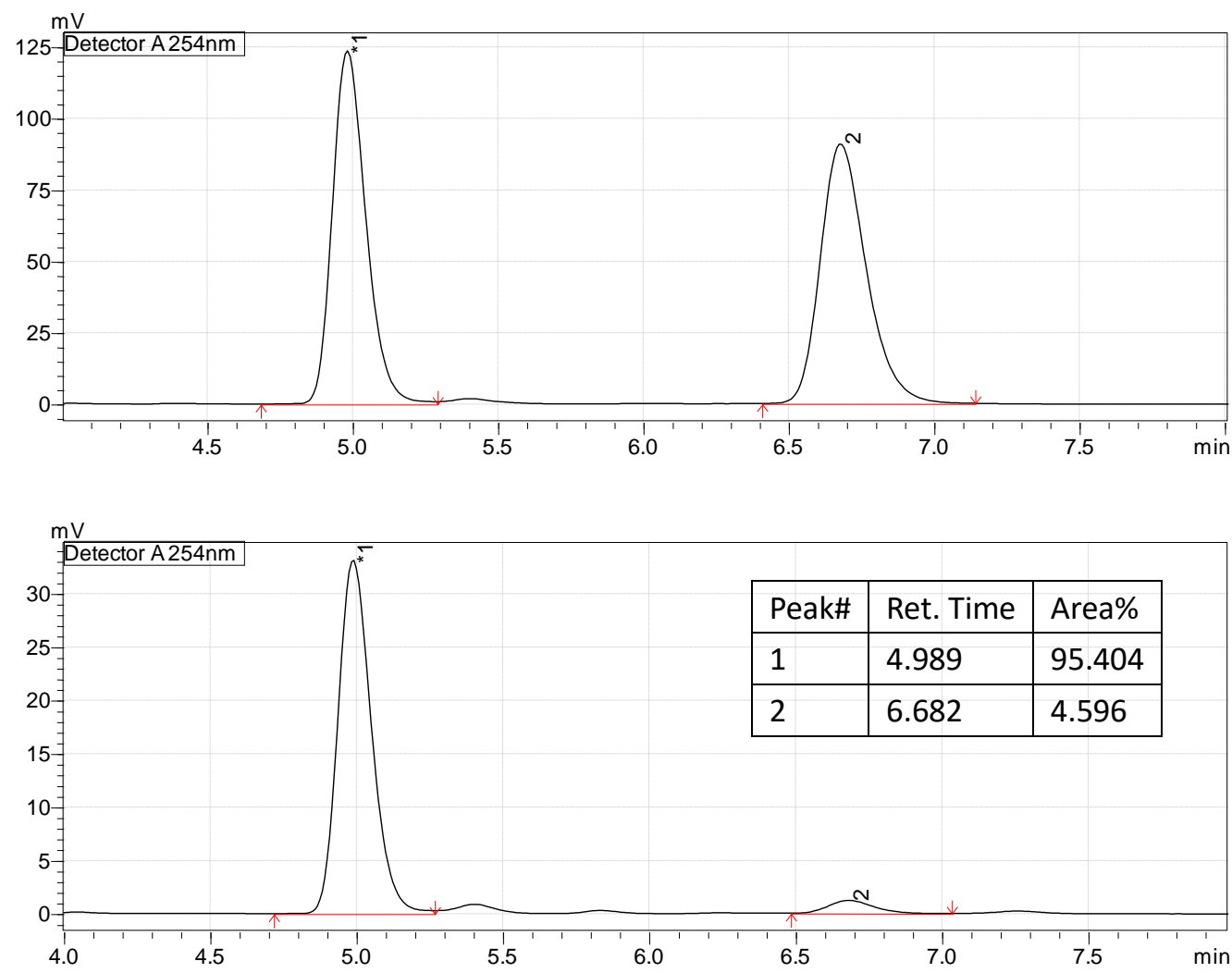


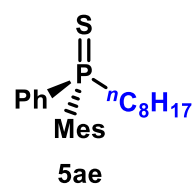

5ae: Procedure B, $70.7 \mathrm{mg}, 95 \%$ yield, yellow liquid.

${ }^{1}$ H NMR (400 MHz, CDCl $)_{3} \delta$ 7.80-7.72 (m, 2H), 7.51-7.38 (m, 3H), $6.83(\mathrm{~d}, J=3.9 \mathrm{~Hz}, 2 \mathrm{H})$, 2.63-2.37 (m, 2H), 2.29 (s, 6H), $2.26(\mathrm{~s}, 3 \mathrm{H}), 1.76-1.59(\mathrm{~m}, 1 \mathrm{H}), 1.36-1.19(\mathrm{~m}, 4 \mathrm{H}), 1.19-1.11$ (m, 6H), $0.83(\mathrm{t}, J=7.0 \mathrm{~Hz}, 3 \mathrm{H}), 0.80-0.70(\mathrm{~m}, 1 \mathrm{H})$.

${ }^{13} \mathbf{C}$ NMR(101 MHz, $\left.\mathbf{C D C l}_{3}\right) \delta 141.46(\mathrm{~d}, J=10.1 \mathrm{~Hz}), 140.65(\mathrm{~d}, J=2.9 \mathrm{~Hz}), 134.69(\mathrm{~d}, J=$ $77.3 \mathrm{~Hz}), 131.22(\mathrm{~d}, J=11.1 \mathrm{~Hz}), 130.64(\mathrm{~d}, J=2.8 \mathrm{~Hz}), 130.60(\mathrm{~d}, J=10.0 \mathrm{~Hz}), 128.93$ (d, $J=$ $81.6 \mathrm{~Hz}), 128.25(\mathrm{~d}, J=11.9 \mathrm{~Hz}), 38.67(\mathrm{~d}, J=53.0 \mathrm{~Hz}), 31.59,30.68(\mathrm{~d}, J=17.2 \mathrm{~Hz}), 28.92$, 28.89, $23.93(\mathrm{~d}, J=5.1 \mathrm{~Hz}), 22.76(\mathrm{~d}, J=3.2 \mathrm{~Hz}), 22.47,20.75(\mathrm{~d}, J=1.5 \mathrm{~Hz}), 13.97$.

${ }^{31}$ P NMR (162 MHz, CDCl $) \delta 43.65$.

HRMS (ESI) $\mathbf{m} / \mathbf{z}[\mathbf{M}+\mathbf{H}]^{+}$: calcd. 373.2113, found. 373.2113.

IR (film): $v_{\max }\left(\mathrm{cm}^{-1}\right)$ 3050, 2924, 2853, 1604, 1455, 1436, 1377, 1100, 1027, 849, 748, 693, 648, 595.

Optical rotation: $[\alpha]_{\mathrm{D}}{ }^{25}=-59.68\left(c=1.000, \mathrm{CHCl}_{3}, 90 \%\right.$ ee $)$.

HPLC: DAICEL CHIRALPAK OD-H, hexane $/ i-\mathrm{PrOH}=97 / 3$, flow rate: $1.0 \mathrm{~mL} / \mathrm{min}, \lambda=254 \mathrm{~nm}$, $\mathrm{t}_{\mathrm{R}}($ major $)=4.9 \mathrm{~min}, \mathrm{t}_{\mathrm{R}}($ minor $)=6.1 \mathrm{~min}, 90 \%$ ee.
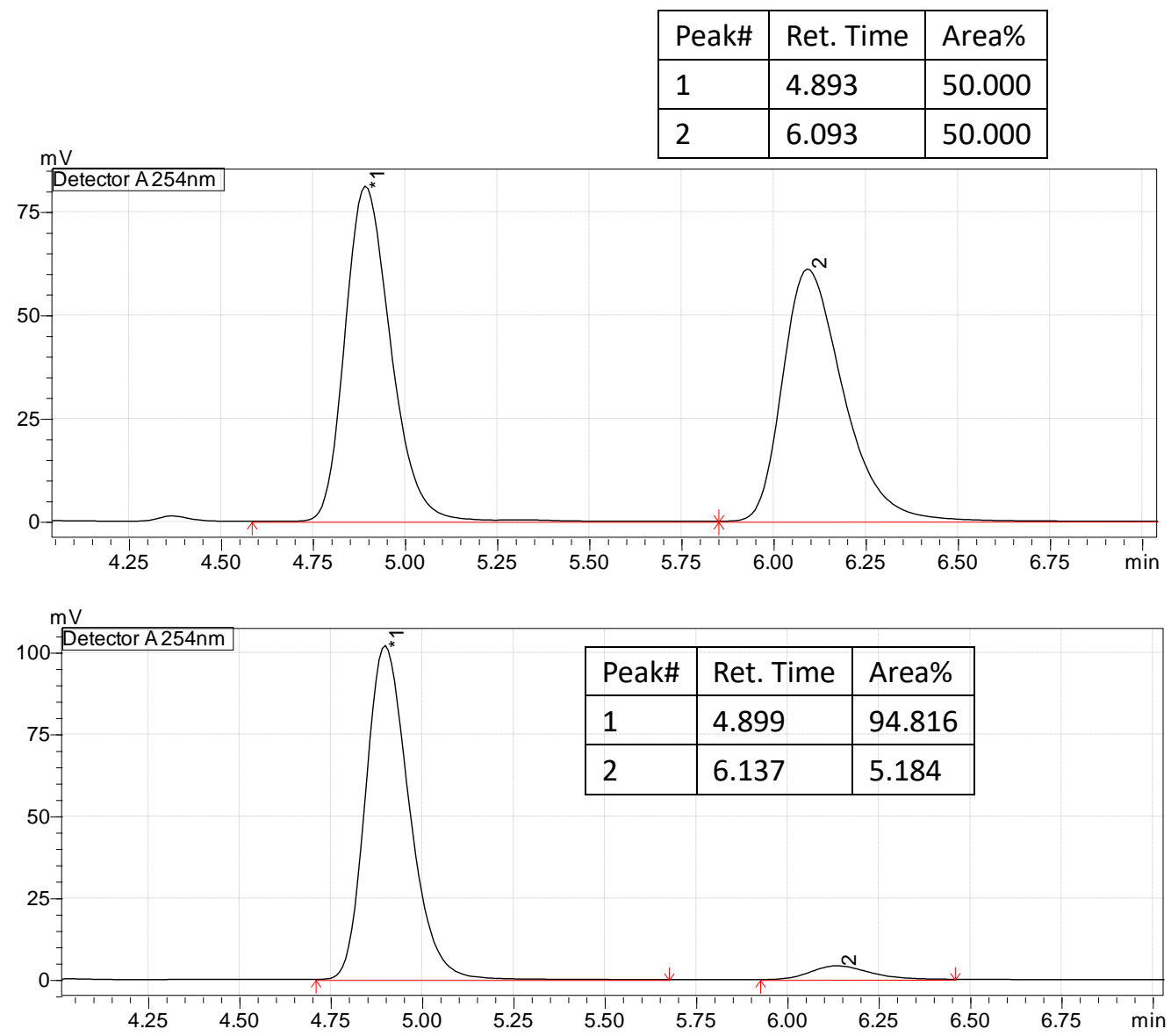


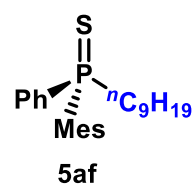

5af: Procedure B, $71.8 \mathrm{mg}, 93 \%$ yield, colorless liquid.

${ }^{1}$ H NMR (400 MHz, CDCl $\left.{ }_{3}\right) \delta 7.81-7.71(\mathrm{~m}, 2 \mathrm{H}), 7.50-7.38(\mathrm{~m}, 3 \mathrm{H}), 6.83(\mathrm{~d}, J=3.9 \mathrm{~Hz}, 2 \mathrm{H})$, $2.62-2.38(\mathrm{~m}, 2 \mathrm{H}), 2.29(\mathrm{~s}, 6 \mathrm{H}), 2.26(\mathrm{~s}, 3 \mathrm{H}), 1.76-1.59(\mathrm{~m}, 1 \mathrm{H}), 1.33-1.21(\mathrm{~m}, 4 \mathrm{H}), 1.20-1.12$ $(\mathrm{m}, 8 \mathrm{H}), 0.85(\mathrm{t}, J=6.9 \mathrm{~Hz}, 3 \mathrm{H}), 0.81-0.70(\mathrm{~m}, 1 \mathrm{H})$.

${ }^{13} \mathbf{C}$ NMR(101 MHz, $\left.\mathbf{C D C l}_{3}\right) \delta 141.45(\mathrm{~d}, J=10.0 \mathrm{~Hz}), 140.64(\mathrm{~d}, J=2.8 \mathrm{~Hz}), 134.67(\mathrm{~d}, J=$ $77.2 \mathrm{~Hz}), 131.21(\mathrm{~d}, J=11.1 \mathrm{~Hz}), 130.63(\mathrm{~d}, J=2.7 \mathrm{~Hz}), 130.59$ (d, $J=10.1 \mathrm{~Hz}), 128.92(\mathrm{~d}, J=$ $81.7 \mathrm{~Hz}), 128.25(\mathrm{~d}, J=11.9 \mathrm{~Hz}), 38.66(\mathrm{~d}, J=53.0 \mathrm{~Hz}), 31.67,30.68(\mathrm{~d}, J=17.2 \mathrm{~Hz}), 29.18$, 29.04, 28.97, 23.93 (d, $J=5.2 \mathrm{~Hz}), 22.76$ (d, $J=3.2 \mathrm{~Hz}), 22.52,20.75$ (d, $J=1.5 \mathrm{~Hz}), 14.00$.

${ }^{31}$ P NMR (162 MHz, $\left.\mathbf{C D C l}_{3}\right) \delta 43.65$.

HRMS (ESI) $\mathbf{m} / \mathbf{z}$ [M+H] $]^{+}$: calcd. 387.2270, found. 387.2270.

IR (film): $v_{\max }\left(\mathrm{cm}^{-1}\right)$ 2958, 2924, 2853, 1604, 1455, 1436, 1100, 1027, 849, 748, 693, 648, 595.

Optical rotation: $[\alpha]_{\mathrm{D}}{ }^{25}=-53.62\left(c=1.460, \mathrm{CHCl}_{3}, 89 \%\right.$ ee $)$.

HPLC: DAICEL CHIRALPAK OD-H, hexane $/ i-\mathrm{PrOH}=97 / 3$, flow rate: $1.0 \mathrm{~mL} / \mathrm{min}, \lambda=254 \mathrm{~nm}$, $\mathrm{t}_{\mathrm{R}}($ major $)=4.9 \mathrm{~min}, \mathrm{t}_{\mathrm{R}}($ minor $)=6.2 \mathrm{~min}, 89 \%$ ee.

\begin{tabular}{|l|l|l|}
\hline Peak\# & Ret. Time & Area\% \\
\hline 1 & 4.867 & 50.081 \\
\hline 2 & 6.160 & 49.919 \\
\hline
\end{tabular}
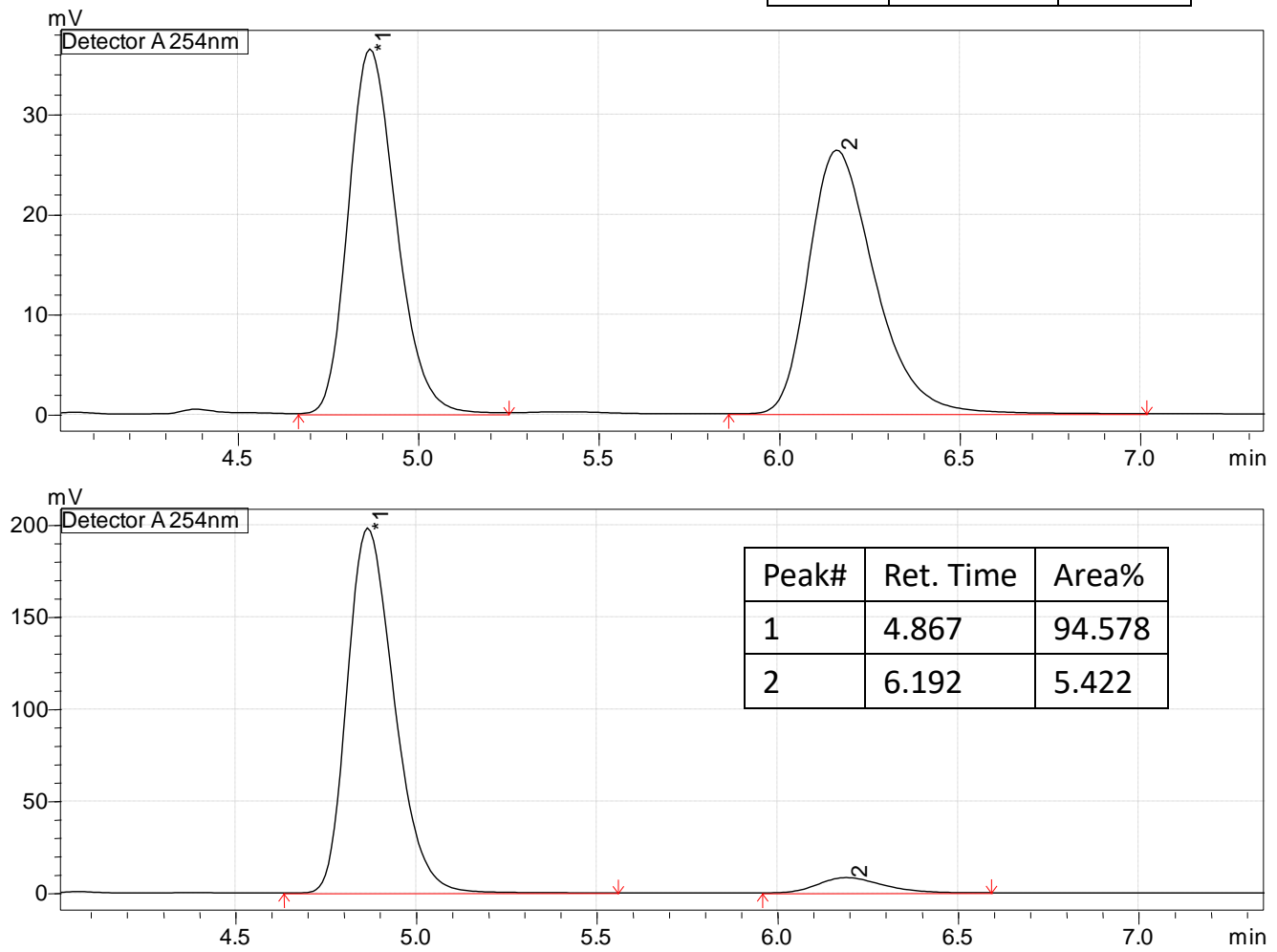


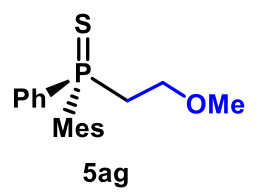

5ag: Procedure B, $62.3 \mathrm{mg}, 98 \%$ yield, light yellow liquid.

${ }^{1}$ H NMR (400 MHz, CDCl M $\delta$ 7.85-7.73 (m, 2H), 7.54-7.39 (m, 3H), $6.84(\mathrm{~d}, J=4.1 \mathrm{~Hz}, 2 \mathrm{H})$, 3.72-3.62 (m, 1H), $3.15(\mathrm{~s}, 3 \mathrm{H}), 3.05-2.87(\mathrm{~m}, 2 \mathrm{H}), 2.85-2.74(\mathrm{~m}, 1 \mathrm{H}), 2.30(\mathrm{~s}, 6 \mathrm{H}), 2.26(\mathrm{~s}, 3 \mathrm{H})$. ${ }^{13}$ C NMR(101 MHz, CDCl $) \delta 141.39(\mathrm{~d}, J=10.3 \mathrm{~Hz}), 140.95(\mathrm{~d}, J=3.0 \mathrm{~Hz}), 134.40(\mathrm{~d}, J=$ $77.6 \mathrm{~Hz}), 131.30$ (d, $J=11.3 \mathrm{~Hz}), 130.93(\mathrm{~d}, J=2.8 \mathrm{~Hz}), 130.40(\mathrm{~d}, J=10.6 \mathrm{~Hz}), 128.45(\mathrm{~d}, J=$ $84.4 \mathrm{~Hz}), 128.45$ (d, $J=12.2 \mathrm{~Hz}), 67.25$ (d, $J=4.3 \mathrm{~Hz}), 58.33,38.23$ (d, $J=54.0 \mathrm{~Hz}), 23.82(\mathrm{~d}, J$ $=5.4 \mathrm{~Hz}), 20.75(\mathrm{~d}, J=1.5 \mathrm{~Hz})$.

${ }^{31}$ P NMR (162 MHz, $\left.\mathbf{C D C l}_{3}\right) \delta 37.63$.

HRMS (ESI) $\mathbf{~ m / z ~ [ M + H ] ~}{ }^{+}$: calcd. 319.1280, found. 319.1280.

IR (film): $v_{\max }\left(\mathrm{cm}^{-1}\right)$ 3049, 2960, 2922, 2829, 1603, 1556, 1455, 1260, 1096, 1026, 850, 797, 749, 692, 648, 594.

Optical rotation: $[\alpha]_{\mathrm{D}}^{25}=-71.34\left(c=1.120, \mathrm{CHCl}_{3}, 82 \%\right.$ ee $)$.

HPLC: DAICEL CHIRALPAK IA, hexane $/ i$-PrOH $=49 / 1$, flow rate: $1.0 \mathrm{~mL} / \mathrm{min}, \lambda=220 \mathrm{~nm}$, $\mathrm{t}_{\mathrm{R}}($ major $)=11.5 \mathrm{~min}, \mathrm{t}_{\mathrm{R}}($ minor $)=13.0 \mathrm{~min}, 82 \%$ ee.

\begin{tabular}{|l|l|l|}
\hline Peak\# & Ret. Time & Area\% \\
\hline 1 & 11.433 & 49.883 \\
\hline 2 & 12.725 & 50.117 \\
\hline
\end{tabular}
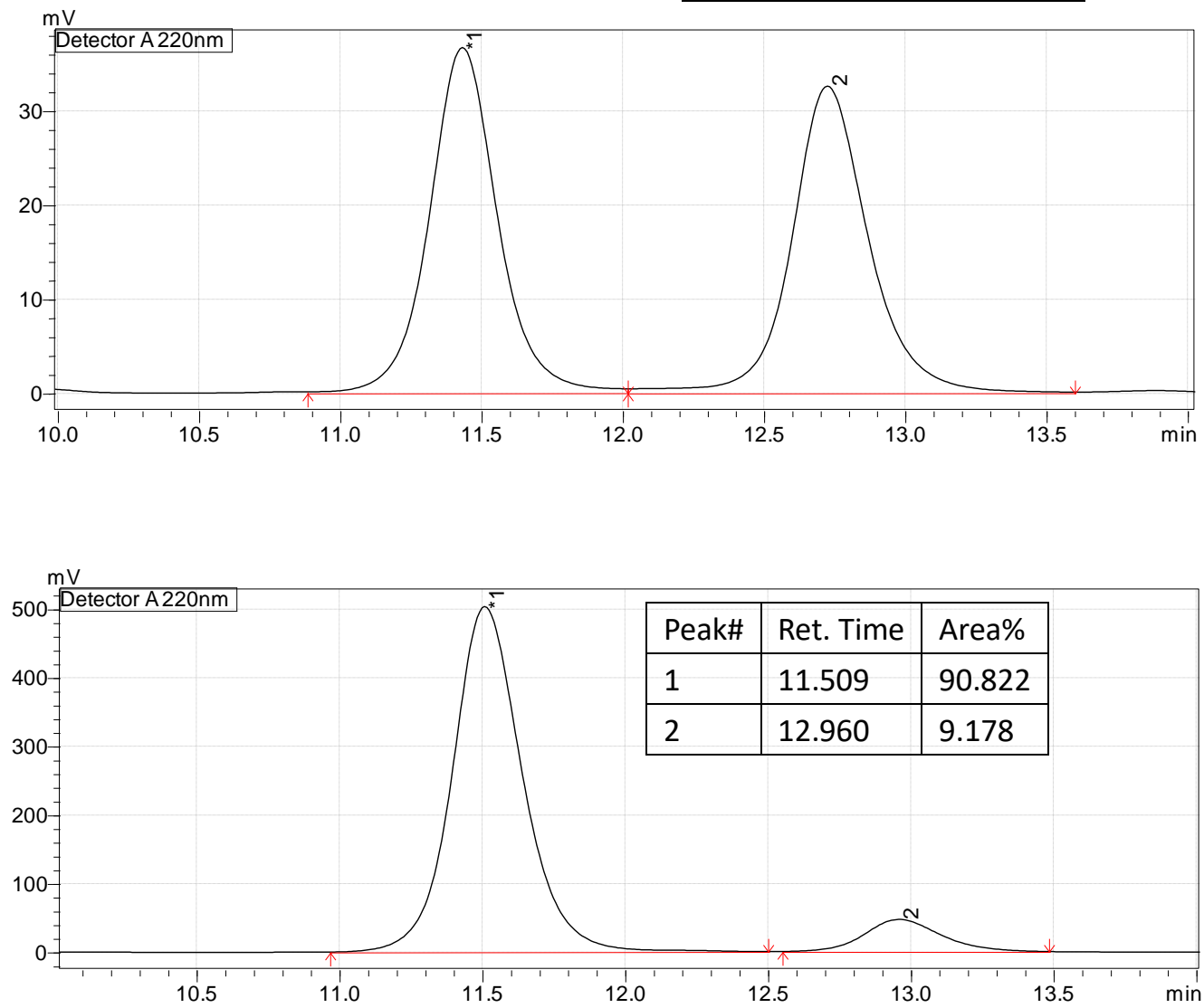


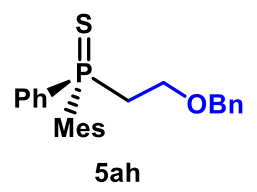

5ah: Procedure B, $74.8 \mathrm{mg}$, 95\% yield, light yellow liquid.

${ }^{1}$ H NMR (400 MHz, CDCl $_{3}$ ) $\delta$ 7.81-7.72 (m, 2H), 7.51-7.36 (m, 3H), 7.30-7.19 (m, 3H), 7.19$7.13(\mathrm{~m}, 2 \mathrm{H}), 6.82(\mathrm{~d}, J=4.1 \mathrm{~Hz}, 2 \mathrm{H}), 4.37-4.21(\mathrm{~m}, 2 \mathrm{H}), 3.90-3.76(\mathrm{~m}, 1 \mathrm{H}), 3.14-2.97(\mathrm{~m}, 2 \mathrm{H})$, 2.92-2.76 (m, 1H), $2.28(\mathrm{~s}, 6 \mathrm{H}), 2.25(\mathrm{~s}, 3 \mathrm{H})$.

${ }^{13} \mathbf{C}$ NMR(101 MHz, CDCl $) \delta 141.38(\mathrm{~d}, J=10.4 \mathrm{~Hz}), 140.89(\mathrm{~d}, J=3.0 \mathrm{~Hz}), 137.64,134.33(\mathrm{~d}$, $J=93.0 \mathrm{~Hz}), 131.26(\mathrm{~d}, J=11.4 \mathrm{~Hz}), 130.89(\mathrm{~d}, J=3.0 \mathrm{~Hz}), 130.40(\mathrm{~d}, J=10.6 \mathrm{~Hz}), 128.46(\mathrm{~d}, J$ $=84.5 \mathrm{~Hz}), 128.42(\mathrm{~d}, J=12.2 \mathrm{~Hz}), 128.19,127.52,72.87,65.33(\mathrm{~d}, J=4.2 \mathrm{~Hz}), 38.45(\mathrm{~d}, J=$ $53.6 \mathrm{~Hz}), 23.83(\mathrm{~d}, J=5.6 \mathrm{~Hz}), 20.73(\mathrm{~d}, J=1.5 \mathrm{~Hz})$.

${ }^{31}$ P NMR (162 MHz, $\left.\mathbf{C D C l}_{\mathbf{3}}\right) \delta 37.81$.

HRMS (ESI) $\mathbf{m} / \mathbf{z}$ [M+H] $]^{+}$: calcd. 395.1593, found. 395.1593 .

IR (film): $v_{\max }\left(\mathrm{cm}^{-1}\right)$ 2954, 2922, 1732, 1603, 1455, 1404, 1377, 1261, 1092, 1027, 850, 796, 749, 692, 648.

Optical rotation: $[\alpha]_{\mathrm{D}}^{25}=-45.25\left(c=1.000, \mathrm{CHCl}_{3}, 86 \%\right.$ ee $)$.

HPLC: DAICEL CHIRALPAK IA, hexane $/ i-\mathrm{PrOH}=49 / 1$, flow rate: $1.0 \mathrm{~mL} / \mathrm{min}, \lambda=220 \mathrm{~nm}$, $\mathrm{t}_{\mathrm{R}}($ major $)=13.5 \mathrm{~min}, \mathrm{t}_{\mathrm{R}}($ minor $)=16.0 \mathrm{~min}, 86 \%$ ee .
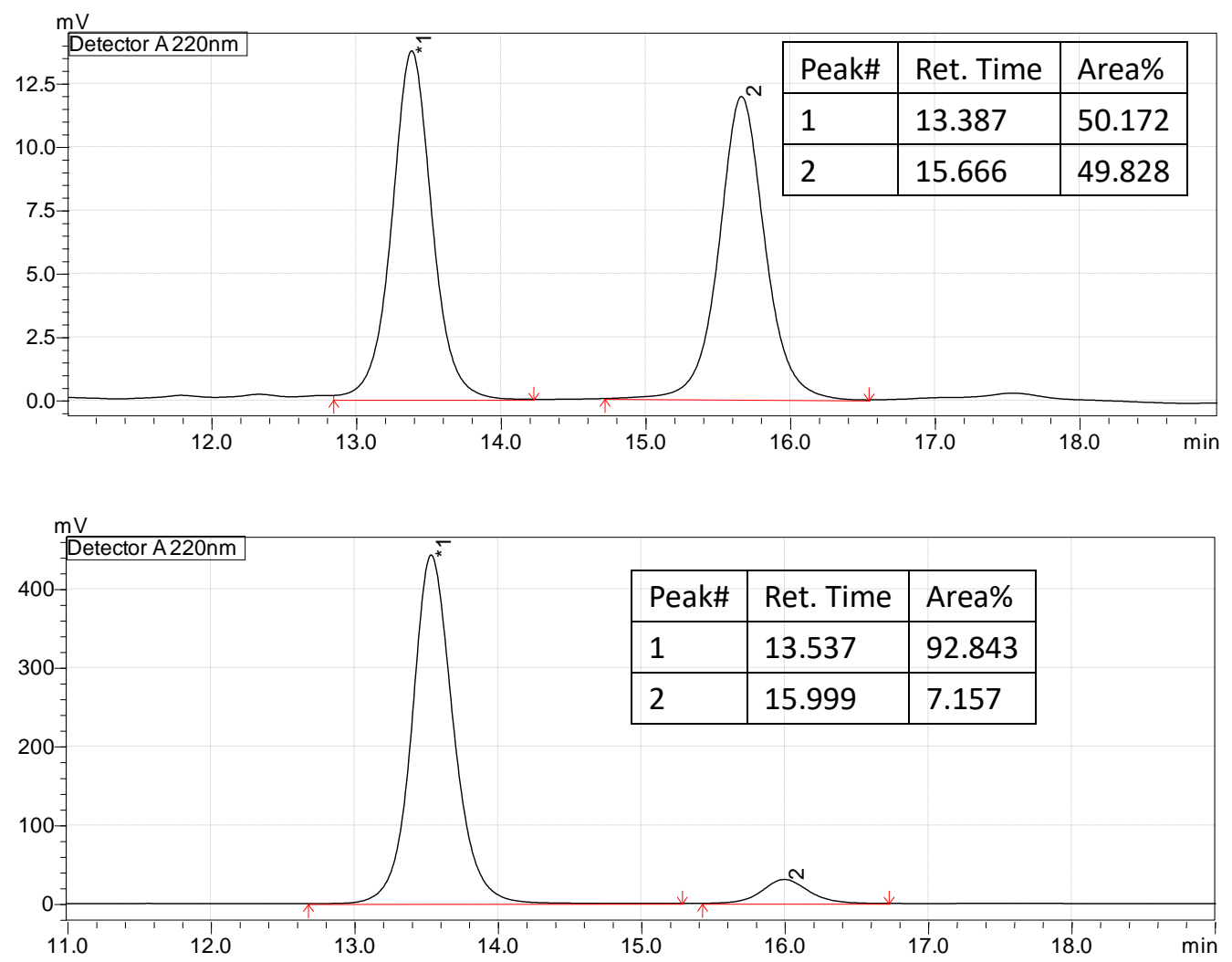


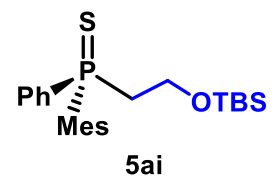

5ai: Procedure B, $76.1 \mathrm{mg}, 91 \%$ yield, yellow solid.

${ }^{1}$ H NMR (400 MHz, $\left.\mathbf{C D C l}_{3}\right) \delta 7.82-7.73(\mathrm{~m}, 2 \mathrm{H}), 7.52-7.39(\mathrm{~m}, 3 \mathrm{H}), 6.83(\mathrm{~d}, J=4.1 \mathrm{~Hz}, 2 \mathrm{H})$, 4.04-3.91 (m, 1H), 3.19-3.09 (m, 1H), 3.00-2.88 (m, 1H), 2.81-2.68 (m, 1H), $2.30(\mathrm{~s}, 6 \mathrm{H}), 2.26$ (s, 3H), $0.78(\mathrm{~s}, 9 \mathrm{H}),-0.09$ (d, $J=8.1 \mathrm{~Hz}, 6 \mathrm{H})$.

${ }^{13} \mathbf{C}$ NMR(101 MHz, $\left.\mathbf{C D C l}_{3}\right) \delta 141.41(\mathrm{~d}, J=10.3 \mathrm{~Hz}), 140.84(\mathrm{~d}, J=3.0 \mathrm{~Hz}), 134.62(\mathrm{~d}, J=$ $77.4 \mathrm{~Hz}), 131.27(\mathrm{~d}, J=11.4 \mathrm{~Hz}), 130.87(\mathrm{~d}, J=3.0 \mathrm{~Hz}), 130.43(\mathrm{~d}, J=10.4 \mathrm{~Hz}), 128.64$ (d, $J=$ $83.5 \mathrm{~Hz}), 128.42(\mathrm{~d}, J=12.1 \mathrm{~Hz}), 58.50(\mathrm{~d}, J=5.1 \mathrm{~Hz}), 41.30(\mathrm{~d}, J=51.7 \mathrm{~Hz}), 25.72,23.86(\mathrm{~d}, J$ $=5.5 \mathrm{~Hz}), 20.76(\mathrm{~d}, J=1.5 \mathrm{~Hz}), 18.04,-5.47(\mathrm{~d}, J=5.1 \mathrm{~Hz})$.

${ }^{31}$ P NMR (162 MHz, $\left.\mathbf{C D C l}_{3}\right) \delta 36.79$.

HRMS (ESI) $\mathbf{m} / \mathbf{z}[\mathbf{M}+\mathbf{H}]^{+}$: calcd. 419.1988 , found. 419.1988 .

IR (film): $v_{\max }\left(\mathrm{cm}^{-1}\right)$ 3054, 2927, 2855, 1604, 1461, 1437, $1404,1255,1212,1086,1006,837$, $777,651,595$.

Optical rotation: $[\alpha]_{\mathrm{D}}{ }^{25}=-54.62\left(c=1.000, \mathrm{CHCl}_{3}, 90 \%\right.$ ee $)$.

HPLC: DAICEL CHIRALPAK OD-H, hexane $/ i-\mathrm{PrOH}=124 / 1$, flow rate: $1.0 \mathrm{~mL} / \mathrm{min}, \lambda=220$ $\mathrm{nm}, \mathrm{t}_{\mathrm{R}}($ major $)=6.0 \mathrm{~min}, \mathrm{t}_{\mathrm{R}}($ minor $)=8.1 \mathrm{~min}, 90 \%$ ee
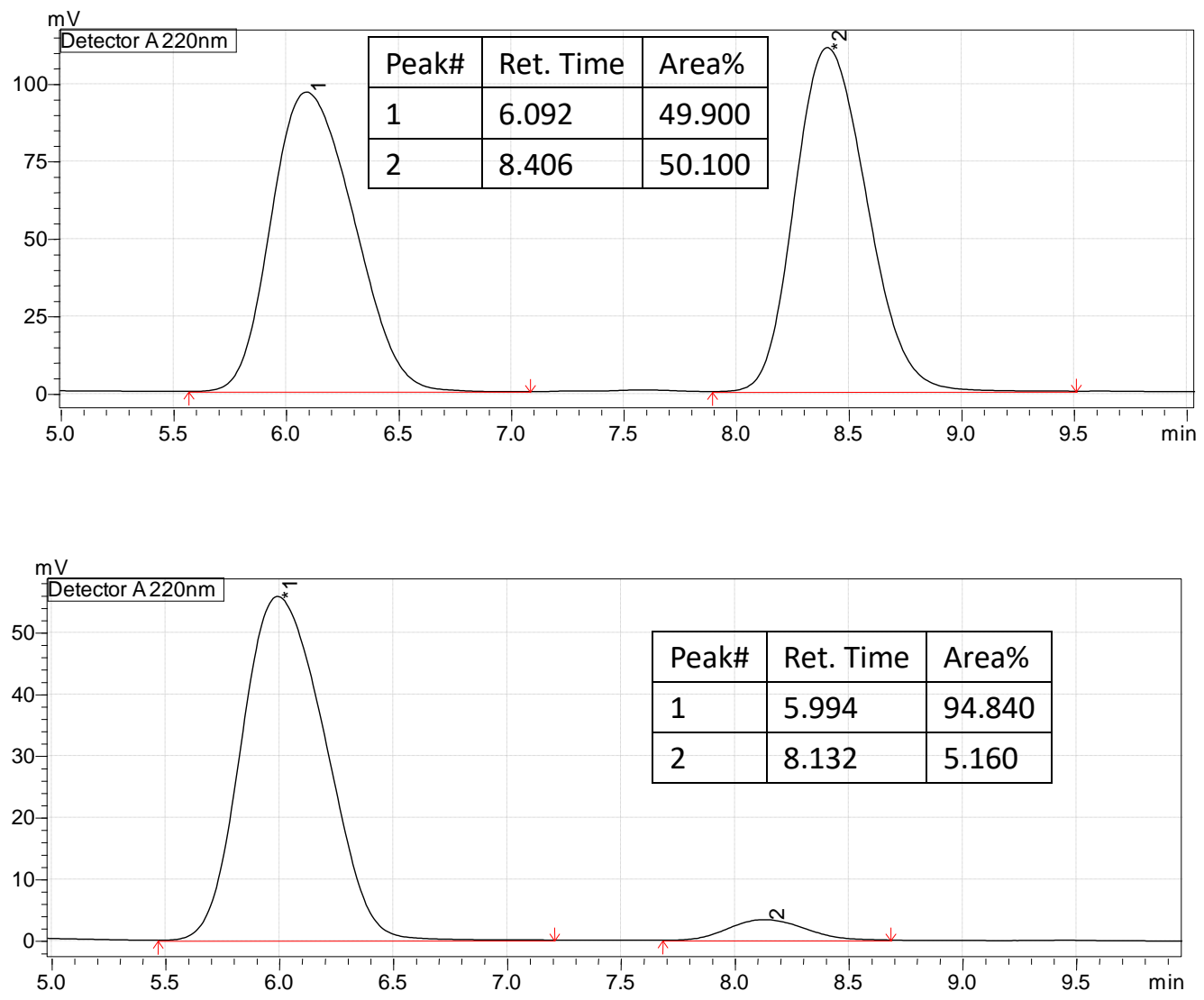


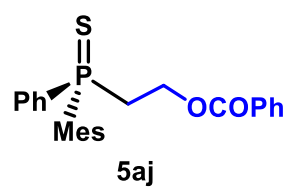

5aj: Procedure B, $80.0 \mathrm{mg}$, 98\% yield, light yellow liquid.

${ }^{1}$ H NMR (400 MHz, $\left.\mathbf{C D C l}_{3}\right) \delta$ 7.88-7.83 (m, 3H), 7.83-7.80 (m, 1H), 7.55-7.48 (m, 1H), 7.47$7.40(\mathrm{~m}, 3 \mathrm{H}), 7.39-7.33(\mathrm{~m}, 2 \mathrm{H}), 6.84(\mathrm{~d}, J=4.1 \mathrm{~Hz}, 2 \mathrm{H}), 4.59-4.47(\mathrm{~m}, 1 \mathrm{H}), 4.17-4.04(\mathrm{~m}, 1 \mathrm{H})$, 3.19-2.99 (m, 2H), $2.31(\mathrm{~s}, 6 \mathrm{H}), 2.26(\mathrm{~s}, 3 \mathrm{H})$.

${ }^{13} \mathbf{C}$ NMR(101 MHz, CDCl $) \delta 166.08,141.40(\mathrm{~d}, J=10.4 \mathrm{~Hz}), 141.07(\mathrm{~d}, J=3.0 \mathrm{~Hz}), 134.16(\mathrm{~d}$, $J=77.9 \mathrm{~Hz}), 132.93,131.36(\mathrm{~d}, J=11.4 \mathrm{~Hz}), 131.13(\mathrm{~d}, J=3.0 \mathrm{~Hz}), 130.48(\mathrm{~d}, J=10.6 \mathrm{~Hz})$, $129.51(\mathrm{~d}, J=8.0 \mathrm{~Hz}), 129.47,128.66(\mathrm{~d}, J=12.3 \mathrm{~Hz}), 128.21(\mathrm{~d}, J=84.9 \mathrm{~Hz}), 128.13,60.04(\mathrm{~d}, J$ $=4.2 \mathrm{~Hz}), 37.54(\mathrm{~d}, J=53.4 \mathrm{~Hz}), 23.86(\mathrm{~d}, J=5.4 \mathrm{~Hz}), 20.76(\mathrm{~d}, J=1.5 \mathrm{~Hz})$.

${ }^{31}$ P NMR (162 MHz, $\mathbf{C D C l}_{3}$ ) $\delta 37.62$.

HRMS (ESI) $\mathbf{m} / \mathbf{z}$ [M+H] $]^{+}$: calcd. 409.1386, found. 409.1386.

IR (film): $v_{\max }\left(\mathrm{cm}^{-1}\right) 3056,2960,2924,2853,1716,1603,1451,1437,1377,1272,1176,1099$, 1070, 976, 852, 710, 650, 598.

Optical rotation: $[\alpha]_{\mathrm{D}}{ }^{25}=-24.12\left(c=1.000, \mathrm{CHCl}_{3}, 90 \%\right.$ ee $)$.

HPLC: DAICEL CHIRALPAK IA, hexane $/ i-\mathrm{PrOH}=99 / 1$, flow rate: $1.0 \mathrm{~mL} / \mathrm{min}, \lambda=220 \mathrm{~nm}$, $\mathrm{t}_{\mathrm{R}}($ minor $)=29.3 \mathrm{~min}, \mathrm{t}_{\mathrm{R}}($ major $)=34.2 \mathrm{~min}, 90 \%$ ee .
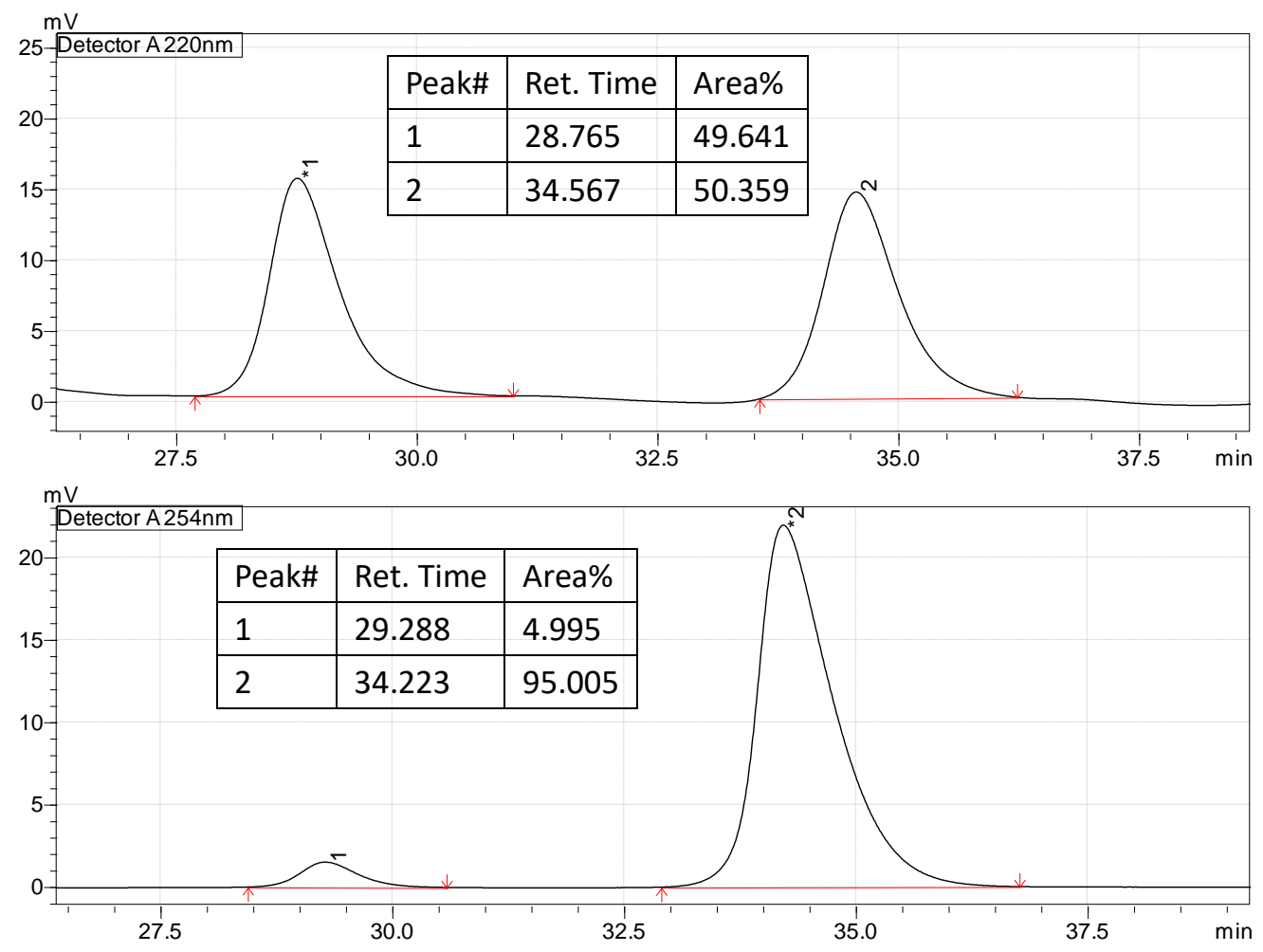


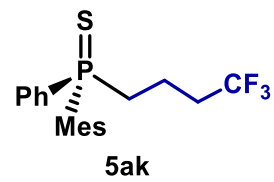

5ak: Procedure B, $76.2 \mathrm{mg}$, 98\% yield, light yellow liquid.

${ }^{1}$ H NMR $\left(400 \mathrm{MHz}, \mathbf{C D C l}_{3}\right) \delta 7.82-7.72(\mathrm{~m}, 2 \mathrm{H}), 7.53-7.41(\mathrm{~m}, 3 \mathrm{H}), 6.85(\mathrm{~d}, J=4.0 \mathrm{~Hz}, 2 \mathrm{H})$, $2.73-2.58(\mathrm{~m}, 1 \mathrm{H}), 2.57-2.43(\mathrm{~m}, 1 \mathrm{H}), 2.29(\mathrm{~s}, 6 \mathrm{H}), 2.26(\mathrm{~s}, 3 \mathrm{H}), 2.23-2.08(\mathrm{~m}, 1 \mathrm{H}), 2.09-1.89$ (m, 2H), 1.17-0.99 (m, 1H).

${ }^{13} \mathbf{C}$ NMR(101 MHz, $\left.\mathbf{C D C l}_{3}\right) \delta 141.39(\mathrm{~d}, J=10.1 \mathrm{~Hz}), 141.03(\mathrm{~d}, J=3.0 \mathrm{~Hz}), 133.97(\mathrm{~d}, J=$ $77.6 \mathrm{~Hz}), 131.36(\mathrm{~d}, J=11.3 \mathrm{~Hz}), 131.08(\mathrm{~d}, J=3.0 \mathrm{~Hz}), 130.57(\mathrm{~d}, J=10.3 \mathrm{~Hz}), 128.56(\mathrm{~d}, J=$ $12.0 \mathrm{~Hz}), 128.47(\mathrm{~d}, J=83.6 \mathrm{~Hz}), 126.50(\mathrm{dd}, J=276.8, J=1.6 \mathrm{~Hz}), 37.21(\mathrm{~d}, J=54.6 \mathrm{~Hz}), 34.20$ $(\mathrm{qd}, J=28.6,17.0 \mathrm{~Hz}), 23.93(\mathrm{~d}, J=5.2 \mathrm{~Hz}), 20.76(\mathrm{~d}, J=1.5 \mathrm{~Hz}), 15.93(\mathrm{~d}, J=2.8 \mathrm{~Hz})$.

${ }^{31}$ P NMR (162 MHz, $\left.\mathbf{C D C l}_{3}\right) \delta 42.80$.

${ }^{19}$ F NMR (376 MHz, $\left.\mathbf{C D C l}_{\mathbf{3}}\right) \delta-65.94(\mathrm{t}, J=10.4 \mathrm{~Hz})$.

HRMS (ESI) $\mathbf{m} / \mathbf{z}[\mathbf{M}+\mathbf{H}]^{+}$: calcd. 371.1205, found. 371.1205 .

IR (film): $v_{\max }\left(\mathrm{cm}^{-1}\right)$ 3062, 2962, 2925, 1604, 1455, 1437, 1387, 1258, 1135, 1099, 1075, 851, 749, 694, 660, 594.

Optical rotation: $[\alpha]_{\mathrm{D}}{ }^{25}=-70.26\left(c=1.000, \mathrm{CHCl}_{3}, 90 \% \mathrm{ee}\right)$.

HPLC: DAICEL CHIRALPAK OD-H, hexane $/ i-\mathrm{PrOH}=97 / 3$, flow rate: $1.0 \mathrm{~mL} / \mathrm{min}, \lambda=254 \mathrm{~nm}$, $\mathrm{t}_{\mathrm{R}}($ major $)=6.8 \mathrm{~min}, \mathrm{t}_{\mathrm{R}}(\operatorname{minor})=10.5 \mathrm{~min}, 90 \%$ ee.
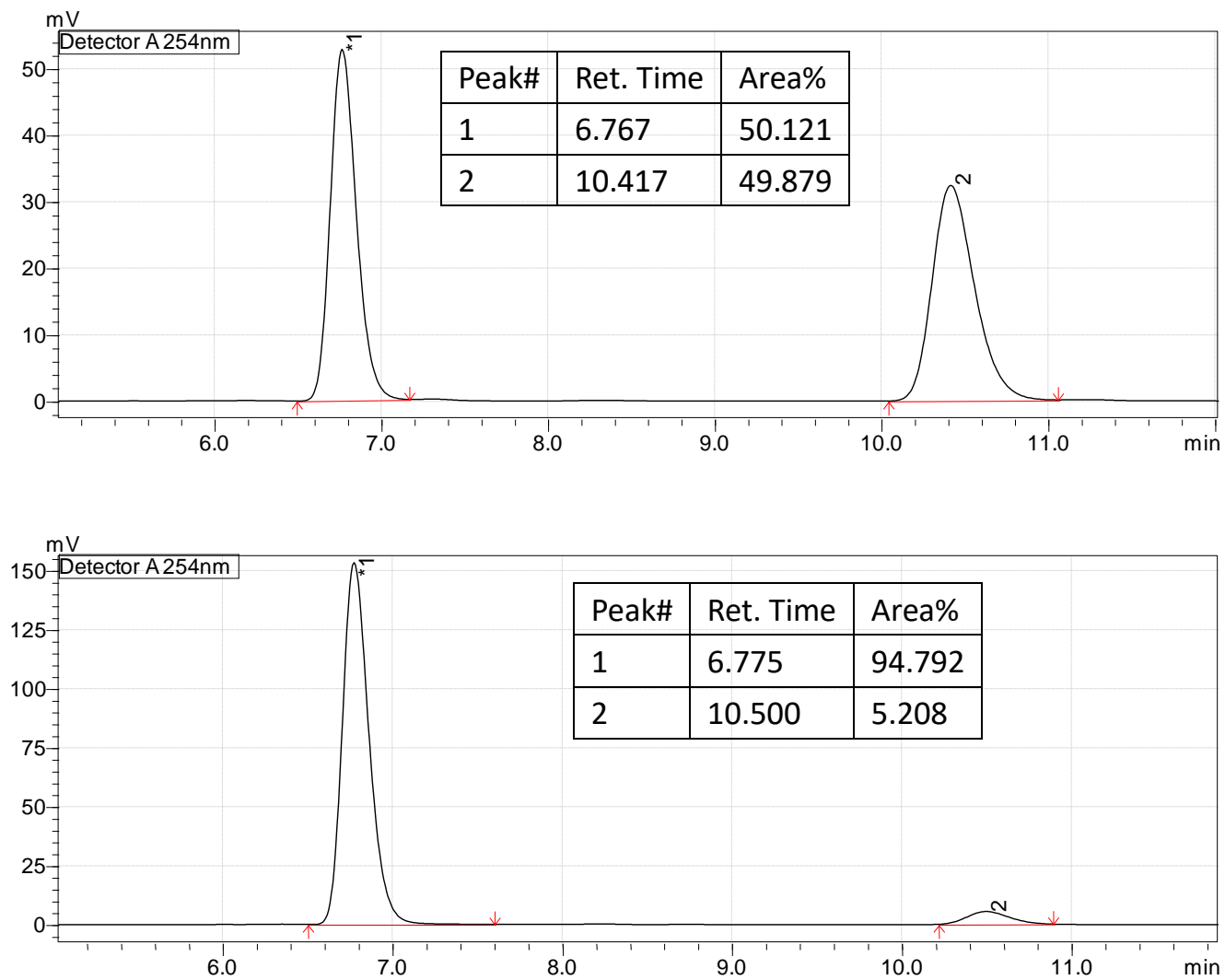


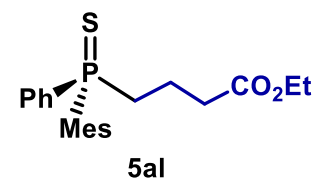

5al: Procedure B, $56.1 \mathrm{mg}, 80 \%$ yield, yellow solid.

${ }^{1}$ H NMR (400 MHz, CDCl $) \delta$ 7.84-7.73 (m, 2H), 7.53-7.39 (m, 3H), $6.83(\mathrm{~d}, J=4.0 \mathrm{~Hz}, 2 \mathrm{H})$, 4.05 (q, $J=7.1 \mathrm{~Hz}, 2 \mathrm{H}), 2.69-2.48(\mathrm{~m}, 2 \mathrm{H}), 2.39-2.22(\mathrm{~m}, 2 \mathrm{H}), 2.29$ (s, 6H), $2.26(\mathrm{~s}, 3 \mathrm{H}), 2.07-$ $1.87(\mathrm{~m}, 1 \mathrm{H}), 1.19(\mathrm{t}, J=7.1 \mathrm{~Hz}, 3 \mathrm{H}), 1.12-1.23(\mathrm{~m}, 1 \mathrm{H})$.

${ }^{13}$ C NMR(101 MHz, CDCl $) \delta 172.51,141.45(\mathrm{~d}, J=10.1 \mathrm{~Hz}), 140.83(\mathrm{~d}, J=2.8 \mathrm{~Hz}), 134.26(\mathrm{~d}$, $J=77.5 \mathrm{~Hz}), 131.26(\mathrm{~d}, J=11.3 \mathrm{~Hz}), 130.87(\mathrm{~d}, J=3.0 \mathrm{~Hz}), 130.66(\mathrm{~d}, J=10.3 \mathrm{~Hz}), 128.62(\mathrm{~d}, J$ $=82.7 \mathrm{~Hz}), 128.42(\mathrm{~d}, J=12.0 \mathrm{~Hz}), 60.28,37.67(\mathrm{~d}, J=54.0 \mathrm{~Hz}), 34.68(\mathrm{~d}, J=17.5 \mathrm{~Hz}), 23.94(\mathrm{~d}$, $J=5.2 \mathrm{~Hz}), 20.76(\mathrm{~d}, J=1.6 \mathrm{~Hz}), 18.60(\mathrm{~d}, J=1.5 \mathrm{~Hz}), 14.10$.

${ }^{31}$ P NMR (162 MHz, $\left.\mathbf{C D C l}_{3}\right) \delta 43.03$.

HRMS (ESI) $\mathbf{m} / \mathbf{z}[\mathbf{M}+\mathbf{H}]^{+}$: calcd. 375.1542 , found. 375.1543 .

IR (film): $v_{\max }\left(\mathrm{cm}^{-1}\right)$ 3058, 2977, 2929, 1731, 1604, 1454, 1437, 1212, 1177, 1099, 1029, 854, 749, 694, 647, 594.

Optical rotation: $[\alpha]_{\mathrm{D}}{ }^{25}=-59.49\left(c=1.000, \mathrm{CHCl}_{3}, 88 \%\right.$ ee $)$.

HPLC: DAICEL CHIRALPAK OD-H, hexane $/ i-\mathrm{PrOH}=97 / 3$, flow rate: $1.0 \mathrm{~mL} / \mathrm{min}, \lambda=254 \mathrm{~nm}$, $\mathrm{t}_{\mathrm{R}}($ major $)=11.4 \mathrm{~min}, \mathrm{t}_{\mathrm{R}}($ minor $)=16.1 \mathrm{~min}, 88 \%$ ee .
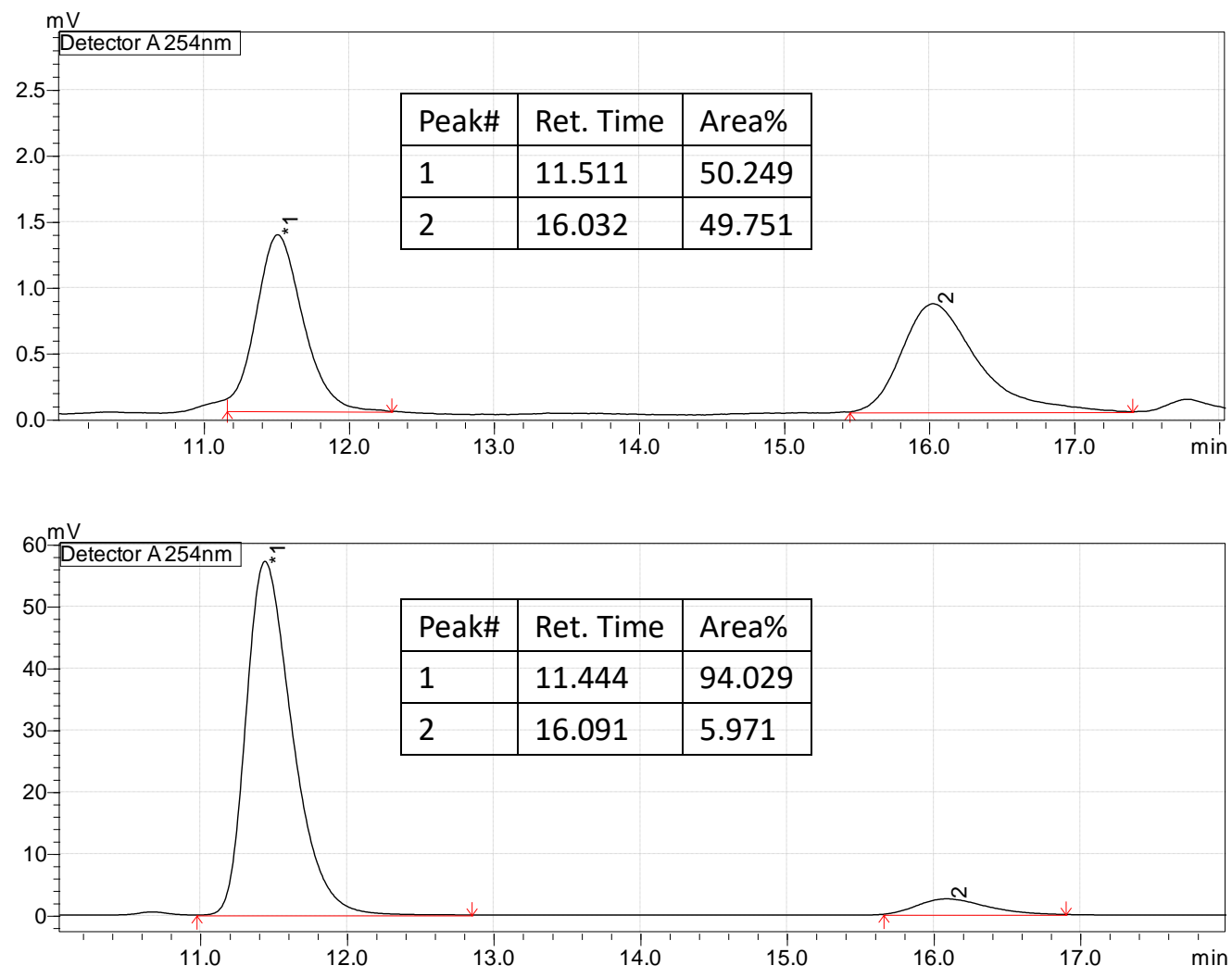


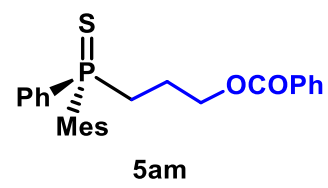

5am: Procedure B, $82.7 \mathrm{mg}, 98 \%$ yield, light yellow solid.

${ }^{1}$ H NMR (400 MHz, CDCl $\left._{3}\right) \delta$ 7.98-7.90 (m, 2H), 7.85-7.73 (m, 2H), 7.58-7.37 (m, 6H), 6.84 (d, $J=4.0 \mathrm{~Hz}, 2 \mathrm{H}), 4.25(\mathrm{t}, J=6.3 \mathrm{~Hz}, 2 \mathrm{H}), 2.82-2.68(\mathrm{~m}, 1 \mathrm{H}), 2.66-2.52(\mathrm{~m}, 1 \mathrm{H}), 2.30(\mathrm{~s}, 6 \mathrm{H}), 2.25$ (s, 3H), 2.23-2.10 (m, 1H), 1.36-1.26 (m, 1H).

${ }^{13}$ C NMR(101 MHz, CDCl $) \delta 166.18,141.40(\mathrm{~d}, J=10.2 \mathrm{~Hz}), 140.88(\mathrm{~d}, J=2.8 \mathrm{~Hz}), 134.26(\mathrm{~d}$, $J=77.6 \mathrm{~Hz}), 132.89,131.27(\mathrm{~d}, J=11.3 \mathrm{~Hz}), 130.92(\mathrm{~d}, J=3.0 \mathrm{~Hz}), 130.57(\mathrm{~d}, J=10.2 \mathrm{~Hz})$, 129.84, 129.38, $128.45(\mathrm{~d}, J=11.9 \mathrm{~Hz}), 128.44(\mathrm{~d}, J=83.2 \mathrm{~Hz}), 128.24,64.61(\mathrm{~d}, J=18.8 \mathrm{~Hz})$, 35.18 (d, $J=55.0 \mathrm{~Hz}), 23.92(\mathrm{~d}, J=5.2 \mathrm{~Hz}), 22.60$ (d, $J=1.8 \mathrm{~Hz}), 20.73(\mathrm{~d}, J=1.5 \mathrm{~Hz})$.

${ }^{31}$ P NMR (162 MHz, $\left.\mathbf{C D C l}_{3}\right) \delta 43.26$.

HRMS (ESI) $\mathbf{m} / \mathbf{z}$ [M+H] $]^{+}$: calcd. 423.1542, found. 423.1542.

IR (film): $v_{\max }\left(\mathrm{cm}^{-1}\right) 3062,2957,2924,2854,1716,1603,1451,1437,1313,1271,1175,1112$, 1069, 850, 749, 649, 595.

Optical rotation: $[\alpha]_{\mathrm{D}}{ }^{25}=-28.11\left(c=1.000, \mathrm{CHCl}_{3}, 88 \%\right.$ ee $)$.

HPLC: DAICEL CHIRALPAK OD-H, hexane $/ i-\mathrm{PrOH}=19 / 1$, flow rate: $1.0 \mathrm{~mL} / \mathrm{min}, \lambda=254 \mathrm{~nm}$, $\mathrm{t}_{\mathrm{R}}($ major $)=12.0 \mathrm{~min}, \mathrm{t}_{\mathrm{R}}($ minor $)=14.4 \mathrm{~min}, 88 \%$ ee

\begin{tabular}{|l|l|l|}
\hline Peak\# & Ret. Time & Area\% \\
\hline 1 & 12.109 & 50.295 \\
\hline 2 & 14.408 & 49.705 \\
\hline
\end{tabular}
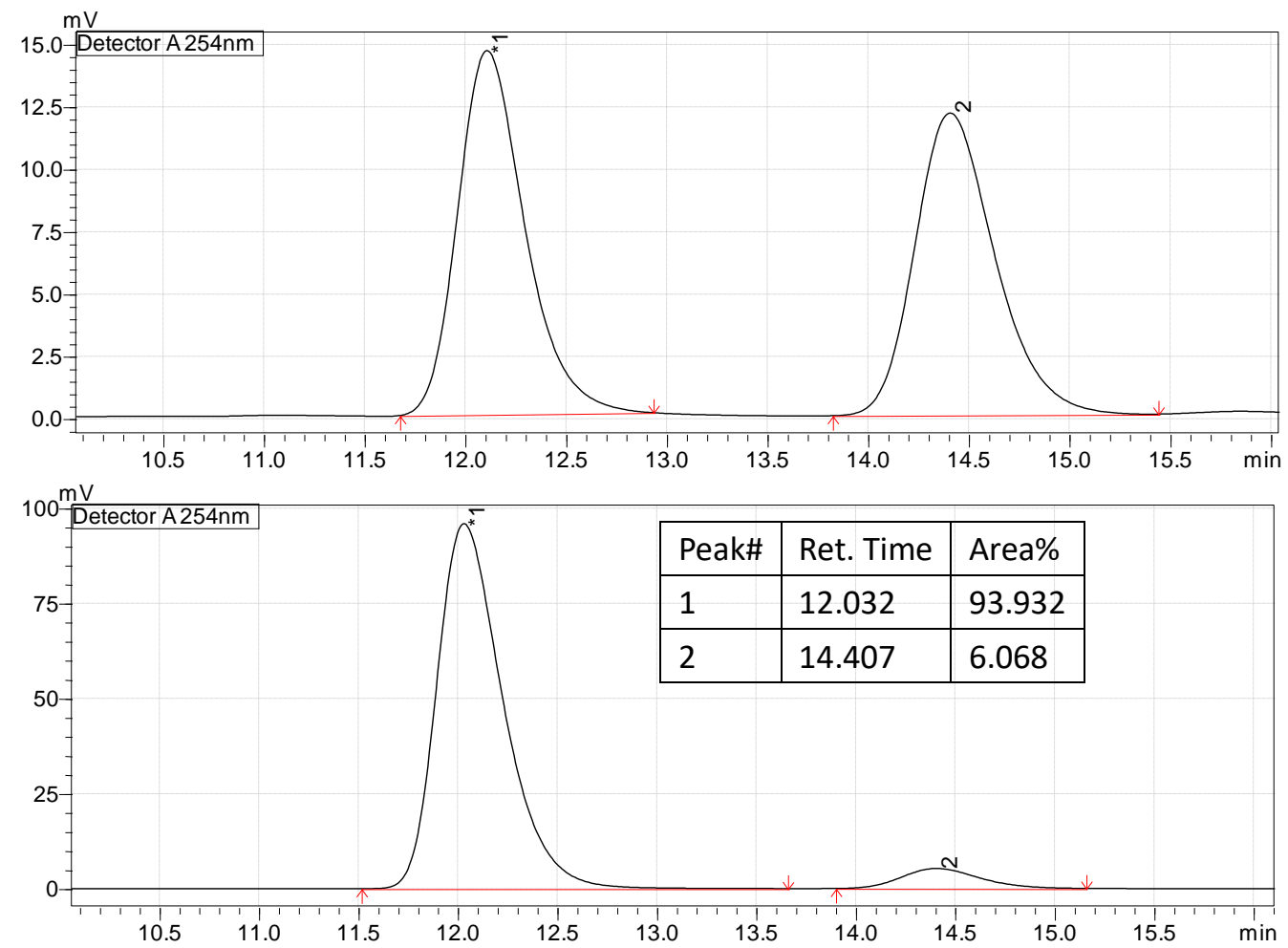


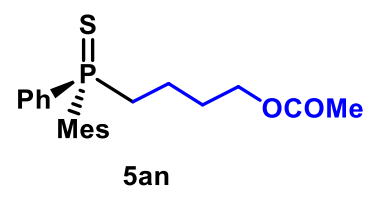

5an: Procedure B, $60.0 \mathrm{mg}, 85 \%$ yield, light yellow liquid.

${ }^{1}$ H NMR (400 MHz, CDCl $)_{3} \delta$ 7.85-7.70 (m, 2H), 7.55-7.35 (m, 3H), $6.84(\mathrm{~d}, J=4.0 \mathrm{~Hz}, 2 \mathrm{H})$, $3.93(\mathrm{t}, J=6.5 \mathrm{~Hz}, 2 \mathrm{H}), 2.69-2.52(\mathrm{~m}, 1 \mathrm{H}), 2.53-2.39(\mathrm{~m}, 1 \mathrm{H}), 2.29(\mathrm{~s}, 6 \mathrm{H}), 2.26(\mathrm{~s}, 3 \mathrm{H}), 1.95(\mathrm{~s}$, $3 \mathrm{H}), 1.87-1.48(\mathrm{~m}, 3 \mathrm{H}), 0.96-0.73(\mathrm{~m}, 1 \mathrm{H})$.

${ }^{13} \mathbf{C}$ NMR(101 MHz, CDCl 3 ) $\delta$ 170.89, $141.40(\mathrm{~d}, J=10.0 \mathrm{~Hz}), 140.82(\mathrm{~d}, J=2.8 \mathrm{~Hz}), 134.42(\mathrm{~d}$, $J=77.6 \mathrm{~Hz}), 131.26(\mathrm{~d}, J=11.2 \mathrm{~Hz}), 130.77(\mathrm{~d}, J=3.0 \mathrm{~Hz}), 130.60(\mathrm{~d}, J=10.1 \mathrm{~Hz}), 128.73(\mathrm{~d}, J$ $=82.5 \mathrm{~Hz}), 128.35(\mathrm{~d}, J=12.0 \mathrm{~Hz}), 63.44,37.99$ (d, $J=53.6 \mathrm{~Hz}), 29.36(\mathrm{~d}, J=17.3 \mathrm{~Hz}), 23.93(\mathrm{~d}$, $J=5.2 \mathrm{~Hz}), 21.09-20.31(\mathrm{~m}), 19.40(\mathrm{~d}, J=2.5 \mathrm{~Hz})$.

${ }^{31}$ P NMR (162 MHz, $\left.\mathbf{C D C l}_{3}\right) \delta 43.39$.

HRMS (ESI) $\mathbf{m} / \mathbf{z}$ [M+H] $]^{+}$: calcd. 375.1542, found. 375.1542 .

IR (film): $v_{\max }\left(\mathrm{cm}^{-1}\right) 3050,2925,1737,1604,1454,1436,1365,1235,1100,1036,850,750,694$, 648, 594.

Optical rotation: $[\alpha]_{\mathrm{D}}{ }^{25}=-74.70\left(c=1.000, \mathrm{CHCl}_{3}, 88 \%\right.$ ee $)$.

HPLC: DAICEL CHIRALPAK OD-H, hexane $/ i-\mathrm{PrOH}=19 / 1$, flow rate: $1.0 \mathrm{~mL} / \mathrm{min}, \lambda=220 \mathrm{~nm}$, $\mathrm{t}_{\mathrm{R}}($ major $)=9.0 \mathrm{~min}, \mathrm{t}_{\mathrm{R}}($ minor $)=11.8 \mathrm{~min}, 88 \%$ ee .
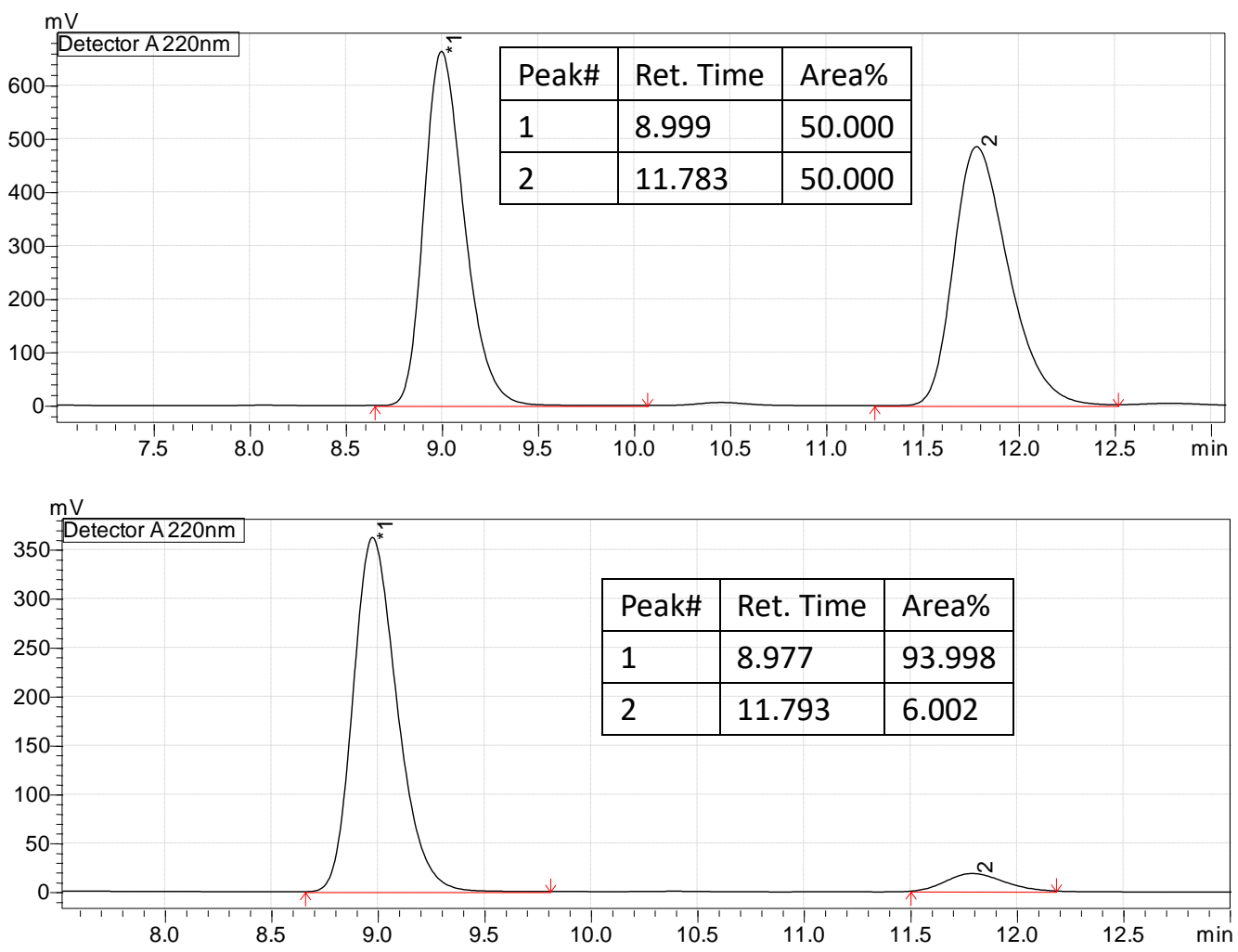


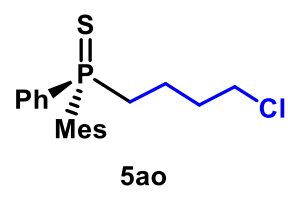

5ao: Procedure B, $67.1 \mathrm{mg}, 96 \%$ yield, light yellow liquid.

${ }^{1} \mathbf{H}$ NMR (400 MHz, $\left.\mathbf{C D C l}_{3}\right) \delta$ 7.83-7.71 (m, 2H), 7.56-7.39 (m, 3H), $6.84(\mathrm{~d}, J=4.0 \mathrm{~Hz}, 2 \mathrm{H})$, 3.51-3.29 (m, 2H), 2.67-2.39 (m, 2H), 2.29 (s, 6H), 2.26 (s, 3H), 1.87-1.66 (m, 3H), 1.07-0.91 $(\mathrm{m}, 1 \mathrm{H})$.

${ }^{13}$ C NMR(101 MHz, CDCl $) \delta 141.39(\mathrm{~d}, J=10.2 \mathrm{~Hz}), 140.82(\mathrm{~d}, J=3.0 \mathrm{~Hz}), 134.29(\mathrm{~d}, J=$ $77.6 \mathrm{~Hz}), 131.26(\mathrm{~d}, J=11.2 \mathrm{~Hz}), 130.85(\mathrm{~d}, J=2.8 \mathrm{~Hz}), 130.56(\mathrm{~d}, J=10.2 \mathrm{~Hz}), 128.63(\mathrm{~d}, J=$ $82.4 \mathrm{~Hz}), 128.39(\mathrm{~d}, J=12.1 \mathrm{~Hz}), 43.97,37.78(\mathrm{~d}, J=53.6 \mathrm{~Hz}), 33.31$ (d, $J=17.2 \mathrm{~Hz}), 23.92(\mathrm{~d}, J$ $=5.1 \mathrm{~Hz}), 20.75(\mathrm{~d}, J=1.5 \mathrm{~Hz}), 20.47(\mathrm{~d}, J=2.4 \mathrm{~Hz})$.

${ }^{31}$ P NMR (162 MHz, $\left.\mathbf{C D C l}_{3}\right) \delta 43.24$.

HRMS (ESI) $\mathbf{m} / \mathbf{z}[\mathbf{M + H}]^{+}$: calcd. 351.1098, found. 351.1098.

IR (film): $v_{\max }\left(\mathrm{cm}^{-1}\right)$ 3054, 2925, 2869, 1604, 1454, 1436, 1289, 1100, 1027, 851, 749, 694, 648, 594.

Optical rotation: $[\alpha]_{\mathrm{D}}^{25}=-64.17\left(c=1.340, \mathrm{CHCl}_{3}, 89 \%\right.$ ee $)$.

HPLC: DAICEL CHIRALPAK OD-H, hexane $/ i-\mathrm{PrOH}=97 / 3$, flow rate: $1.0 \mathrm{~mL} / \mathrm{min}, \lambda=254 \mathrm{~nm}$, $\mathrm{t}_{\mathrm{R}}($ major $)=8.5 \mathrm{~min}, \mathrm{t}_{\mathrm{R}}($ minor $)=12.1 \mathrm{~min}, 89 \%$ ee.
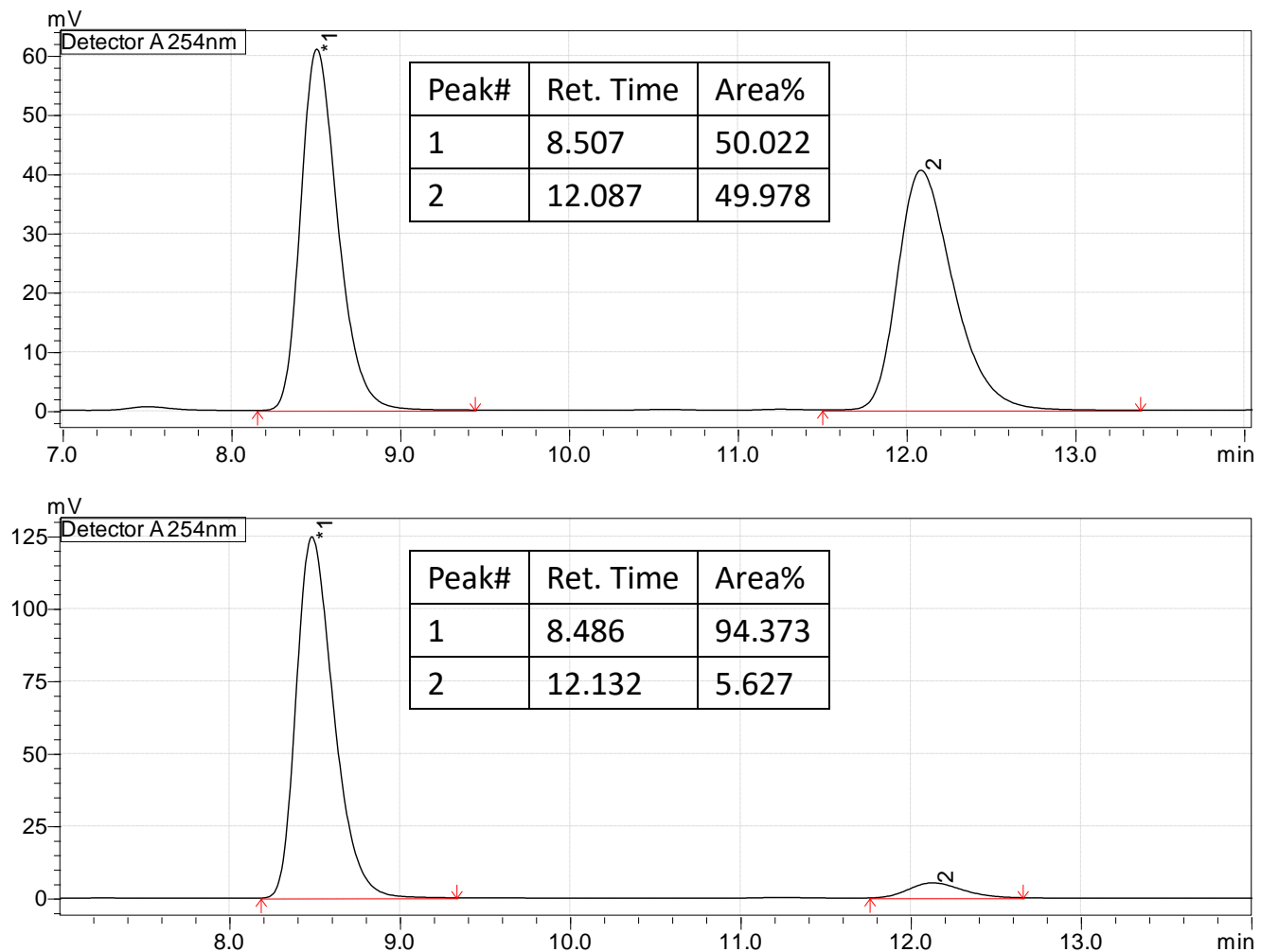


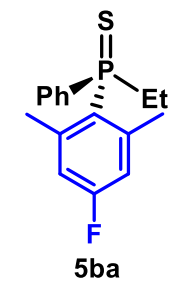

5ba: Procedure B, $54.3 \mathrm{mg}$, 95\% yield, light yellow solid.

${ }^{1}$ H NMR (500 MHz, $\left.\mathbf{C D C l}_{3}\right) \delta 7.74(\mathrm{dd}, J=13.0,7.5 \mathrm{~Hz}, 2 \mathrm{H}), 7.54-7.40(\mathrm{~m}, 3 \mathrm{H}), 6.75(\mathrm{dd}, J=$ 9.3, 3.0 Hz, 2H), 2.68-2.54 (m, 1H), 2.52-2.40 (m, 1H), $2.34(\mathrm{~s}, 6 \mathrm{H}), 0.89(\mathrm{dt}, J=21.0,7.3 \mathrm{~Hz}$, $3 \mathrm{H})$.

${ }^{13} \mathbf{C}$ NMR(101 MHz, $\left.\mathbf{C D C l}_{3}\right) \delta 163.25(\mathrm{dd}, J=251.9,3.5 \mathrm{~Hz}), 144.80(\mathrm{dd}, J=11.3,8.5 \mathrm{~Hz})$, $133.65(\mathrm{~d}, J=77.8 \mathrm{~Hz}), 130.92(\mathrm{~d}, J=3.0 \mathrm{~Hz}), 130.62(\mathrm{~d}, J=10.0 \mathrm{~Hz}), 128.40(\mathrm{~d}, J=12.2 \mathrm{~Hz})$, $128.23(\mathrm{dd}, J=83.1,3.4 \mathrm{~Hz}), 117.04(\mathrm{dd}, J=20.3,11.8 \mathrm{~Hz}), 31.72(\mathrm{~d}, J=54.6 \mathrm{~Hz}), 24.22(\mathrm{dd}, J=$ $5.0,1.5 \mathrm{~Hz}), 6.95(\mathrm{~d}, J=3.8 \mathrm{~Hz})$.

${ }^{31}$ P NMR (162 MHz, $\left.\mathbf{C D C l}_{3}\right) \delta 46.07$.

${ }^{19}$ F NMR (376 MHz, $\left.\mathrm{CDCl}_{3}\right) \delta-107.00$.

HRMS (ESI) $\mathbf{m} / \mathbf{z}$ [M+H] $]^{+}$: calcd. 293.0924, found. 293.0923.

IR (film): $v_{\max }\left(\mathrm{cm}^{-1}\right)$ 2974, 2933, 1584, 1455, 1293, 1101, 1030, 860, 695, 665, 592.

Optical rotation: $[\alpha]_{\mathrm{D}}{ }^{25}=-93.88\left(c=1.086, \mathrm{CHCl}_{3}, 94 \%\right.$ ee $)$.

HPLC: DAICEL CHIRALPAK AD-H, hexane $/ i-\mathrm{PrOH}=49 / 1$, flow rate: $1.0 \mathrm{~mL} / \mathrm{min}, \lambda=254 \mathrm{~nm}$, $\mathrm{t}_{\mathrm{R}}($ major $)=9.7 \mathrm{~min}, \mathrm{t}_{\mathrm{R}}($ minor $)=9.0 \mathrm{~min}, 94 \%$ ee .

\begin{tabular}{|l|l|l|}
\hline Peak\# & Ret. Time & Area\% \\
\hline 1 & 9.026 & 50.021 \\
\hline 2 & 9.714 & 49.979 \\
\hline
\end{tabular}

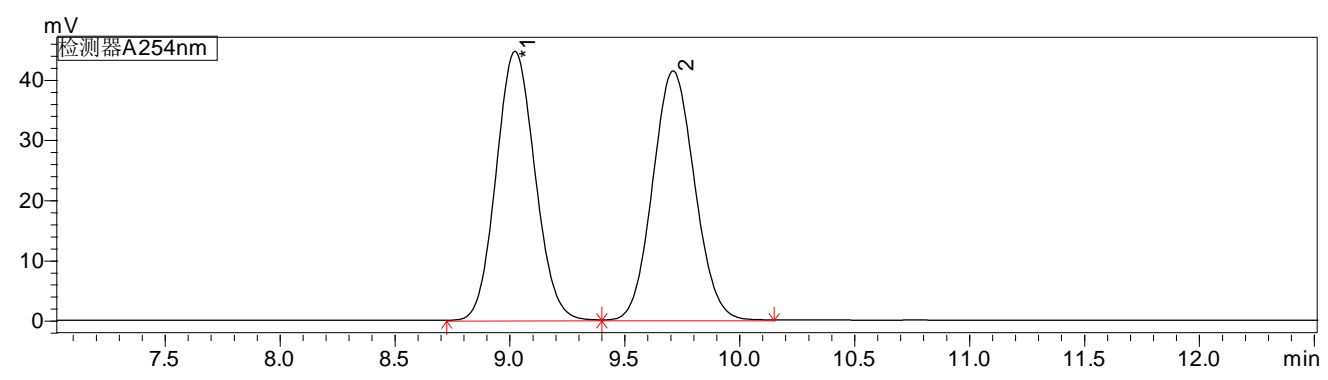

\begin{tabular}{|l|l|l|}
\hline Peak\# & Ret. Time & Area\% \\
\hline 1 & 9.041 & 2.971 \\
\hline 2 & 9.726 & 97.029 \\
\hline
\end{tabular}

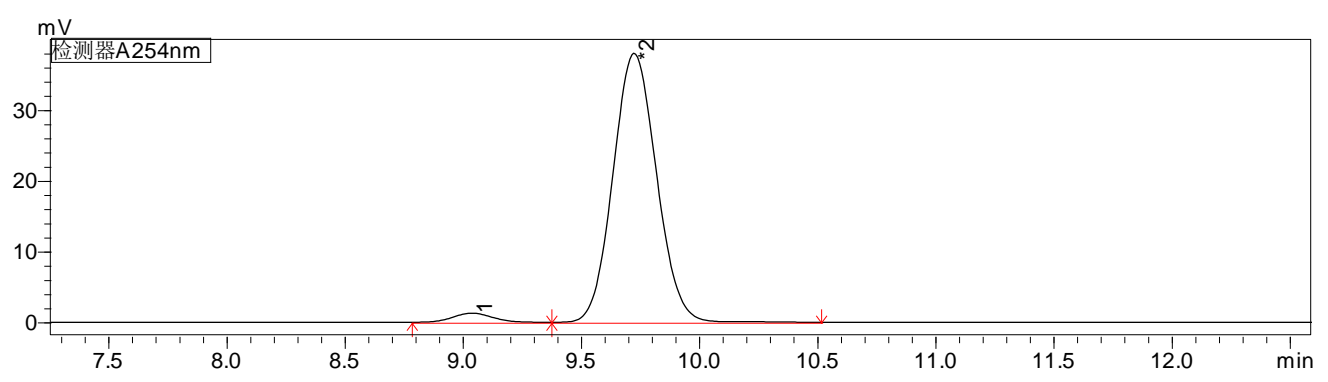




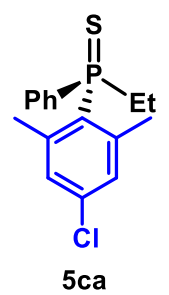

5ca: Procedure B, $53.0 \mathrm{mg}, 86 \%$ yield, light yellow solid.

${ }^{1}$ H NMR (500 MHz, CDCl $)$ S 7.78-7.68 (m, 2H), 7.54-7.47 (m, 1H), 7.47-7.41 (m, 2H), 7.03 (d, $J=3.3 \mathrm{~Hz}, 2 \mathrm{H}), 2.68-2.53(\mathrm{~m}, 1 \mathrm{H}), 2.52-2.39(\mathrm{~m}, 1 \mathrm{H}), 2.32(\mathrm{~s}, 6 \mathrm{H}), 0.89(\mathrm{dt}, J=21.0,7.3 \mathrm{~Hz}$, $3 \mathrm{H})$.

${ }^{13} \mathbf{C ~ N M R}\left(101 \mathrm{MHz}, \mathbf{C D C l}_{3}\right) \delta 143.41(\mathrm{~d}, J=10.7 \mathrm{~Hz}), 136.39(\mathrm{~d}, J=3.5 \mathrm{~Hz}), 133.40(\mathrm{~d}, J=$ $77.6 \mathrm{~Hz}), 130.98(\mathrm{~d}, J=2.9 \mathrm{~Hz}), 130.91(\mathrm{~d}, J=81.2 \mathrm{~Hz}), 130.61(\mathrm{~d}, J=10.0 \mathrm{~Hz}), 130.03(\mathrm{~d}, J=$ $11.3 \mathrm{~Hz}), 128.42(\mathrm{~d}, J=12.0 \mathrm{~Hz}), 31.67(\mathrm{~d}, J=54.3 \mathrm{~Hz}), 23.90(\mathrm{~d}, J=5.0 \mathrm{~Hz}), 6.91(\mathrm{~d}, J=3.8$ $\mathrm{Hz})$.

${ }^{31}$ P NMR (162 MHz, $\left.\mathrm{CDCl}_{3}\right) \delta 46.21$.

HRMS (ESI) $\mathbf{m} / \mathbf{z}$ [M+H] $]^{+}$: calcd. 309.0628, found. 309.0628 .

IR (film): $v_{\max }\left(\mathrm{cm}^{-1}\right)$ 2973, 2932, 1574, 1455, 1399, 1247, 1055, 1100, 874, 776, 574.

Optical rotation: $[\alpha]_{\mathrm{D}}{ }^{25}=-93.41\left(c=1.060, \mathrm{CHCl}_{3}, 90 \%\right.$ ee $)$.

HPLC: DAICEL CHIRALPAK ID, hexane $/ i-\mathrm{PrOH}=19 / 1$, flow rate: $1.0 \mathrm{~mL} / \mathrm{min}, \lambda=254 \mathrm{~nm}$, $\mathrm{t}_{\mathrm{R}}($ major $)=10.0 \mathrm{~min}, \mathrm{t}_{\mathrm{R}}($ minor $)=10.6 \mathrm{~min}, 90 \%$ ee .

\begin{tabular}{|l|l|l|}
\hline Peak\# & Ret. Time & Area\% \\
\hline 1 & 9.998 & 50.084 \\
\hline 2 & 10.596 & 49.916 \\
\hline
\end{tabular}

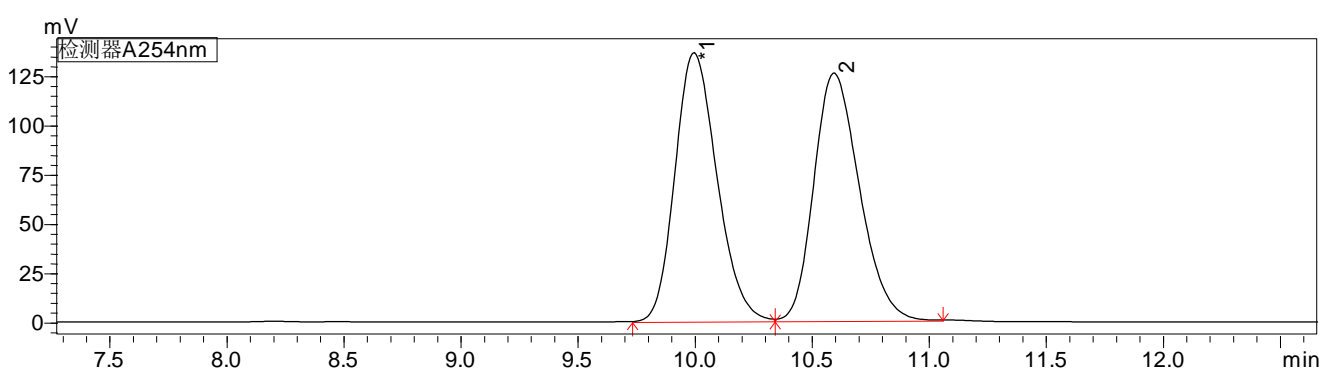

\begin{tabular}{|l|l|l|}
\hline Peak\# & Ret. Time & Area\% \\
\hline 1 & 9.985 & 94.806 \\
\hline 2 & 10.613 & 5.194 \\
\hline
\end{tabular}

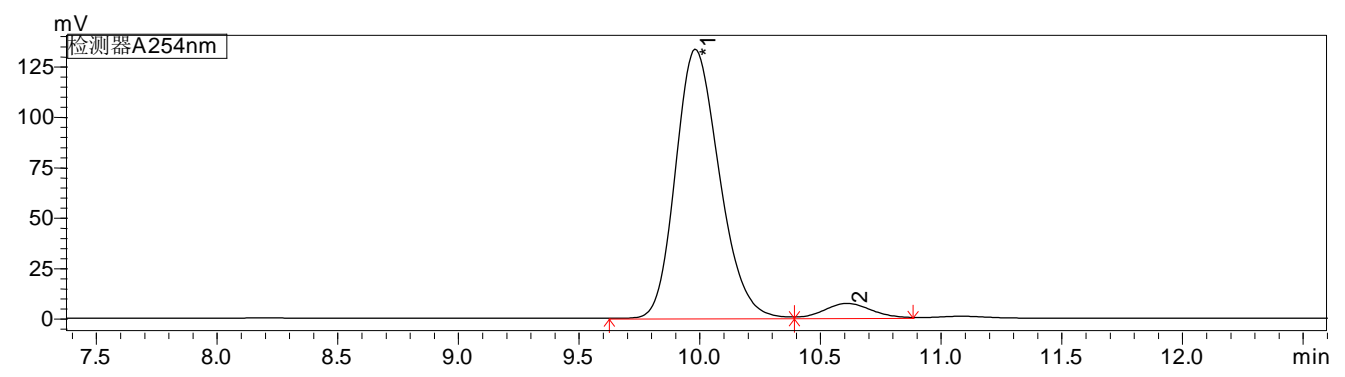




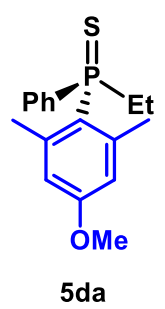

5da: Procedure B, $51.0 \mathrm{mg}, 84 \%$ yield, light yellow solid.

${ }^{1}$ H NMR (500 MHz, $\left.\mathbf{C D C l}_{3}\right) \delta$ 7.81-7.70 (m, 2H), 7.51-7.39 (m, 3H), $6.56(\mathrm{~d}, J=3.3 \mathrm{~Hz}, 2 \mathrm{H})$, $3.78(\mathrm{~s}, 3 \mathrm{H}), 2.65-2.51(\mathrm{~m}, 1 \mathrm{H}), 2.51-2.38(\mathrm{~m}, 1 \mathrm{H}), 2.32(\mathrm{~s}, 6 \mathrm{H}), 0.88(\mathrm{dt}, J=20.7,7.3 \mathrm{~Hz}, 3 \mathrm{H})$.

${ }^{13} \mathbf{C ~ N M R}\left(101 \mathrm{MHz}, \mathbf{C D C l}_{3}\right) \delta 160.67(\mathrm{~d}, J=3.1 \mathrm{~Hz}), 143.77(\mathrm{~d}, J=11.3 \mathrm{~Hz}), 134.28(\mathrm{~d}, J=$ $77.7 \mathrm{~Hz}), 130.68(\mathrm{~d}, J=9.9 \mathrm{~Hz}), 130.65(\mathrm{~d}, J=3.1 \mathrm{~Hz}), 128.25(\mathrm{~d}, J=11.9 \mathrm{~Hz}), 123.56(\mathrm{~d}, J=$ $85.8 \mathrm{~Hz}), 115.64(\mathrm{~d}, J=11.5 \mathrm{~Hz}), 54.98,31.80(\mathrm{~d}, J=54.7 \mathrm{~Hz}), 24.34(\mathrm{~d}, J=4.7 \mathrm{~Hz}), 7.03$ (d, $J=$ $3.9 \mathrm{~Hz})$.

${ }^{31}$ P NMR (162 MHz, $\left.\mathrm{CDCl}_{3}\right) \delta 45.87$.

HRMS (ESI) $\mathbf{m} / \mathbf{z}[\mathbf{M}+\mathbf{H}]^{+}$: calcd. 305.1123 , found. 305.1124 .

IR (film): $v_{\max }\left(\mathrm{cm}^{-1}\right)$ 2968, 2934, 1593, 1455, 1306, 1168, 1090, 856, 700, 651.

Optical rotation: $[\alpha]_{\mathrm{D}}{ }^{25}=-80.33\left(c=1.020, \mathrm{CHCl}_{3}, 90 \% \mathrm{ee}\right)$.

HPLC: DAICEL CHIRALPAK AD-H, hexane $/ i-\mathrm{PrOH}=49 / 1$, flow rate: $1.0 \mathrm{~mL} / \mathrm{min}, \lambda=254 \mathrm{~nm}$, $\mathrm{t}_{\mathrm{R}}($ major $)=22.6 \mathrm{~min}, \mathrm{t}_{\mathrm{R}}($ minor $)=20.9 \mathrm{~min}, 90 \%$ ee .

\begin{tabular}{|l|l|l|}
\hline Peak\# & Ret. Time & Area\% \\
\hline 1 & 20.917 & 50.011 \\
\hline 2 & 22.684 & 49.989 \\
\hline
\end{tabular}

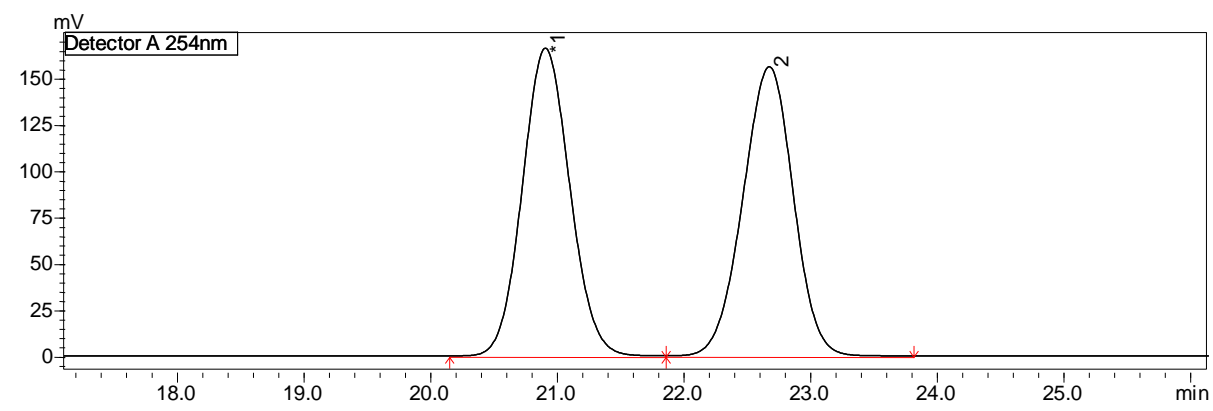

\begin{tabular}{|l|l|l|}
\hline Peak\# & Ret. Time & Area\% \\
\hline 1 & 20.915 & 5.183 \\
\hline 2 & 22.637 & 94.817 \\
\hline
\end{tabular}

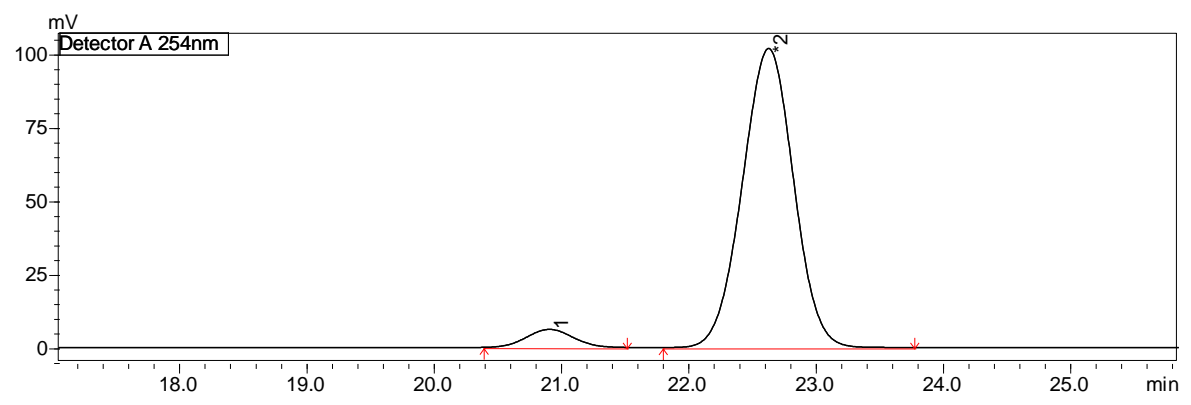




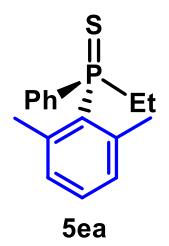

5ea: Procedure B, $52.1 \mathrm{mg}$, 95\% yield, light yellow solid.

${ }^{1} \mathbf{H}$ NMR (500 MHz, $\left.\mathbf{C D C l}_{3}\right) \delta 7.76(\mathrm{dd}, J=12.9,7.5 \mathrm{~Hz}, 2 \mathrm{H}), 7.54-7.36(\mathrm{~m}, 3 \mathrm{H}), 7.25-7.17(\mathrm{~m}$, $1 \mathrm{H}), 7.01$ (dd, $J=7.7,4.1 \mathrm{~Hz}, 2 \mathrm{H}), 2.70-2.55(\mathrm{~m}, 1 \mathrm{H}), 2.54-2.41$ (m, 1H), 2.34 (s, 6H), 0.89 (dt, $J$ $=20.8,7.3 \mathrm{~Hz}, 3 \mathrm{H})$.

${ }^{13} \mathbf{C}$ NMR(101 MHz, $\left.\mathbf{C D C l}_{3}\right) \delta 141.55(\mathrm{~d}, J=9.6 \mathrm{~Hz}), 133.89(\mathrm{~d}, J=77.1 \mathrm{~Hz}), 132.04(\mathrm{~d}, J=$ $80.0 \mathrm{~Hz}), 130.77$ (d, $J=3.0 \mathrm{~Hz}), 130.68$ (d, $J=10.0 \mathrm{~Hz}), 130.56,130.37$ (d, $J=10.7 \mathrm{~Hz}), 128.32$ $(\mathrm{d}, J=11.9 \mathrm{~Hz}), 31.75(\mathrm{~d}, J=54.1 \mathrm{~Hz}), 24.07(\mathrm{~d}, J=5.0 \mathrm{~Hz}), 7.00(\mathrm{~d}, J=3.9 \mathrm{~Hz})$.

${ }^{31}$ P NMR (162 MHz, $\left.\mathbf{C D C l}_{3}\right) \delta 46.43$.

HRMS (ESI) $\mathbf{m} / \mathbf{z}$ [M+H] $]^{+}$: calcd. 275.1018, found. 275.1018.

IR (film): $v_{\max }\left(\mathrm{cm}^{-1}\right)$ 3054, 2972, 1478, 1453, 1102, 1028, 773, 705, 625, 569.

Optical rotation: $[\alpha]_{\mathrm{D}}{ }^{25}=-93.41\left(c=0.954, \mathrm{CHCl}_{3}, 93 \%\right.$ ee $)$.

HPLC: DAICEL CHIRALPAK ID, hexane $/ i-\mathrm{PrOH}=9 / 1$, flow rate: $1.0 \mathrm{~mL} / \mathrm{min}, \lambda=254 \mathrm{~nm}$, $\mathrm{t}_{\mathrm{R}}($ major $)=8.5 \mathrm{~min}, \mathrm{t}_{\mathrm{R}}($ minor $)=10.1 \mathrm{~min}, 93 \%$ ee

\begin{tabular}{|l|l|l|}
\hline Peak\# & Ret. Time & Area\% \\
\hline 1 & 8.525 & 49.945 \\
\hline 2 & 10.198 & 50.055 \\
\hline
\end{tabular}

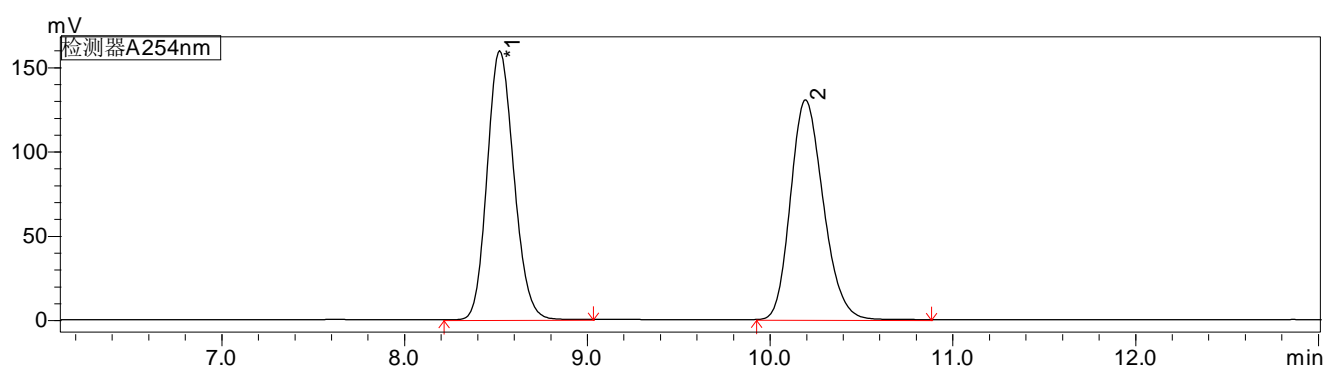

\begin{tabular}{|l|l|l|}
\hline Peak\# & Ret. Time & Area\% \\
\hline 1 & 8.479 & 96.251 \\
\hline 2 & 10.121 & 3.749 \\
\hline
\end{tabular}

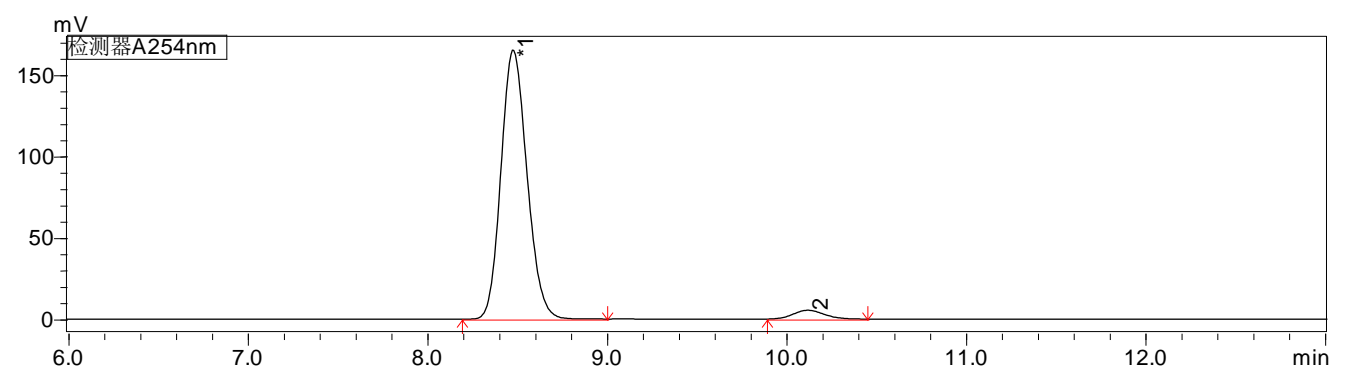




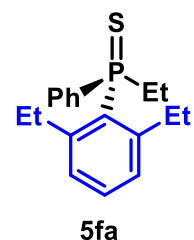

5fa: Procedure B, $47.2 \mathrm{mg}, 78 \%$ yield, light yellow solid.

${ }^{1}$ H NMR (500 MHz, $\left.\mathbf{C D C l}_{3}\right) \delta$ 7.76-7.64 (m, 2H), 7.50-7.37 (m, 3H), 7.37-7.29 (m, 1H), 7.16$7.06(\mathrm{~m}, 2 \mathrm{H}), 2.87-2.71(\mathrm{~m}, 4 \mathrm{H}), 2.67-2.57(\mathrm{~m}, 1 \mathrm{H}), 2.53-2.42(\mathrm{~m}, 1 \mathrm{H}), 1.04(\mathrm{t}, J=7.4 \mathrm{~Hz}, 6 \mathrm{H})$, $0.89(\mathrm{dt}, J=20.8,7.3 \mathrm{~Hz}, 3 \mathrm{H})$.

${ }^{13} \mathbf{C}$ NMR(101 MHz, $\left.\mathbf{C D C l}_{3}\right) \delta 147.93(\mathrm{~d}, J=10.2 \mathrm{~Hz}), 134.96(\mathrm{~d}, J=77.1 \mathrm{~Hz}), 131.51(\mathrm{~d}, J=$ $80.5 \mathrm{~Hz}), 130.96(\mathrm{~d}, J=2.8 \mathrm{~Hz}), 130.68(\mathrm{~d}, J=9.9 \mathrm{~Hz}), 130.61(\mathrm{~d}, J=2.8 \mathrm{~Hz}), 128.19(\mathrm{~d}, J=$ $11.9 \mathrm{~Hz}), 128.05(\mathrm{~d}, J=10.7 \mathrm{~Hz}), 31.73(\mathrm{~d}, J=53.8 \mathrm{~Hz}), 28.73(\mathrm{~d}, J=5.7 \mathrm{~Hz}), 15.48,7.14(\mathrm{~d}, J=$ $3.9 \mathrm{~Hz})$.

${ }^{31}$ P NMR (162 MHz, $\left.\mathbf{C D C l}_{3}\right) \delta 45.53$.

HRMS (ESI) $\mathbf{m} / \mathbf{z}[\mathbf{M}+\mathbf{H}]^{+}$: calcd. 303.1331, found. 303.1331 .

IR (film): $v_{\max }\left(\mathrm{cm}^{-1}\right)$ 2967, 2932, 1456, 1436, 1375, 1100, 1026, 750, 706, 628 .

Optical rotation: $[\alpha]_{\mathrm{D}}{ }^{25}=-79.07\left(c=0.626, \mathrm{CHCl}_{3}, 88 \%\right.$ ee $)$.

HPLC: DAICEL CHIRALPAK OD-H, hexane $/ i-\mathrm{PrOH}=249 / 1$, flow rate: $1.0 \mathrm{~mL} / \mathrm{min}, \lambda=220$ $\mathrm{nm}, \mathrm{t}_{\mathrm{R}}($ major $)=10.3 \mathrm{~min}, \mathrm{t}_{\mathrm{R}}($ minor $)=16.1 \mathrm{~min}, 88 \%$ ee .

\begin{tabular}{|l|l|l|}
\hline Peak\# & Ret. Time & Area\% \\
\hline 1 & 10.285 & 49.981 \\
\hline 2 & 16.115 & 50.019 \\
\hline
\end{tabular}

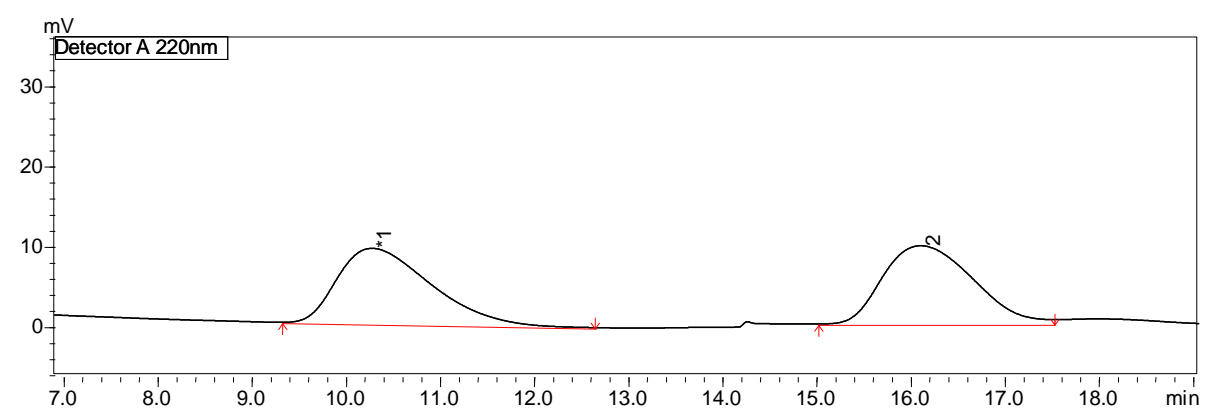

\begin{tabular}{|l|l|l|}
\hline Peak\# & Ret. Time & Area\% \\
\hline 1 & 10.699 & 94.077 \\
\hline 2 & 14.928 & 5.923 \\
\hline
\end{tabular}

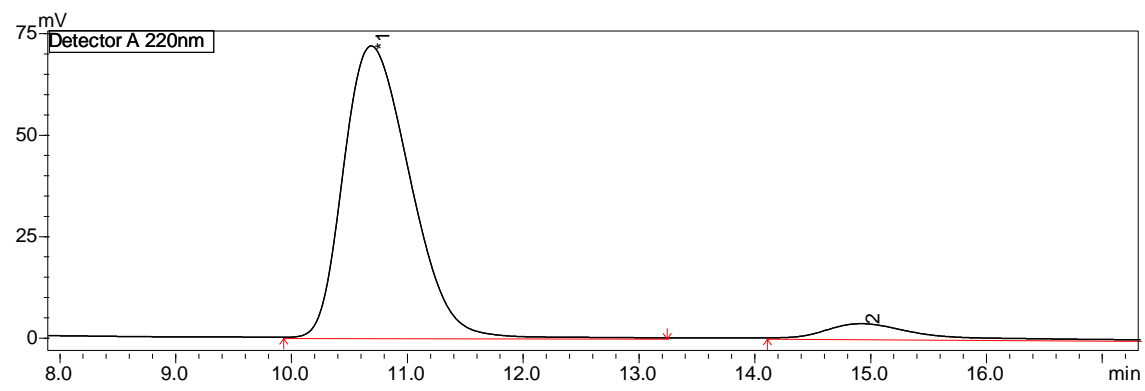




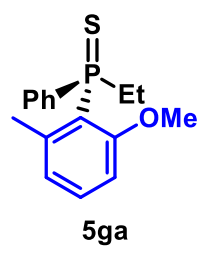

5ga: Procedure B, $44.4 \mathrm{mg}$, 93\% yield, light yellow solid.

${ }^{1}$ H NMR (500 MHz, $\left.\mathbf{C D C l}_{3}\right) \delta$ 7.84-7.72 (m, 2H), 7.50-7.37 (m, 3H), 7.34-7.28 (m, 1H), 6.82 $(\mathrm{dd}, J=8.4,5.0 \mathrm{~Hz}, 1 \mathrm{H}), 6.74(\mathrm{dd}, J=7.6,3.6 \mathrm{~Hz}, 1 \mathrm{H}), 3.84(\mathrm{~s}, 3 \mathrm{H}), 2.79-2.64(\mathrm{~m}, 1 \mathrm{H}), 2.57-$ $2.43(\mathrm{~m}, 1 \mathrm{H}), 2.08(\mathrm{~s}, 3 \mathrm{H}), 0.97(\mathrm{dt}, J=21.7,7.4 \mathrm{~Hz}, 3 \mathrm{H})$.

${ }^{13} \mathbf{C}$ NMR(101 MHz, CDCl $) \delta 160.91(\mathrm{~d}, J=3.4 \mathrm{~Hz}), 144.28(\mathrm{~d}, J=6.6 \mathrm{~Hz}), 134.26(\mathrm{~d}, J=77.9$ $\mathrm{Hz}), 132.21(\mathrm{~d}, J=2.1 \mathrm{~Hz}), 130.63(\mathrm{~d}, J=10.2 \mathrm{~Hz}), 130.61(\mathrm{~d}, J=2.8 \mathrm{~Hz}), 128.18(\mathrm{~d}, J=12.1$ $\mathrm{Hz}), 125.40(\mathrm{~d}, J=9.9 \mathrm{~Hz}), 120.19(\mathrm{~d}, J=79.9 \mathrm{~Hz}), 108.92(\mathrm{~d}, J=6.5 \mathrm{~Hz}), 55.32,31.91(\mathrm{~d}, J=$ $56.3 \mathrm{~Hz}), 22.47(\mathrm{~d}, J=6.5 \mathrm{~Hz}), 6.93(\mathrm{~d}, J=4.0 \mathrm{~Hz})$.

${ }^{31}$ P NMR (162 MHz, $\left.\mathbf{C D C l}_{3}\right) \delta 45.05$.

HRMS (ESI) $\mathbf{m} / \mathbf{z}$ [M+H] $]^{+}$: calcd. 291.0967, found. 291.0967.

IR (film): $v_{\max }\left(\mathrm{cm}^{-1}\right)$ 3054, 2934, 1570, 1464, 1436, 1266, 1102, 1077, 776, 712, 598.

Optical rotation: $[\alpha]_{\mathrm{D}}{ }^{25}=-135.42\left(c=0.888, \mathrm{CHCl}_{3}, 83 \%\right.$ ee $)$.

HPLC: DAICEL CHIRALPAK ID, hexane $/ i-\mathrm{PrOH}=9 / 1$, flow rate: $1.0 \mathrm{~mL} / \mathrm{min}, \lambda=254 \mathrm{~nm}$, $\mathrm{t}_{\mathrm{R}}($ major $)=12.5 \mathrm{~min}, \mathrm{t}_{\mathrm{R}}($ minor $)=13.7 \mathrm{~min}, 83 \%$ ee.

\begin{tabular}{|l|l|l|}
\hline Peak\# & Ret. Time & Area\% \\
\hline 1 & 12.507 & 49.830 \\
\hline 2 & 13.655 & 50.170 \\
\hline
\end{tabular}

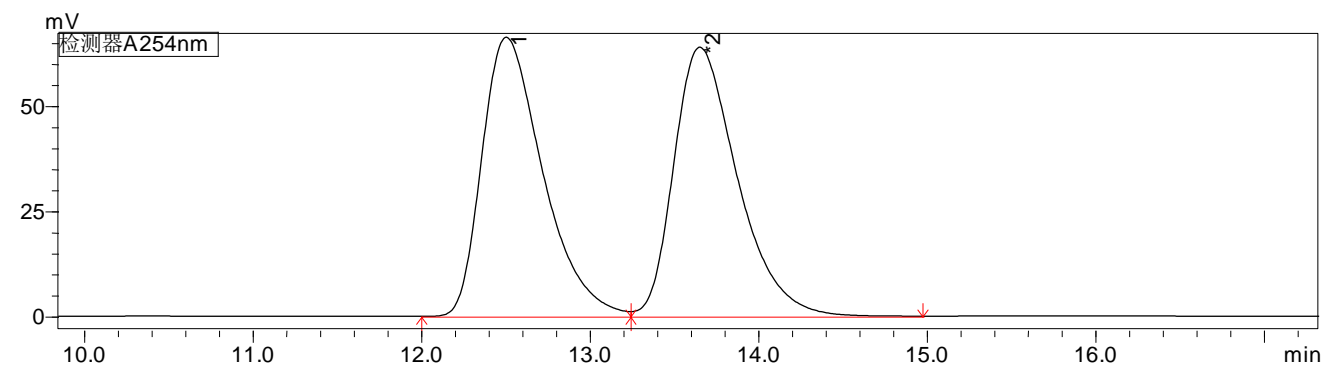

\begin{tabular}{|l|l|l|}
\hline Peak\# & Ret. Time & Area\% \\
\hline 1 & 12.509 & 91.255 \\
\hline 2 & 13.662 & 8.745 \\
\hline
\end{tabular}

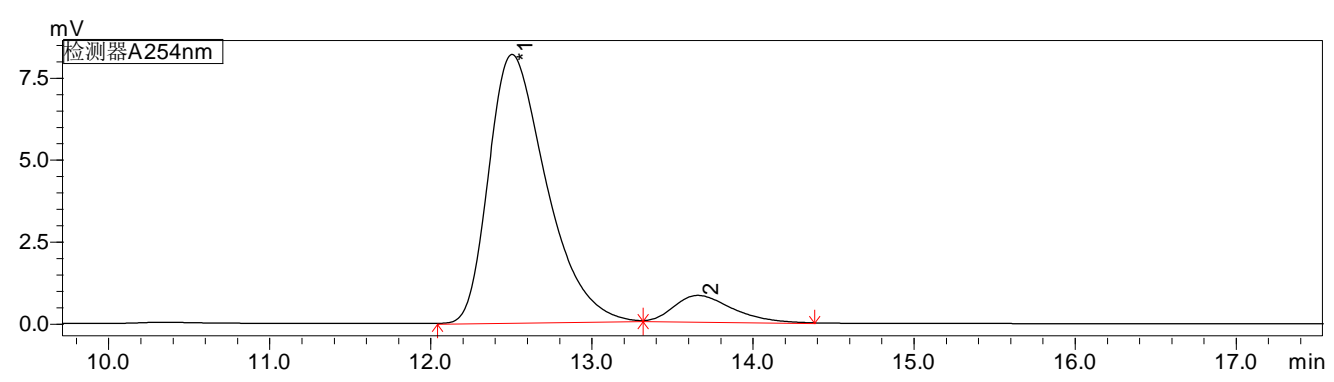




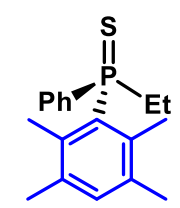

5ha

5ha: Procedure B, $55.6 \mathrm{mg}, 92 \%$ yield, light yellow solid.

${ }^{1}$ H NMR (500 MHz, $\mathbf{C D C l}_{\mathbf{3}}$ ) $\delta$ 7.76-7.66 (m, 2H), 7.50-7.36 (m, 3H), $7.05(\mathrm{~s}, 1 \mathrm{H}), 2.69-2.55(\mathrm{~m}$, $1 \mathrm{H}), 2.44-2.32(\mathrm{~m}, 1 \mathrm{H}), 2.17(\mathrm{~s}, 6 \mathrm{H}), 2.15(\mathrm{~s}, 6 \mathrm{H}), 0.81(\mathrm{dt}, J=20.5,7.4 \mathrm{~Hz}, 3 \mathrm{H})$.

${ }^{13} \mathbf{C}$ NMR(101 MHz, $\left.\mathbf{C D C l}_{3}\right) \delta 137.85(\mathrm{~d}, J=9.9 \mathrm{~Hz}), 135.39(\mathrm{~d}, J=11.8 \mathrm{~Hz}), 134.76(\mathrm{~d}, J=$ $77.7 \mathrm{~Hz}), 134.20$ (d, $J=3.0 \mathrm{~Hz}), 132.56(\mathrm{~d}, J=83.5 \mathrm{~Hz}), 130.59$ (d, $J=9.6 \mathrm{~Hz}), 130.44$ (d, $J=$ $2.8 \mathrm{~Hz}), 128.15(\mathrm{~d}, J=12.0 \mathrm{~Hz}), 31.70(\mathrm{~d}, J=55.3 \mathrm{~Hz}), 20.50(\mathrm{~d}, J=6.5 \mathrm{~Hz}), 20.05(\mathrm{~d}, J=1.8$ $\mathrm{Hz}), 7.13(\mathrm{~d}, J=3.8 \mathrm{~Hz})$.

${ }^{31}$ P NMR (162 MHz, $\left.\mathbf{C D C l}_{3}\right) \delta 45.62$.

HRMS (ESI) $\mathbf{m} / \mathbf{z}$ [M+H] ${ }^{+}$: calcd. 303.1331, found. 303.1331.

IR (film): $v_{\max }\left(\mathrm{cm}^{-1}\right)$ 2968, 2930, 1458, 1437, 1100, 1024, 830, 617, 606.

Optical rotation: $[\alpha]_{\mathrm{D}}{ }^{25}=-41.57\left(c=1.026, \mathrm{CHCl}_{3}, 87 \%\right.$ ee $)$.

HPLC: DAICEL CHIRALPAK ID, hexane $/ i-\mathrm{PrOH}=9 / 1$, flow rate: $1.0 \mathrm{~mL} / \mathrm{min}, \lambda=254 \mathrm{~nm}$, $\mathrm{t}_{\mathrm{R}}($ major $)=8.4 \mathrm{~min}, \mathrm{t}_{\mathrm{R}}($ minor $)=7.9 \mathrm{~min}, 87 \%$ ee.

\begin{tabular}{|l|l|l|}
\hline Peak\# & Ret. Time & Area\% \\
\hline 1 & 7.942 & 49.813 \\
\hline 2 & 8.427 & 50.187 \\
\hline
\end{tabular}

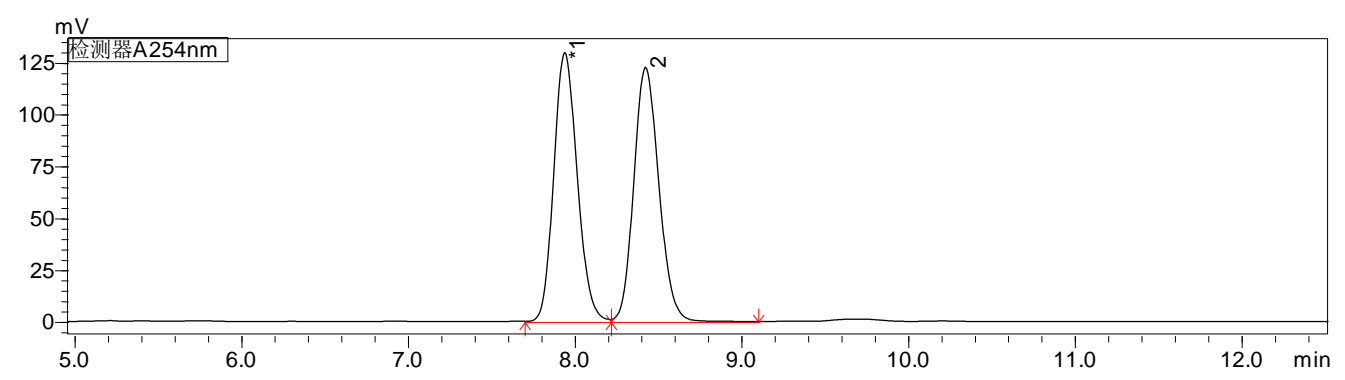

\begin{tabular}{|l|l|l|}
\hline Peak\# & Ret. Time & Area\% \\
\hline 1 & 7.935 & 6.723 \\
\hline 2 & 8.408 & 93.277 \\
\hline
\end{tabular}

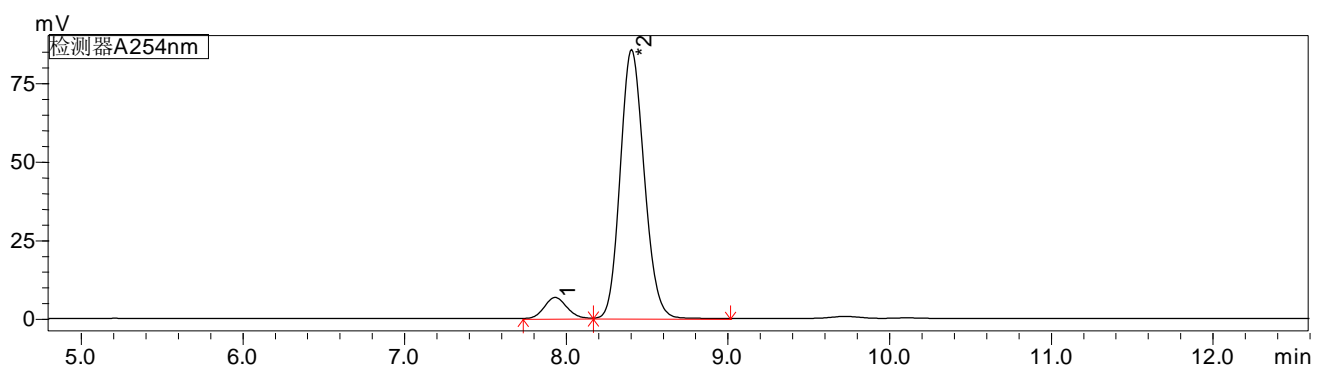




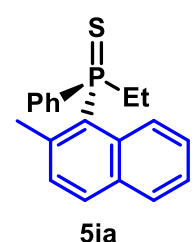

5ia: Procedure B, $61.4 \mathrm{mg}$, 99\% yield, light yellow solid.

${ }^{1}$ H NMR (500 MHz, $\left.\mathbf{C D C l}_{3}\right) \delta 8.40(\mathrm{~d}, J=8.7 \mathrm{~Hz}, 1 \mathrm{H}), 7.85-7.68(\mathrm{~m}, 4 \mathrm{H}), 7.49-7.33(\mathrm{~m}, 4 \mathrm{H})$, $7.28(\mathrm{dd}, J=8.7,6.8 \mathrm{~Hz}, 1 \mathrm{H}), 7.22(\mathrm{dd}, J=8.4,4.1 \mathrm{~Hz}, 1 \mathrm{H}), 2.89-2.76(\mathrm{~m}, 1 \mathrm{H}), 2.53-2.45(\mathrm{~m}$, $1 \mathrm{H}), 2.44(\mathrm{~s}, 3 \mathrm{H}), 0.92(\mathrm{dt}, J=20.8,7.4 \mathrm{~Hz}, 3 \mathrm{H})$.

${ }^{13} \mathbf{C}$ NMR(101 MHz, CDCl $) \delta 141.53(\mathrm{~d}, J=8.1 \mathrm{~Hz}), 134.08(\mathrm{~d}, J=77.7 \mathrm{~Hz}), 133.12(\mathrm{~d}, J=9.6$ $\mathrm{Hz}), 132.43(\mathrm{~d}, J=9.1 \mathrm{~Hz}), 131.82(\mathrm{~d}, J=3.1 \mathrm{~Hz}), 130.84(\mathrm{~d}, J=10.2 \mathrm{~Hz}), 130.80(\mathrm{~d}, J=2.3$ Hz)130.11 (d, $J=12.3 \mathrm{~Hz}), 128.46(\mathrm{~d}, J=1.3 \mathrm{~Hz}), 128.33(\mathrm{~d}, J=12.1 \mathrm{~Hz}), 127.47$ (d, $J=80.8$ $\mathrm{Hz}), 126.79$ (d, $J=7.6 \mathrm{~Hz}), 125.80,125.20,31.78$ (d, $J=55.4 \mathrm{~Hz}), 24.55(\mathrm{~d}, J=5.7 \mathrm{~Hz}), 6.97$ (d, $J=3.7 \mathrm{~Hz})$.

${ }^{31}$ P NMR (162 MHz, CDCl $\left.{ }_{3}\right) \delta 43.76$.

HRMS (ESI) $\mathbf{m} / \mathbf{z}[\mathbf{M}+\mathbf{H}]^{+}$: calcd. 311.1018, found. 311.1018.

IR (film): $v_{\max }\left(\mathrm{cm}^{-1}\right) 3050,2932,1507,1455,1100,1027,815,656,600,529$.

Optical rotation: $[\alpha]_{\mathrm{D}}{ }^{25}=-76.66\left(c=1.170, \mathrm{CHCl}_{3}, 90 \%\right.$ ee $)$.

HPLC: DAICEL CHIRALPAK ID, hexane $/ i-\mathrm{PrOH}=9 / 1$, flow rate: $1.0 \mathrm{~mL} / \mathrm{min}, \lambda=254 \mathrm{~nm}$, $\mathrm{t}_{\mathrm{R}}($ major $)=10.1 \mathrm{~min}, \mathrm{t}_{\mathrm{R}}($ minor $)=11.6 \mathrm{~min}, 90 \%$ ee

\begin{tabular}{|l|l|l|}
\hline Peak\# & Ret. Time & Area\% \\
\hline 1 & 10.144 & 49.904 \\
\hline 2 & 11.625 & 50.096 \\
\hline
\end{tabular}

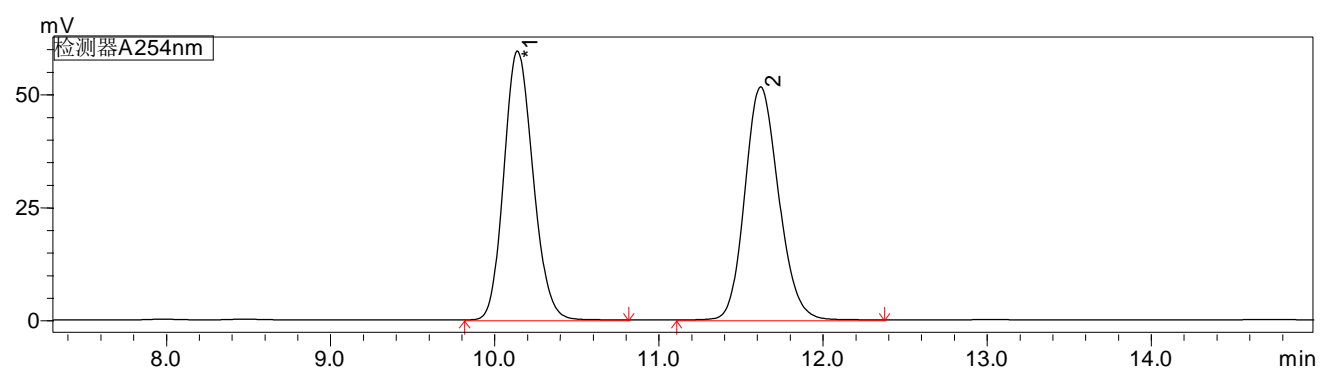

\begin{tabular}{|l|l|l|}
\hline Peak\# & Ret. Time & Area\% \\
\hline 1 & 10.106 & 94.851 \\
\hline 2 & 11.605 & 5.149 \\
\hline
\end{tabular}

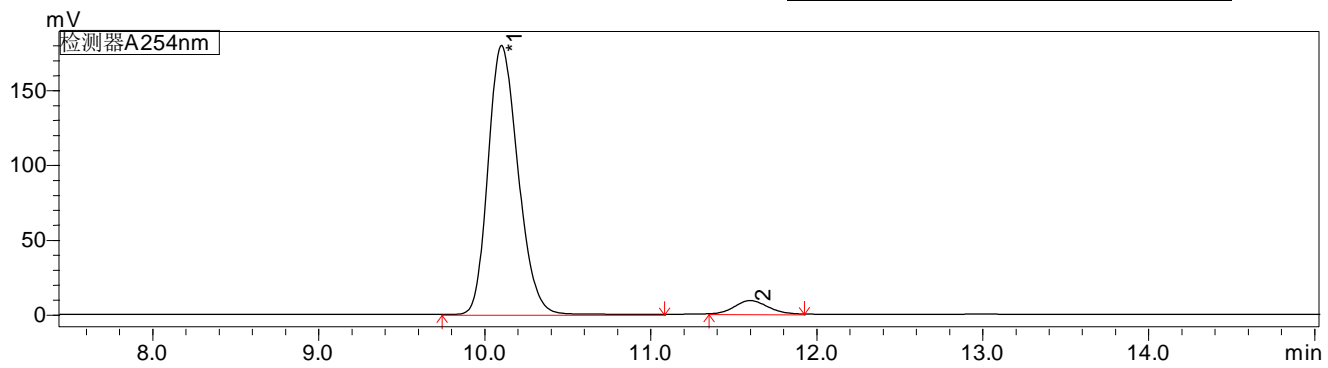




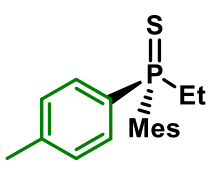

5 ja

5ja: Procedure B, $46.6 \mathrm{mg}$, 77\% yield, light yellow solid.

${ }^{1}$ H NMR (400 MHz, $\left.\mathbf{C D C l}_{3}\right)$ 87.69-7.54 (m, 2H), 7.26-7.17 (m, 2H), 6.83 (d, J = 3.9 Hz, 2H), 2.65-2.52 (m, 1H), 2.50-2.37 (m, 4H), $2.30(\mathrm{~s}, 6 \mathrm{H}), 2.26(\mathrm{~s}, 3 \mathrm{H}), 0.89(\mathrm{dt}, J=20.7,7.3 \mathrm{~Hz}, 3 \mathrm{H})$.

${ }^{13} \mathbf{C}$ NMR(101 MHz, CDCl $) \delta 141.49(\mathrm{~d}, J=9.9 \mathrm{~Hz}), 141.05(\mathrm{~d}, J=2.8 \mathrm{~Hz}), 140.61(\mathrm{~d}, J=2.9$ Hz), 131.19 (d, $J=11.0 \mathrm{~Hz}), 130.75$ (d, $J=10.3 \mathrm{~Hz}), 130.67$ (d, $J=79.4 \mathrm{~Hz}), 129.04$ (d, $J=12.4$ $\mathrm{Hz}), 128.93(\mathrm{~d}, J=82.0 \mathrm{~Hz}), 31.78(\mathrm{~d}, J=54.4 \mathrm{~Hz}), 23.97(\mathrm{~d}, J=5.0 \mathrm{~Hz}), 21.37(\mathrm{~d}, J=1.5 \mathrm{~Hz})$, $20.76(\mathrm{~d}, J=1.5 \mathrm{~Hz}), 7.02(\mathrm{~d}, J=3.9 \mathrm{~Hz})$.

${ }^{31}$ P NMR (162 MHz, $\left.\mathbf{C D C l}_{3}\right) \delta 45.93$.

HRMS (ESI) $\mathbf{m} / \mathbf{z}$ [M+H] $]^{+}$: calcd. 303.1331, found. 303.1331.

IR (film): $v_{\max }\left(\mathrm{cm}^{-1}\right)$ 2970, 2922, 1603, 1455, 1398, 1100, 1030, 851, 811, 767, 650 .

Optical rotation: $[\alpha]_{\mathrm{D}}{ }^{25}=-89.97\left(c=0.898, \mathrm{CHCl}_{3}, 93 \%\right.$ ee $)$.

HPLC: DAICEL CHIRALPAK AD-H, hexane $/ i-\mathrm{PrOH}=19 / 1$, flow rate: $1.0 \mathrm{~mL} / \mathrm{min}, \lambda=254 \mathrm{~nm}$, $\mathrm{t}_{\mathrm{R}}($ major $)=6.8 \mathrm{~min}, \mathrm{t}_{\mathrm{R}}($ minor $)=8.7 \mathrm{~min}, 93 \%$ ee.

\begin{tabular}{|l|l|l|}
\hline Peak\# & Ret. Time & Area\% \\
\hline 1 & 6.845 & 50.052 \\
\hline 2 & 8.705 & 49.948 \\
\hline
\end{tabular}

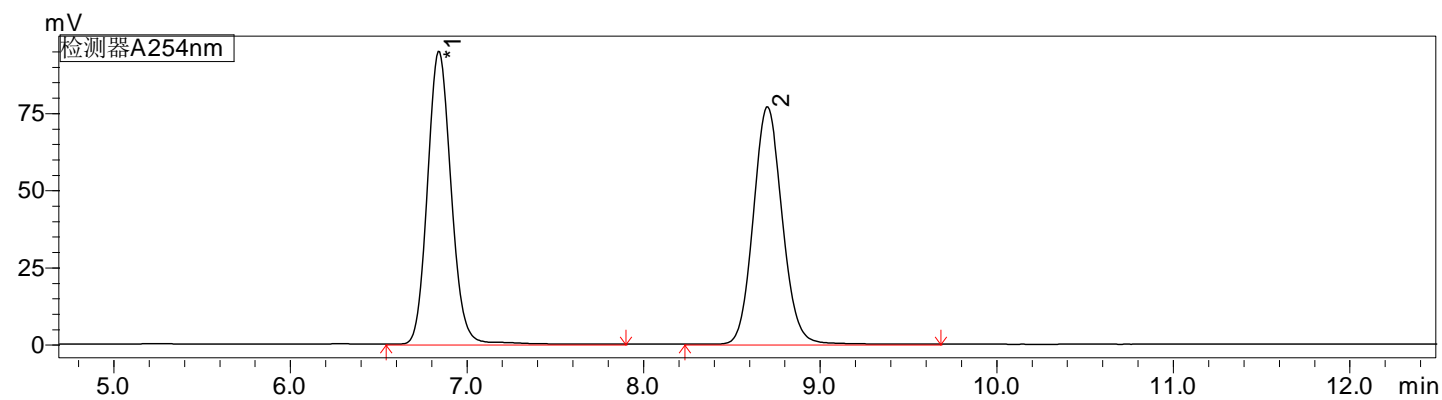

\begin{tabular}{|l|l|l|}
\hline Peak\# & Ret. Time & Area\% \\
\hline 1 & 6.860 & 96.622 \\
\hline 2 & 8.745 & 3.378 \\
\hline
\end{tabular}

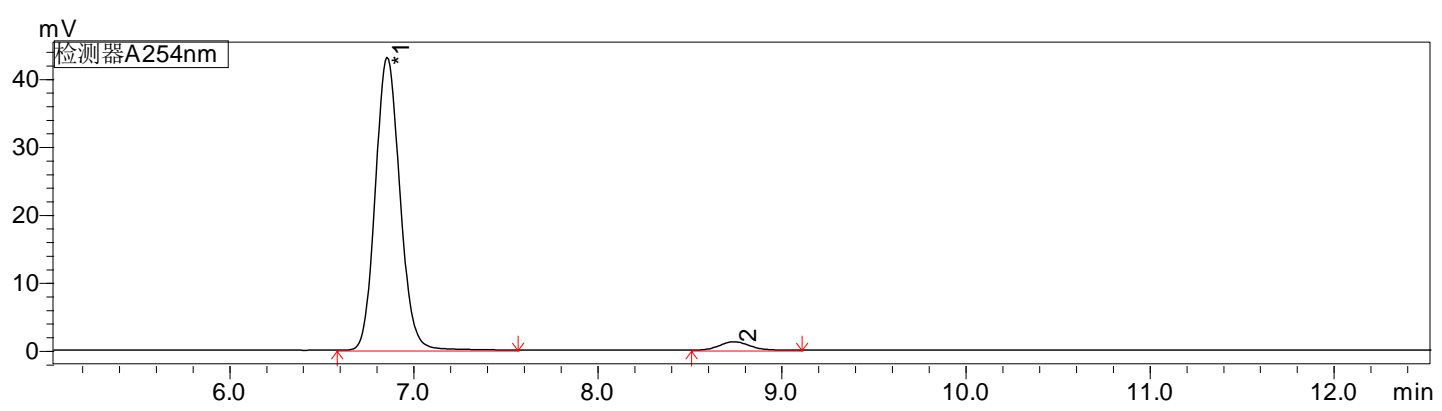




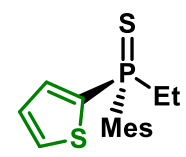

$5 \mathrm{ka}$

5ka: Procedure B, $67.4 \mathrm{mg}$, 99\% yield, light yellow solid.

${ }^{1}$ H NMR (500 MHz, CDCl $)_{3}$ ) 7.71-7.63 (m, 1H), 7.55-7.47 (m, 1H), 7.17-7.10 (m, 1H), $6.85(\mathrm{~d}$ $J=4.1 \mathrm{~Hz}, 2 \mathrm{H}), 2.71-2.57(\mathrm{~m}, 1 \mathrm{H}), 2.55-2.43(\mathrm{~m}, 1 \mathrm{H}), 2.38(\mathrm{~s}, 6 \mathrm{H}), 2.26(\mathrm{~s}, 3 \mathrm{H}), 1.05(\mathrm{dt}, J=$ $21.4,7.3 \mathrm{~Hz}, 3 \mathrm{H})$.

${ }^{13} \mathbf{C}$ NMR(101 MHz, $\left.\mathbf{C D C l}_{3}\right) \delta 141.47(\mathrm{~d}, J=10.6 \mathrm{~Hz}), 140.93(\mathrm{~d}, J=2.8 \mathrm{~Hz}), 137.58(\mathrm{~d}, J=$ $83.6 \mathrm{~Hz}), 135.55$ (d, $J=9.4 \mathrm{~Hz}), 132.97(\mathrm{~d}, J=4.3 \mathrm{~Hz}), 131.27$ (d, $J=11.5 \mathrm{~Hz}), 129.08$ (d, $J=$ $84.5 \mathrm{~Hz}), 127.99$ (d, $J=13.4 \mathrm{~Hz}), 33.15(\mathrm{~d}, J=57.5 \mathrm{~Hz}), 23.55$ (d, $J=5.0 \mathrm{~Hz}), 20.77$ (d, $J=1.5$ $\mathrm{Hz}), 7.10(\mathrm{~d}, J=4.6 \mathrm{~Hz})$.

${ }^{31}$ P NMR (162 MHz, $\left.\mathbf{C D C l}_{3}\right) \delta 38.06$.

HRMS (ESI) $\mathbf{m} / \mathbf{z}$ [M+H] ${ }^{+}$: calcd. 295.0739, found. 295.0739.

IR (film): $v_{\max }\left(\mathrm{cm}^{-1}\right)$ 2970, 2931, 1604, 1455, 1405, 1214, 1010, 851, 772, 681, 646.

Optical rotation: $[\alpha]_{\mathrm{D}}{ }^{25}=-20.31\left(c=1.402, \mathrm{CHCl}_{3}, 91 \%\right.$ ee $)$.

HPLC: DAICEL CHIRALPAK OD-H, hexane $/ i-\mathrm{PrOH}=19 / 1$, flow rate: $1.0 \mathrm{~mL} / \mathrm{min}, \lambda=254 \mathrm{~nm}$, $\mathrm{t}_{\mathrm{R}}($ major $)=7.5 \mathrm{~min}, \mathrm{t}_{\mathrm{R}}($ minor $)=9.7 \mathrm{~min}, 91 \%$ ee.

\begin{tabular}{|l|l|l|}
\hline Peak\# & Ret. Time & Area\% \\
\hline 1 & 7.483 & 50.002 \\
\hline 2 & 9.684 & 49.998 \\
\hline
\end{tabular}

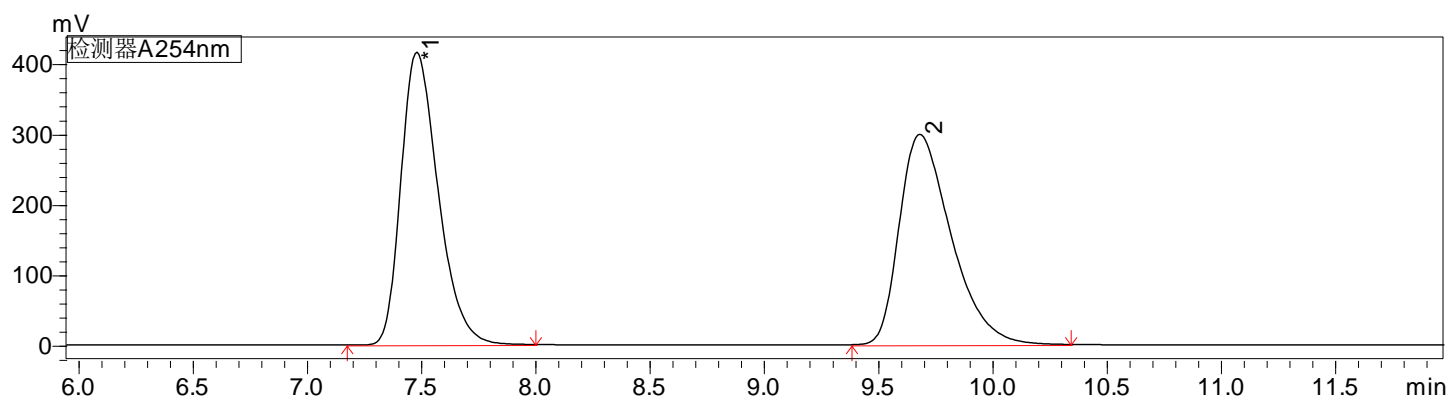

\begin{tabular}{|l|l|l|}
\hline Peak\# & Ret. Time & Area\% \\
\hline 1 & 7.440 & 95.448 \\
\hline 2 & 9.728 & 4.552 \\
\hline
\end{tabular}

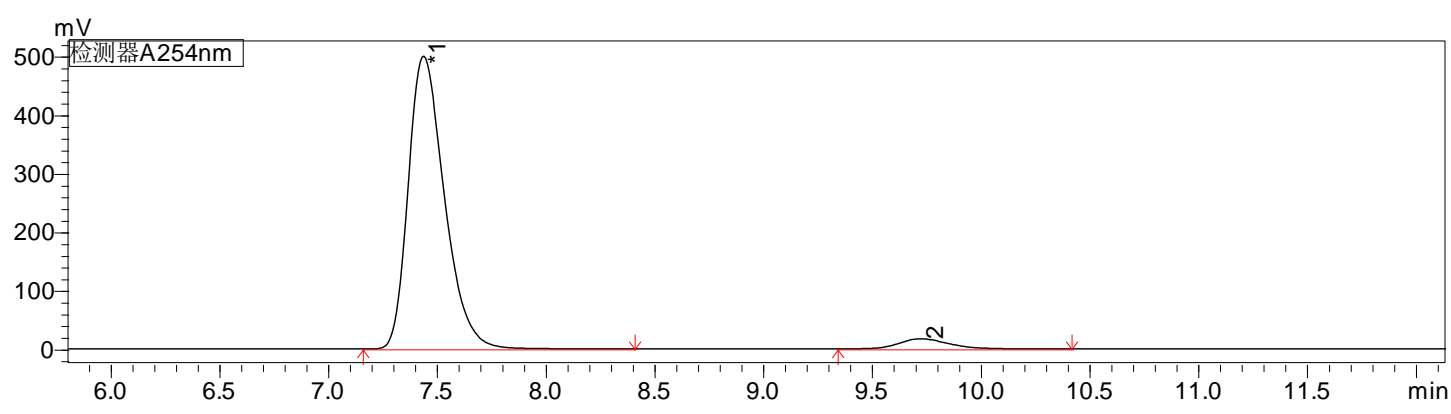




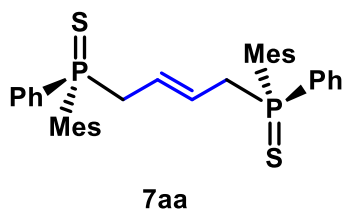

7aa: Procedure C, $55.2 \mathrm{mg}$, 95\% yield, light yellow solid.

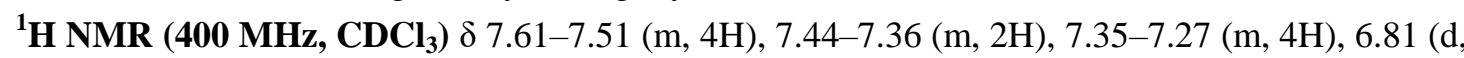
$J=3.2 \mathrm{~Hz}, 4 \mathrm{H}), 4.82(\mathrm{t}, J=4.1 \mathrm{~Hz}, 2 \mathrm{H}), 3.29-3.09$ (m, 2H), 3.06-2.88 (m, 2H), $2.25(\mathrm{~s}, 6 \mathrm{H}), 2.21$ (s, 12H).

${ }^{13} \mathbf{C}$ NMR(101 MHz, $\left.\mathbf{C D C l}_{3}\right) \delta$ 141.70-141.24 (m), 140.92-140.83 (m), 135.09-133.60 (m), 131.33-131.05 (m), 130.70-130.53 (m), $128.32(\mathrm{dd}, J=83.0,2.5 \mathrm{~Hz}), 128.21-127.91(\mathrm{~m})$, $126.15-125.60(\mathrm{~m}), 43.85(\mathrm{dd}, J=54.9,6.4 \mathrm{~Hz}), 23.89(\mathrm{t}, J=2.5 \mathrm{~Hz}), 20.78$.

${ }^{31}$ P NMR (162 MHz, $\left.\mathrm{CDCl}_{3}\right) \delta 40.87$.

HRMS (ESI) $\mathbf{m} / \mathbf{z}$ [M+H] $]^{+}$: calcd. 573.1963, found. 573.1959.

IR (film): $v_{\max }\left(\mathrm{cm}^{-1}\right)$ 3029, 2963, 2922, 2853, 1603, 1555, 1454, 1436, 1397, 1263, 1099, 1061, 845, 737, 694, 647, 597.

Optical rotation: $[\alpha]_{\mathrm{D}}^{25}=-65.64\left(c=1.000, \mathrm{CHCl}_{3}, 10.7 / 1 \mathrm{dr}, 96 \%\right.$ ee $)$.

HPLC: DAICEL CHIRALPAK IA, hexane $/ i-\mathrm{PrOH}=9 / 1$, flow rate: $1.0 \mathrm{~mL} / \mathrm{min}, \lambda=220 \mathrm{~nm}$, $\mathrm{t}_{\mathrm{R}}($ major $)=15.3 \mathrm{~min}, \mathrm{t}_{\mathrm{R}}($ minor $)=16.8 \mathrm{~min}, 10.7 / 1 \mathrm{dr}, 96 \%$ ee .
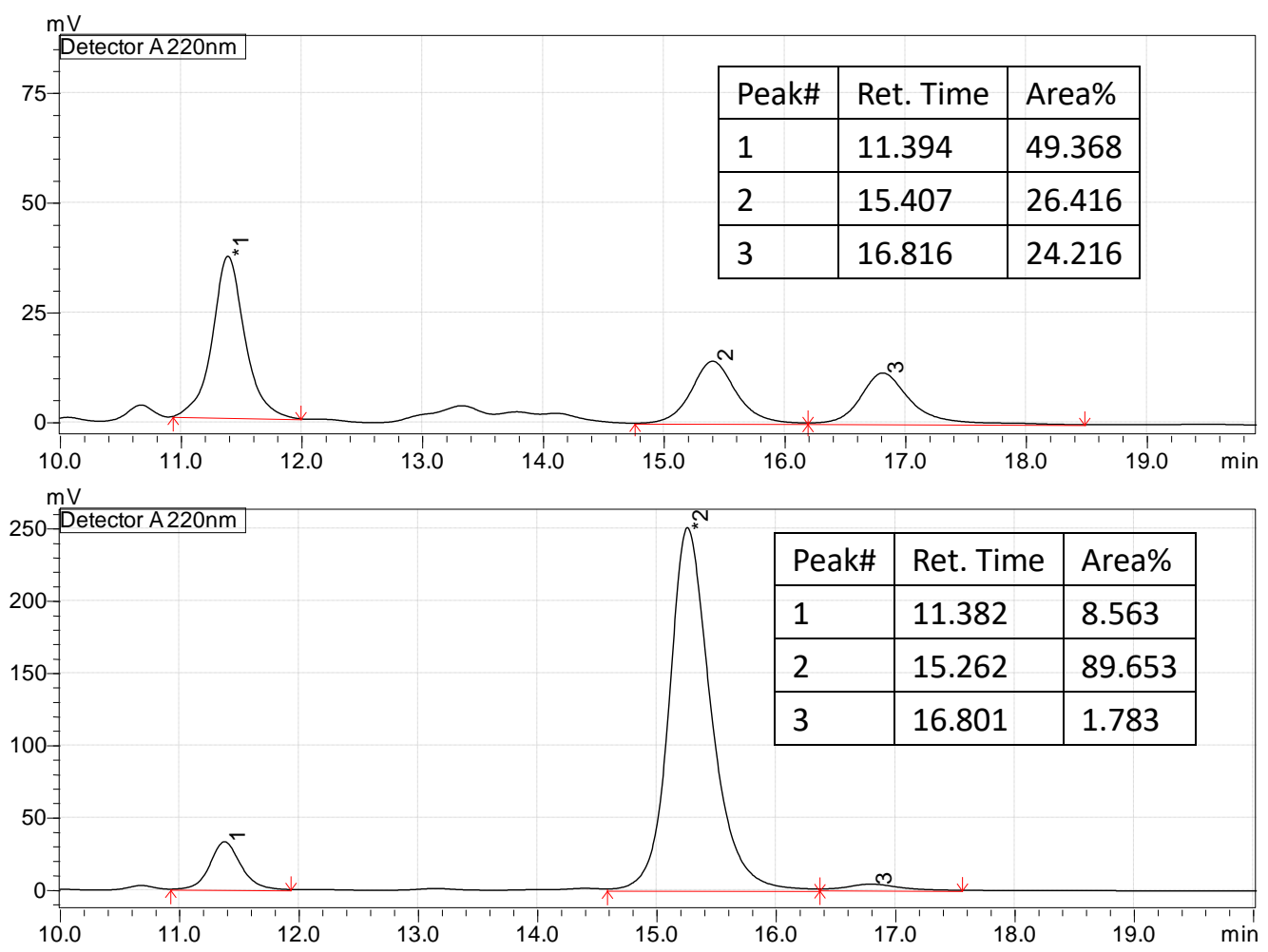


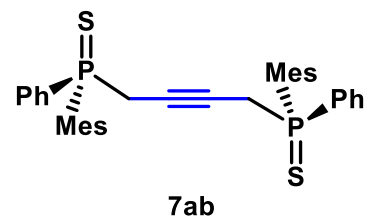

7ab: Procedure C, $43.5 \mathrm{mg}, 80 \%$ yield, white solid.

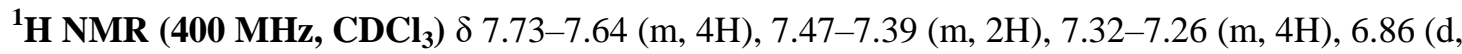
$J=3.8 \mathrm{~Hz}, 4 \mathrm{H}), 3.52-3.38(\mathrm{~m}, 2 \mathrm{H}), 3.14-2.97(\mathrm{~m}, 2 \mathrm{H}), 2.31$ (s, 6H), $2.22(\mathrm{~s}, 12 \mathrm{H})$.

${ }^{13} \mathbf{C}$ NMR(101 MHz, CDCl 3 ) $\delta 141.95-141.50(\mathrm{~m}), 141.21(\mathrm{t}, J=1.5 \mathrm{~Hz}), 134.33(\mathrm{~d}, J=81.6 \mathrm{~Hz})$, 131.48-131.19 (m), 131.23 (d, $J=1.9 \mathrm{~Hz}), 130.82-130.19(\mathrm{~m}), 128.44-128.02(\mathrm{~m}), 126.85$ (d, $J=$ $85.0 \mathrm{~Hz}$ ), 34.27 (dd, $J=54.5,4.6 \mathrm{~Hz}$ ), 23.74-23.12 (m), 20.97.

${ }^{31}$ P NMR (162 MHz, $\left.\mathrm{CDCl}_{3}\right) \delta 38.82$.

HRMS (ESI) $\mathbf{m} / \mathbf{z}[\mathbf{M}+\mathbf{H}]^{+}$: calcd. 571.1806, found. 571.1807.

IR (film): $v_{\max }\left(\mathrm{cm}^{-1}\right) 3050,2957,2924,2853,1603,1455,1436,1377,1263,1155,1099,1027$, 825, 724, 694, 669, 598.

Optical rotation: $[\alpha]_{\mathrm{D}}^{25}=61.24\left(c=0.950, \mathrm{CHCl}_{3}, 4.8 / 1 \mathrm{dr}, 97 \%\right.$ ee $)$.

HPLC: DAICEL CHIRALPAK IG-3, hexane $/ i-\operatorname{PrOH}=2 / 1$, flow rate: $0.6 \mathrm{~mL} / \mathrm{min}, \lambda=220 \mathrm{~nm}$, $\mathrm{t}_{\mathrm{R}}($ major $)=27.1 \mathrm{~min}, \mathrm{t}_{\mathrm{R}}(\operatorname{minor})=45.7 \mathrm{~min}, 4.8 / 1 \mathrm{dr}, 97 \%$ ee .
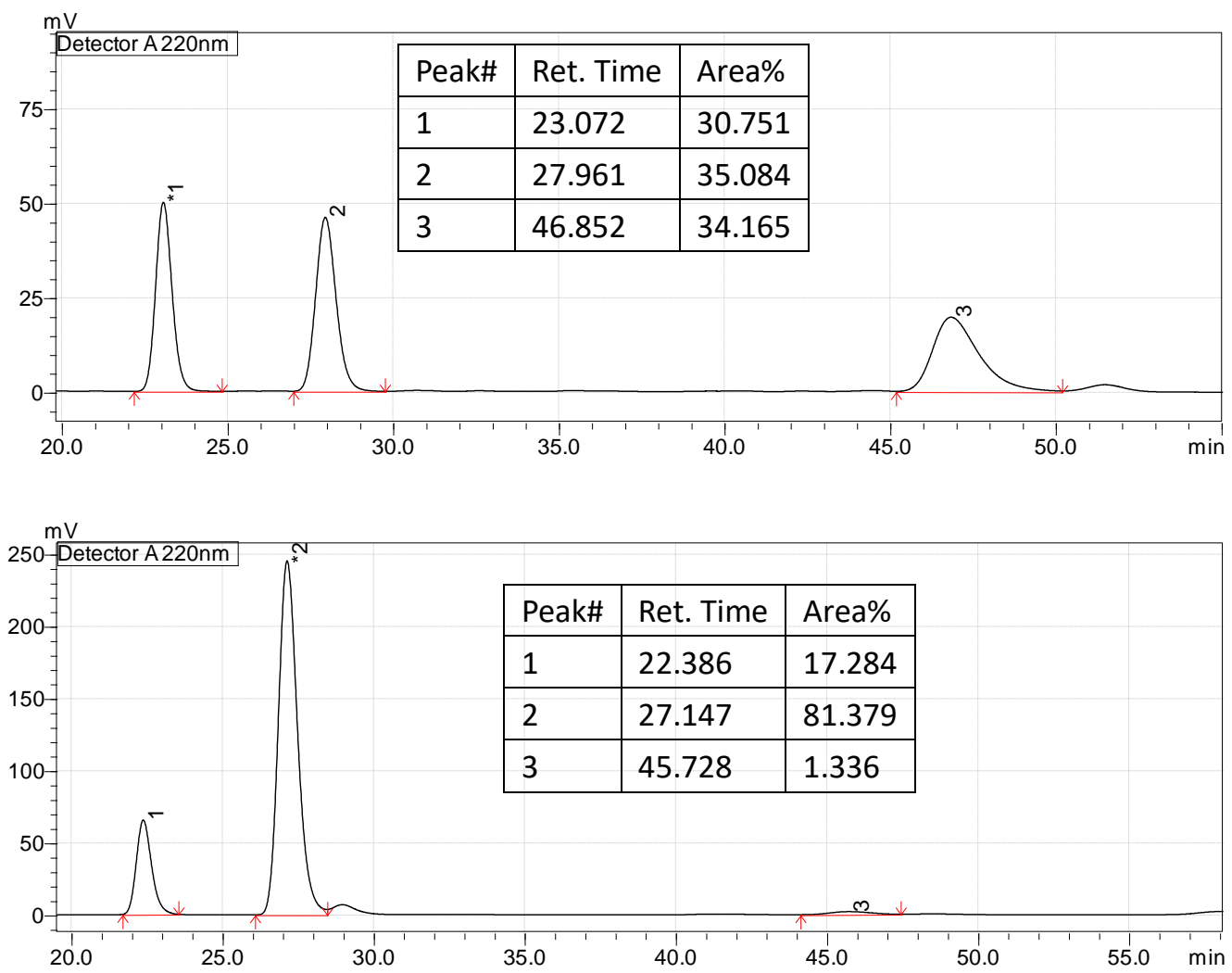


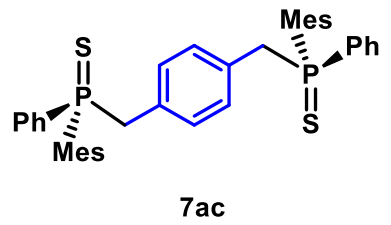

7ac: Procedure C, $56.2 \mathrm{mg}, 93 \%$ yield, white solid.

${ }^{1}$ H NMR (400 MHz, CDCl $) \delta$ 7.44-7.31 (m, 10H), $6.87(\mathrm{~d}, J=3.4 \mathrm{~Hz}, 4 \mathrm{H}), 6.34(\mathrm{~d}, J=1.2 \mathrm{~Hz}$, $4 \mathrm{H}$ ), 3.92-3.84 (m, 2H), 3.57 (dd, $J=12.8,9.3 \mathrm{~Hz}, 2 \mathrm{H}), 2.34(\mathrm{~s}, 12 \mathrm{H}), 2.28(\mathrm{~s}, 6 \mathrm{H})$.

${ }^{13} \mathbf{C}$ NMR(101 MHz, $\left.\mathbf{C D C l}_{3}\right) \delta$ 141.64-141.33 (m), $140.78(\mathrm{~d}, J=1.7 \mathrm{~Hz}), 133.73(\mathrm{~d}, J=82.2$ Hz), 131.62-131.13 (m), 130.79, 130.72-130.58 (m), 129.80, 129.34-129.13 (m), 129.19 (d, $J=$ $81.4 \mathrm{~Hz}), 128.24-127.94(\mathrm{~m}), 45.26(\mathrm{dd}, J=48.9,3.6 \mathrm{~Hz}), 24.57-23.82(\mathrm{~m}), 20.82$.

${ }^{31}$ P NMR (162 MHz, $\left.\mathbf{C D C l}_{3}\right) \delta 42.95$.

HRMS (ESI) $\mathbf{m} / \mathbf{z}[\mathbf{M}+\mathbf{H}]^{+}$: calcd. 623.2119 , found. 623.2122 .

IR (film): $v_{\max }\left(\mathrm{cm}^{-1}\right)$ 3050, 2959, 2923, 2853, 1603, 1510, 1455, 1437, 1264, 1101, 1024, 841, 746, 693, 643, 593.

Optical rotation: $[\alpha]_{D}{ }^{25}=-261.27\left(c=1.170, \mathrm{CHCl}_{3}, 6.1 / 1 \mathrm{dr}, 99 \%\right.$ ee $)$.

HPLC: DAICEL CHIRALPAK AD-H, hexane $/ i-\mathrm{PrOH}=19 / 1$, flow rate: $1.0 \mathrm{~mL} / \mathrm{min}, \lambda=220 \mathrm{~nm}$, $\mathrm{t}_{\mathrm{R}}($ major $)=21.9 \mathrm{~min}, \mathrm{t}_{\mathrm{R}}($ minor $)=27.6 \mathrm{~min}, 6.1 / 1 \mathrm{dr}, 99 \%$ ee .
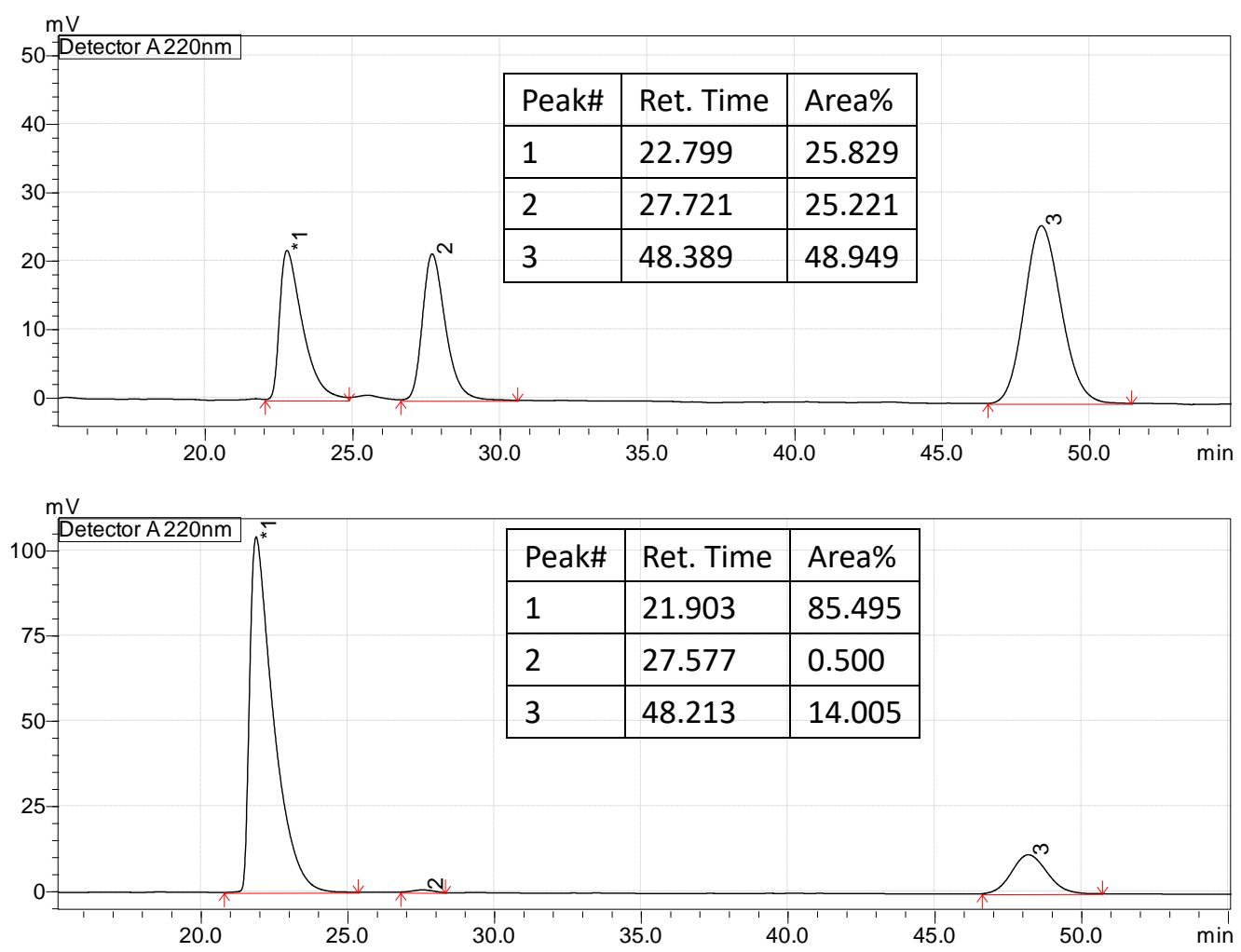


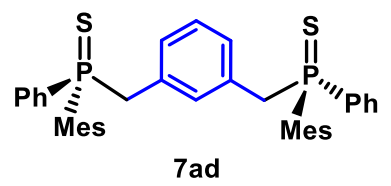

7ad: Procedure C, $51.9 \mathrm{mg}, 88 \%$ yield, white solid.

${ }^{1}$ H NMR $\left(400 \mathrm{MHz}, \mathbf{C D C l}_{3}\right) \delta$ 7.37-7.30 (m, 6H), 7.25-7.18 (m, 4H), $6.86(\mathrm{~d}, J=3.8 \mathrm{~Hz}, 4 \mathrm{H})$, 6.67-6.61 (m, 1H), 6.56-6.49 (m, 2H), 6.01-5.95 (m, 1H), $3.85(\mathrm{dd}, J=15.9,13.1 \mathrm{~Hz}, 2 \mathrm{H}), 3.39$ (dd, $J=13.1,10.6 \mathrm{~Hz}, 2 \mathrm{H}), 2.30(\mathrm{~s}, 12 \mathrm{H}), 2.28(\mathrm{~s}, 6 \mathrm{H})$.

${ }^{13} \mathbf{C}$ NMR(101 MHz, CDCl 3 ) $\delta$ 141.62-141.34 (m), $140.78(\mathrm{t}, J=1.4 \mathrm{~Hz}), 134.05(\mathrm{~d}, J=80.0 \mathrm{~Hz})$, 133.45 (t, $J=5.7 \mathrm{~Hz}), 131.32(\mathrm{~d}, J=11.6 \mathrm{~Hz}), 130.99-130.70(\mathrm{~m}), 130.39$ (d, $J=1.6 \mathrm{~Hz})$, 130.28-129.99 (m), $129.16(\mathrm{~d}, J=80.3 \mathrm{~Hz}), 128.99$ (t, $J=4.6 \mathrm{~Hz}), 127.84(\mathrm{~d}, J=12.9 \mathrm{~Hz})$, $126.36(\mathrm{t}, J=3.4 \mathrm{~Hz}), 45.10$ (d, $J=47.4 \mathrm{~Hz}), 24.33$ (dd, $J=4.5,2.2 \mathrm{~Hz}), 20.82$.

${ }^{31}$ P NMR (162 MHz, $\mathbf{C D C l}_{3}$ ) $\delta 42.69$.

HRMS (ESI) $\mathbf{m} / \mathbf{z}[\mathbf{M}+\mathrm{H}]^{+}$: calcd. 623.2119 , found. 623.2119 .

IR (film): $v_{\max }\left(\mathrm{cm}^{-1}\right)$ 3050, 3028, 2965, 2922, 1603, 1454, 1437, 1404, 1265, 1100, 1027, 841, 797, 737, 699, 646, 592.

Optical rotation: $[\alpha]_{\mathrm{D}}^{25}=-150.02\left(c=1.170, \mathrm{CHCl}_{3}, 5.6 / 1 \mathrm{dr}, 99 \%\right.$ ee $)$.

HPLC: DAICEL CHIRALPAK OD-H, hexane $/ i-\mathrm{PrOH}=19 / 1$, flow rate: $1.0 \mathrm{~mL} / \mathrm{min}, \lambda=220 \mathrm{~nm}$, $\mathrm{t}_{\mathrm{R}}($ major $)=18.3 \mathrm{~min}, \mathrm{t}_{\mathrm{R}}($ minor $)=37.4 \mathrm{~min}, 5.6 / 1 \mathrm{dr}, 99 \%$ ee .
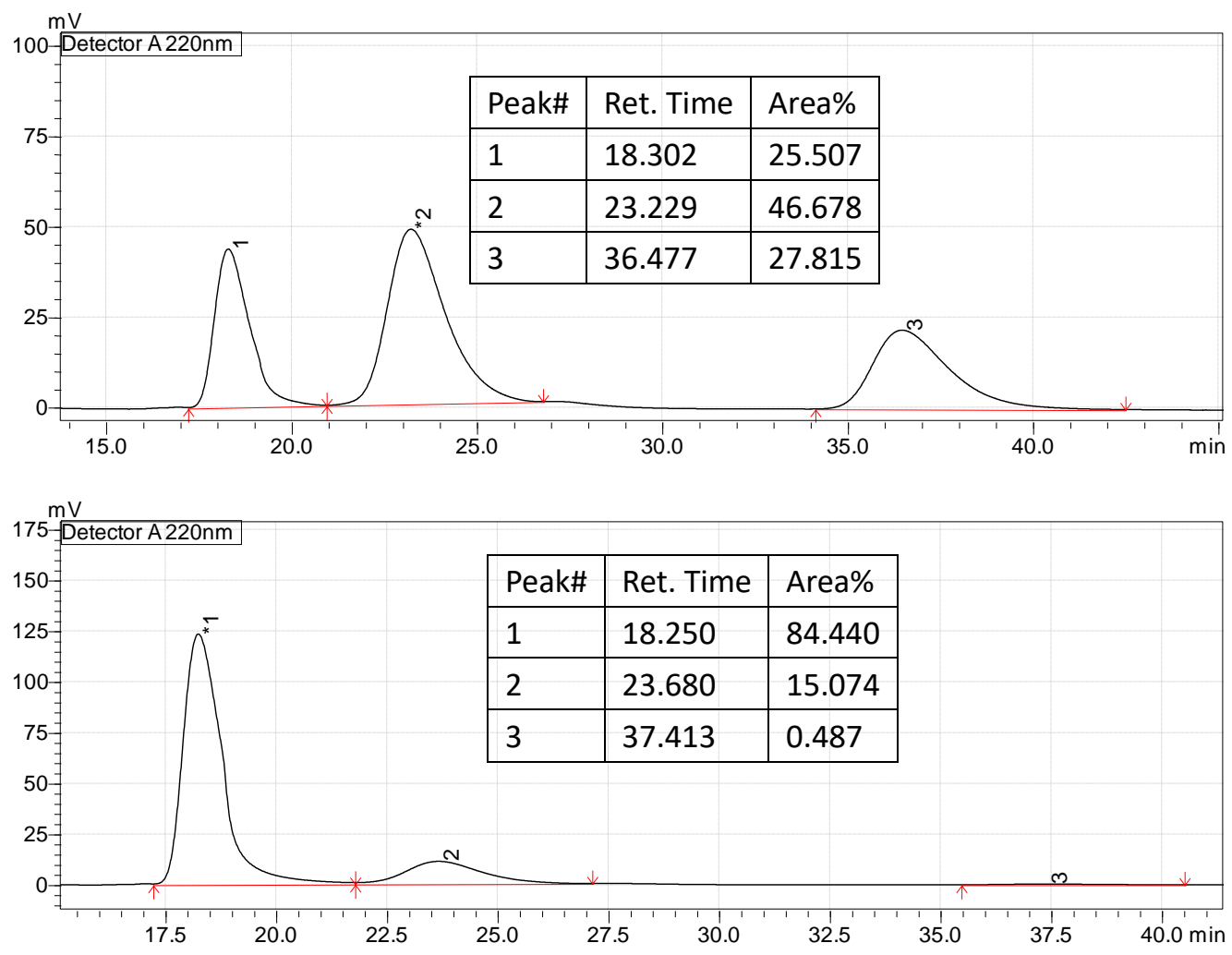


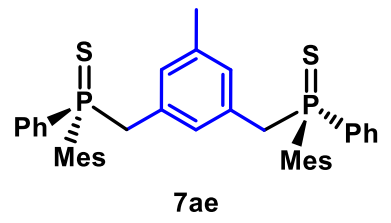

7ae: Procedure C, $61.0 \mathrm{mg}, 96 \%$ yield, white solid.

${ }^{1}$ H NMR (400 MHz, CDCl $\left.)_{3}\right) \delta 7.39-7.29(\mathrm{~m}, 6 \mathrm{H}), 7.25-7.18(\mathrm{~m}, 4 \mathrm{H}), 6.86(\mathrm{~d}, J=3.8 \mathrm{~Hz}, 4 \mathrm{H})$, 6.12-6.02 (m, 3H), $3.83(\mathrm{dd}, J=15.8,13.0 \mathrm{~Hz}, 2 \mathrm{H}), 3.41(\mathrm{dd}, J=13.0,10.6 \mathrm{~Hz}, 2 \mathrm{H}), 2.31$ (s, $12 \mathrm{H}), 2.28(\mathrm{~s}, 6 \mathrm{H}), 1.77(\mathrm{~s}, 3 \mathrm{H})$.

${ }^{13}$ C NMR(101 MHz, CDCl 3 ) $\delta$ 141.67-141.34 (m), 140.88-140.59 (m), $135.66(\mathrm{t}, J=3.5 \mathrm{~Hz})$, $134.59,133.79,131.31(\mathrm{~d}, J=11.5 \mathrm{~Hz}), 131.08-130.72(\mathrm{~m}), 130.34(\mathrm{~d}, J=1.6 \mathrm{~Hz}), 129.93-$ $129.70(\mathrm{~m}), 129.25(\mathrm{~d}, J=80.0 \mathrm{~Hz}), 127.72(\mathrm{~d}, J=12.8 \mathrm{~Hz}), 45.22(\mathrm{~d}, J=47.4 \mathrm{~Hz}), 24.59-23.90$ (m), 20.82, 20.70.

${ }^{31}$ P NMR (162 MHz, CDCl $) \delta 42.74$.

HRMS (ESI) $\mathbf{m} / \mathbf{z}[\mathbf{M}+\mathbf{H}]^{+}$: calcd. 637.2276, found. 637.2277 .

IR (film): $v_{\max }\left(\mathrm{cm}^{-1}\right)$ 3054, 2960, 2921, 2854, 1603, 1556, 1452, 1436, 1265, 1100, 1027, 848, 735, 698, 646, 596.

Optical rotation: $[\alpha]_{\mathrm{D}}^{25}=-190.94\left(c=1.067, \mathrm{CHCl}_{3}, 8.9 / 1 \mathrm{dr},>99 \%\right.$ ee $)$.

HPLC: DAICEL CHIRALPAK OD-H, hexane $/ i-\mathrm{PrOH}=19 / 1$, flow rate: $1.0 \mathrm{~mL} / \mathrm{min}, \lambda=254 \mathrm{~nm}$, $\mathrm{t}_{\mathrm{R}}($ major $)=15.5 \mathrm{~min}, \mathrm{t}_{\mathrm{R}}($ minor $)=39.6 \mathrm{~min}, 8.9 / 1 \mathrm{dr},>99 \%$ ee .
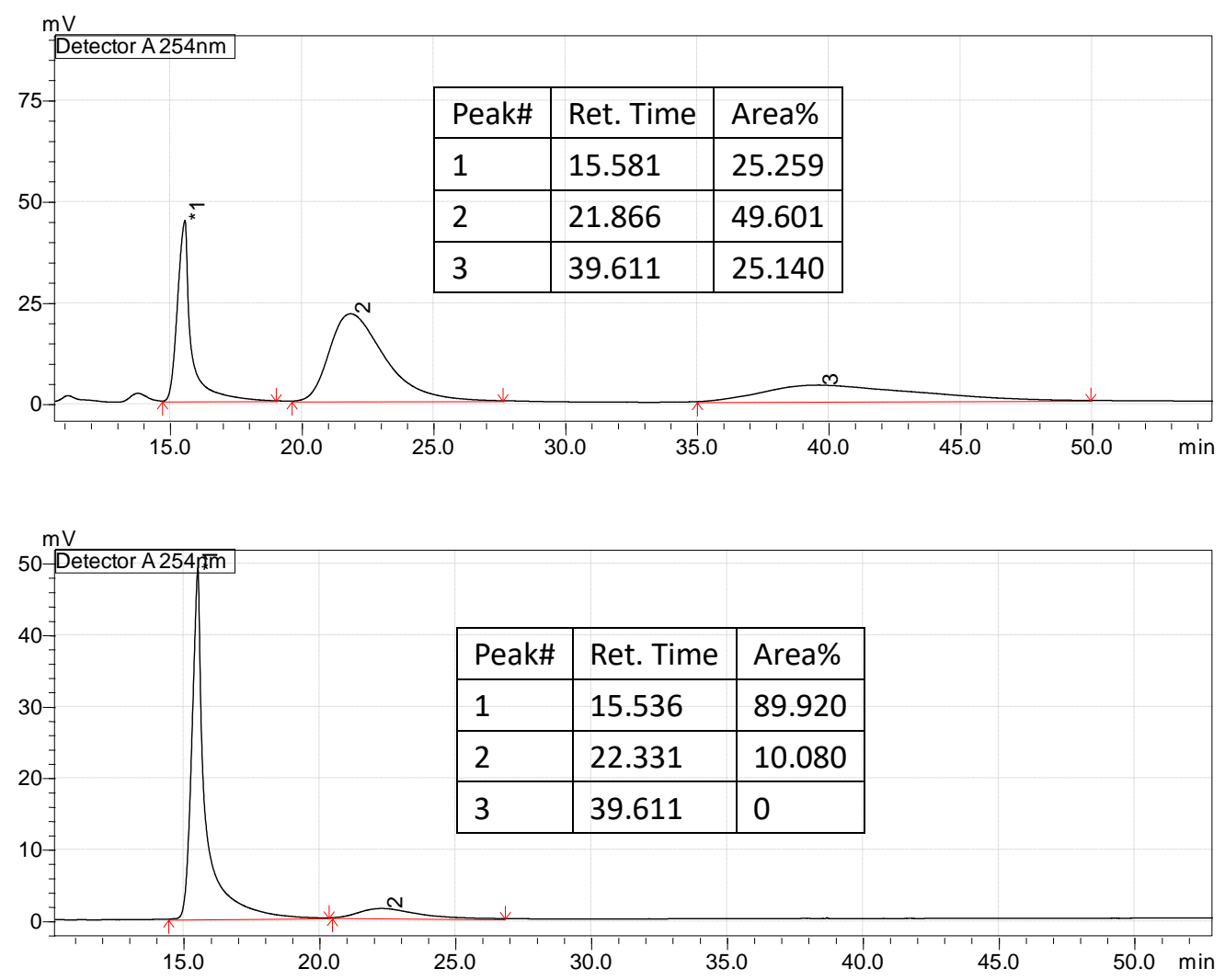


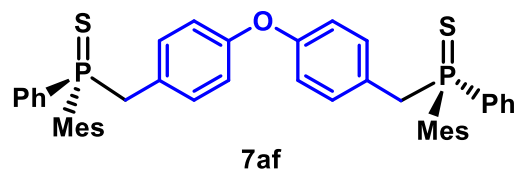

7af: Procedure C, $66.4 \mathrm{mg}, 93 \%$ yield, light yellow solid.

${ }^{1} \mathbf{H}$ NMR (400 MHz, $\mathbf{C D C l}_{3}$ ) $\delta$ 7.49-7.40 (m, 4H), 7.39-7.33 (m, 2H), 7.28-7.22 (m, 4H), 6.88 (d, $J=3.9 \mathrm{~Hz}, 4 \mathrm{H}), 6.75-6.68(\mathrm{~m}, 4 \mathrm{H}), 6.55-6.48(\mathrm{~m}, 4 \mathrm{H}), 3.99(\mathrm{dd}, J=15.6,13.1 \mathrm{~Hz}, 2 \mathrm{H}), 3.70(\mathrm{dd}$, $J=13.1,10.3 \mathrm{~Hz}, 2 \mathrm{H}), 2.35$ (s, 12H), 2.29 (s, 6H).

${ }^{13}$ C NMR(101 MHz, CDCl $) \delta 155.92(\mathrm{~d}, J=4.3 \mathrm{~Hz}), 141.50(\mathrm{~d}, J=10.0 \mathrm{~Hz}), 140.87(\mathrm{~d}, J=2.9$ $\mathrm{Hz}), 133.90$ (d, $J=79.9 \mathrm{~Hz}), 131.70(\mathrm{~d}, J=5.5 \mathrm{~Hz}), 131.31(\mathrm{~d}, J=11.1 \mathrm{~Hz}), 130.66(\mathrm{~d}, J=9.8$ $\mathrm{Hz}), 130.54$ (d, $J=3.0 \mathrm{~Hz}), 129.08$ (d, $J=79.8 \mathrm{~Hz}), 127.90$ (d, $J=12.2 \mathrm{~Hz}), 125.81(\mathrm{~d}, J=7.3$ $\mathrm{Hz}), 117.66(\mathrm{~d}, J=3.4 \mathrm{~Hz}), 44.93(\mathrm{~d}, J=47.2 \mathrm{~Hz}), 24.30(\mathrm{~d}, J=4.7 \mathrm{~Hz}), 20.83(\mathrm{~d}, J=1.5 \mathrm{~Hz})$.

${ }^{31}$ P NMR (162 MHz, $\left.\mathbf{C D C l}_{3}\right) \delta 43.28$.

HRMS (ESI) $\mathbf{m} / \mathbf{z}[\mathbf{M}+\mathbf{H}]^{+}$: calcd. 715.2382, found. 715.2378.

IR (film): $v_{\max }\left(\mathrm{cm}^{-1}\right)$ 3058, 2958, 2924, 2853, 1602, 1498, 1437, 1238, 1170, 1100, 1027, 845, 737, 692, 646, 594.

Optical rotation: $[\alpha]_{\mathrm{D}}{ }^{25}=-253.35\left(c=1.000, \mathrm{CHCl}_{3}, 8.8 / 1 \mathrm{dr}, 99 \%\right.$ ee $)$.

HPLC: DAICEL CHIRALPAK IA, hexane $/ i-\mathrm{PrOH}=9 / 1$, flow rate: $1.0 \mathrm{~mL} / \mathrm{min}, \lambda=220 \mathrm{~nm}$, $\mathrm{t}_{\mathrm{R}}($ major $)=24.4 \mathrm{~min}, \mathrm{t}_{\mathrm{R}}($ minor $)=31.3 \mathrm{~min}, 8.8 / 1 \mathrm{dr}, 99 \%$ ee.
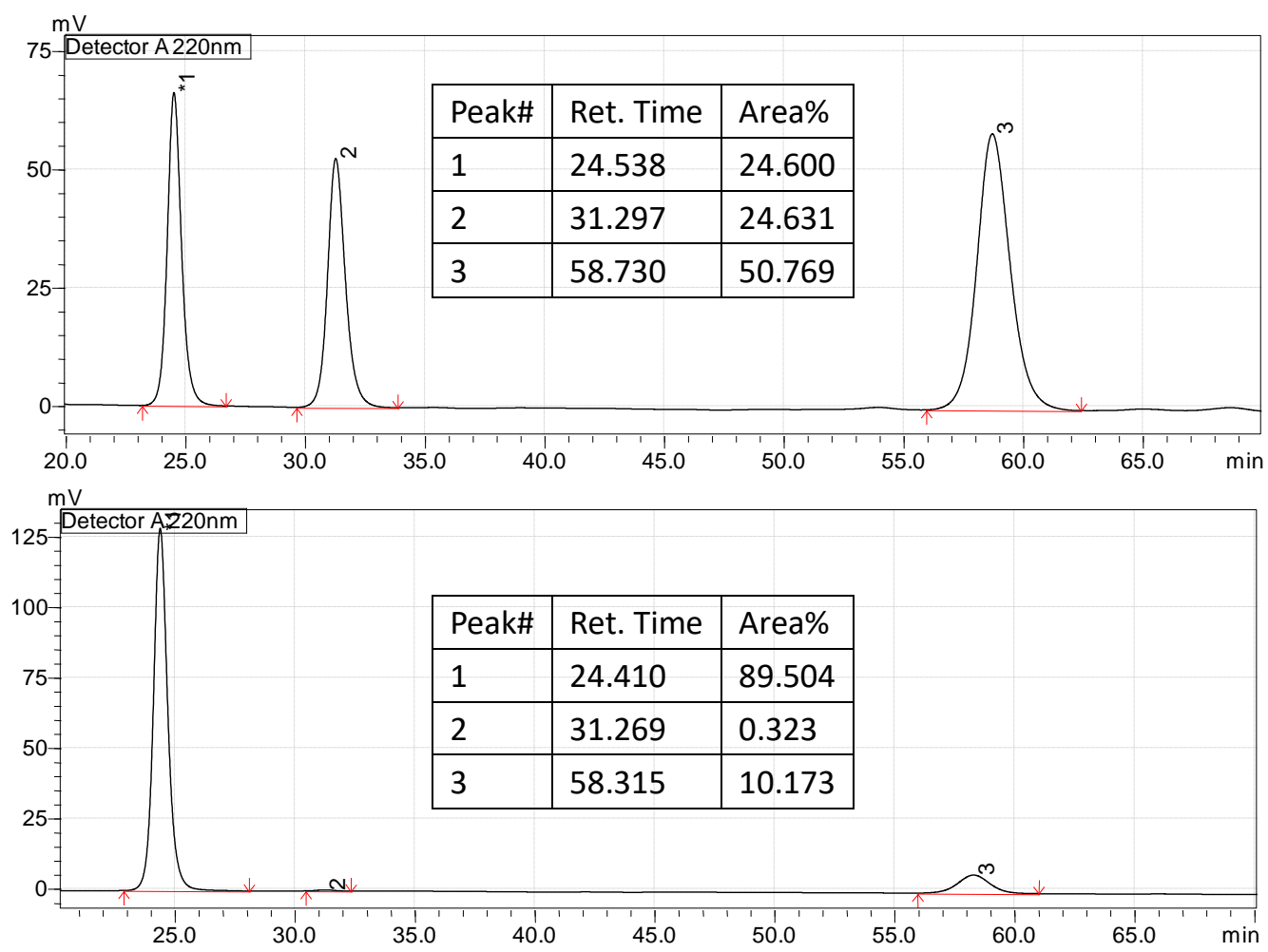


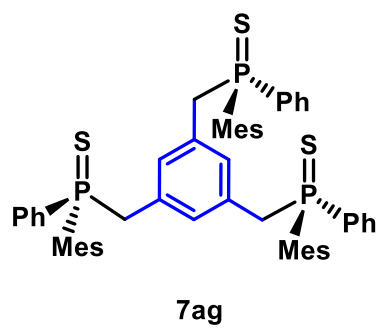

7ag: Procedure D, $77.8 \mathrm{mg}, 88 \%$ yield, white solid.

${ }^{1}$ H NMR (400 MHz, CDCl $) \delta$ 7.40-7.19 (m, 15H), $6.84(\mathrm{~d}, J=3.6 \mathrm{~Hz}, 6 \mathrm{H}), 5.92(\mathrm{q}, J=2.6 \mathrm{~Hz}$, $3 \mathrm{H}$ ), 3.60 (dd, $J=15.8,13.2 \mathrm{~Hz}, 3 \mathrm{H}), 3.23(\mathrm{dd}, J=13.3,11.0 \mathrm{~Hz}, 3 \mathrm{H}), 2.27$ (s, 9H), 2.25 (s, 18H). ${ }^{13}$ C NMR(101 MHz, CDCl $) \delta$ 141.74-141.30 (m), 140.87-140.63 (m), 134.23 (d, $\left.J=79.6 \mathrm{~Hz}\right)$, 131.74-131.51 (m), 131.52-131.26 (m), 131.21-130.96 (m), 130.38, 130.12-129.87 (m), 129.16 (d, $J=80.1 \mathrm{~Hz}), 128.12-127.75(\mathrm{~m}), 44.79$ (d, $J=47.7 \mathrm{~Hz}), 24.80-23.64$ (m), 20.86.

${ }^{31}$ P NMR (162 MHz, CDCl 3 ) $\delta 41.71$.

HRMS (ESI) $\mathbf{m} / \mathbf{z}$ [M+H] $]^{+}$: calcd. 895.2908, found. 895.2909.

IR (film): $v_{\max }\left(\mathrm{cm}^{-1}\right) 3049,2960,2923,2853,1603,1554,1451,1436,1403,1265,1100,1027$, 834, 737, 703, 646, 598.

Optical rotation: $[\alpha]_{D}{ }^{25}=-177.64\left(c=1.000, \mathrm{CHCl}_{3}, 3.2 / 1 \mathrm{dr}, 99 \%\right.$ ee $)$.

HPLC: DAICEL CHIRALPAK IA, hexane $/ i-\mathrm{PrOH}=3 / 1$, flow rate: $0.8 \mathrm{~mL} / \mathrm{min}, \lambda=220 \mathrm{~nm}$, $\mathrm{t}_{\mathrm{R}}($ minor $)=15.3 \mathrm{~min}, \mathrm{t}_{\mathrm{R}}($ major $)=23.2 \mathrm{~min}, 3.2 / 1 \mathrm{dr}, 99 \%$ ee .
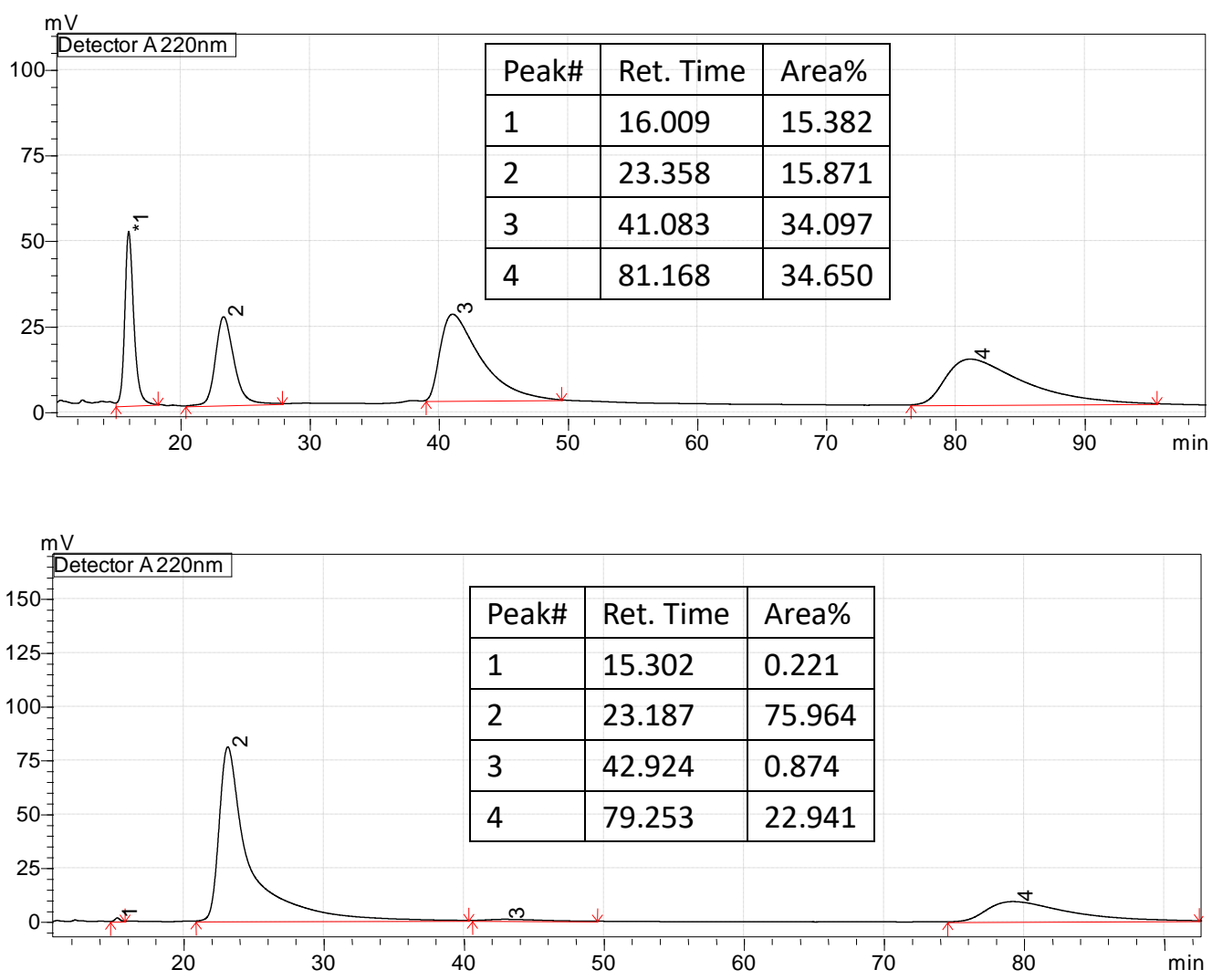


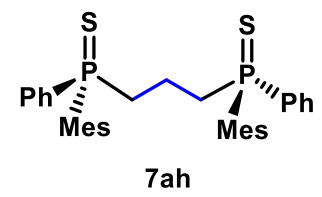

7ah: Procedure C, $44.8 \mathrm{mg}, 80 \%$ yield, white solid.

${ }^{1}$ H NMR (400 MHz, CDCl $_{3}$ ) $\delta$ 7.55-7.45 (m, 4H), 7.43-7.36 (m, 2H), 7.32-7.24 (m, 5H), 6.80 (d, $J=3.9 \mathrm{~Hz}, 4 \mathrm{H}), 2.87-2.69(\mathrm{~m}, 2 \mathrm{H}), 2.64-2.46(\mathrm{~m}, 2 \mathrm{H}), 2.25$ (s, 7H), $2.23(\mathrm{~s}, 12 \mathrm{H}), 1.22-1.05(\mathrm{~m}$, $2 \mathrm{H})$.

${ }^{13} \mathbf{C}$ NMR(101 MHz, $\left.\mathbf{C D C l}_{3}\right) \delta 141.37(\mathrm{~d}, J=10.5 \mathrm{~Hz}), 140.83(\mathrm{~d}, J=2.6 \mathrm{~Hz}), 133.79(\mathrm{~d}, J=$ $77.4 \mathrm{~Hz}), 131.26(\mathrm{~d}, J=11.5 \mathrm{~Hz}), 130.61(\mathrm{~d}, J=2.5 \mathrm{~Hz}), 130.48(\mathrm{~d}, J=10.6 \mathrm{~Hz}), 128.71$ (d, $J=$ $82.6 \mathrm{~Hz}), 128.33(\mathrm{~d}, J=12.4 \mathrm{~Hz}), 39.10(\mathrm{dd}, J=52.7,13.0 \mathrm{~Hz}), 24.42-23.57(\mathrm{~m}), 20.79,17.75$.

${ }^{31}$ P NMR (162 MHz, CDCl $) \delta 42.67$.

HRMS (ESI) $\mathbf{m} / \mathbf{z}$ [M+H] $]^{+}$: calcd. 561.1963, found. 561.1964.

IR (film): $v_{\max }\left(\mathrm{cm}^{-1}\right)$ 3026, 2964, 2922, 1603, 1555, 1454, 1436, 1404, 1263, 1099, 1028, 850, 737, 693, 647, 588.

Optical rotation: $[\alpha]_{D}^{25}=-123.54\left(c=1.150, \mathrm{CHCl}_{3}, 6.2 / 1 \mathrm{dr}, 98 \%\right.$ ee $)$.

HPLC: DAICEL CHIRALPAK IA, hexane $/ i-\mathrm{PrOH}=19 / 1$, flow rate: $1.0 \mathrm{~mL} / \mathrm{min}, \lambda=254 \mathrm{~nm}$, $\mathrm{t}_{\mathrm{R}}($ major $)=21.1 \mathrm{~min}, \mathrm{t}_{\mathrm{R}}($ minor $)=28.1 \mathrm{~min}, 6.2 / 1 \mathrm{dr}, 98 \%$ ee .
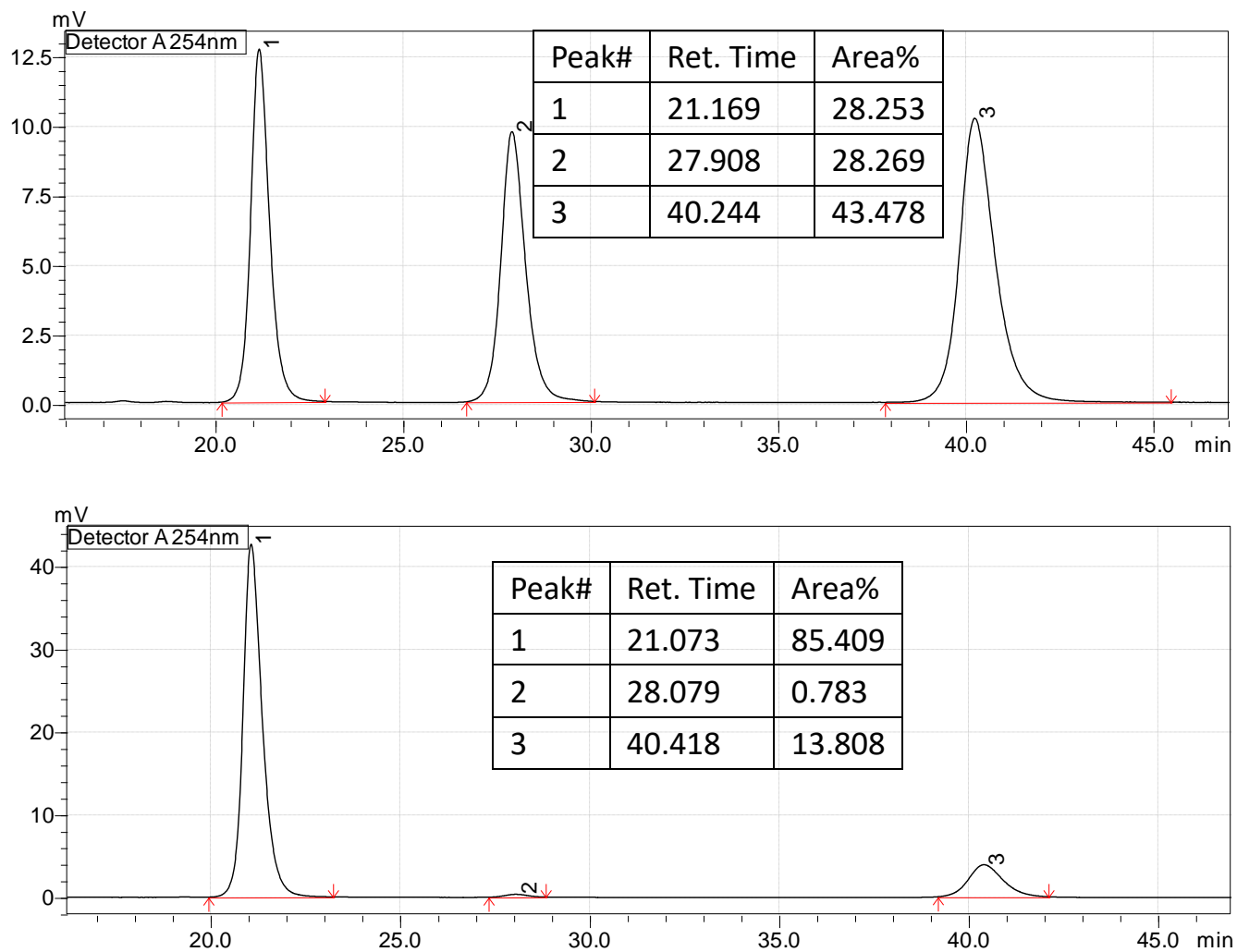


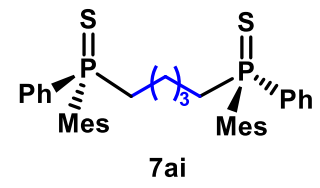

7ai: Procedure C, $50.0 \mathrm{mg}, 85 \%$ yield, white solid.

${ }^{1}$ H NMR (400 MHz, CDCl $)$ ) $7.74-7.64(\mathrm{~m}, 4 \mathrm{H}), 7.50-7.43(\mathrm{~m}, 2 \mathrm{H}), 7.42-7.34(\mathrm{~m}, 4 \mathrm{H}), 6.81(\mathrm{~d}$ $J=3.9 \mathrm{~Hz}, 4 \mathrm{H}), 2.49-2.37(\mathrm{~m}, 2 \mathrm{H}), 2.34-2.27(\mathrm{~m}, 2 \mathrm{H}), 2.25(\mathrm{~s}, 6 \mathrm{H}), 2.23(\mathrm{~s}, 12 \mathrm{H}), 1.63-1.49(\mathrm{~m}$, $2 \mathrm{H}), 1.32-1.22(\mathrm{~m}, 2 \mathrm{H}), 0.68-0.51(\mathrm{~m}, 2 \mathrm{H})$.

${ }^{13} \mathbf{C}$ NMR(101 MHz, $\left.\mathbf{C D C l}_{3}\right) \delta 141.40(\mathrm{~d}, J=10.2 \mathrm{~Hz}), 140.78(\mathrm{~d}, J=3.0 \mathrm{~Hz}), 134.37(\mathrm{~d}, J=$ $77.4 \mathrm{~Hz}), 131.24(\mathrm{~d}, J=11.2 \mathrm{~Hz}), 130.77(\mathrm{~d}, J=2.9 \mathrm{~Hz}), 130.52(\mathrm{~d}, J=10.2 \mathrm{~Hz}), 128.77$ (d, $J=$ $82.1 \mathrm{~Hz}), 128.33(\mathrm{~d}, J=12.1 \mathrm{~Hz}), 38.11(\mathrm{~d}, J=53.2 \mathrm{~Hz}), 31.86(\mathrm{t}, J=17.5 \mathrm{~Hz}), 23.95(\mathrm{~d}, J=5.3$ $\mathrm{Hz}), 22.27,20.77$.

${ }^{31}$ P NMR (162 MHz, $\left.\mathbf{C D C l}_{3}\right) \delta 43.38$.

HRMS (ESI) $\mathbf{m} / \mathbf{z}[\mathbf{M}+\mathbf{H}]^{+}$: calcd. 589.2276, found. 589.2279.

IR (film): $v_{\max }\left(\mathrm{cm}^{-1}\right)$ 3050, 2930, 2864, 1603, 1557, 1455, 1436, 1398, 1099, 1027, 850, 749, 693, 647, 595.

Optical rotation: $[\alpha]_{\mathrm{D}}{ }^{25}=-103.82\left(c=1.100, \mathrm{CHCl}_{3}, 6.4 / 1 \mathrm{dr},>99 \%\right.$ ee $)$.

HPLC: DAICEL CHIRALPAK IC, hexane $/ i-\mathrm{PrOH}=4 / 1$, flow rate: $1.0 \mathrm{~mL} / \mathrm{min}, \lambda=220 \mathrm{~nm}$, $\mathrm{t}_{\mathrm{R}}($ major $)=29.6 \mathrm{~min}, \mathrm{t}_{\mathrm{R}}($ minor $)=38.7 \mathrm{~min}, 6.4 / 1 \mathrm{dr},>99 \%$ ee .
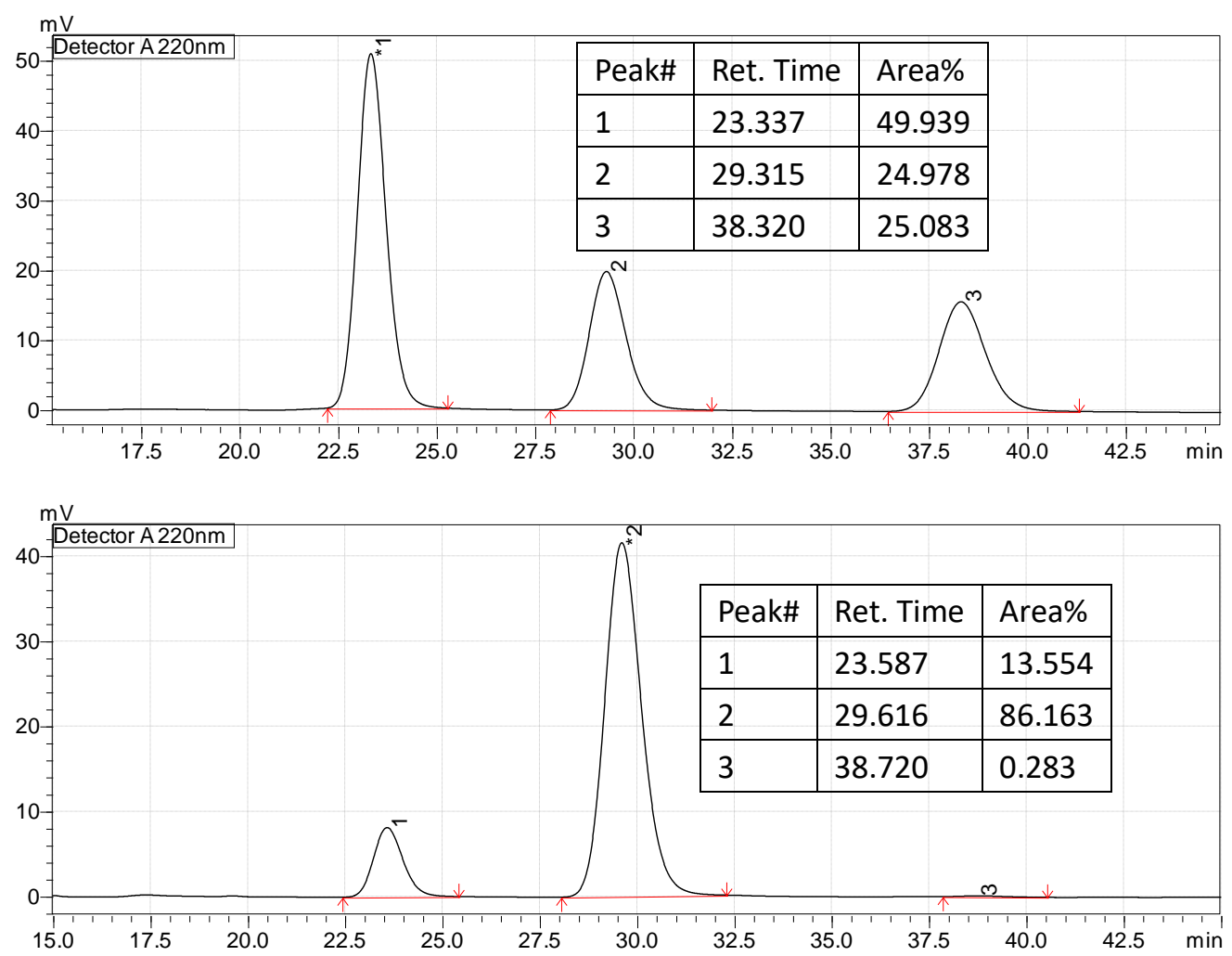


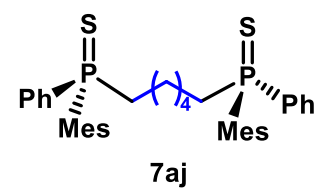

7aj: Procedure C, $49.9 \mathrm{mg}, 83 \%$ yield, light yellow solid.

${ }^{1}$ H NMR (400 MHz, CDCl $)_{3} \delta$ 7.76-7.66 (m, 4H), 7.48-7.37 (m, 6H), $6.82(\mathrm{~d}, J=3.9 \mathrm{~Hz}, 4 \mathrm{H})$, $2.52-2.33(\mathrm{~m}, 4 \mathrm{H}), 2.28-2.23(\mathrm{~m}, 18 \mathrm{H}), 1.61-1.48(\mathrm{~m}, 2 \mathrm{H}), 1.23-1.03(\mathrm{~m}, 4 \mathrm{H}), 0.74-0.54(\mathrm{~m}$, $2 \mathrm{H})$.

${ }^{13} \mathbf{C}$ NMR(101 MHz, $\left.\mathbf{C D C l}_{3}\right) \delta 141.39(\mathrm{~d}, J=10.2 \mathrm{~Hz}), 140.71(\mathrm{~d}, J=2.9 \mathrm{~Hz}), 134.47(\mathrm{~d}, J=$ $77.4 \mathrm{~Hz}), 131.21(\mathrm{~d}, J=11.1 \mathrm{~Hz}), 130.65(\mathrm{~d}, J=3.0 \mathrm{~Hz}), 130.52(\mathrm{~d}, J=10.1 \mathrm{~Hz}), 128.79$ (d, $J=$ $81.9 \mathrm{~Hz}), 128.28(\mathrm{~d}, J=12.0 \mathrm{~Hz}), 38.35(\mathrm{~d}, J=53.1 \mathrm{~Hz}), 30.07(\mathrm{~d}, J=17.3 \mathrm{~Hz}), 23.93(\mathrm{~d}, J=5.1$ $\mathrm{Hz}), 22.51(\mathrm{~d}, J=2.9 \mathrm{~Hz}), 20.75(\mathrm{~d}, J=1.5 \mathrm{~Hz})$.

${ }^{31}$ P NMR (162 MHz, $\left.\mathbf{C D C l}_{3}\right) \delta 43.55$.

HRMS (ESI) $\mathbf{m} / \mathbf{z}$ [M+H] $]^{+}$: calcd. 603.2432, found. 603.2436 .

IR (film): $v_{\max }\left(\mathrm{cm}^{-1}\right)$ 3050, 3020, 2926, 2853, 1603, 1455, 1436, 1398, 1265, 1100, 1027, 851, 749, 693, 647, 594.

Optical rotation: $[\alpha]_{\mathrm{D}}{ }^{25}=-66.71\left(c=1.000, \mathrm{CHCl}_{3}, 7.6 / 1 \mathrm{dr},>99 \%\right.$ ee $)$.

HPLC: DAICEL CHIRALPAK IC, hexane $/ i-\mathrm{PrOH}=4 / 1$, flow rate: $1.0 \mathrm{~mL} / \mathrm{min}, \lambda=220 \mathrm{~nm}$, $\mathrm{t}_{\mathrm{R}}($ major $)=44.2 \mathrm{~min}, \mathrm{t}_{\mathrm{R}}($ minor $)=57.1 \mathrm{~min}, 7.6 / 1 \mathrm{dr},>99 \%$ ee.
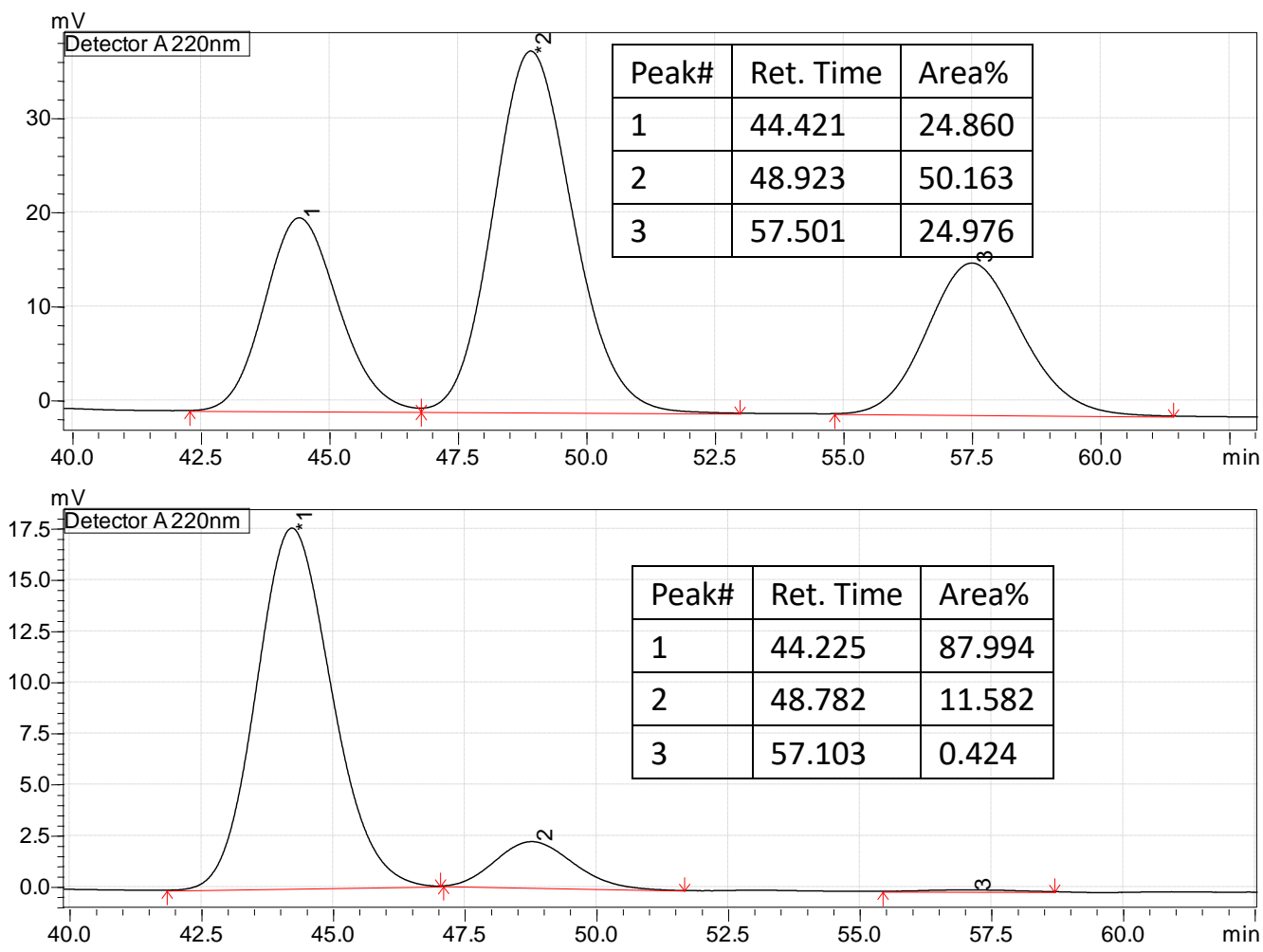


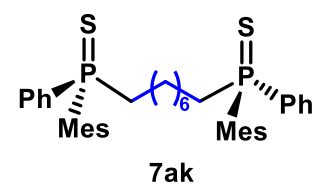

7ak: Procedure C, $61.7 \mathrm{mg}$, 98\% yield, brown yellow liquid.

${ }^{1}$ H NMR (400 MHz, CDCl $\left.)_{3}\right) \delta$ 7.79-7.67 (m, 4H), 7.50-7.35 (m, 6H), $6.83(\mathrm{~d}, J=3.9 \mathrm{~Hz}, 4 \mathrm{H})$, $2.56-2.34(\mathrm{~m}, 4 \mathrm{H}), 2.28(\mathrm{~s}, 12 \mathrm{H}), 2.26(\mathrm{~s}, 6 \mathrm{H}), 1.67-1.54(\mathrm{~m}, 2 \mathrm{H}), 1.24-1.07$ (m, 4H), 1.06-0.95 (m, 4H), 0.78-0.58 (m, 2H).

${ }^{13} \mathbf{C}$ NMR(101 MHz, $\left.\mathbf{C D C l}_{3}\right) \delta 141.43(\mathrm{~d}, J=10.0 \mathrm{~Hz}), 140.69(\mathrm{~d}, J=2.8 \mathrm{~Hz}), 134.62(\mathrm{~d}, J=$ $77.2 \mathrm{~Hz}), 131.23(\mathrm{~d}, J=11.1 \mathrm{~Hz}), 130.67(\mathrm{~d}, J=2.9 \mathrm{~Hz}), 130.58(\mathrm{~d}, J=10.1 \mathrm{~Hz}), 128.87$ (d, $J=$ $81.8 \mathrm{~Hz}), 128.27(\mathrm{~d}, J=12.1 \mathrm{~Hz}), 38.51(\mathrm{~d}, J=53.0 \mathrm{~Hz}), 30.43(\mathrm{~d}, J=17.2 \mathrm{~Hz}), 28.54,23.94(\mathrm{~d}, J$ $=5.0 \mathrm{~Hz}), 22.60(\mathrm{~d}, J=3.1 \mathrm{~Hz}), 20.76(\mathrm{~d}, J=1.5 \mathrm{~Hz})$.

${ }^{31}$ P NMR (162 MHz, $\left.\mathbf{C D C l}_{3}\right) \delta 43.62$.

HRMS (ESI) $\mathbf{m} / \mathbf{z}$ [M+H] $]^{+}$: calcd. 631.2745 , found. 631.2746 .

IR (film): $v_{\max }\left(\mathrm{cm}^{-1}\right)$ 3058, 3025, 2925, 2853, 1603, 1455, 1436, 1398, 1377, 1100, 1027, 850, 748, 693, 647, 594.

Optical rotation: $[\alpha]_{\mathrm{D}}{ }^{25}=-61.46\left(c=1.000, \mathrm{CHCl}_{3}, 9.7 / 1 \mathrm{dr},>99 \%\right.$ ee $)$.

HPLC: DAICEL CHIRALPAK OD-H, hexane $/ i-\mathrm{PrOH}=19 / 1$, flow rate: $1.0 \mathrm{~mL} / \mathrm{min}, \lambda=220 \mathrm{~nm}$, $\mathrm{t}_{\mathrm{R}}($ major $)=14.3 \mathrm{~min}, \mathrm{t}_{\mathrm{R}}($ minor $)=25.4 \mathrm{~min}, 9.7 / 1 \mathrm{dr},>99 \%$ ee .
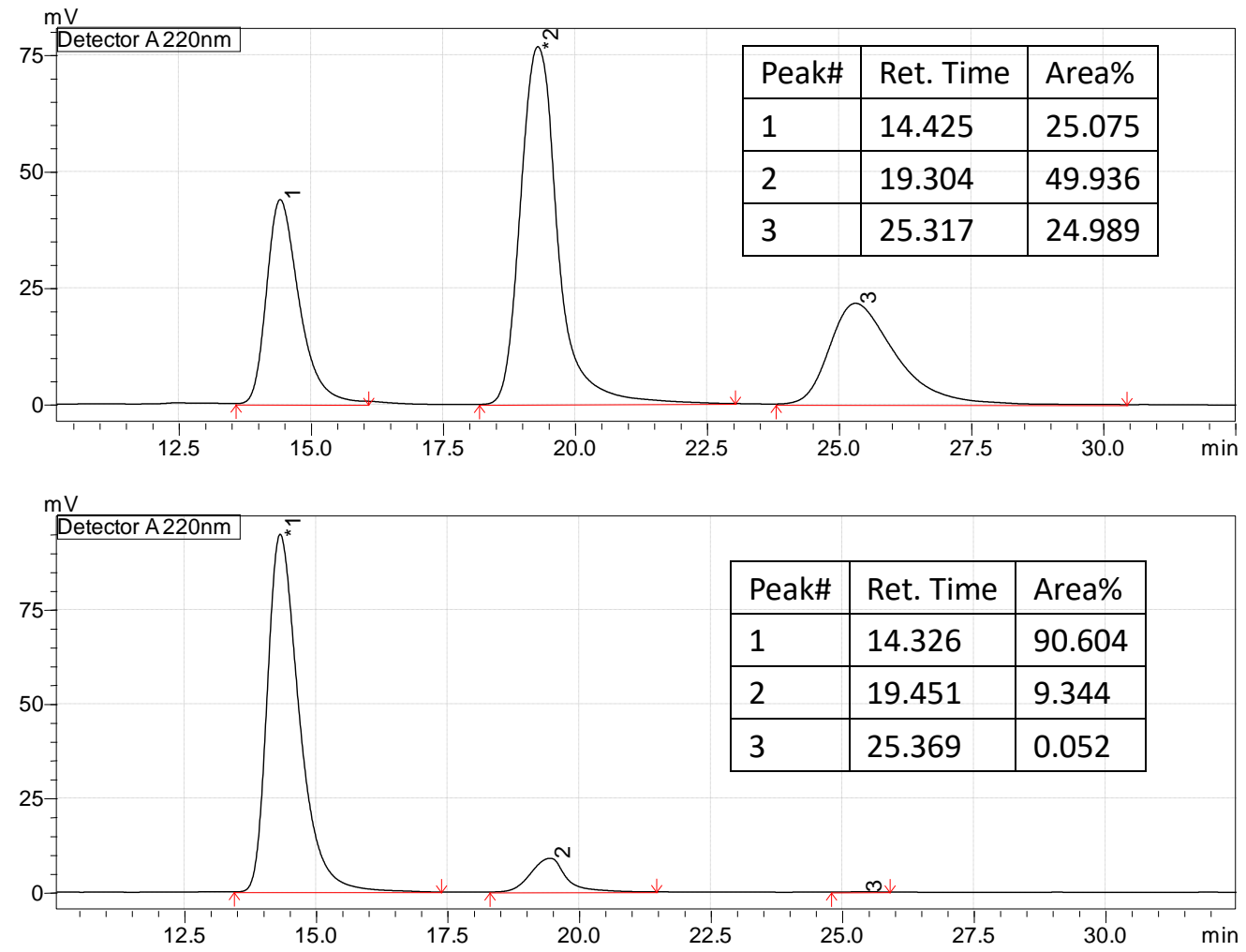


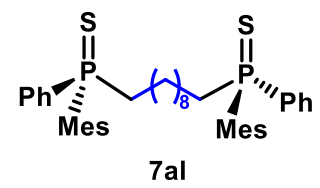

7al: Procedure C, $55.9 \mathrm{mg}, 85 \%$ yield, light yellow liquid.

${ }^{1}$ H NMR (400 MHz, CDCl $)_{3} \delta$ 7.81-7.68 (m, 4H), 7.49-7.37 (m, 6H), $6.83(\mathrm{~d}, J=3.9 \mathrm{~Hz}, 4 \mathrm{H})$, $2.60-2.46(\mathrm{~m}, 2 \mathrm{H}), 2.46-2.36(\mathrm{~m}, 2 \mathrm{H}), 2.29(\mathrm{~s}, 12 \mathrm{H}), 2.26(\mathrm{~s}, 6 \mathrm{H}), 1.70-1.57(\mathrm{~m}, 2 \mathrm{H}), 1.26-1.13$ (m, 4H), 1.11-1.00 (m, 8H), 0.81-0.66 (m, 2H).

${ }^{13} \mathbf{C}$ NMR(101 MHz, $\left.\mathbf{C D C l}_{3}\right) \delta 141.52(\mathrm{~d}, J=10.0 \mathrm{~Hz}), 140.75(\mathrm{~d}, J=2.7 \mathrm{~Hz}), 134.73(\mathrm{~d}, J=$ $77.3 \mathrm{~Hz}), 131.30$ (d, $J=11.0 \mathrm{~Hz}), 130.73$ (d, $J=2.9 \mathrm{~Hz}), 130.67$ (d, $J=10.0 \mathrm{~Hz}), 128.97$ (d, $J=$ $81.7 \mathrm{~Hz}), 128.34(\mathrm{~d}, J=12.1 \mathrm{~Hz}), 38.68(\mathrm{~d}, J=52.9 \mathrm{~Hz}), 30.68(\mathrm{~d}, J=17.2 \mathrm{~Hz}), 29.08,28.92$, $24.01(\mathrm{~d}, J=5.2 \mathrm{~Hz}), 22.79(\mathrm{~d}, J=3.2 \mathrm{~Hz}), 20.83(\mathrm{~d}, J=1.5 \mathrm{~Hz})$.

${ }^{31}$ P NMR (162 MHz, $\left.\mathbf{C D C l}_{3}\right) \delta 43.65$.

HRMS (ESI) $\mathbf{m} / \mathbf{z}[\mathbf{M}+\mathbf{H}]^{+}$: calcd. 659.3058 , found. 659.3058 .

IR (film): $v_{\max }\left(\mathrm{cm}^{-1}\right)$ 3048, 2925, 2852, 1604, 1455, 1436, 1403, 1263, 1100, 1027, 850, 749, 693, 647, 594.

Optical rotation: $[\alpha]_{\mathrm{D}}{ }^{25}=-65.45\left(c=1.000, \mathrm{CHCl}_{3}, 9.3 / 1 \mathrm{dr},>99 \%\right.$ ee $)$.

HPLC: DAICEL CHIRALPAK IA, hexane $/ i-\mathrm{PrOH}=9 / 1$, flow rate: $1.0 \mathrm{~mL} / \mathrm{min}, \lambda=220 \mathrm{~nm}$, $\mathrm{t}_{\mathrm{R}}($ major $)=14.3 \mathrm{~min}, \mathrm{t}_{\mathrm{R}}($ minor $)=21.6 \mathrm{~min}, 9.3 / 1 \mathrm{dr},>99 \%$ ee .
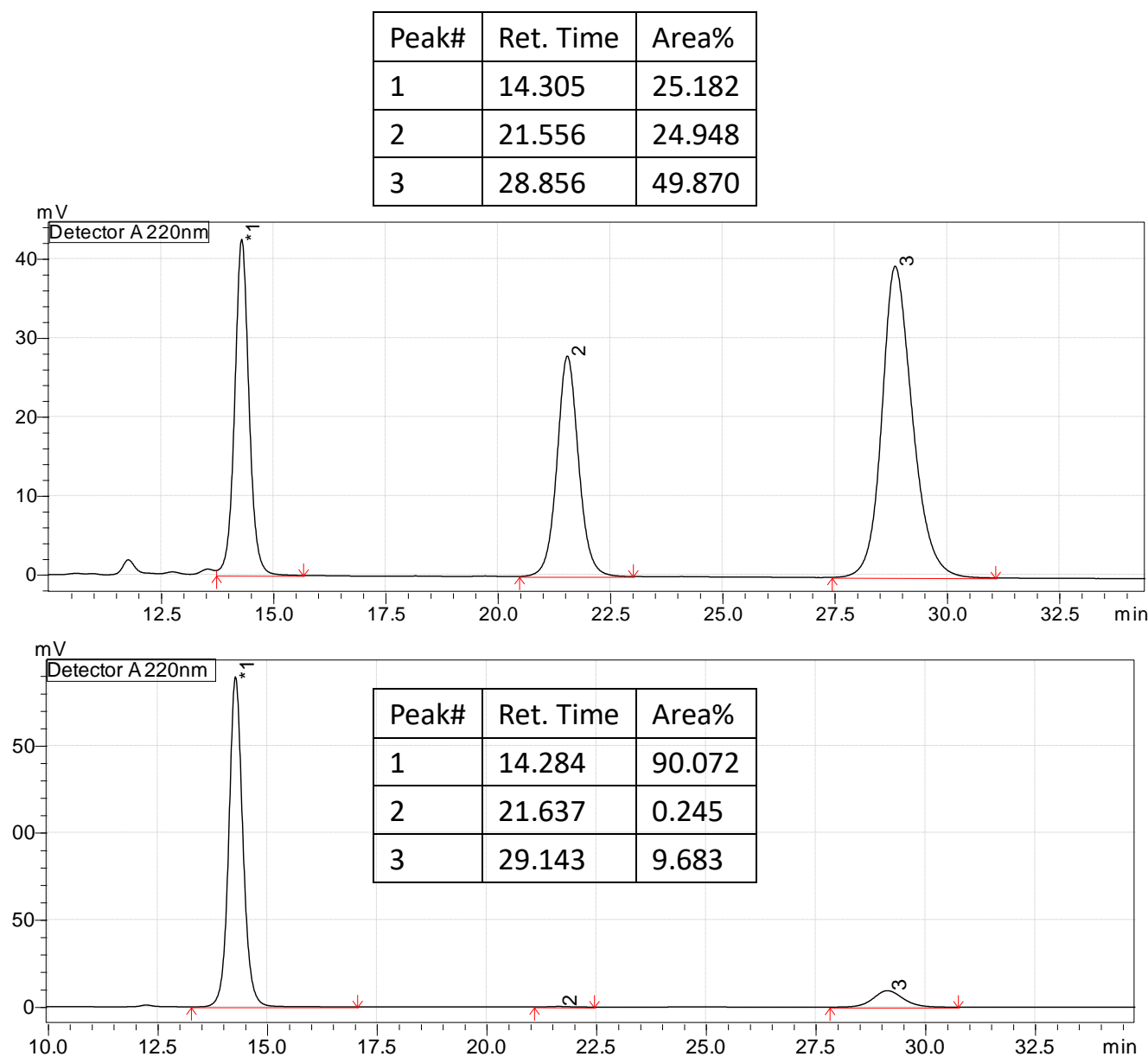


\section{0.5 mmol-Scale Reaction}

A dried $25 \mathrm{~mL}$ Schlenk tube equipped with a magnetic stirring bar was charged with $\left[\mathrm{Cu}\left(\mathrm{CH}_{3} \mathrm{CN}\right)_{4}\right] \mathrm{PF}_{6}\left(18.6 \mathrm{mg}, 0.05 \mathrm{mmol}, 0.1\right.$ equiv) and $\left(R, R_{\mathrm{P}}\right)$-TANIAPHOS (34.4 mg, 0.05 mmol, 0.1 equiv) in a glove box under Ar atmosphere. Anhydrous DME $(5.0 \mathrm{~mL})$ was added via a syringe. The mixture was stirred at room temperature for 15 minutes to give a yellow catalyst solution. Then $1 \mathrm{a}$ (1.0 mmol, 2.0 equiv) and $\mathrm{Cs}_{2} \mathrm{CO}_{3}(977.0 \mathrm{mg}, 3.0 \mathrm{mmol}, 6.0$ equiv) were added sequentially and the Schlenk tube was taken out of the glove box. After the mixture was cooled to $0{ }^{\circ} \mathrm{C}, 6 \mathbf{6 d}(0.5 \mathrm{mmol}, 1.0$ equiv) and Barton's Base (200 $\mu \mathrm{L}$ ( $0.5 \mathrm{M}$ in DME), $0.1 \mathrm{mmol}, 0.2$ equiv) was added sequentially. The resulting reaction mixture was stirred at $0{ }^{\circ} \mathrm{C}$ for 48 hours. Then the reaction mixture was quenched by acetic acid $(200 \mu \mathrm{L})$ and $\mathrm{S}_{8}$ and the reaction mixture was stirred for additional 30 minutes at room temperature. After solvent was removed under reduced pressure, the residue was purified by silica gel column chromatography (petroleum ether/ethyl acetate $=$ 20/1-10/1) to give the desired product 7ad (253.1 mg, white solid, $82 \%$ yield, $99 \%$ ee, 5.6/1 dr).

A dried $25 \mathrm{~mL}$ Schlenk tube equipped with a magnetic stirring bar was charged with $\left[\mathrm{Cu}\left(\mathrm{CH}_{3} \mathrm{CN}\right)_{4}\right] \mathrm{PF}_{6}\left(18.6 \mathrm{mg}, 0.05 \mathrm{mmol}, 0.1\right.$ equiv) and $\left(R, R_{\mathrm{P}}\right)$-TANIAPHOS (34.4 mg, 0.05 mmol, 0.1 equiv) in a glove box under Ar atmosphere. Anhydrous DME $(5.0 \mathrm{~mL})$ was added via a syringe. The mixture was stirred at room temperature for 15 minutes to give a yellow catalyst solution. Then $1 \mathrm{a}$ (1.0 mmol, 2.0 equiv) and $\mathrm{Cs}_{2} \mathrm{CO}_{3}(977.0 \mathrm{mg}, 3.0 \mathrm{mmol}, 6.0$ equiv) were added sequentially and the Schlenk tube was taken out of the glove box. After the mixture was cooled to $0{ }^{\circ} \mathrm{C}, \boldsymbol{6} \mathbf{h}(0.5 \mathrm{mmol}, 1.0$ equiv) and Barton's Base ( $200 \mu \mathrm{L}$ ( $0.5 \mathrm{M}$ in DME), $0.1 \mathrm{mmol}, 0.2$ equiv) was added sequentially. The resulting reaction mixture was stirred at $0{ }^{\circ} \mathrm{C}$ for 48 hours. Then the reaction mixture was quenched by acetic acid $(200 \mu \mathrm{L})$ and $\mathrm{S}_{8}$ and the reaction mixture was stirred for additional 30 minutes at room temperature. After solvent was removed under reduced pressure, the residue was purified by silica gel column chromatography (petroleum ether/ethyl acetate $=$ 20/1-10/1) to give the desired product 7 ah $(238.1 \mathrm{mg}$, white solid, $85 \%$ yield, $98 \%$ ee, $6.2 / 1 \mathrm{dr}$ ). 


\section{Preparation of Copper(I)-Complexes 16, 17, and 18}

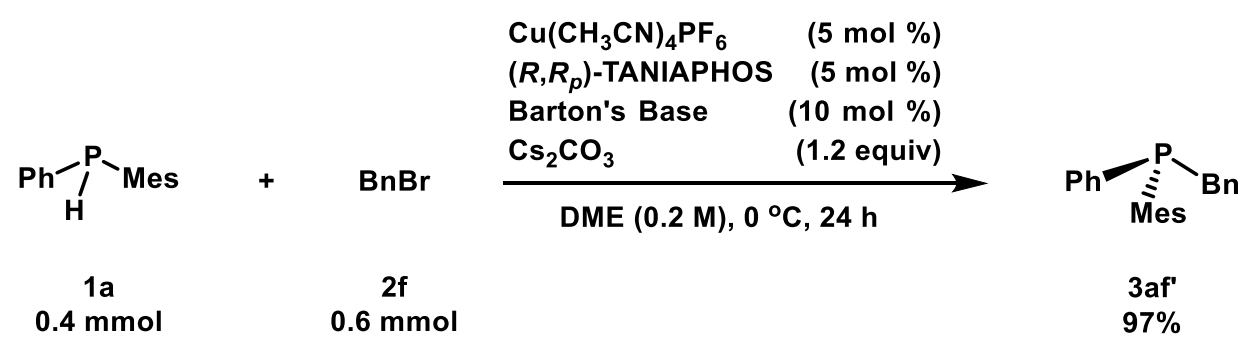

Preparation of 3af': A dried $25 \mathrm{~mL}$ Schlenk tube equipped with a magnetic stirring bar was charged with $\left[\mathrm{Cu}\left(\mathrm{CH}_{3} \mathrm{CN}\right)_{4}\right] \mathrm{PF}_{6}\left(7.4 \mathrm{mg}, 0.02 \mathrm{mmol}, 0.05\right.$ equiv) and $\left(R, R_{P}\right)$-TANIAPHOS (13.8 $\mathrm{mg}, 0.02 \mathrm{mmol}, 0.05$ equiv) in a glove box under $\mathrm{Ar}$ atmosphere. Anhydrous DME (2.0 mL) was added via a syringe. The mixture was stirred at room temperature for 15 minutes to give a yellow catalyst solution. Then 1a ( $92 \mu \mathrm{L}, 0.4 \mathrm{mmol}, 1.0$ equiv) and $\mathrm{Cs}_{2} \mathrm{CO}_{3}(156.0 \mathrm{mg}, 0.48 \mathrm{mmol}, 1.2$ equiv) were added sequentially and the Schlenk was taken out of the glove box. After the mixture was cooled to $0{ }^{\circ} \mathrm{C}, \mathbf{2 f}(48 \mu \mathrm{L}, 0.6 \mathrm{mmol}, 1.5$ equiv) and Barton's Base ( $80 \mu \mathrm{L}$ (0.5 M in DME), $0.04 \mathrm{mmol}, 0.1$ equiv) was added sequentially. The resulting reaction mixture was stirred at $0{ }^{\circ} \mathrm{C}$ for 24 hours. Then the reaction mixture was subjected to silica gel column chromatography under $\mathrm{N}_{2}$ atmosphere with degassed eluent (petroleum ether/ethyl acetate $\left.=20 / 1\right)$ to give 3af' $(123.5 \mathrm{mg}$ ) in $97 \%$ yield, which was stocked in a glove box.

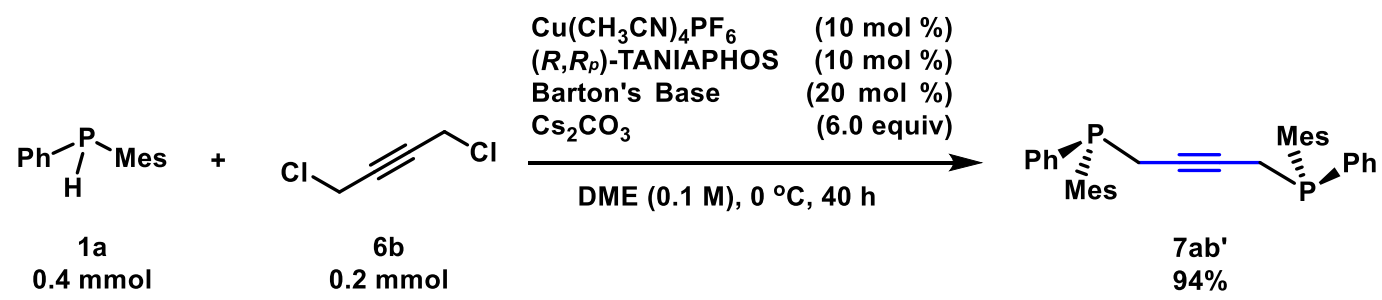

Preparation of 7ab': A dried $25 \mathrm{~mL}$ Schlenk tube equipped with a magnetic stirring bar was charged with $\left[\mathrm{Cu}\left(\mathrm{CH}_{3} \mathrm{CN}\right)_{4}\right] \mathrm{PF}_{6}\left(7.4 \mathrm{mg}, 0.02 \mathrm{mmol}, 0.1\right.$ equiv) and $\left(R, R_{P}\right)$-TANIAPHOS (13.8 $\mathrm{mg}, 0.02 \mathrm{mmol}, 0.1$ equiv) in a glove box under Ar atmosphere. Anhydrous DME (2.0 mL) was added via a syringe. The mixture was stirred at room temperature for 15 minutes to give a yellow catalyst solution. Then 1a ( $92 \mu \mathrm{L}, 0.4 \mathrm{mmol}, 2.0$ equiv) and $\mathrm{Cs}_{2} \mathrm{CO}_{3}(390.0 \mathrm{mg}, 1.2 \mathrm{mmol}, 6.0$ equiv) were added sequentially and the Schlenk was taken out of the glove box. After the mixture was cooled to $0{ }^{\circ} \mathrm{C}, \mathbf{6 b}(20 \mu \mathrm{L}, 0.2 \mathrm{mmol}, 1.0$ equiv) and Barton's Base ( $80 \mu \mathrm{L}$ (0.5 M in DME), $0.04 \mathrm{mmol}, 0.2$ equiv) was added sequentially. The resulting reaction mixture was stirred at $0{ }^{\circ} \mathrm{C}$ for 40 hours. Then the reaction mixture was subjected to silica gel column chromatography under $\mathrm{N}_{2}$ atmosphere with degassed eluent (petroleum ether/ethyl acetate $\left.=20 / 1\right)$ to give $\mathbf{7} \mathbf{a b}^{\prime}(95.2 \mathrm{mg}$ ) in $94 \%$ yield, which was stocked in a glove box.

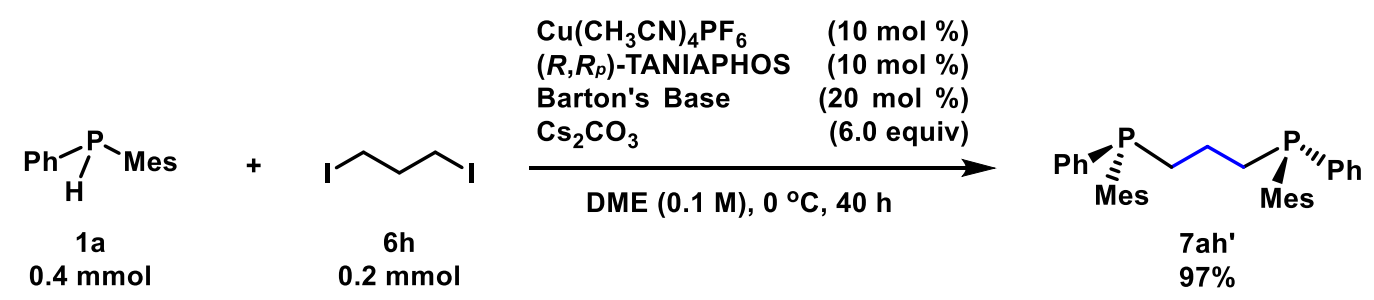

Preparation of 7ah': A dried $25 \mathrm{~mL}$ Schlenk tube equipped with a magnetic stirring bar was 
charged with $\left[\mathrm{Cu}\left(\mathrm{CH}_{3} \mathrm{CN}\right)_{4}\right] \mathrm{PF}_{6}\left(7.4 \mathrm{mg}, 0.02 \mathrm{mmol}, 0.1\right.$ equiv) and $\left(R, R_{P}\right)$-TANIAPHOS (13.8 $\mathrm{mg}, 0.02 \mathrm{mmol}, 0.1$ equiv) in a glove box under Ar atmosphere. Anhydrous DME (2.0 mL) was added via a syringe. The mixture was stirred at room temperature for 15 minutes to give a yellow catalyst solution. Then 1a ( $92 \mu \mathrm{L}, 0.4 \mathrm{mmol}, 2.0$ equiv) and $\mathrm{Cs}_{2} \mathrm{CO}_{3}(390.0 \mathrm{mg}, 1.2 \mathrm{mmol}, 6.0$ equiv) were added sequentially and the Schlenk was taken out of the glove box. After the mixture was cooled to $0{ }^{\circ} \mathrm{C}, \mathbf{6 h}(24 \mu \mathrm{L}, 0.2 \mathrm{mmol}, 1.0$ equiv) and Barton's Base (80 $\mu \mathrm{L}$ (0.5 M in DME), $0.04 \mathrm{mmol}, 0.2$ equiv) was added sequentially. The resulting reaction mixture was stirred at $0{ }^{\circ} \mathrm{C}$ for 40 hours. Then the reaction mixture was subjected to silica gel column chromatography under $\mathrm{N}_{2}$ atmosphere with degassed eluent (petroleum ether/ethyl acetate $\left.=20 / 1\right)$ to give $\mathbf{7} \mathbf{a h}^{\prime}(96.3 \mathrm{mg})$ in $97 \%$ yield, which was stocked in a glove box.

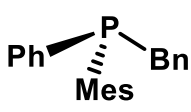

3af'

$0.38 \mathrm{mmol}$

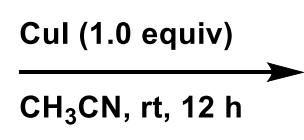

$\mathrm{CH}_{3} \mathrm{CN}, \mathrm{rt}, 12 \mathrm{~h}$

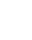

Preparation of 16: A dried $25 \mathrm{~mL}$ round-bottom flask equipped with a magnetic stirring bar was charged with $\mathrm{CuI}$ (72.0 mg, $0.38 \mathrm{mmol}, 1.0$ equiv), chiral monophosphine 3af' (120.4 mg, 0.38 mmol, 1.0 equiv), and anhydrous $\mathrm{CH}_{3} \mathrm{CN}(5 \mathrm{~mL})$ in a glove box under $\mathrm{Ar}$ atmosphere. The resulting mixture was stirred at room temperature for $12 \mathrm{~h}$. Then, the mixture was filtered to give a clear solution and ${ }^{n}$ pentane $(5 \mathrm{~mL})$ was added. The resulting solution was allowed to evaporate at room temperature to grow crystals of $\mathbf{1 6}(170.6 \mathrm{mg})$ in $89 \%$ yield. Crystals of $\mathbf{1 6}$ were submitted to single crystal X-ray diffraction for the determination of the exact structure. CCDC 2025208 contains the supplementary crystallographic date of $\mathbf{1 6}$.

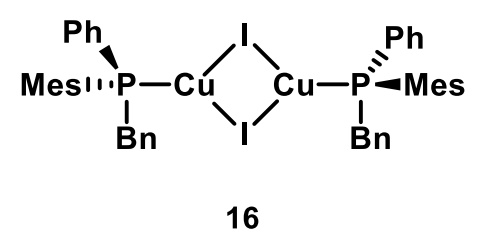

${ }^{1} \mathbf{H}$ NMR (500 MHz, $\left.\mathbf{C D}_{2} \mathbf{C l}_{2}\right) \delta$ 7.54-7.30 (m, 10H), 7.25-7.01 (m, 10H), 6.92-6.73 (m, 4H), $3.97-3.83(\mathrm{~m}, 2 \mathrm{H}), 3.81-3.67(\mathrm{~m}, 2 \mathrm{H}), 2.26(\mathrm{~s}, 6 \mathrm{H}), 2.11(\mathrm{~s}, 12 \mathrm{H})$.

${ }^{13}$ C NMR (126 MHz, $\left.\mathbf{C D}_{2} \mathbf{C l}_{2}\right) \delta 144.31(\mathrm{~d}, J=10.4 \mathrm{~Hz}), 140.89,134.80(\mathrm{~d}, J=29.3 \mathrm{~Hz}), 134.30$, $130.70(\mathrm{~d}, J=11.2 \mathrm{~Hz}), 130.37(\mathrm{~d}, J=6.8 \mathrm{~Hz}), 130.22(\mathrm{~d}, J=5.2 \mathrm{~Hz}), 128.63,128.56,128.01(\mathrm{~d}$, $J=2.8 \mathrm{~Hz}), 126.45(\mathrm{~d}, J=3.5 \mathrm{~Hz}), 123.45(\mathrm{~d}, J=27.8 \mathrm{~Hz}), 32.54(\mathrm{~d}, J=14.7 \mathrm{~Hz}), 23.87(\mathrm{~d}, J=$ $11.5 \mathrm{~Hz}), 20.74$.

${ }^{31}$ P NMR (162 MHz, $\left.\mathbf{C D}_{2} \mathrm{Cl}_{2}\right) \delta-19.58$.

IR (film): $v_{\max }\left(\mathrm{cm}^{-1}\right)$ 3028, 2920, 1602, 1495, 1453, 1435, 1065, 1028, 846, 739, 698, 611. 


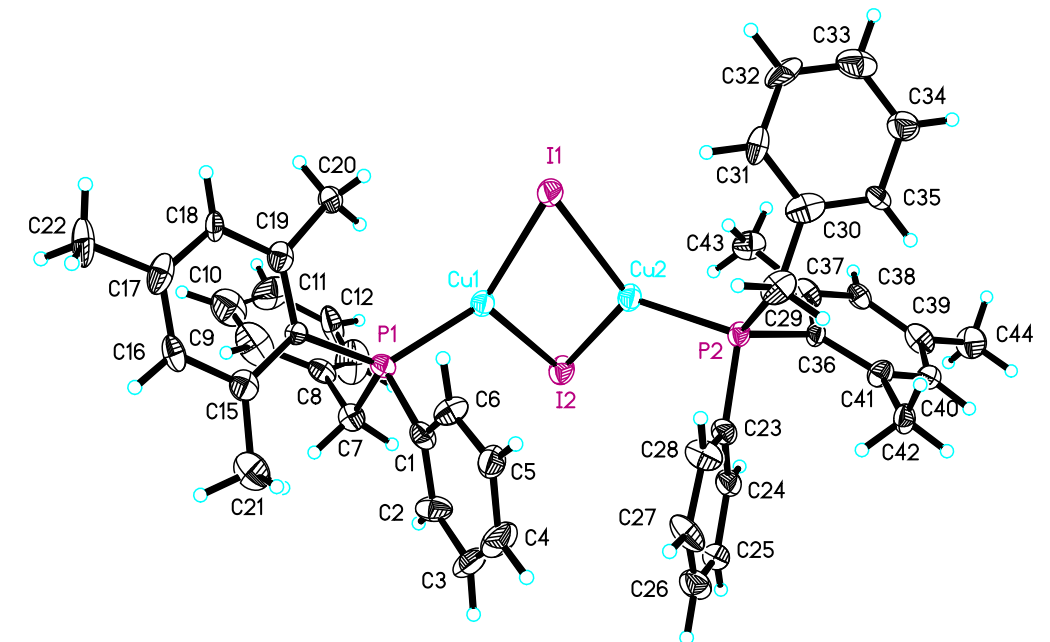

Table 1. Crystal data and structure refinement for CCDC 2025208.

Identification code

Empirical formula

Formula weight

Temperature

Wavelength

Crystal system

Space group

Unit cell dimensions

Volume

$\mathrm{Z}$

Density (calculated)

Absorption coefficient

$\mathrm{F}(000)$

Crystal size

Theta range for data collection

Index ranges

Reflections collected

Independent reflections

Completeness to theta $=25.242^{\circ}$

Absorption correction

Max. and min. transmission

Refinement method mo_d8v20474_0m

C44 H46 Cu2 I2 P2

1017.63

193(2) K

$0.71073 \AA$

Orthorhombic

P 212121

$\mathrm{a}=10.6681(6) \AA \quad \alpha=90^{\circ}$.

$\mathrm{b}=20.7498(10) \AA \quad \beta=90^{\circ}$.

$\mathrm{c}=37.6186(19) \AA \quad \gamma=90^{\circ}$.

8327.3(7) $\AA^{3}$

8

$1.623 \mathrm{Mg} / \mathrm{m}^{3}$

$2.611 \mathrm{~mm}^{-1}$

4032

$0.150 \times 0.130 \times 0.100 \mathrm{~mm}^{3}$

1.461 to $24.999^{\circ}$.

$-12<=\mathrm{h}<=12,-22<=\mathrm{k}<=24,-44<=1<=40$

38063

$14619[\mathrm{R}(\mathrm{int})=0.0639]$

$97.4 \%$

Semi-empirical from equivalents

0.7456 and 0.4428

Full-matrix least-squares on $\mathrm{F}^{2}$ 
Data / restraints / parameters

Goodness-of-fit on $\mathrm{F}^{2}$

Final R indices [I>2sigma(I)]

$\mathrm{R}$ indices (all data)

Absolute structure parameter

Extinction coefficient

Largest diff. peak and hole
14619 / 60 / 902

1.080

$\mathrm{R} 1=0.0846, \mathrm{wR} 2=0.2084$

$\mathrm{R} 1=0.1016, \mathrm{wR} 2=0.2194$

$0.089(14)$

$0.00121(16)$

3.306 and -1.543 e. $\AA^{-3}$ 


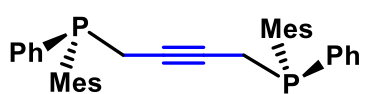

$7 a^{\prime}$

$0.18 \mathrm{mmol}$
CuCl (2.0 equiv)

Toluene, rt, $12 \mathrm{~h}$

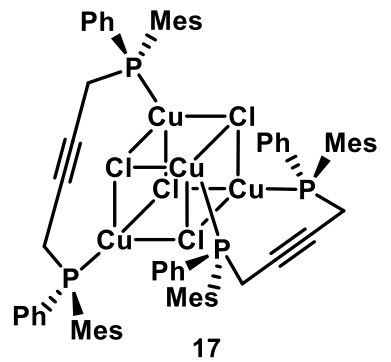

$77.4 \mathrm{mg}, 61 \%$

Preparation of 17: A dried $25 \mathrm{~mL}$ round-bottom flask equipped with a magnetic stirring bar was charged with $\mathrm{CuCl}$ (36.1 mg, $0.36 \mathrm{mmol}, 2.0$ equiv), chiral monophosphine 7ab' (93.0 mg, $0.18 \mathrm{mmol}, 1.0$ equiv), and anhydrous toluene $(5 \mathrm{~mL})$ in a glove box under Ar atmosphere. The resulting mixture was stirred at room temperature for $12 \mathrm{~h}$. Then, the mixture was filtered to give a clear solution, which was evaporated under reduced pressure to give crude complex. The crude complex was recrystallized with $\mathrm{CH}_{2} \mathrm{Cl}_{2}$ and petroleum ether to give crystals of $\mathbf{1 7}(77.4 \mathrm{mg}$ ) in $61 \%$ yield. Crystals of $\mathbf{1 7}$ were submitted to single crystal X-ray diffraction for the determination of the exact structure. CCDC 2021216 contains the supplementary crystallographic date of $\mathbf{1 7 .}$

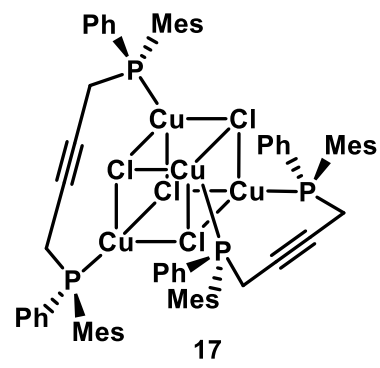

${ }^{1} \mathbf{H}$ NMR (500 MHz, $\left.\mathbf{C D}_{2} \mathbf{C l}_{2}\right) \delta$ 7.43-7.33 (m, 8H), 7.33-7.28 (m, 4H), 7.28-7.22 (m, 8H), 6.86 (s, 8H), 3.39 (d, $J=15.3 \mathrm{~Hz}, 4 \mathrm{H}), 3.15(\mathrm{~d}, J=15.9 \mathrm{~Hz}, 4 \mathrm{H}), 2.37$ (s, 24H), 2.28 (s, 12H).

${ }^{13}$ C NMR (126 MHz, $\left.\mathbf{C D}_{2} \mathbf{C l}_{2}\right) \delta 143.94(\mathrm{~d}, J=10.8 \mathrm{~Hz}), 141.00,134.52(\mathrm{~d}, J=29.3 \mathrm{~Hz}), 130.75$, $130.57(\mathrm{~d}, J=12.5 \mathrm{~Hz}), 128.92,128.61(\mathrm{~d}, J=8.0 \mathrm{~Hz}), 123.99(\mathrm{~d}, J=27.0 \mathrm{~Hz}), 82.48,24.17$, 24.07, 20.75 .

${ }^{31} \mathbf{P}$ NMR (162 MHz, $\left.\mathbf{C D}_{2} \mathrm{Cl}_{2}\right) \delta-15.47$.

IR (film): $v_{\max }\left(\mathrm{cm}^{-1}\right)$ 3050, 2962, 2919, 1603, 1555, 1435, 1266, 1097, 1026, 850, 742, 693, 613. 


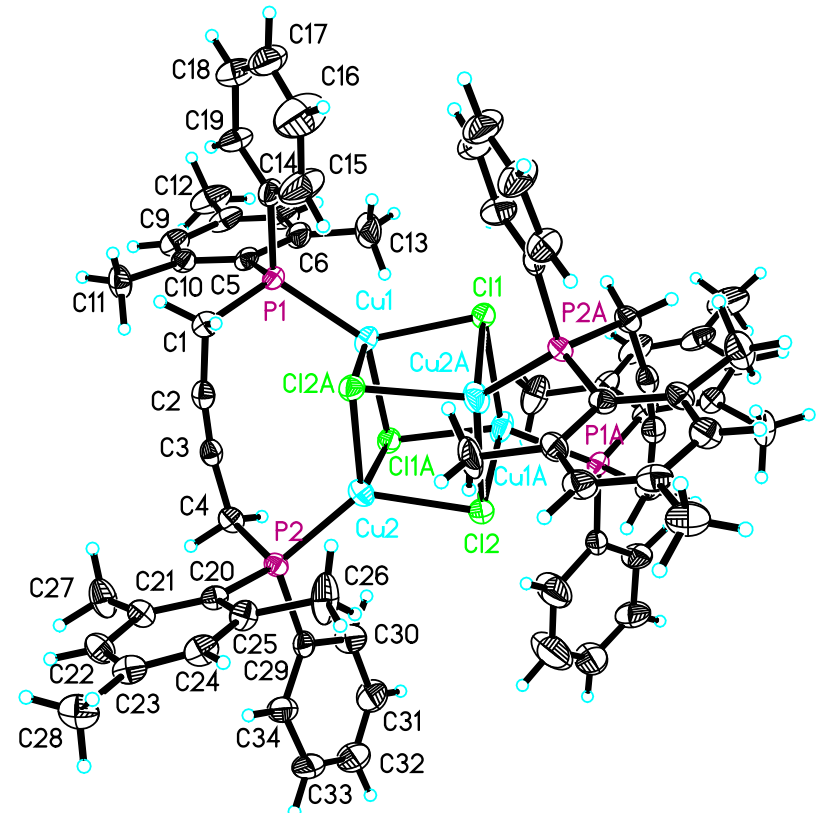

Table 1. Crystal data and structure refinement for CCDC 2021216.

Identification code

Empirical formula

Formula weight

Temperature

Wavelength

Crystal system

Space group

Unit cell dimensions

Volume

$\mathrm{Z}$

Density (calculated)

Absorption coefficient

$\mathrm{F}(000)$

Crystal size

Theta range for data collection

Index ranges

Reflections collected

Independent reflections

Completeness to theta $=25.242^{\circ}$ d8v20389

C68 $\mathrm{H} 72 \mathrm{Cl} 4 \mathrm{Cu} 4 \mathrm{P} 4$

1409.09

193(2) K

$0.71073 \AA$

Orthorhombic

P 21212

$\mathrm{a}=15.4106(5) \AA$

$\alpha=90^{\circ}$.

$\mathrm{b}=23.5689(7) \AA$

$\beta=90^{\circ}$.

$\mathrm{c}=10.5868(3) \AA$

$\gamma=90^{\circ}$.
$1.217 \mathrm{Mg} / \mathrm{m}^{3}$

$1.347 \mathrm{~mm}^{-1}$

1448

$0.170 \times 0.140 \times 0.110 \mathrm{~mm}^{3}$

2.489 to $25.995^{\circ}$.

$-18<=\mathrm{h}<=18,-25<=\mathrm{k}<=29,-11<=1<=13$

19405

$7512[\mathrm{R}($ int $)=0.0456]$

$99.5 \%$ 
Absorption correction

Max. and min. transmission

Refinement method

Data / restraints / parameters

Goodness-of-fit on $\mathrm{F}^{2}$

Final $\mathrm{R}$ indices [I $>2 \operatorname{sigma}(\mathrm{I})]$

$\mathrm{R}$ indices (all data)

Absolute structure parameter

Extinction coefficient

Largest diff. peak and hole
Semi-empirical from equivalents

0.7456 and 0.5479

Full-matrix least-squares on $\mathrm{F}^{2}$

$7512 / 18 / 368$

1.003

$\mathrm{R} 1=0.0499, \mathrm{wR} 2=0.1300$

$\mathrm{R} 1=0.0664, \mathrm{wR} 2=0.1411$

$0.035(10)$

$0.0025(8)$

0.452 and -0.368 e. $\AA^{-3}$ 

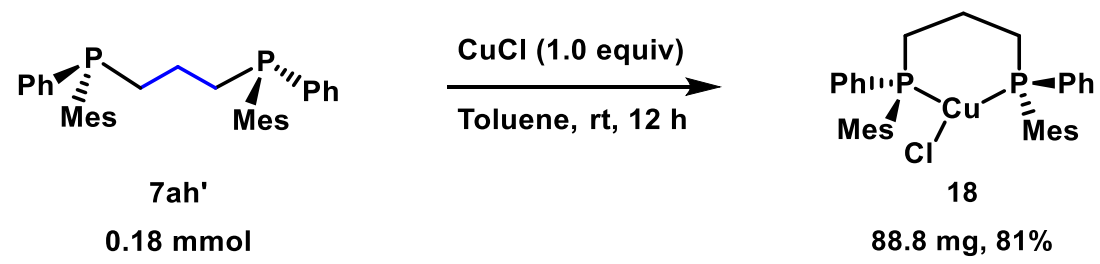

Preparation of 18: A dried $25 \mathrm{~mL}$ round-bottom flask equipped with a magnetic stirring bar was charged with $\mathrm{CuCl}$ (18.1 mg, $0.18 \mathrm{mmol}, 1.0$ equiv), chiral monophosphine 7ah' (91.0 mg, $0.18 \mathrm{mmol}, 1.0$ equiv), and anhydrous toluene $(5 \mathrm{~mL})$ in a glove box under Ar atmosphere. The resulting mixture was stirred at room temperature for $12 \mathrm{~h}$. Then, the mixture was filtered to give a clear solution, which was evaporated under reduced pressure to give crude complex. The crude complex was recrystallized with $\mathrm{CH}_{2} \mathrm{Cl}_{2}$ and petroleum ether to give crystals of $\mathbf{1 8}(88.8 \mathrm{mg})$ in $81 \%$ yield. Crystals of $\mathbf{1 8}$ were submitted to single crystal X-ray diffraction for the determination of the exact structure. CCDC 2020490 contains the supplementary crystallographic date of $\mathbf{1 8 .}$

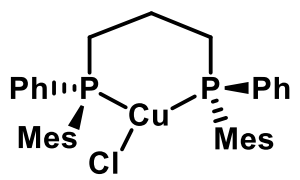

18

${ }^{1}$ H NMR (500 MHz, $\left.\mathbf{C D}_{2} \mathbf{C l}_{2}\right) \delta$ 7.49-7.38 (m, 4H), 7.38-7.28 (m, 6H), $6.83(\mathrm{~s}, 4 \mathrm{H}), 2.81-2.64$ (m, 2H), 2.49-2.37 (m, 2H), 2.26 (s, 12H), 2.24 (s, 6H), 2.16-1.97 (m, 2H).

${ }^{13}$ C NMR (126 MHz, $\left.\mathbf{C D}_{2} \mathbf{C l}_{2}\right) \delta$ 144.08, 141.02, 135.11, 130.81-130.35 (m), 130.05, 129.06128.81 (m), 128.51, 125.90-125.19 (m), 26.99, 23.93, 23.88, 23.83, 20.69.

${ }^{31}$ P NMR (162 MHz, $\left.\mathbf{C D}_{\mathbf{2}} \mathbf{C l}_{2}\right) \delta-29.28$.

IR (film): $v_{\max }\left(\mathrm{cm}^{-1}\right)$ 2957, 2923, 2854, 1603, 1434, 1412, 1270, 1027, 850, 744, 693, 619. 


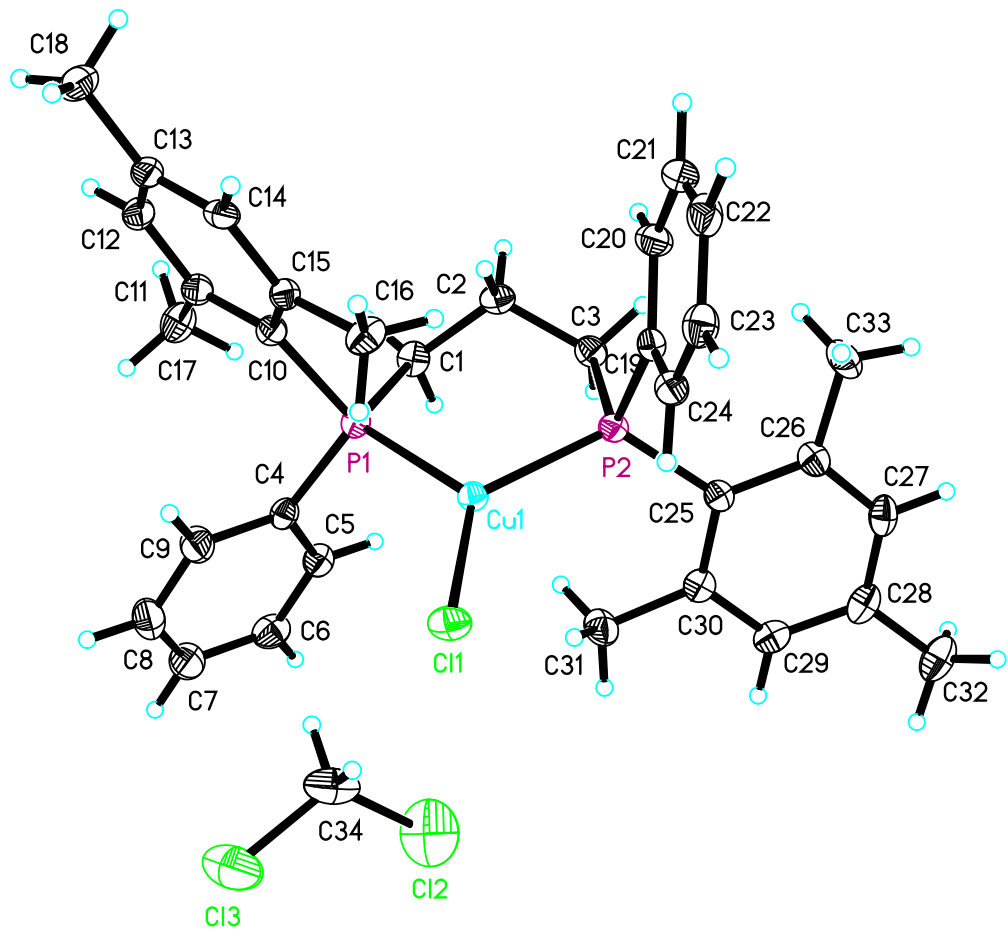

Table 1. Crystal data and structure refinement for CCDC 2020490.

Identification code

Empirical formula

Formula weight

Temperature

Wavelength

Crystal system

Space group

Unit cell dimensions

Volume

$\mathrm{Z}$

Density (calculated)

Absorption coefficient

$\mathrm{F}(000)$

Crystal size

Theta range for data collection

Index ranges

Reflections collected mo_d8v20344_0m

$\mathrm{C} 34 \mathrm{H} 40 \mathrm{Cl} 3 \mathrm{Cu} \mathrm{P} 2$

680.49

193(2) K

$0.71073 \AA$

Orthorhombic

P 212121

$\mathrm{a}=10.8838(3) \AA$

$\alpha=90^{\circ}$.

$\mathrm{b}=13.8373(3) \AA$

$\beta=90^{\circ}$.

$\mathrm{c}=22.5019(6) \AA$ $\gamma=90^{\circ}$

3388.84(15) $\AA^{3}$

4

$1.334 \mathrm{Mg} / \mathrm{m}^{3}$

$0.997 \mathrm{~mm}^{-1}$

1416

$0.170 \times 0.140 \times 0.120 \mathrm{~mm}^{3}$

2.547 to $25.998^{\circ}$.

$-13<=\mathrm{h}<=13,-17<=\mathrm{k}<=17,-19<=\mathrm{l}<=27$

17242 
Independent reflections

Completeness to theta $=25.242^{\circ}$

Absorption correction

Max. and min. transmission

Refinement method

Data / restraints / parameters

Goodness-of-fit on $\mathrm{F}^{2}$

Final R indices [I $>2 \operatorname{sigma}(\mathrm{I})]$

$\mathrm{R}$ indices (all data)

Absolute structure parameter

Extinction coefficient

Largest diff. peak and hole
$6644[\mathrm{R}(\mathrm{int})=0.0315]$

$99.7 \%$

Semi-empirical from equivalents

0.7456 and 0.5426

Full-matrix least-squares on $\mathrm{F}^{2}$

6644 / 0 / 367

1.034

$\mathrm{R} 1=0.0343, \mathrm{wR} 2=0.0746$

$\mathrm{R} 1=0.0417, \mathrm{wR} 2=0.0787$

$0.008(6)$

$\mathrm{n} / \mathrm{a}$

0.449 and -0.486 e. $\AA^{-3}$ 


\section{Determination of the Absolute Configurations of the Products}

7.1 Determination of the absolute configuration of $\mathbf{3}$

A solution of 3af in $\mathrm{CH}_{2} \mathrm{Cl}_{2}$ /petroleum ether in the round bottom flask was left at room temperature to grow single crystals. The absolute configuration of 3af was determined by X-ray analysis of its single crystals. CCDC 2020486 contains the supplementary crystallographic data of 3af. The stereochemistry in other products (3) was assigned by analogy.

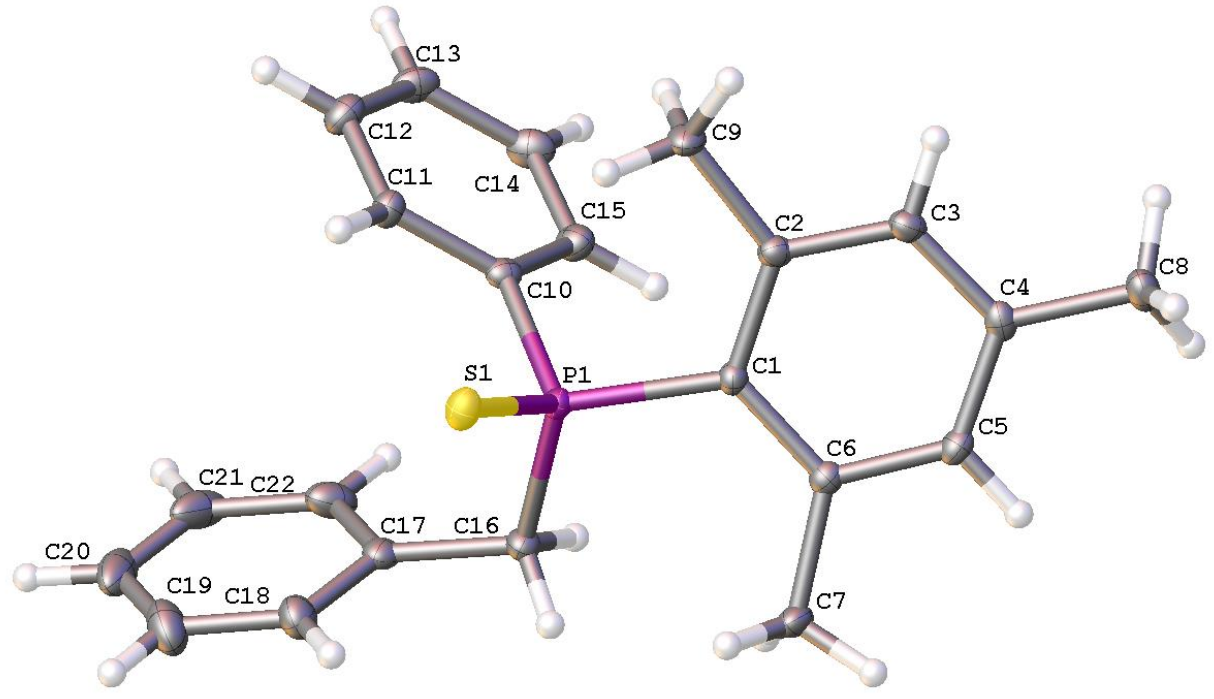

Table 1. Crystal data and structure refinement for CCDC 2020486.

Identification code

Empirical formula

Formula weight

Temperature

Wavelength

Crystal system

Space group

Unit cell dimensions

Volume

$\mathrm{Z}$

Density (calculated)

Absorption coefficient

$\mathrm{F}(000)$

Crystal size

Theta range for data collection

Index ranges mj20124_0m

C22 H23 P S

350.43

$169.99 \mathrm{~K}$

$1.34139 \AA$

Orthorhombic

$\mathrm{P} 2{ }_{1} 2_{1} 2_{1}$

$\mathrm{a}=7.6605(4) \AA$

$\alpha=90^{\circ}$.

$\mathrm{b}=15.3695(7) \AA$

$\beta=90^{\circ}$.

$\mathrm{c}=16.2627(7) \AA$

$\gamma=90^{\circ}$.

1914.74(16) $\AA^{3}$

4

$1.216 \mathrm{Mg} / \mathrm{m}^{3}$

$1.473 \mathrm{~mm}^{-1}$

744

$0.15 \times 0.12 \times 0.1 \mathrm{~mm}^{3}$

3.442 to $54.847^{\circ}$.

$-6<=\mathrm{h}<=9,-18<=\mathrm{k}<=13,-19<=\mathrm{l}<=14$ 
Reflections collected

Independent reflections

Completeness to theta $=53.594^{\circ}$

Absorption correction

Max. and min. transmission

Refinement method

Data / restraints / parameters

Goodness-of-fit on $\mathrm{F}^{2}$

Final R indices [I $>2 \operatorname{sigma}(\mathrm{I})]$

$\mathrm{R}$ indices (all data)

Absolute structure parameter

Extinction coefficient

Largest diff. peak and hole
11707

$3415[\mathrm{R}(\mathrm{int})=0.0485]$

$98.6 \%$

Semi-empirical from equivalents

0.7508 and 0.5462

Full-matrix least-squares on $\mathrm{F}^{2}$

3415 / 0 / 220

1.060

$\mathrm{R} 1=0.0324, \mathrm{wR} 2=0.0764$

$\mathrm{R} 1=0.0341, \mathrm{wR} 2=0.0781$

$0.038(12)$

$\mathrm{n} / \mathrm{a}$

0.363 and -0.288 e. $\AA^{-3}$ 
7.2 Determination of the absolute configuration of $\mathbf{5}$ and $\mathbf{7}$

A solution of 7ah in $\mathrm{CH}_{2} \mathrm{Cl}_{2}$ /petroleum ether in the round bottom flask was left at room temperature to grow single crystals. The absolute configuration of $\mathbf{7 a h}$ was determined by X-ray analysis of its single crystals. CCDC 2020488 contains the supplementary crystallographic data of 7ah. Analogically, the absolute configurations of $\mathbf{5}$ and 7ah-7al were assigned tentatively. The absolute configurations of 7aa-7ag were assigned by their analogy to the exact structure of $\mathbf{3 a f}$.

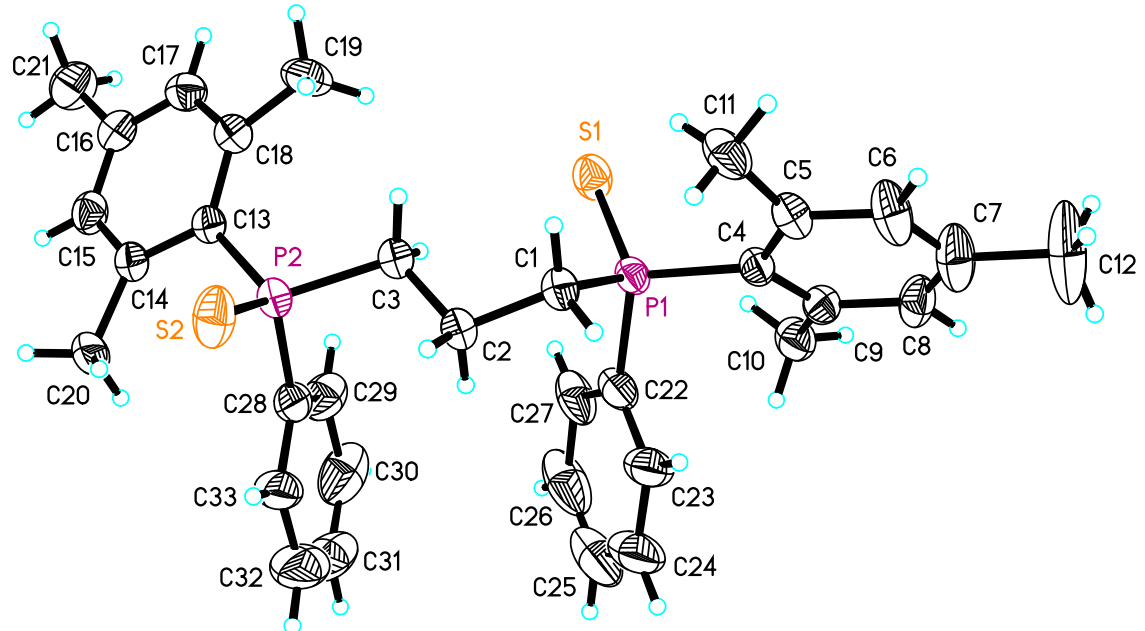

Table 1. Crystal data and structure refinement for CCDC 2020488.

Identification code

Empirical formula

Formula weight

Temperature

Wavelength

Crystal system

Space group

Unit cell dimensions

Volume

Z

Density (calculated)

Absorption coefficient

$\mathrm{F}(000)$

Crystal size

Theta range for data collection

Index ranges

Reflections collected mo_d8v20302 0m

C33 H38 P2 S2

560.69

293(2) K

$0.71073 \AA$

Tetragonal

P 43

$\mathrm{a}=18.7625(5) \AA$

$\alpha=90^{\circ}$.

$\mathrm{b}=18.7625(5) \AA$

$\beta=90^{\circ}$.

$\mathrm{c}=8.8596(3) \AA$

$\gamma=90^{\circ}$

3118.9(2) $\AA^{3}$

4

$1.194 \mathrm{Mg} / \mathrm{m}^{3}$

$0.293 \mathrm{~mm}^{-1}$

1192

$0.180 \times 0.150 \times 0.120 \mathrm{~mm}^{3}$

2.542 to $25.968^{\circ}$.

$-23<=\mathrm{h}<=20,-23<=\mathrm{k}<=15,-10<=\mathrm{l}<=10$

15782 
Independent reflections

Completeness to theta $=25.242^{\circ}$

Absorption correction

Max. and min. transmission

Refinement method

Data / restraints / parameters

Goodness-of-fit on $\mathrm{F}^{2}$

Final $\mathrm{R}$ indices [I $>2 \operatorname{sigma}(\mathrm{I})]$

$\mathrm{R}$ indices (all data)

Absolute structure parameter

Extinction coefficient

Largest diff. peak and hole
$6093[\mathrm{R}(\mathrm{int})=0.0288]$

$99.5 \%$

Semi-empirical from equivalents

0.7456 and 0.6487

Full-matrix least-squares on $\mathrm{F}^{2}$

6093 / 1 / 340

1.026

$\mathrm{R} 1=0.0427, \mathrm{wR} 2=0.0949$

$\mathrm{R} 1=0.0569, \mathrm{wR} 2=0.1052$

$-0.02(3)$

$\mathrm{n} / \mathrm{a}$

0.219 and -0.199 e. $\AA^{-3}$ 


\section{Ligand-Accelerating Effect}

The ligand accelerating effect in this reaction was roughly investigated as shown in Figure 1. Without an external ligand, the copper(I)-catalyzed non-enantioselective reaction proceeded slowly and provided the product in $40 \%$ yield in $24 \mathrm{~h}$. In this reaction, HPPhMes itself might serve as a ligand to $\mathrm{Cu}(\mathrm{I})$. In the reaction with $(R)$-TOL-BINAP, the yield in $24 \mathrm{~h}$ increased to $73 \%$, indicating that the reaction catalyzed by $\mathrm{Cu}(\mathrm{I})-(R)$-TOL-BINAP complex was not accelerated significantly. However, the reactions with $(R, R)$-Ph-BPE and $\left(R, R_{P}\right)$-TANIAPHOS were much faster than the background reaction. Evidently, the moderate enantioselectivity of the reaction with $(R, R)$-Ph-BPE was largely due to its unsatisfactory performance in the asymmetric induction. Such an interesting ligand accelerating effect was the key to achieve high enantioselectivity in the present reaction.

Figure 1. Ligand-Accelerating Effect $\left({ }^{1} \mathrm{H}\right.$ NMR Yields Are Given)

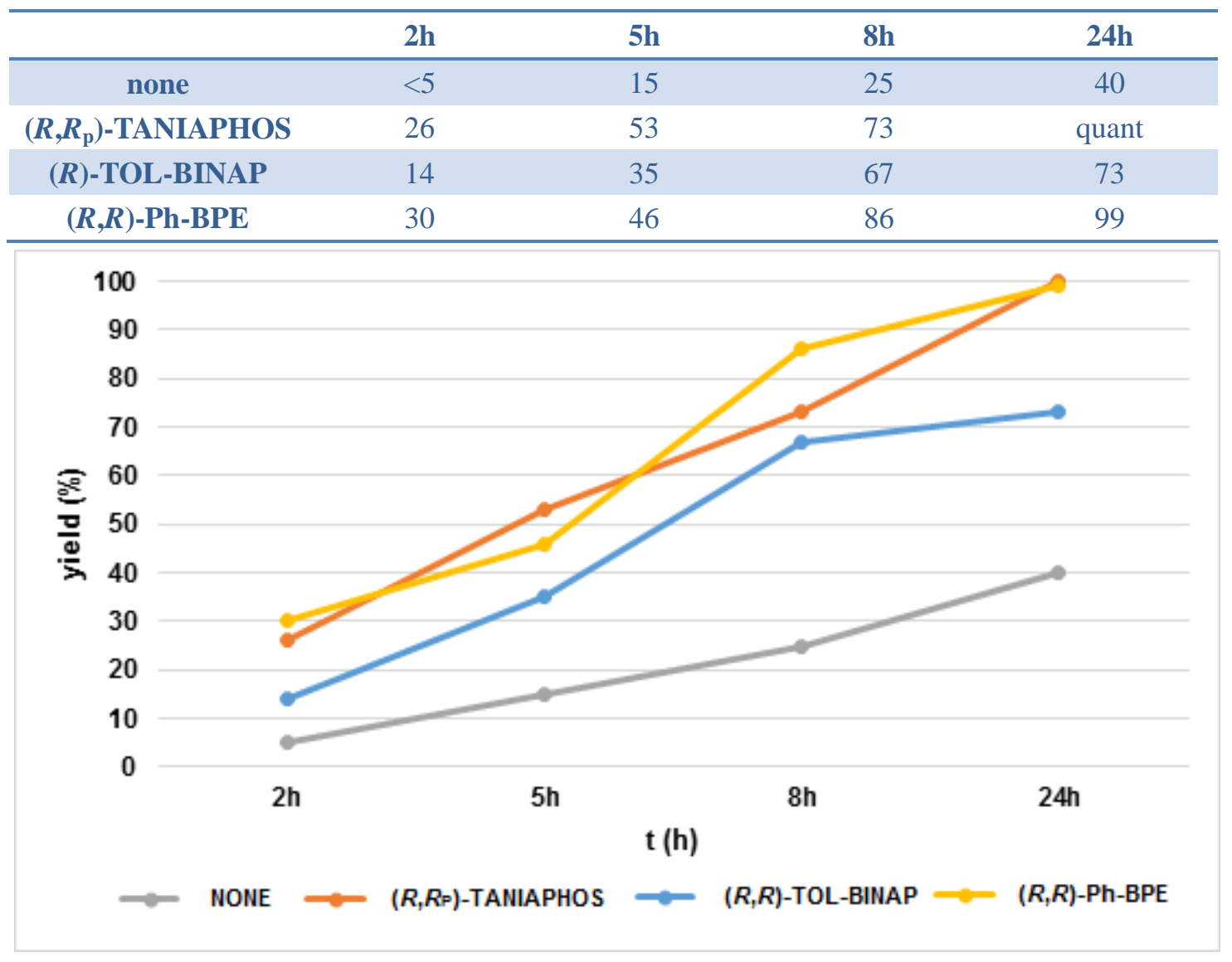




\section{References}

[1] Huang, Y.; Li, Y.; Leung, P.-H.; Hayashi, T. J. Am. Chem. Soc. 2014, 136, 4865-4868.

[2] Foubelo, F.; Lloret, F.; Yus, M. Tetrahedron 1994, 50, 5131-5138.

[3] Miura, K.; Tomita, M.; Yamada, Y.; Hosomi, A. J. Org. Chem. 2007, 72, 787-792.

[4] Kitabayashi, Y.; Yokoshima, S.; Fukuyama, T. Org. Lett. 2014, 16, $2862-2864$.

[5] Rocaboy, R.; Dailler, D.; Zellweger, F.; Neuburger, M.; Salomé, C.; Clot, E.; Baudoin, O. Angew. Chem. Int. Ed. 2018, 57, 12131-12135.

[6] McGowan, M. A.; Perreault, D. M.; Thornton, N. B.; Garaas, S. D.; Gribble, G. W. Arkivoc 2018, 2018, 334-347.

[7] Zon, G.; DeBruin, K. E.; Naumann, K.; Mislow, K. J. Am. Chem. Soc. 1969, 91, 7023-7027. 
10.NMR Spectra Data

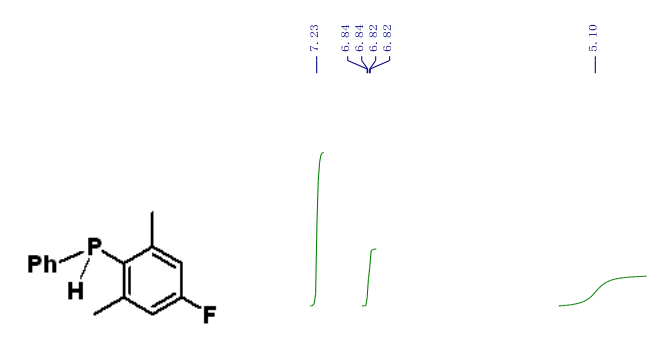

$1 b$
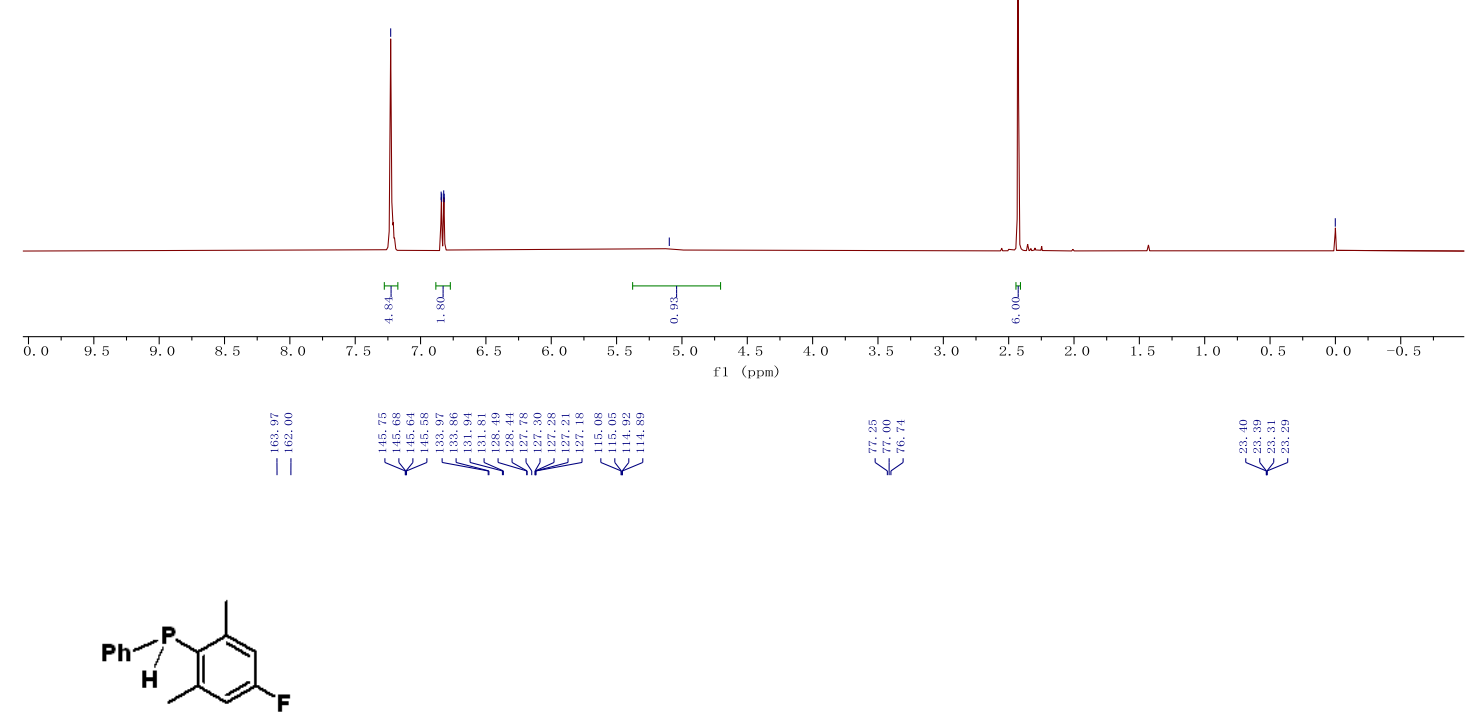

$1 b$

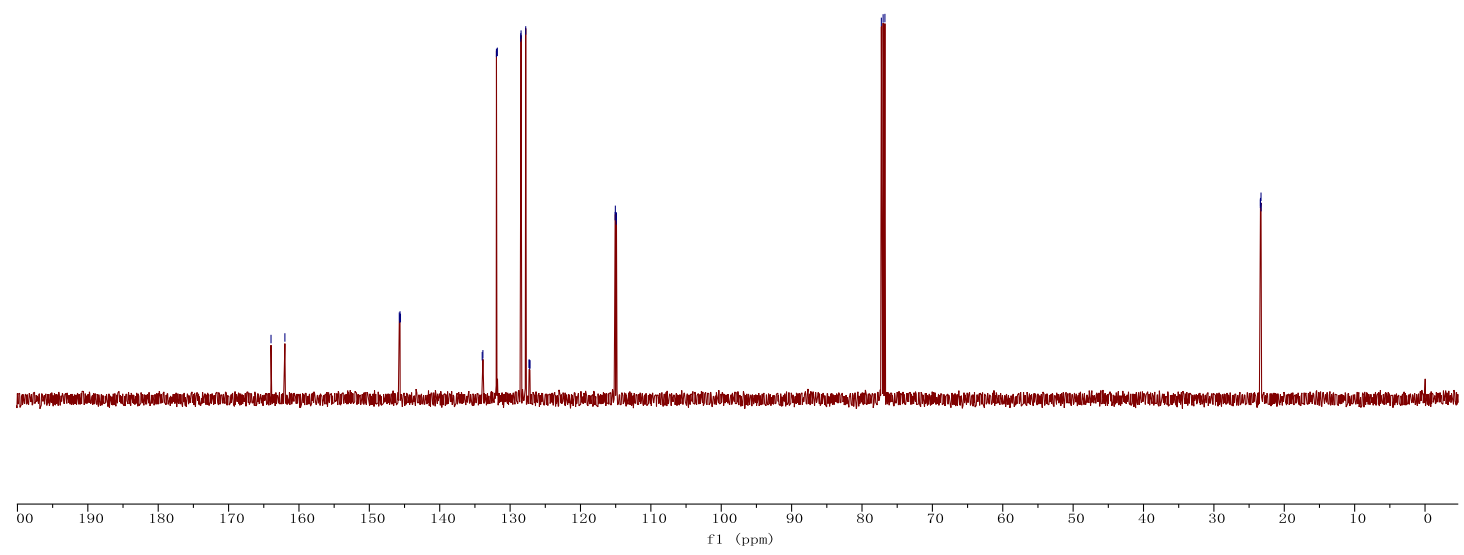

121 


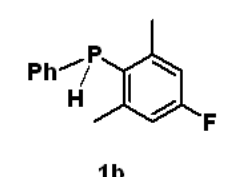

1b

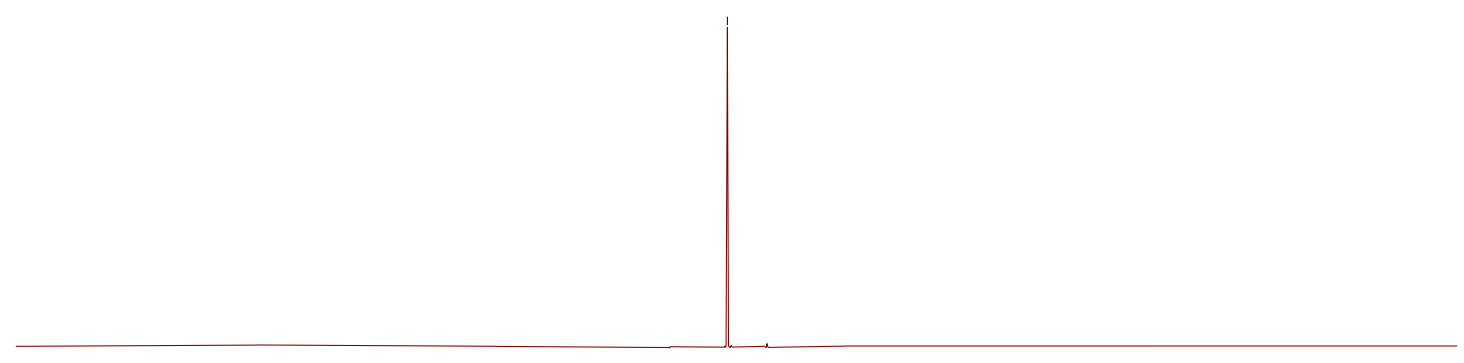

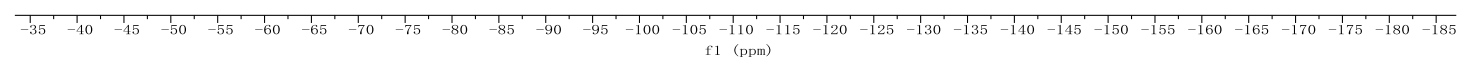
$\vec{a}$
$\infty$
$\substack{\infty \\ \mid}$

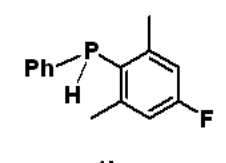

$1 \mathbf{b}$

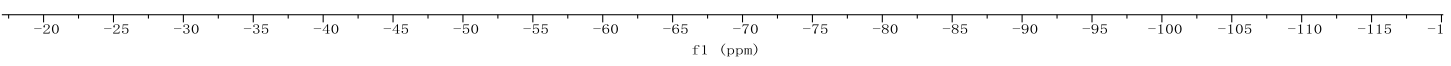



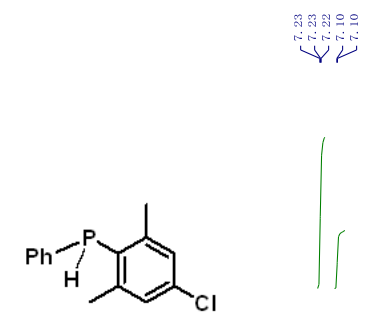

$1 \mathrm{c}$
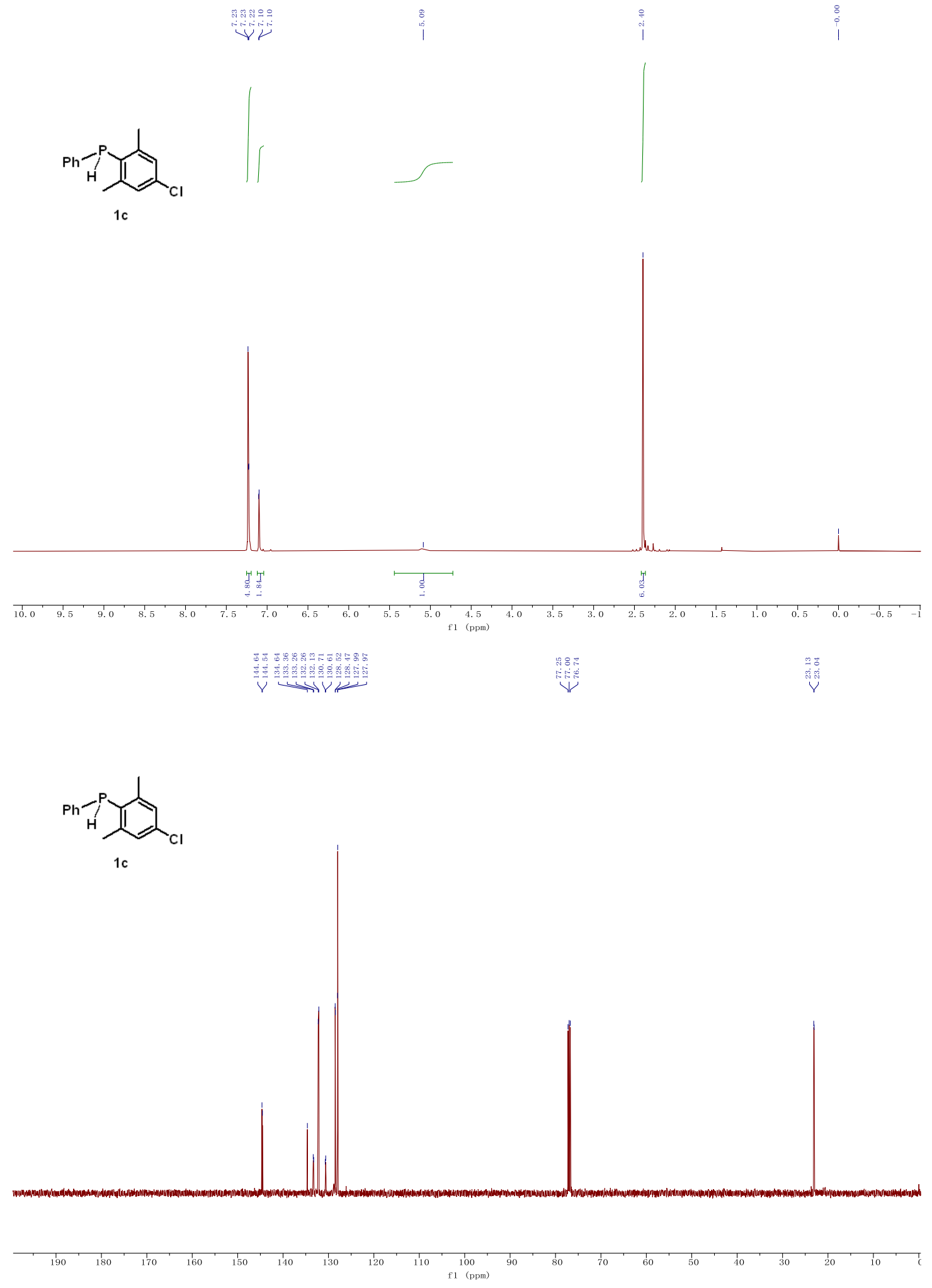

123 

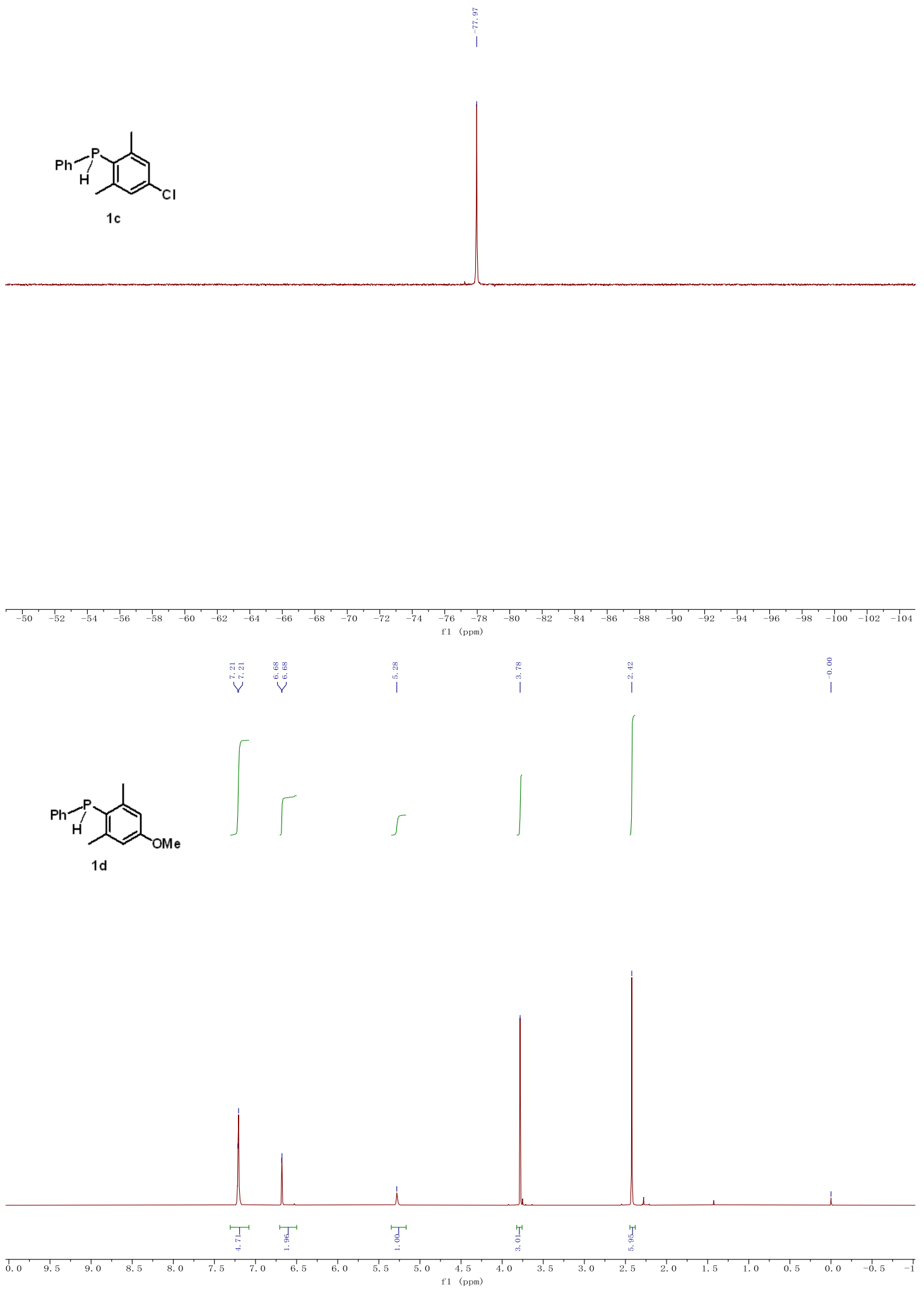

124 


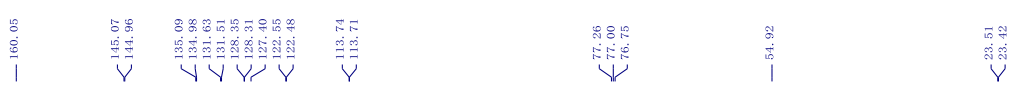
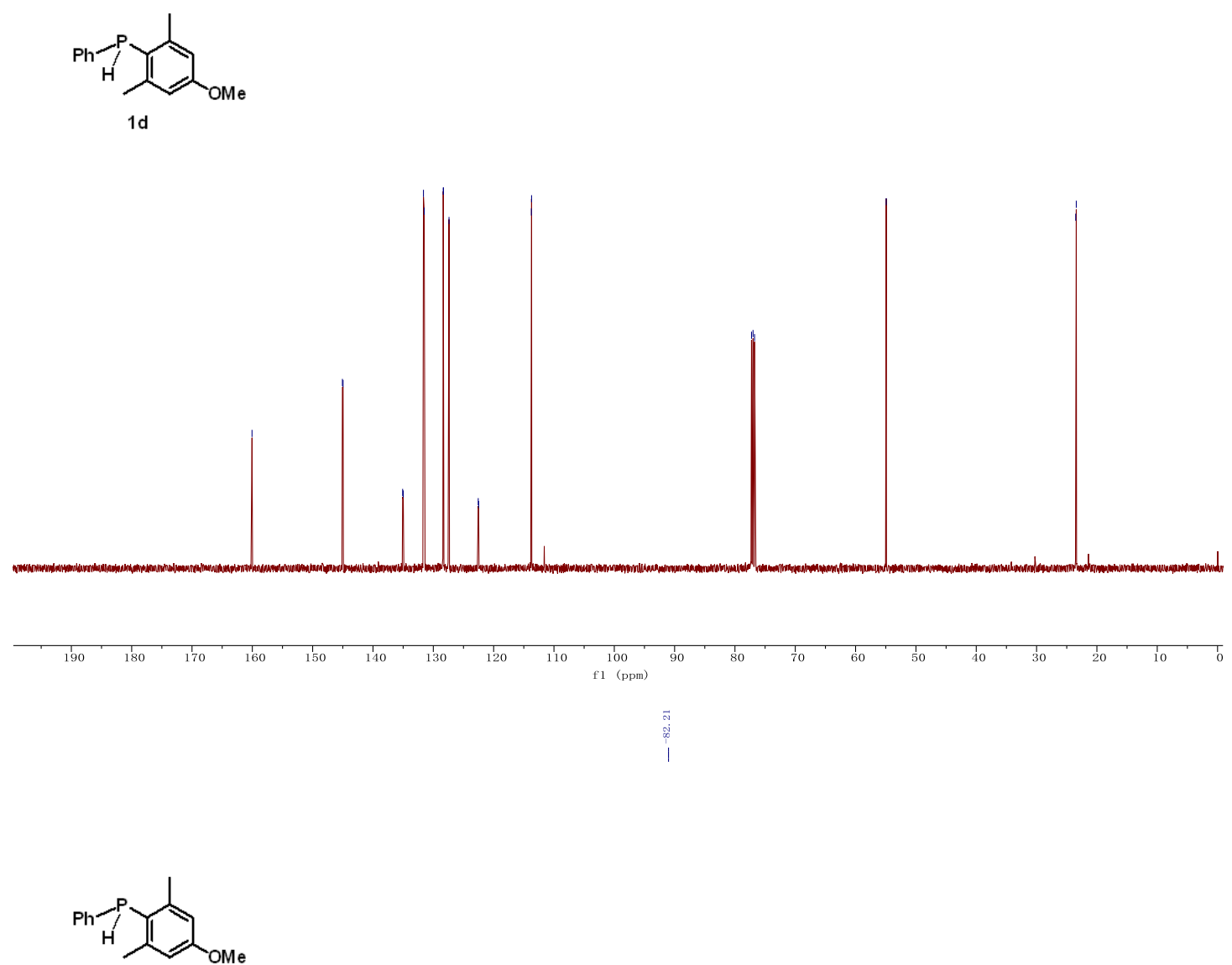

$1 \mathrm{~d}$

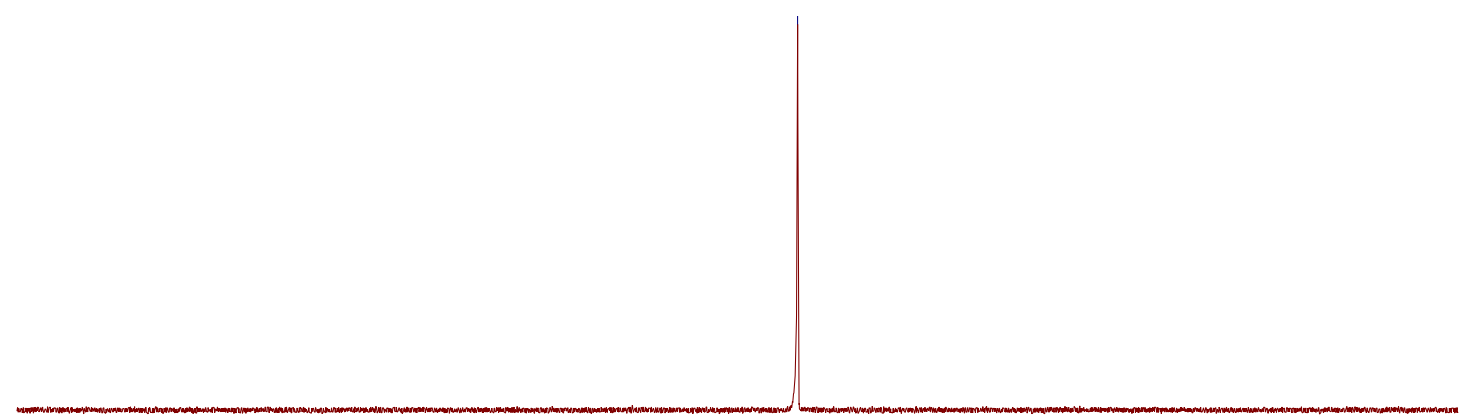

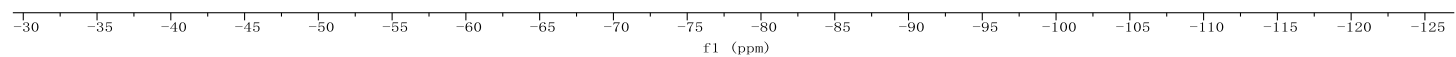



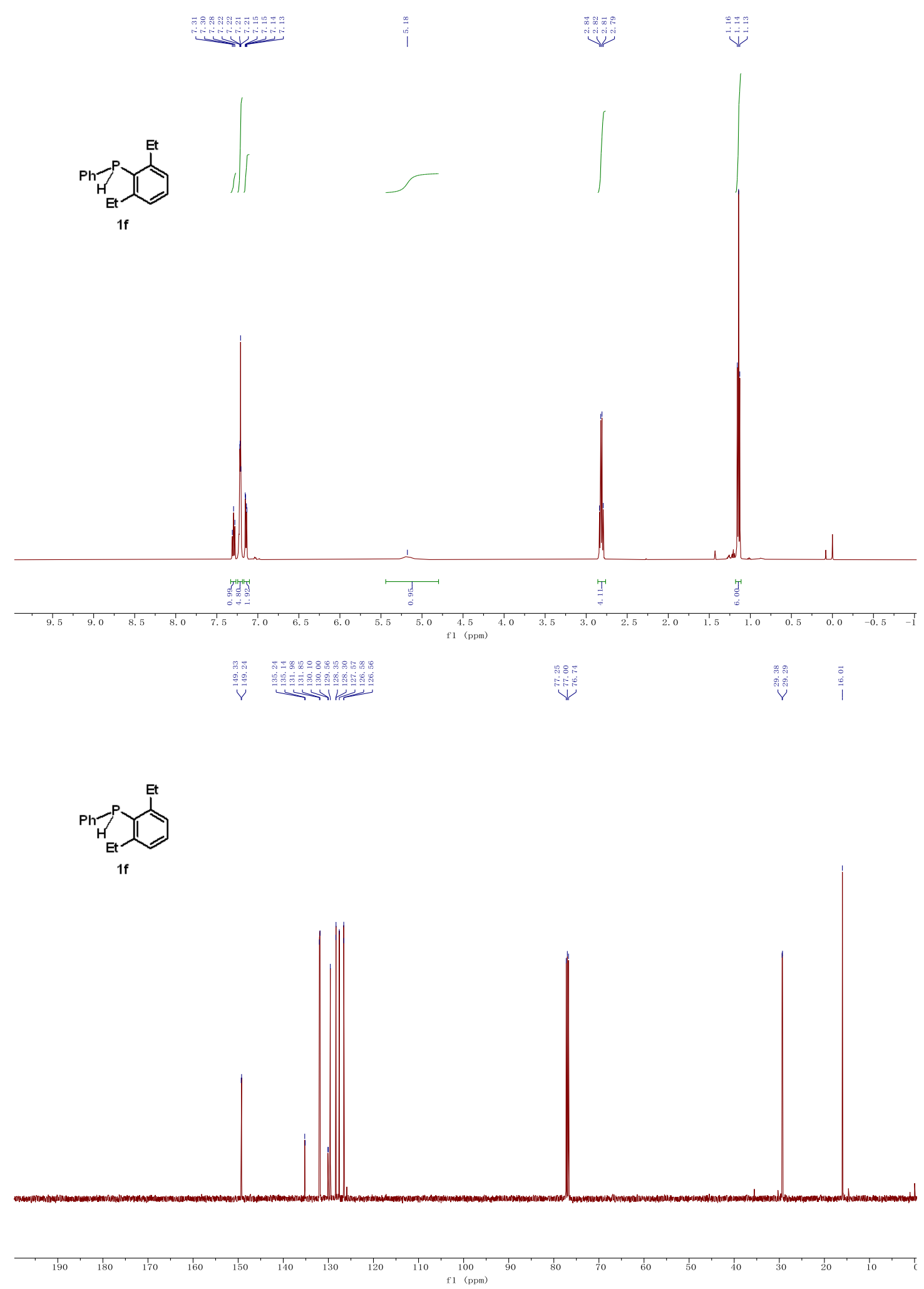

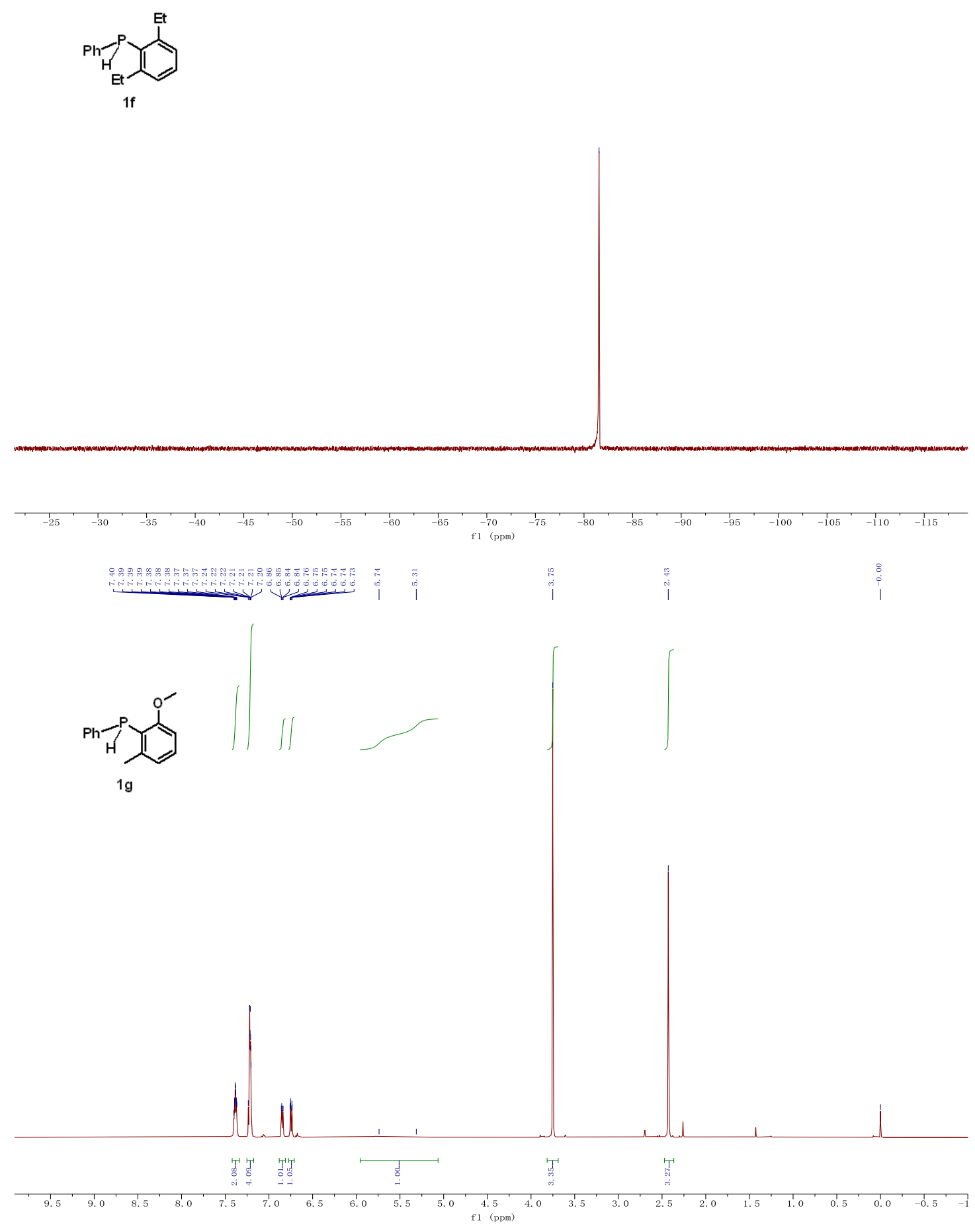


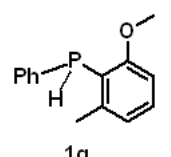

$1 \mathrm{~g}$
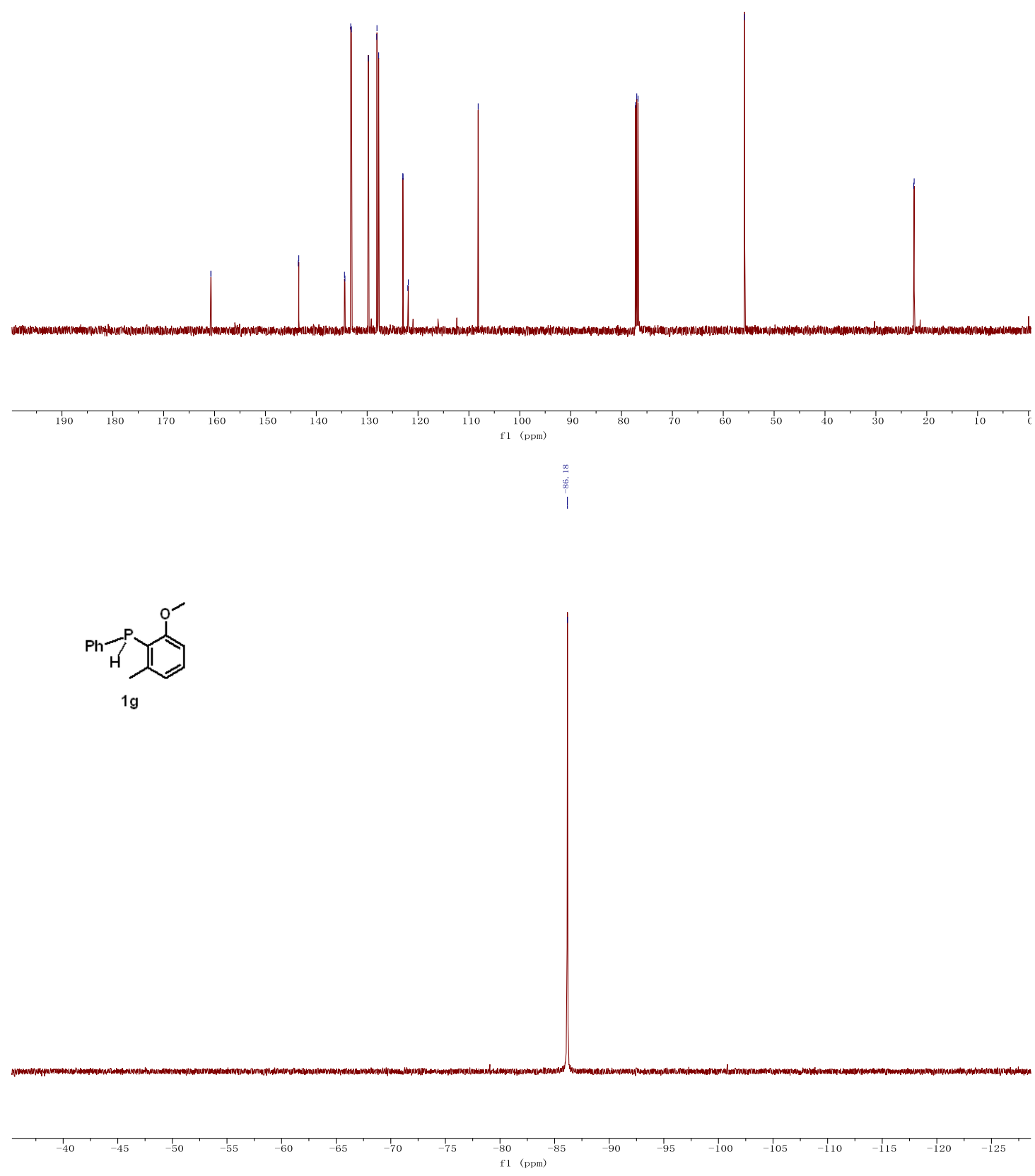

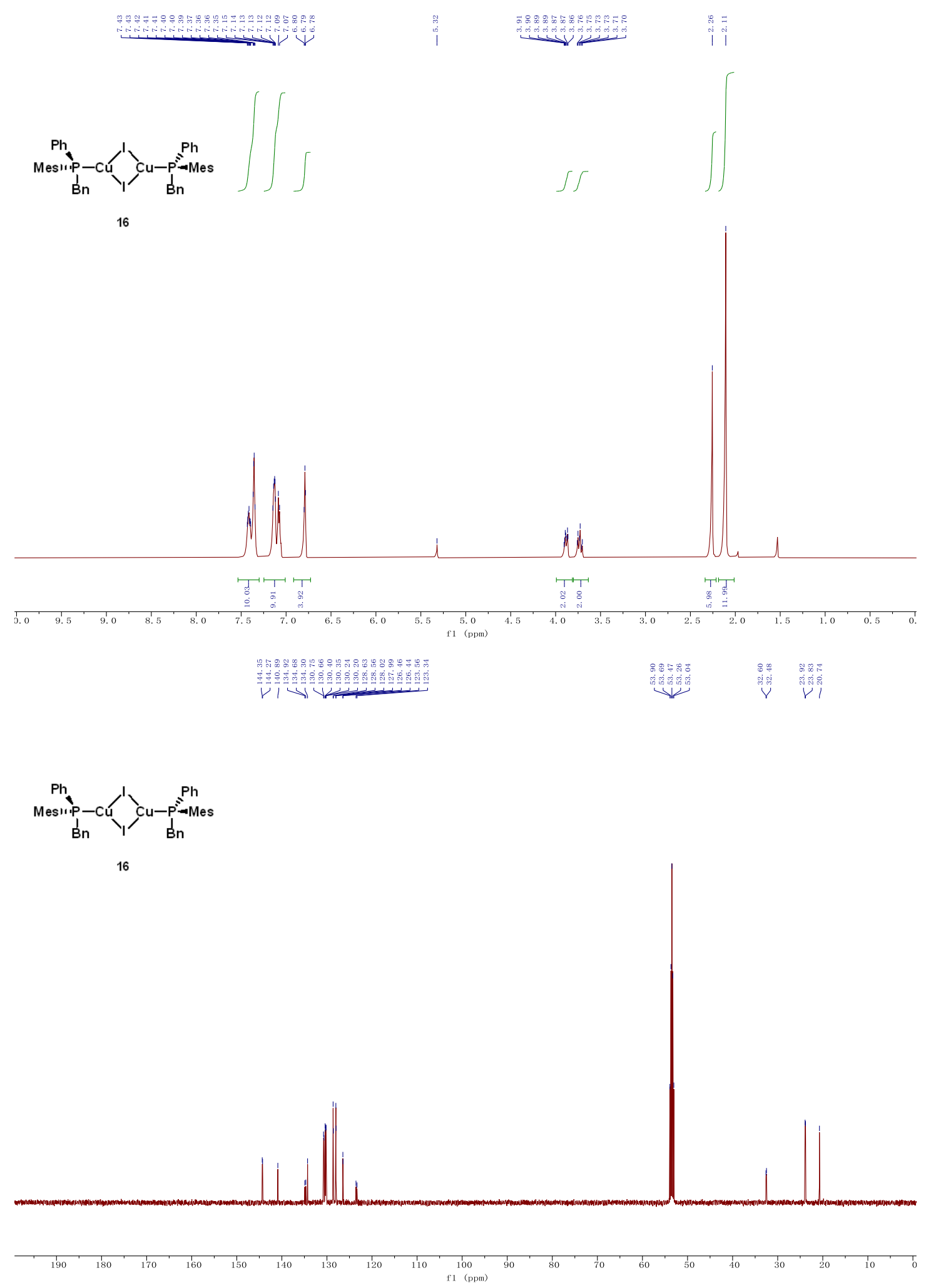

129 

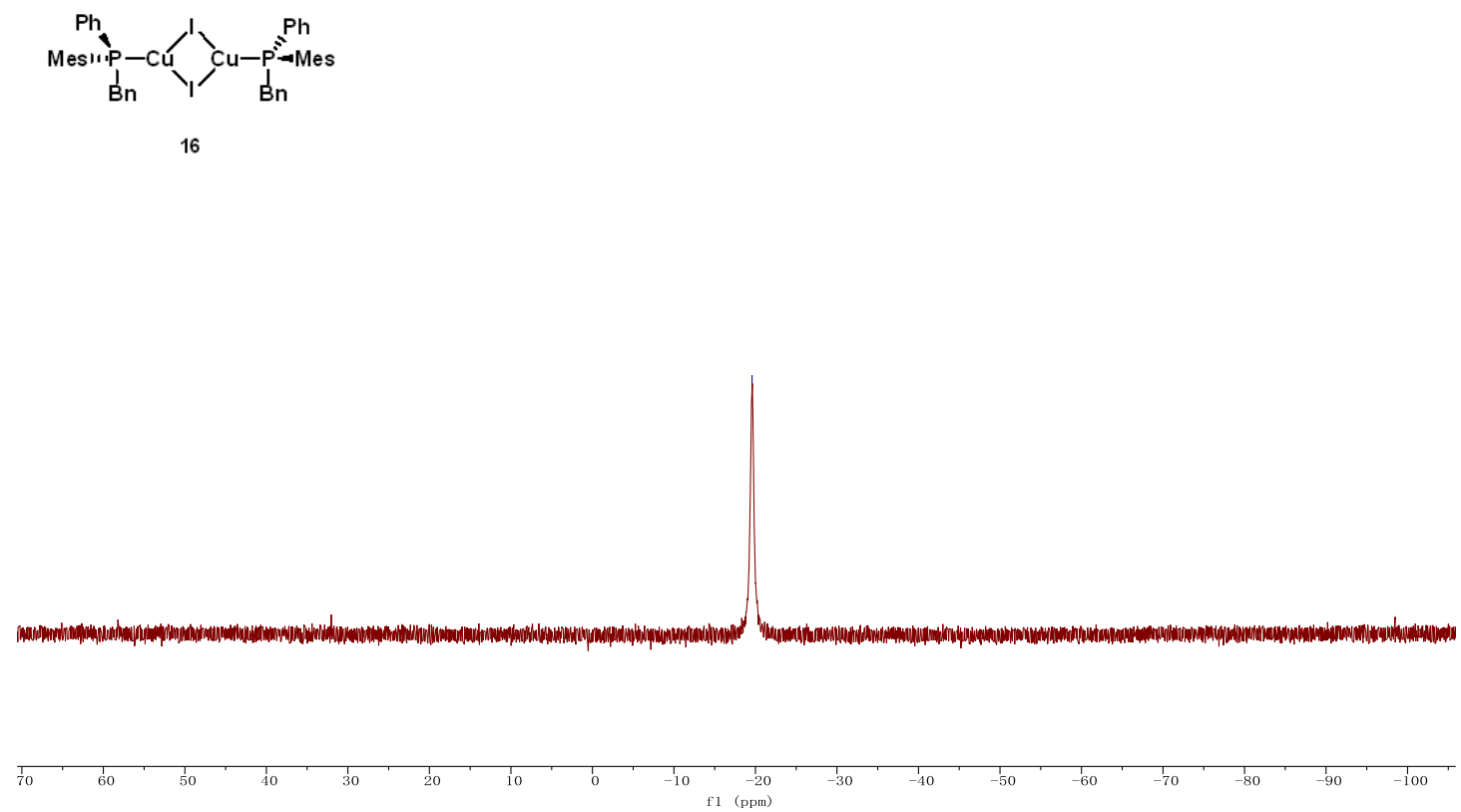

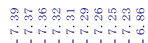
ฟjin
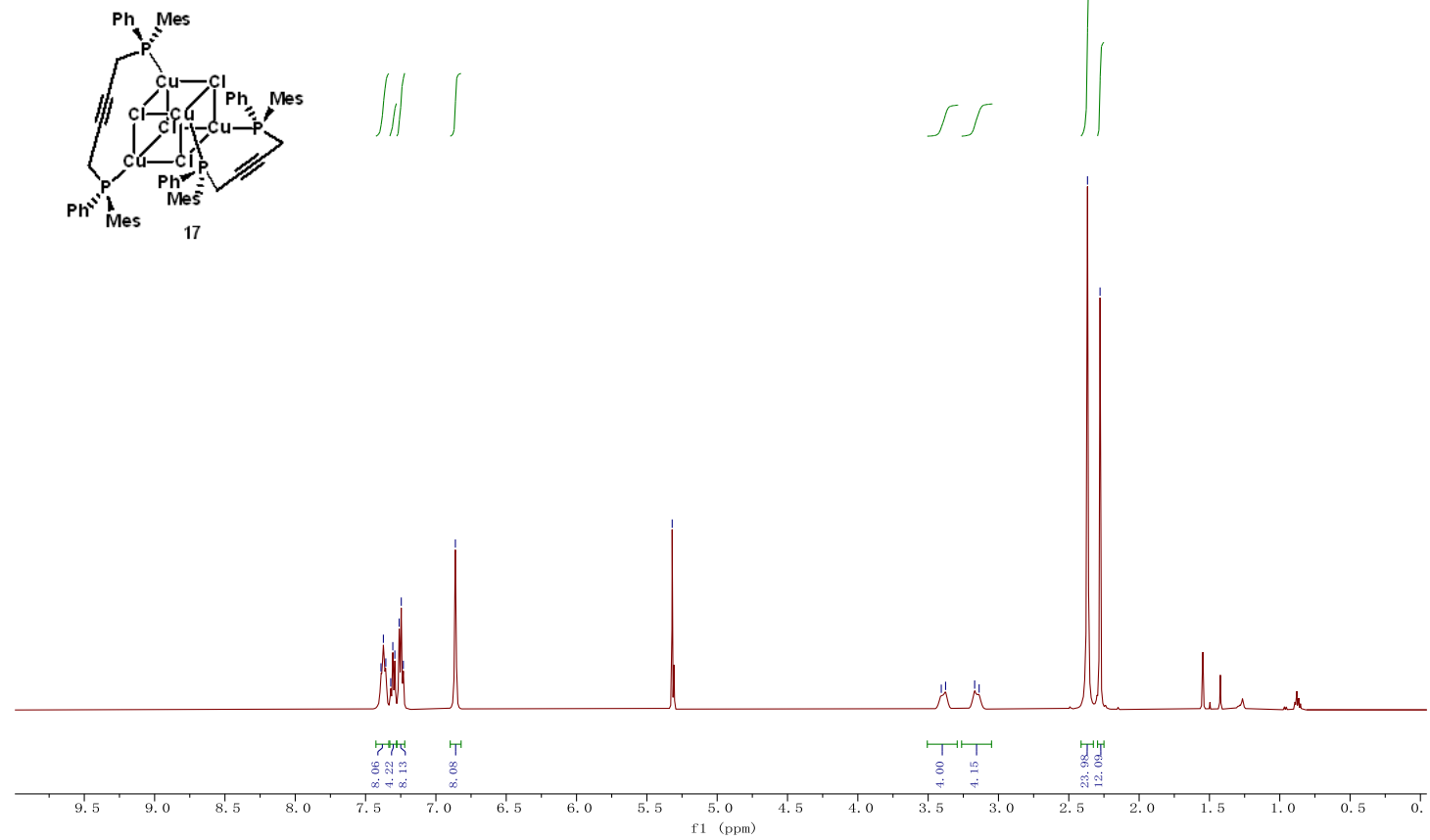

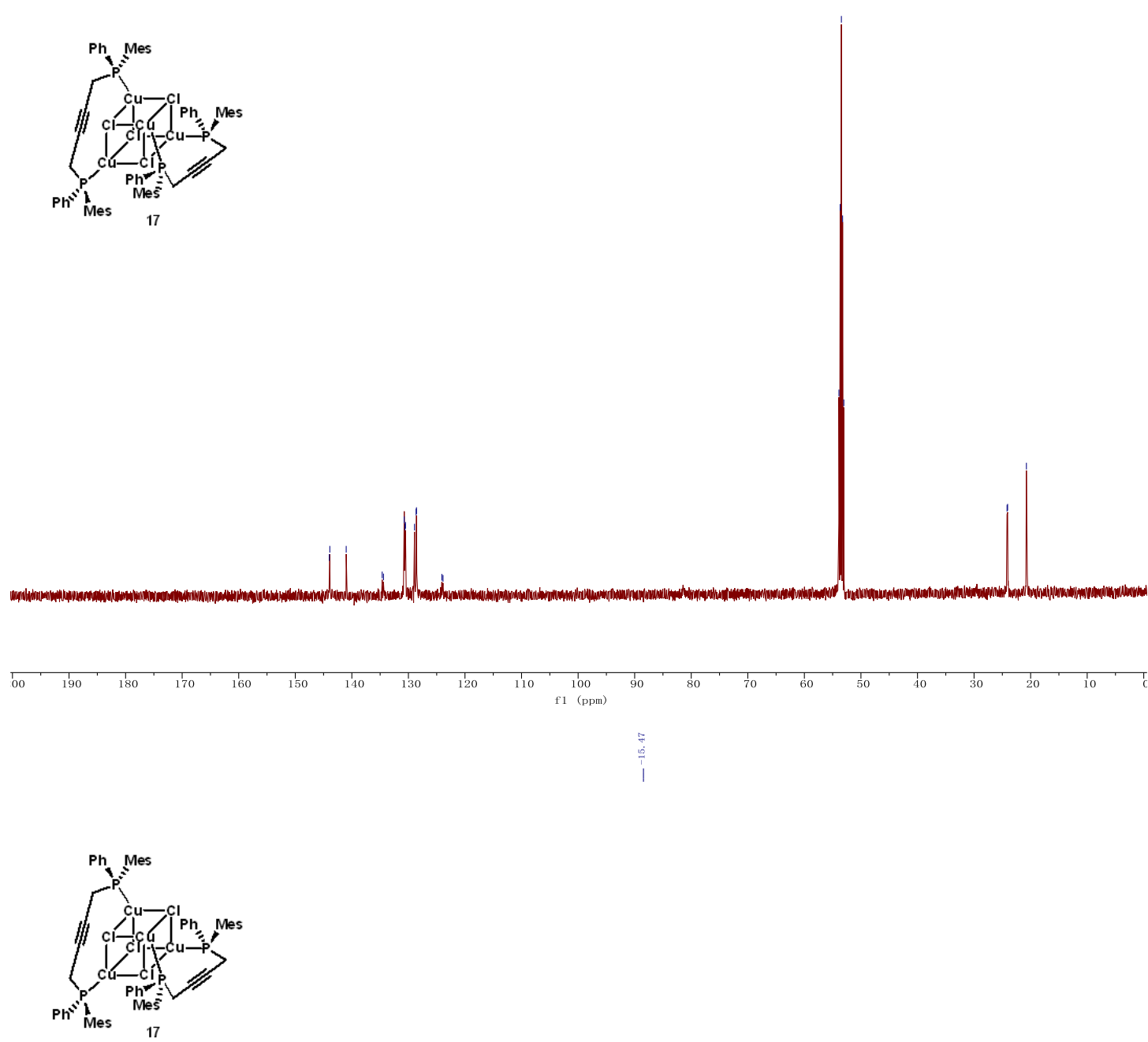

.

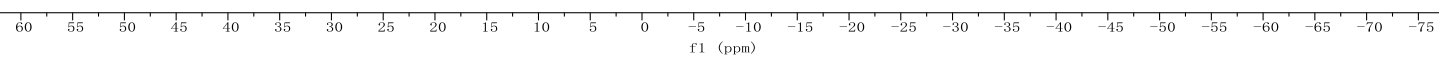


琶照照

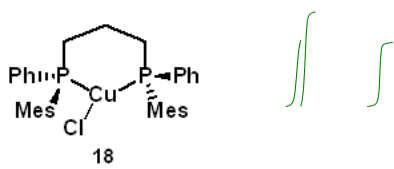

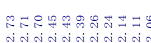

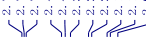

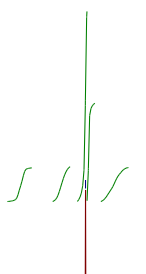

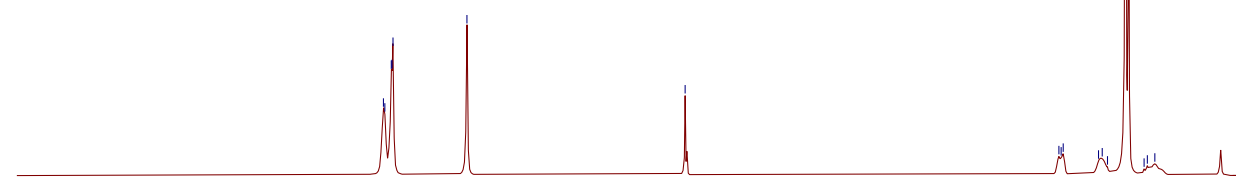

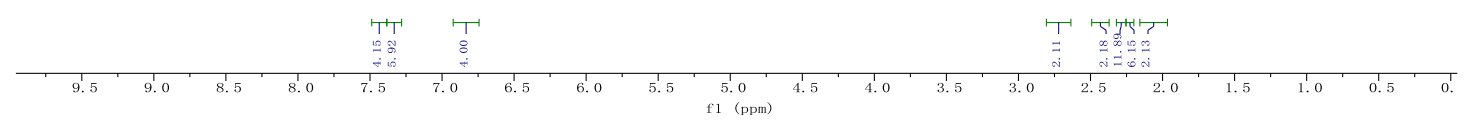

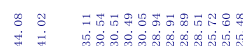

|

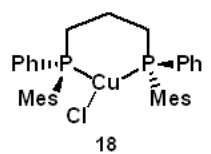

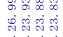

เพ้

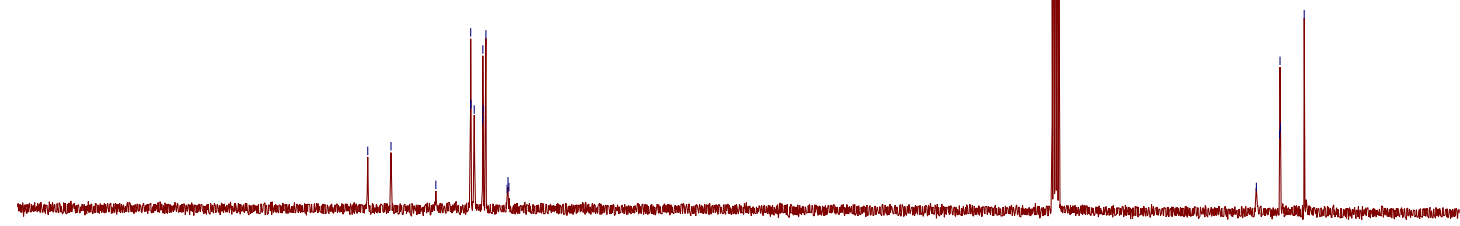

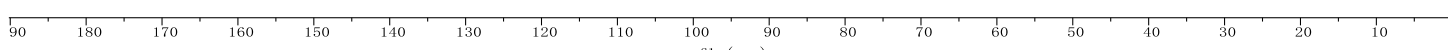


${ }_{18}^{\mathrm{Mes}_{\mathrm{C}}^{\prime} \mathrm{Cl}^{\prime}}$

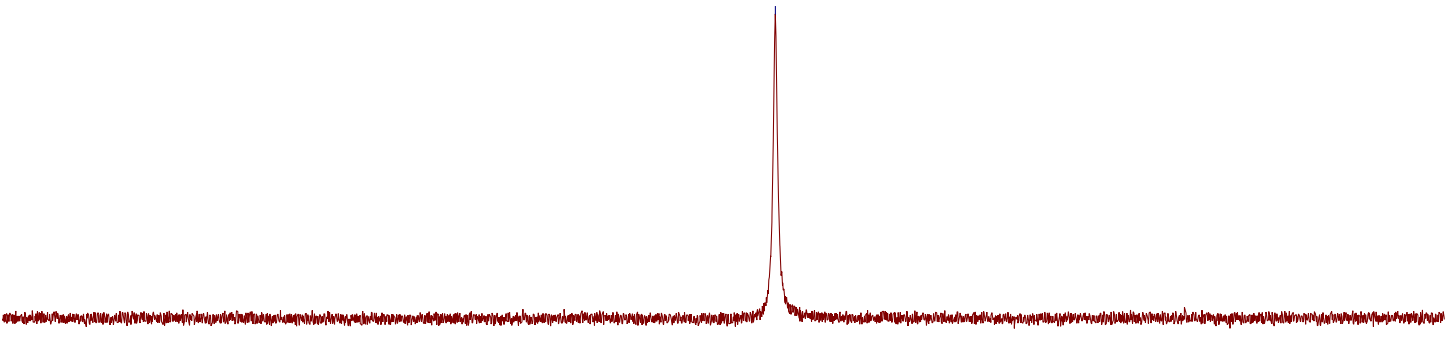



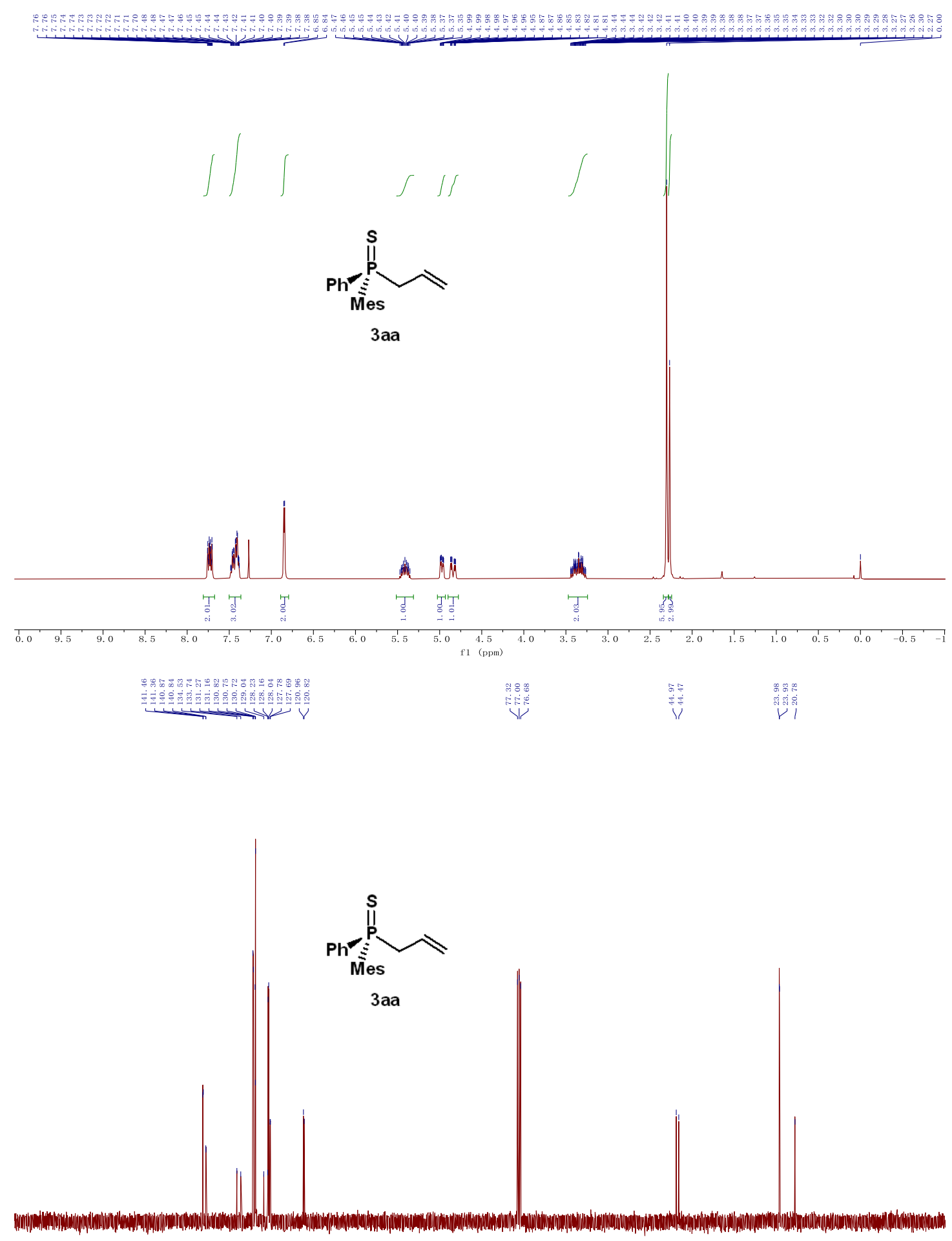


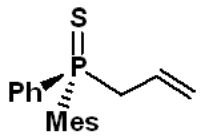

$3 a a$

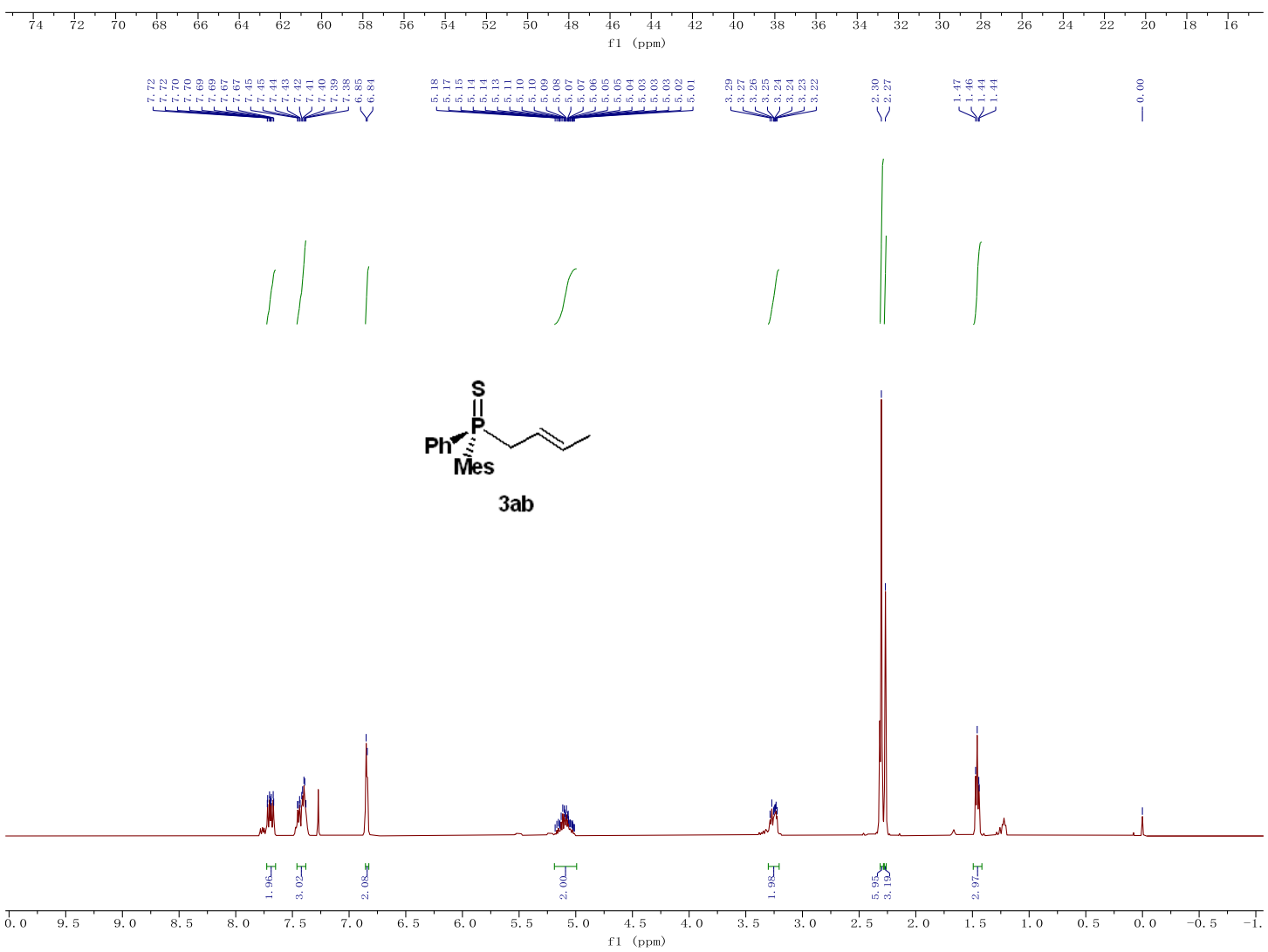




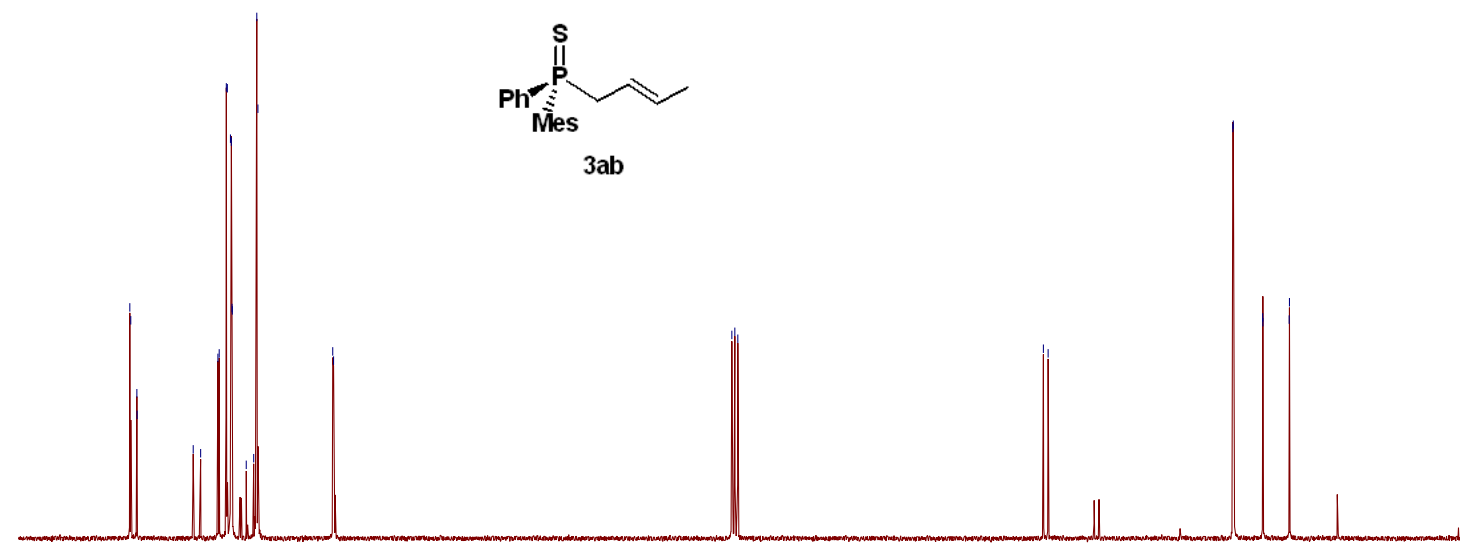

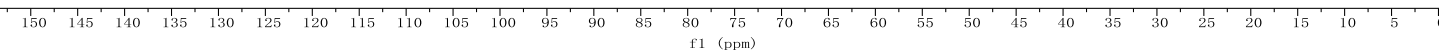<smiles>C=C(C)CC=CC</smiles>

$3 a b$ 

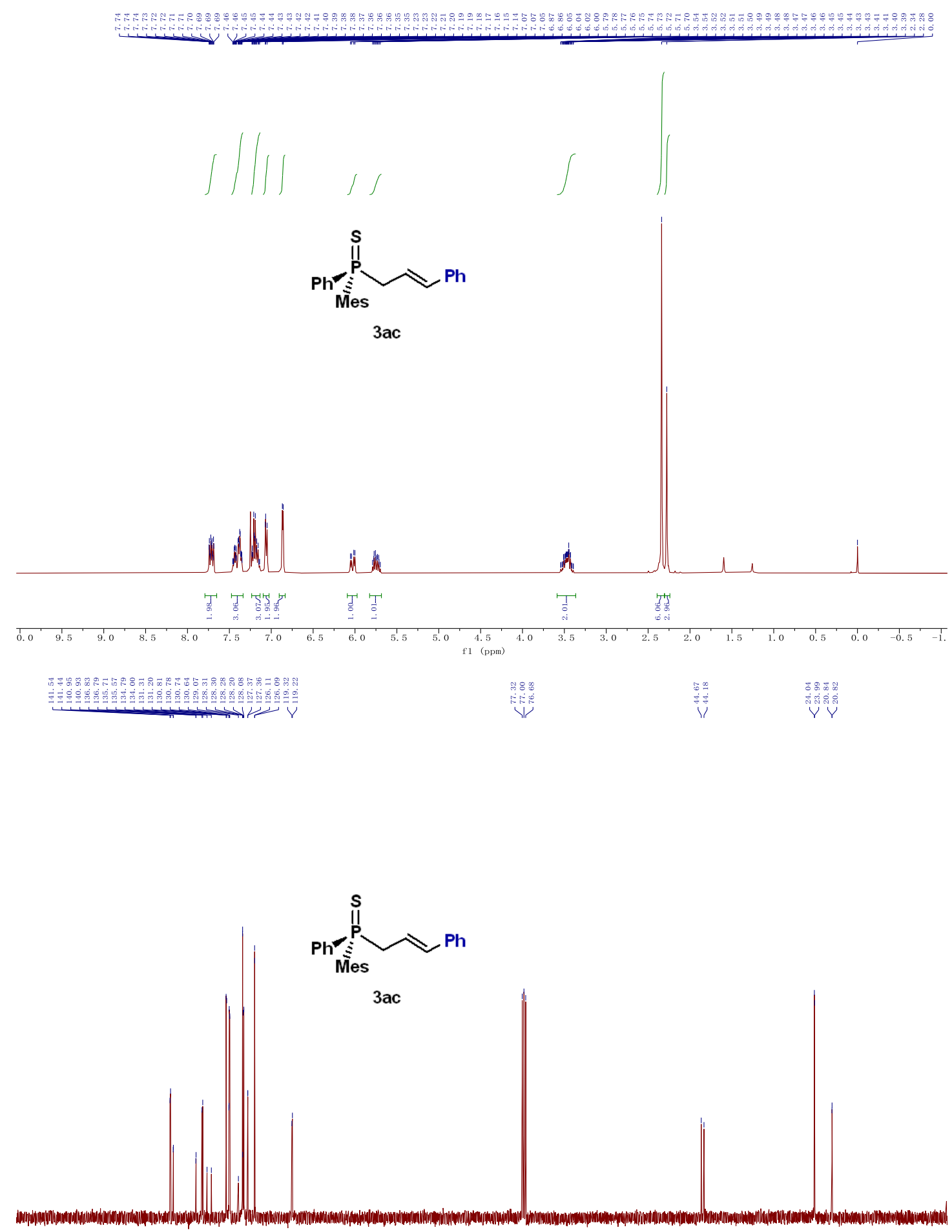


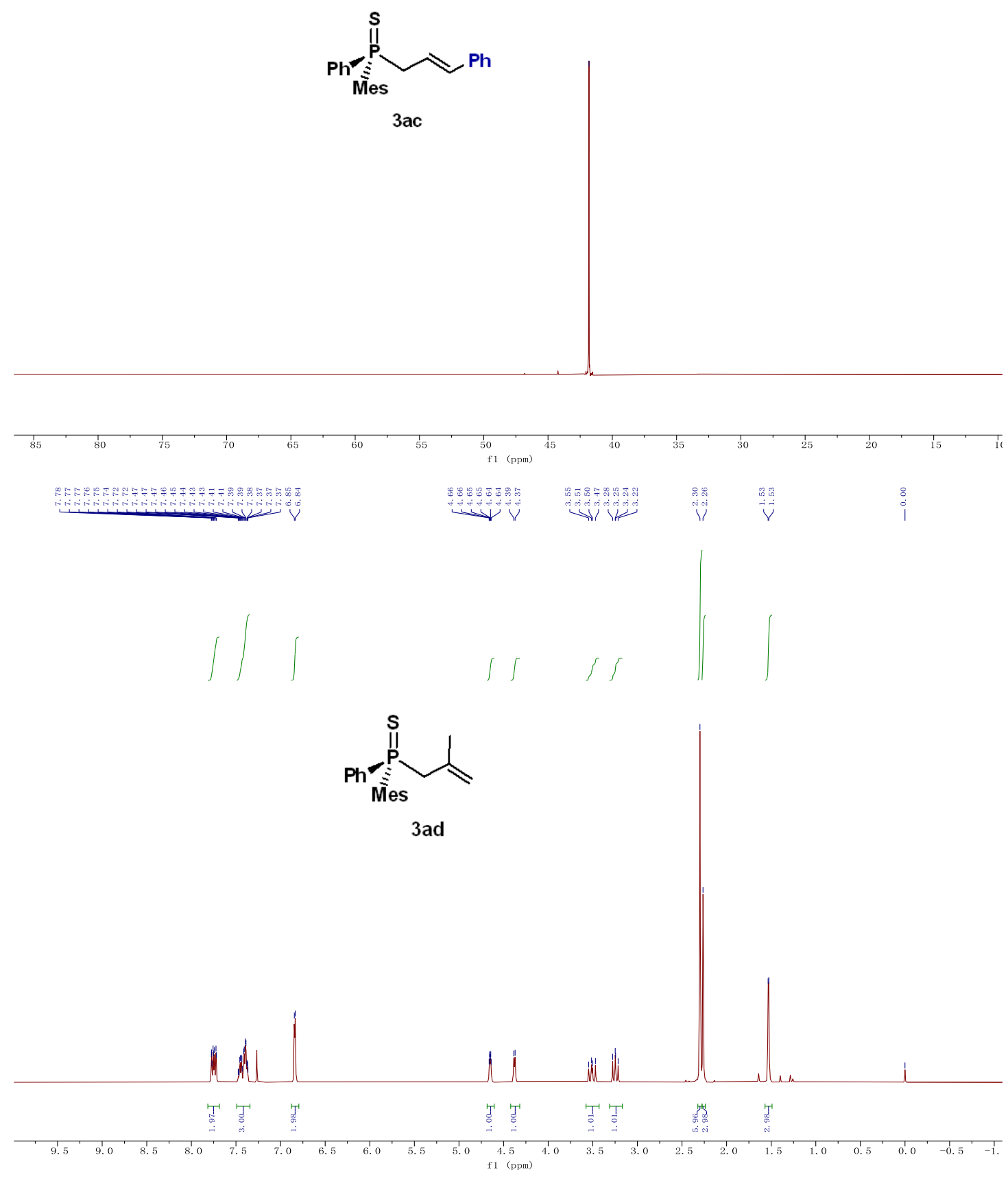



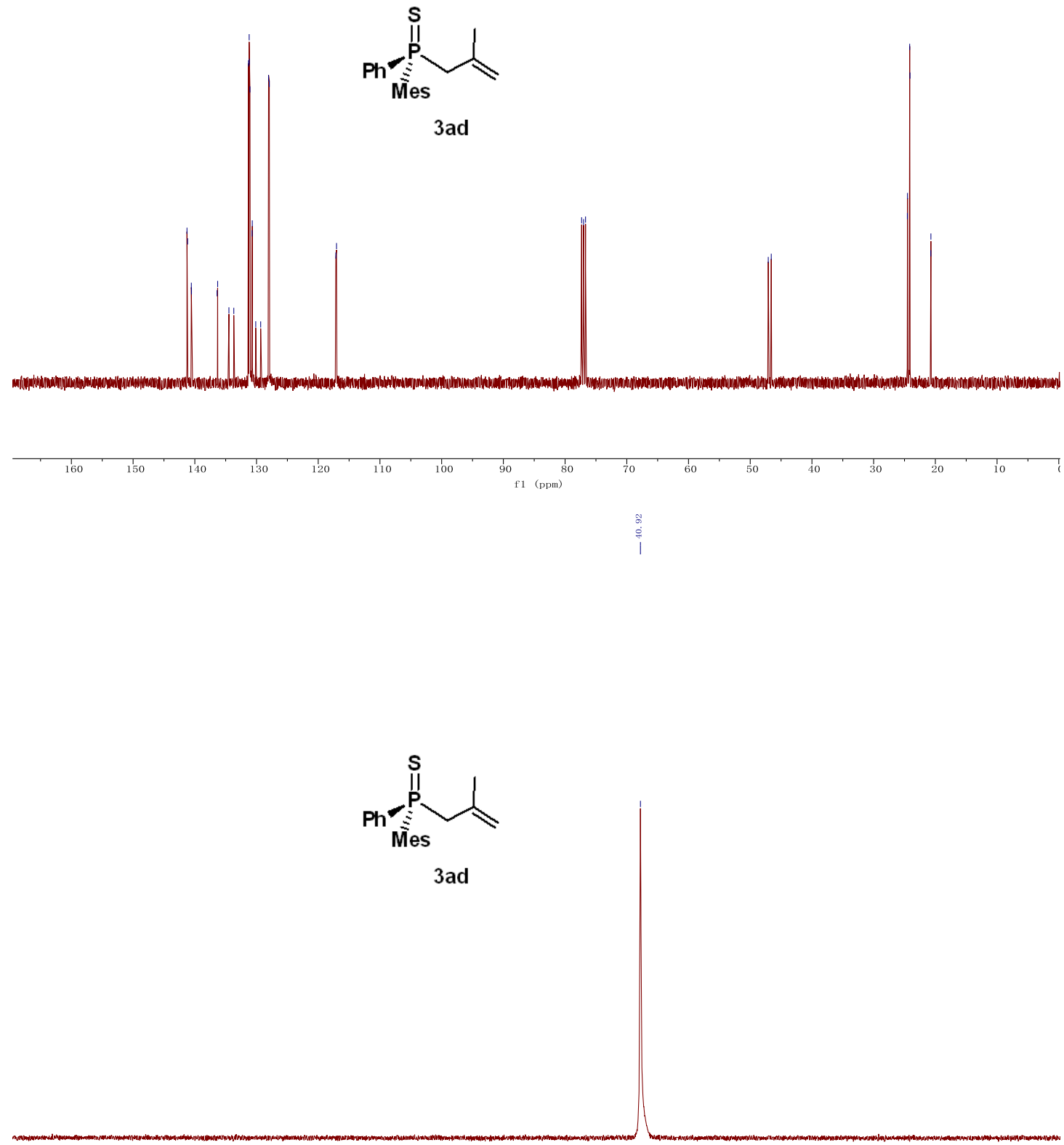

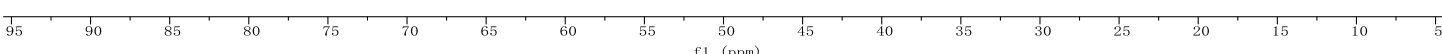



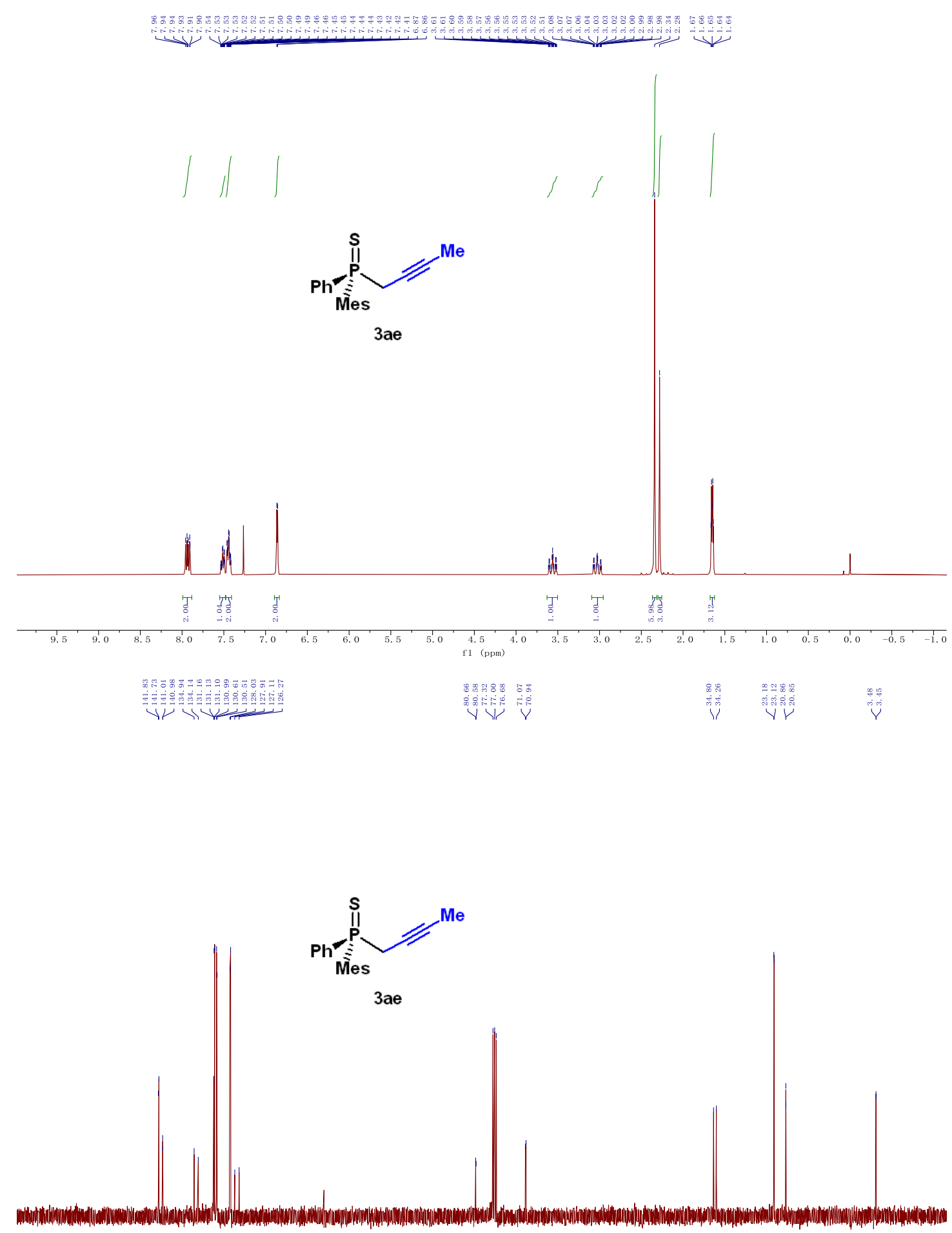

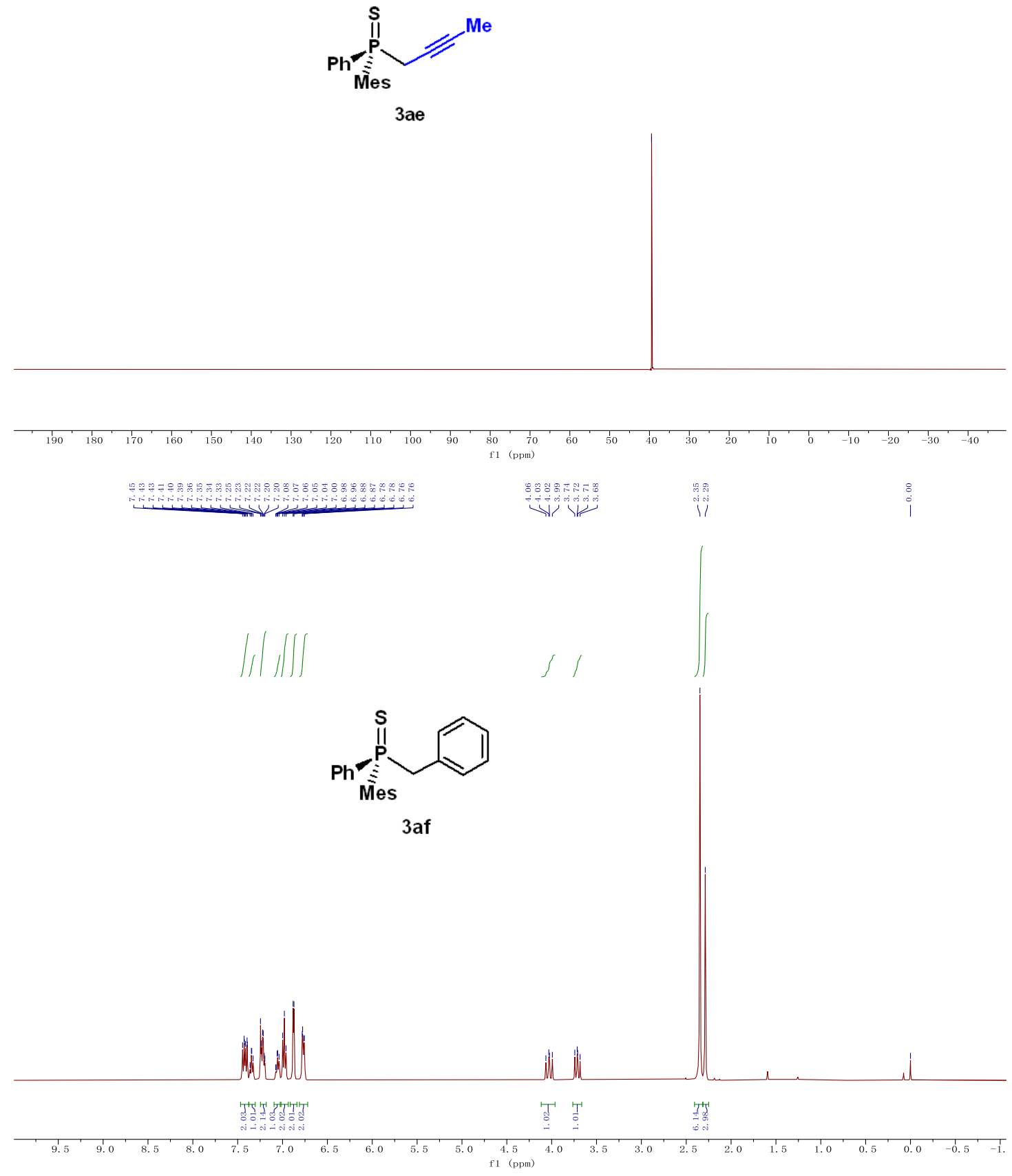


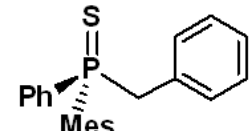

M̂es

3af
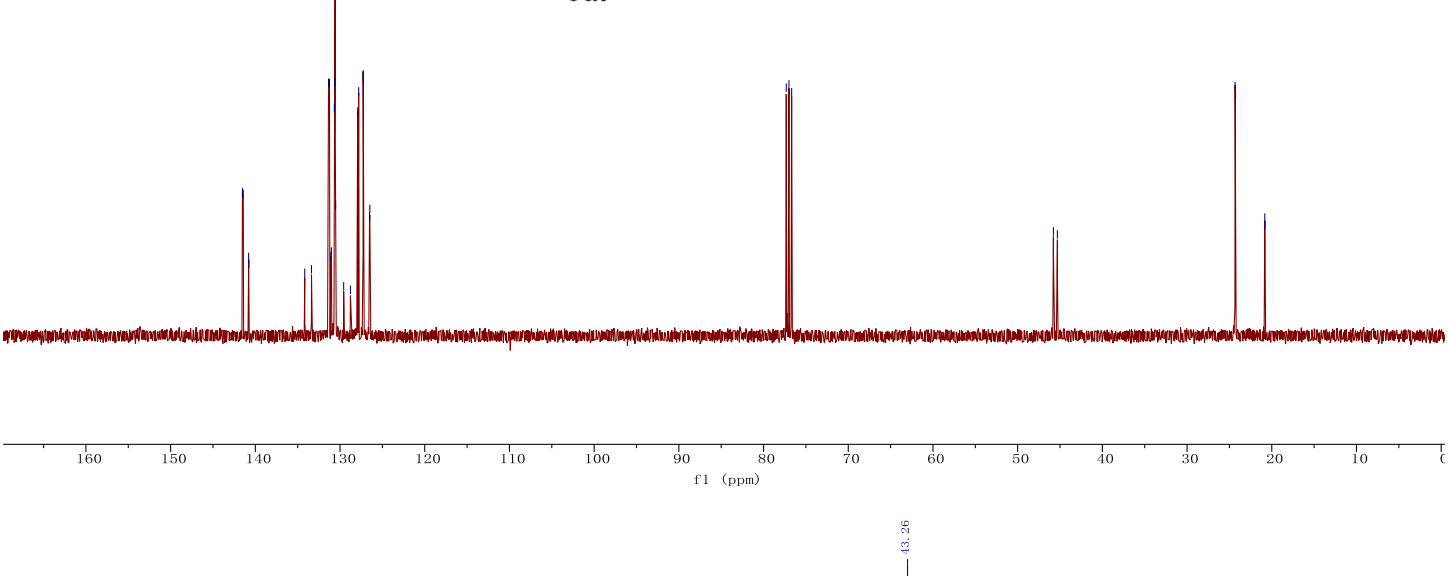

Mes

3af 

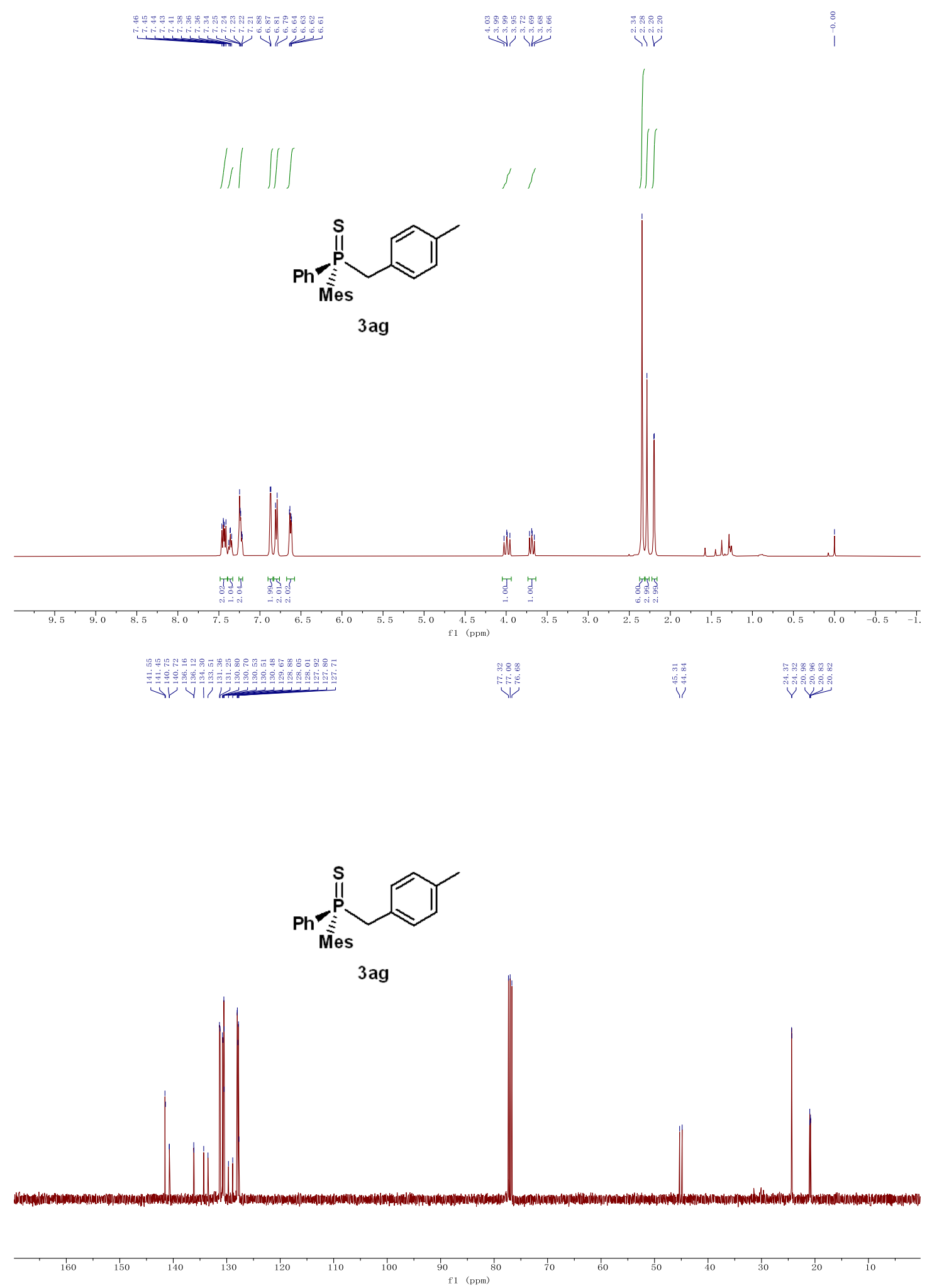


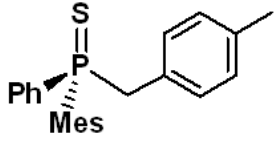

$3 \mathrm{ag}$

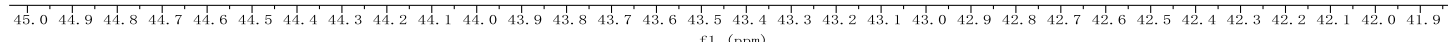

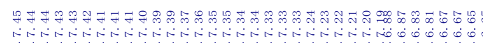

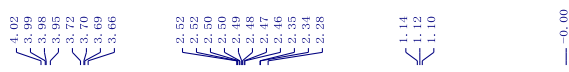

11111
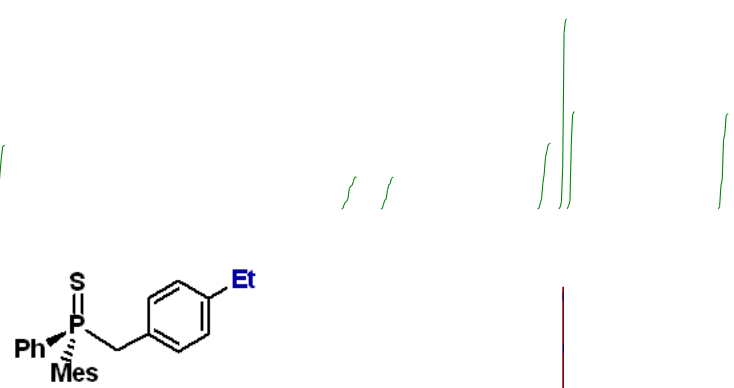

3ah

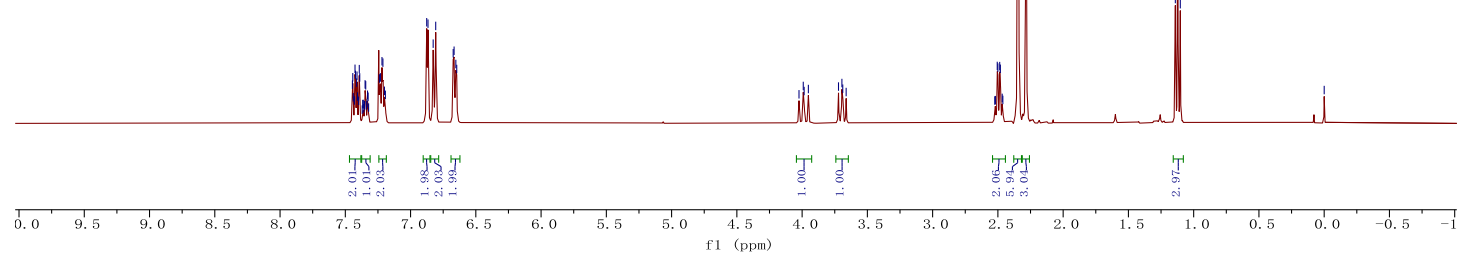



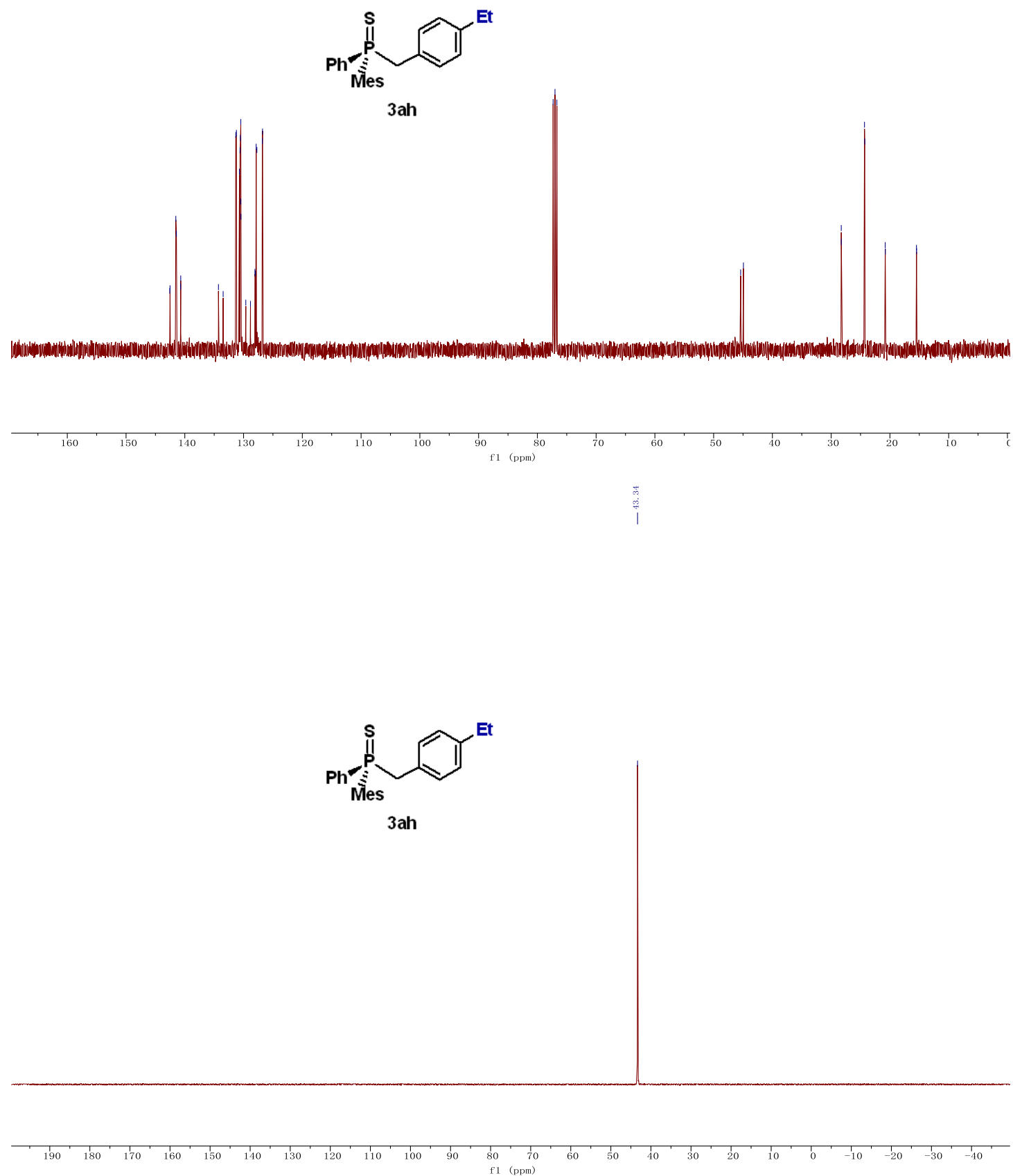


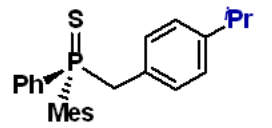

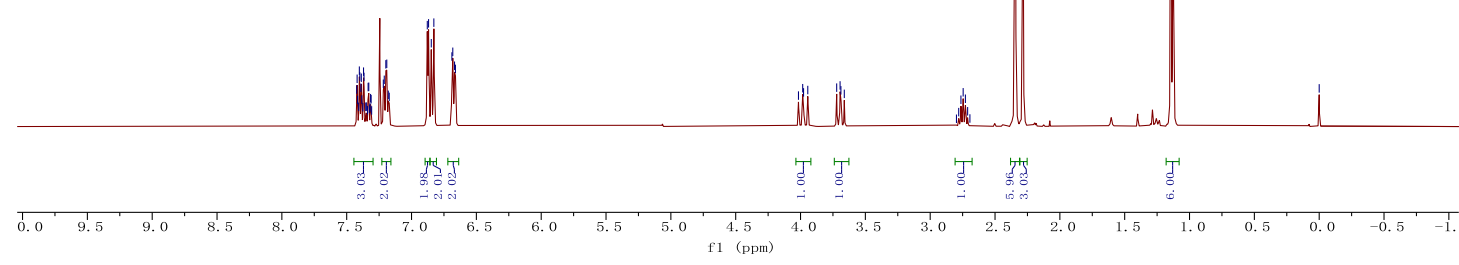

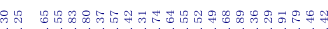

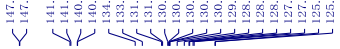

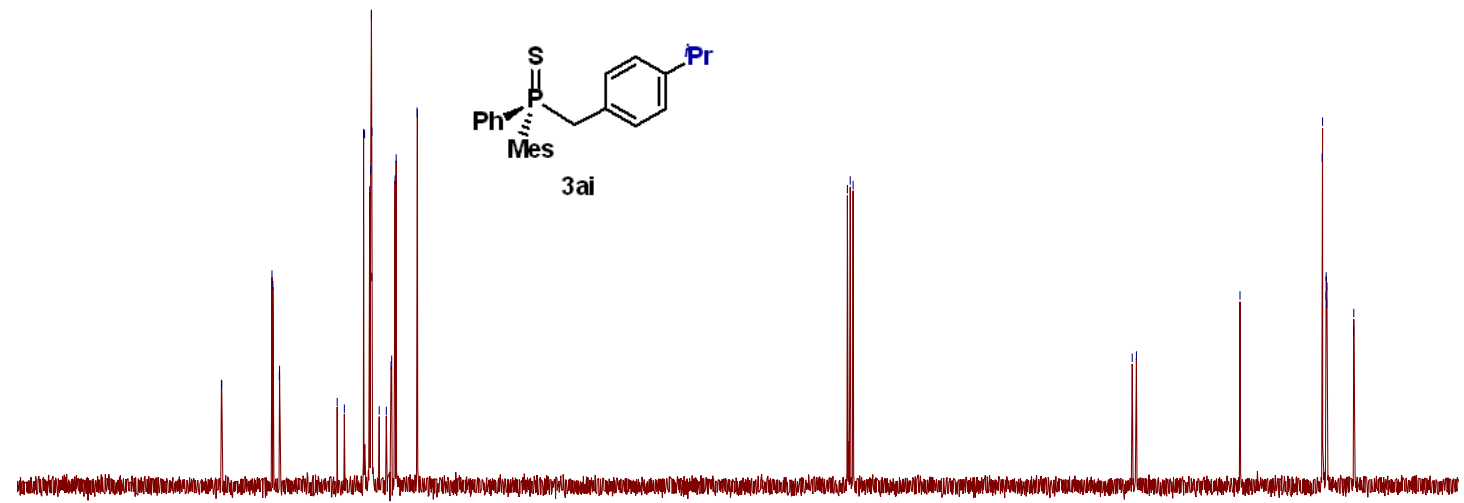

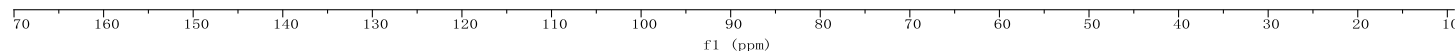




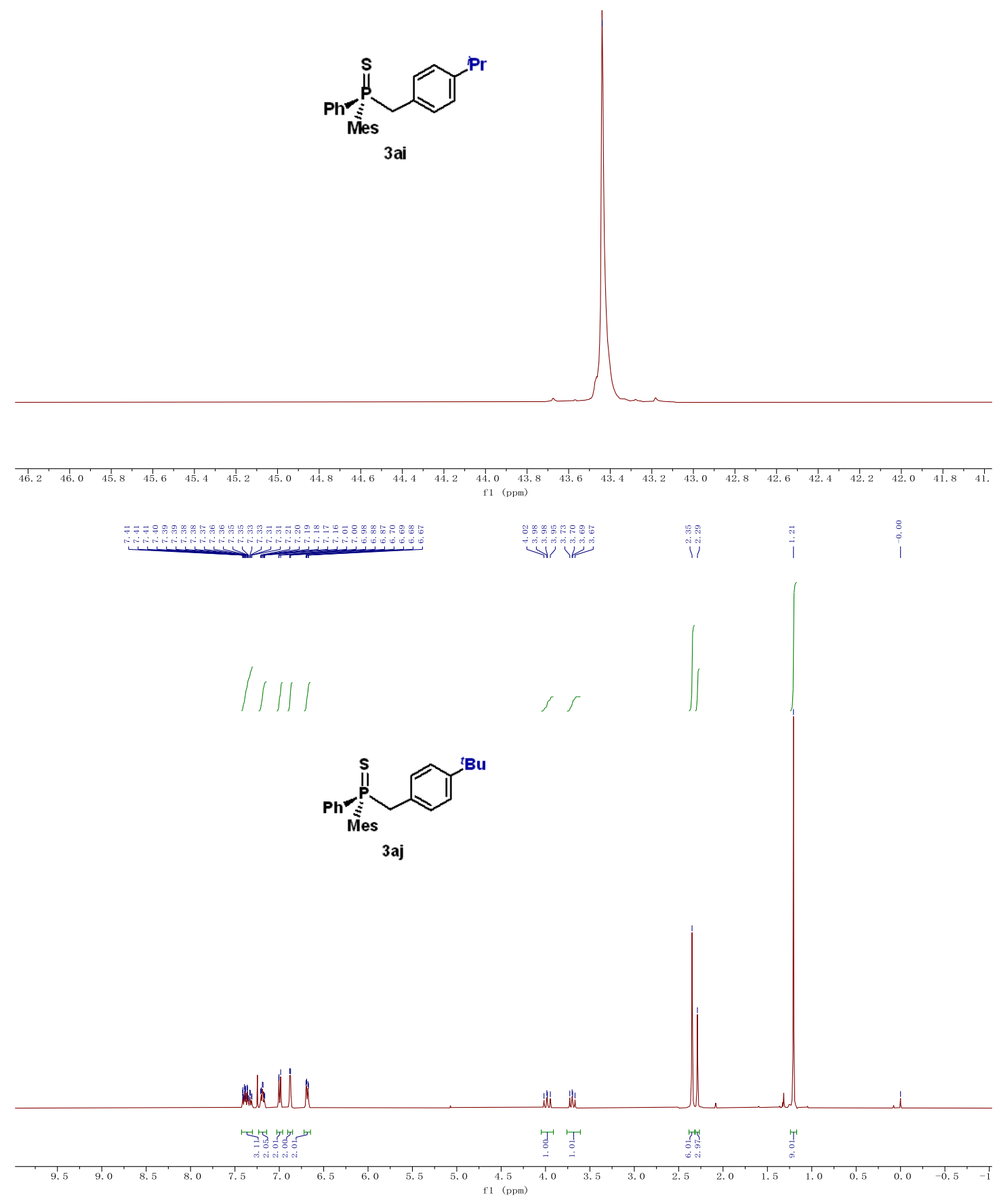




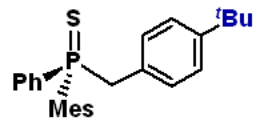

3aj
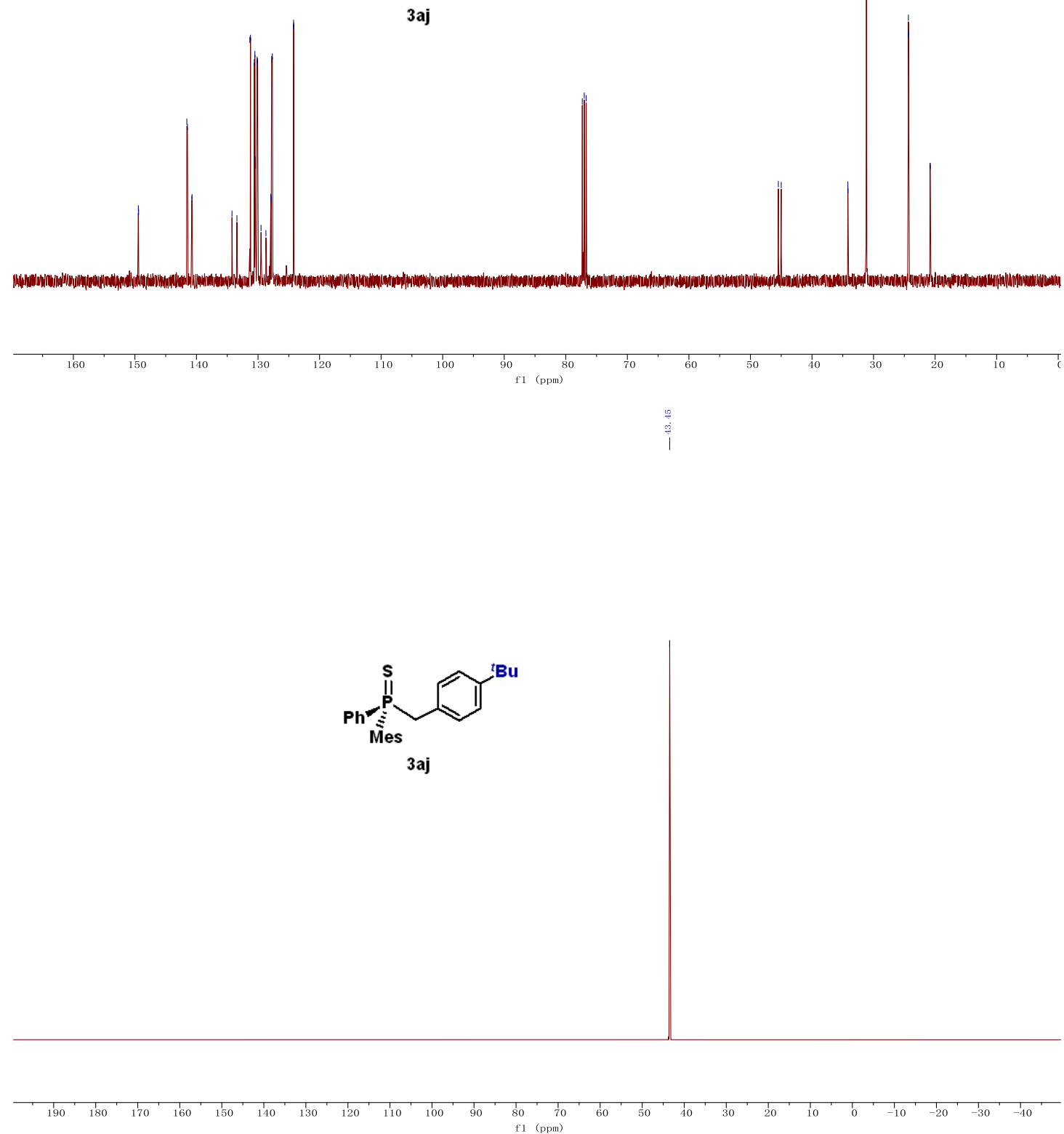


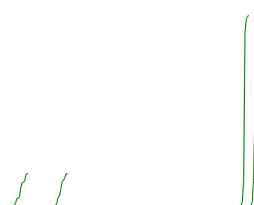<smiles></smiles>
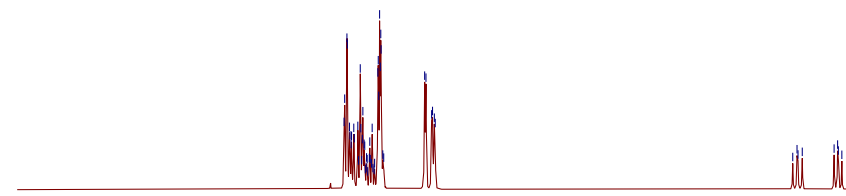

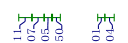

गู่

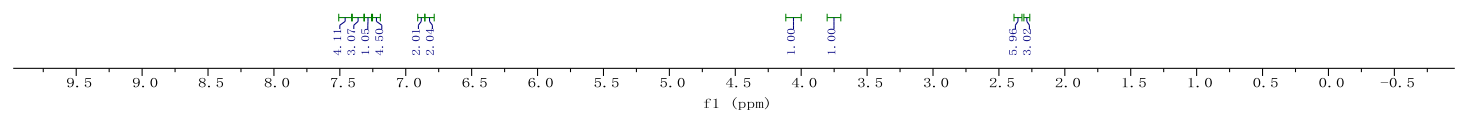

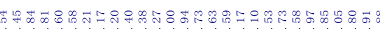

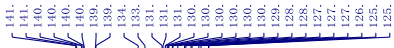
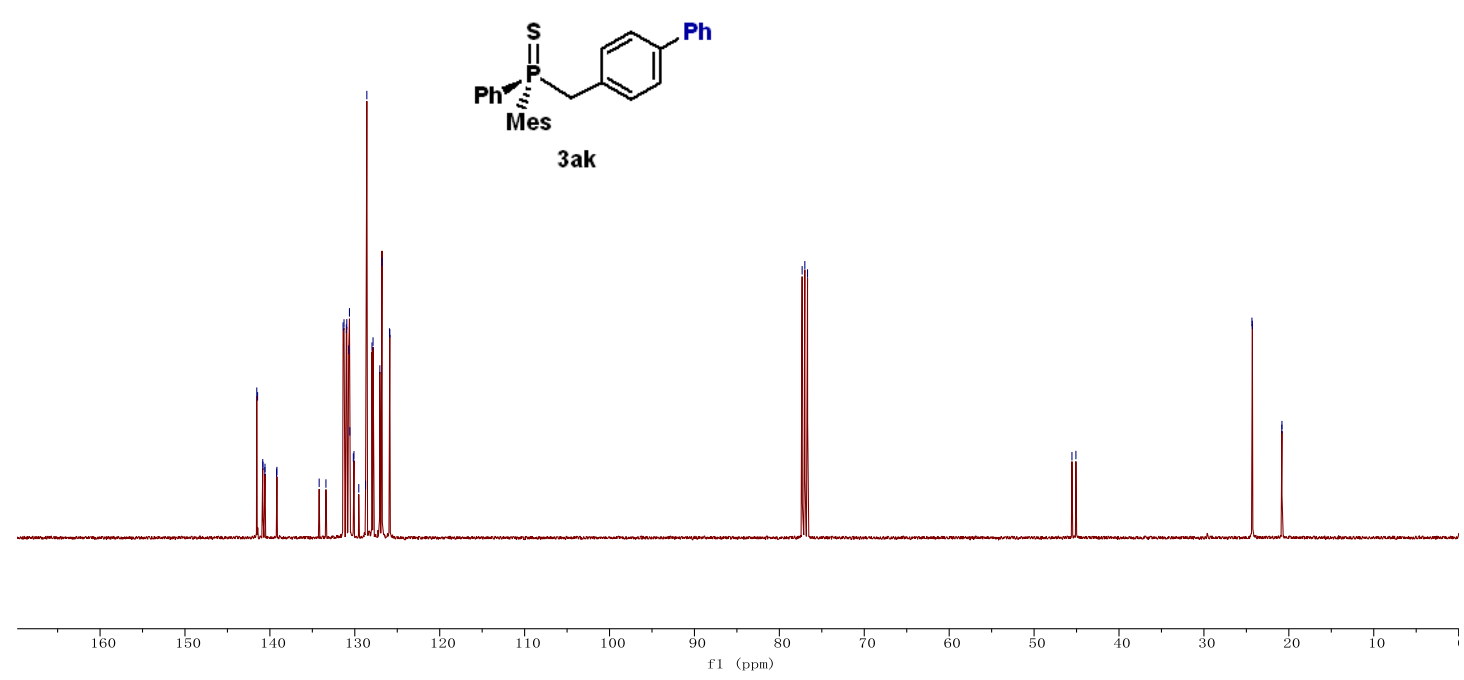

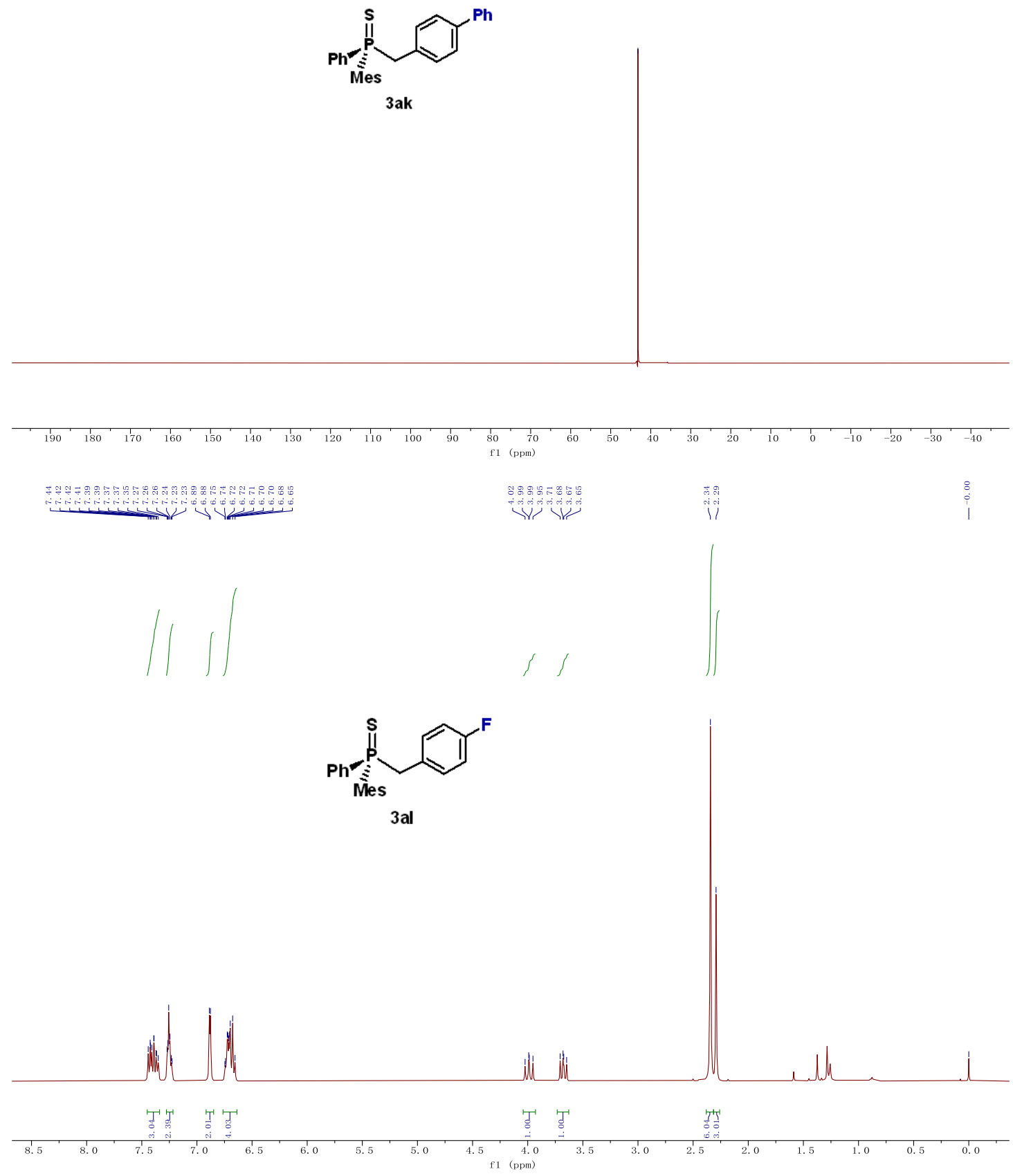

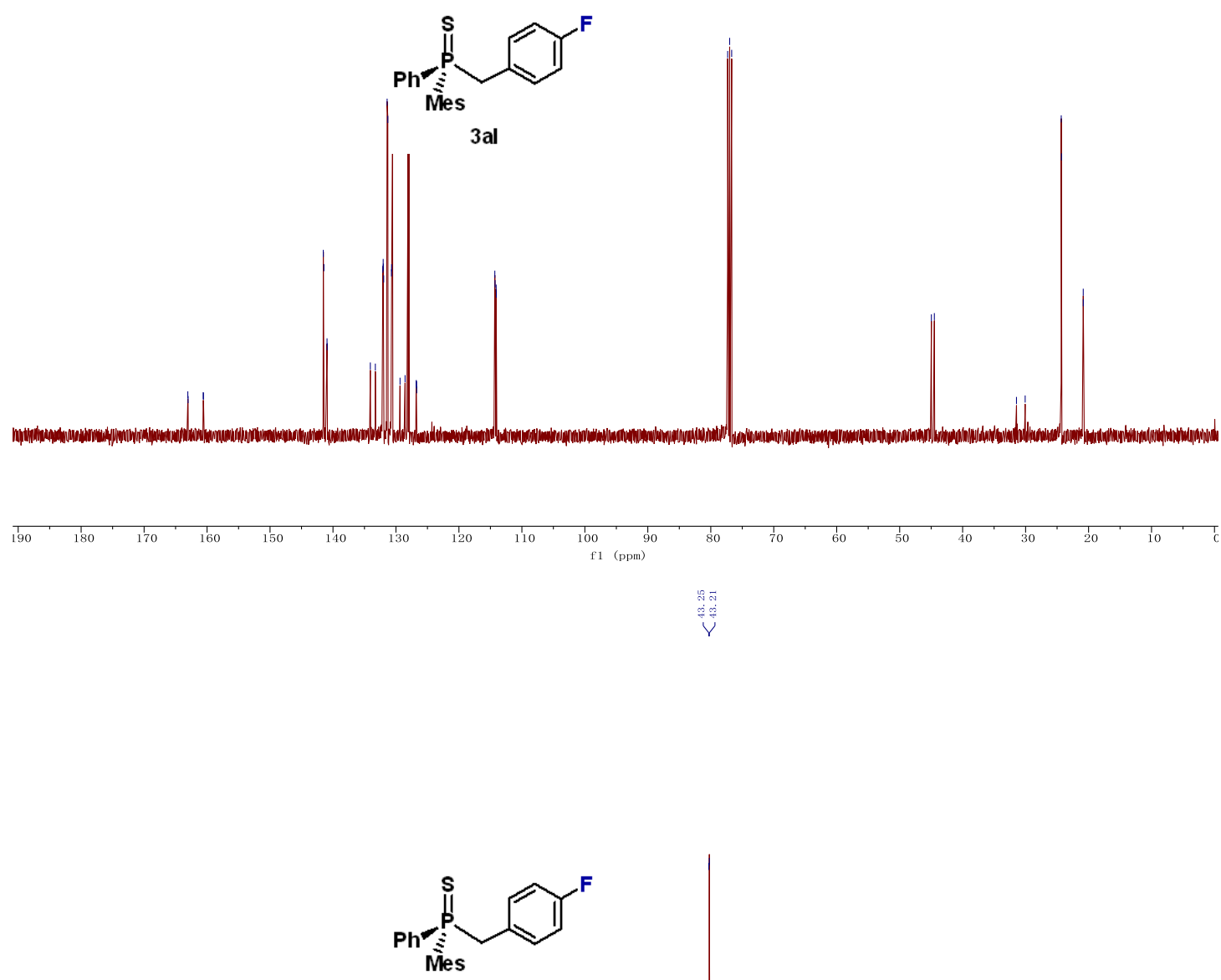

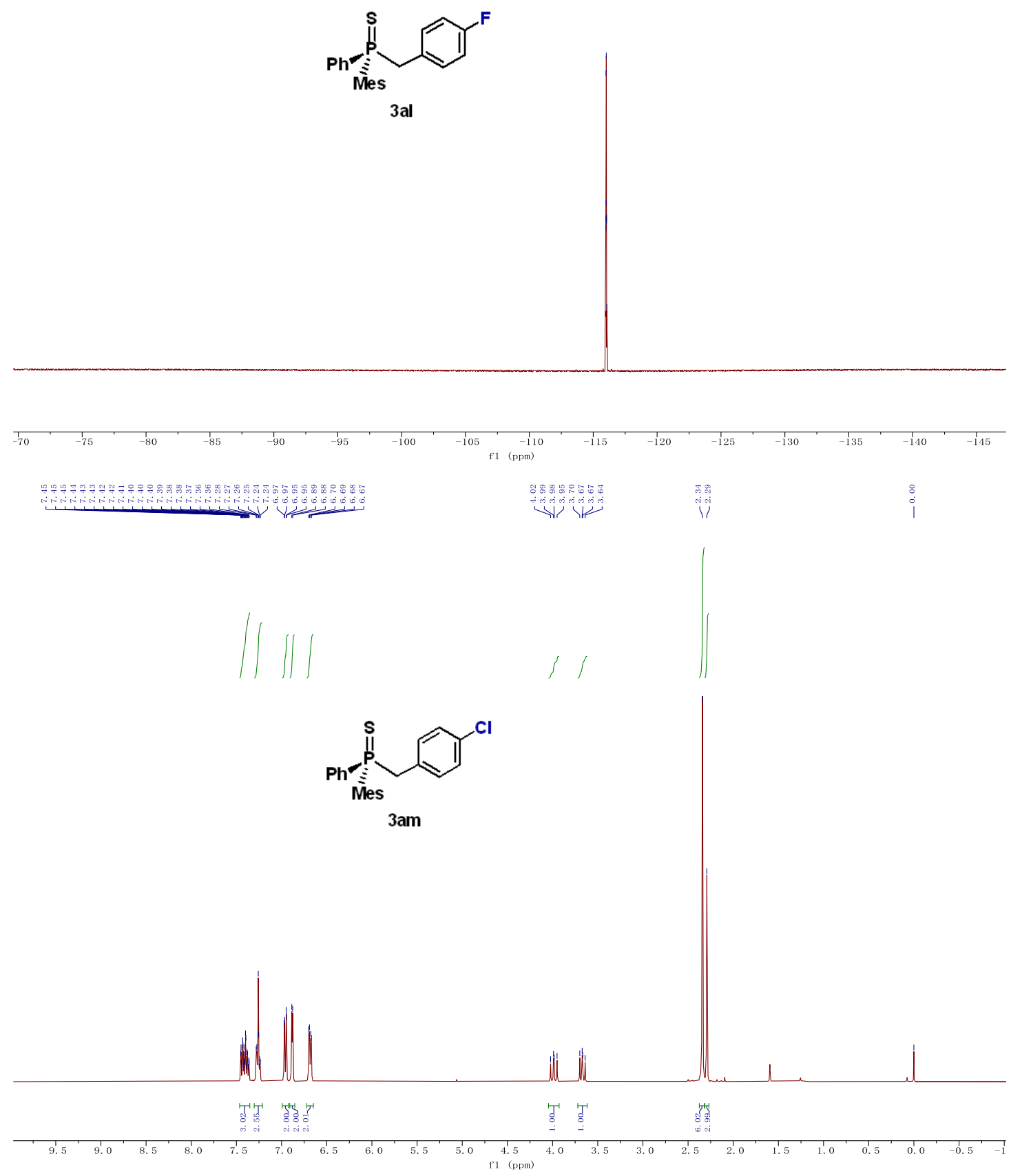


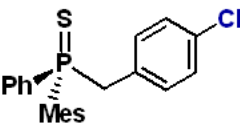

3am

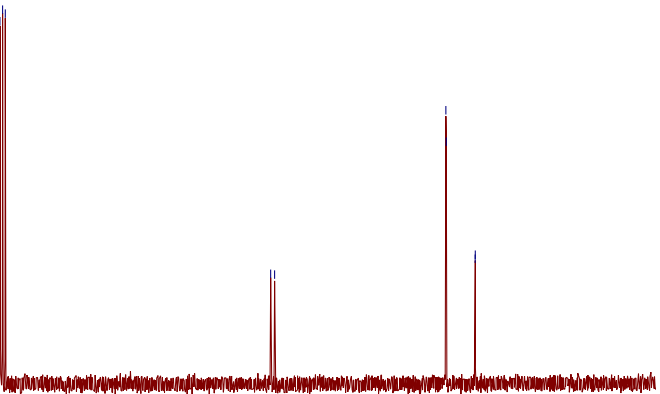

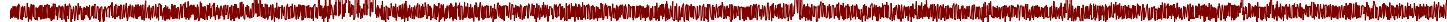
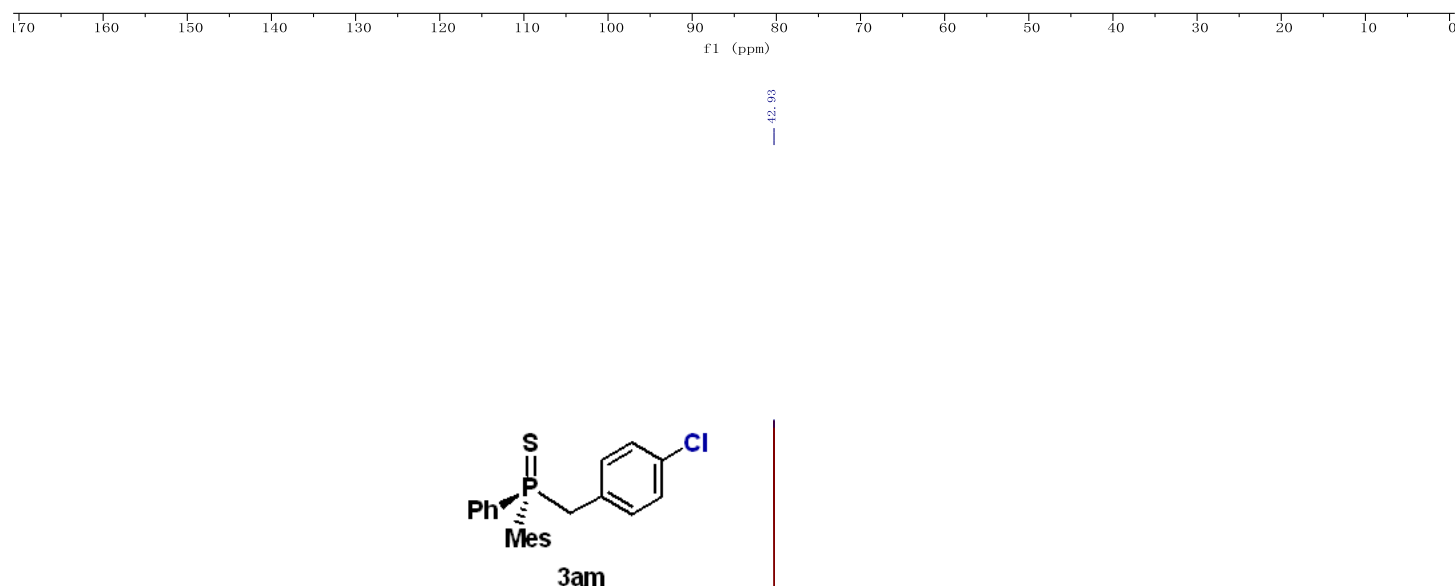

3am

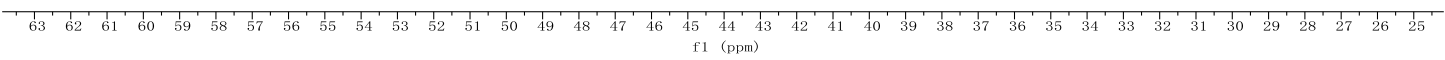



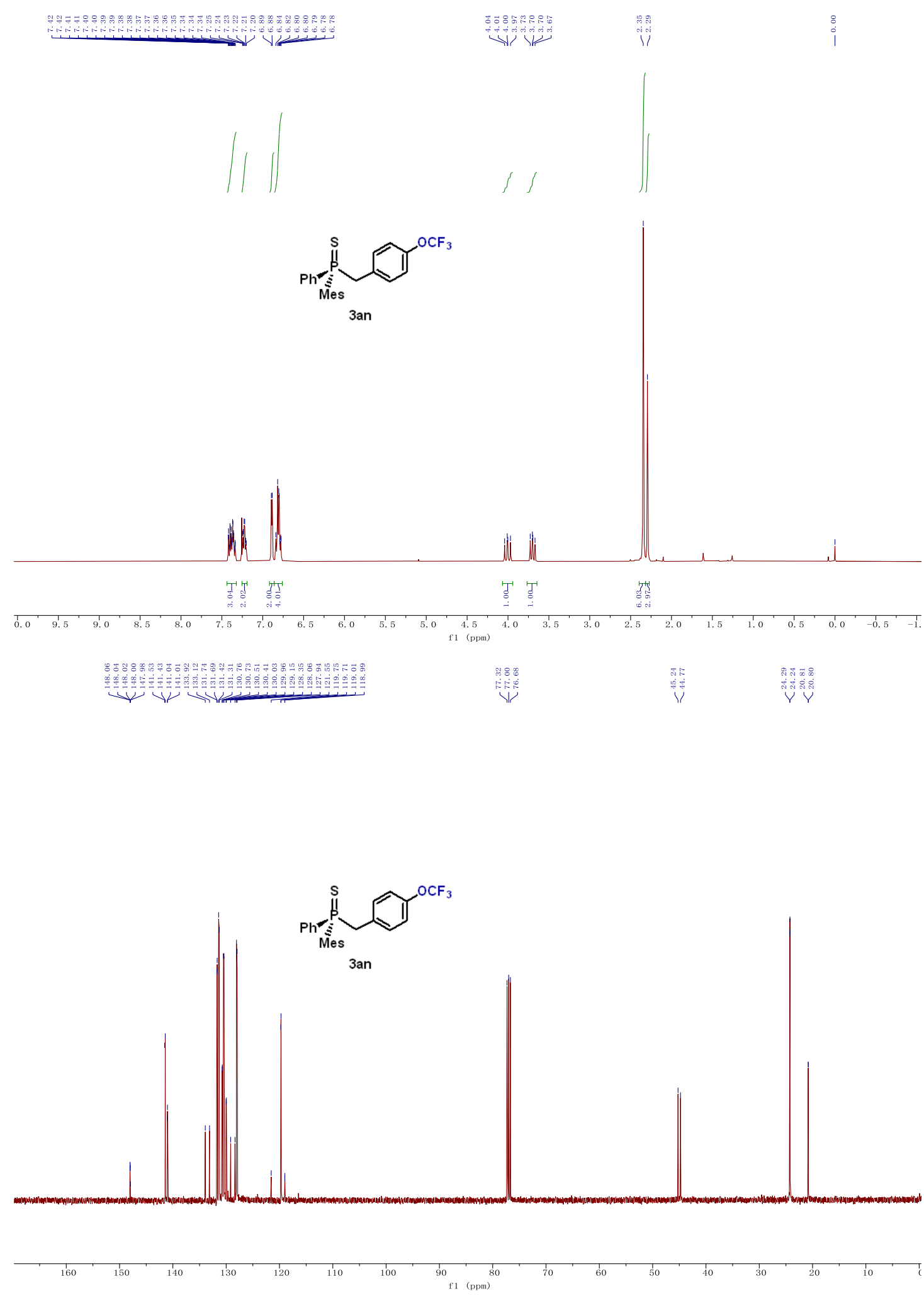


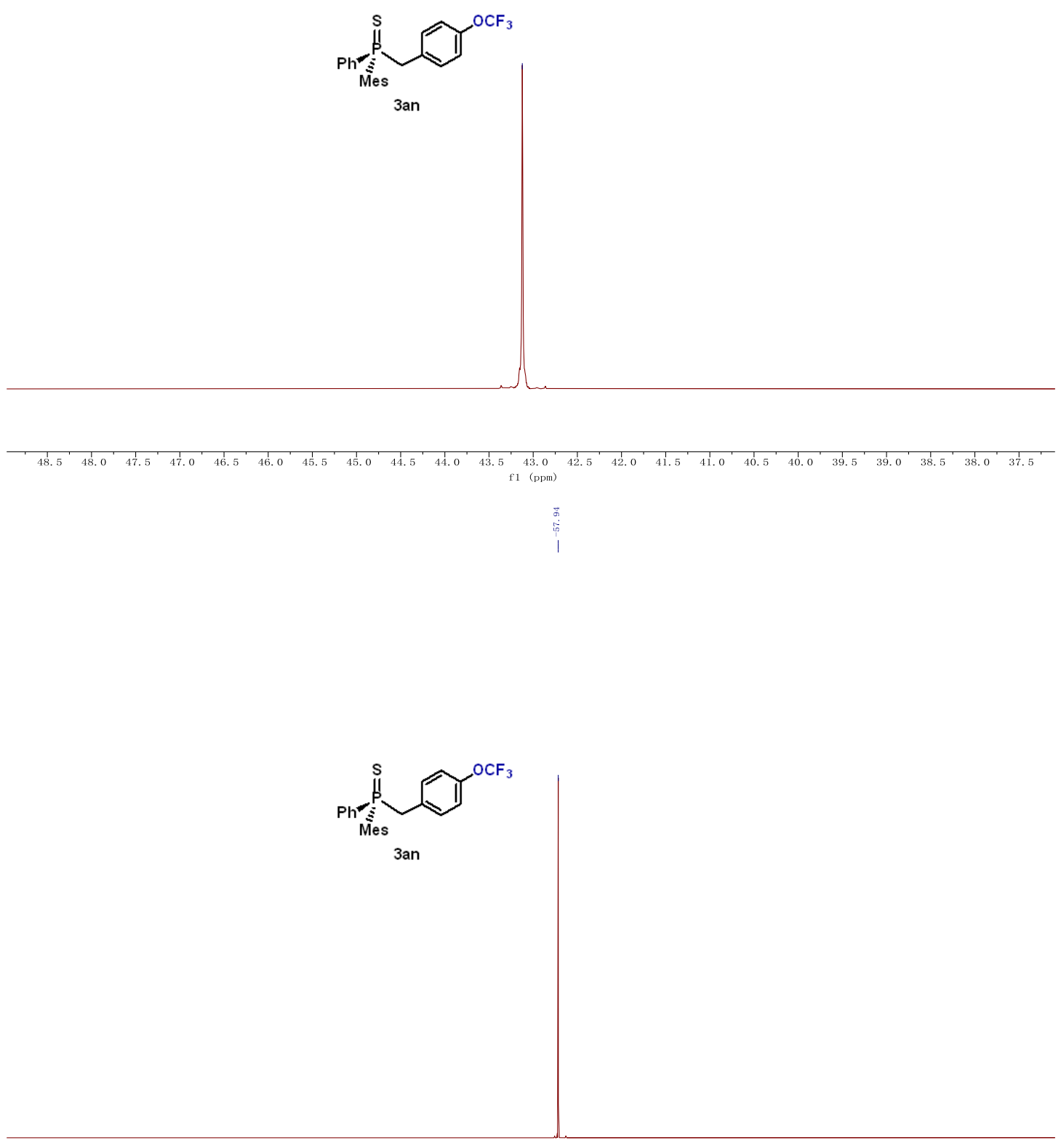

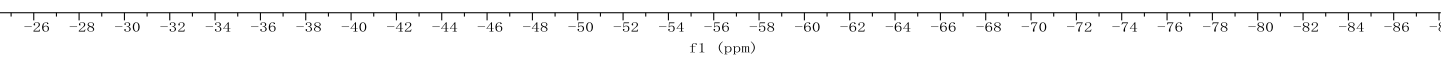




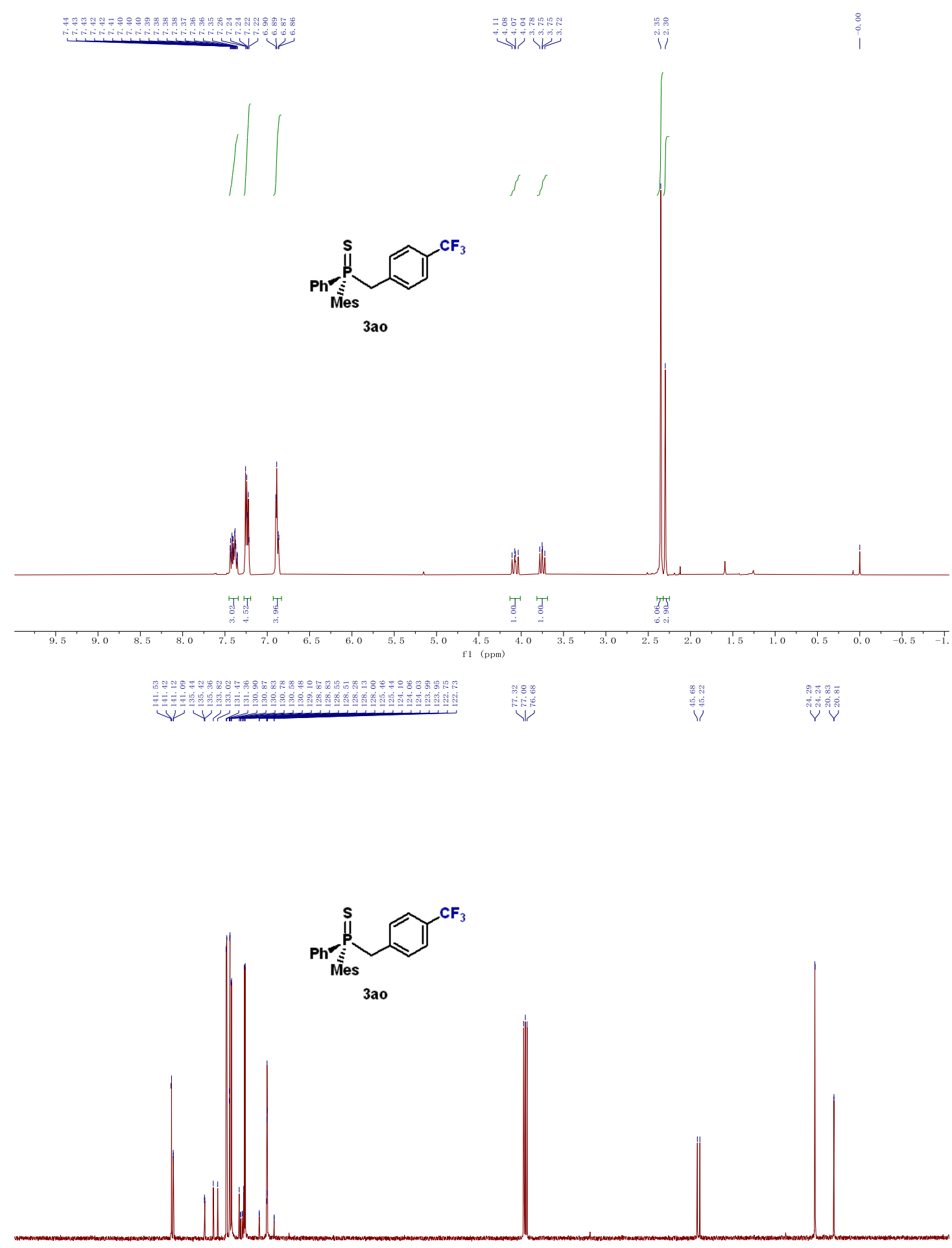

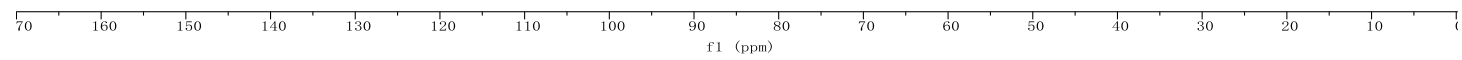




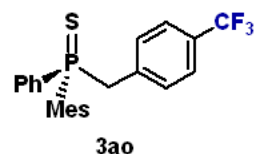

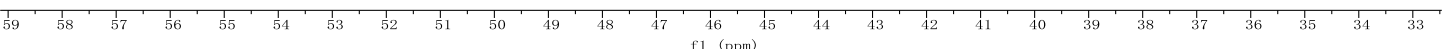

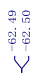

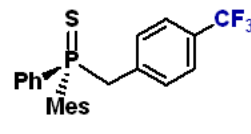

3ao
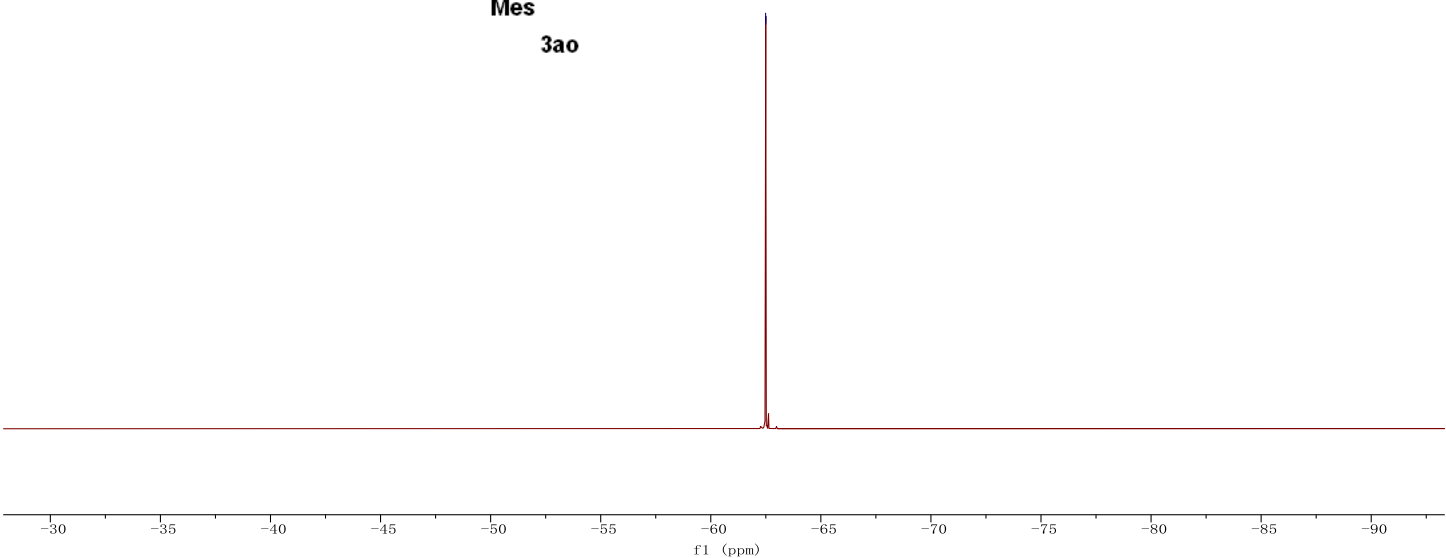

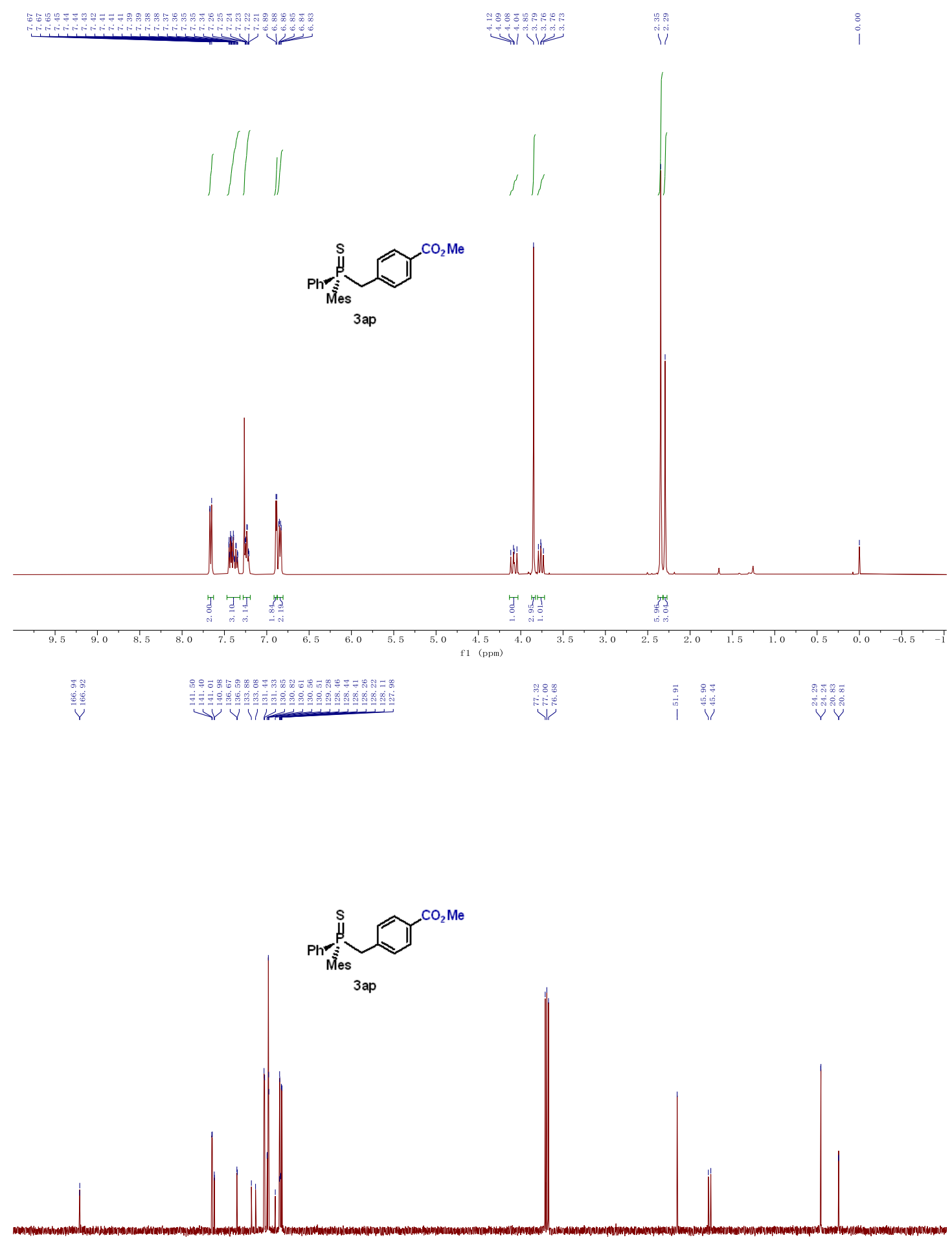

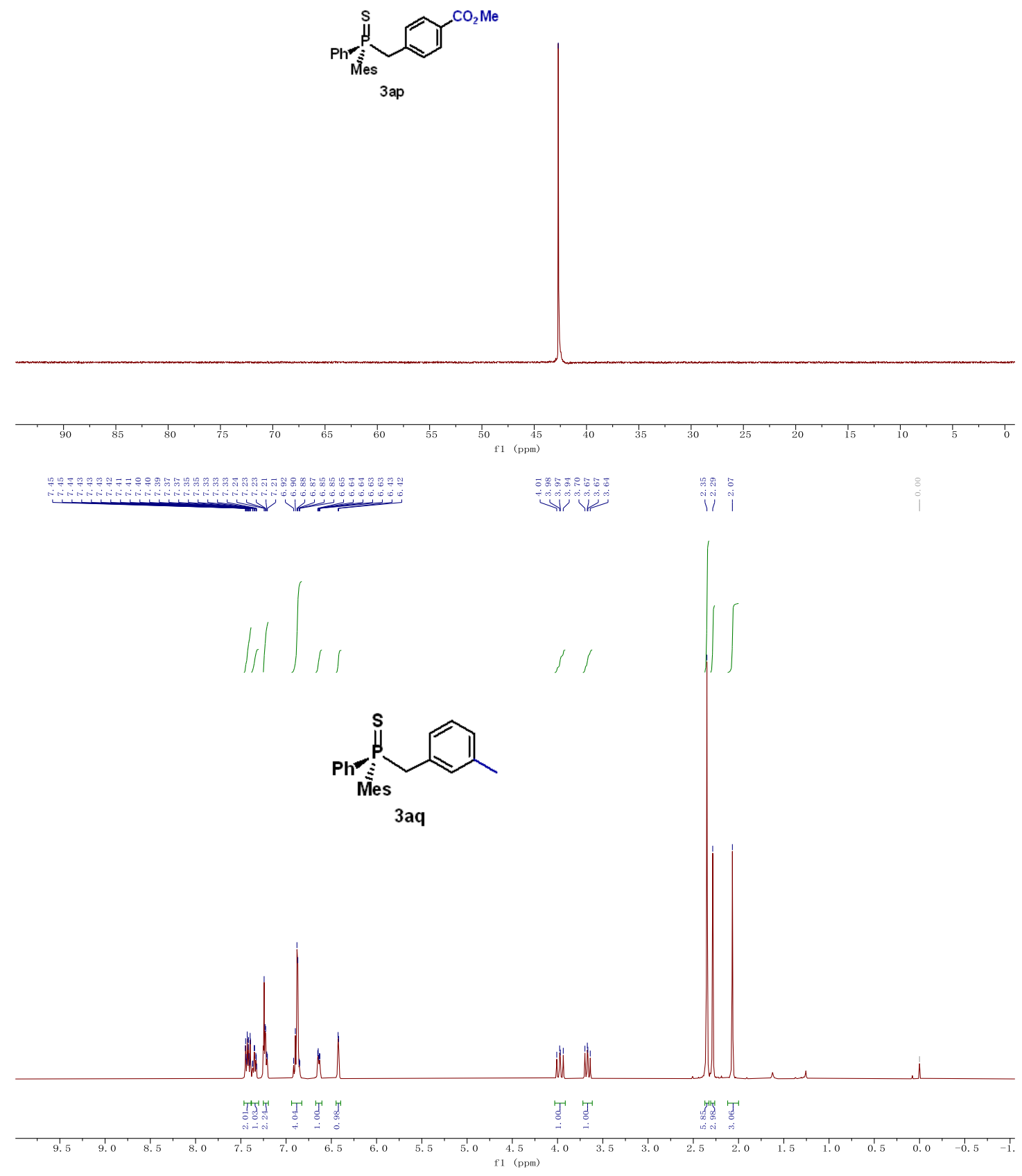

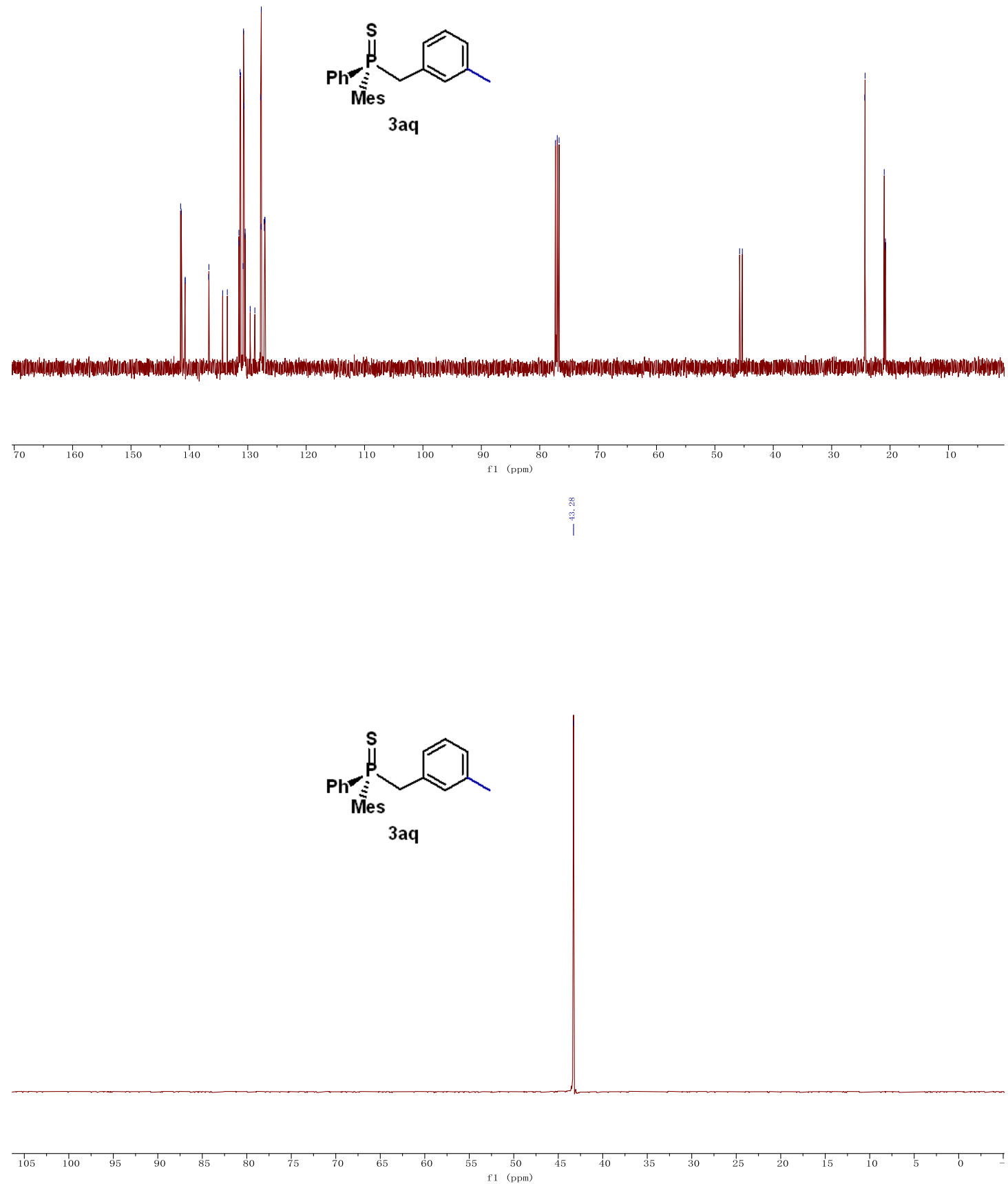

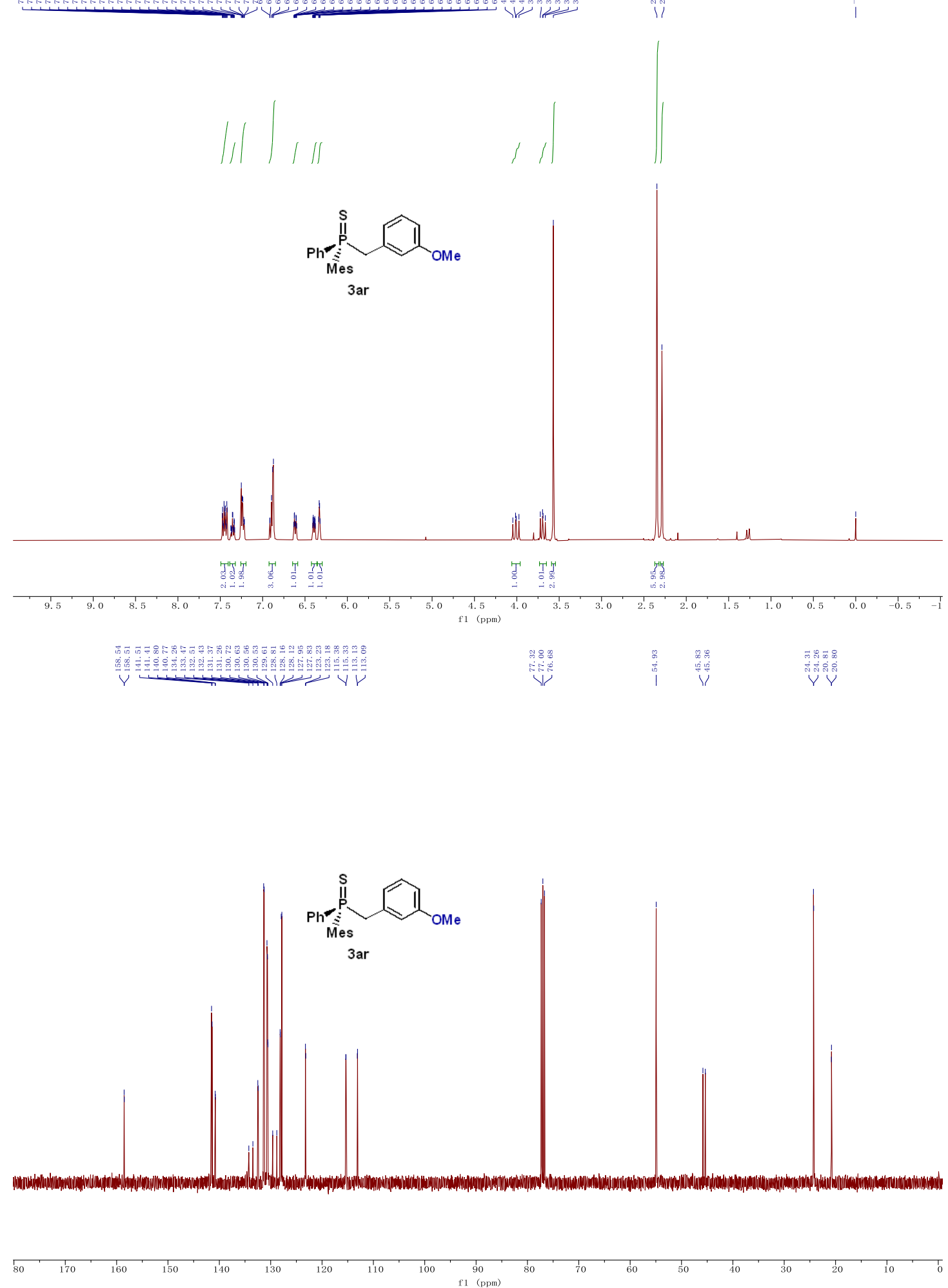


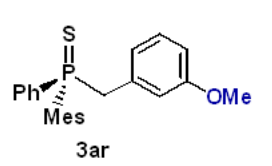

e 

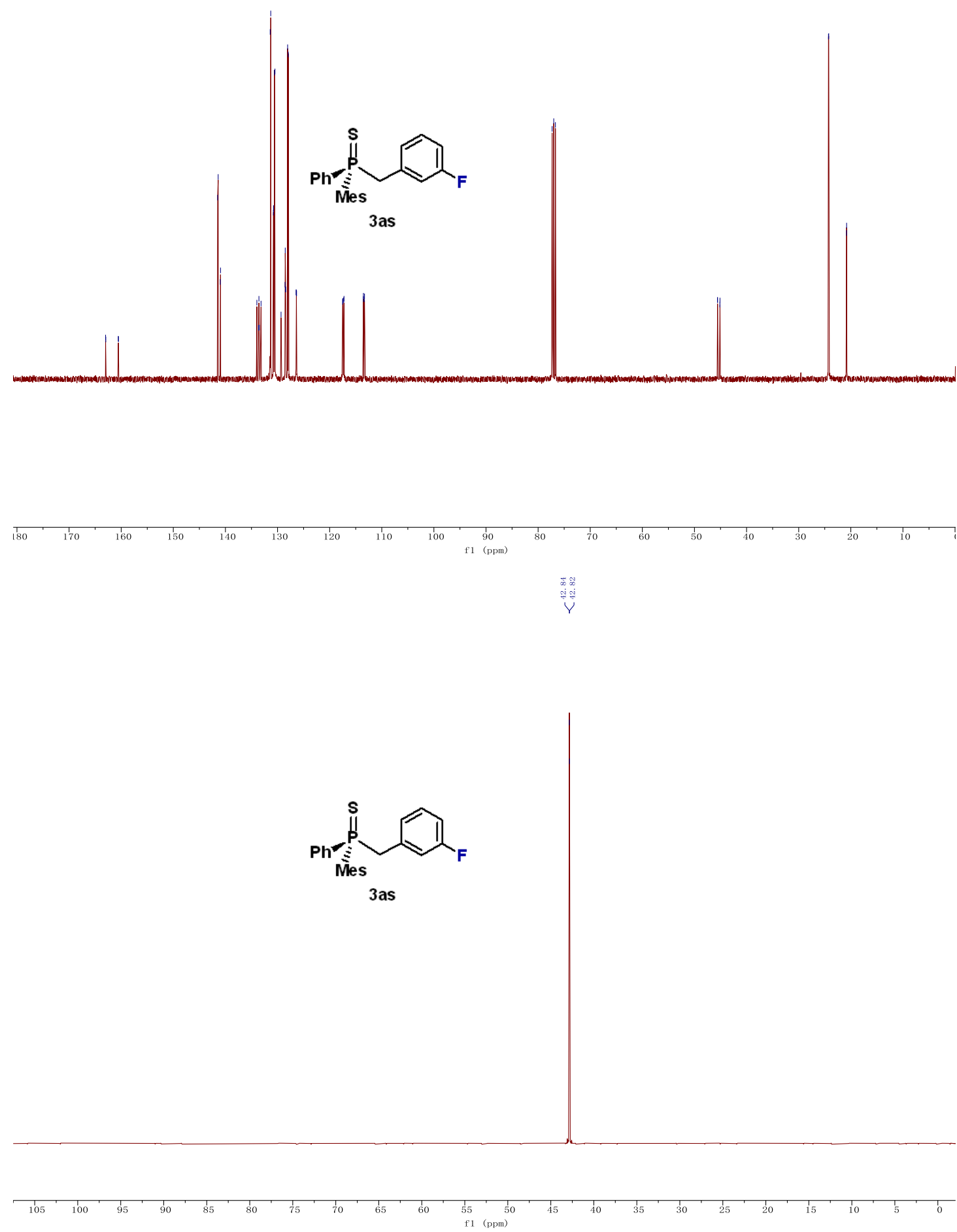


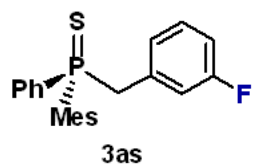

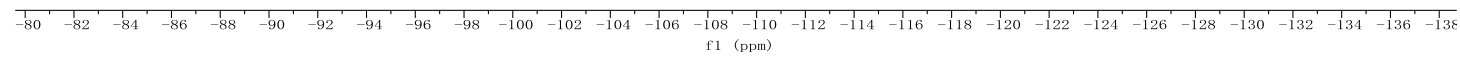

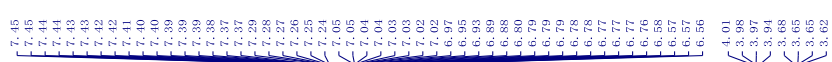

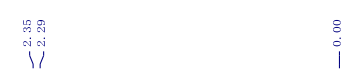

$\int 1 d \int 1$

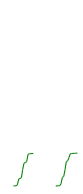<smiles>[Y5][PH](=S)(=S)Cc1cccc(Cl)c1</smiles>

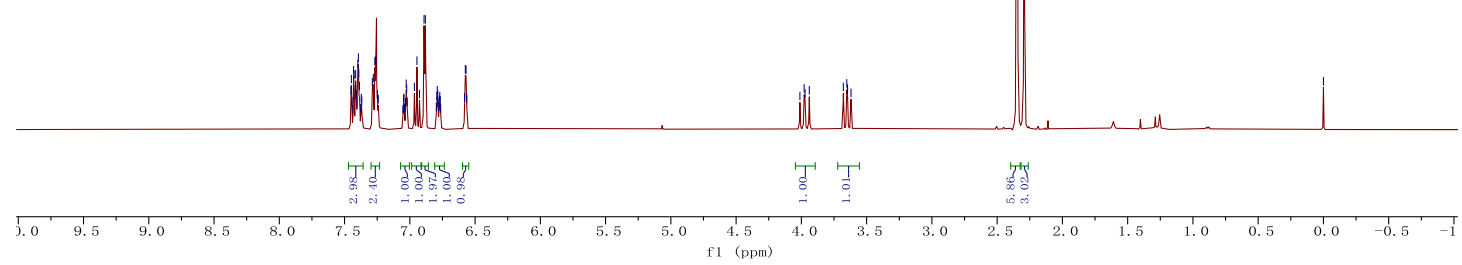



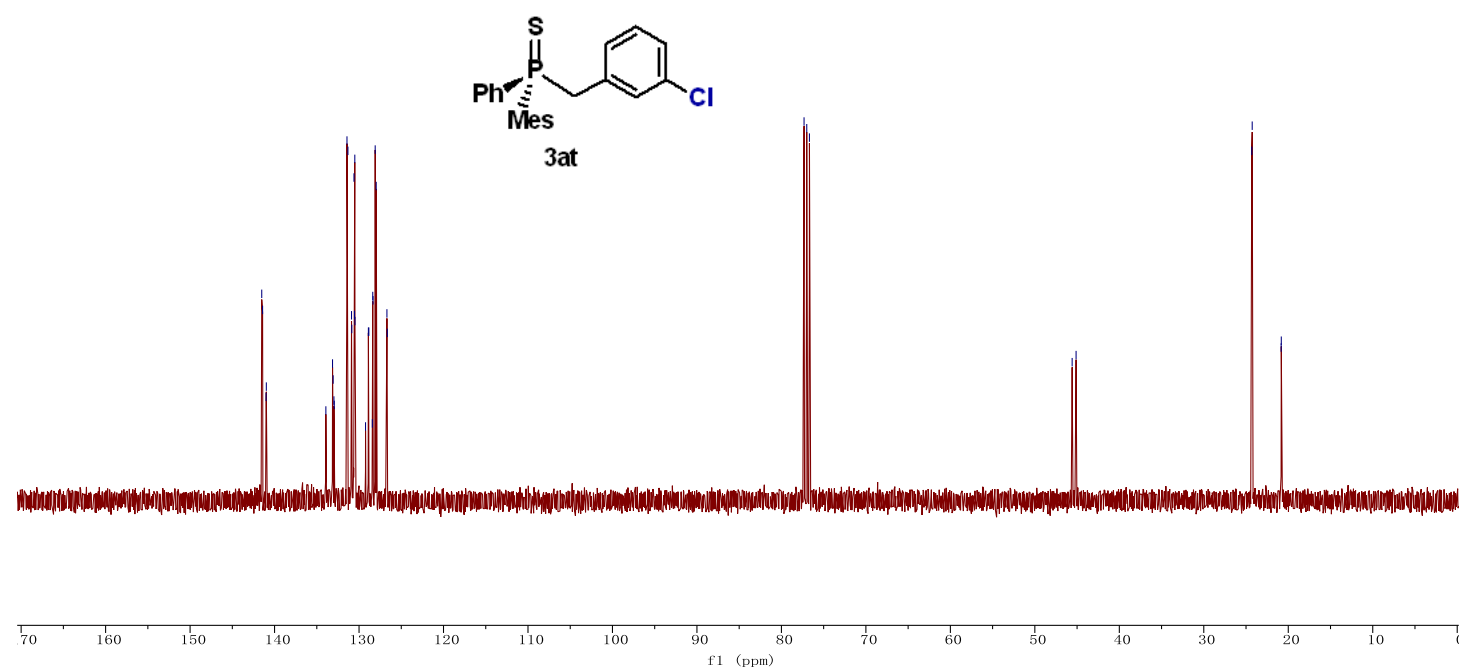<smiles>C[SH](=S)(Cc1cccc(Cl)c1)c1ccccc1</smiles> 

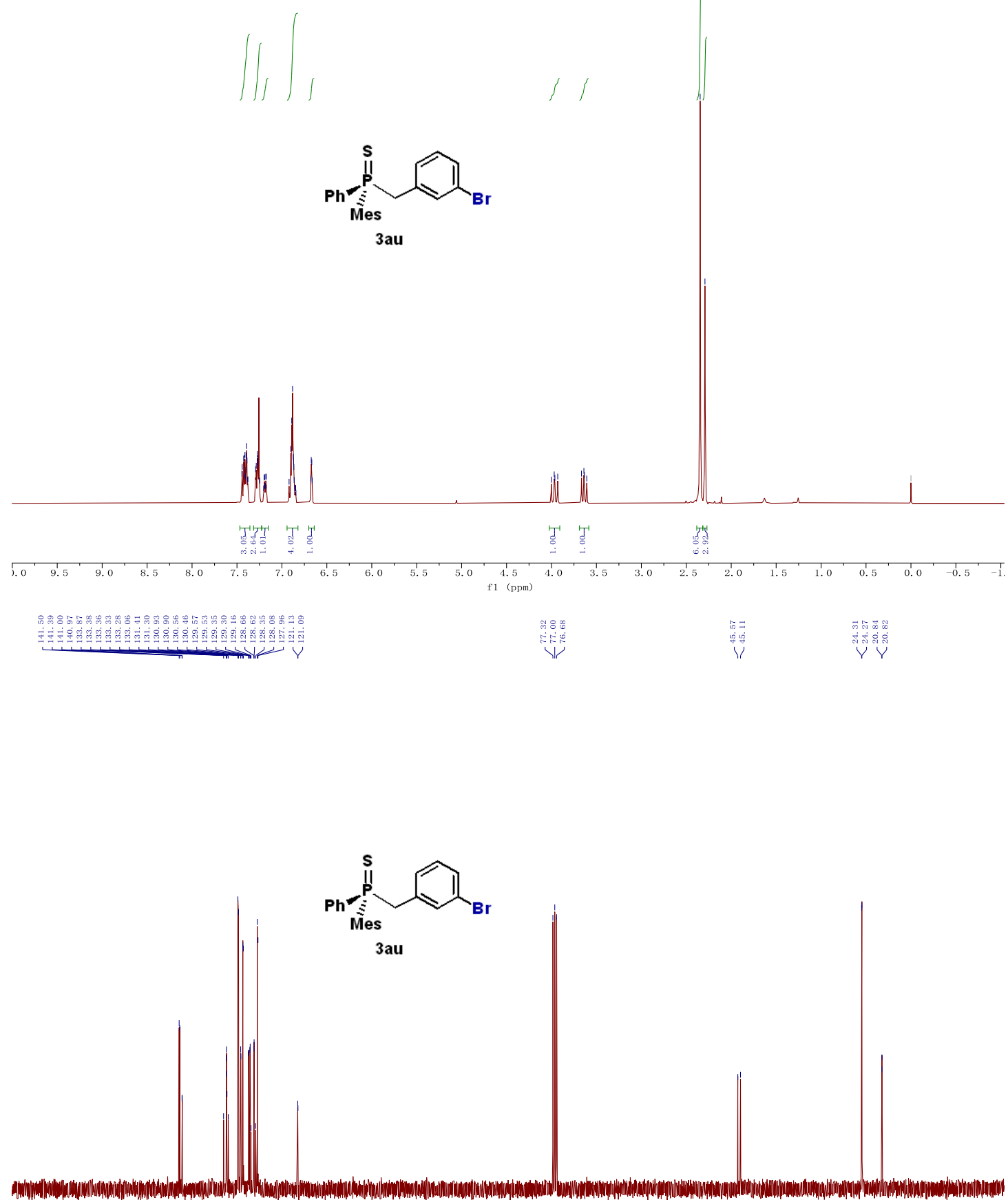

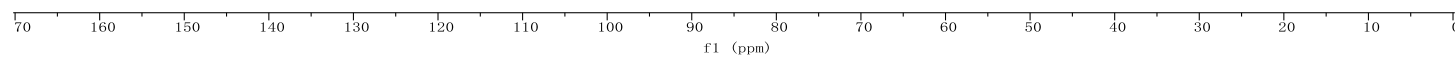



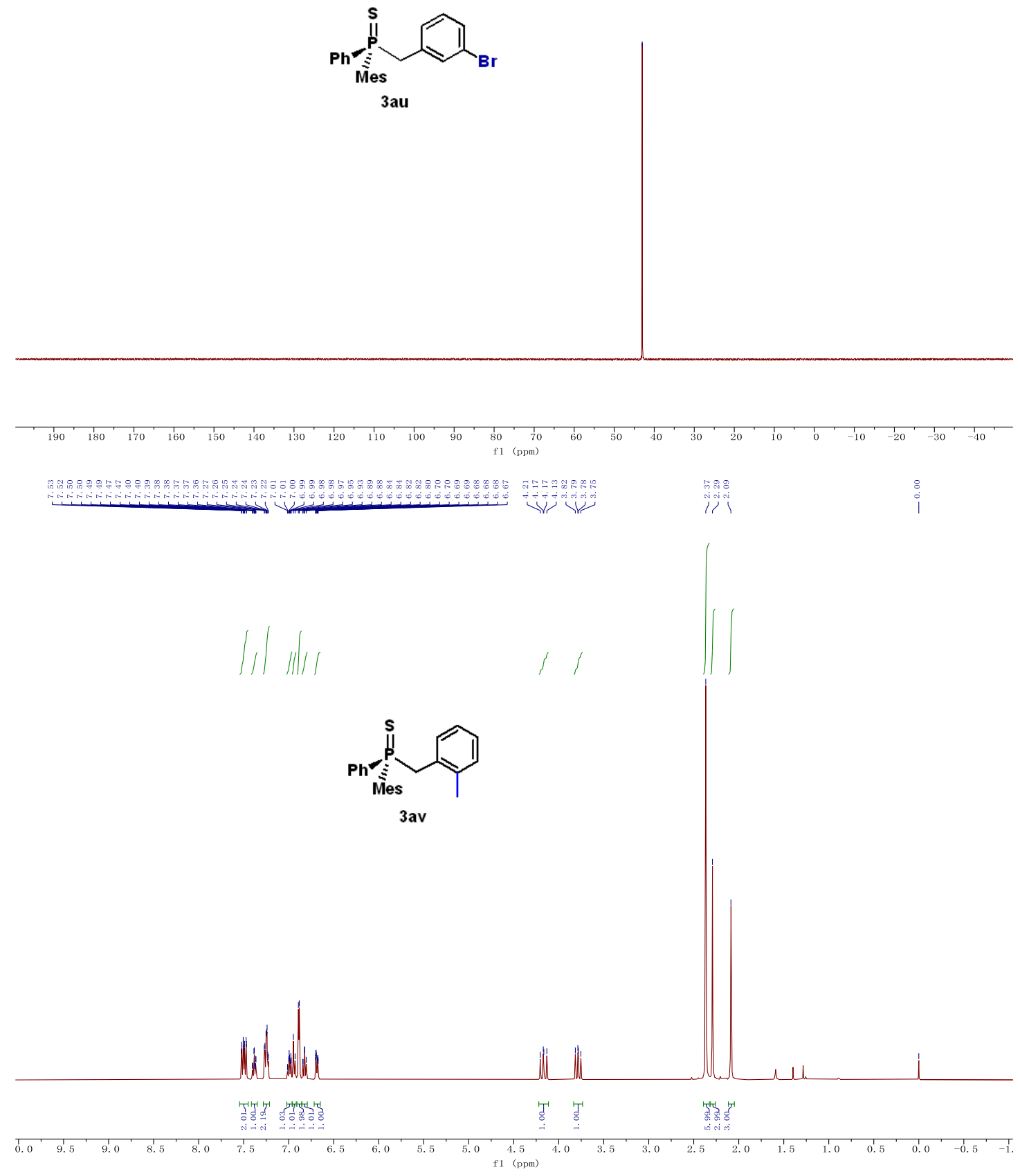

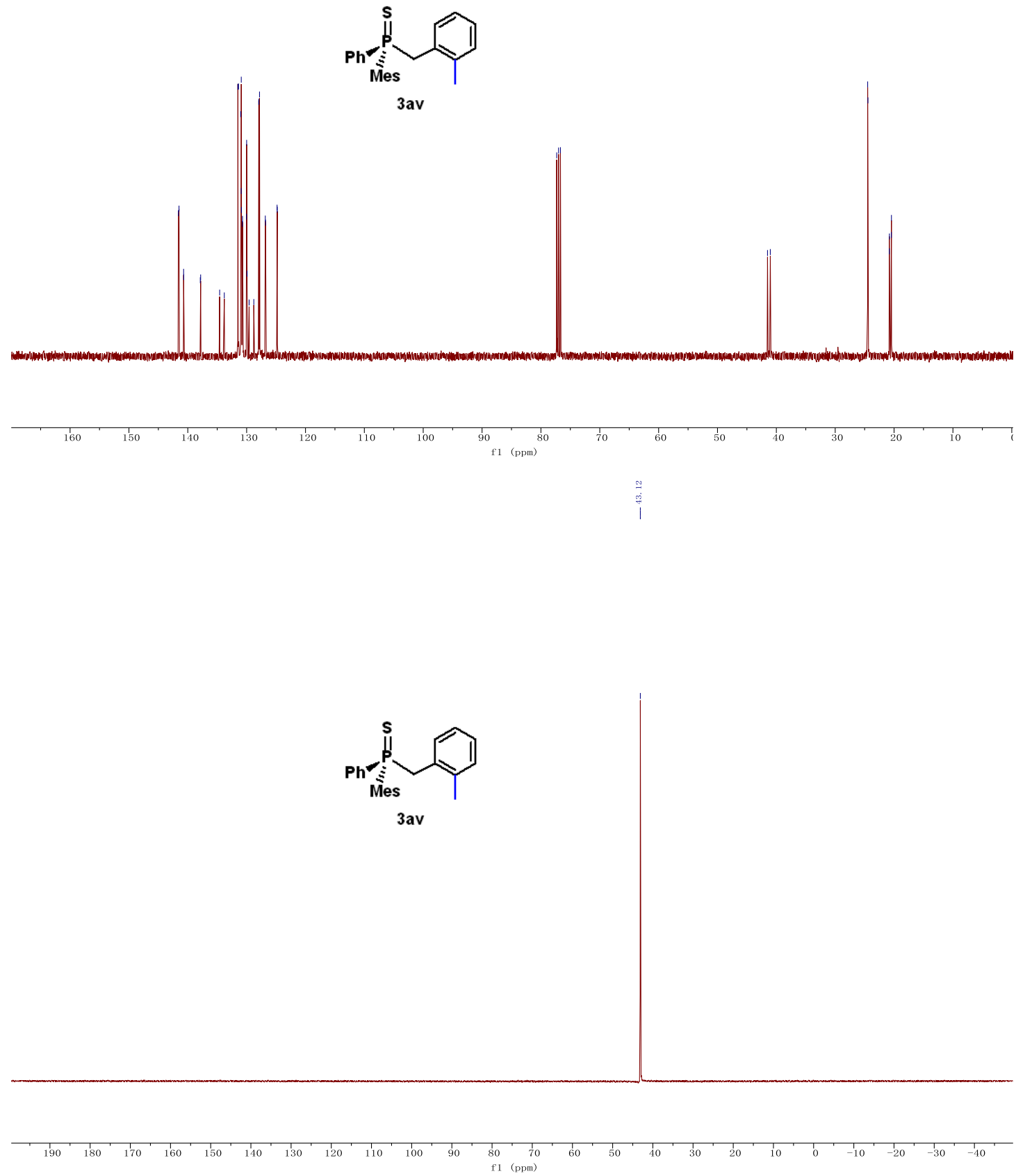

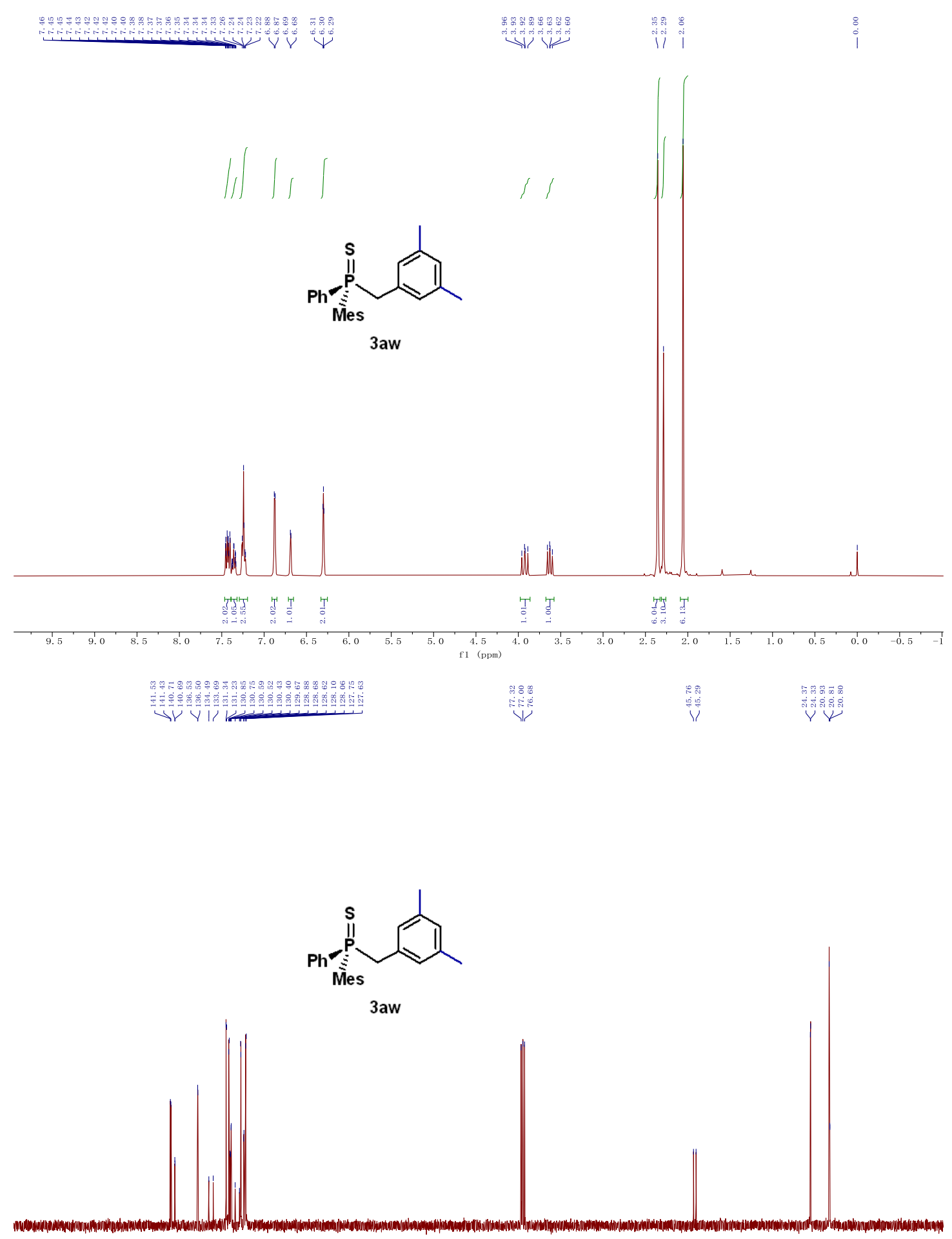

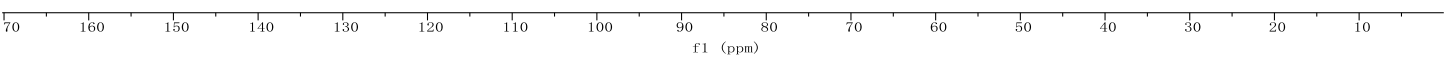



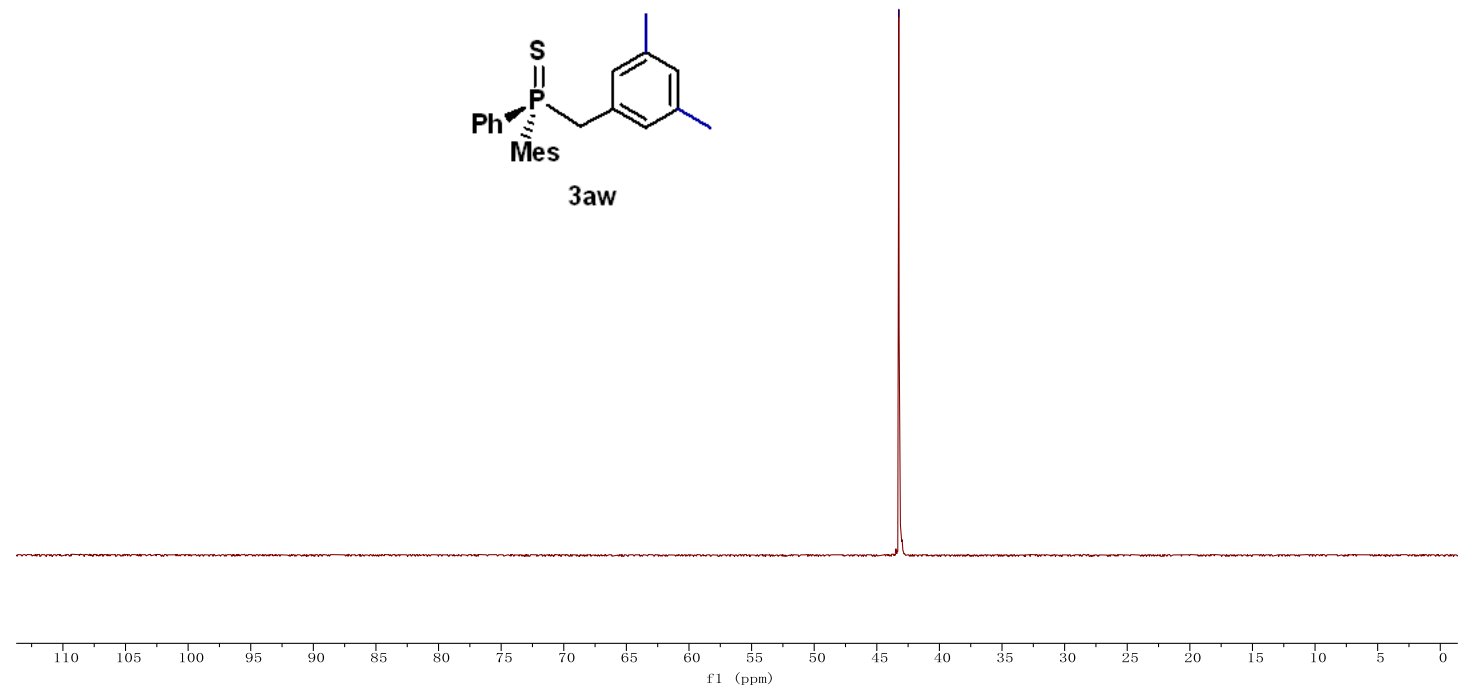

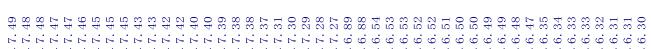

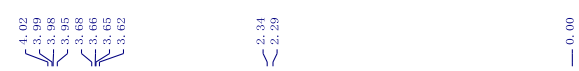

$\int 1 \int$
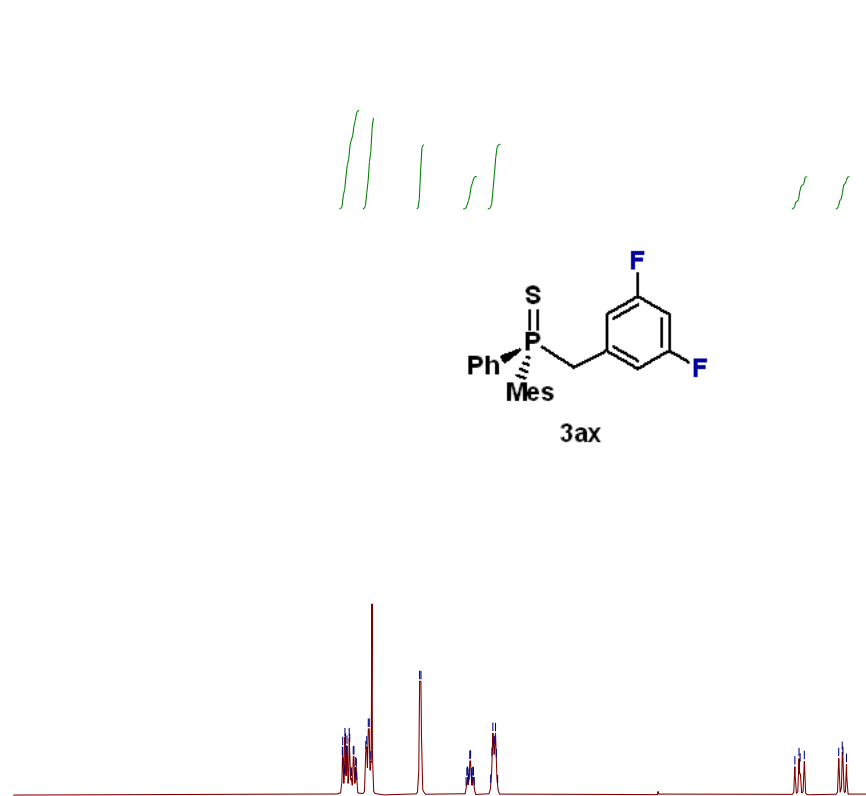

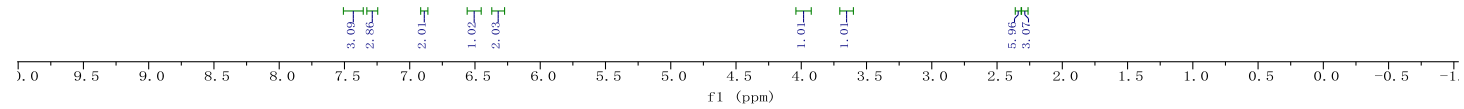



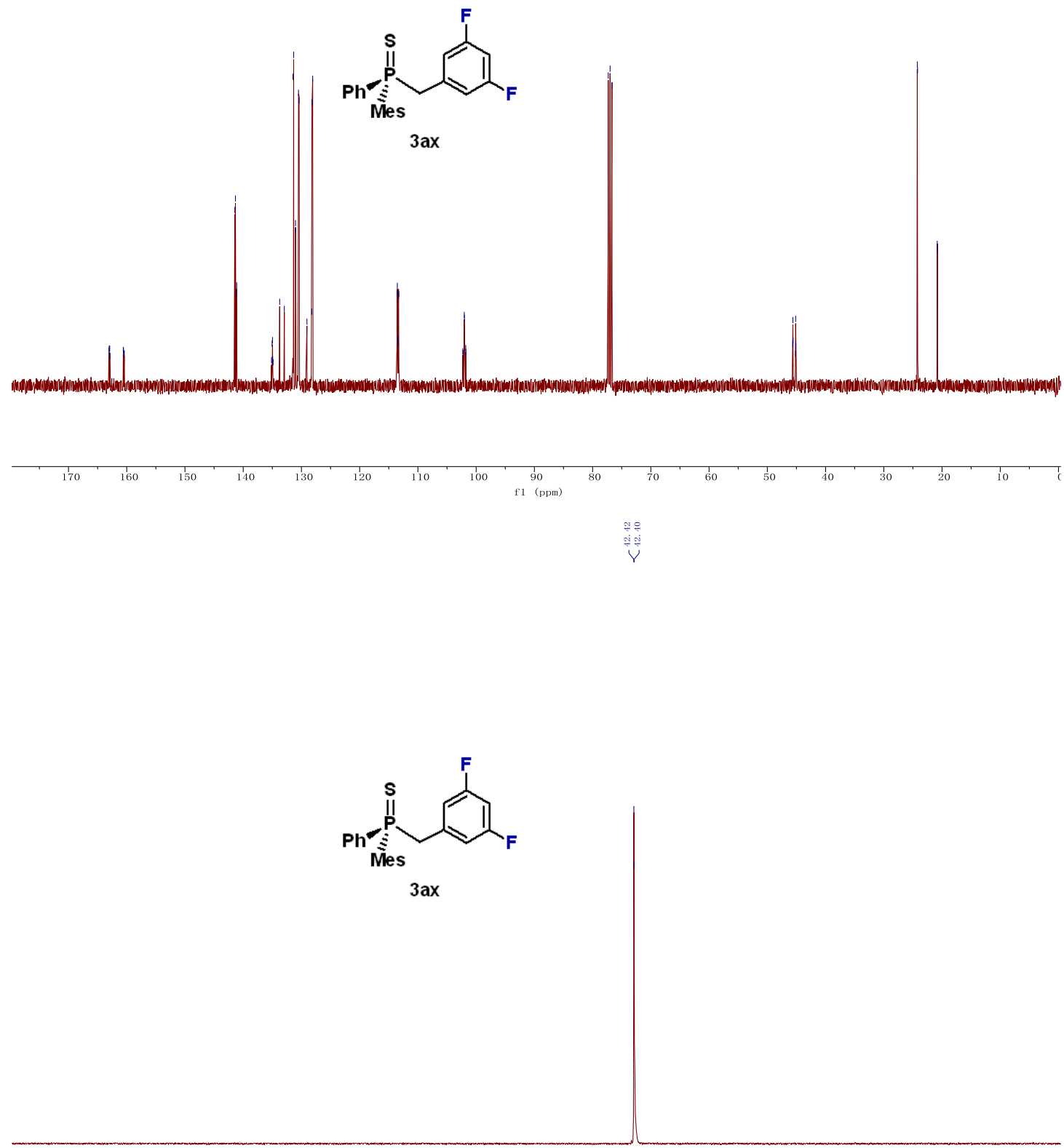

$\stackrel{1}{100}$

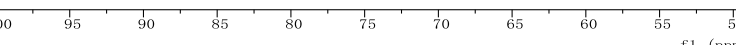



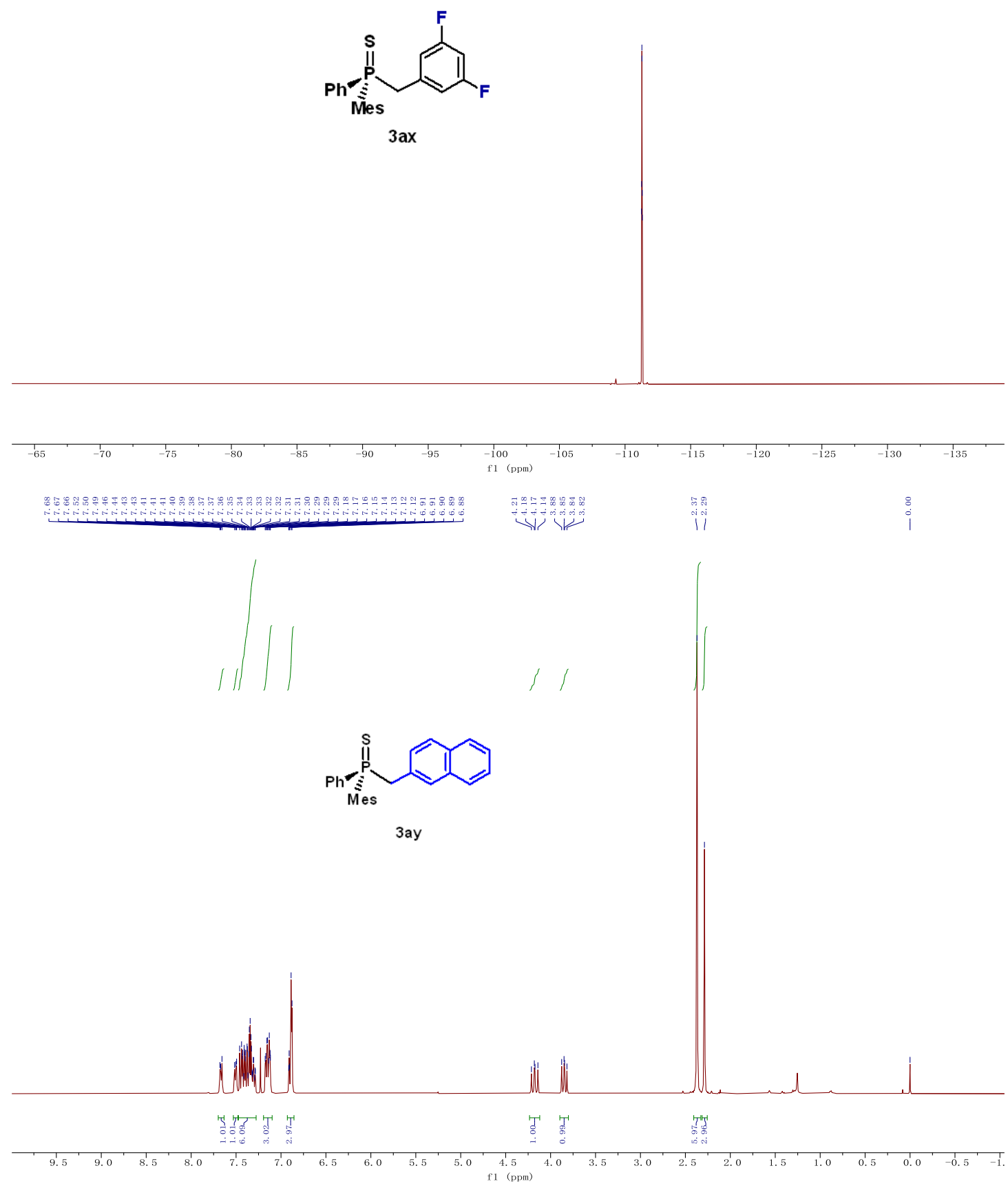

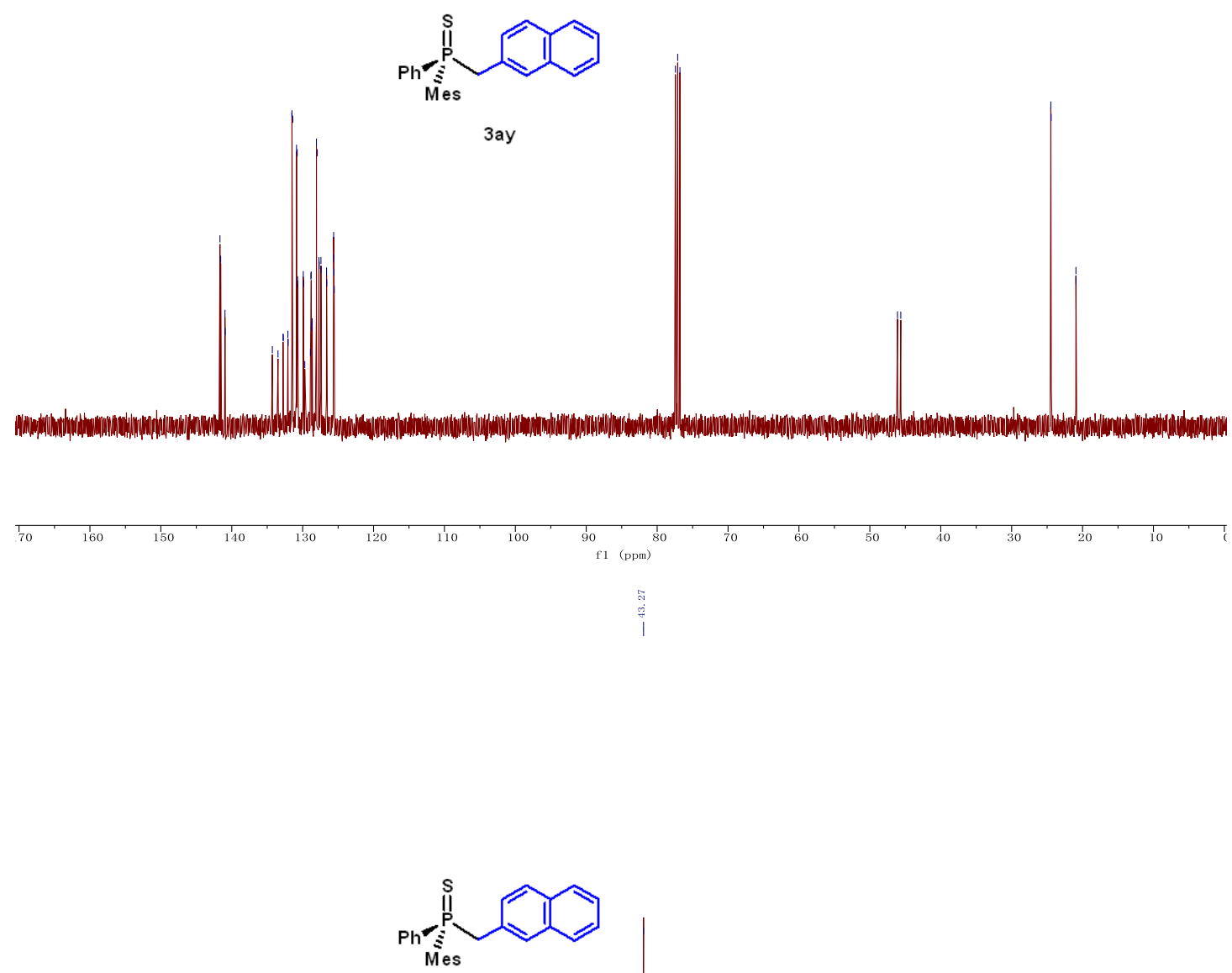

3ay

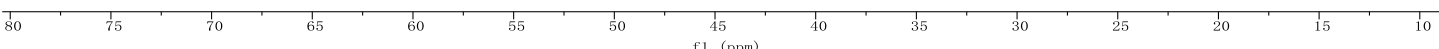



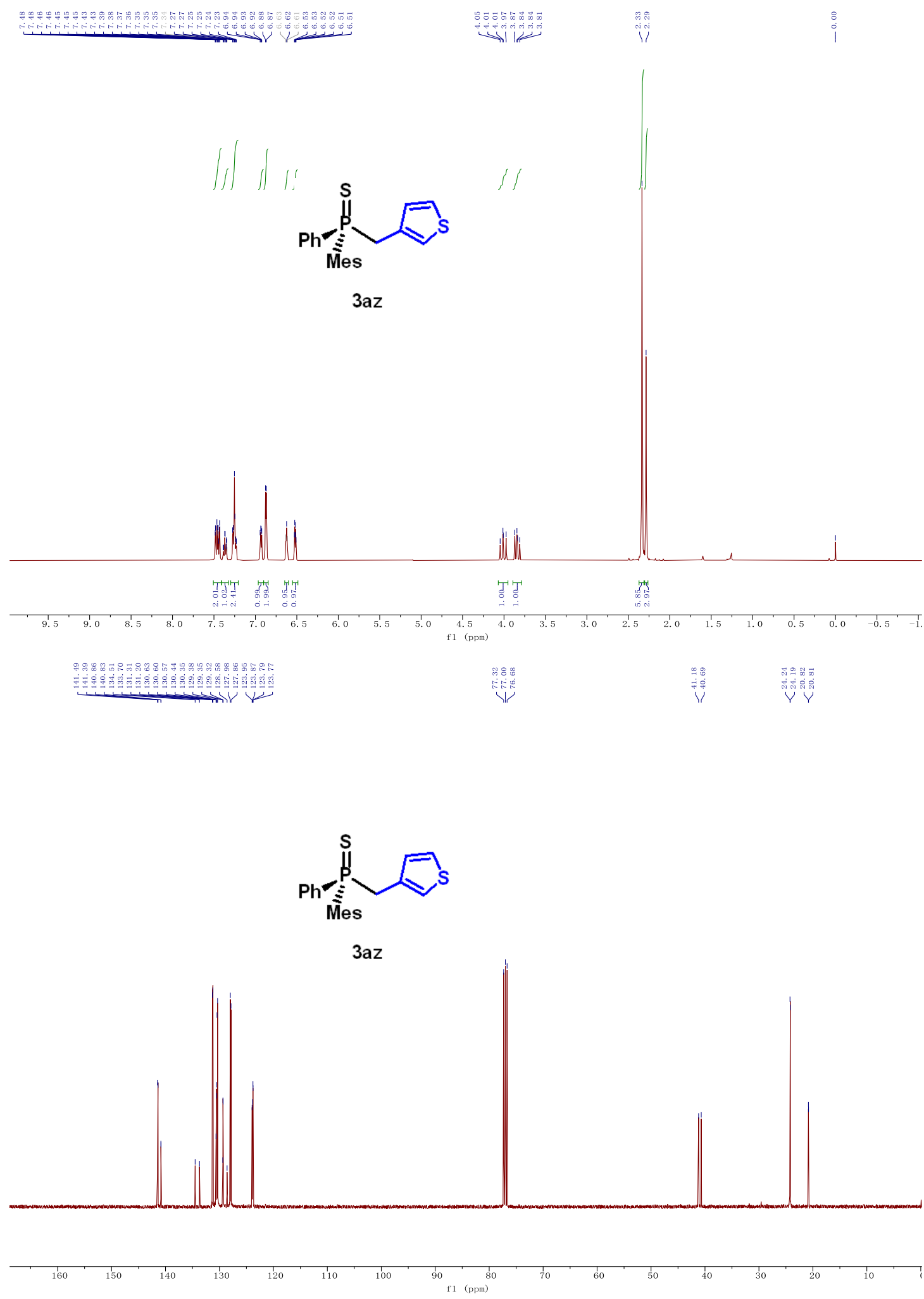


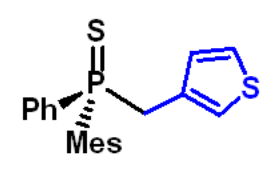

$3 a z$

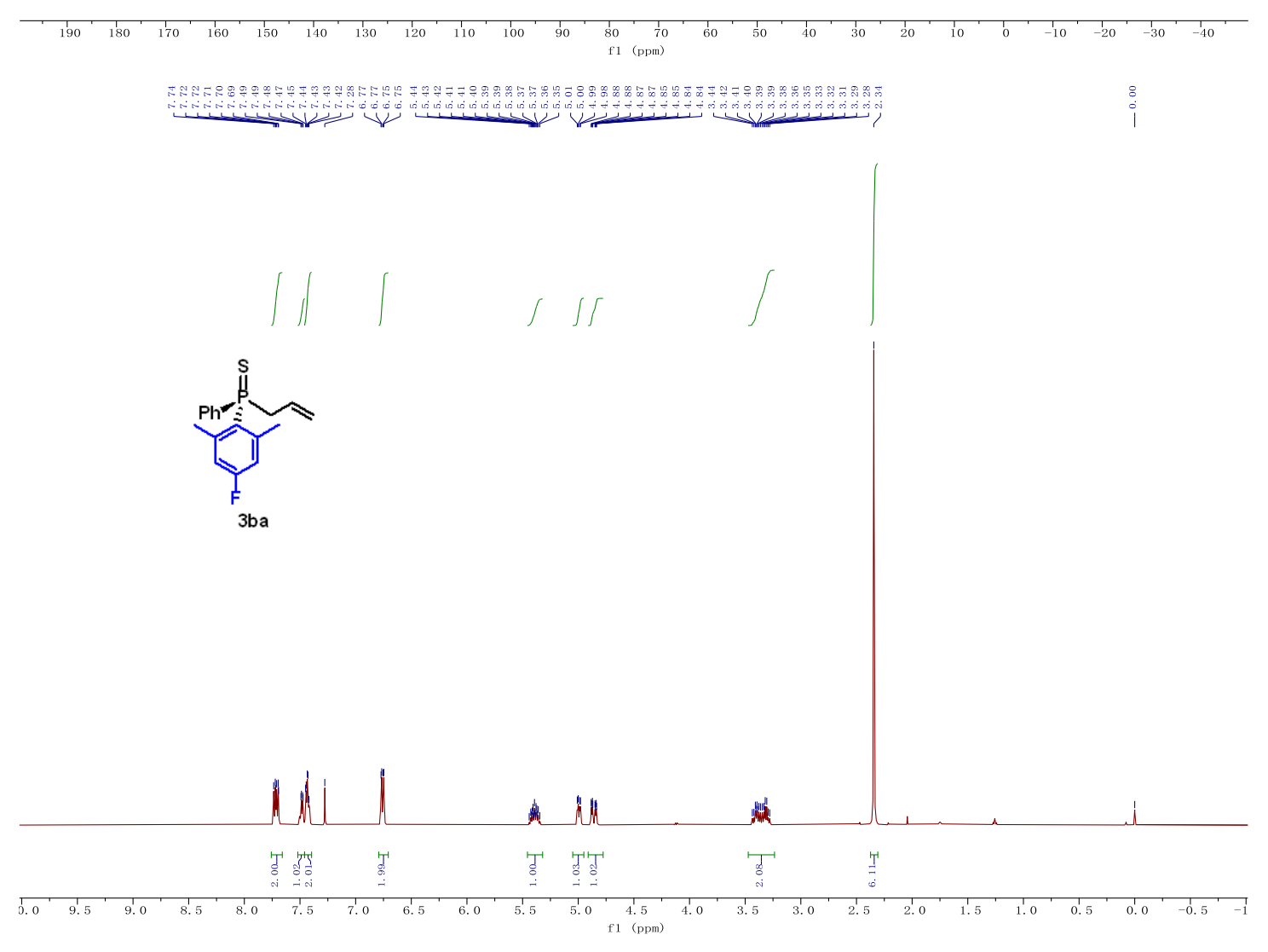



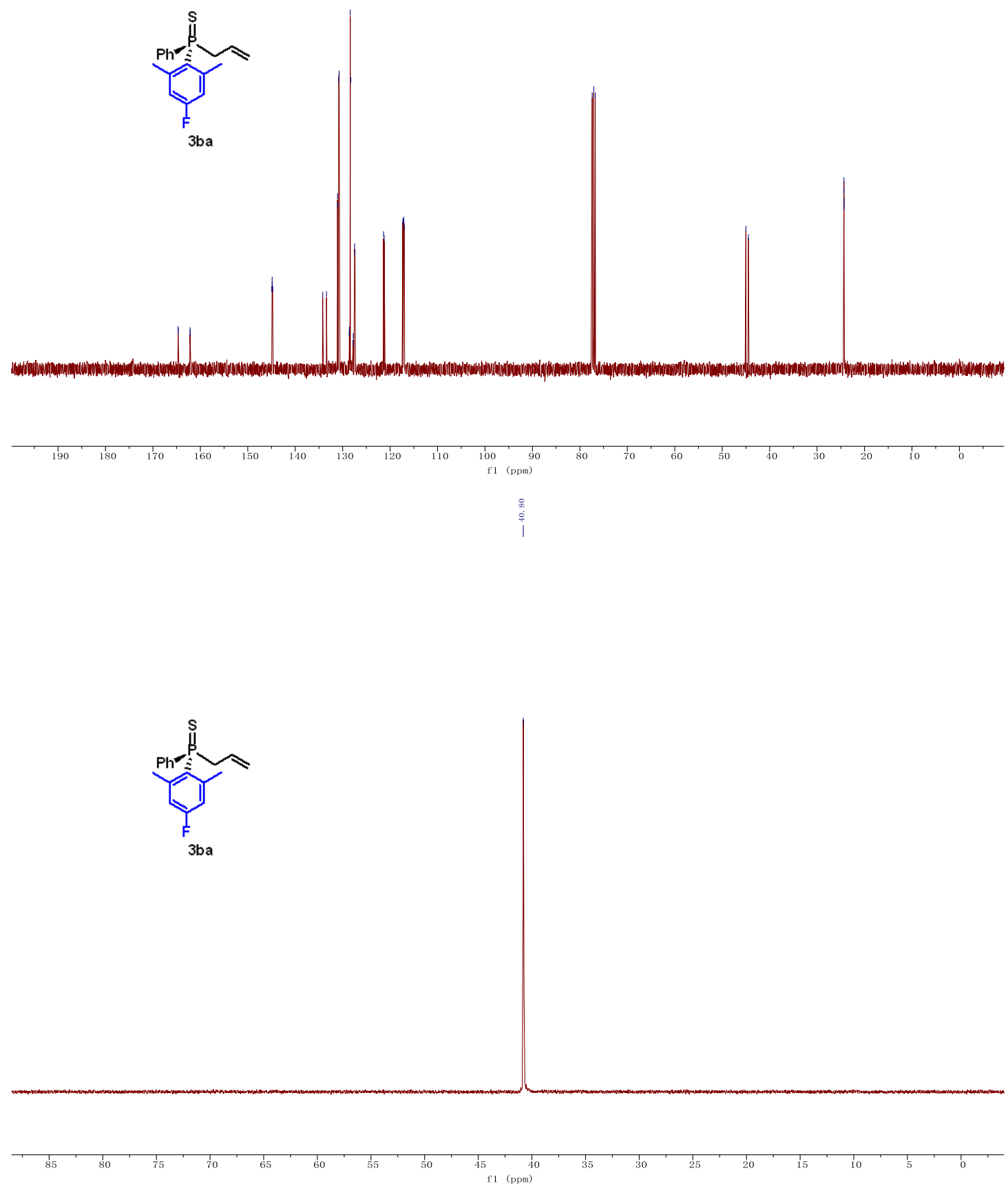

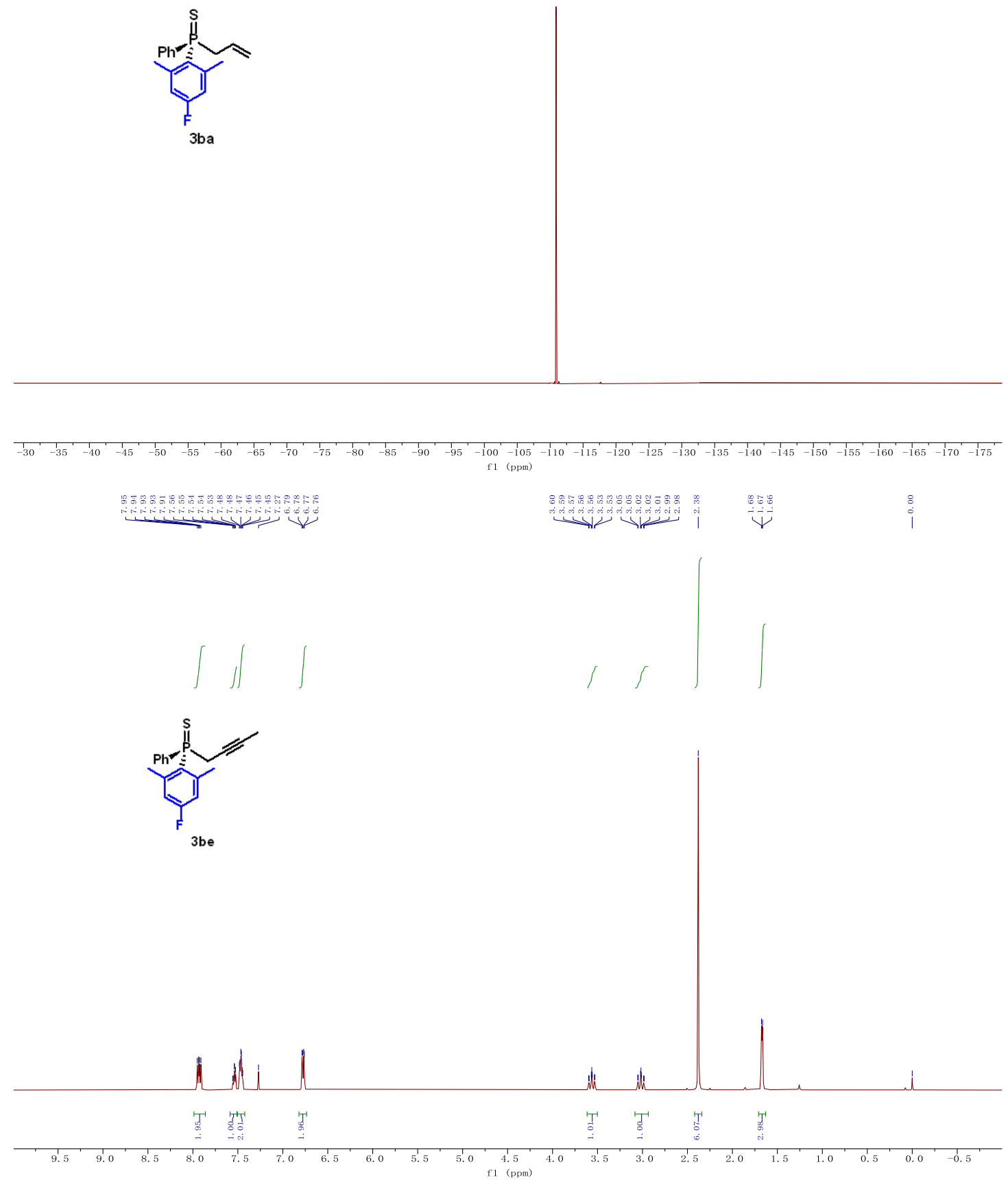


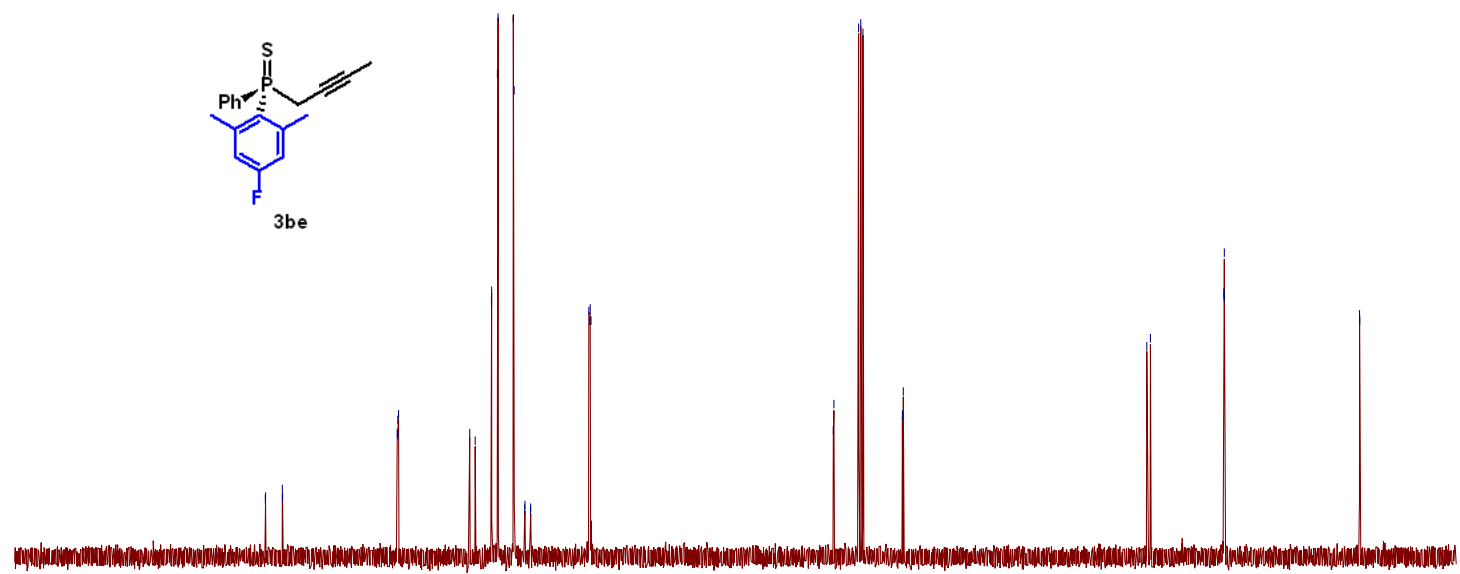

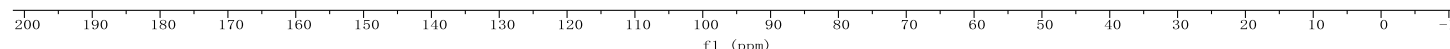
$\overrightarrow{5}$
0
0
0
$j$

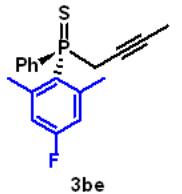

3be 

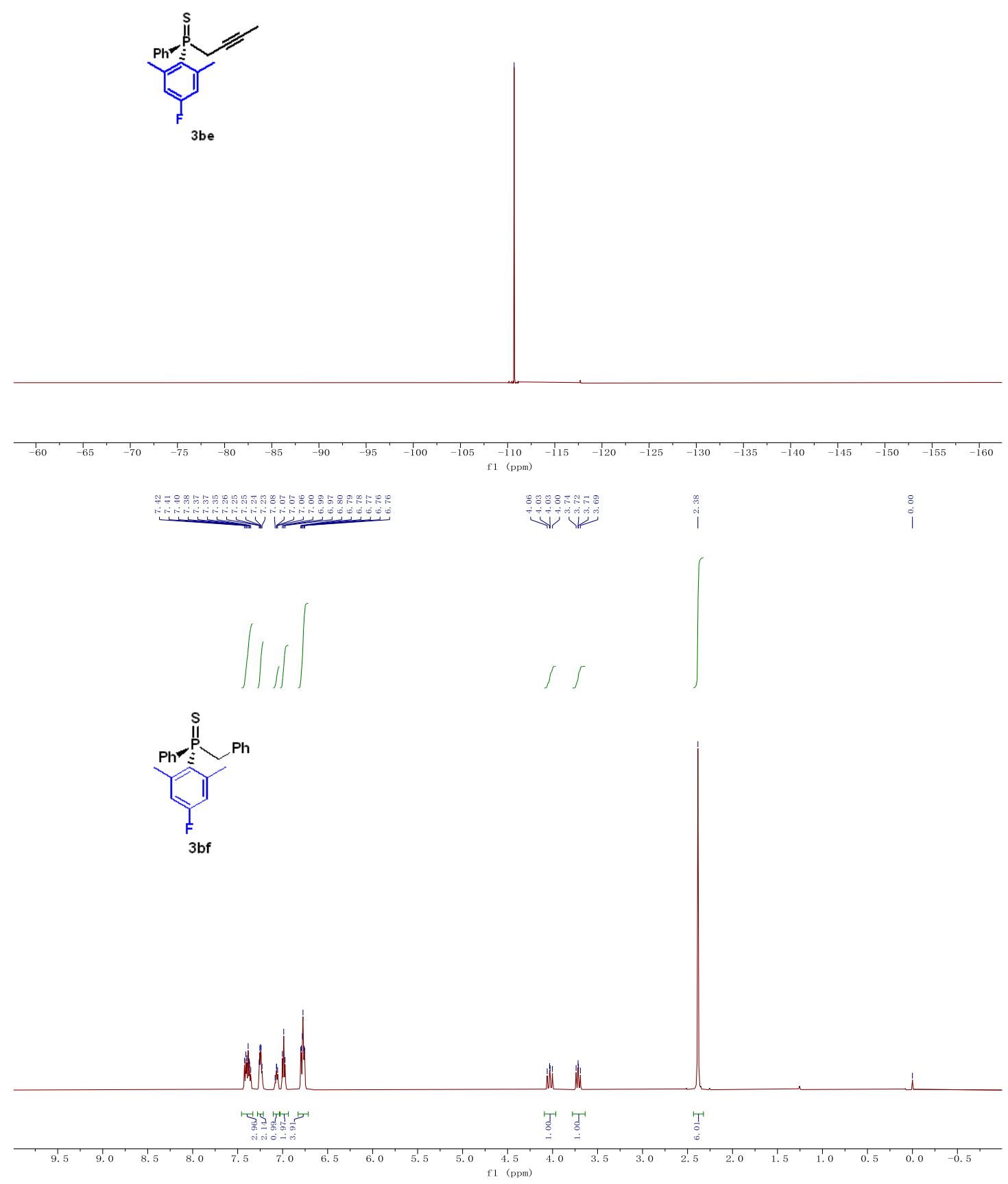


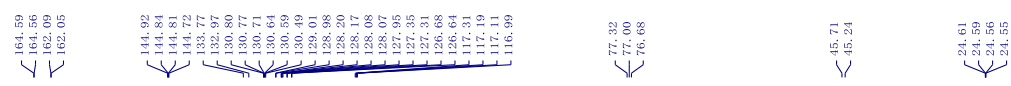

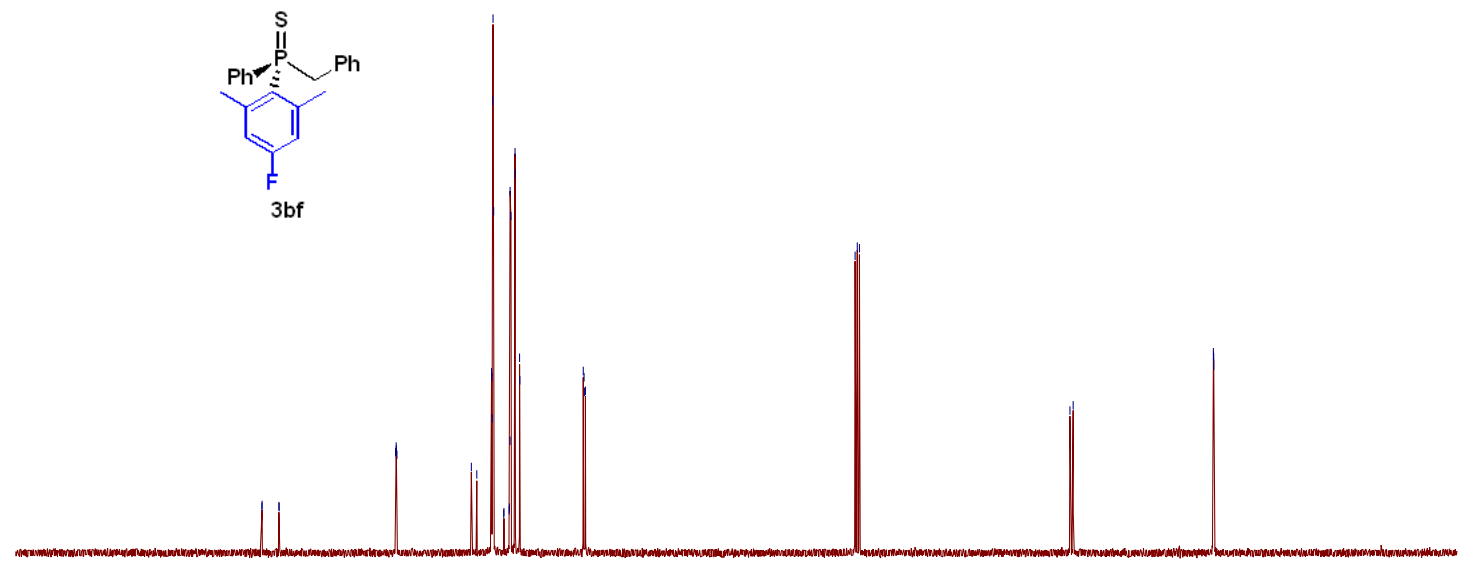

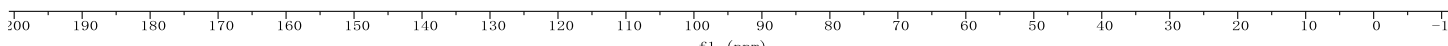
|

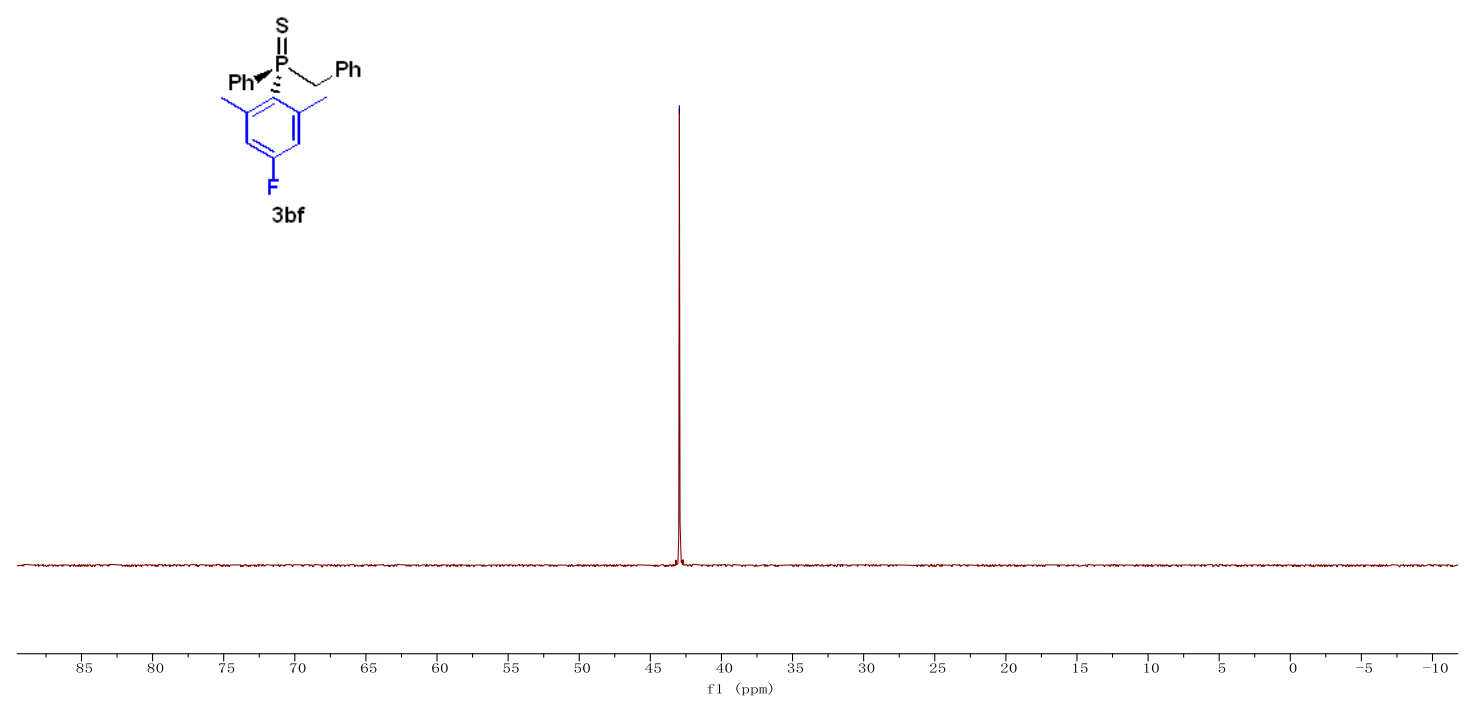



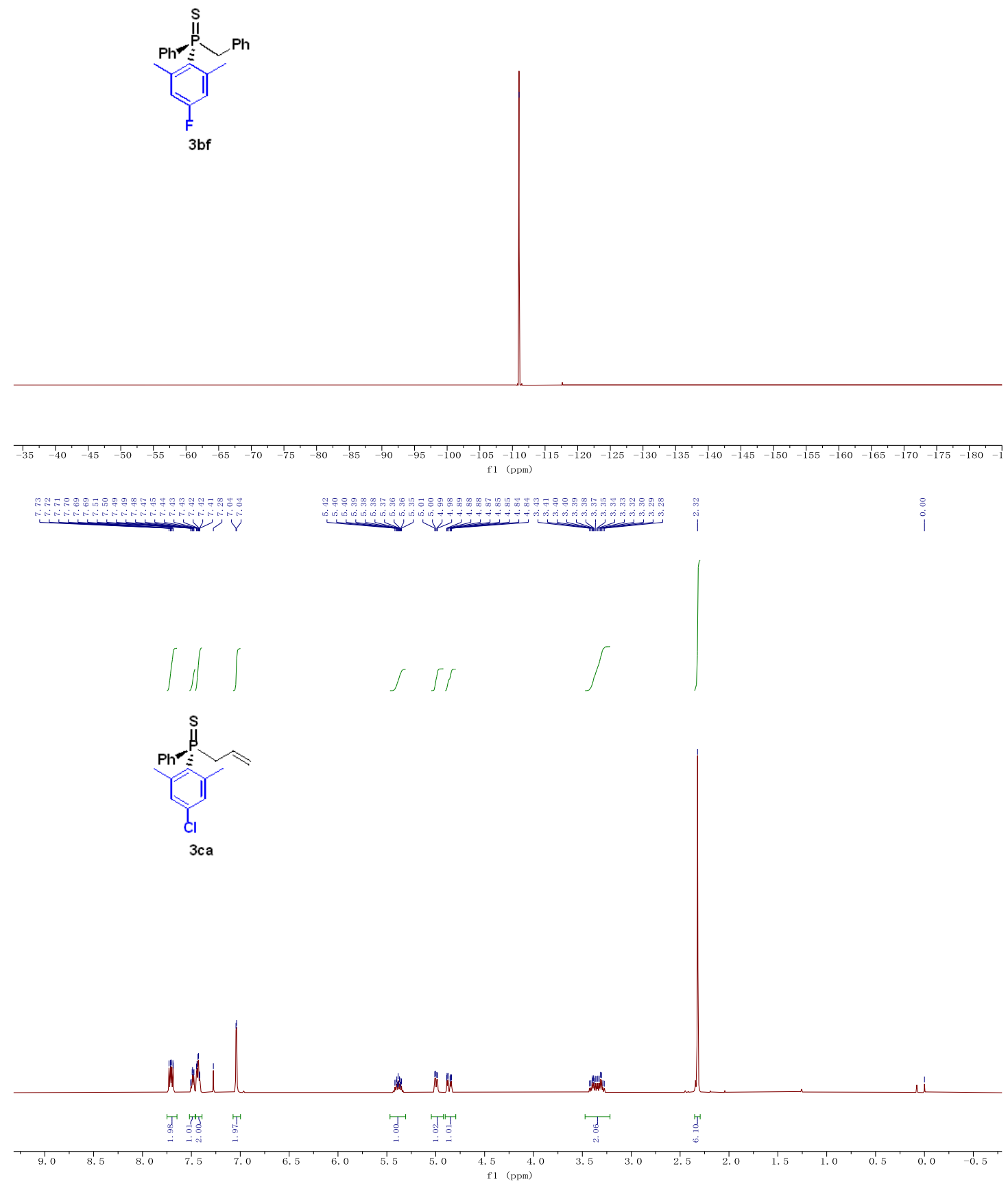

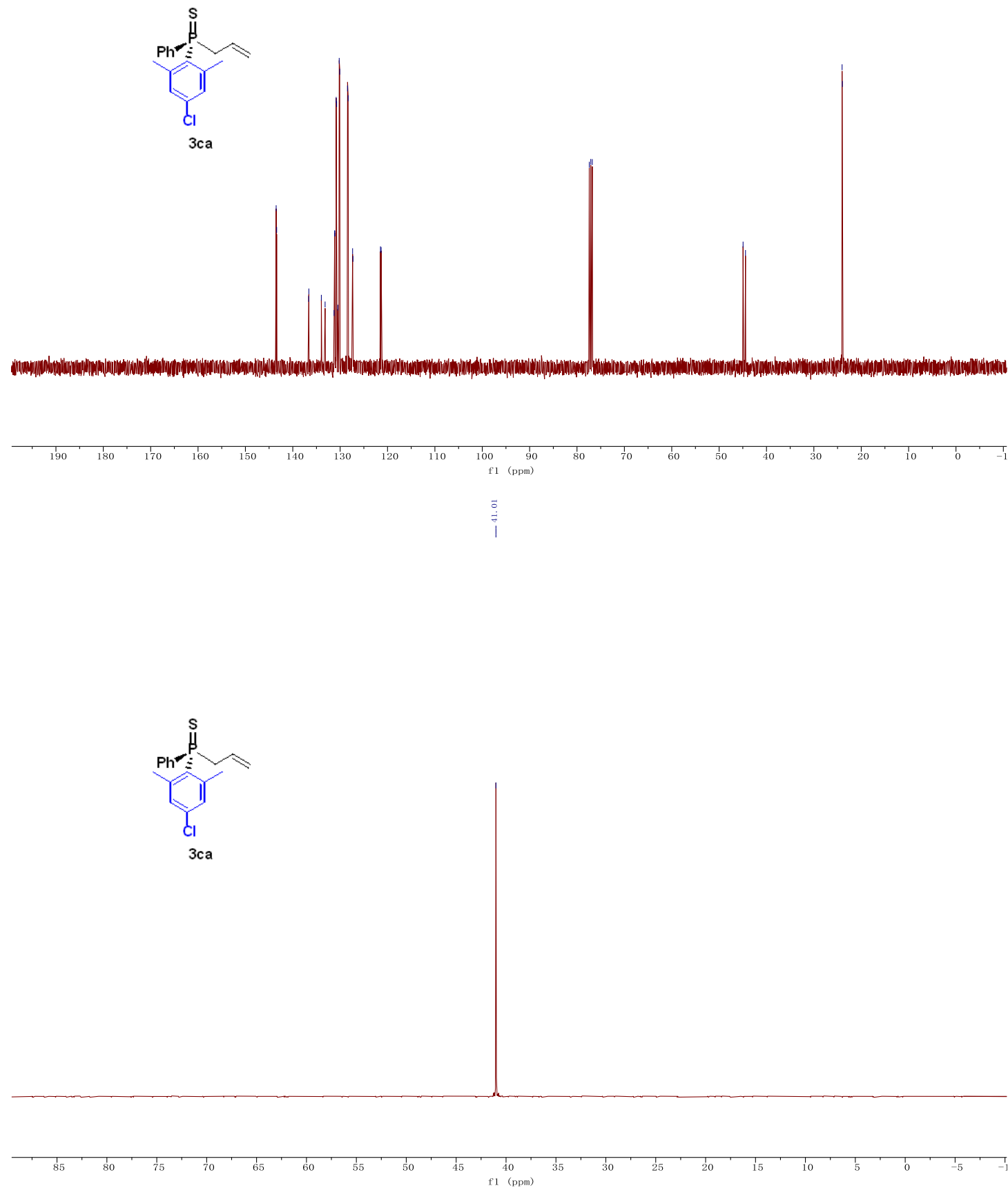

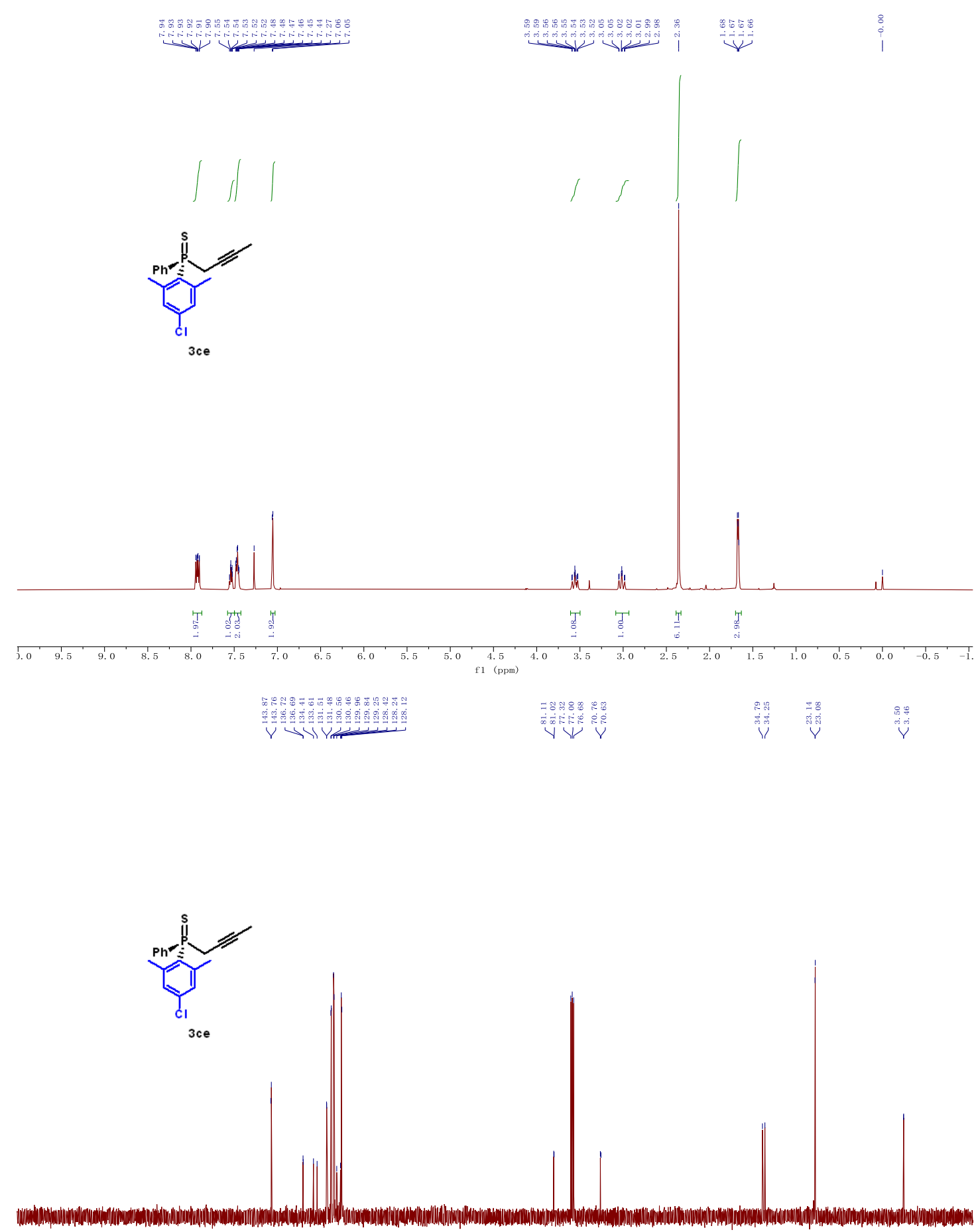

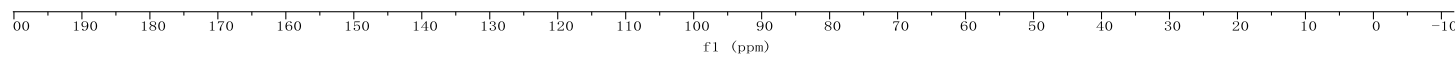



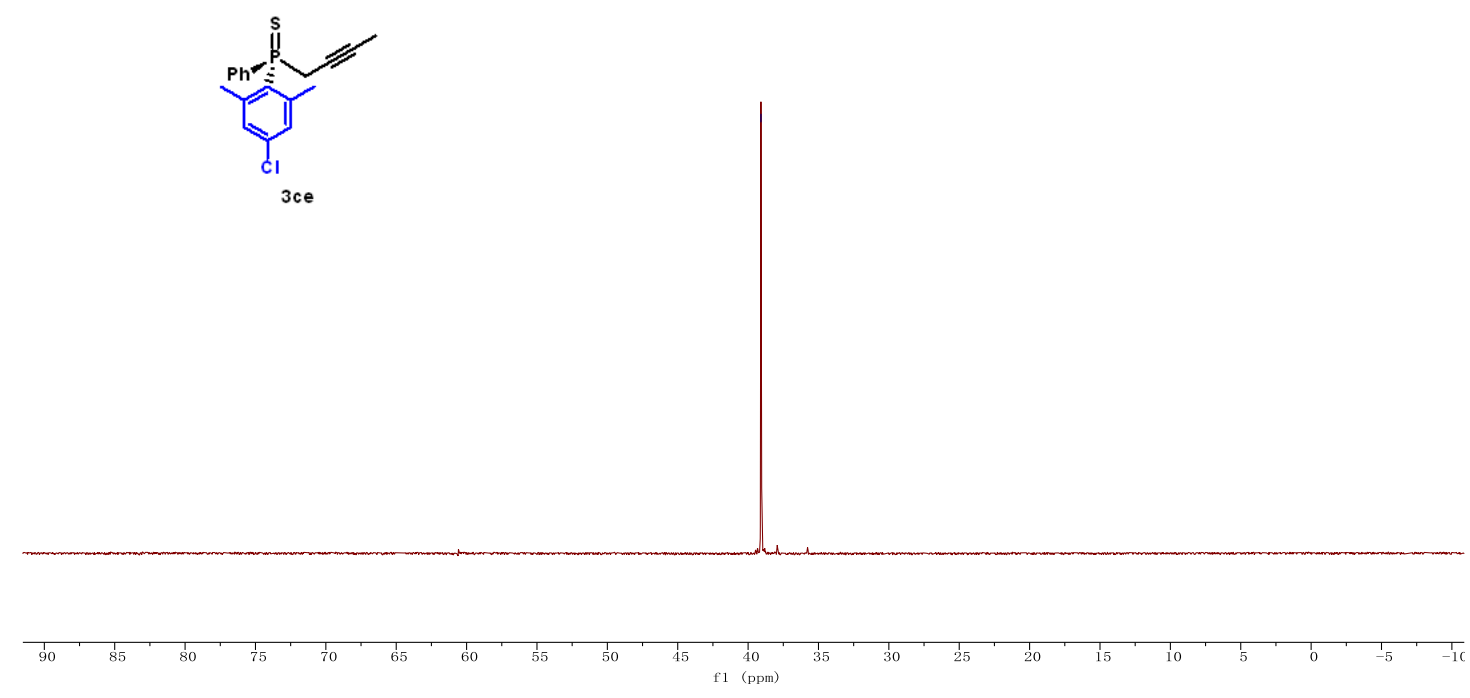

\begin{tabular}{l}
2 \\
\hline 9
\end{tabular}

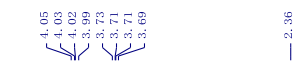

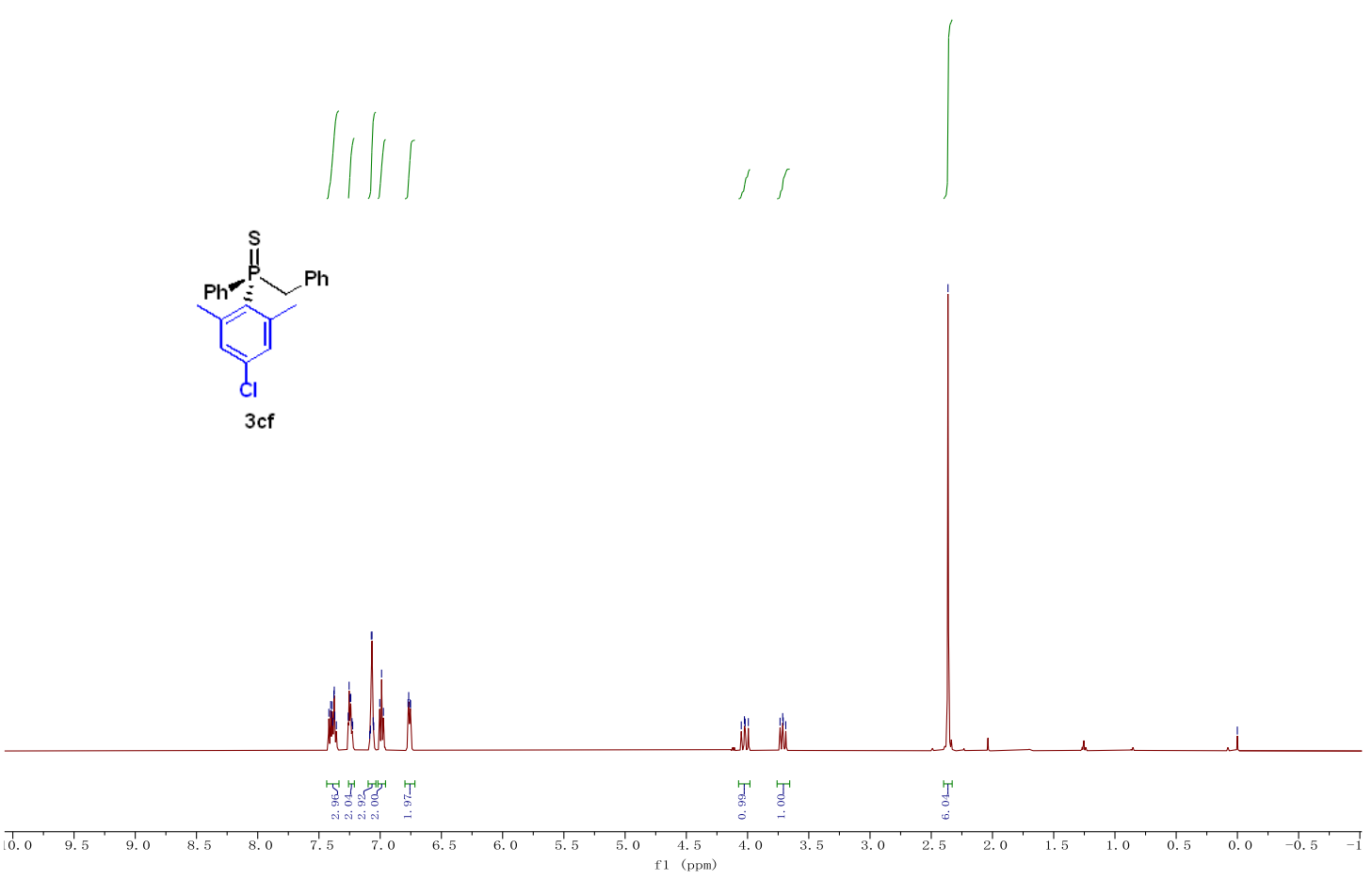



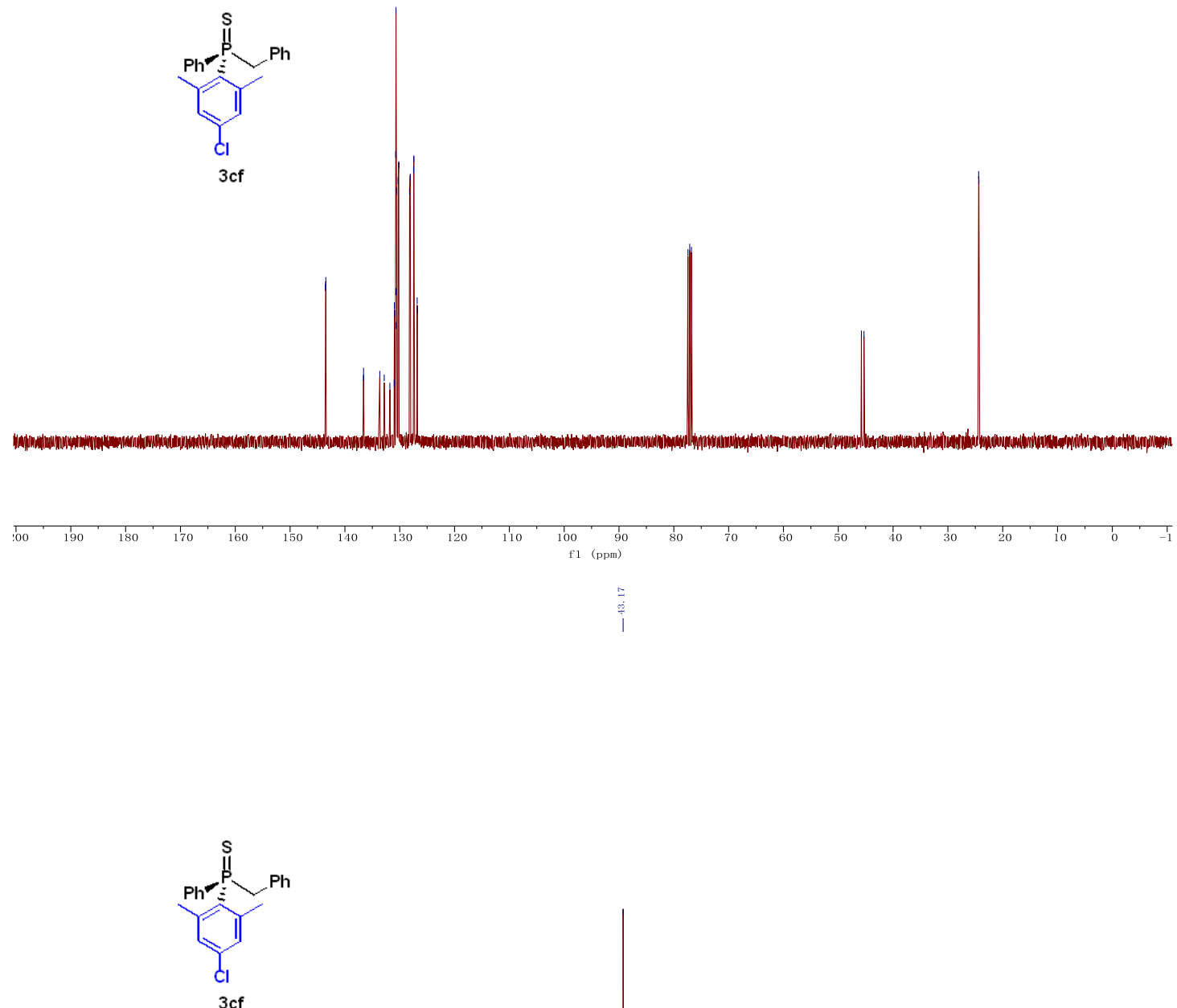


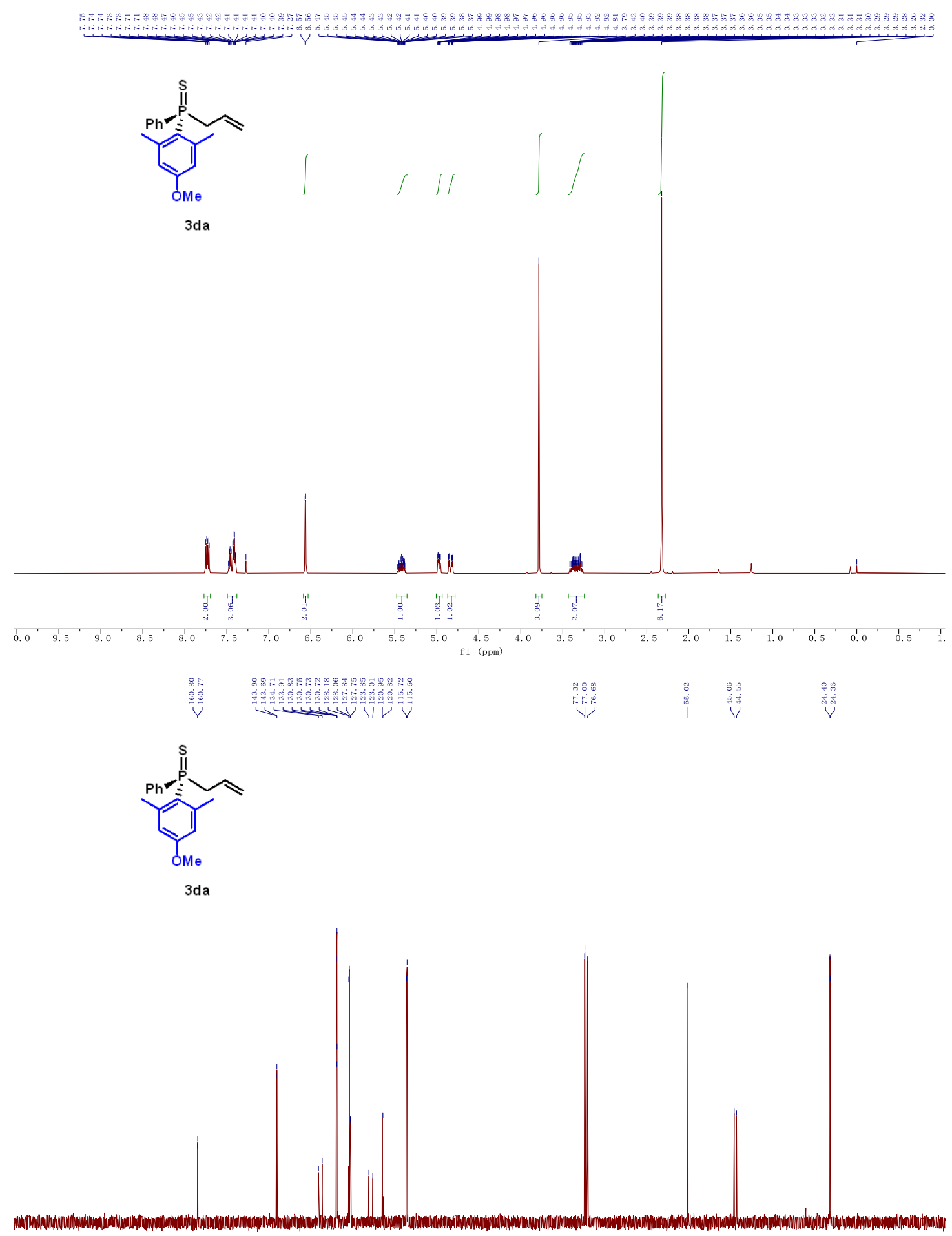

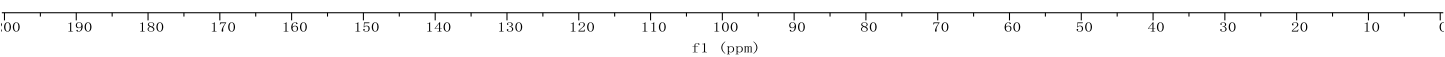




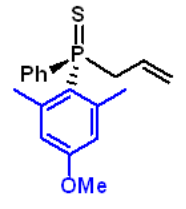

$3 d a$

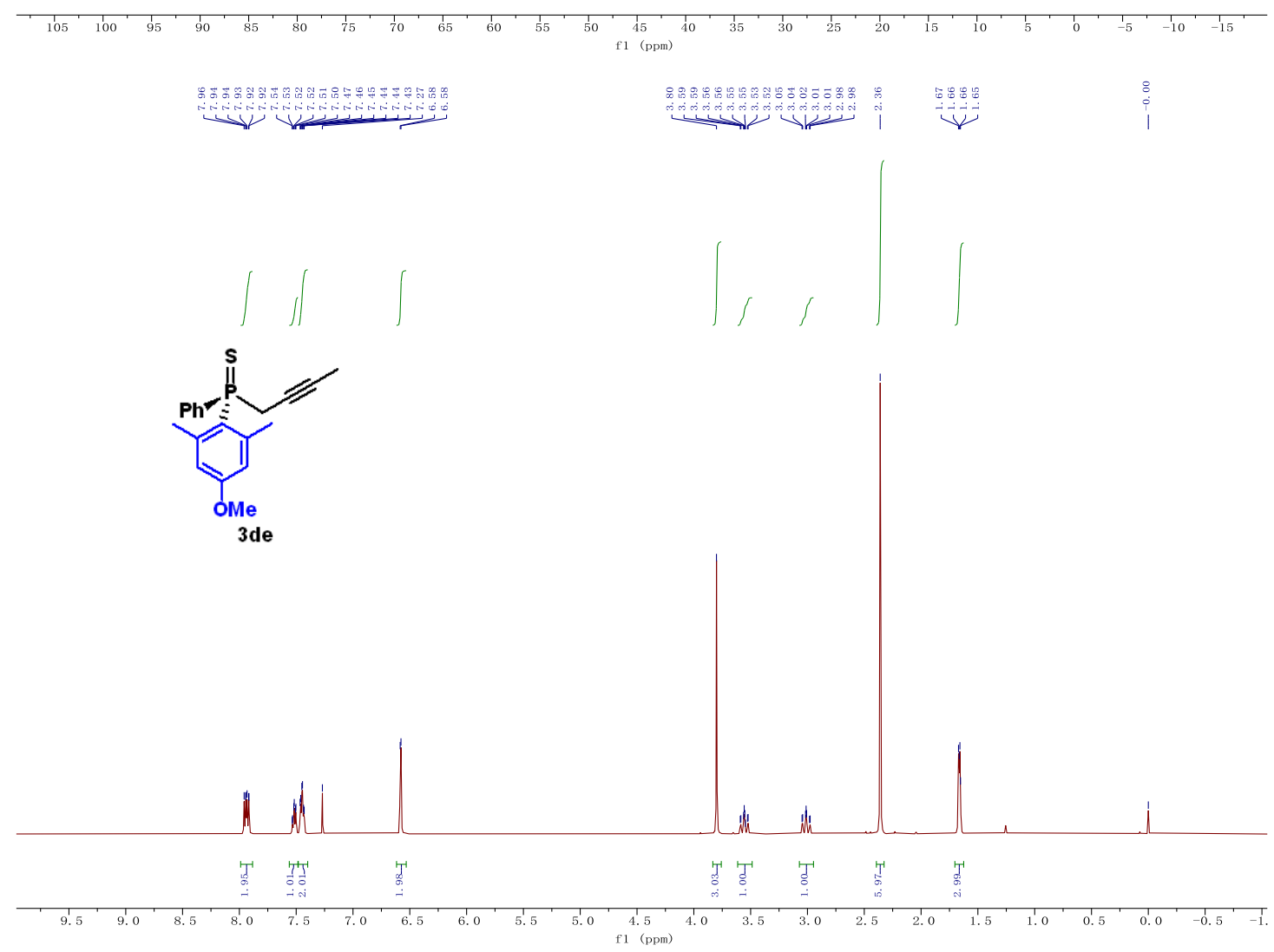




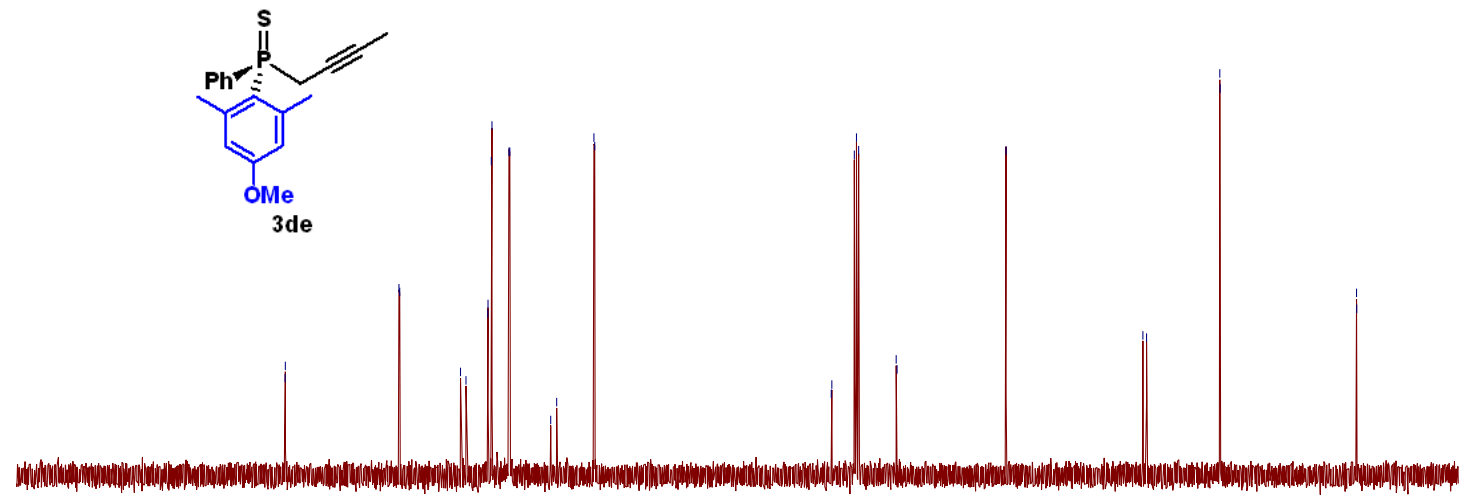

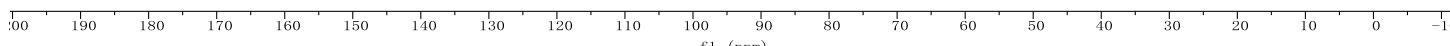

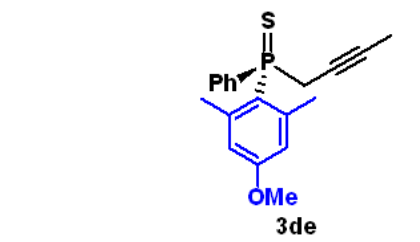

3 de

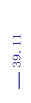

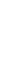
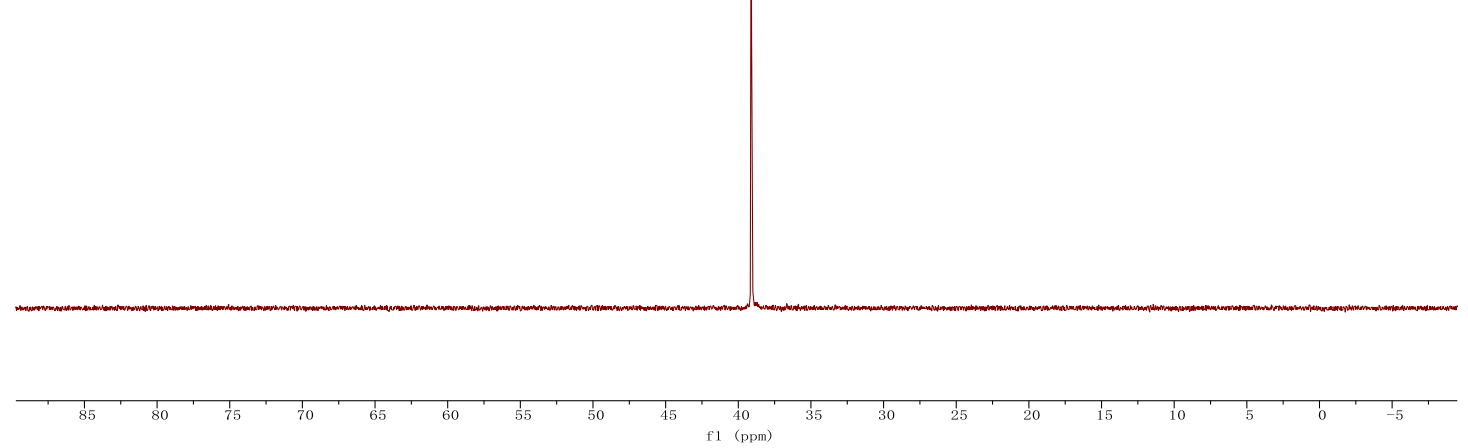


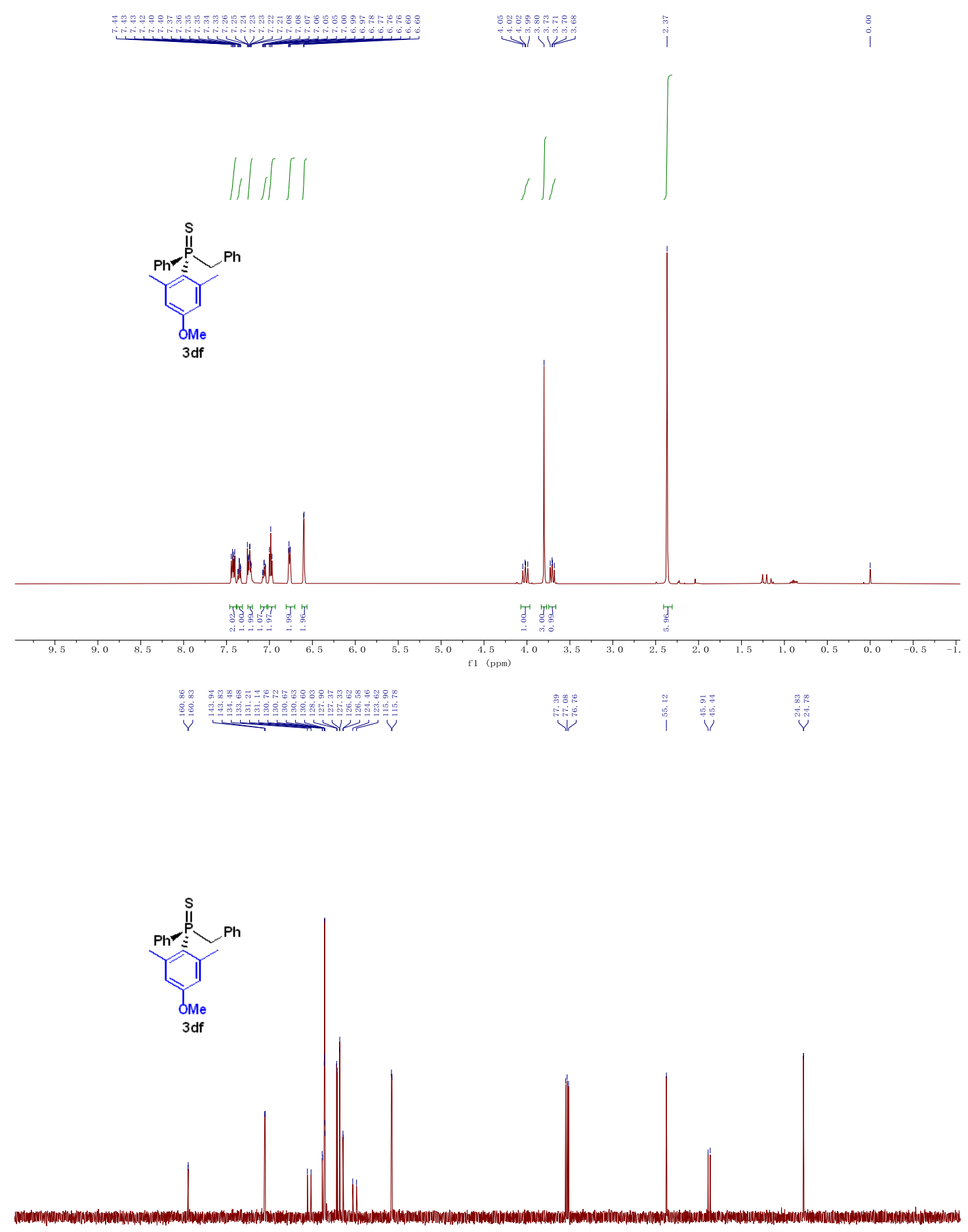



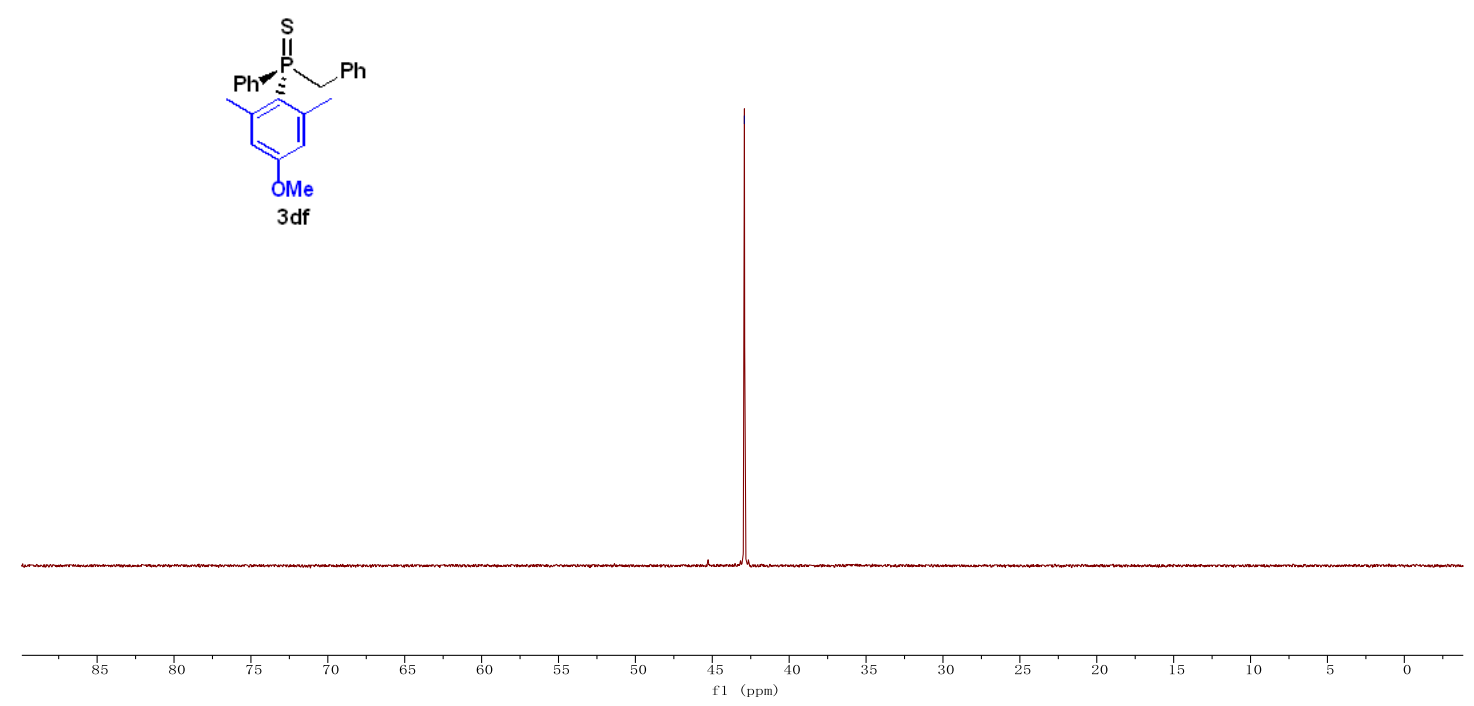

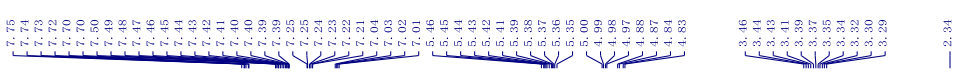



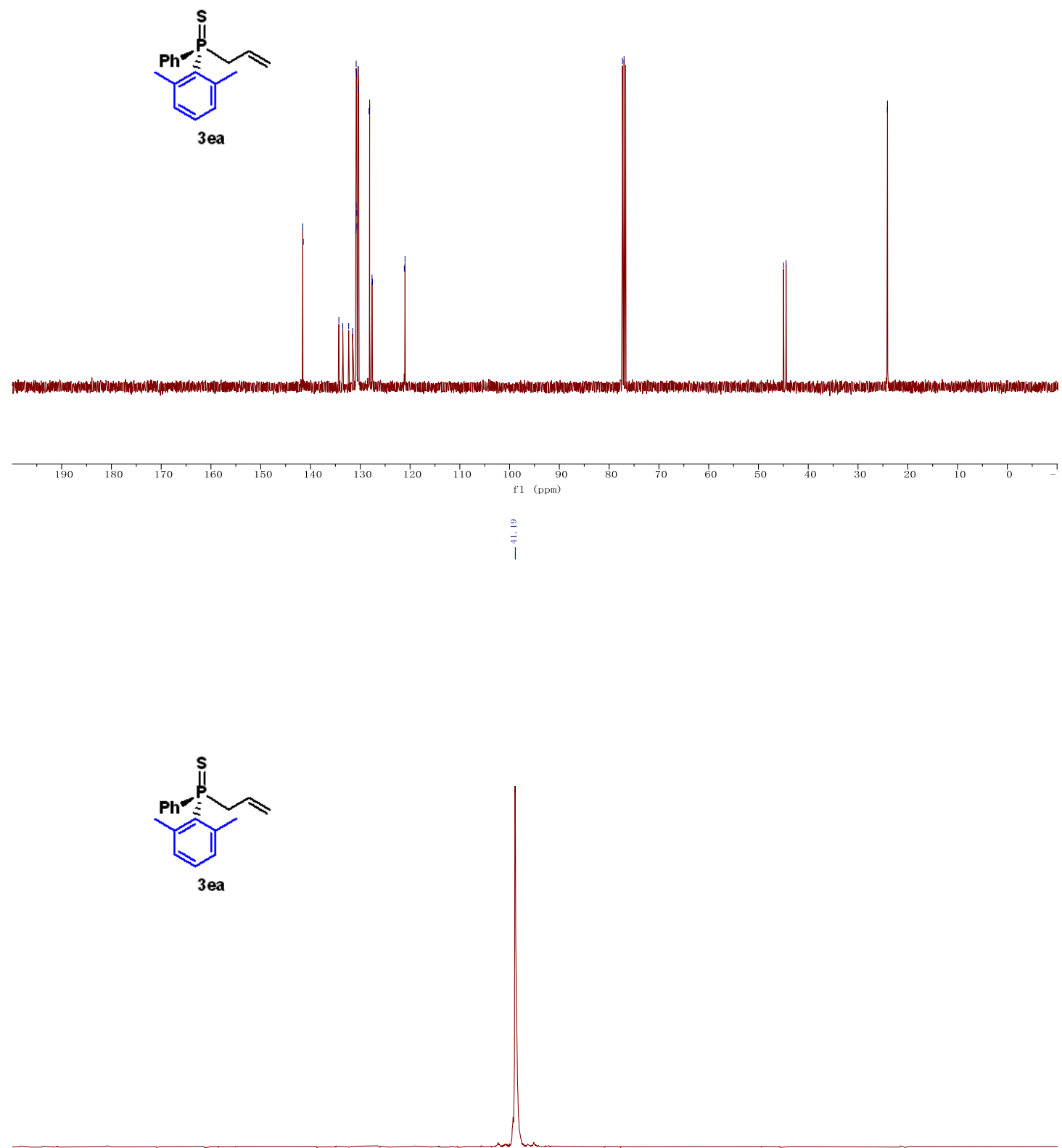

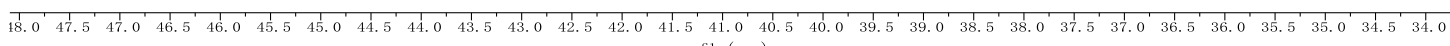
1 (ppm) 

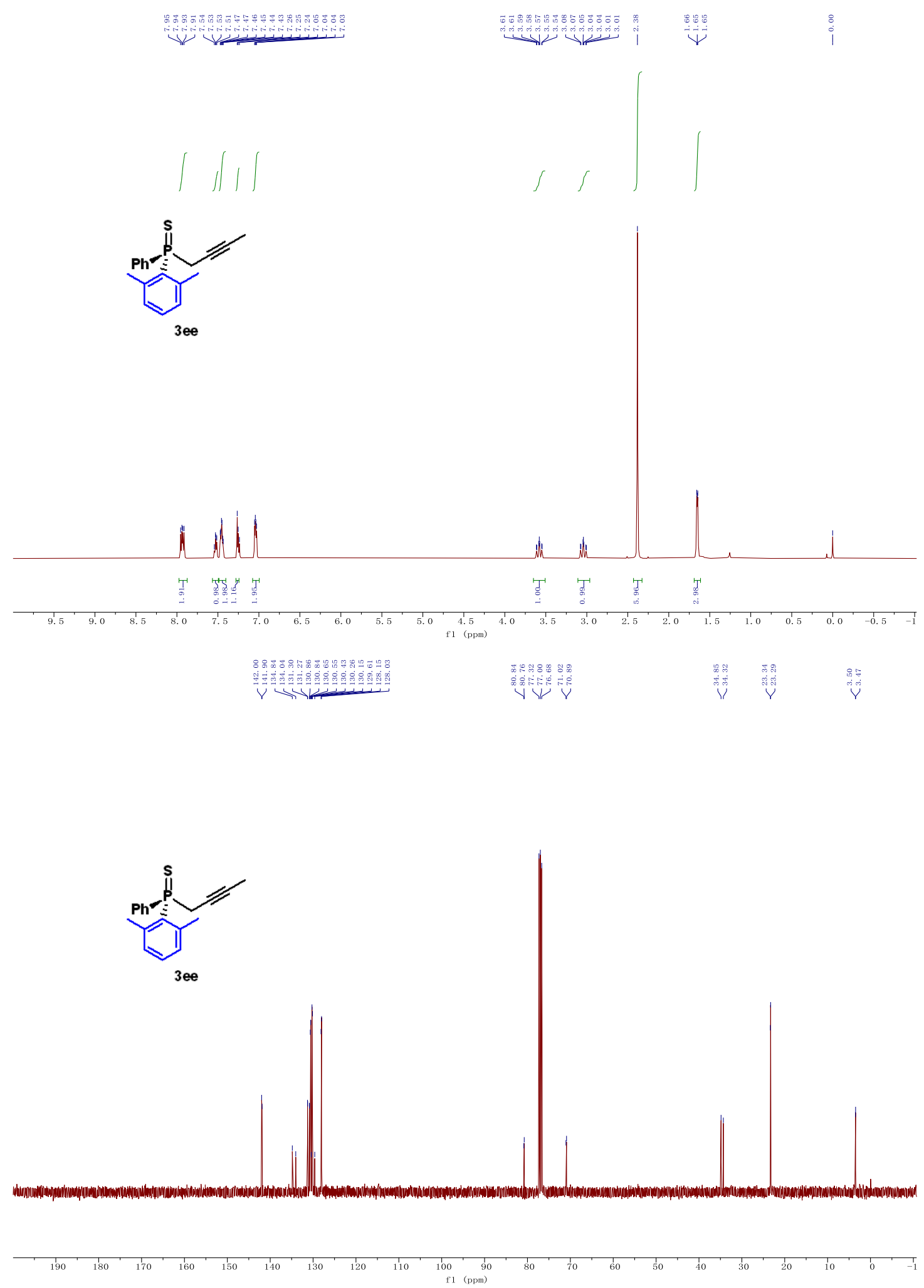

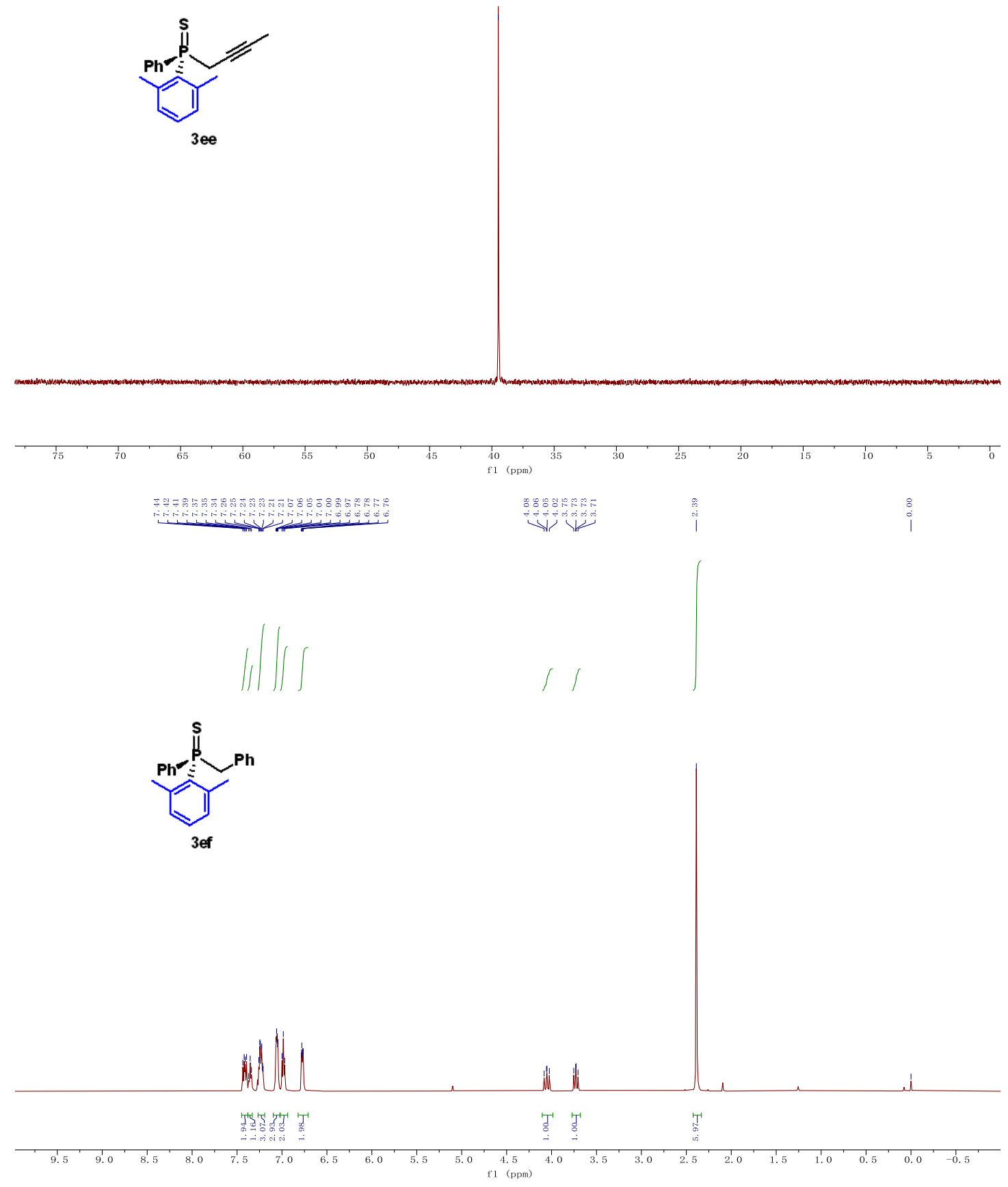


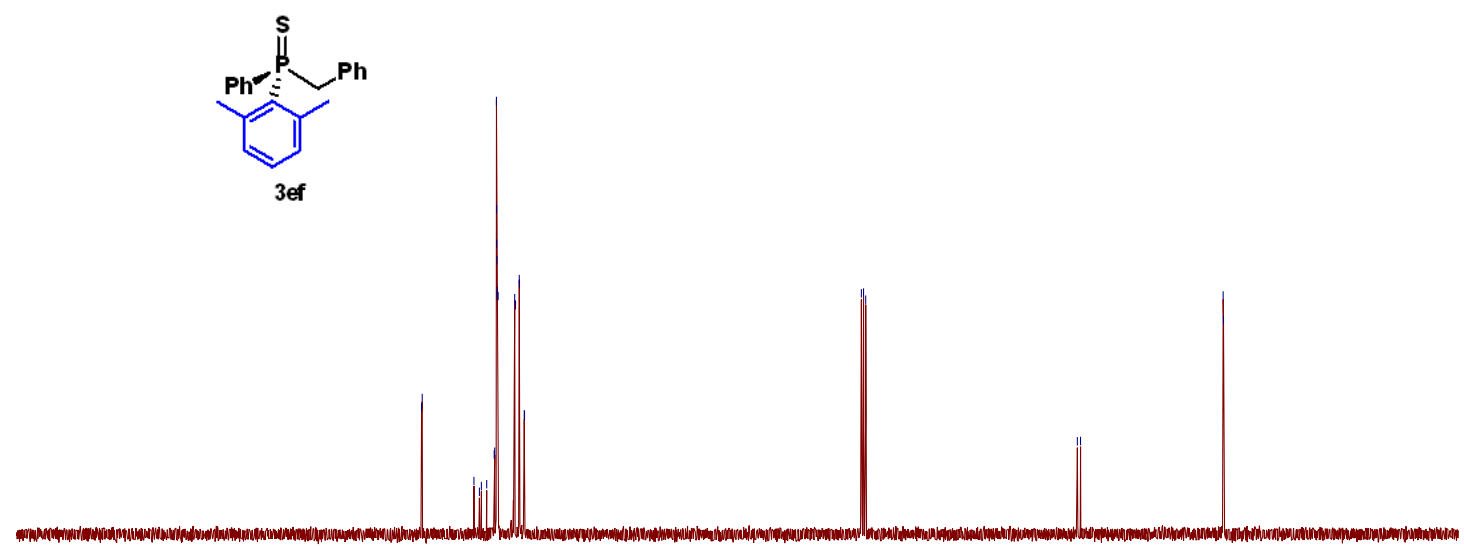

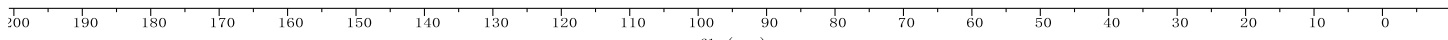
$\underset{\substack{\infty \\ m}}{\substack{j \\ j}}$<smiles>C=P1(c2ccccc2)c2ccccc2C1c1ccccc1</smiles>

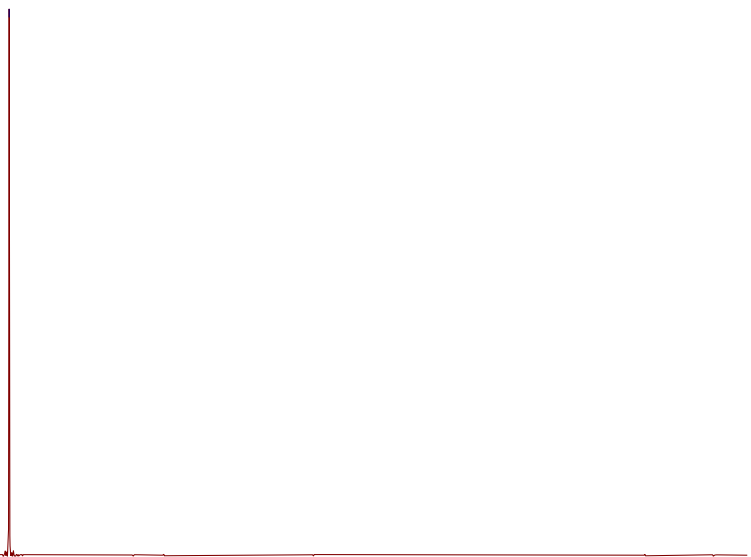

85 1
70 $45 \underset{f 1(\mathrm{ppm})}{40}$ 


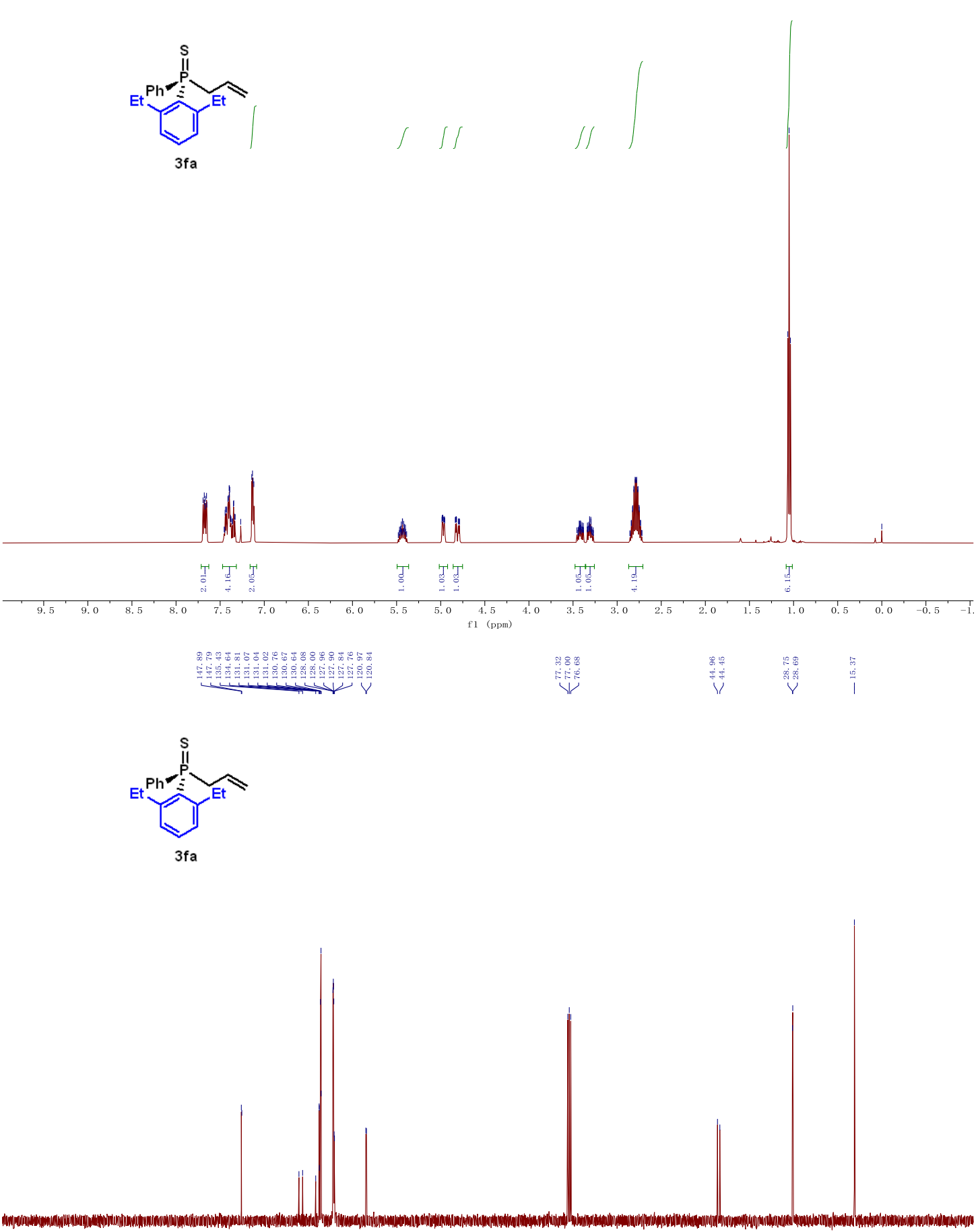

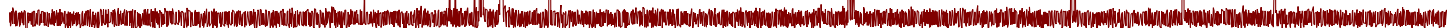

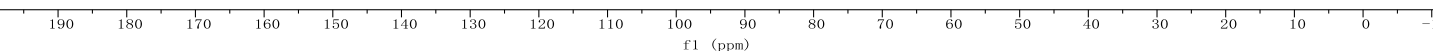



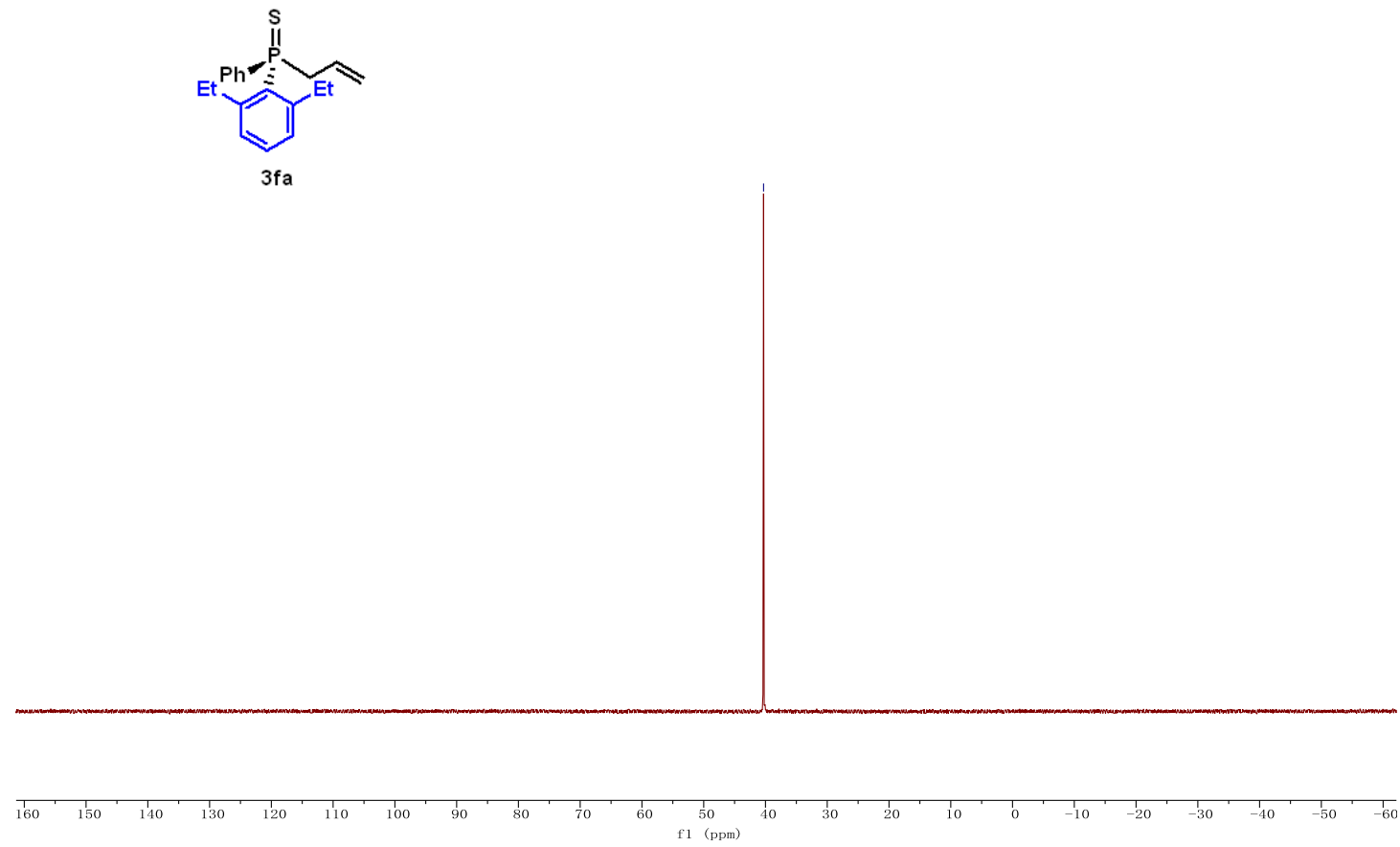

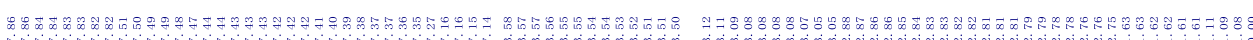
$\underbrace{2}_{1}$

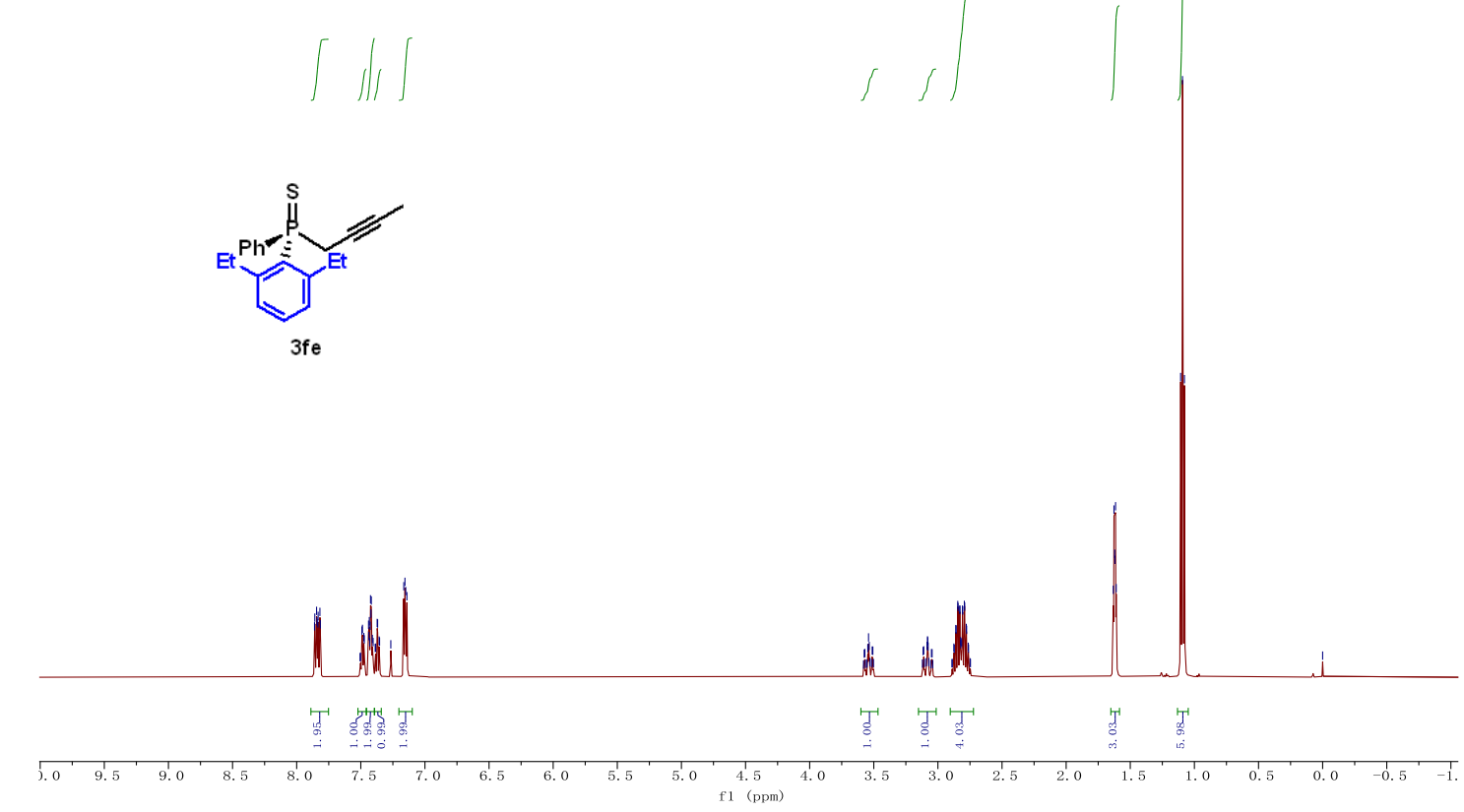



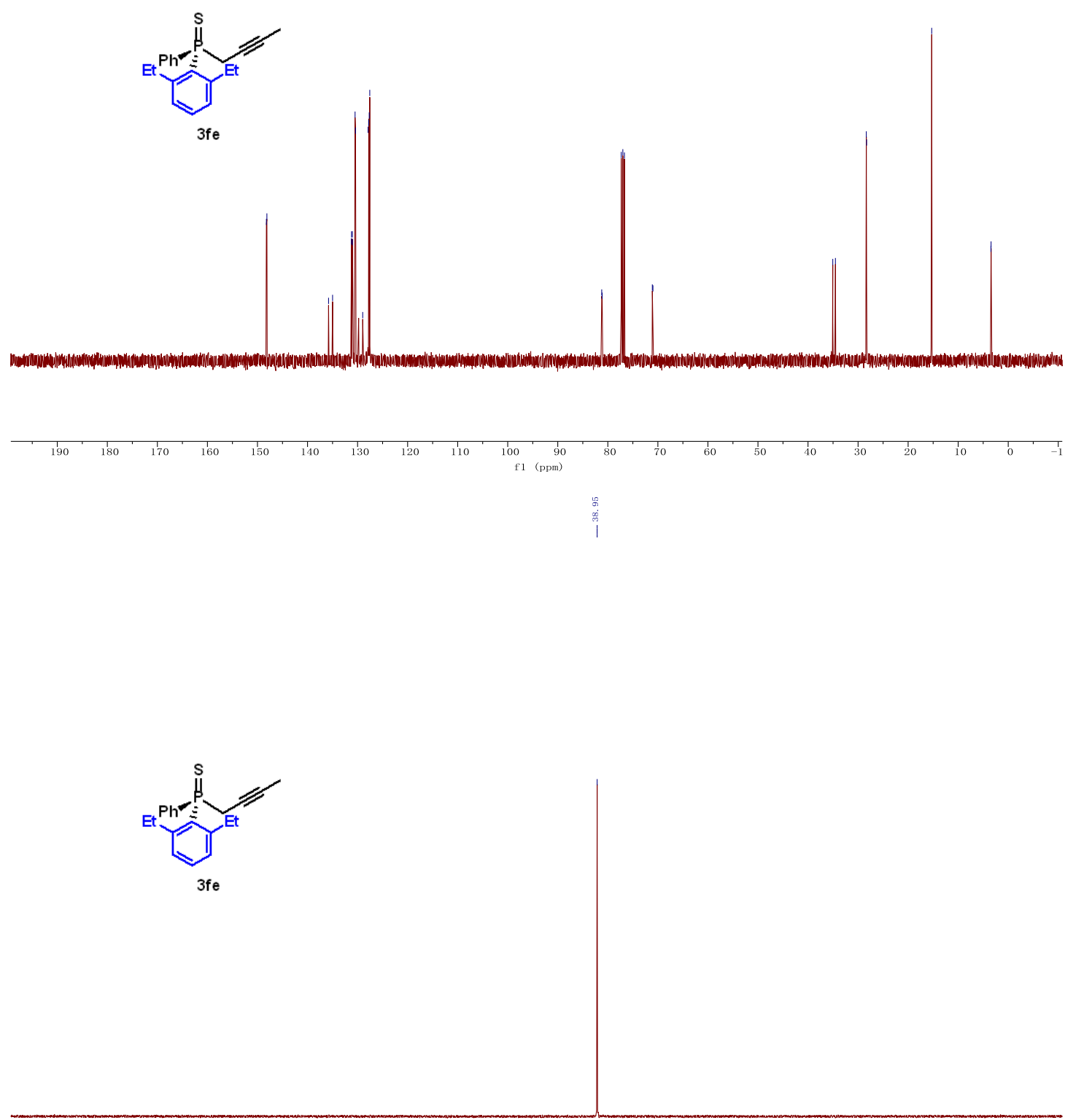

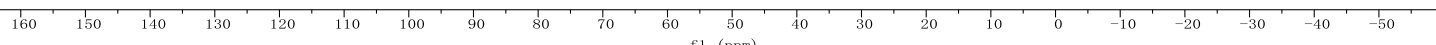



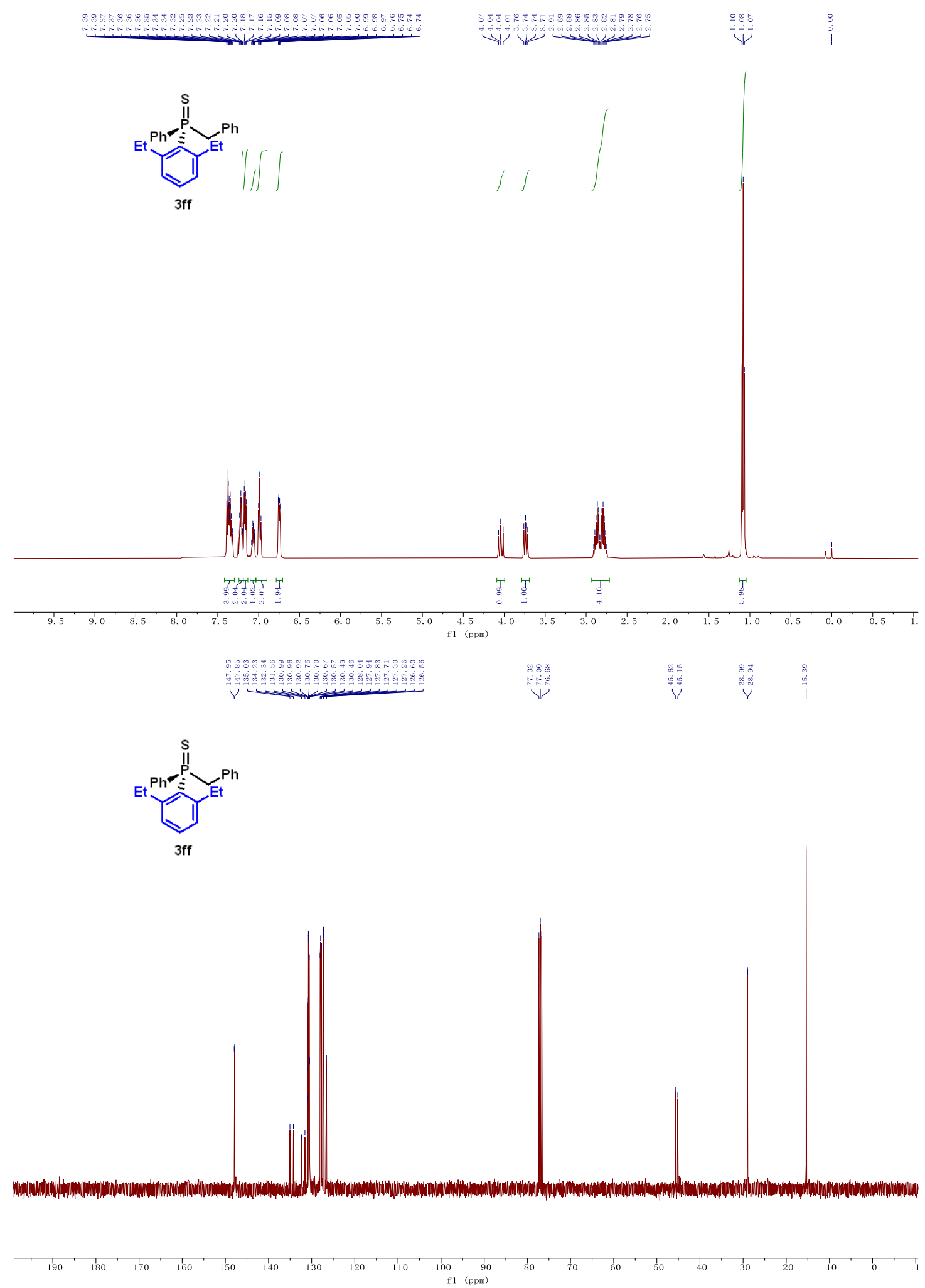

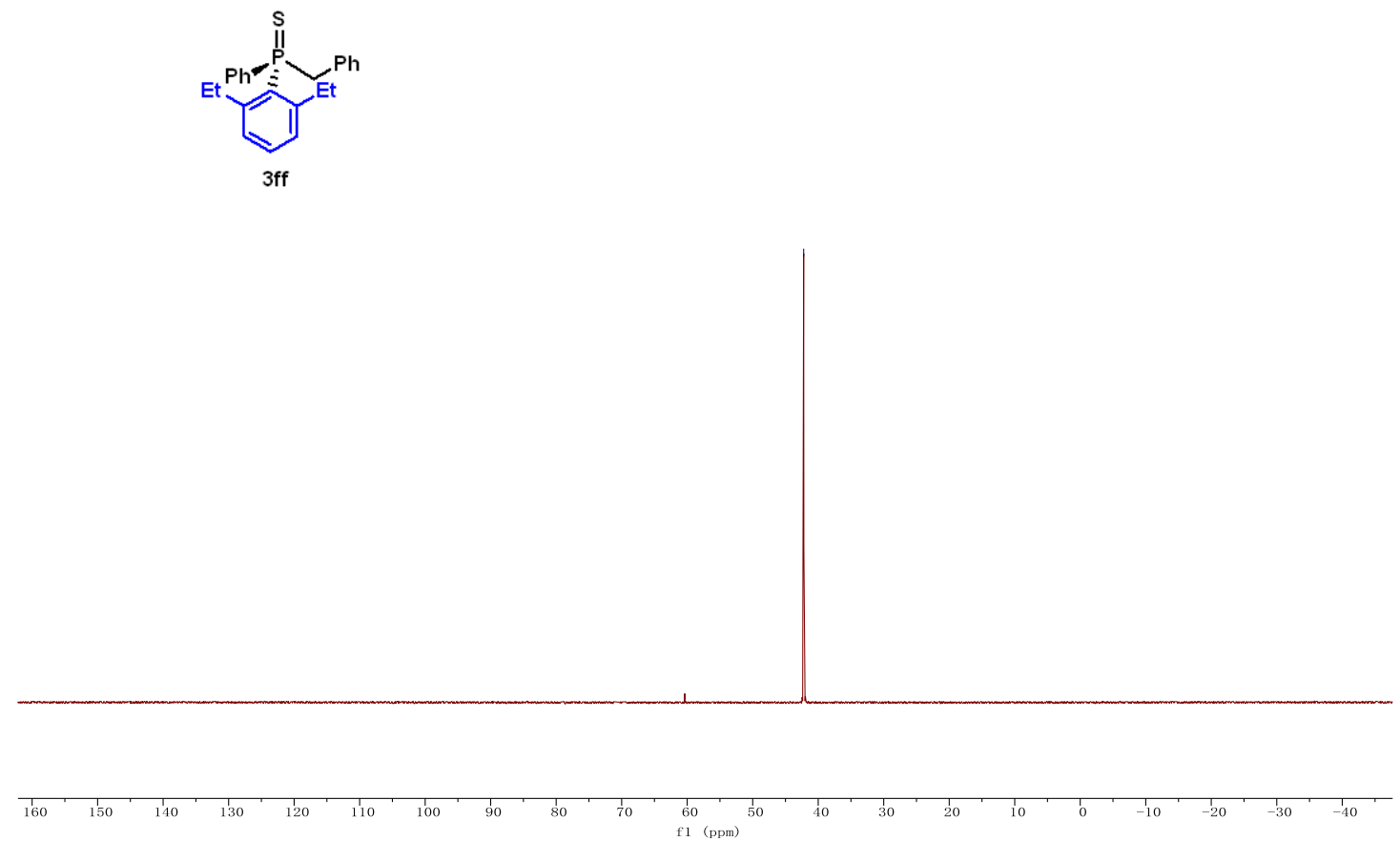

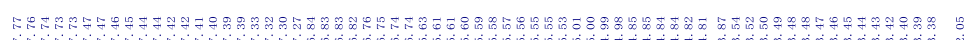

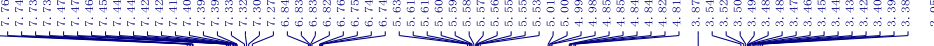
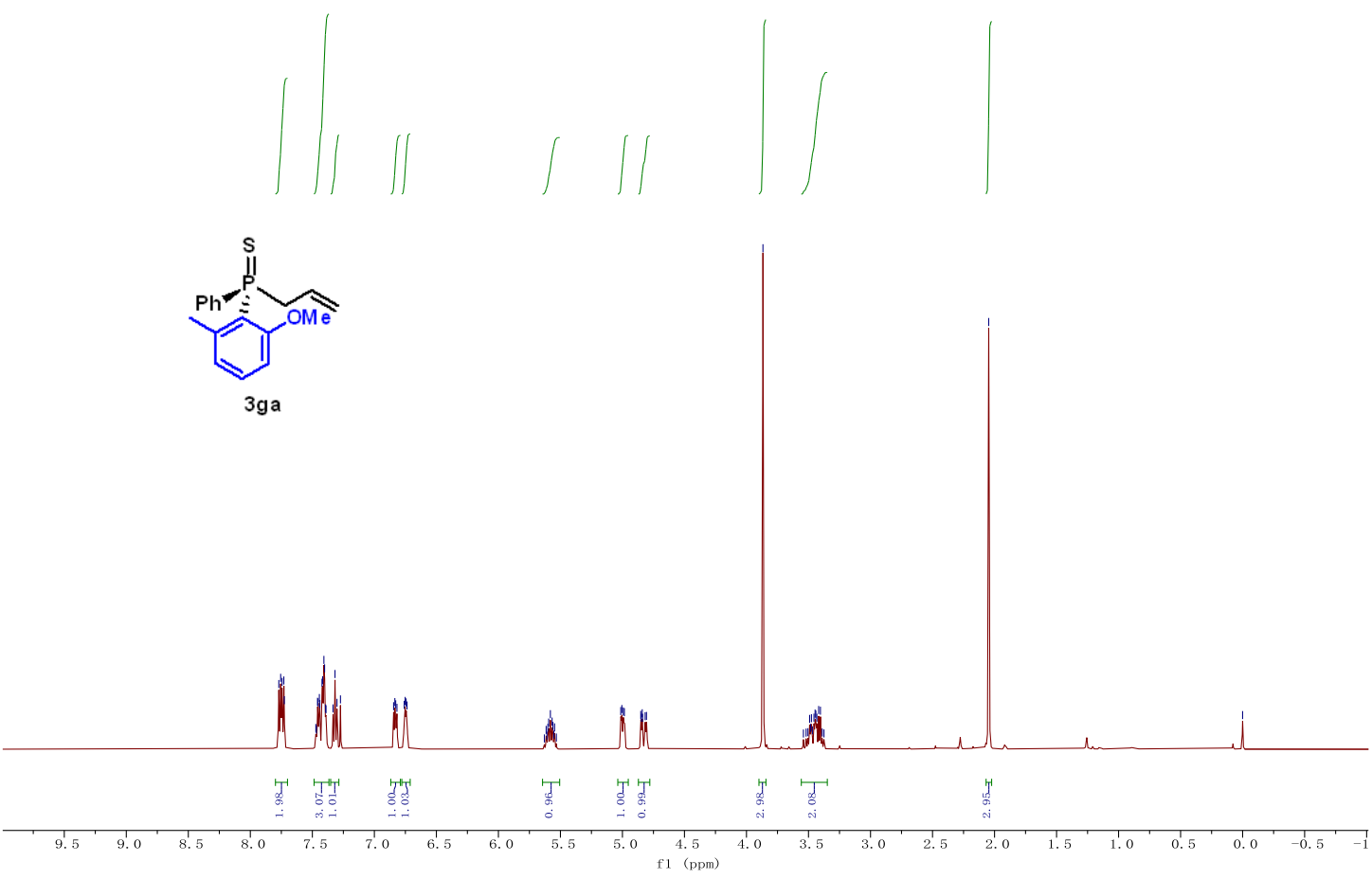

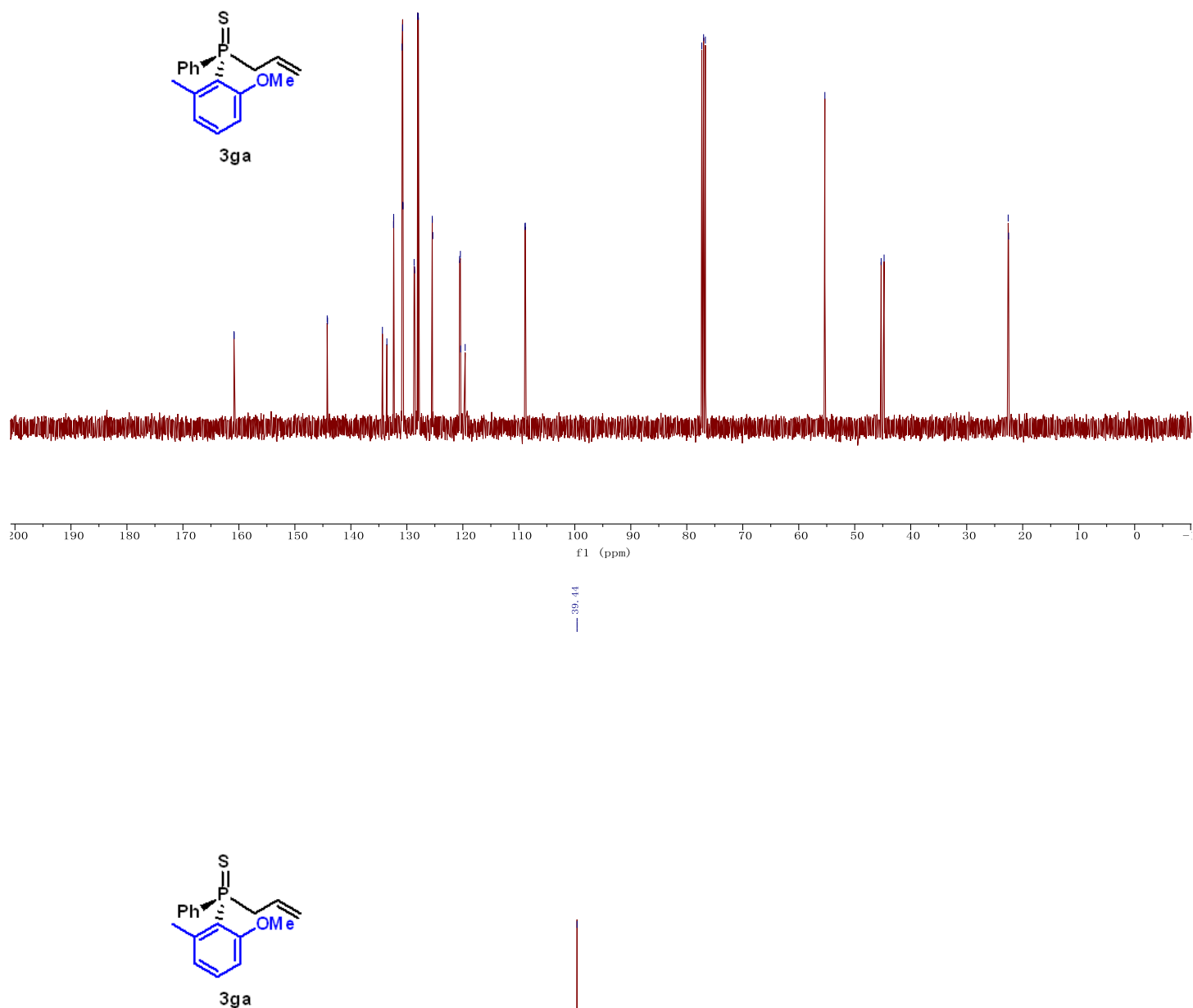

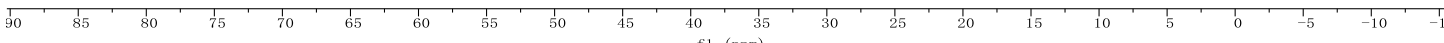




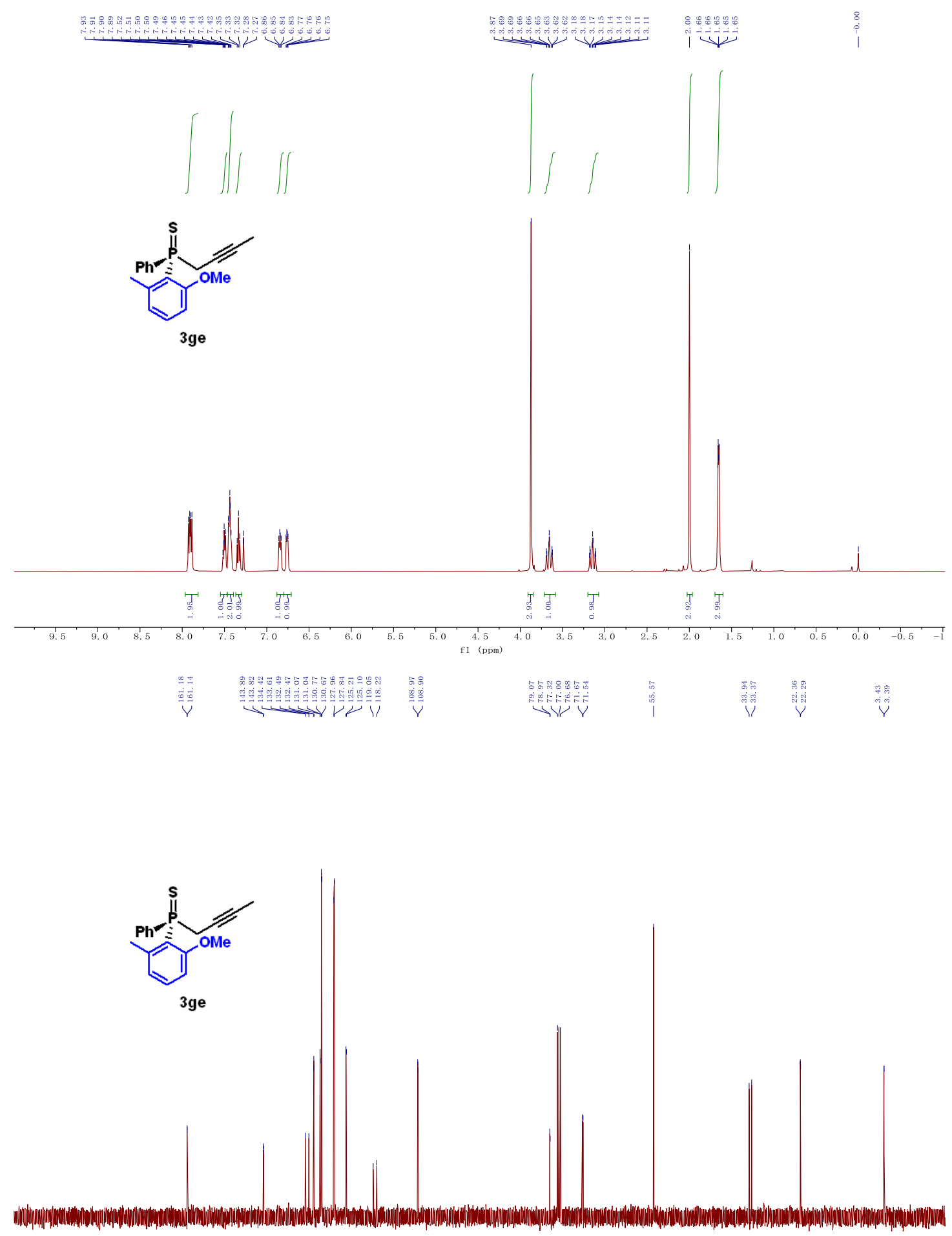

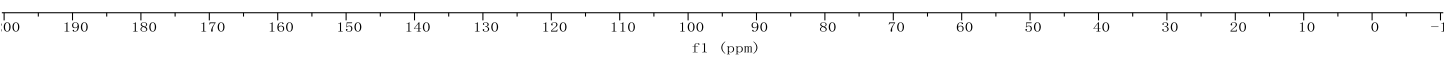



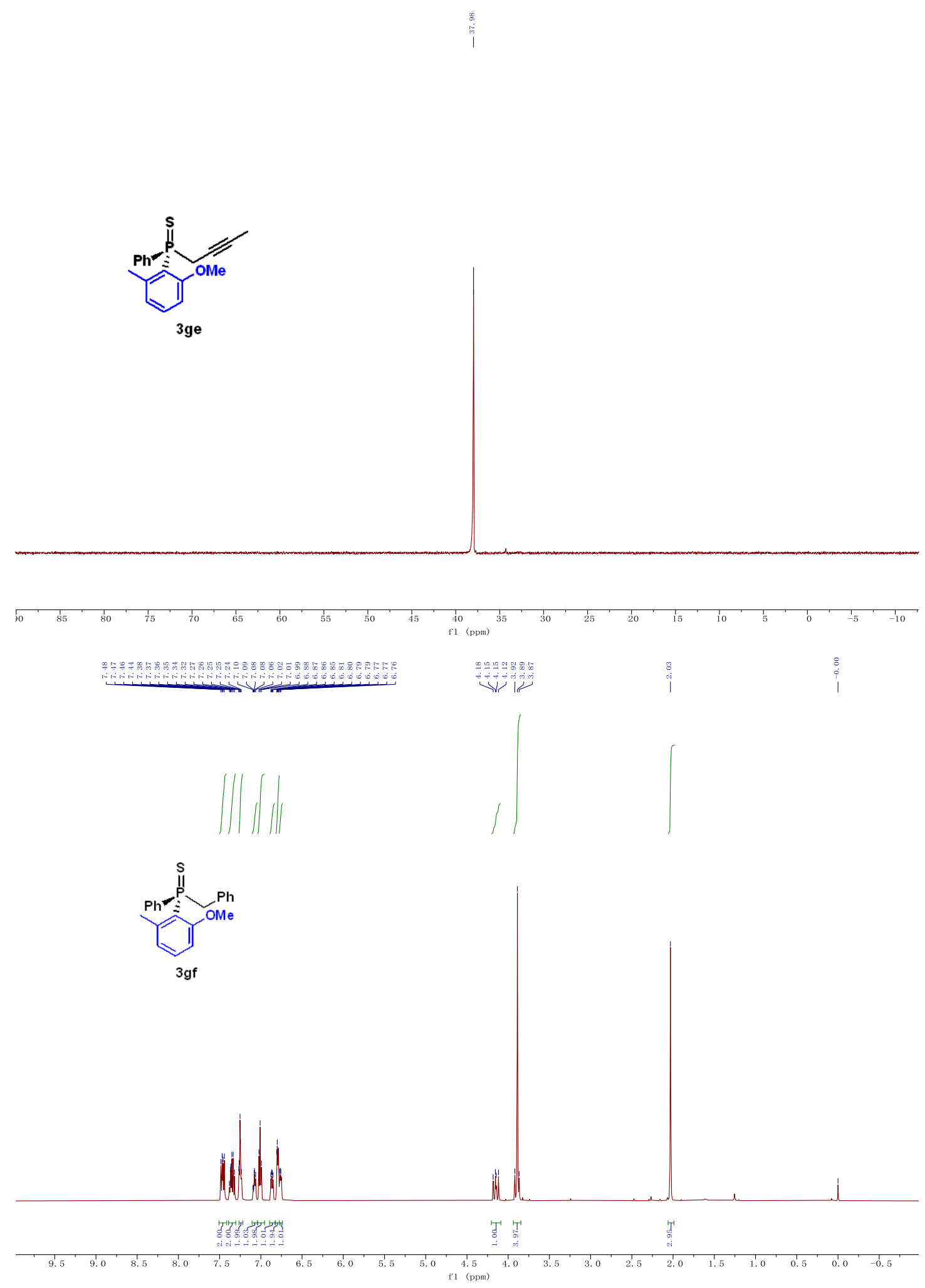

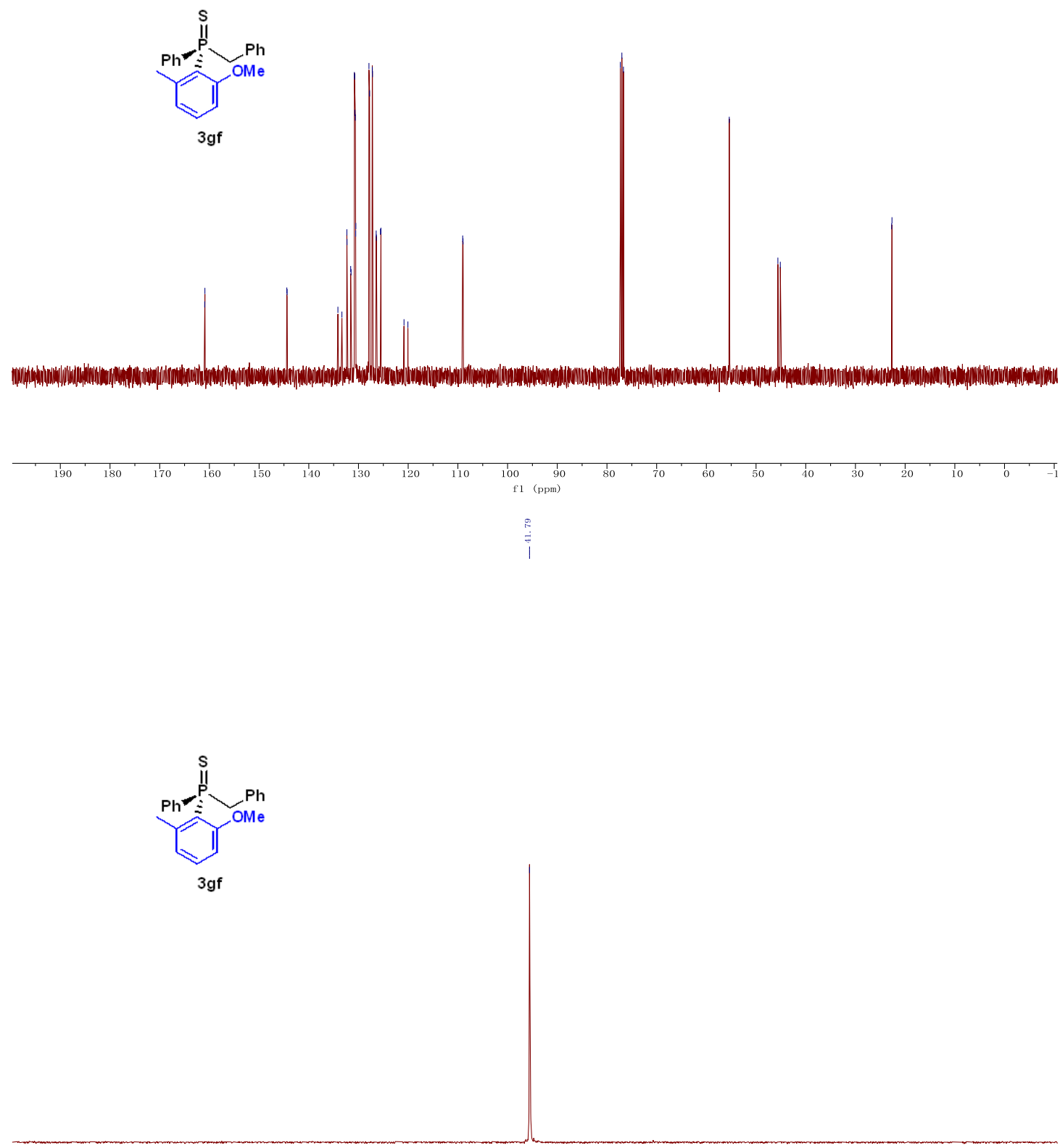

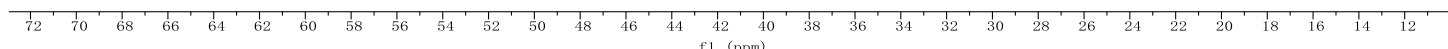



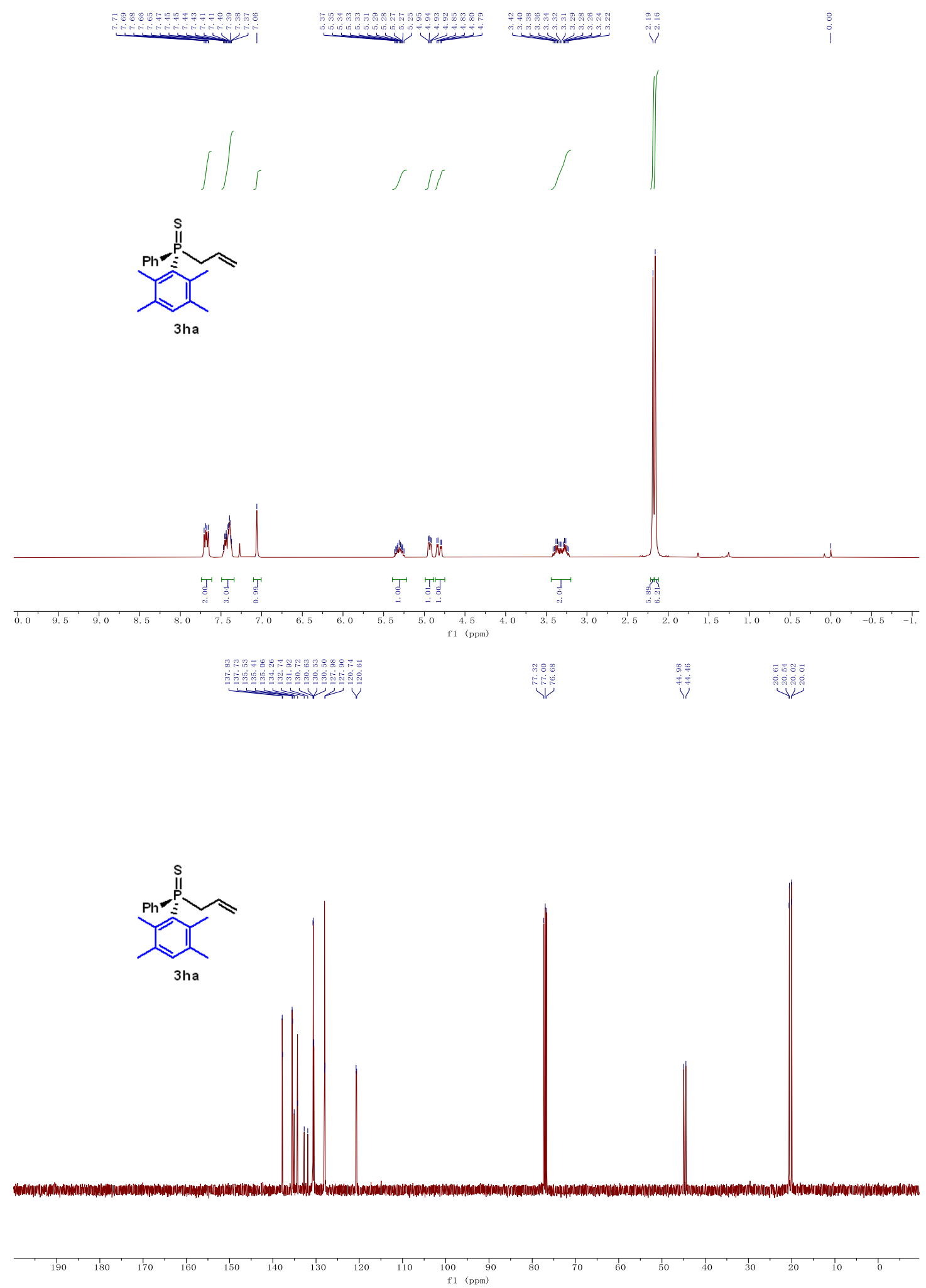

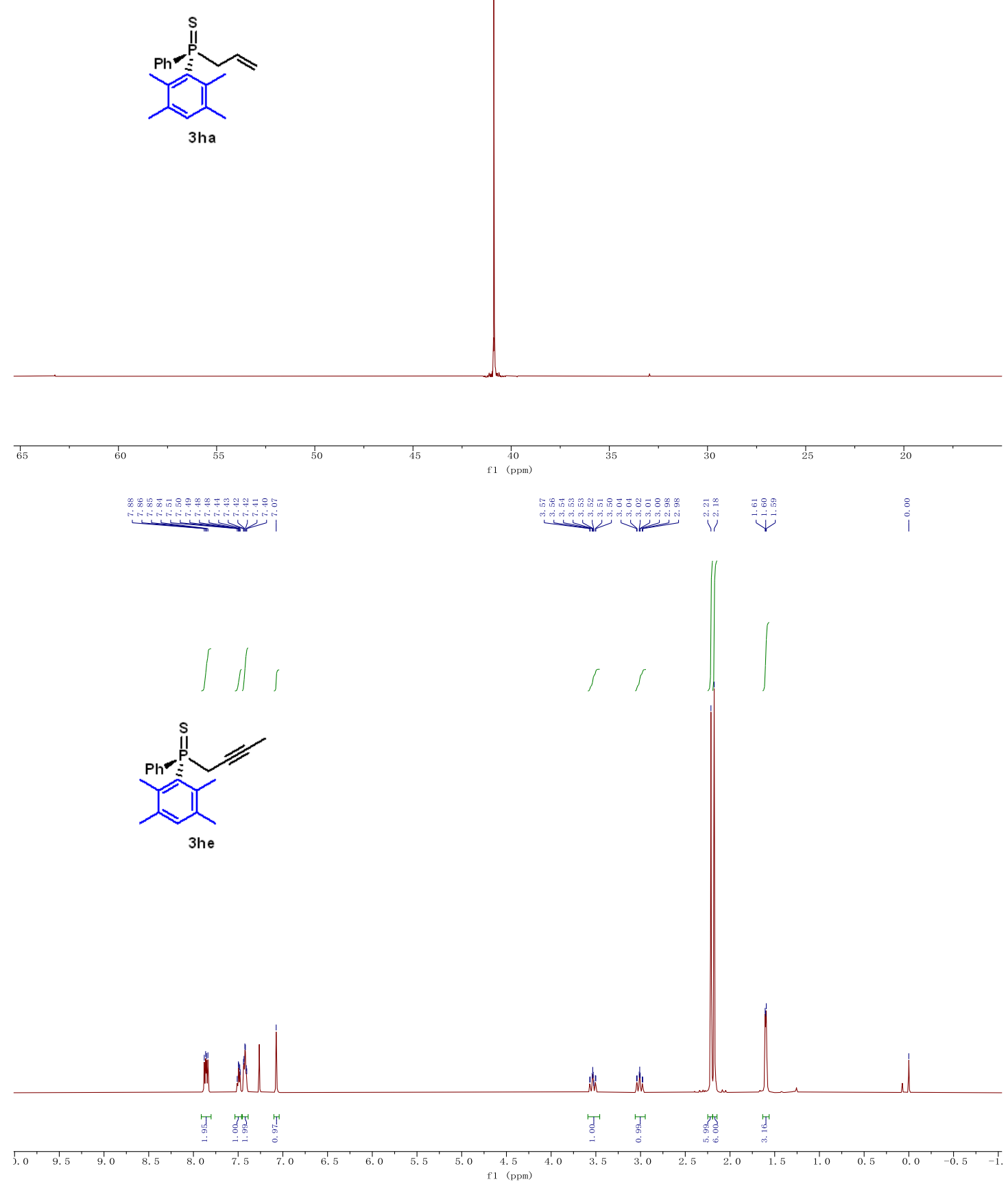


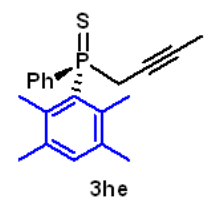

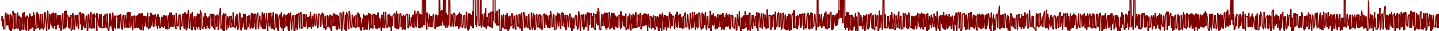
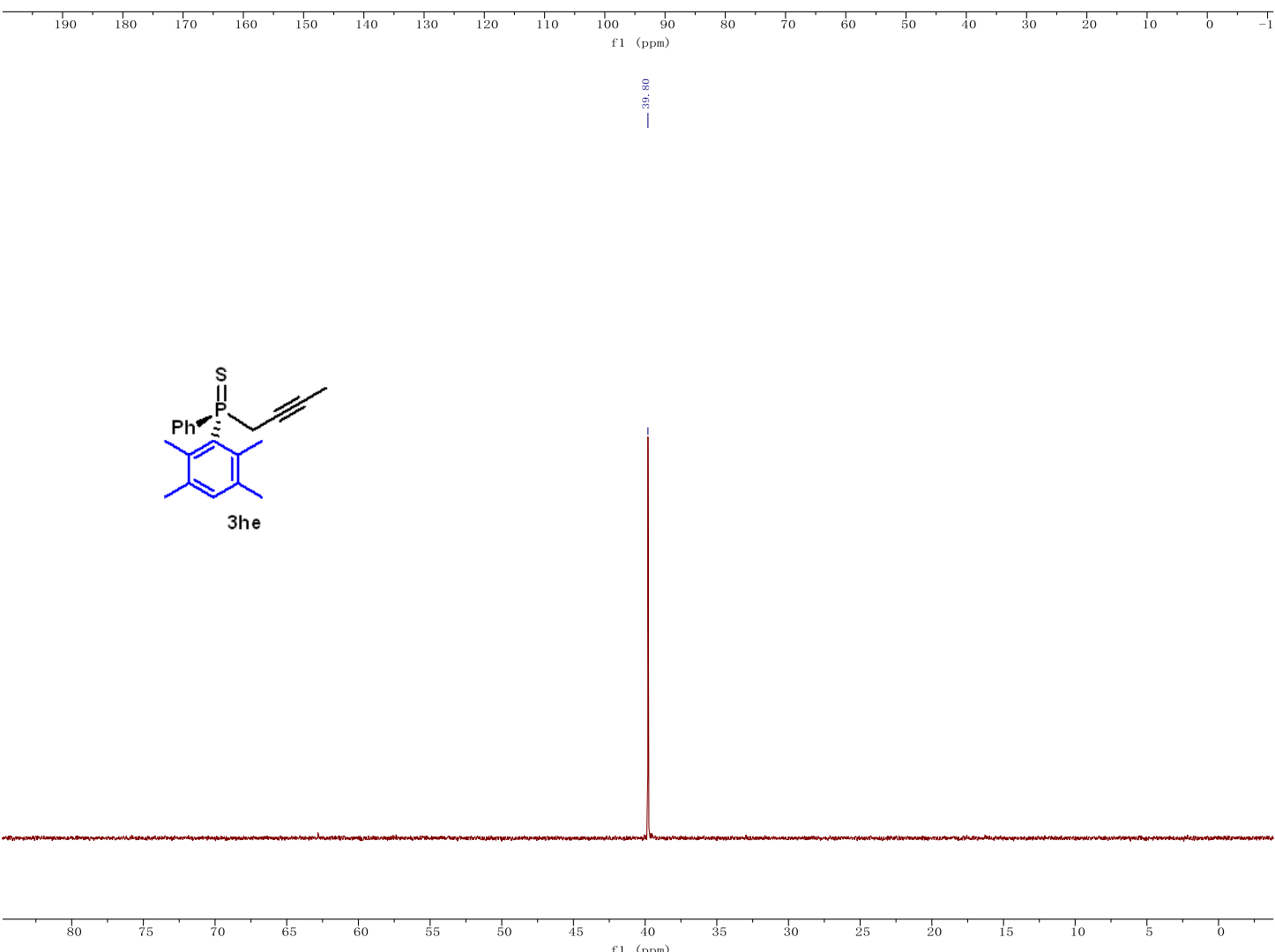


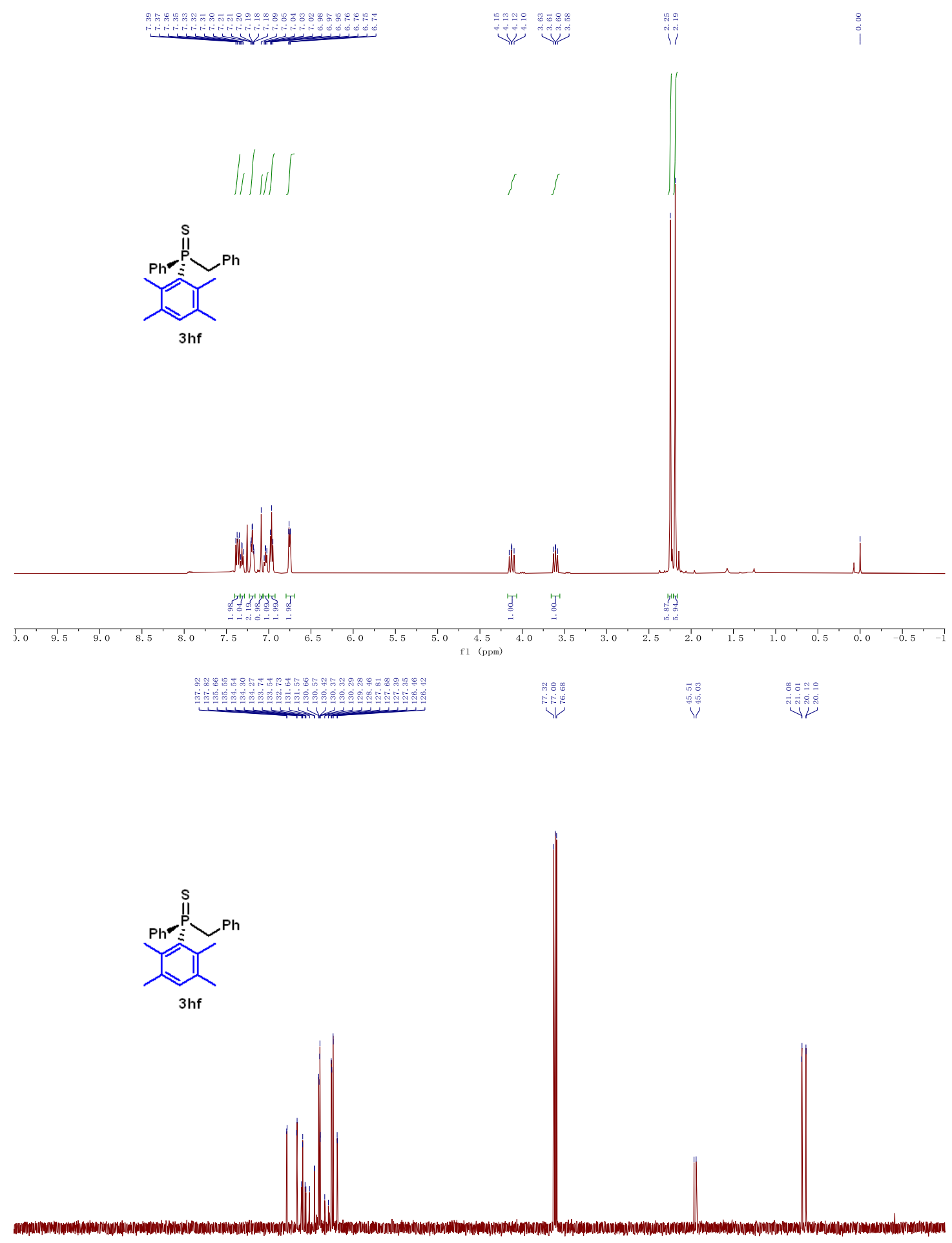



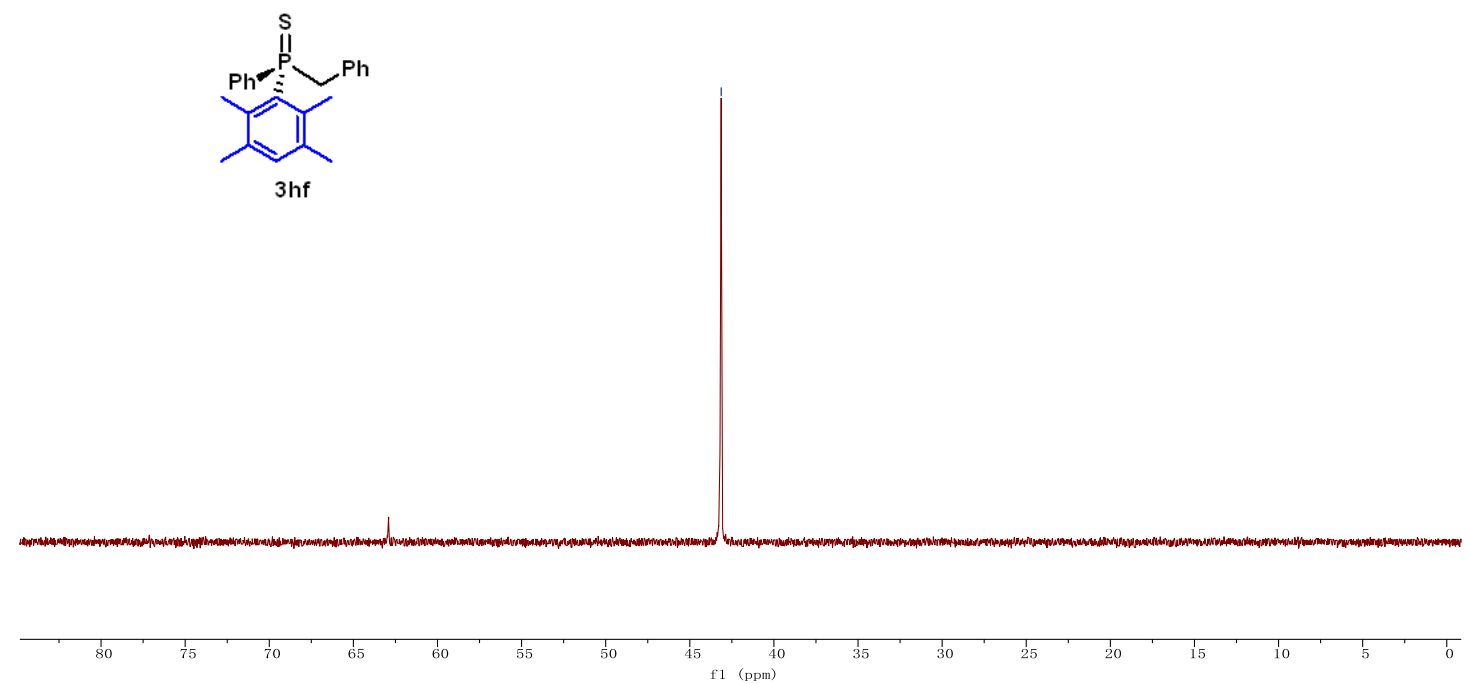

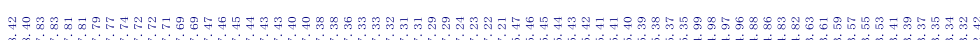

(1)
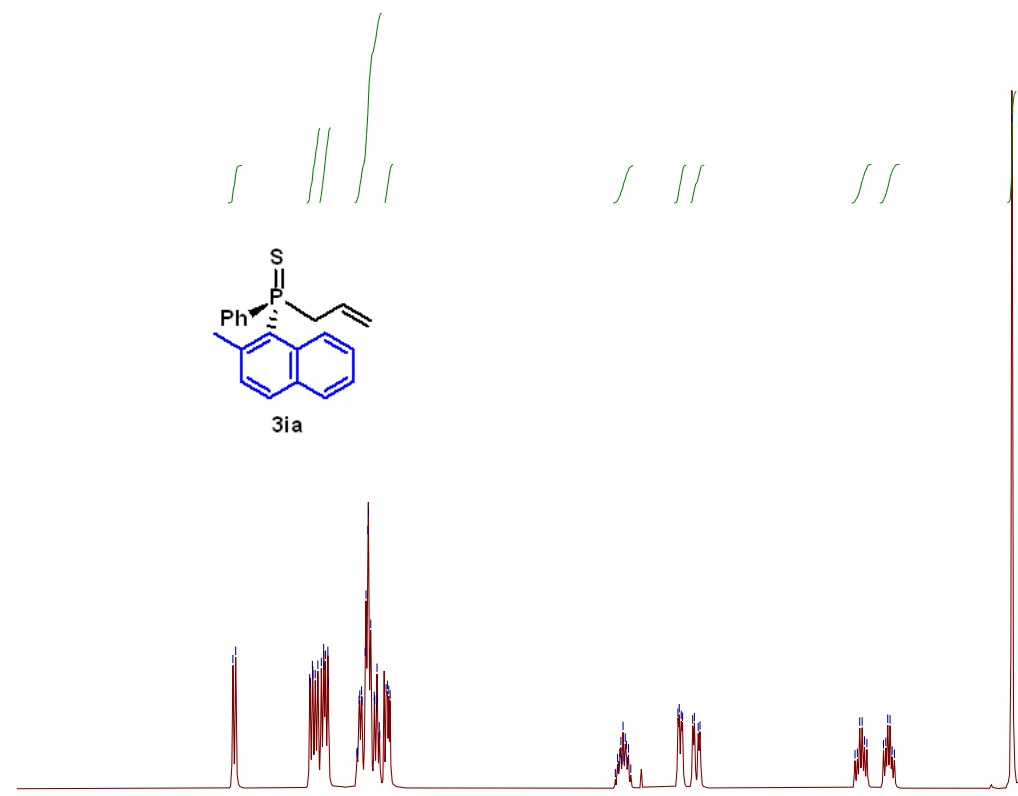

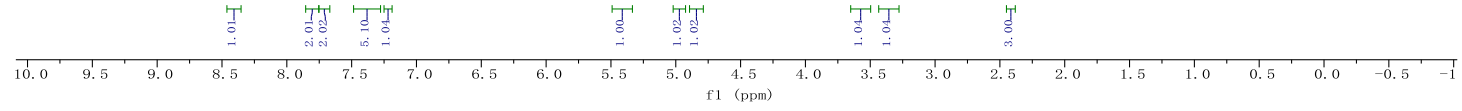



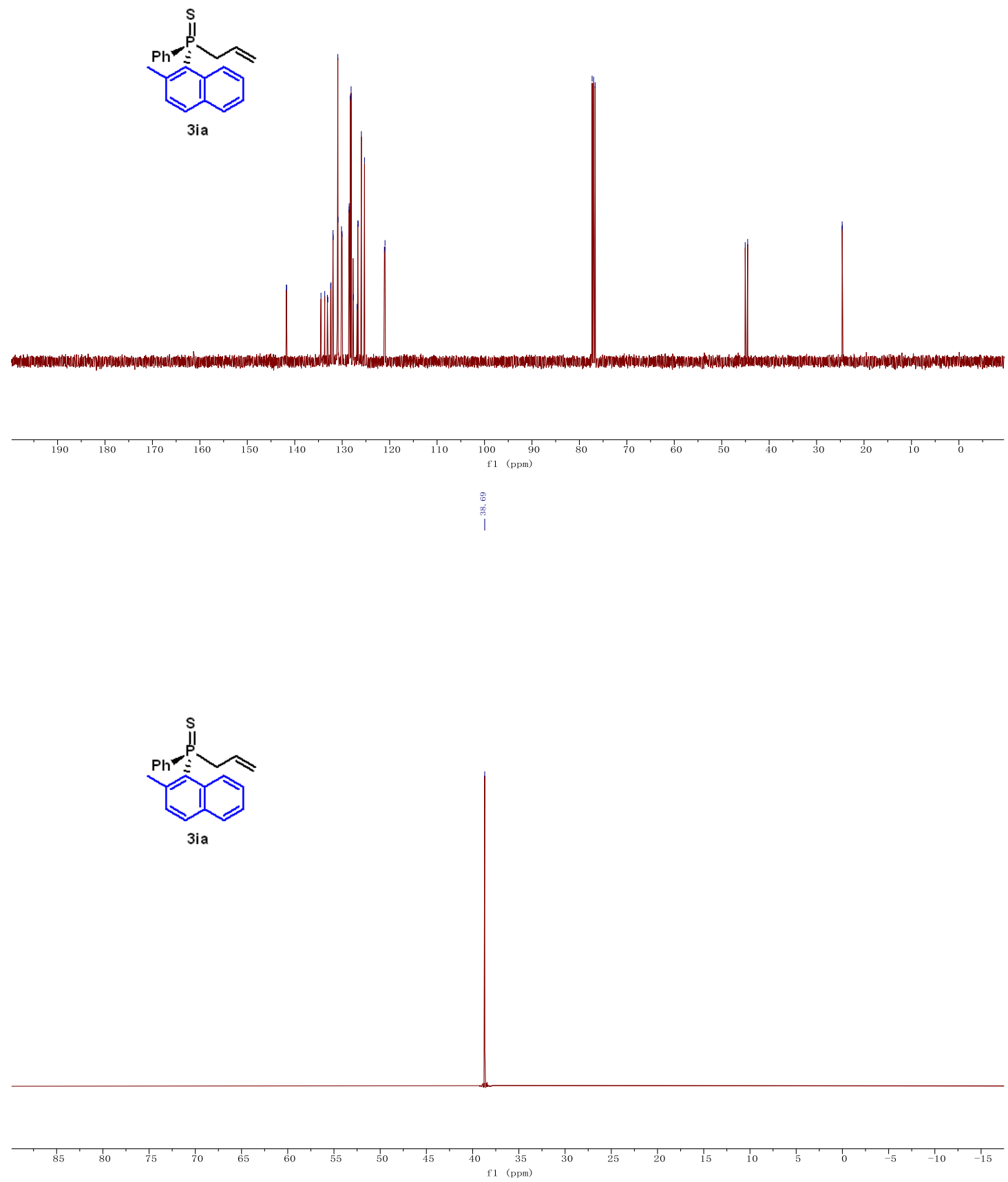

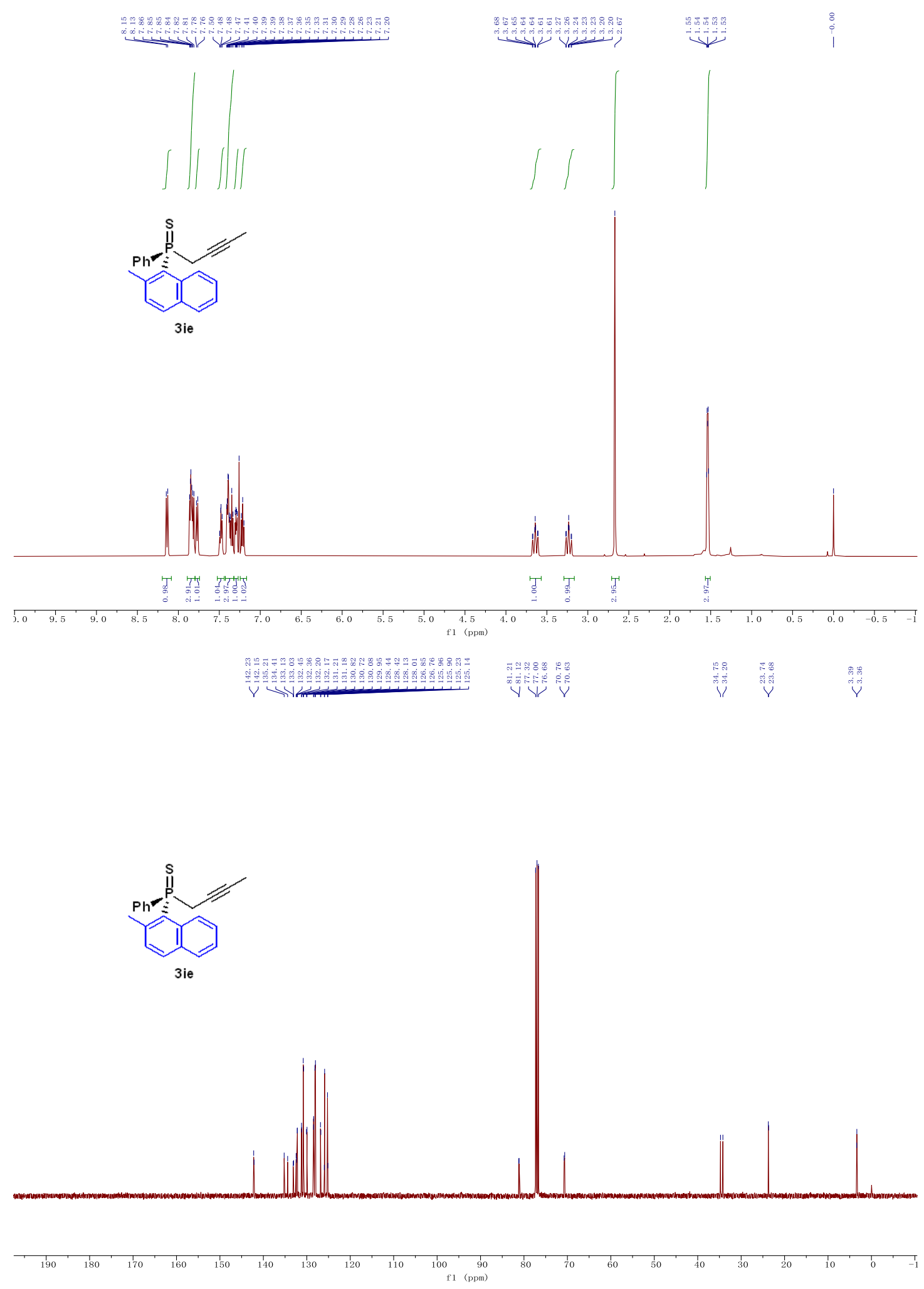

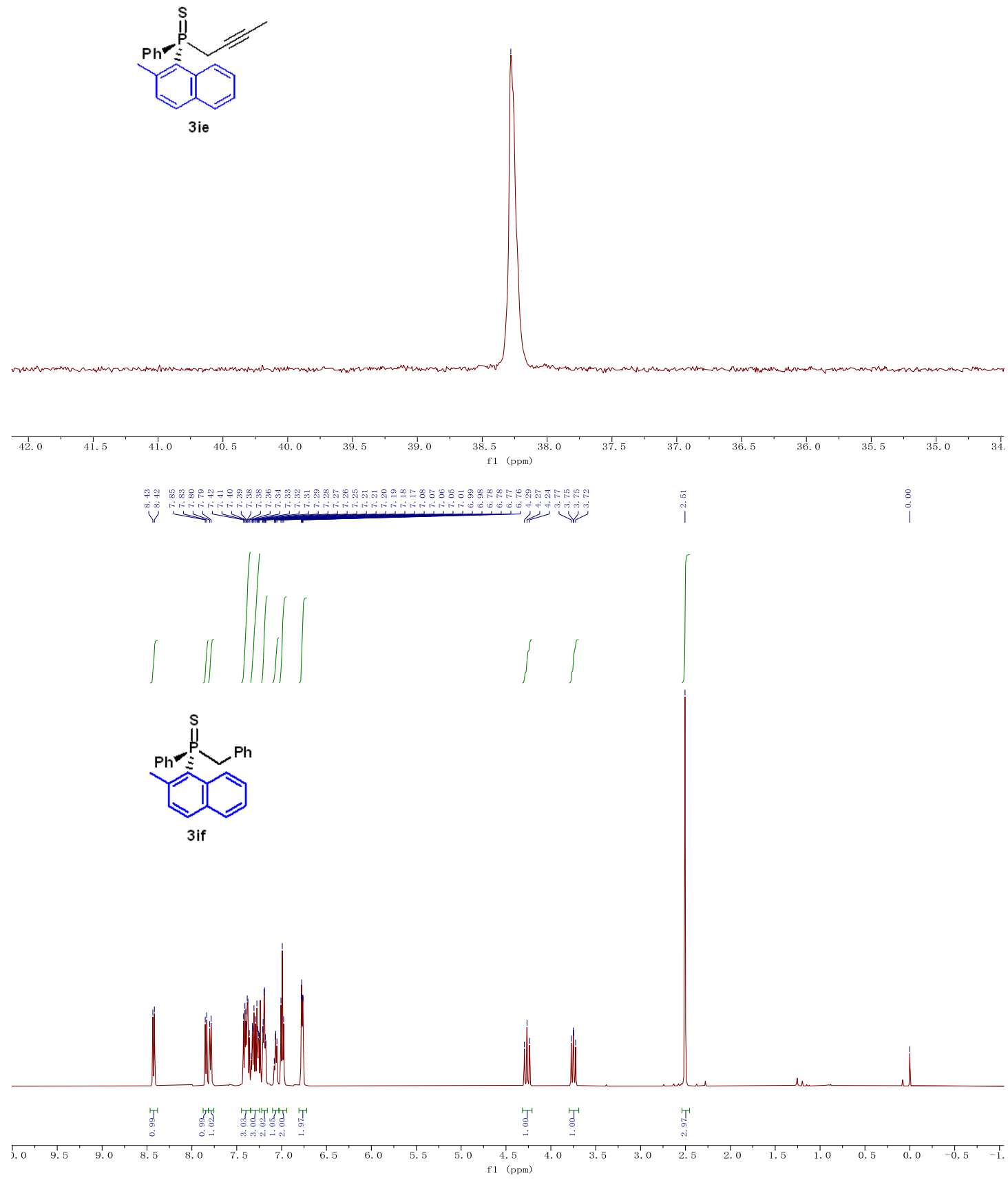

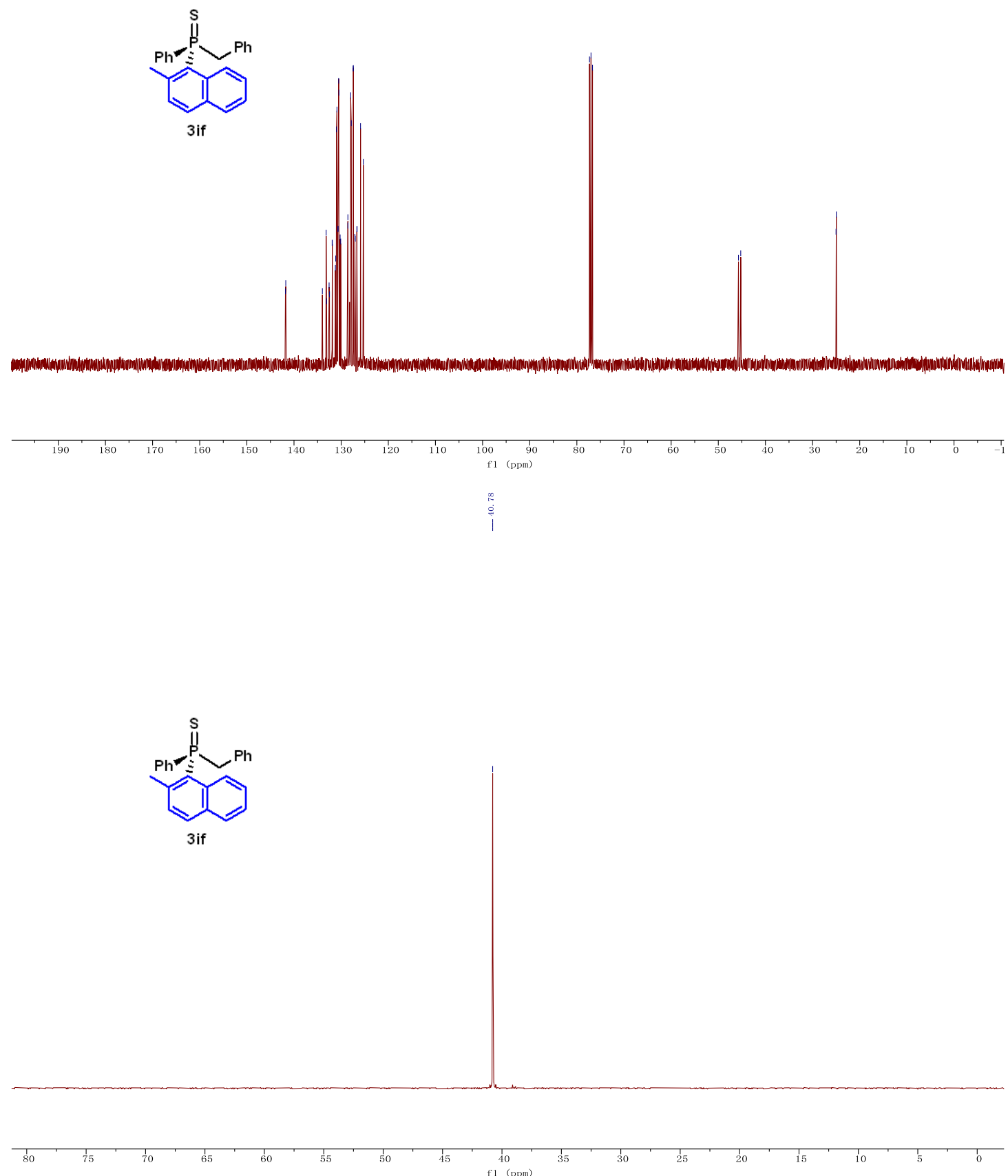


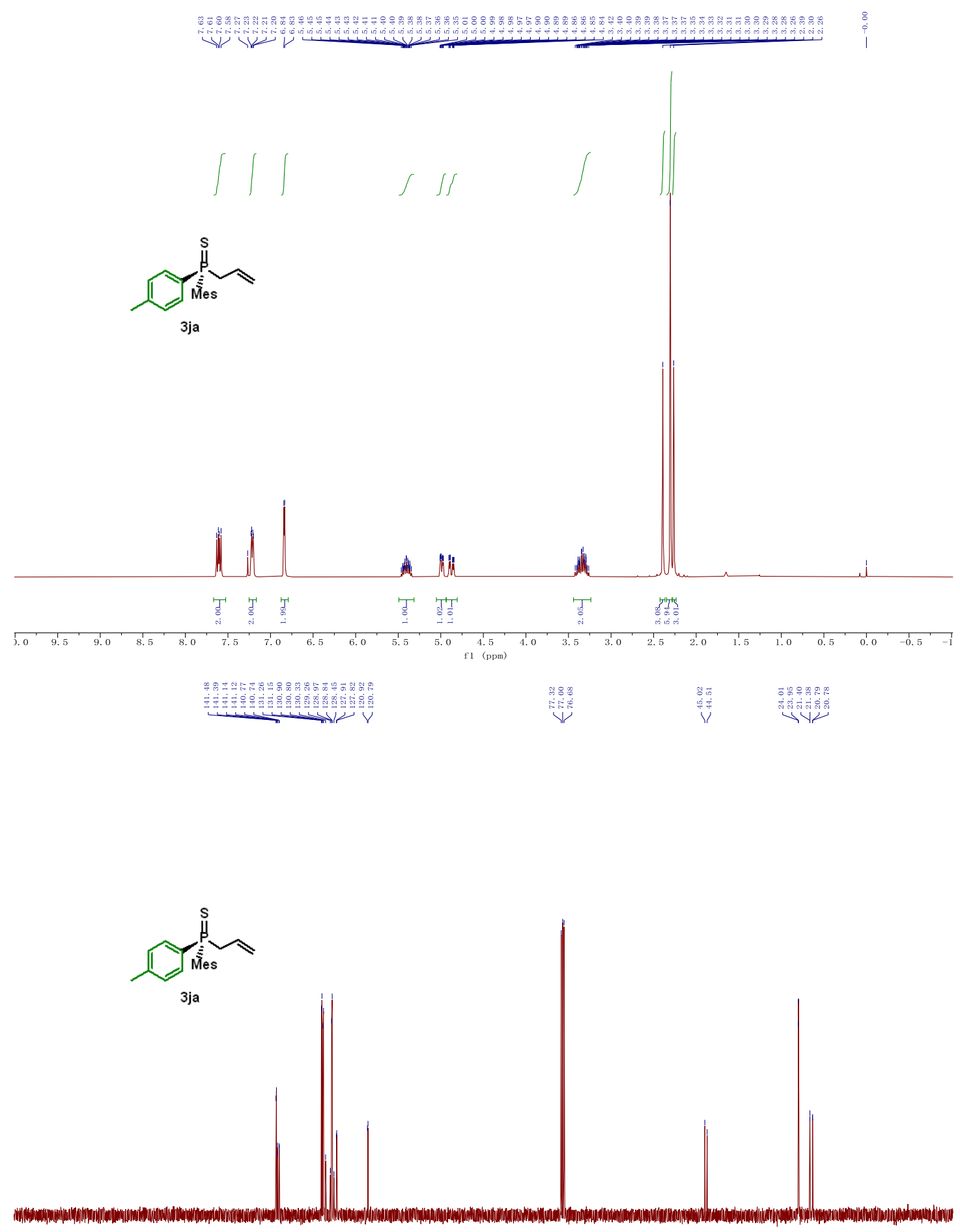

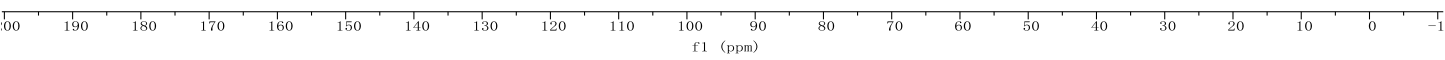



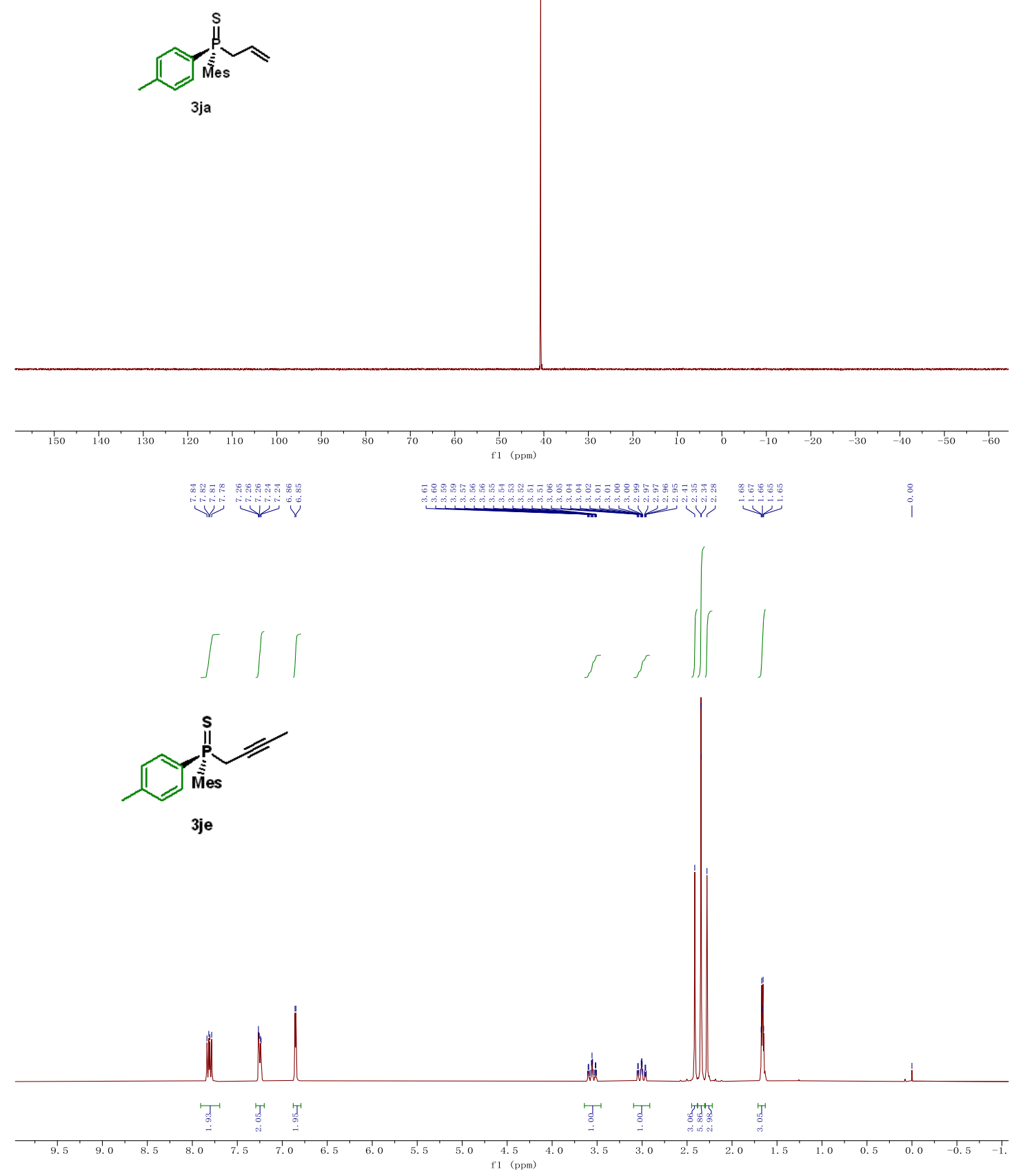

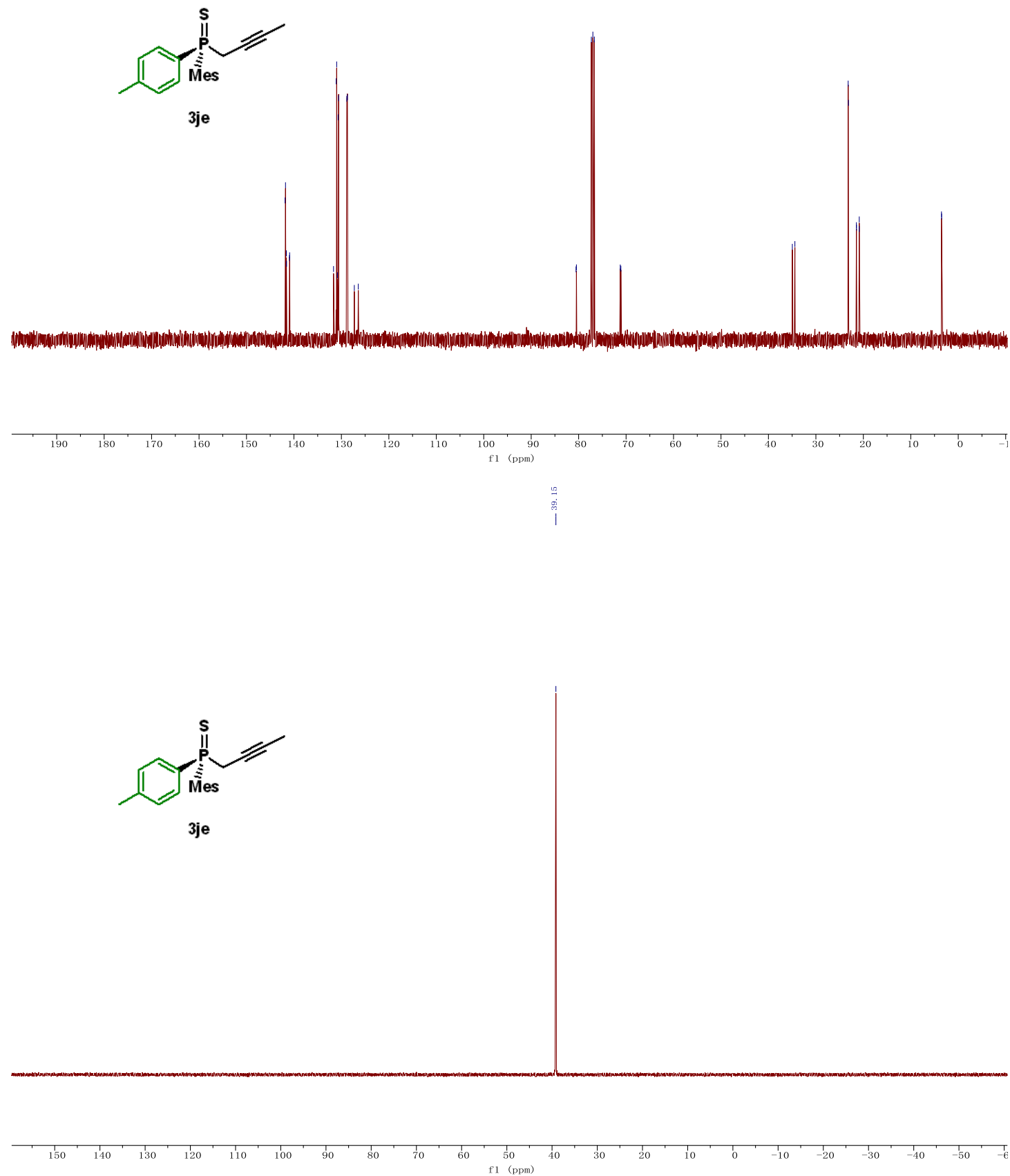


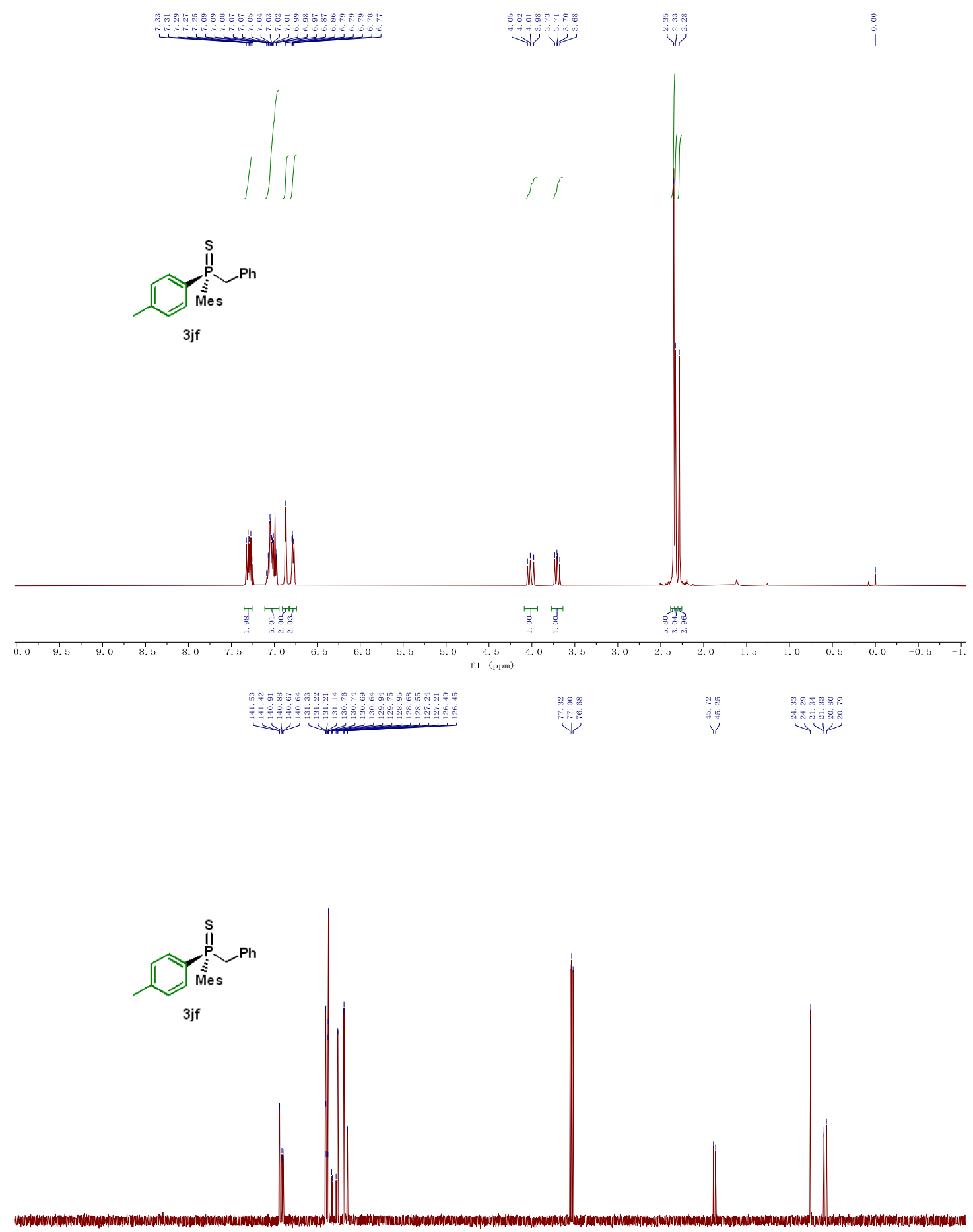

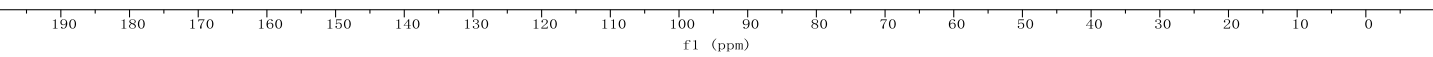




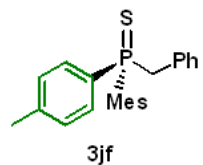

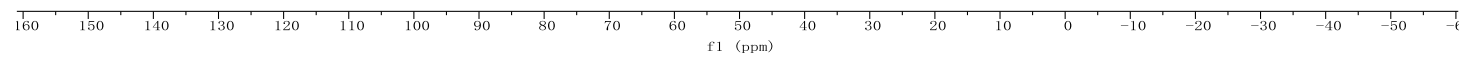

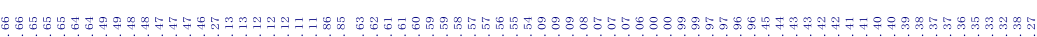

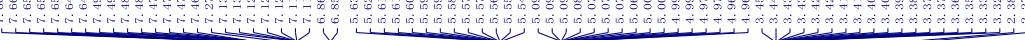
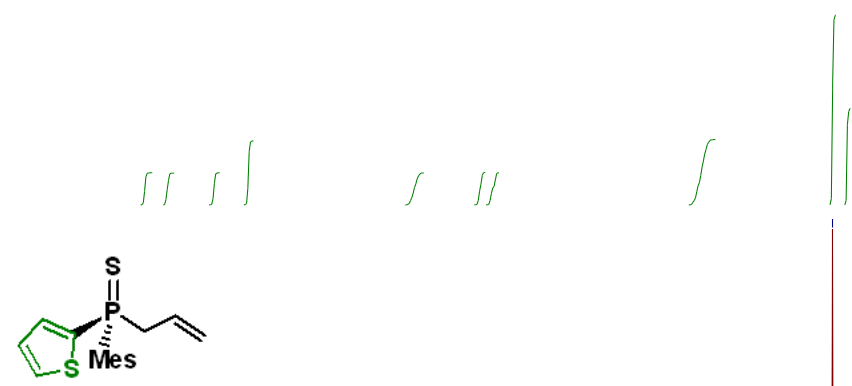

3ka
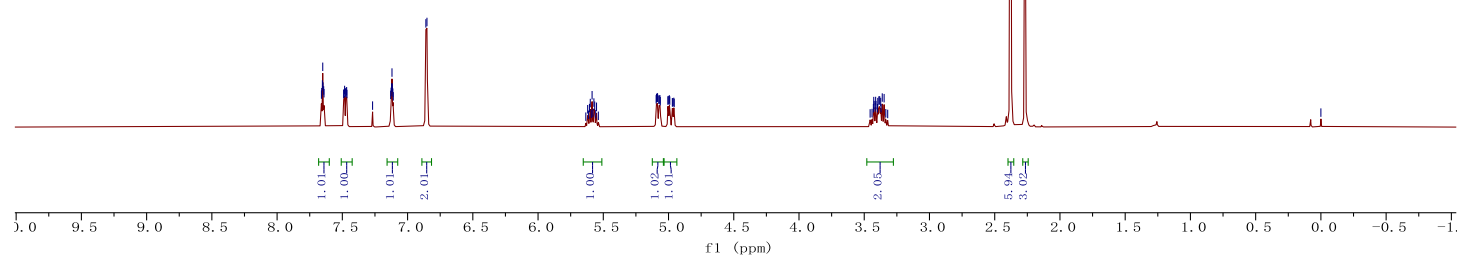

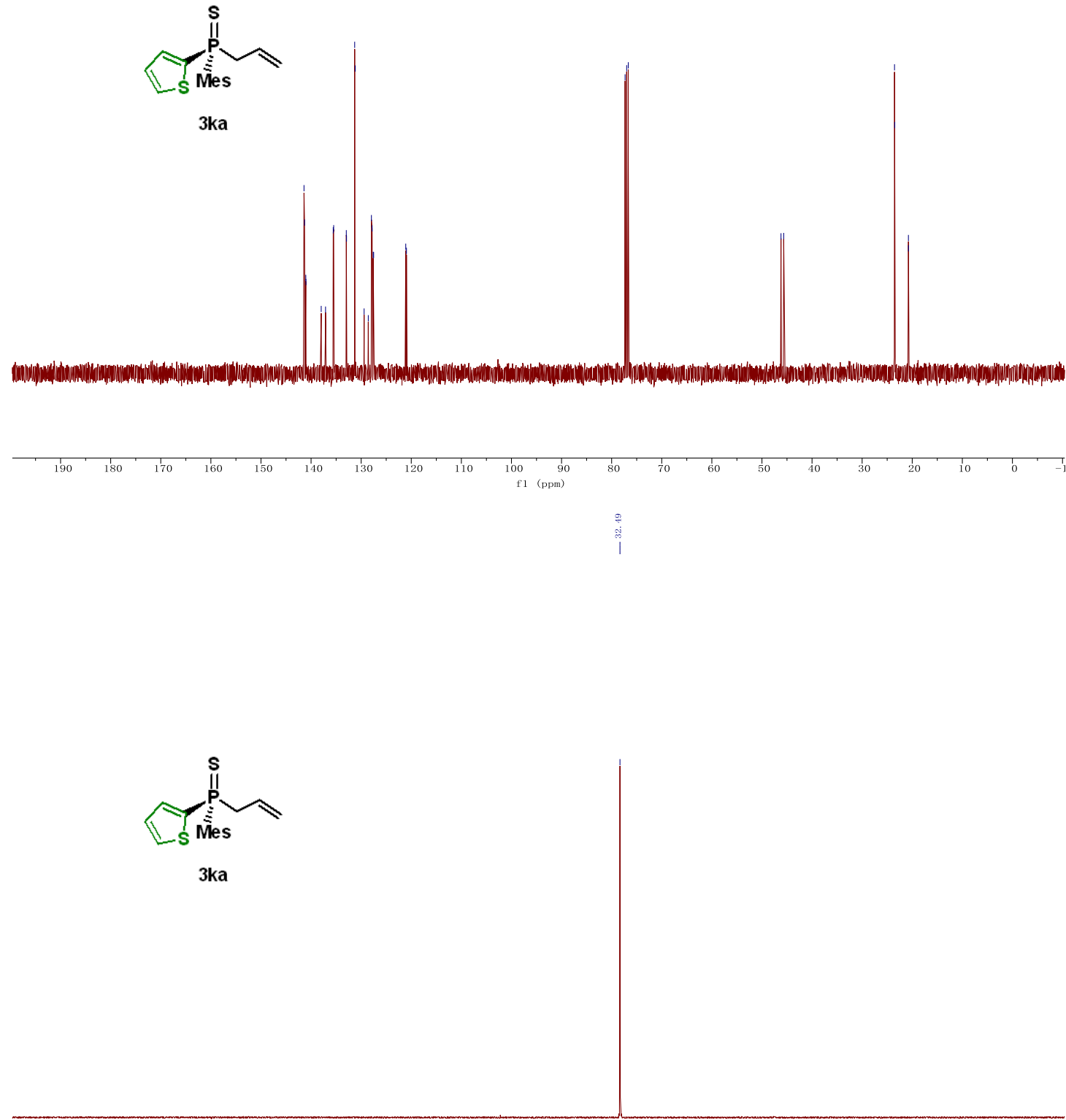

$\frac{7}{160}$

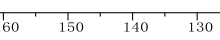



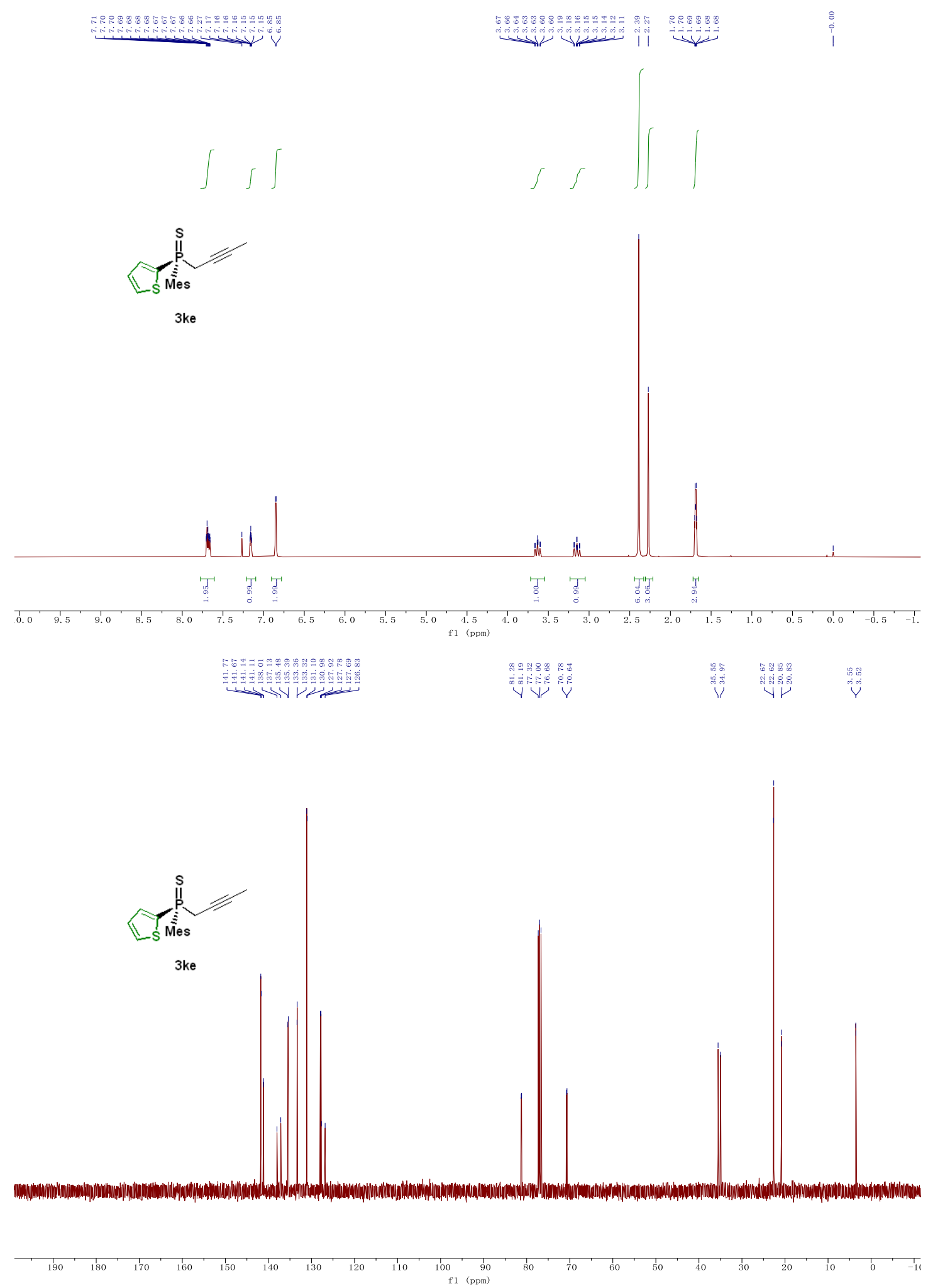

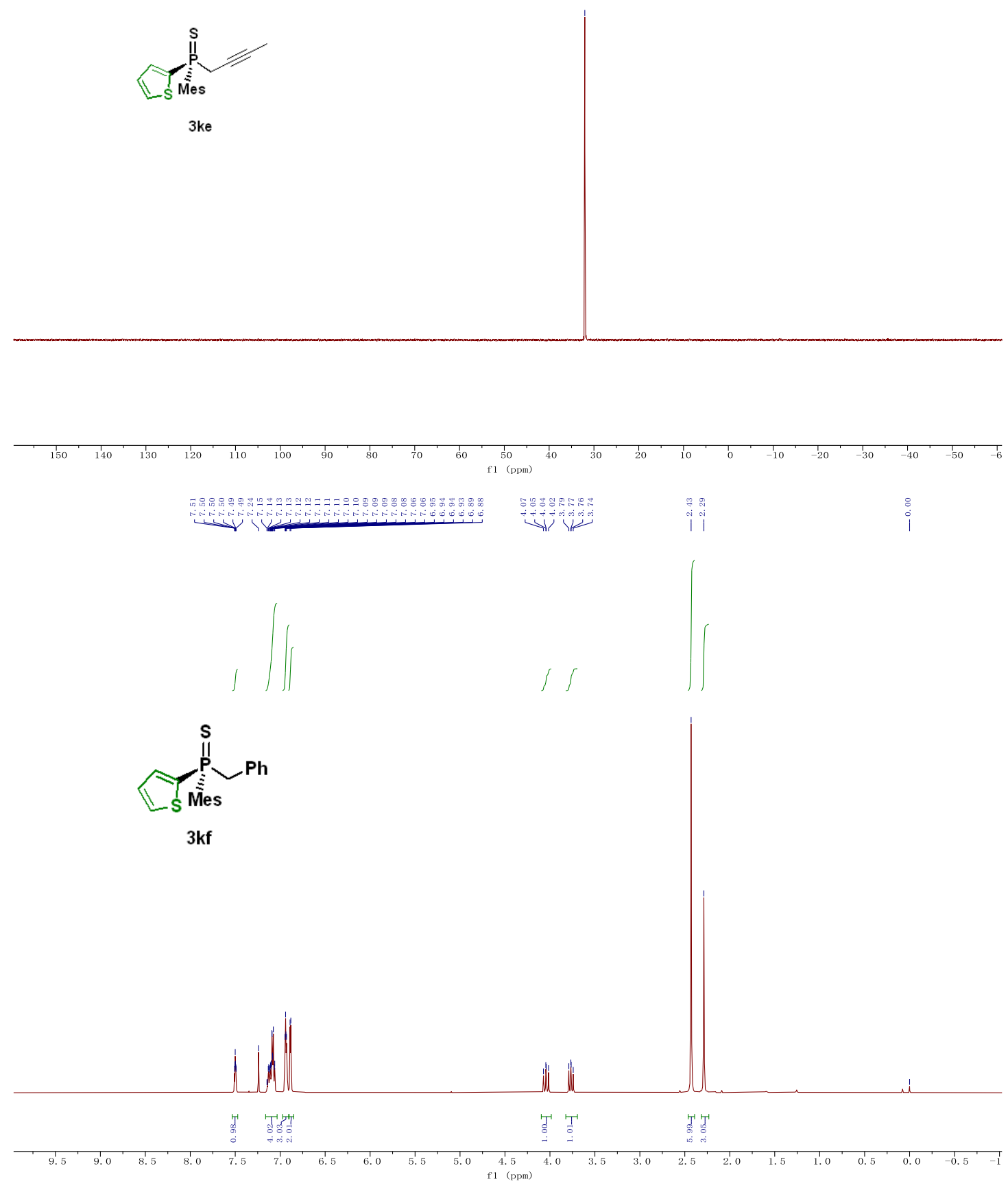

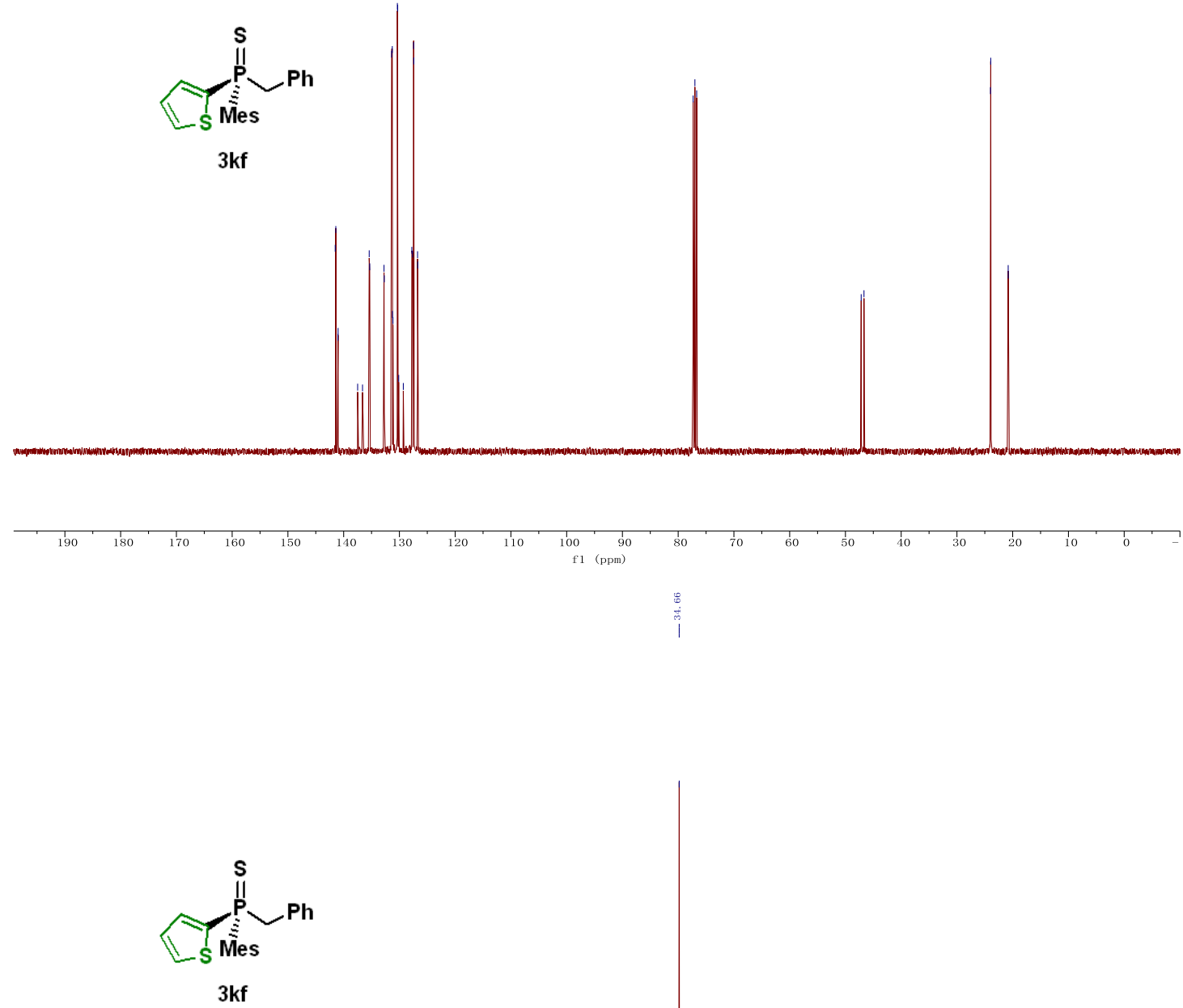

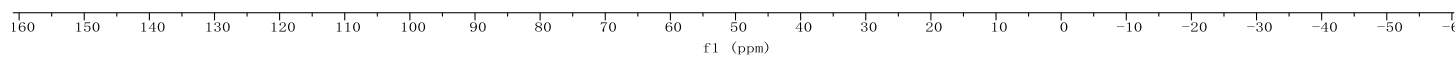




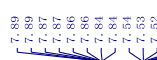

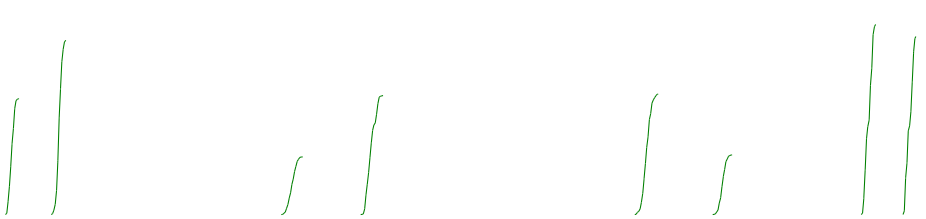

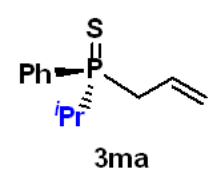
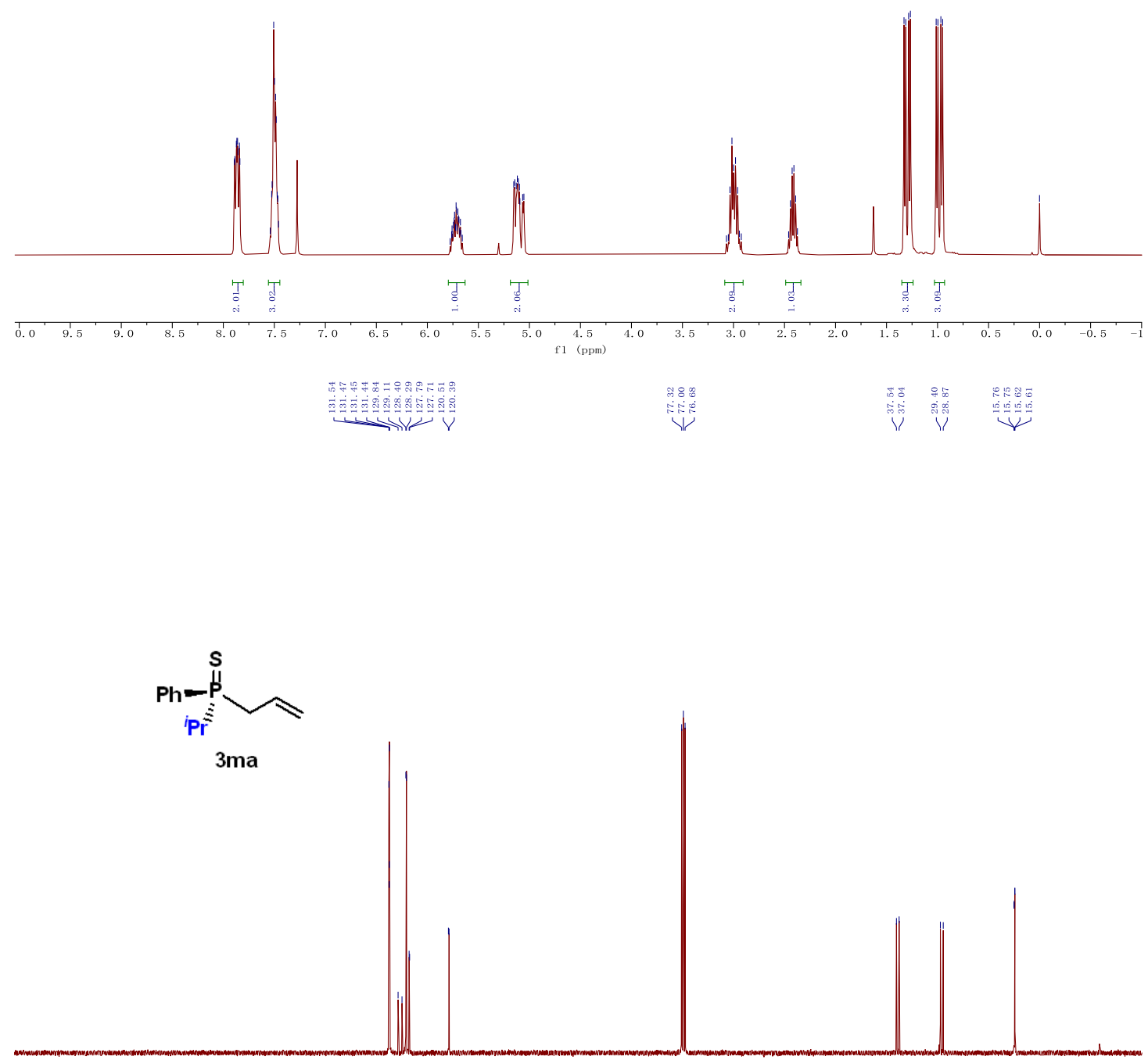

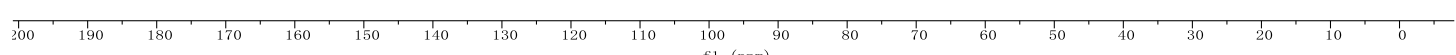




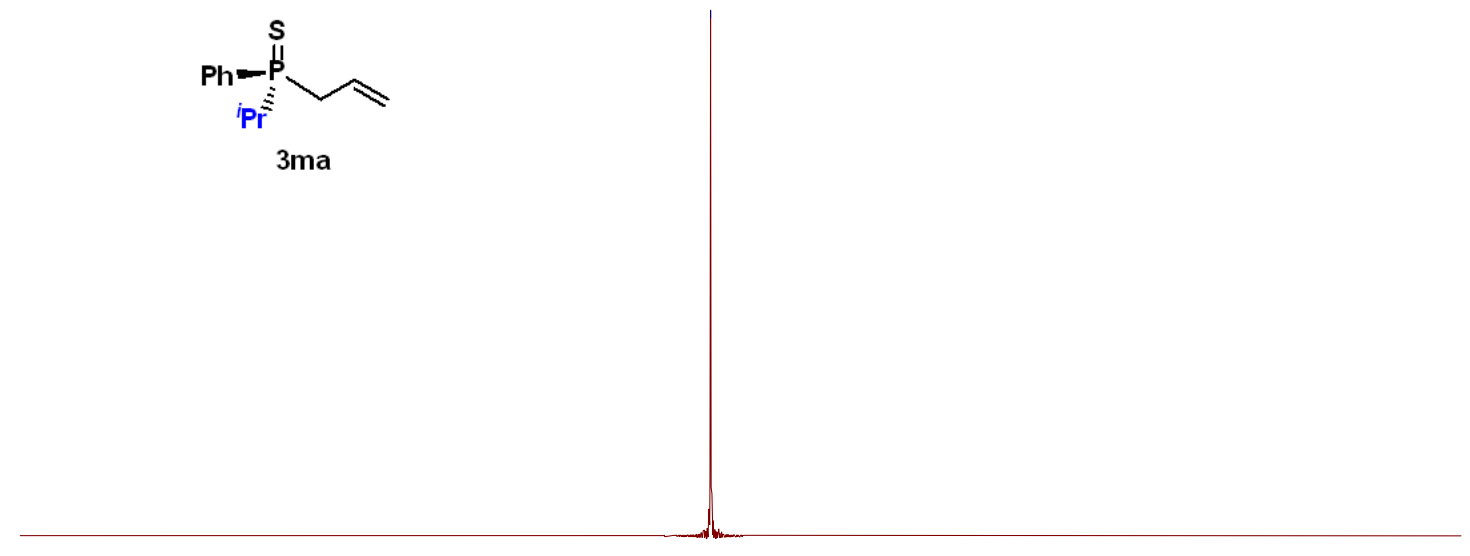

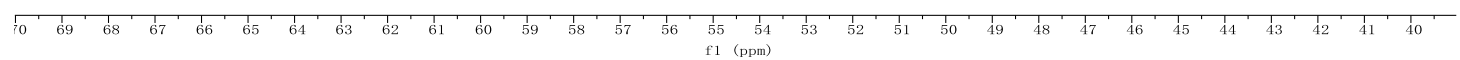



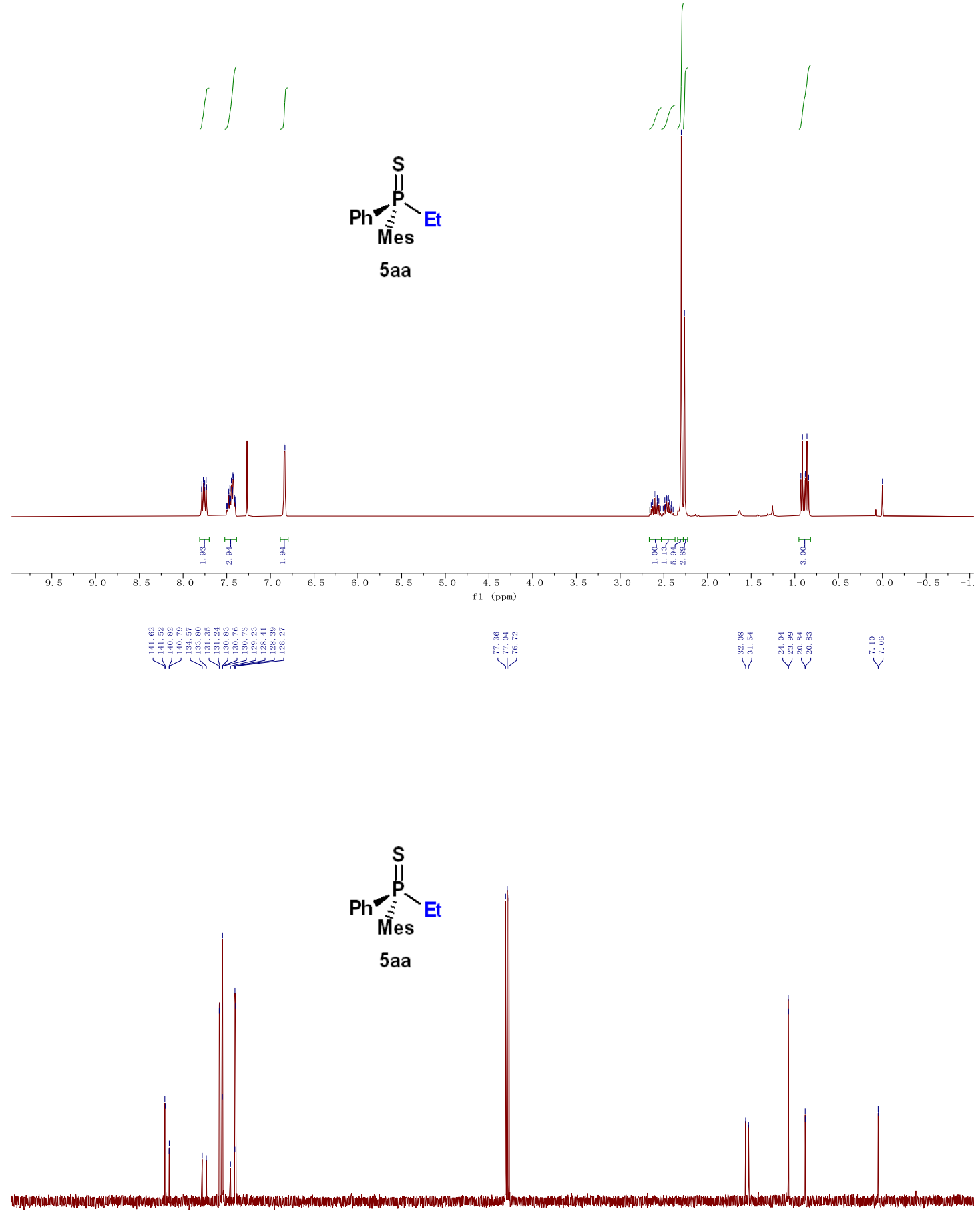

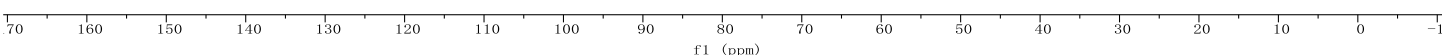




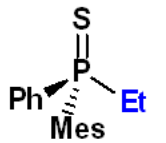

5 aa

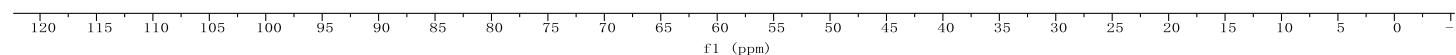

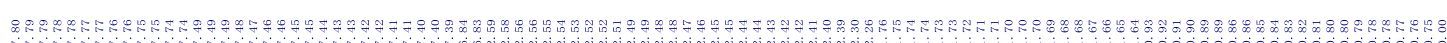
$\longrightarrow$

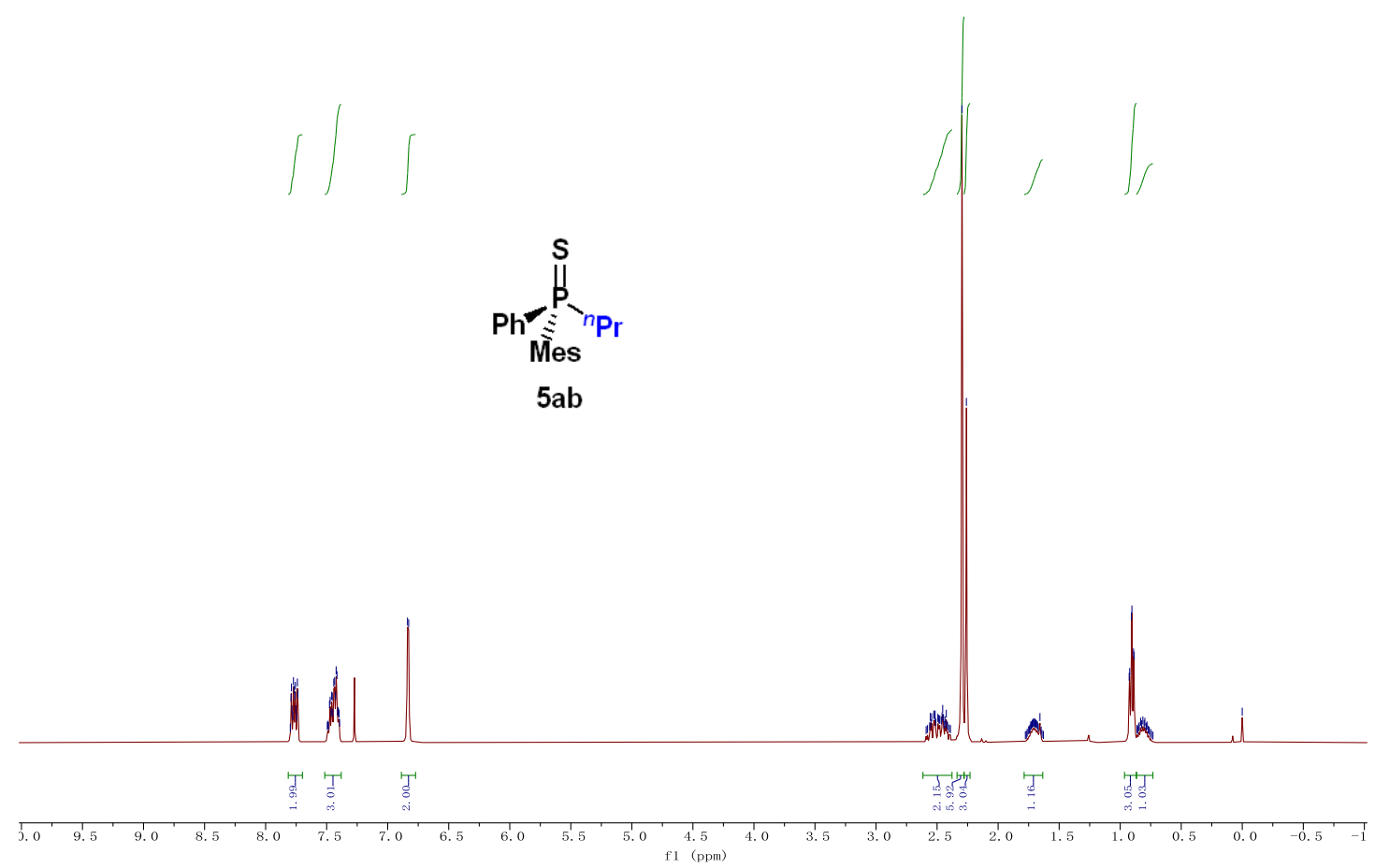



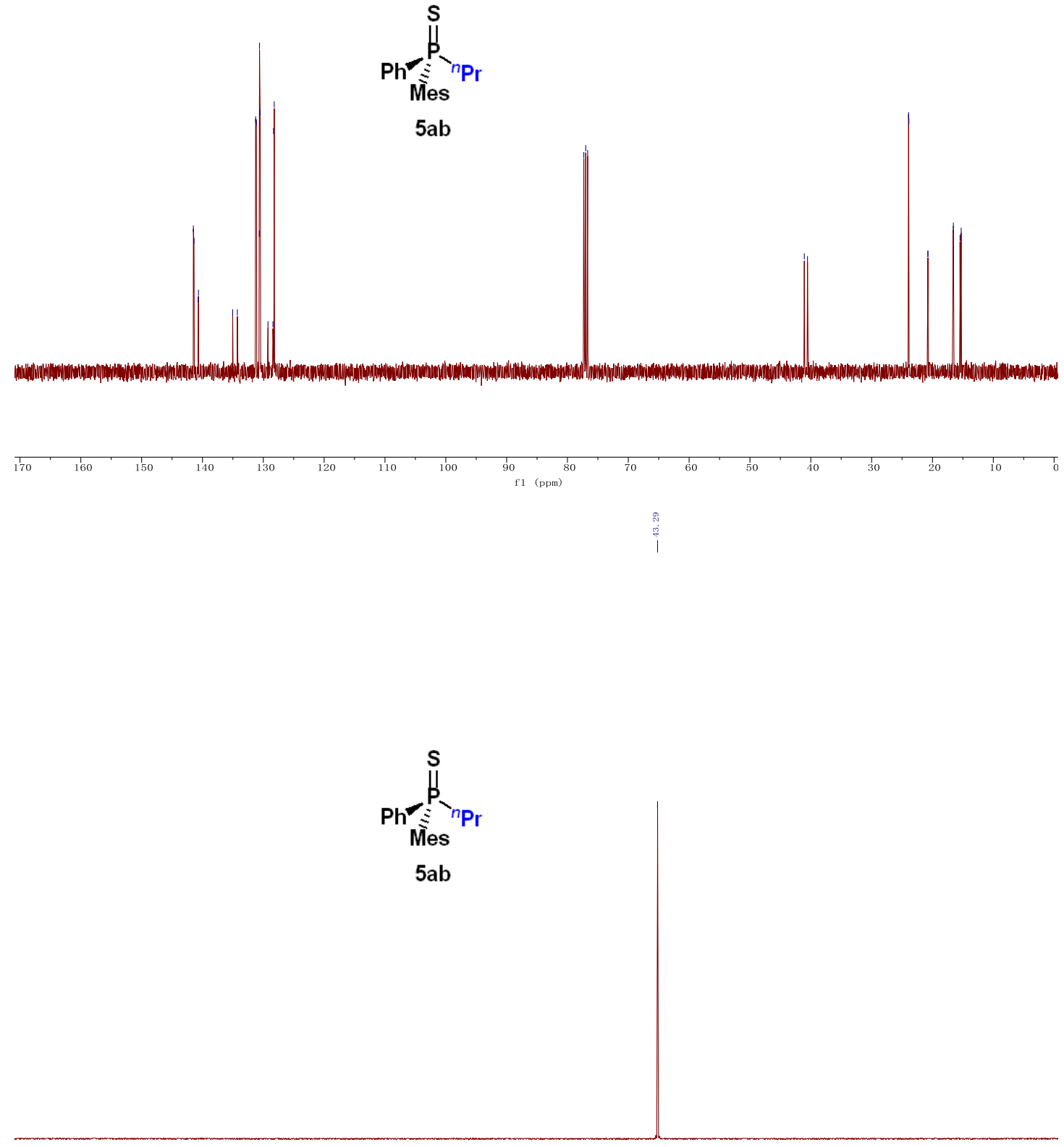

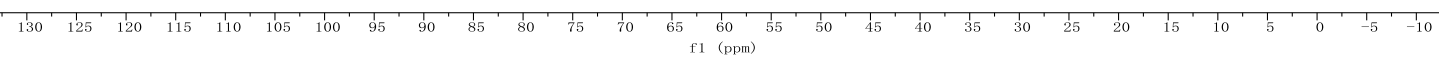



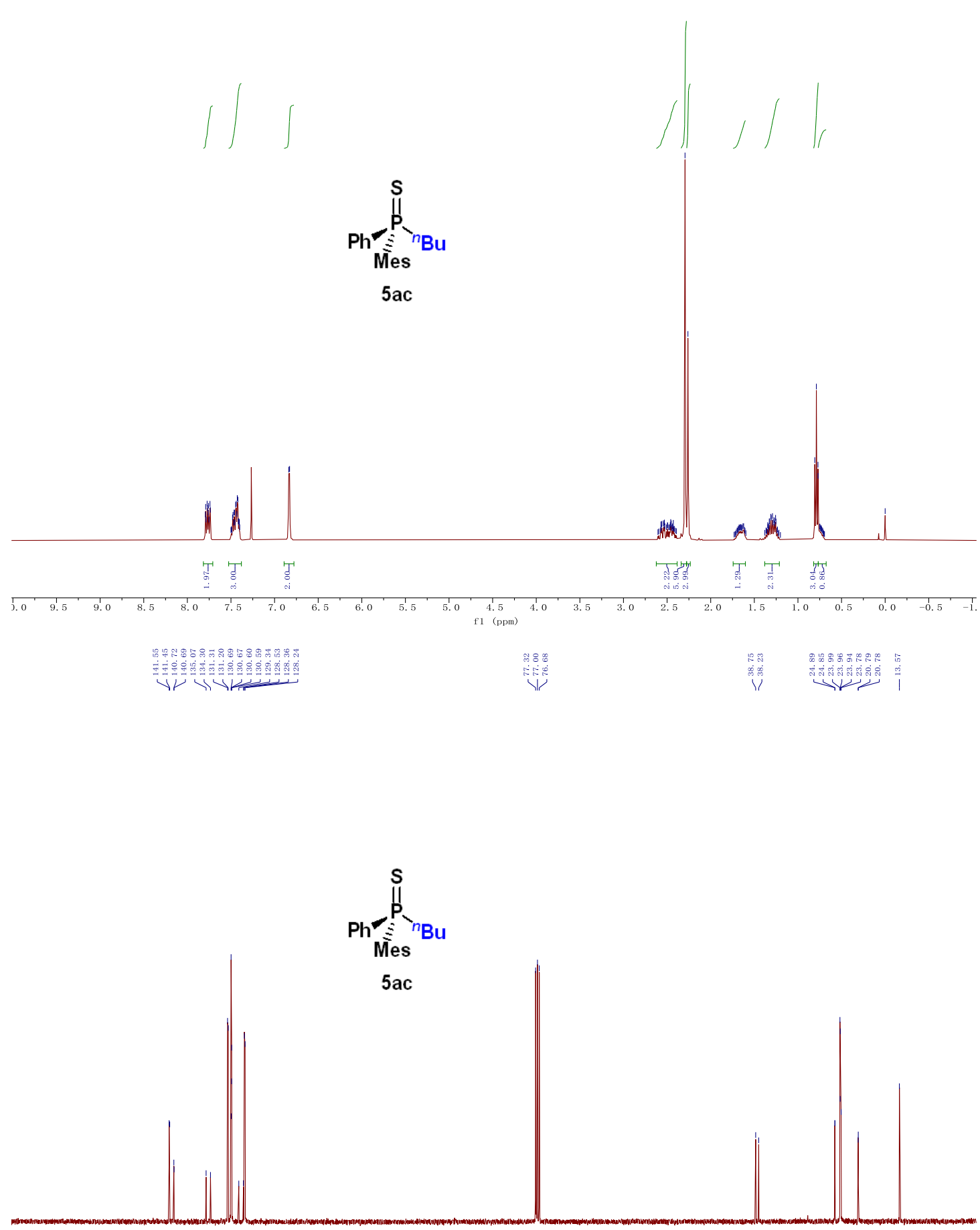
$\mathrm{Ph}_{\mathrm{Mes}}^{\mathrm{S}}$

5 ac

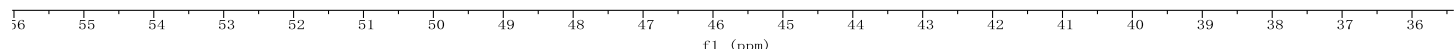

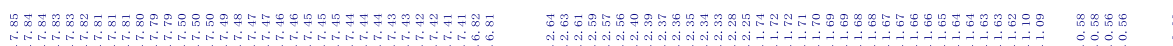

$\longrightarrow$ y

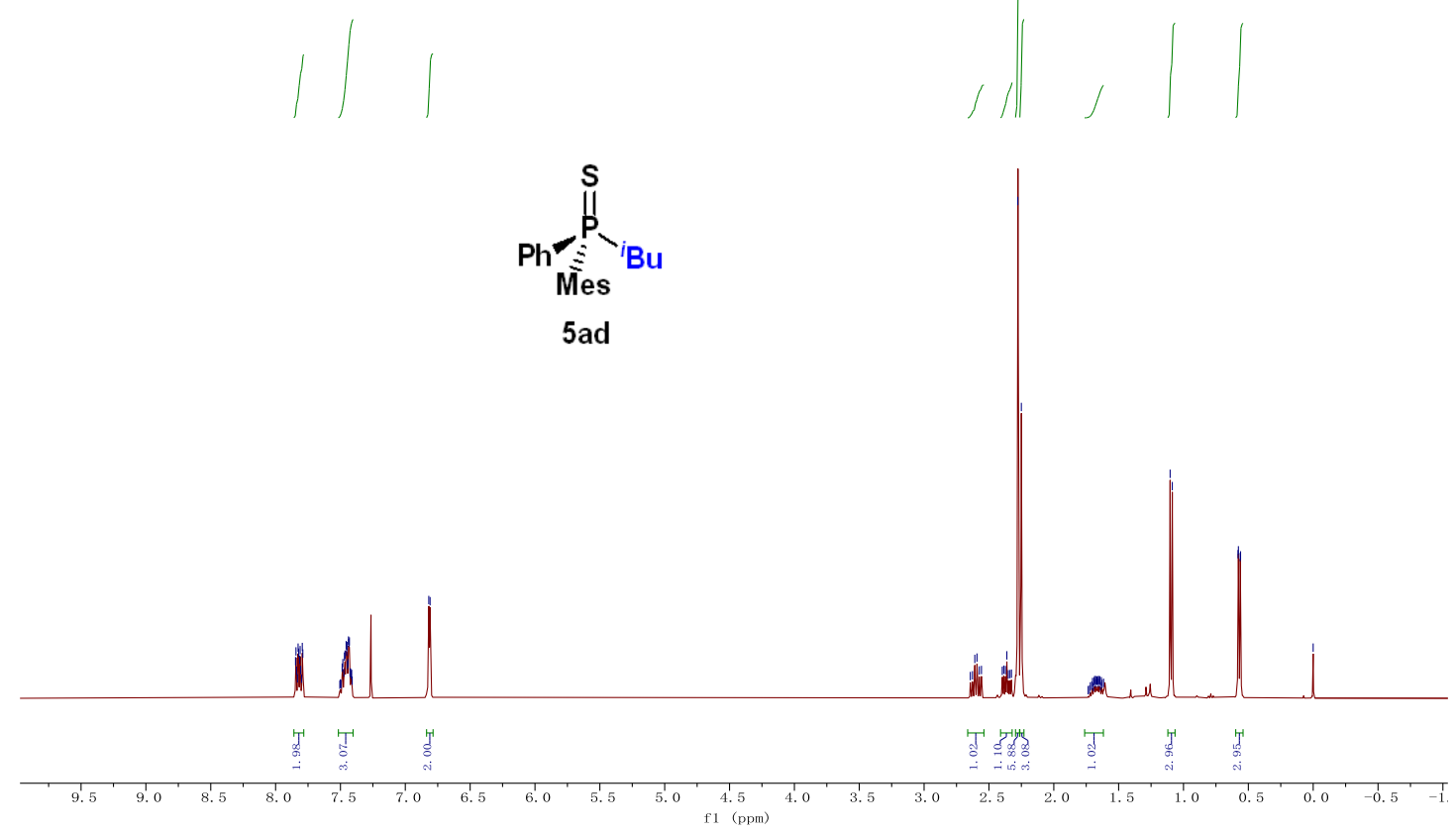



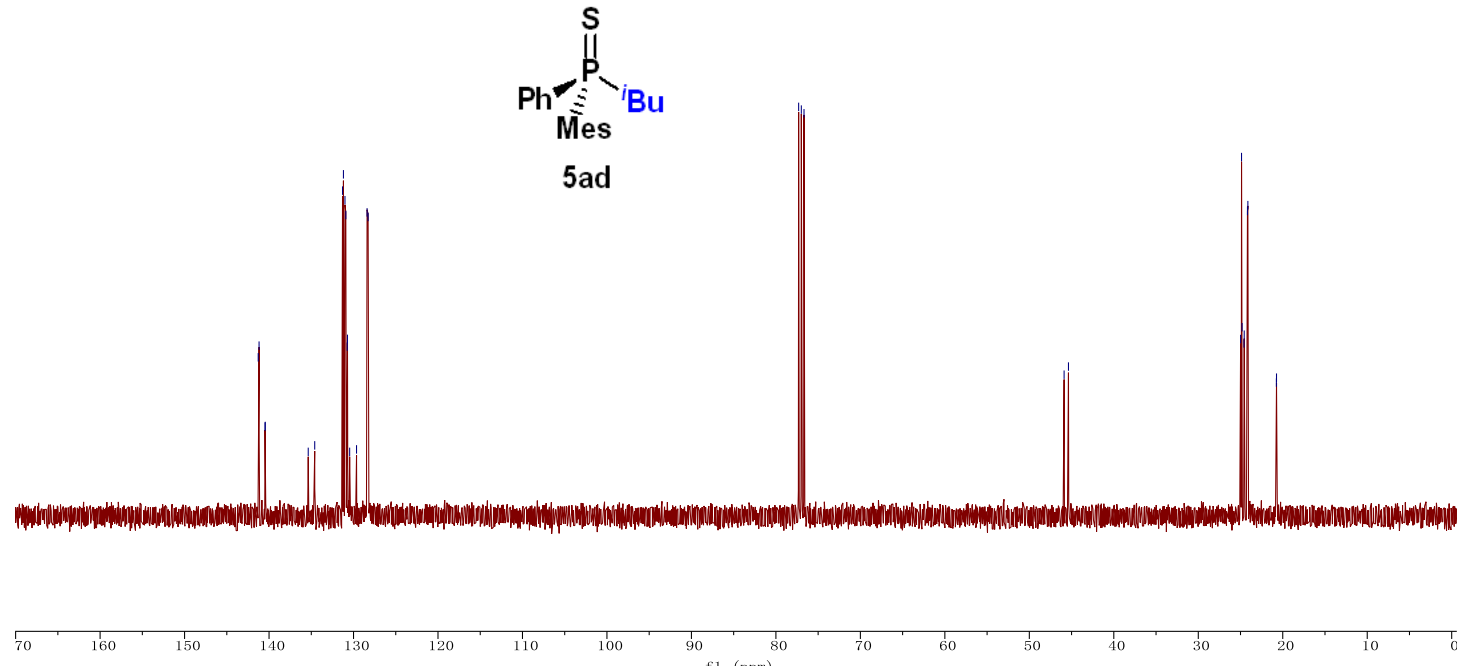

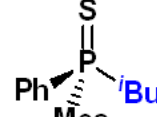

Mes

5 ad

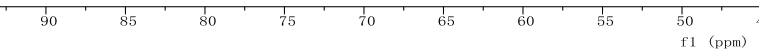




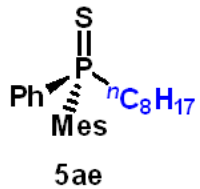

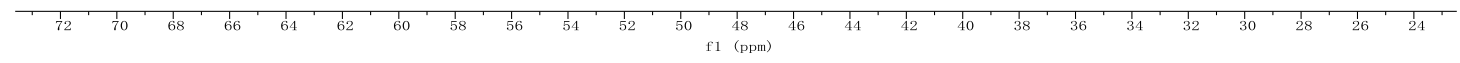

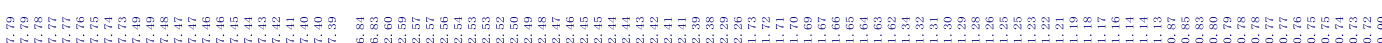

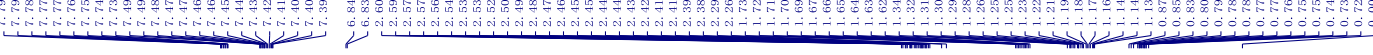

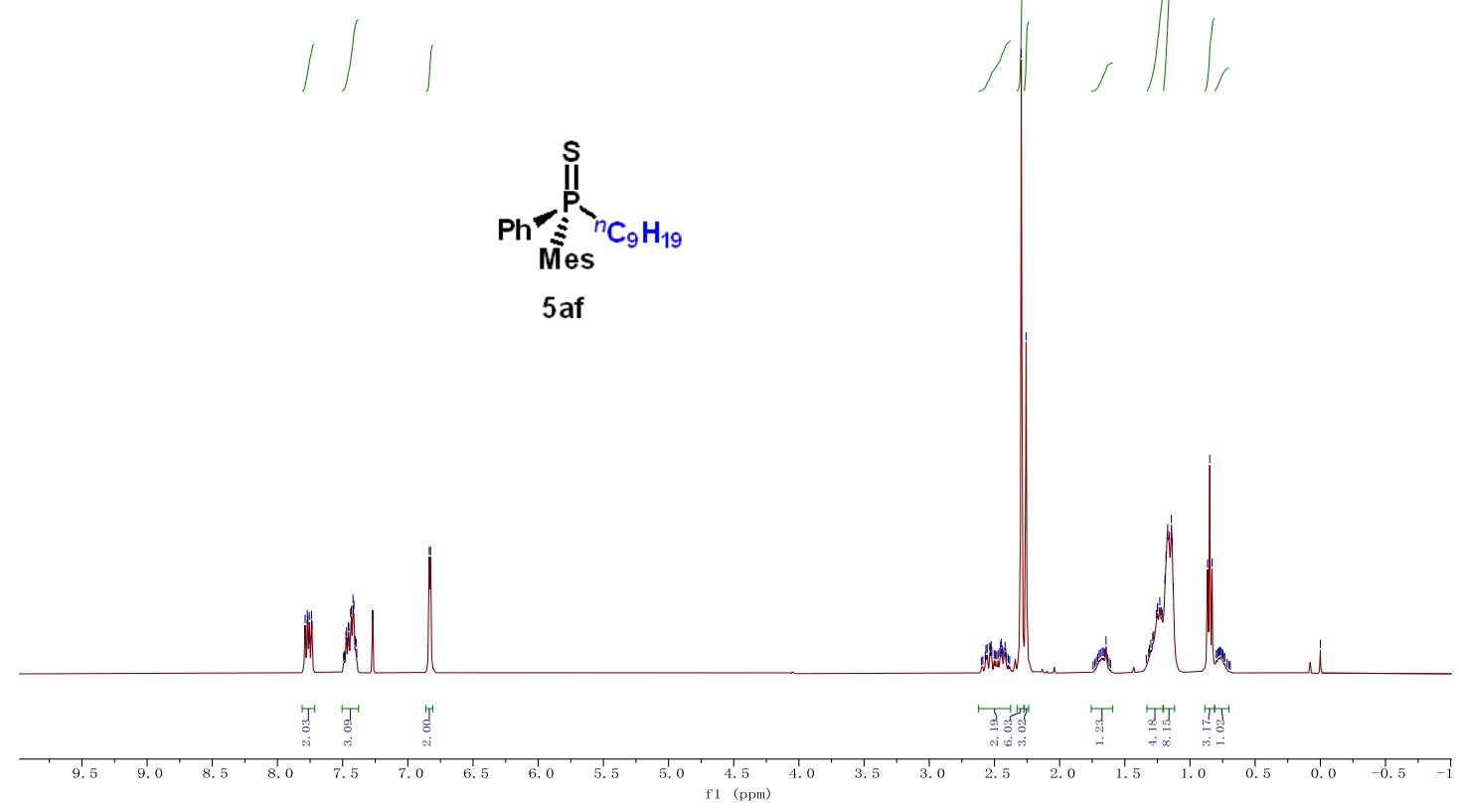



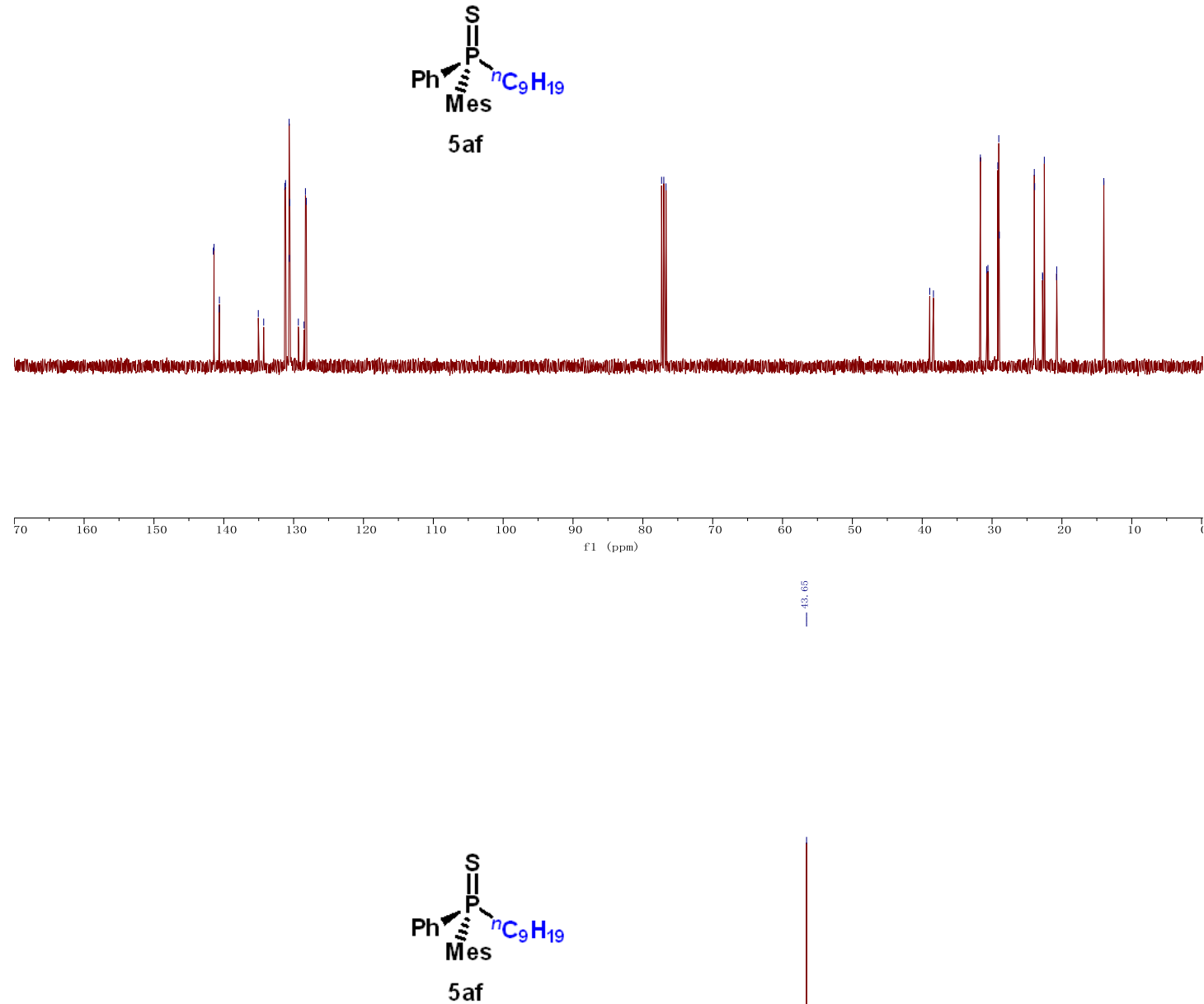

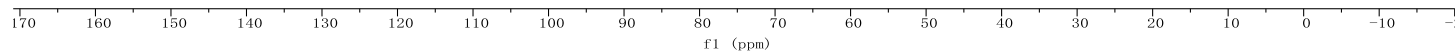




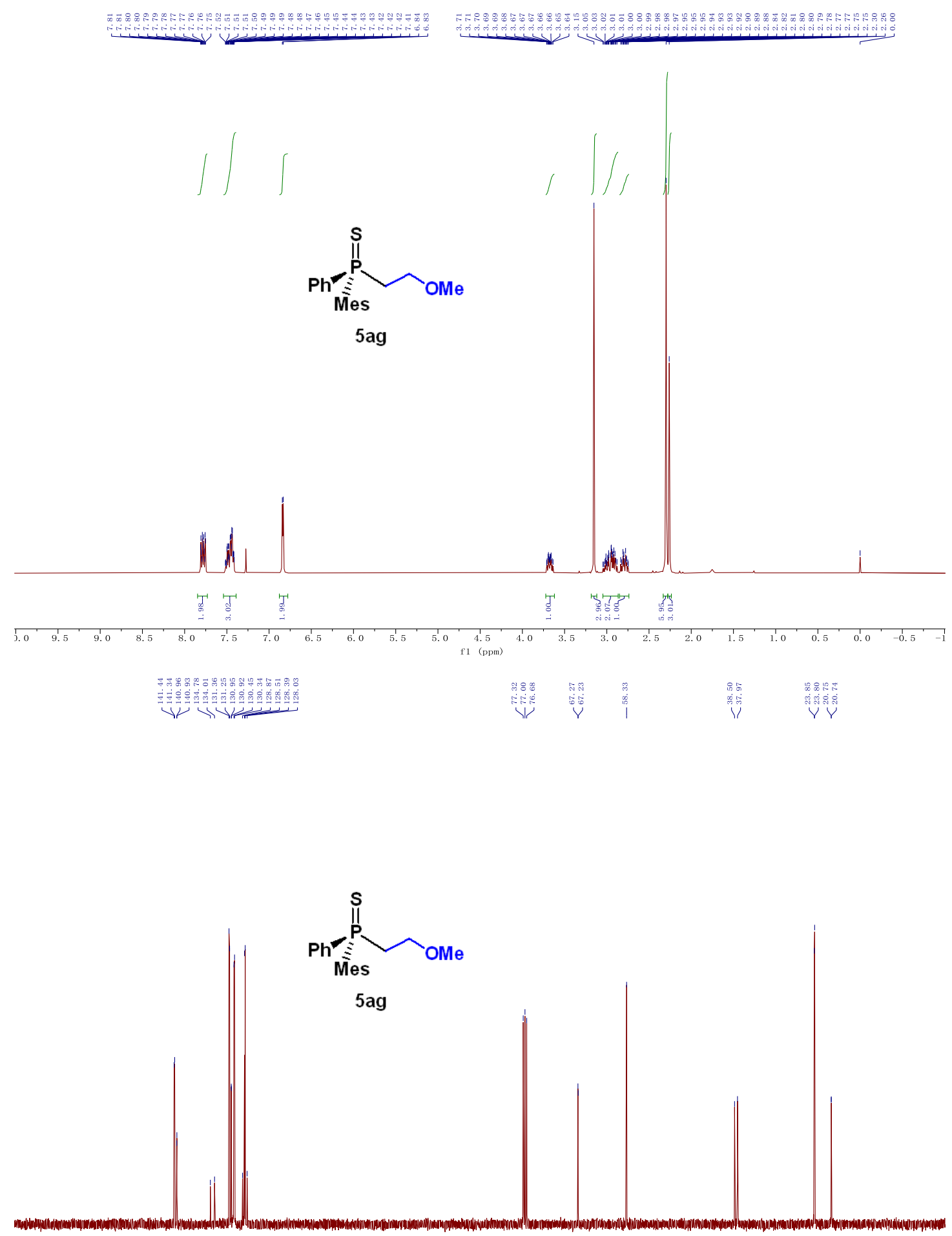

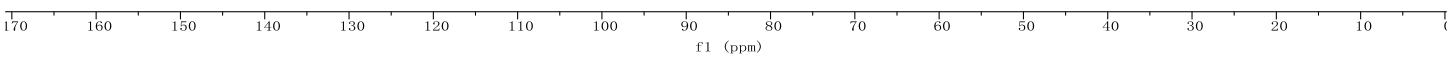




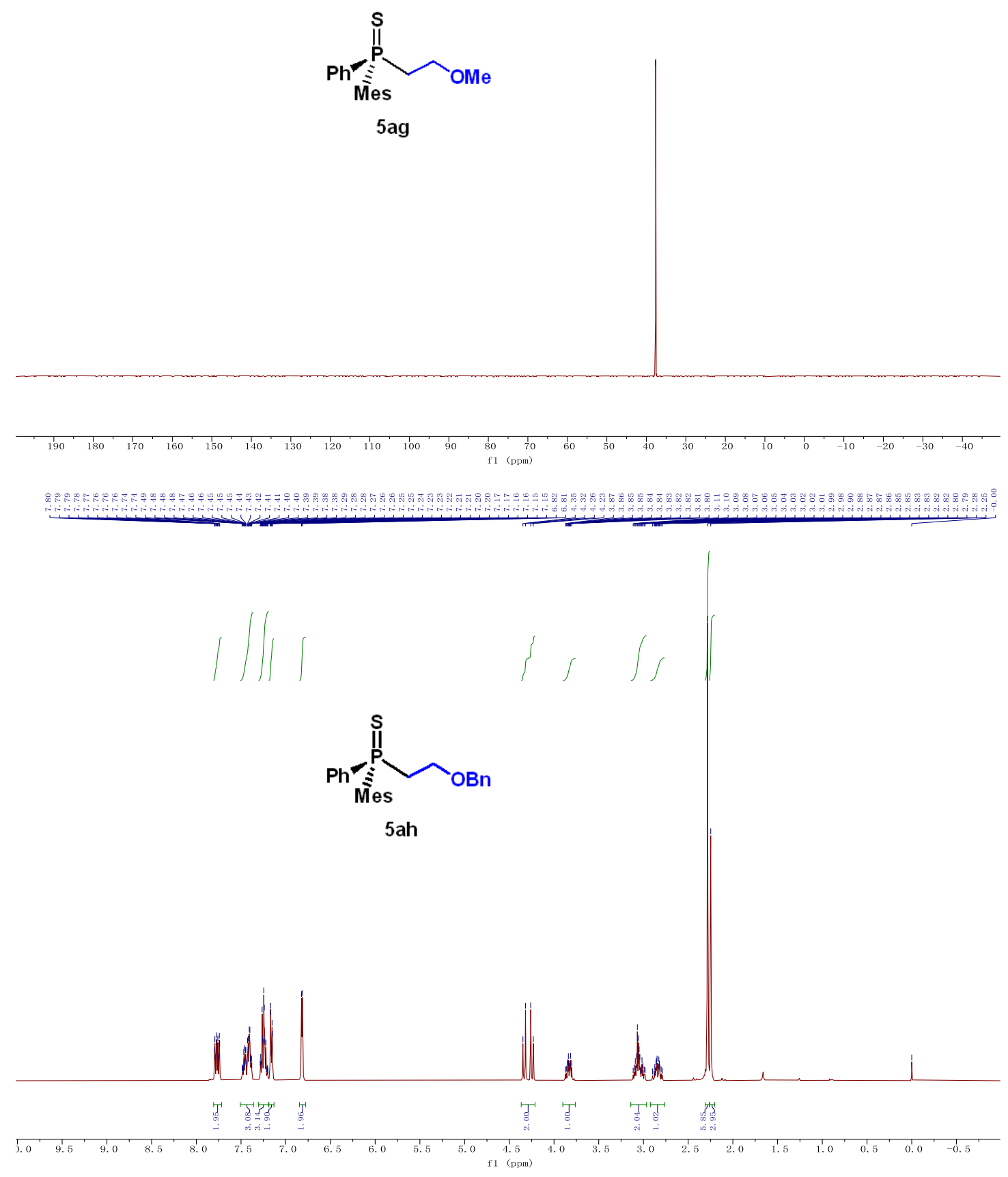



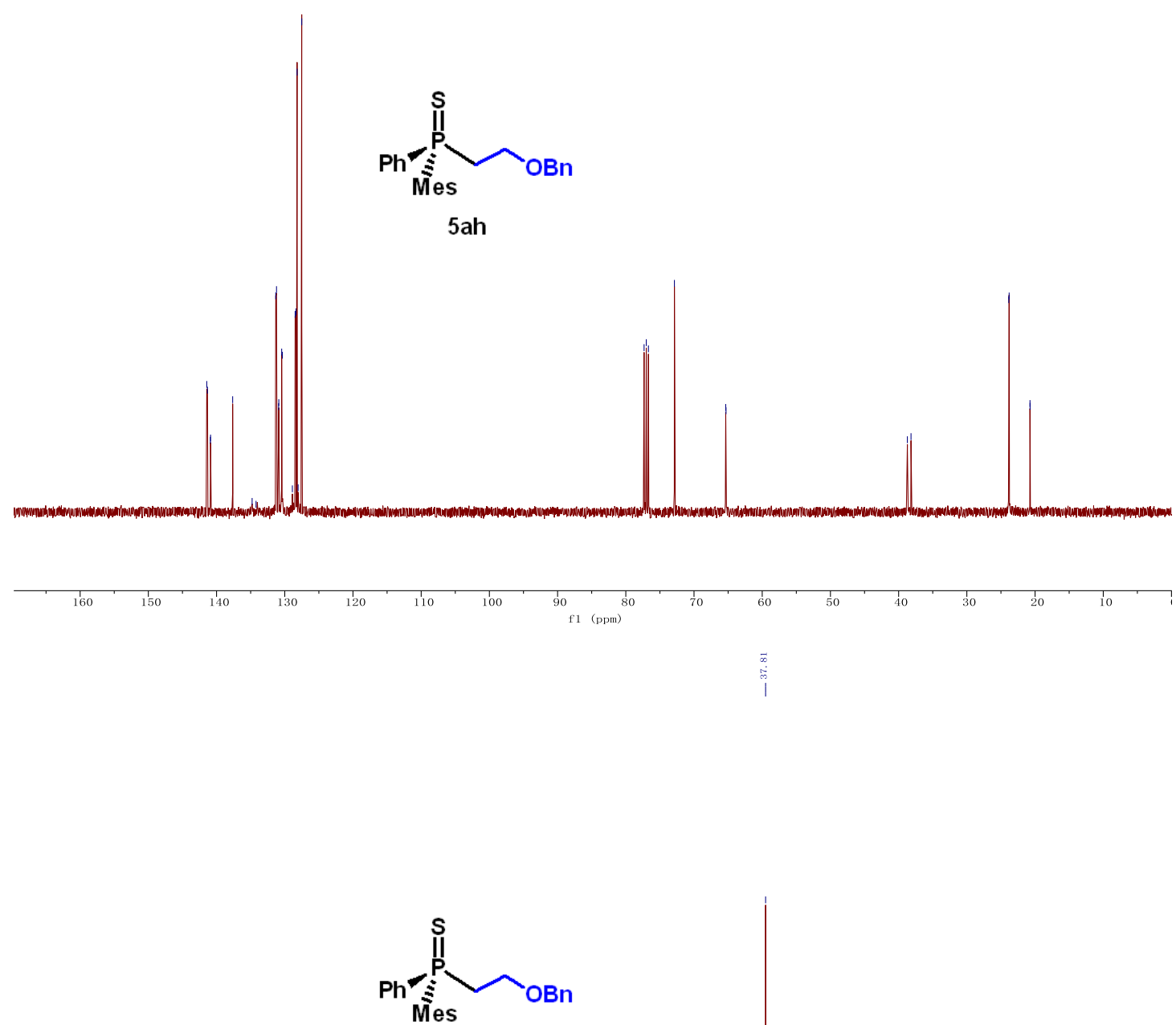

5 ah

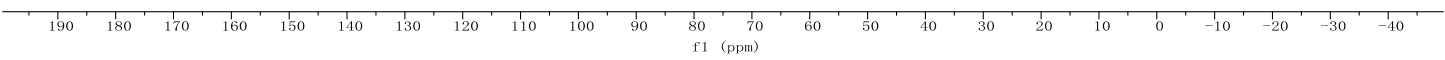



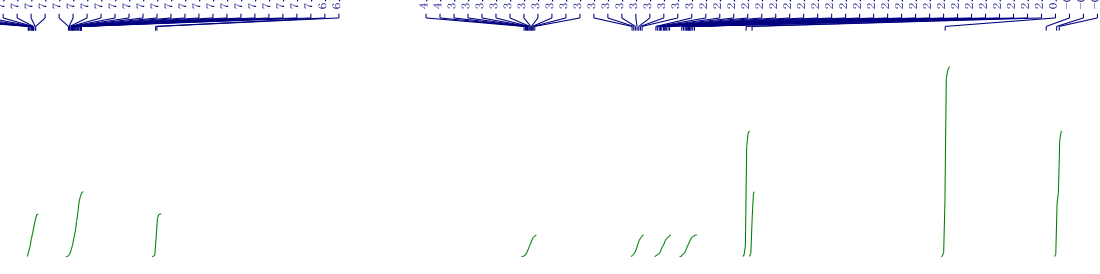

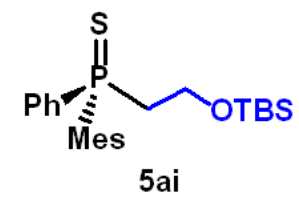

5 ai

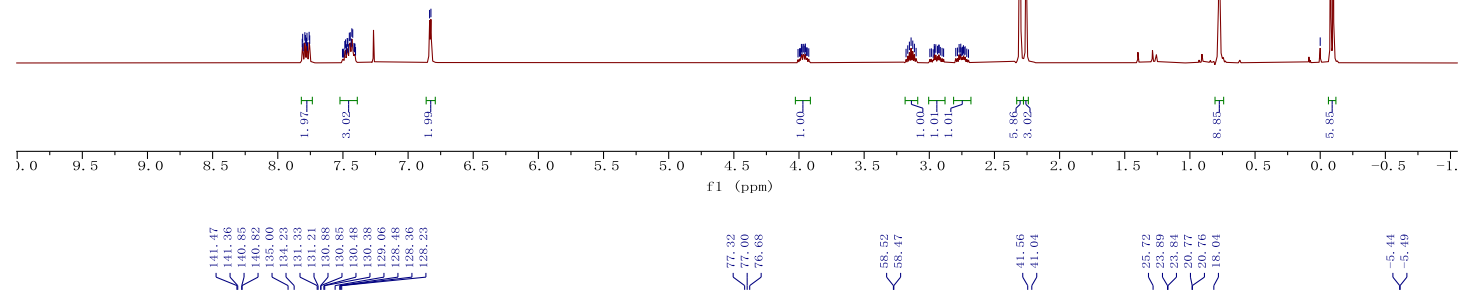<smiles>C[As](=S)(CCO[Sb])P(=S)(c1ccccc1)c1ccccc1</smiles>

$5 a i$
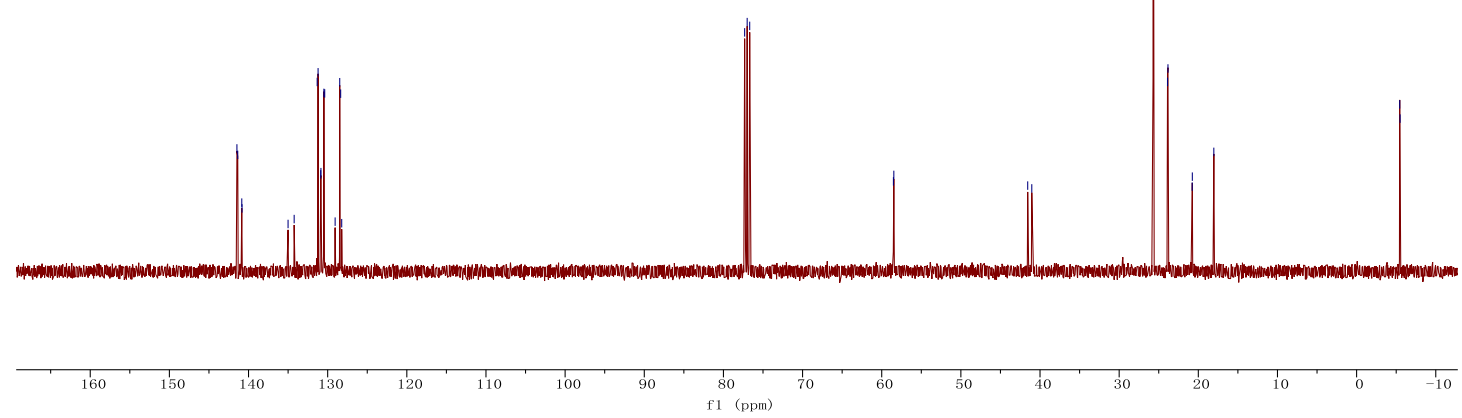

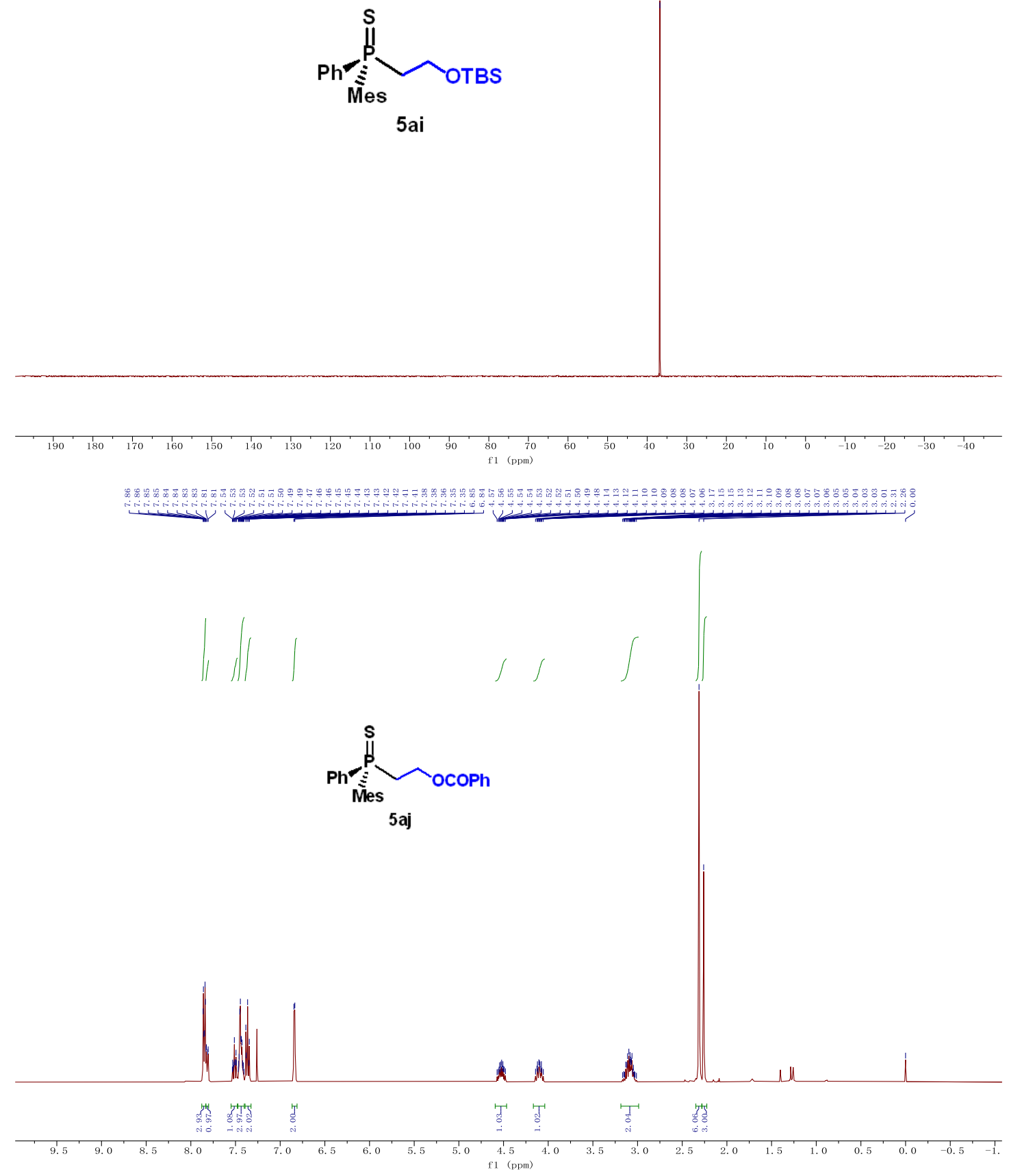

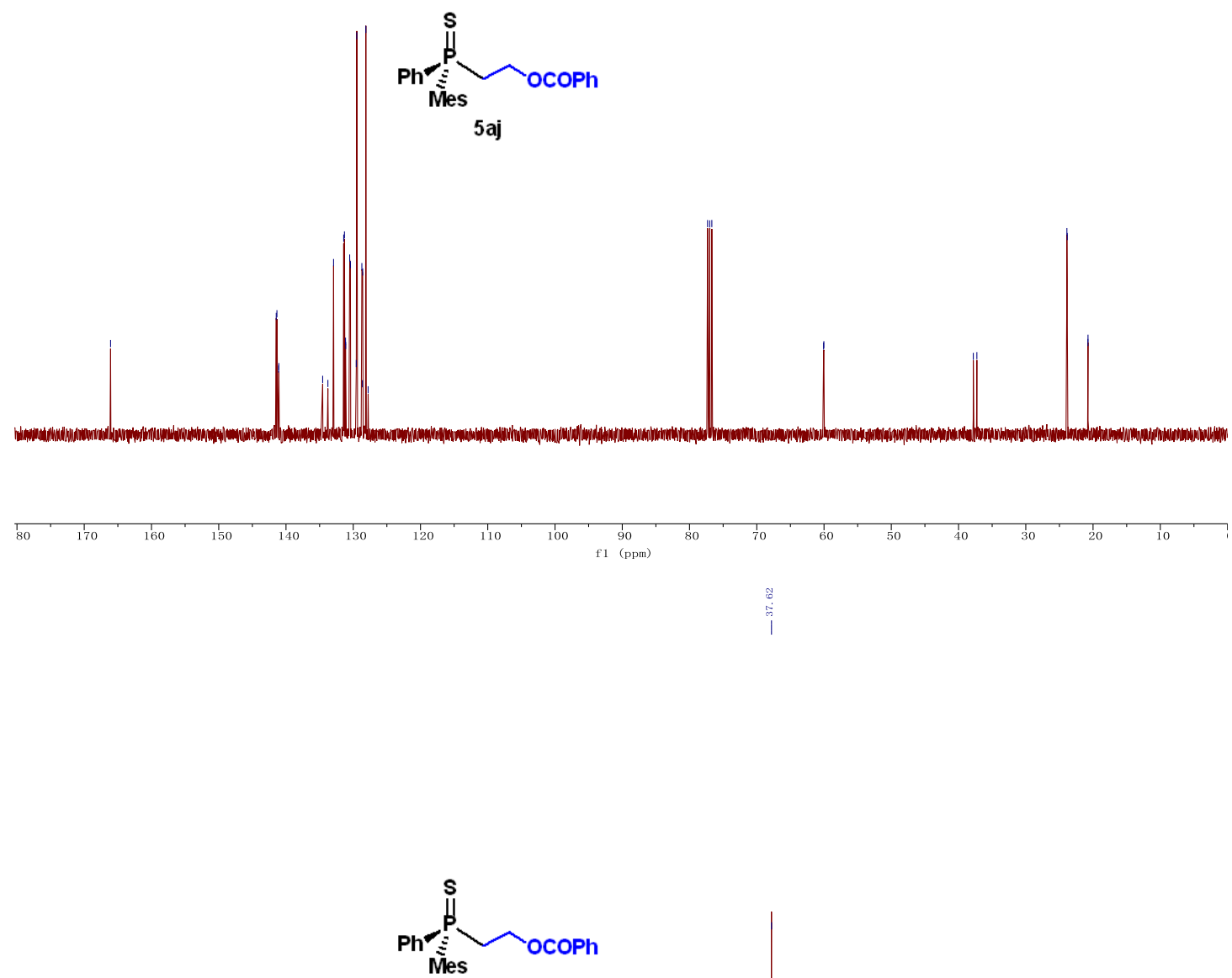

5aj

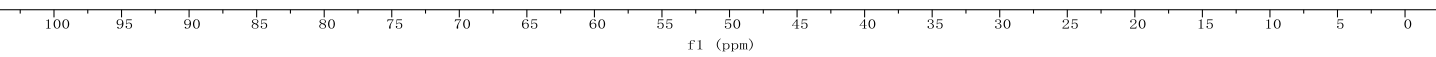




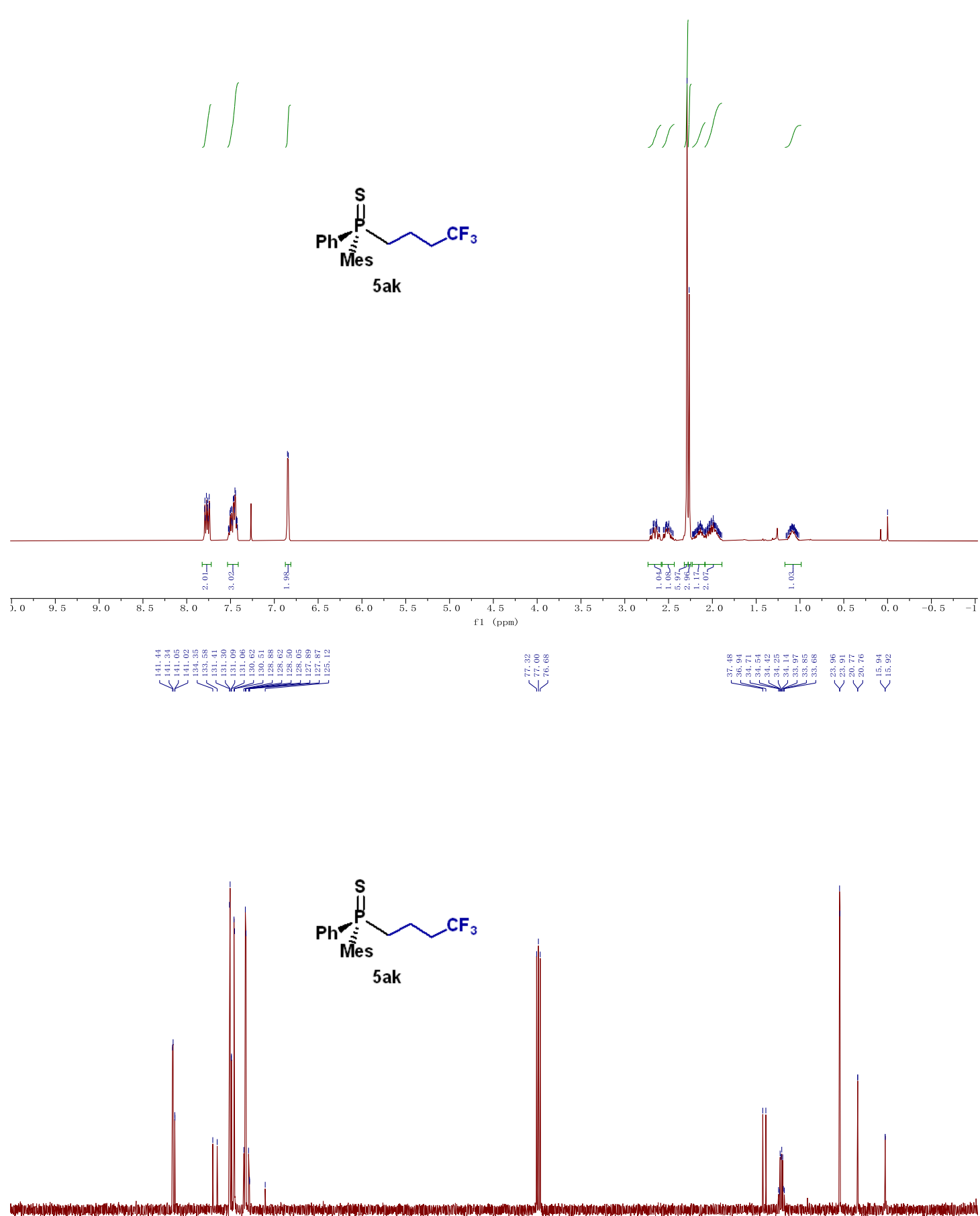

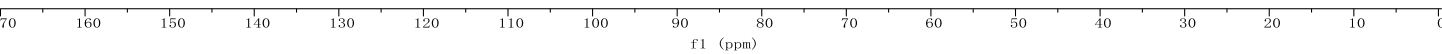



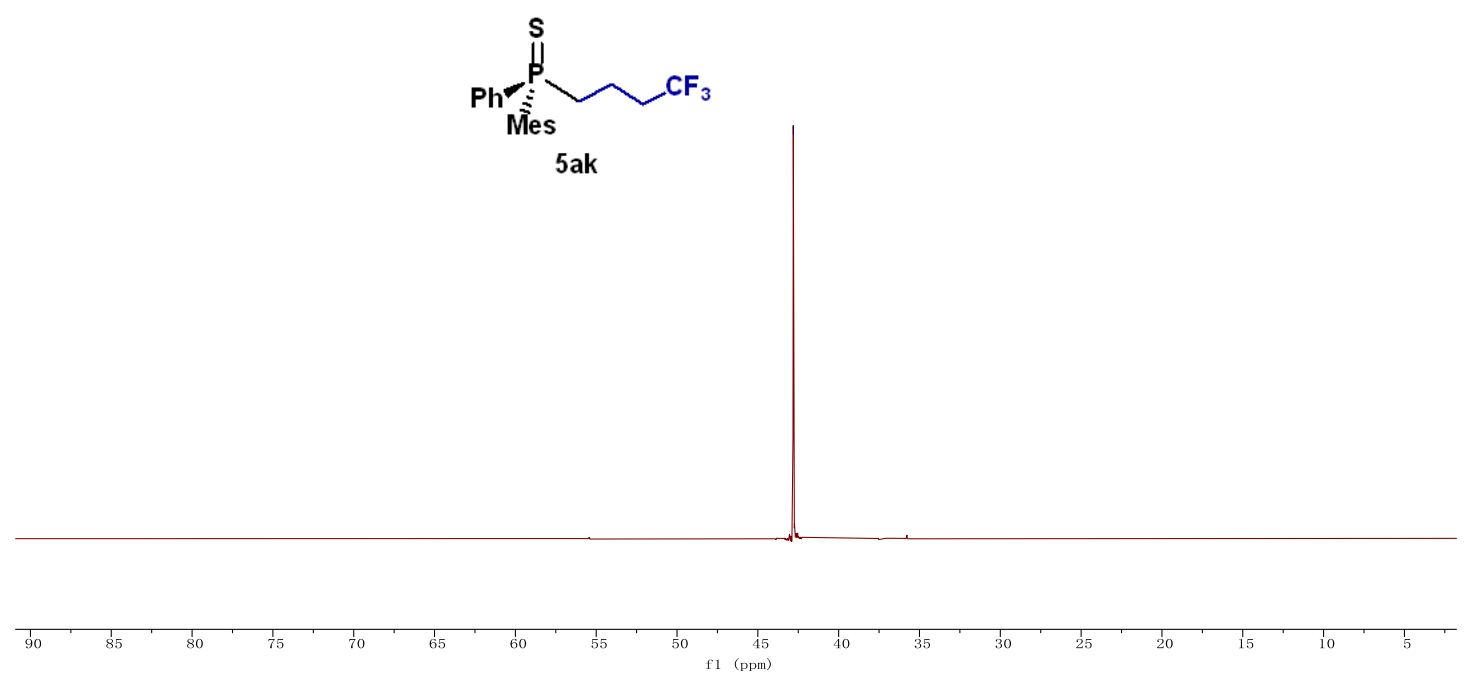

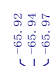

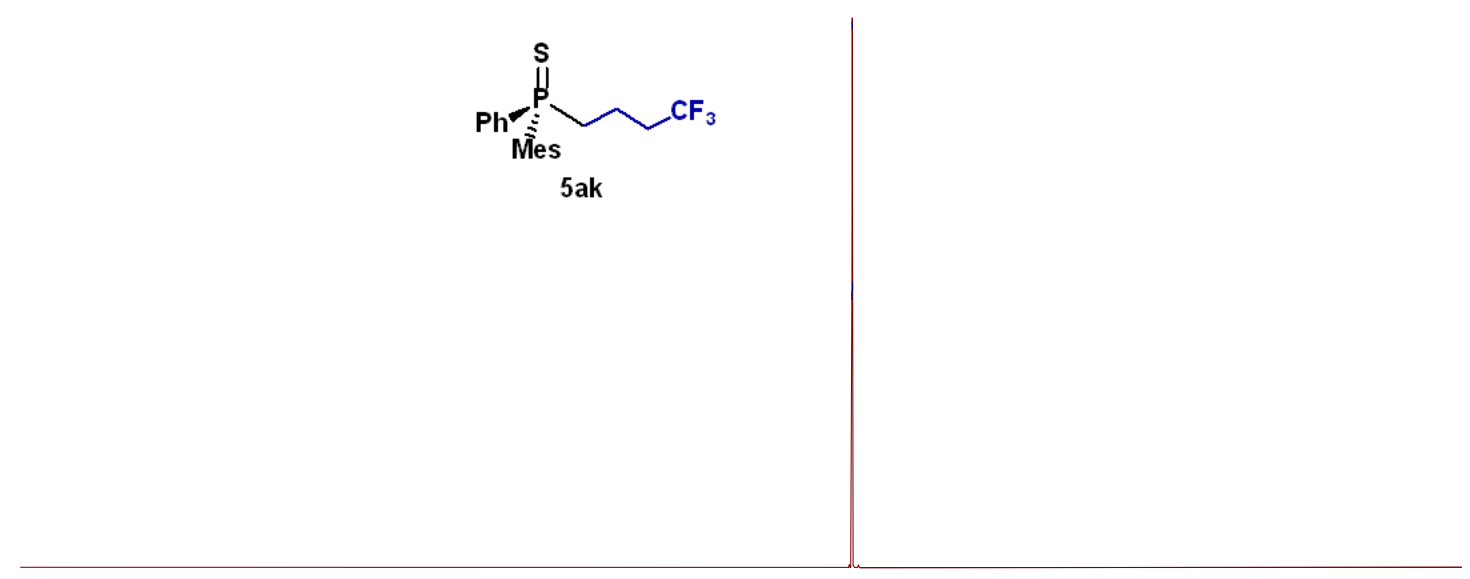

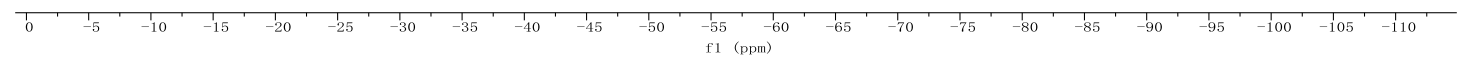




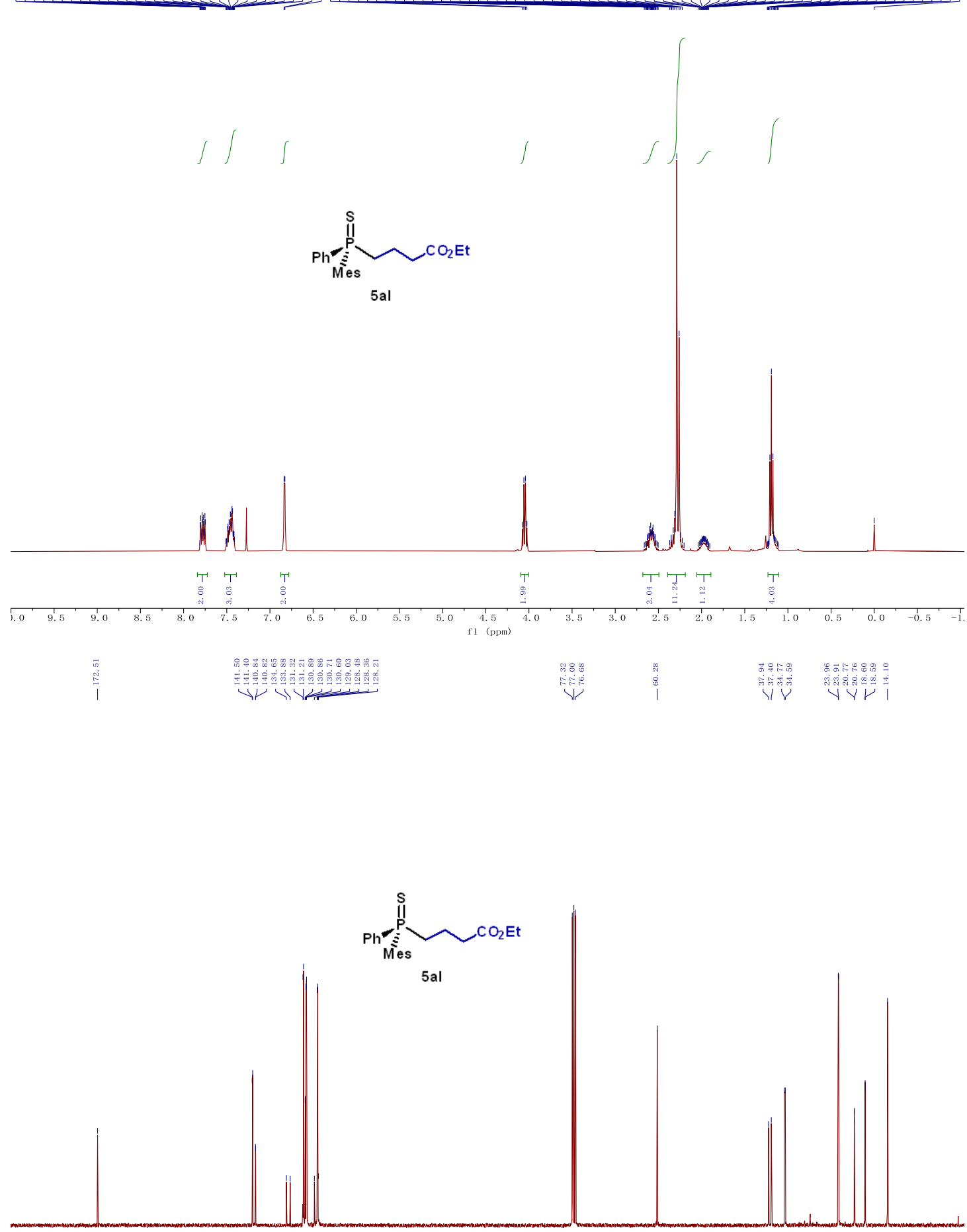

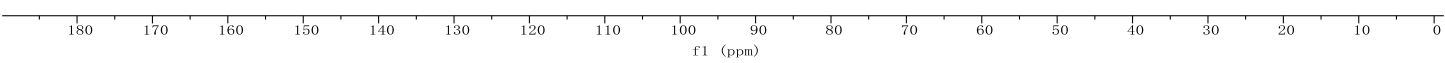



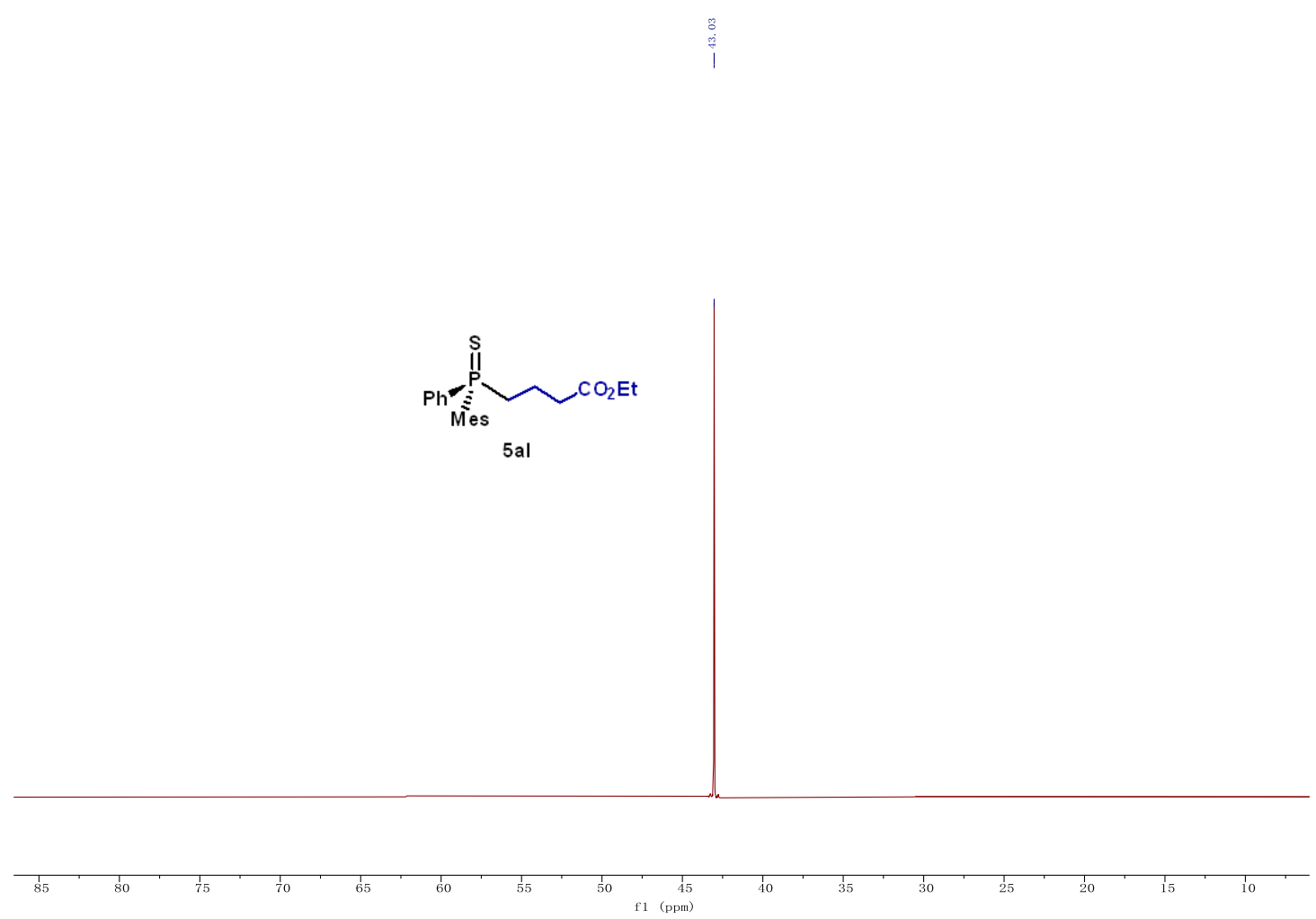

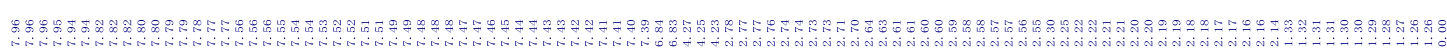
$\longrightarrow$
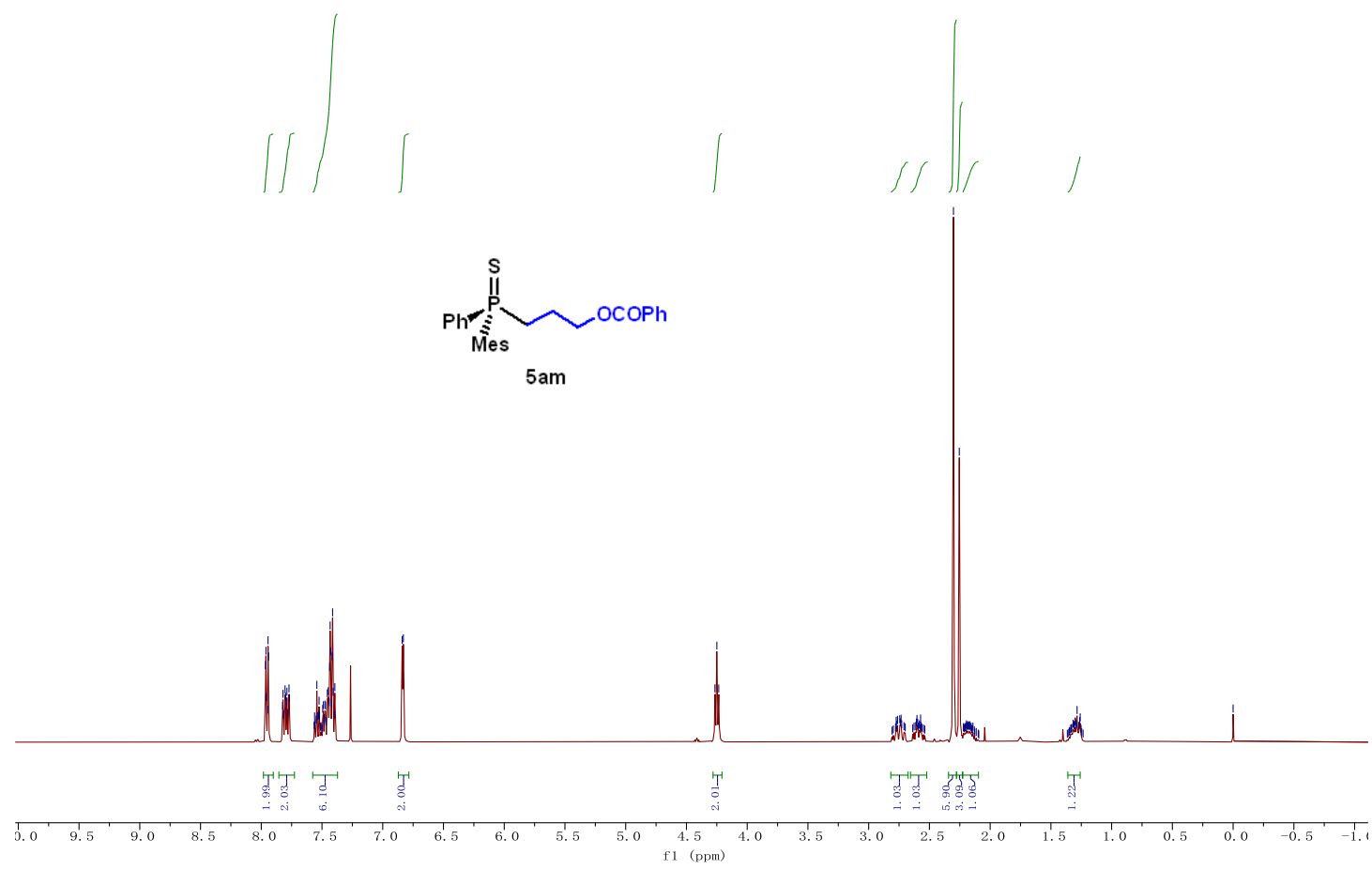

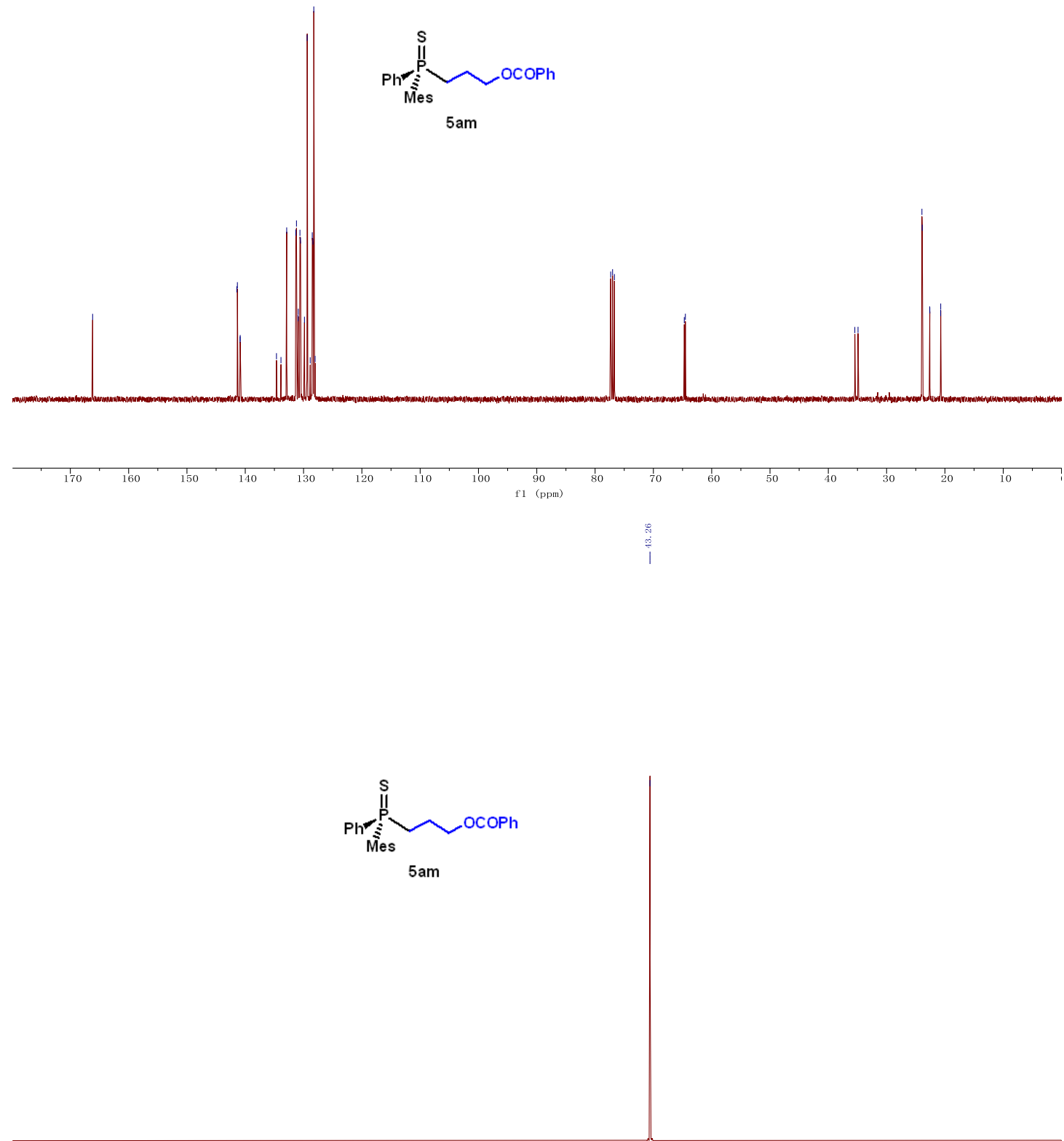

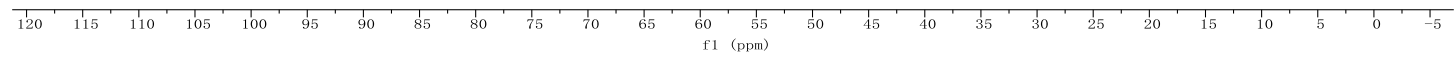



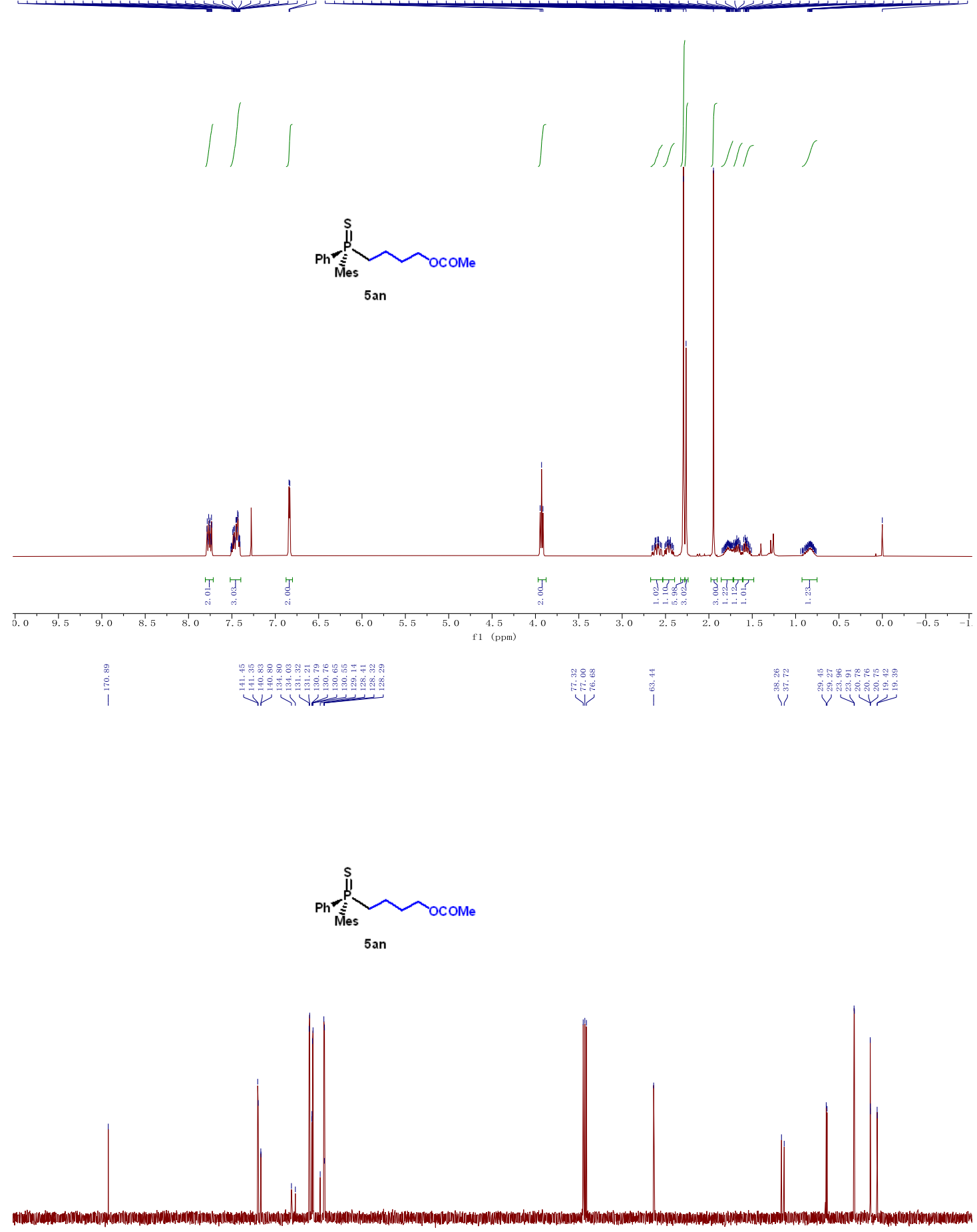

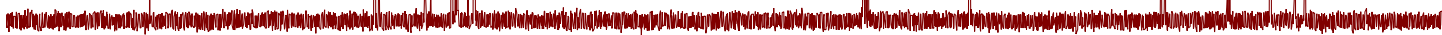

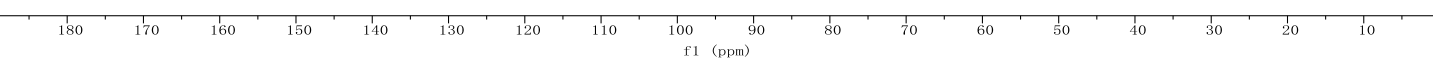




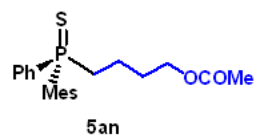

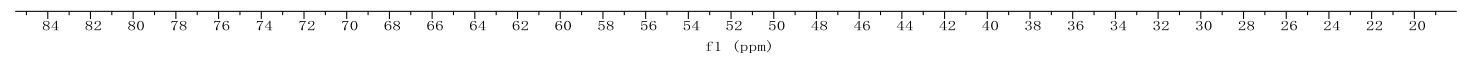

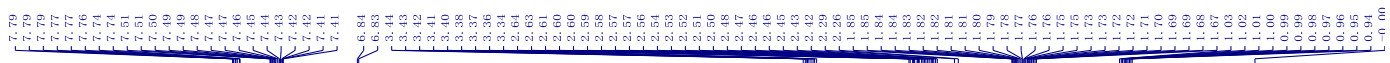<smiles>CC=CC(C)C</smiles><smiles>C=P(N)(CCCCCl)P([NH3+])(=O)P</smiles>
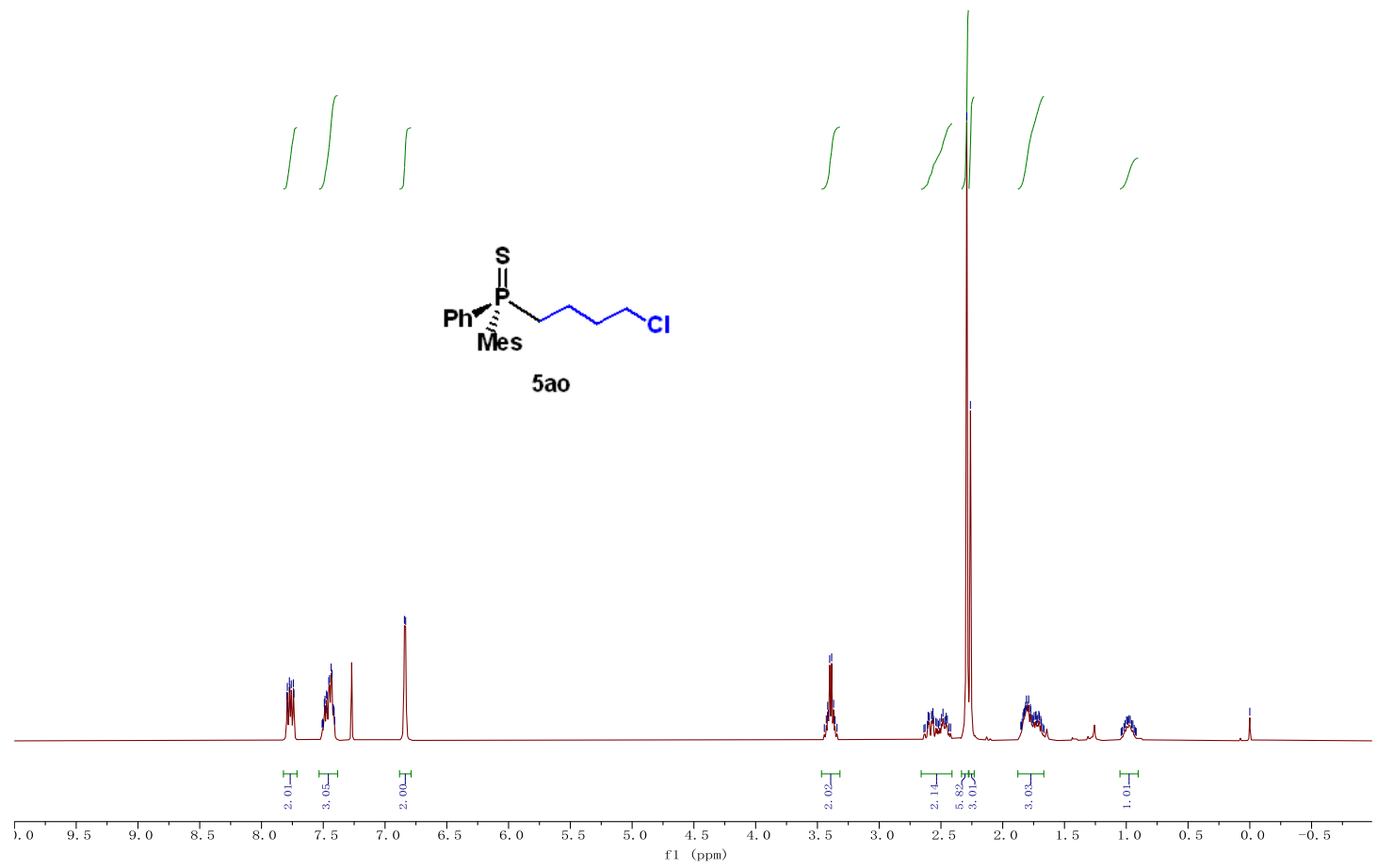

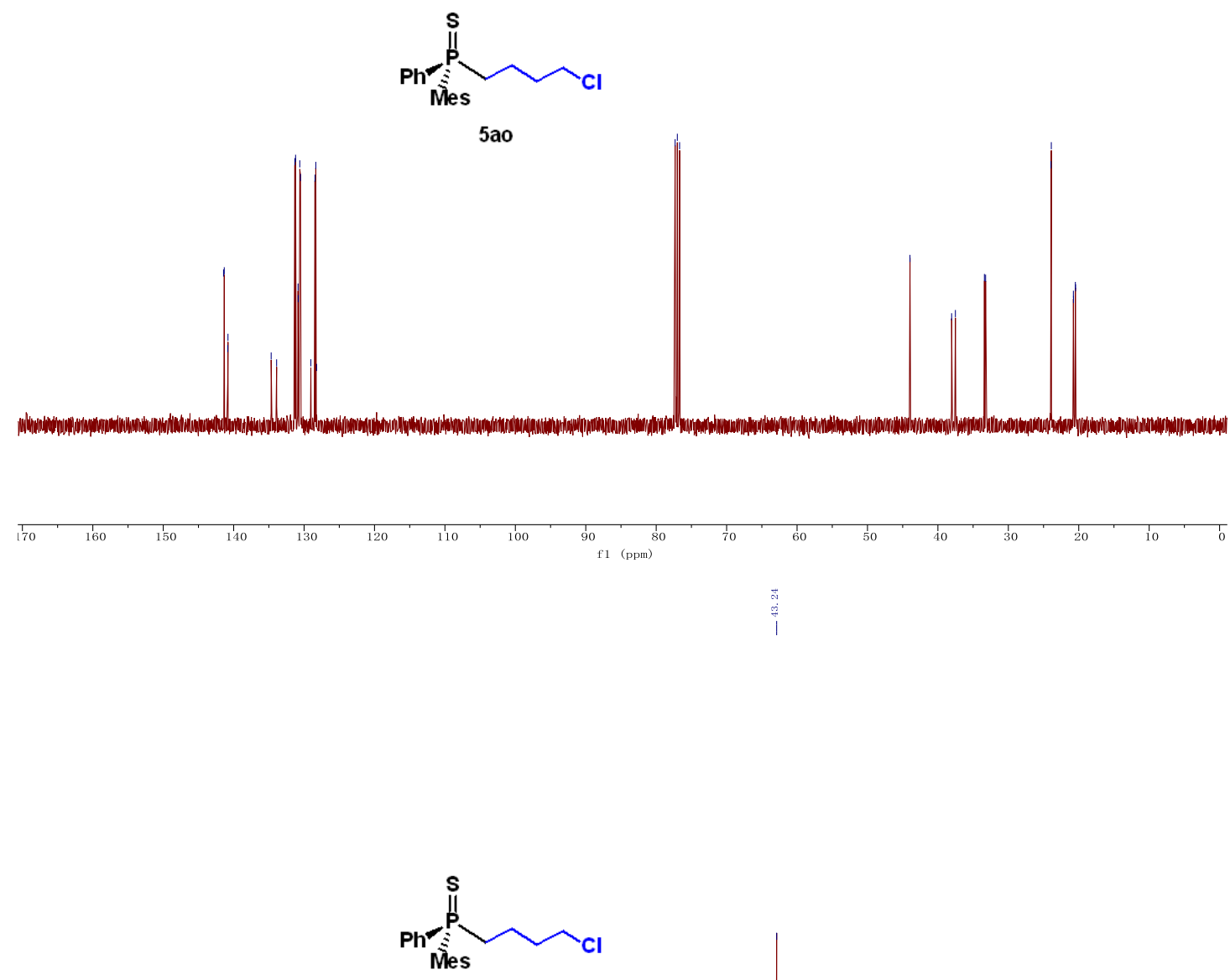

5 ao

12. 

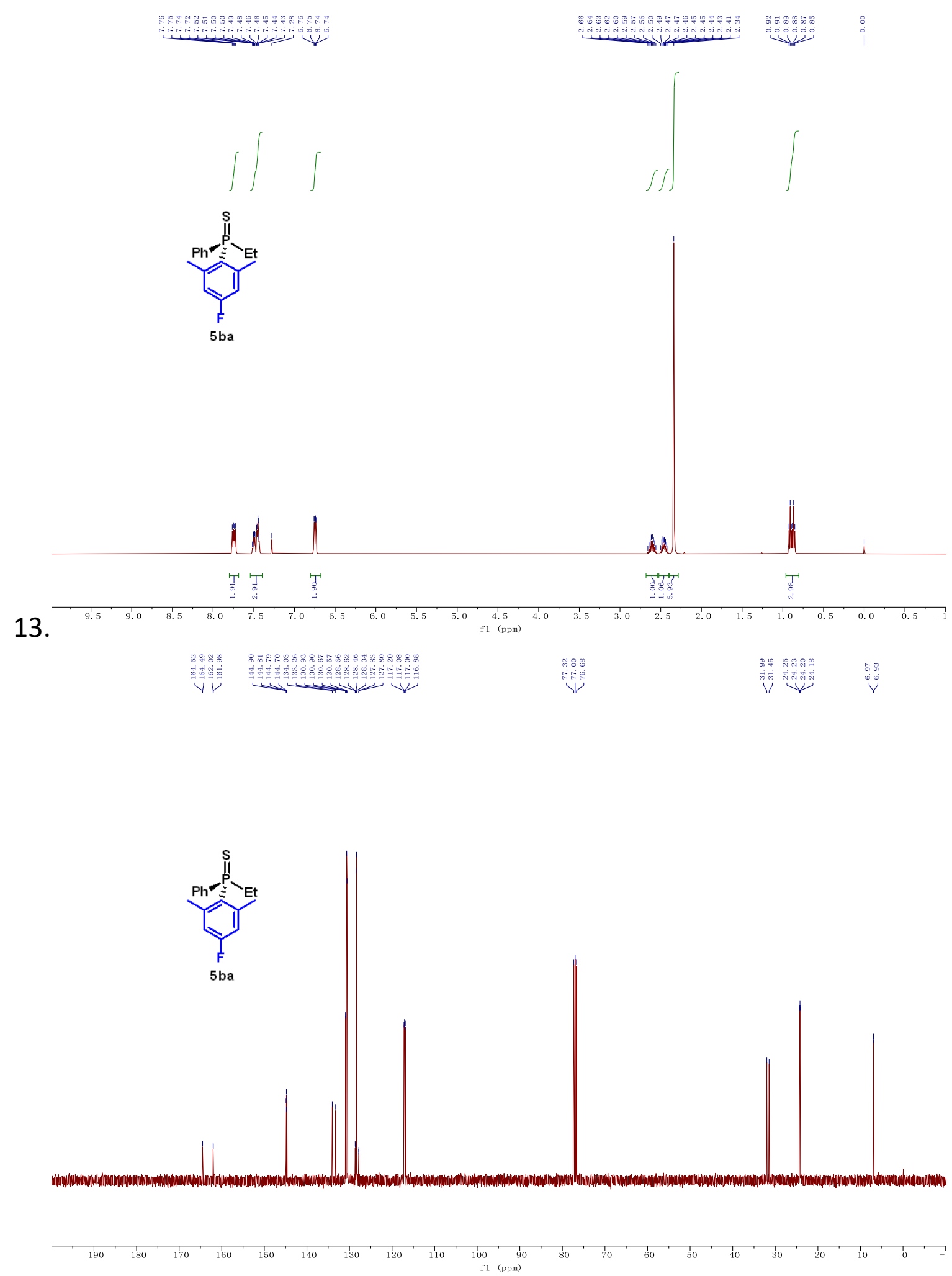


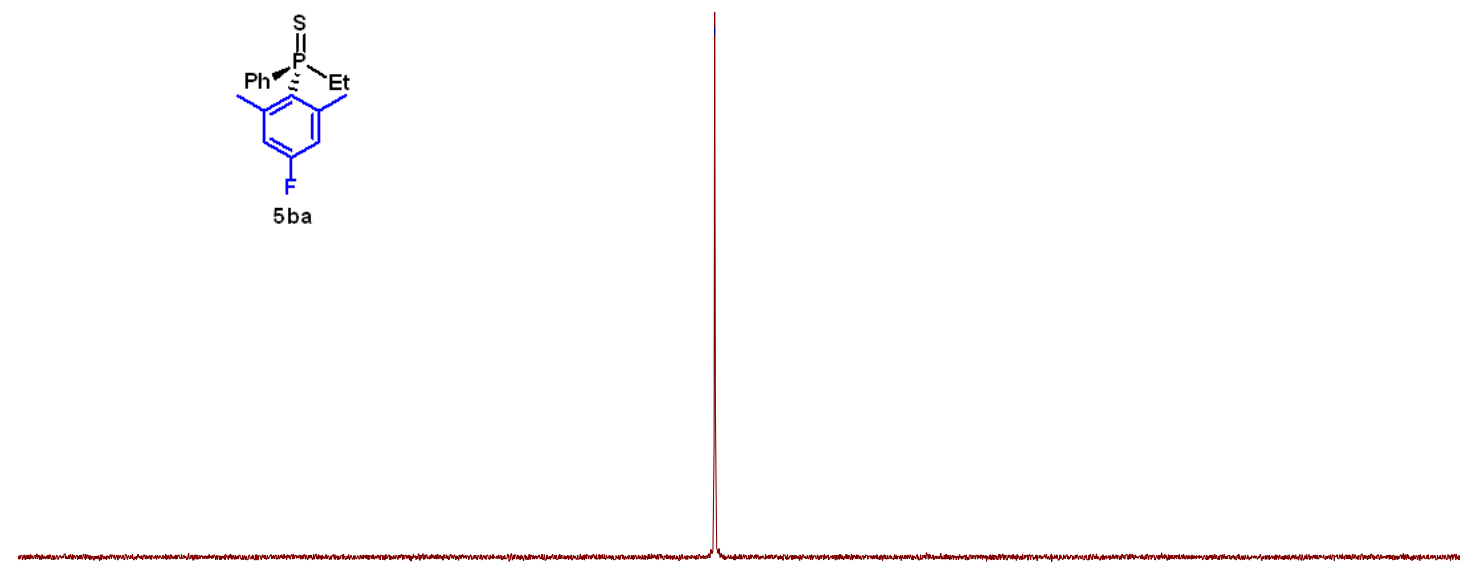

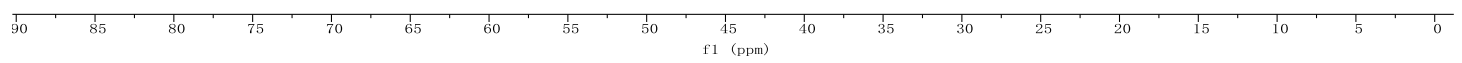

8
$\stackrel{8}{\circ}$
$\stackrel{0}{0}$

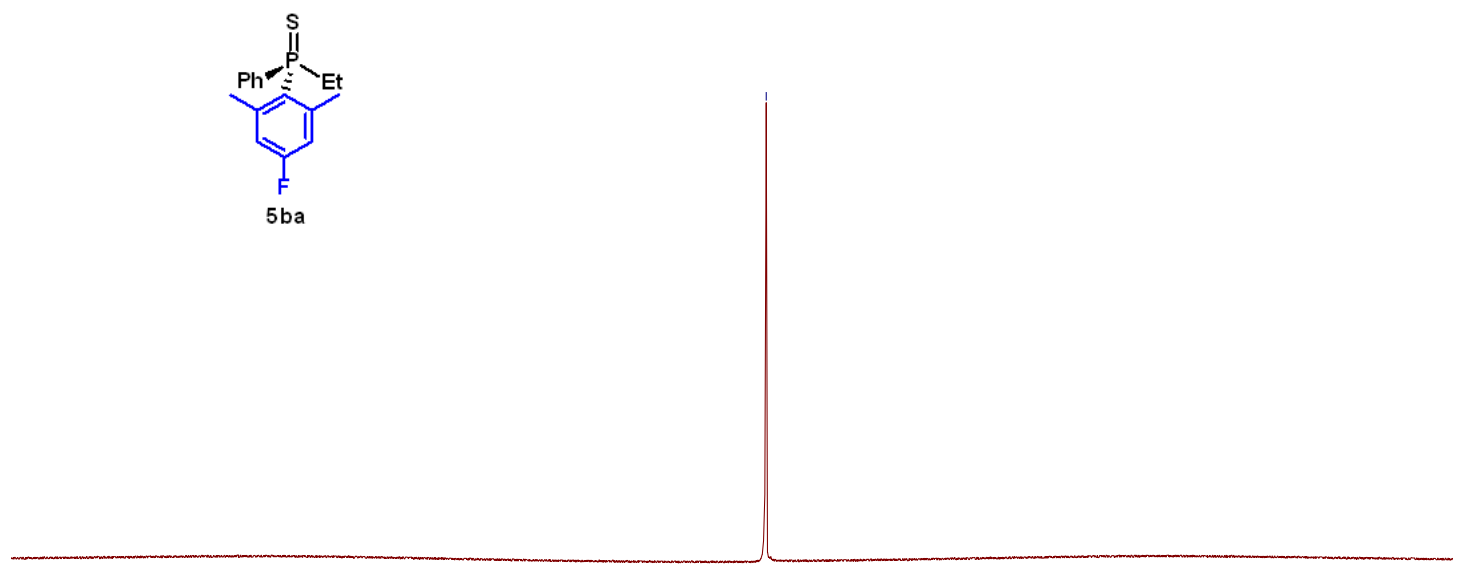

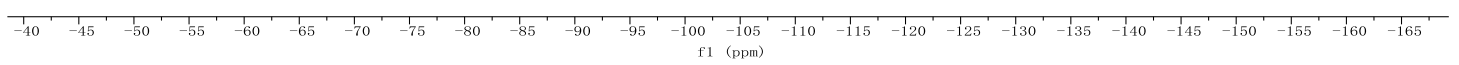




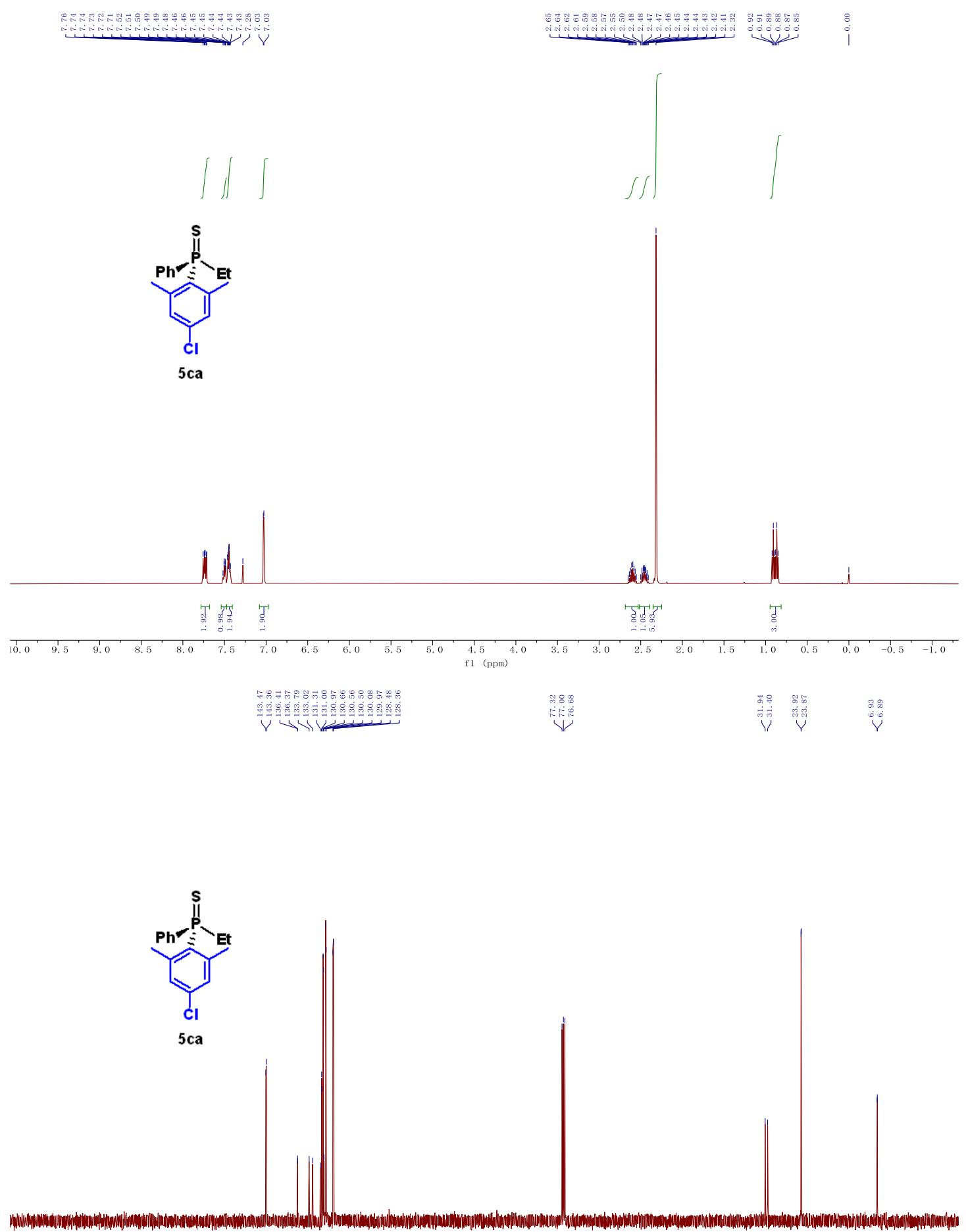

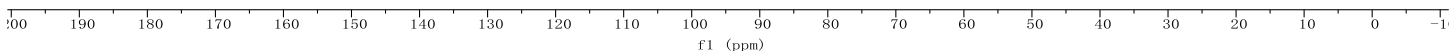



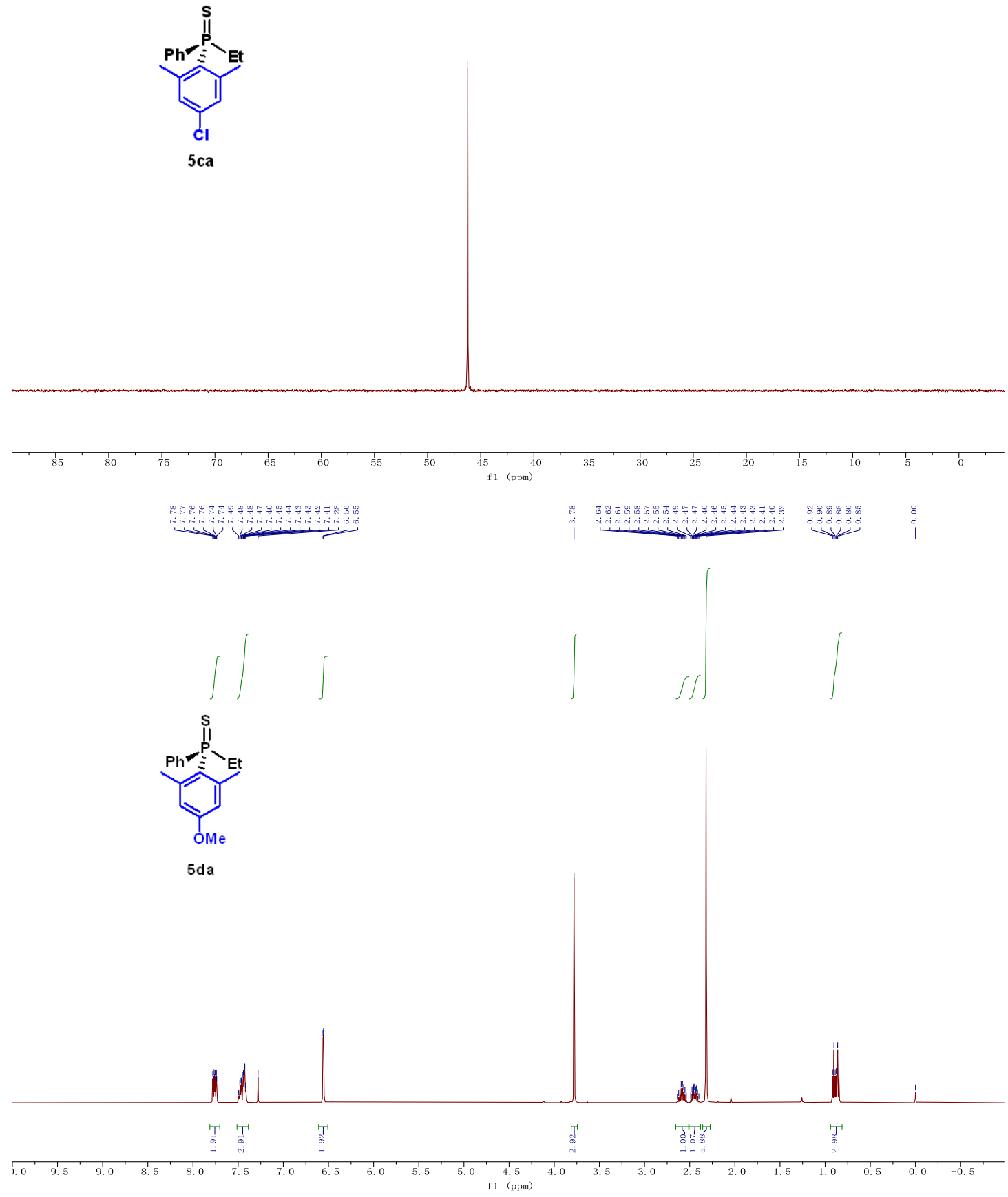

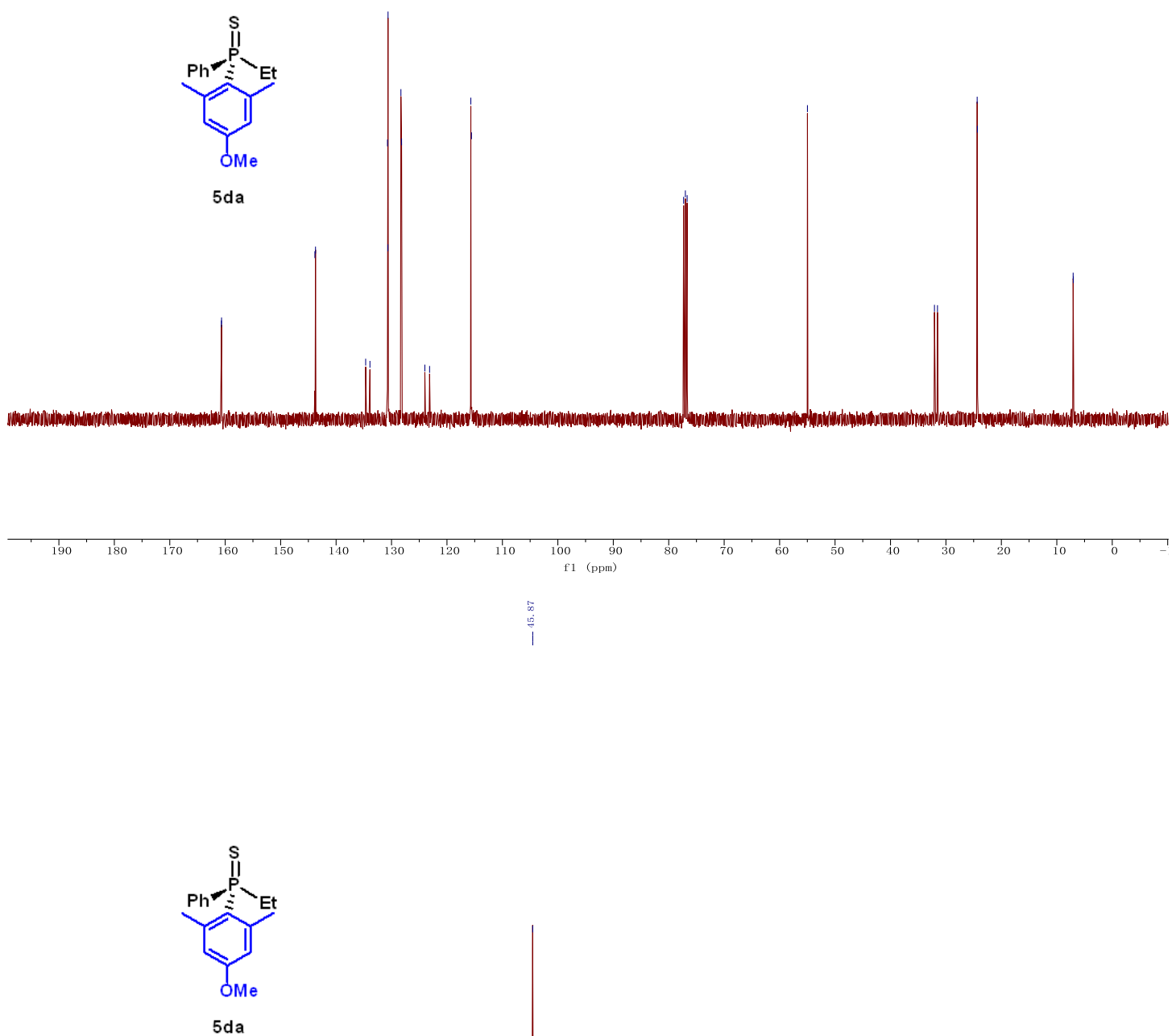


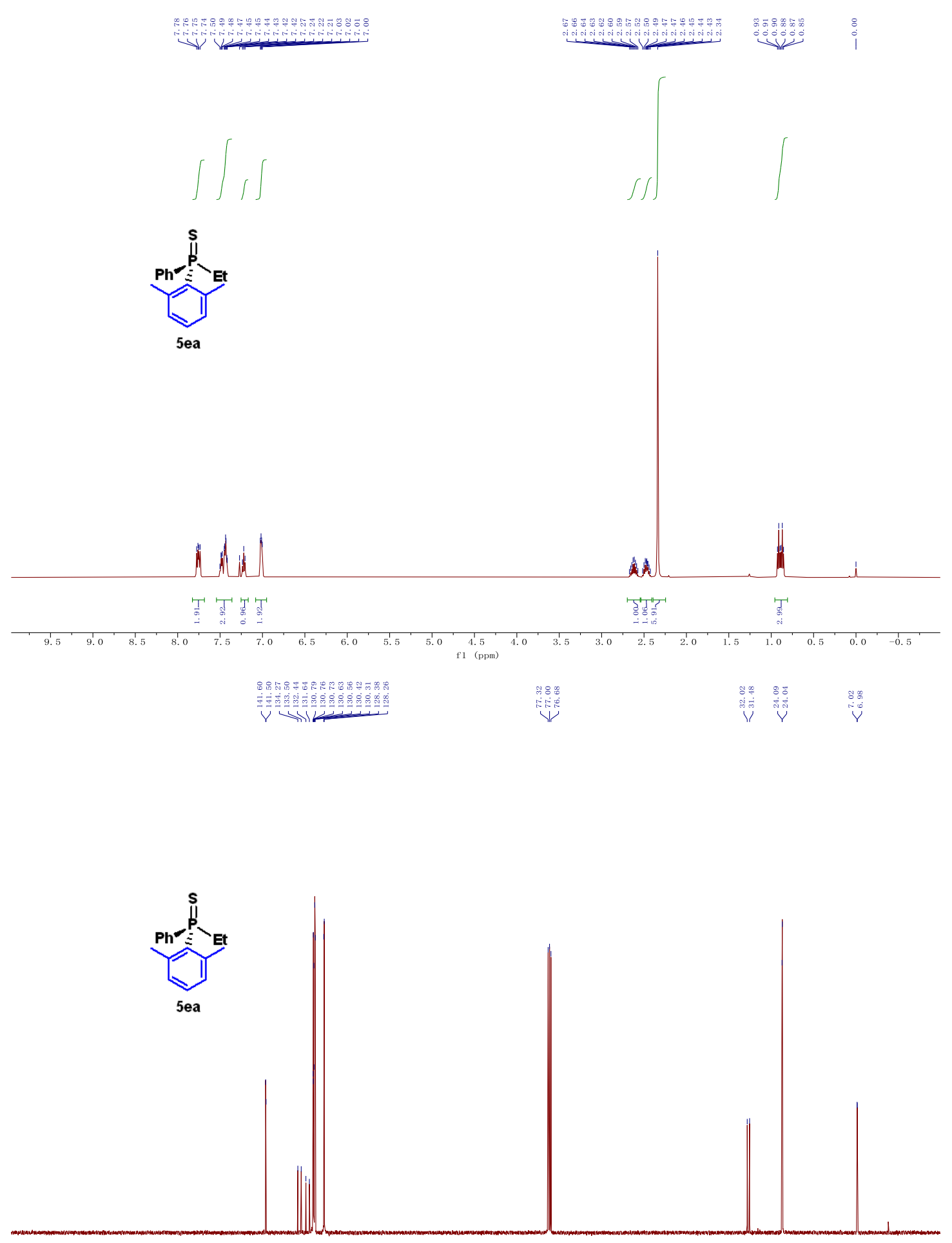

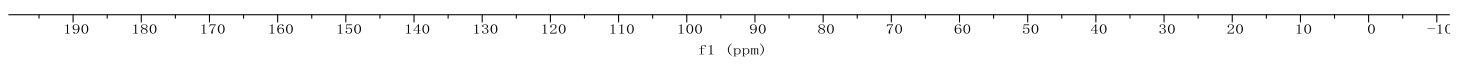



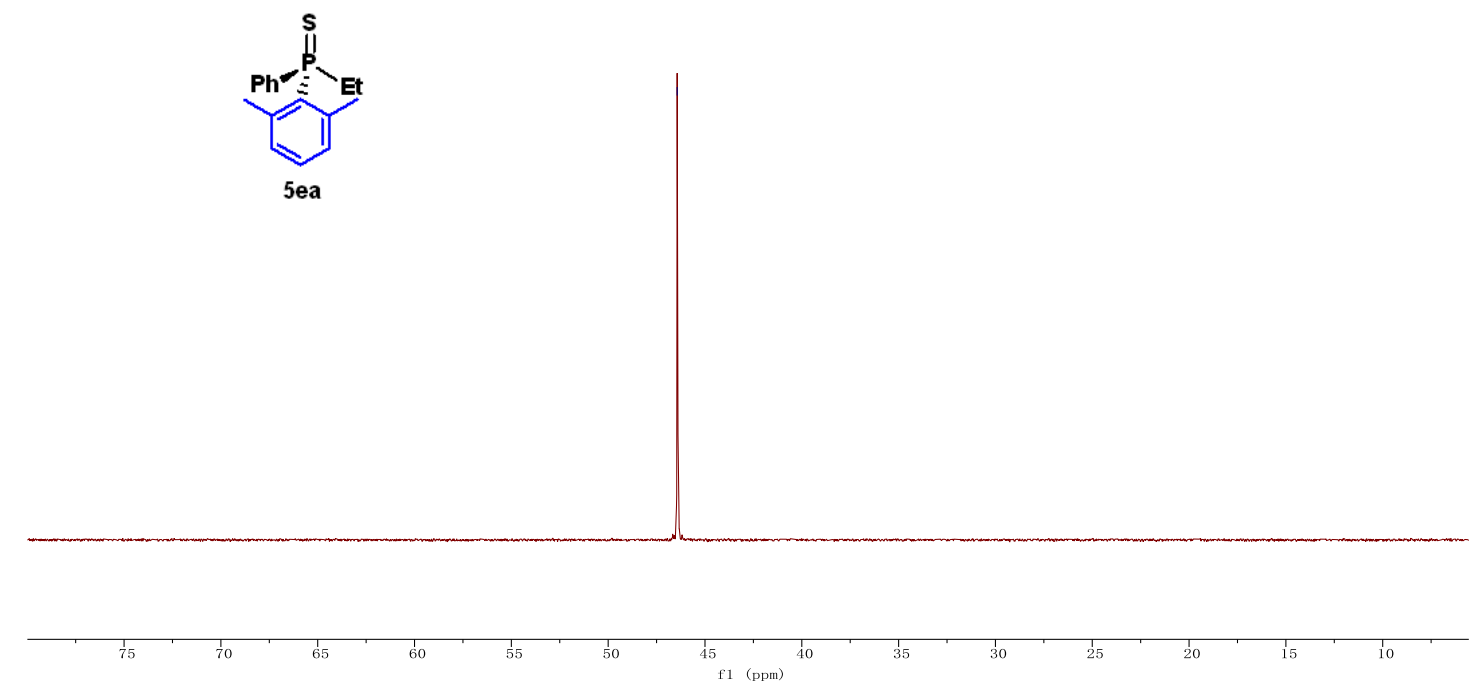

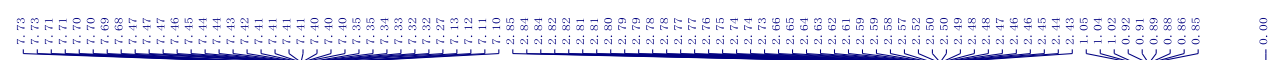

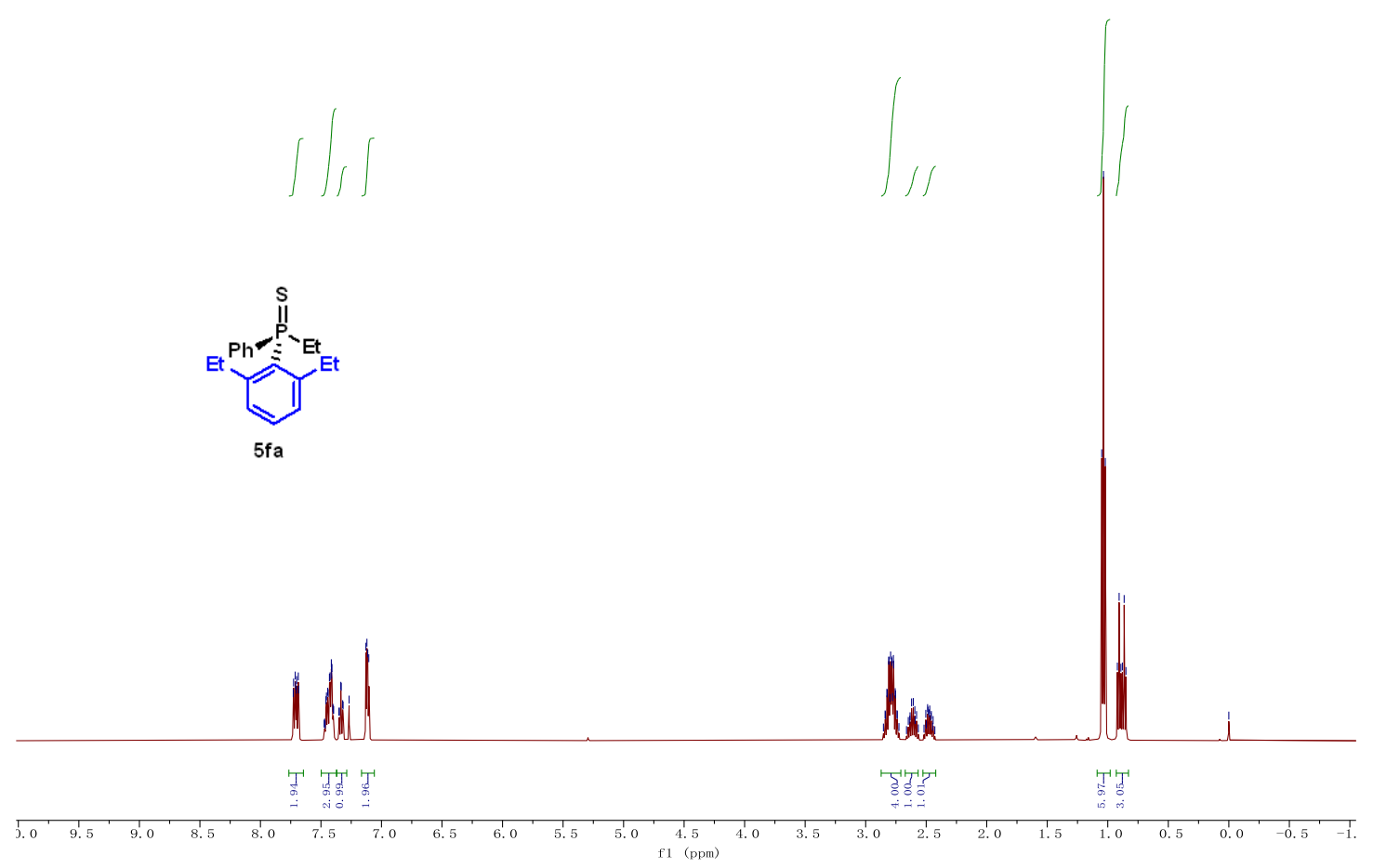



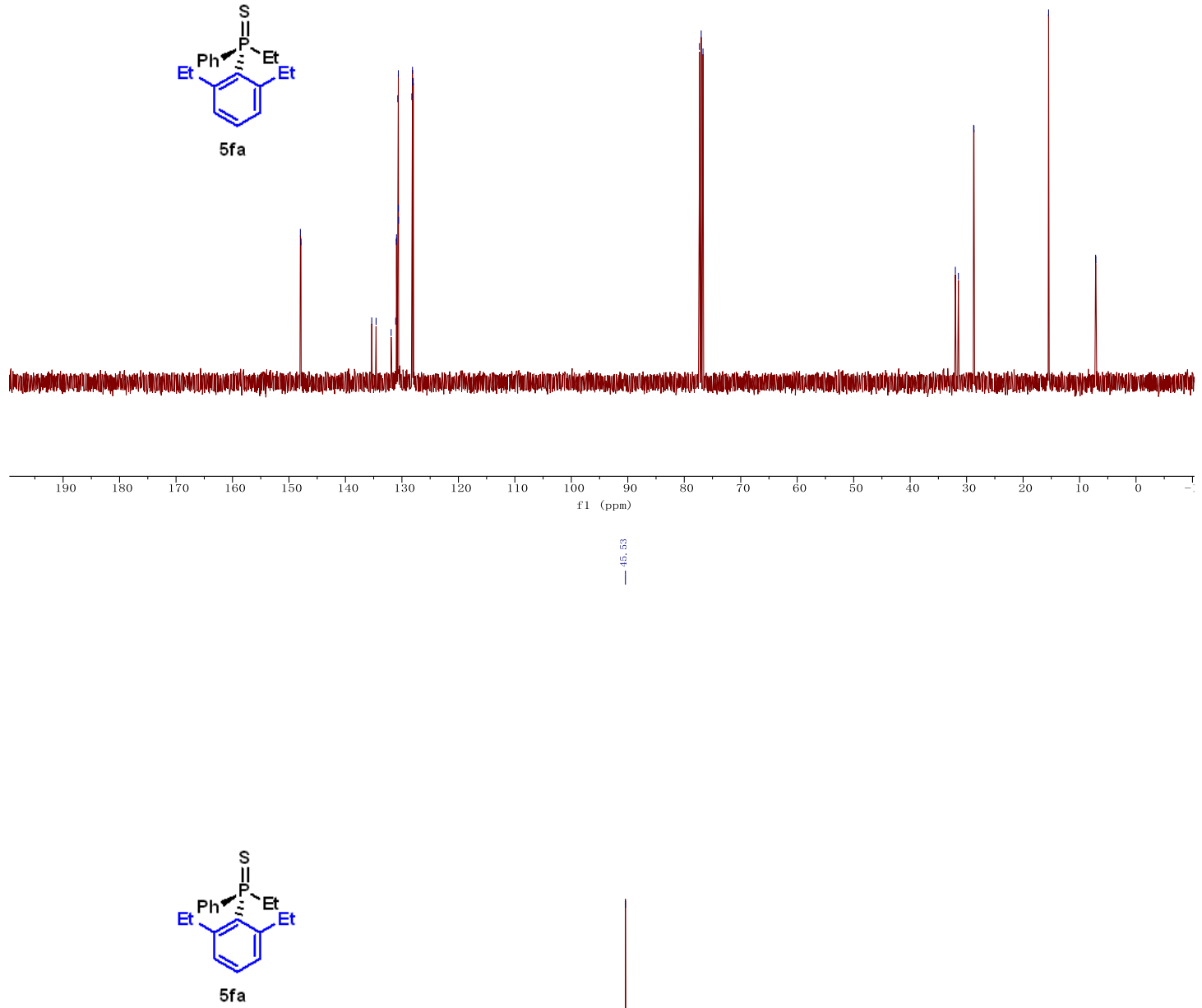

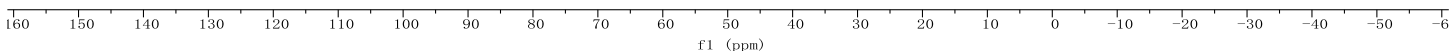



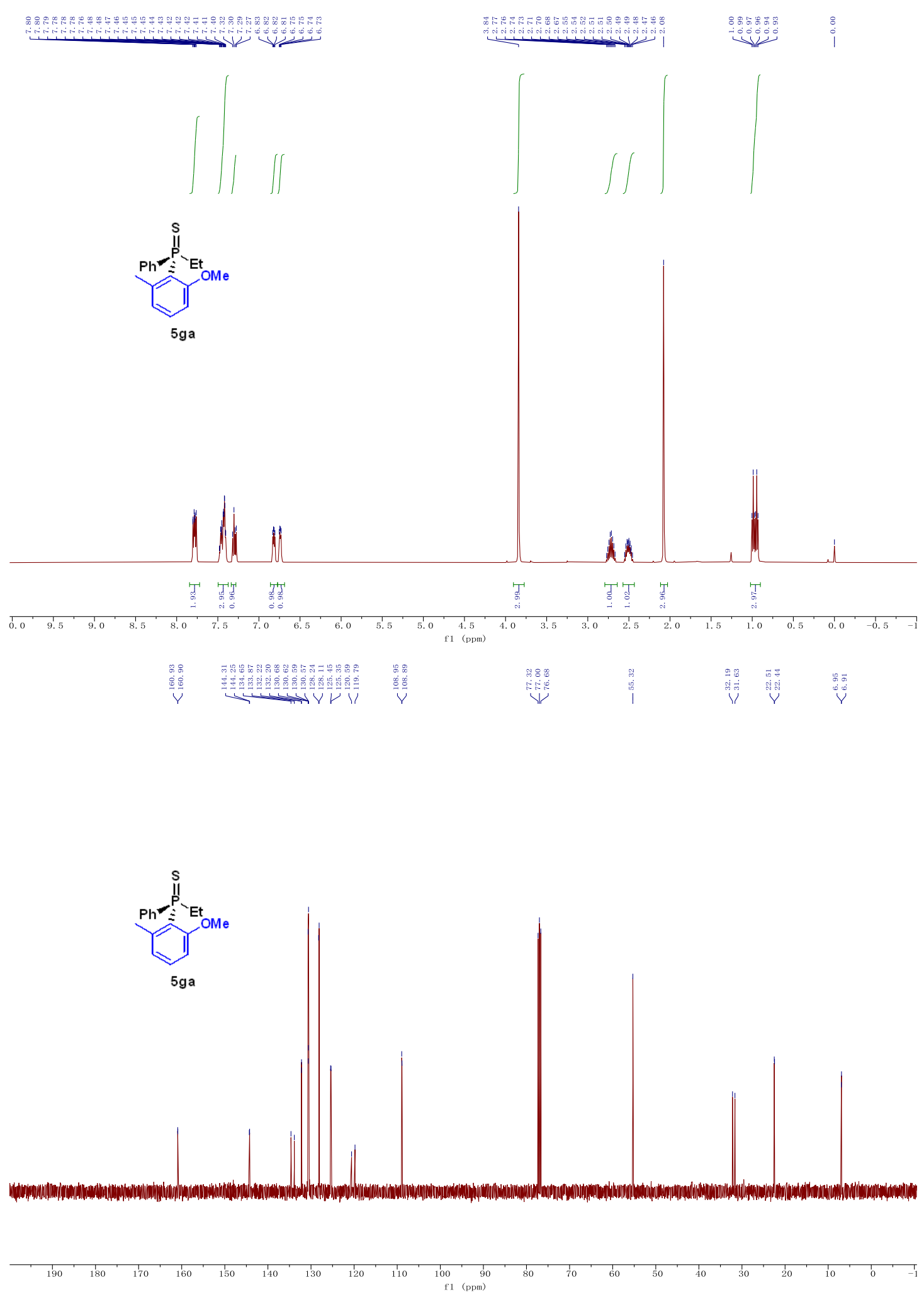

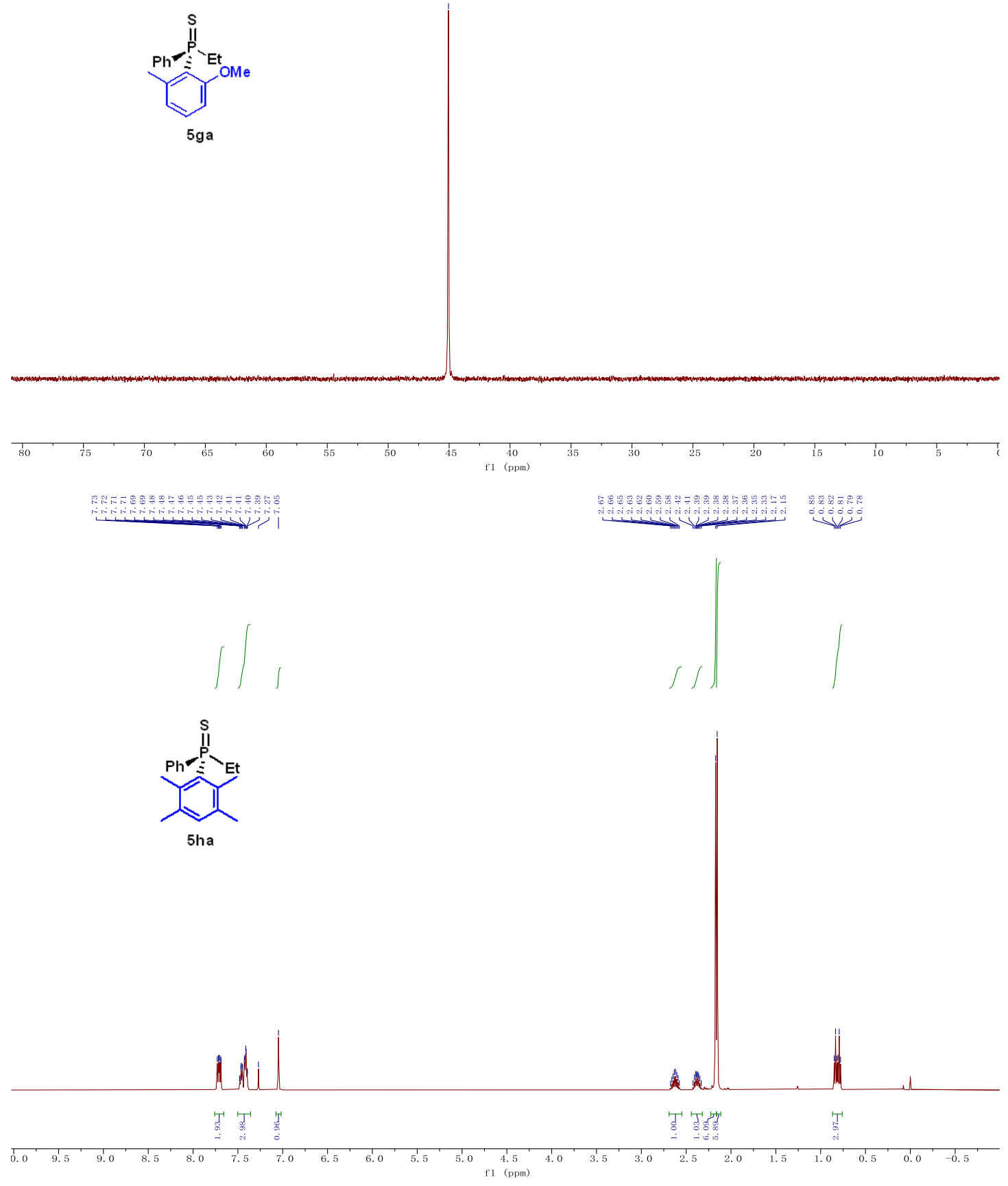

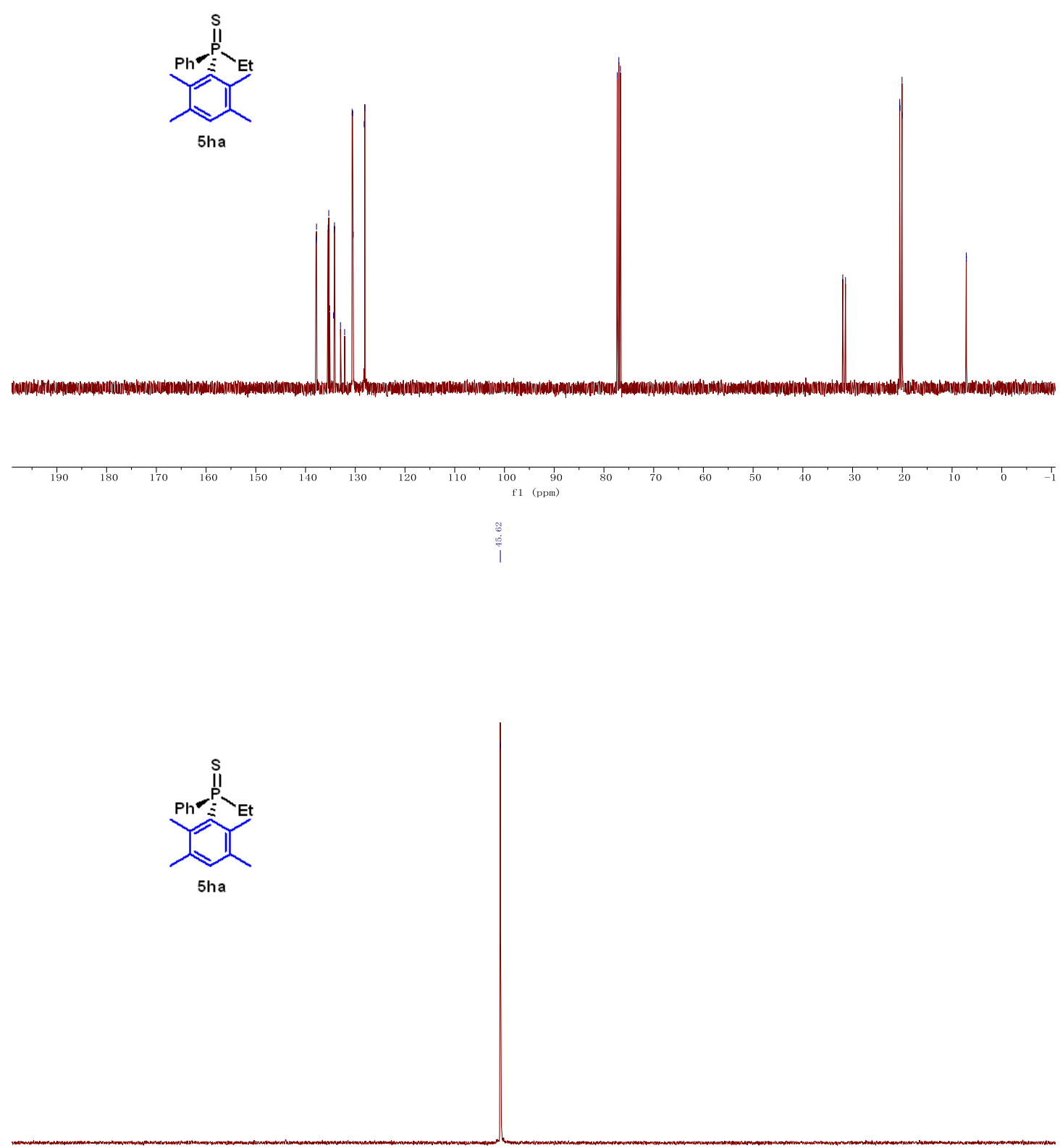

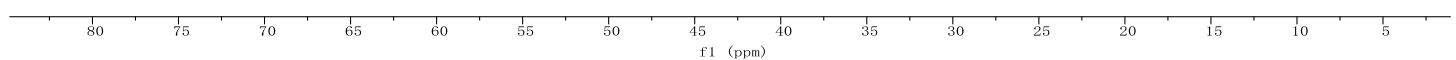



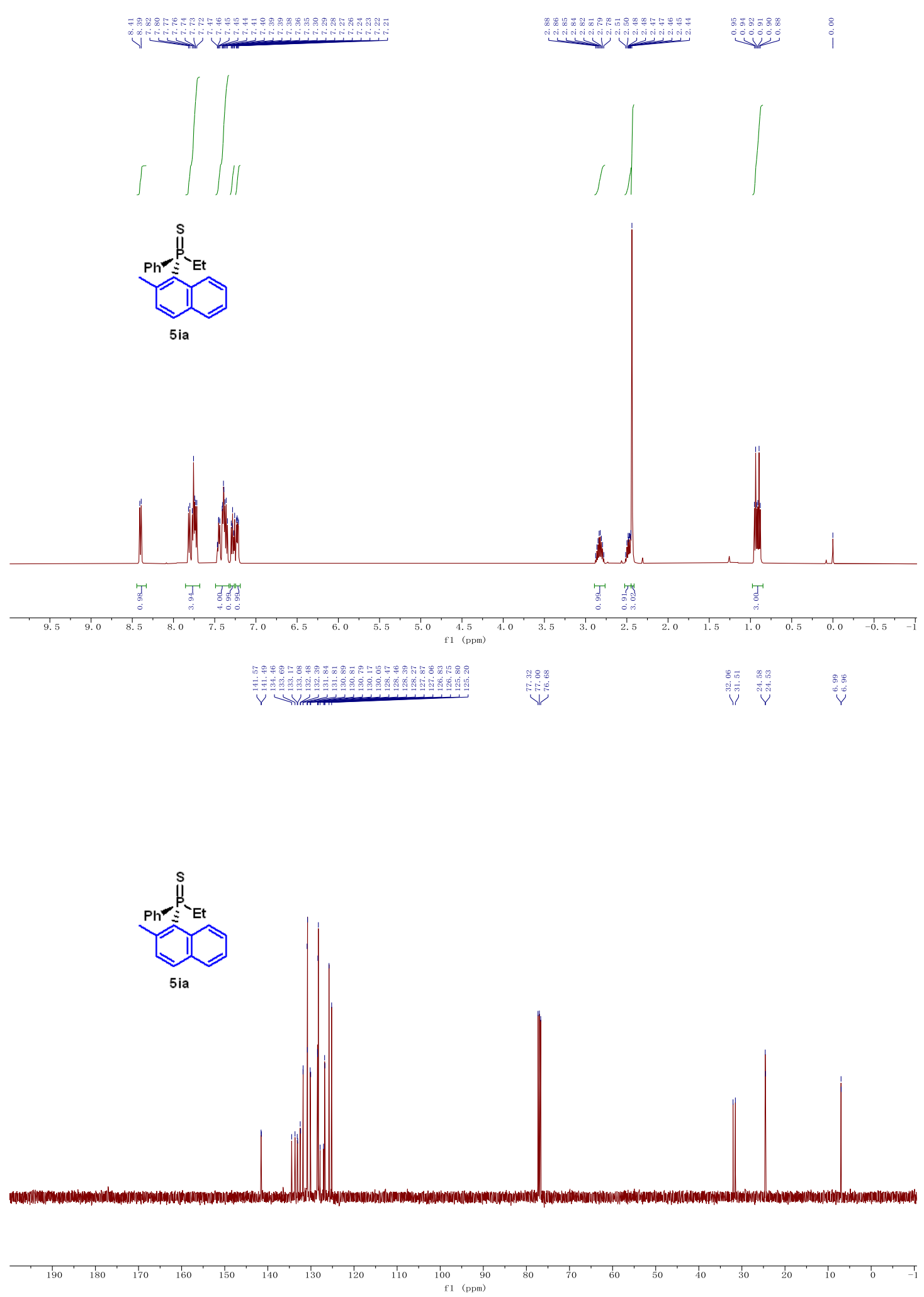

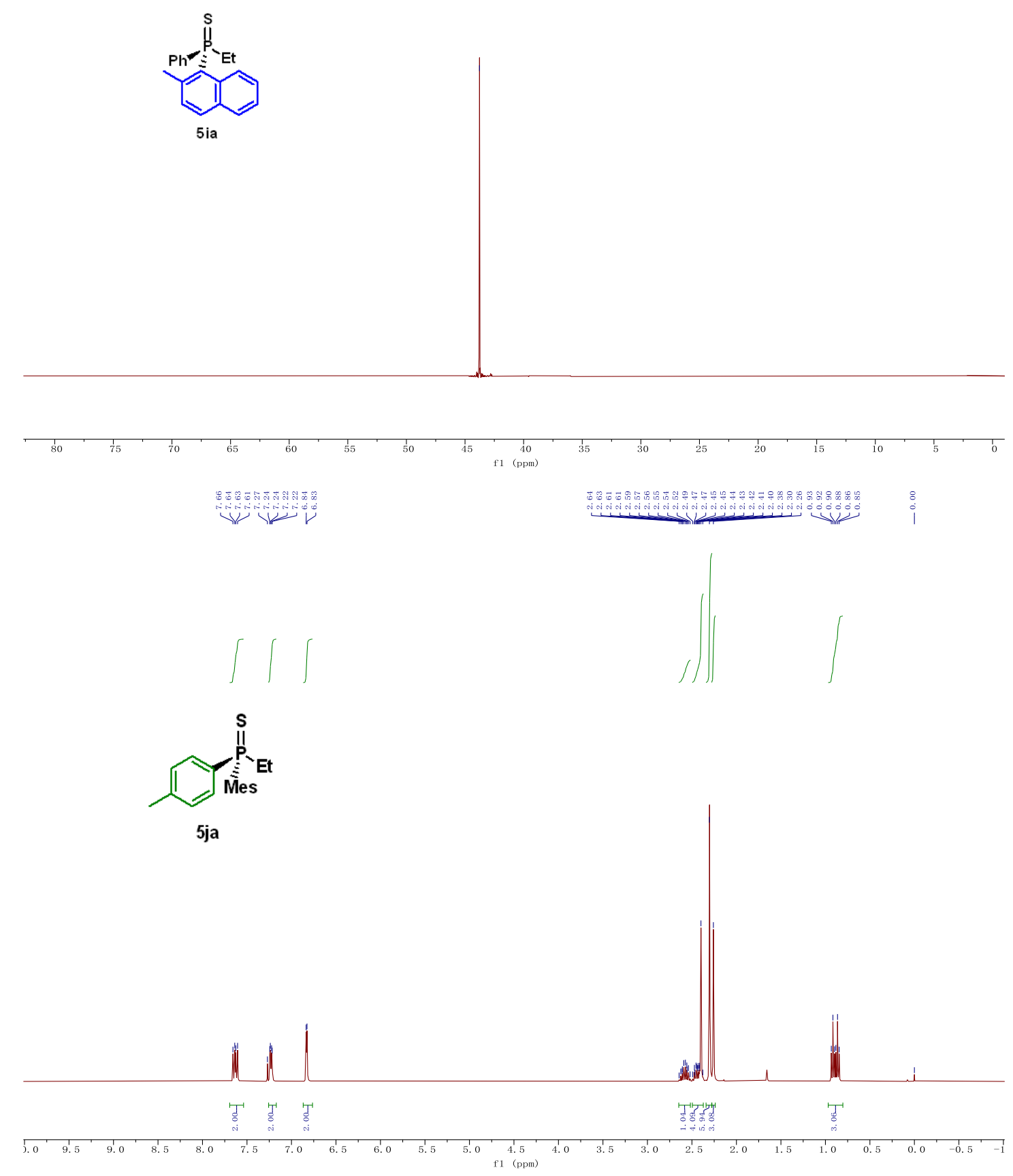

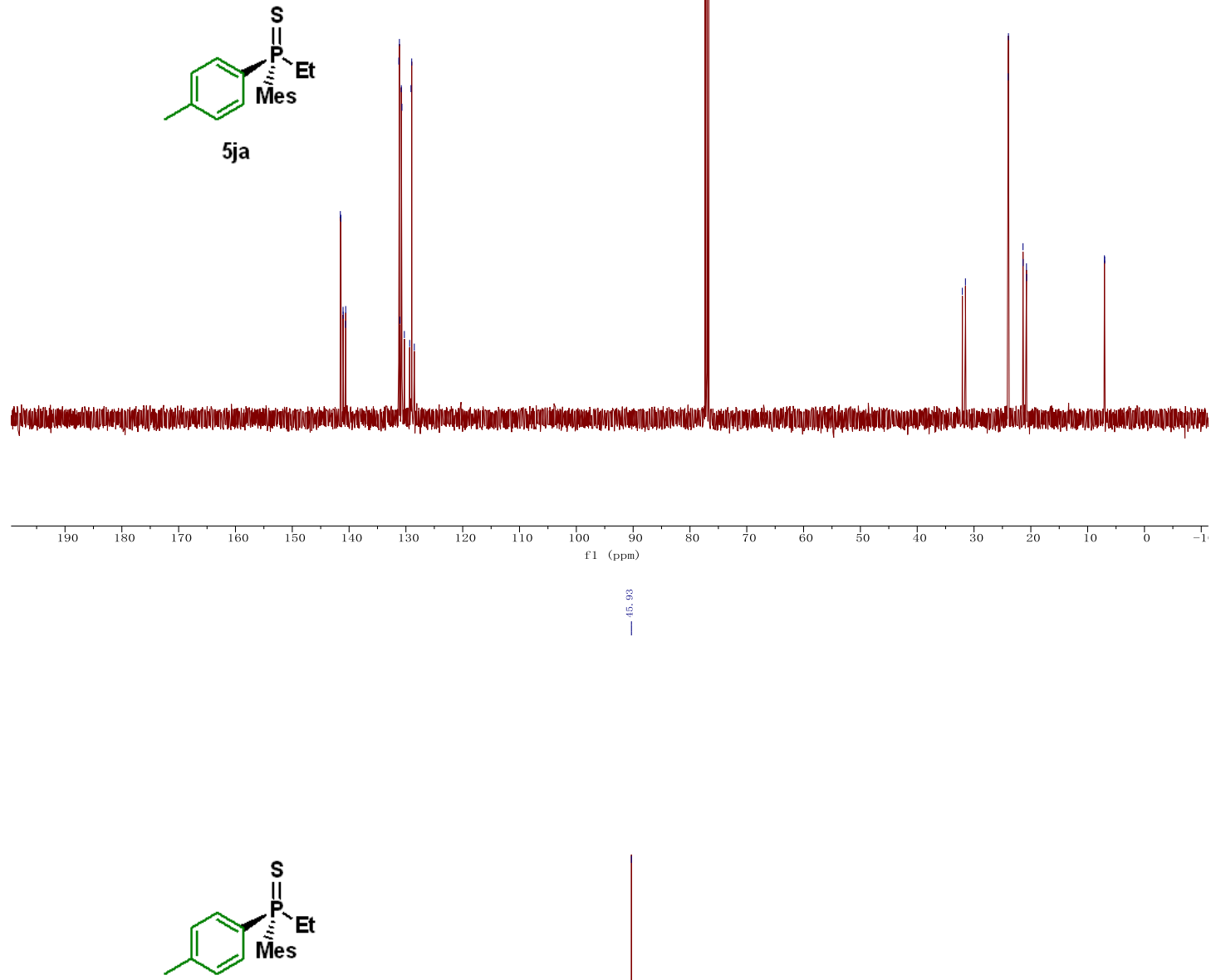

$5 j a$

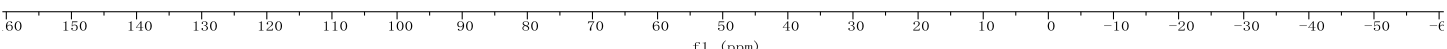




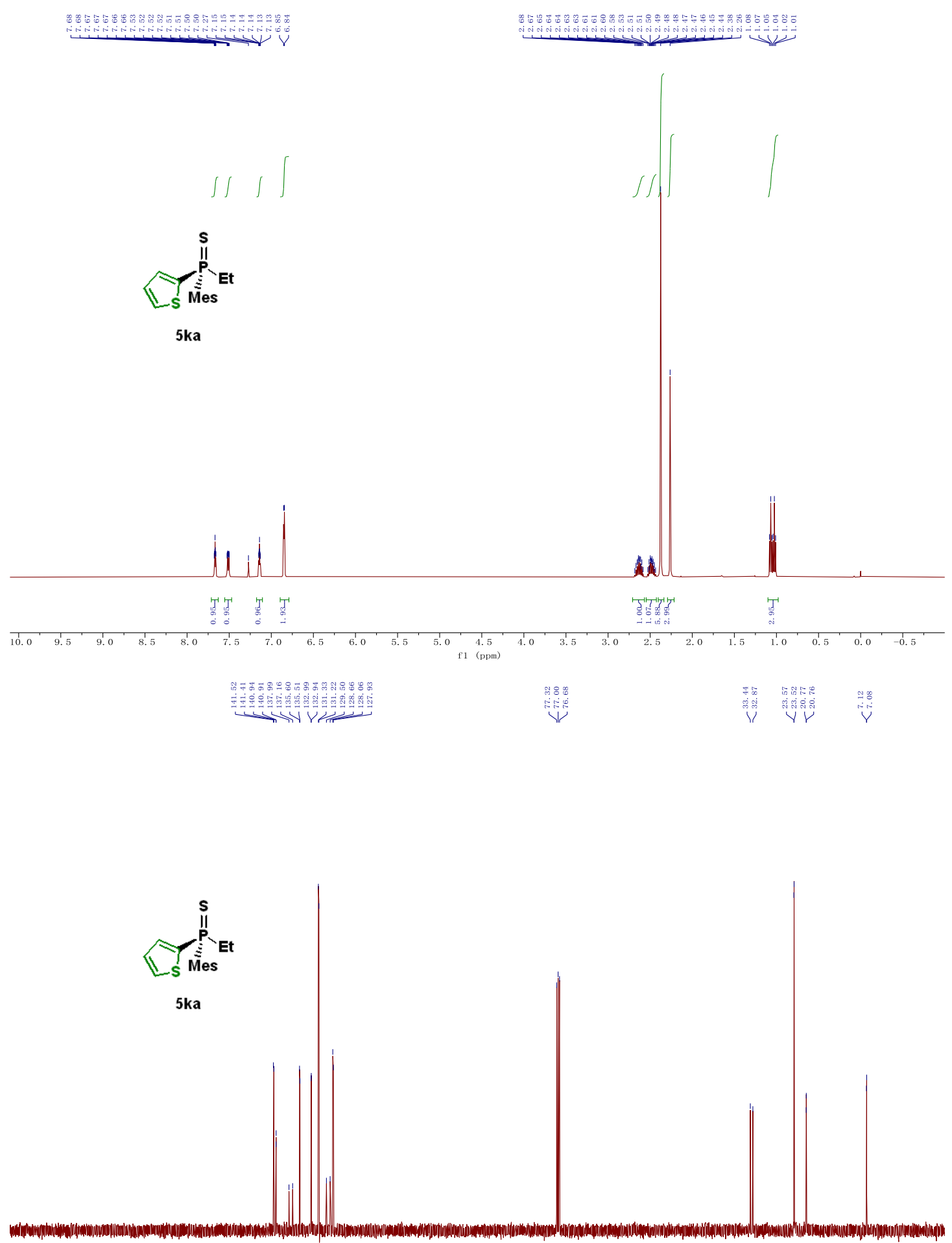

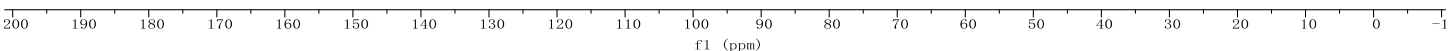




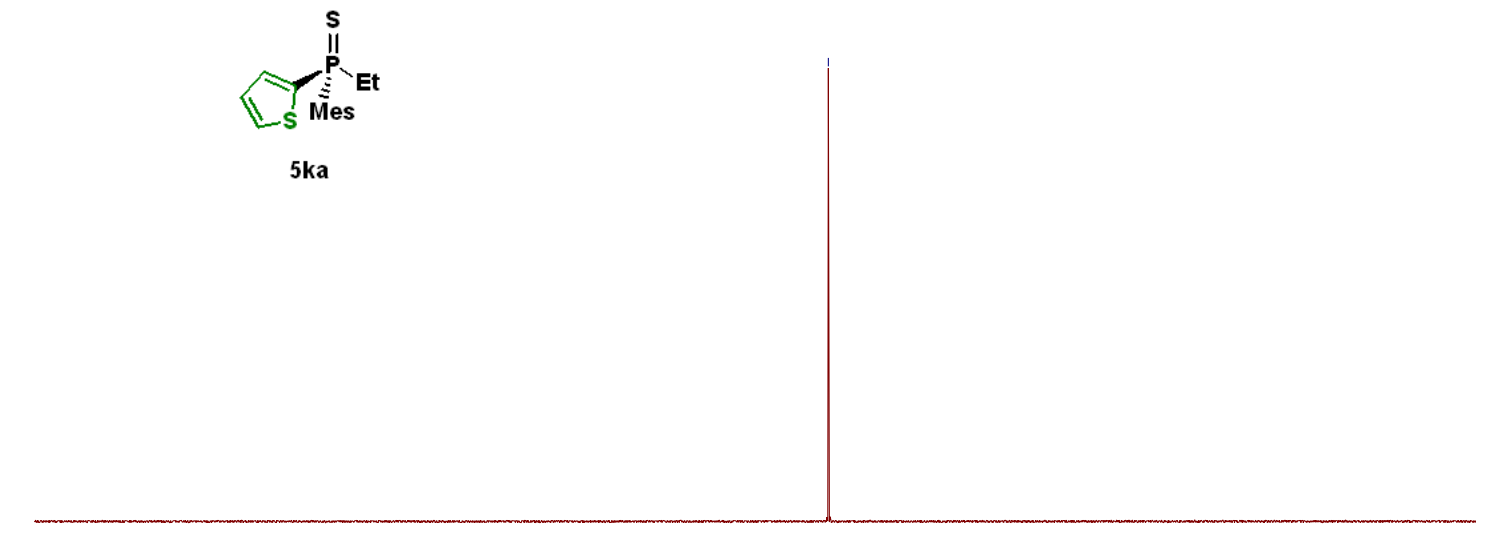

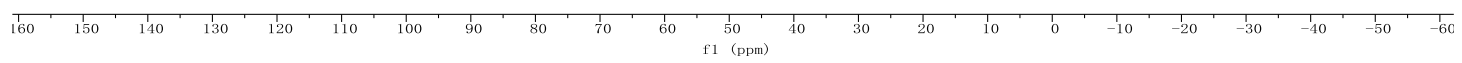

员

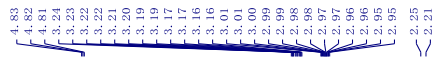

$\stackrel{8}{\stackrel{0}{0}}$

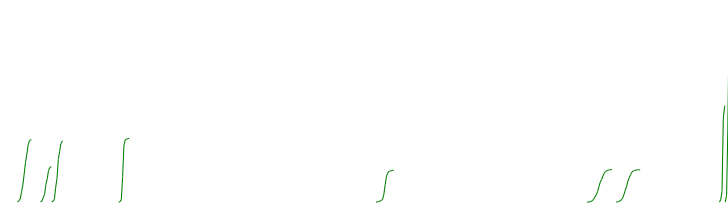

$\underbrace{\text { Mhes }}_{7 \text { aa }}$

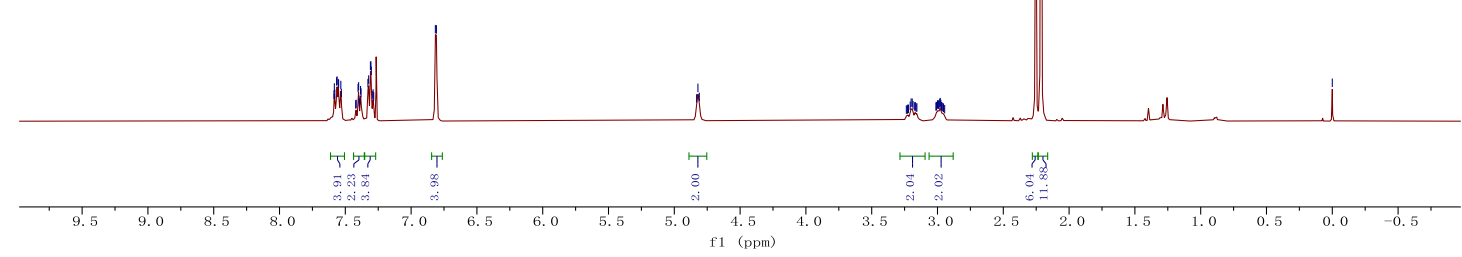



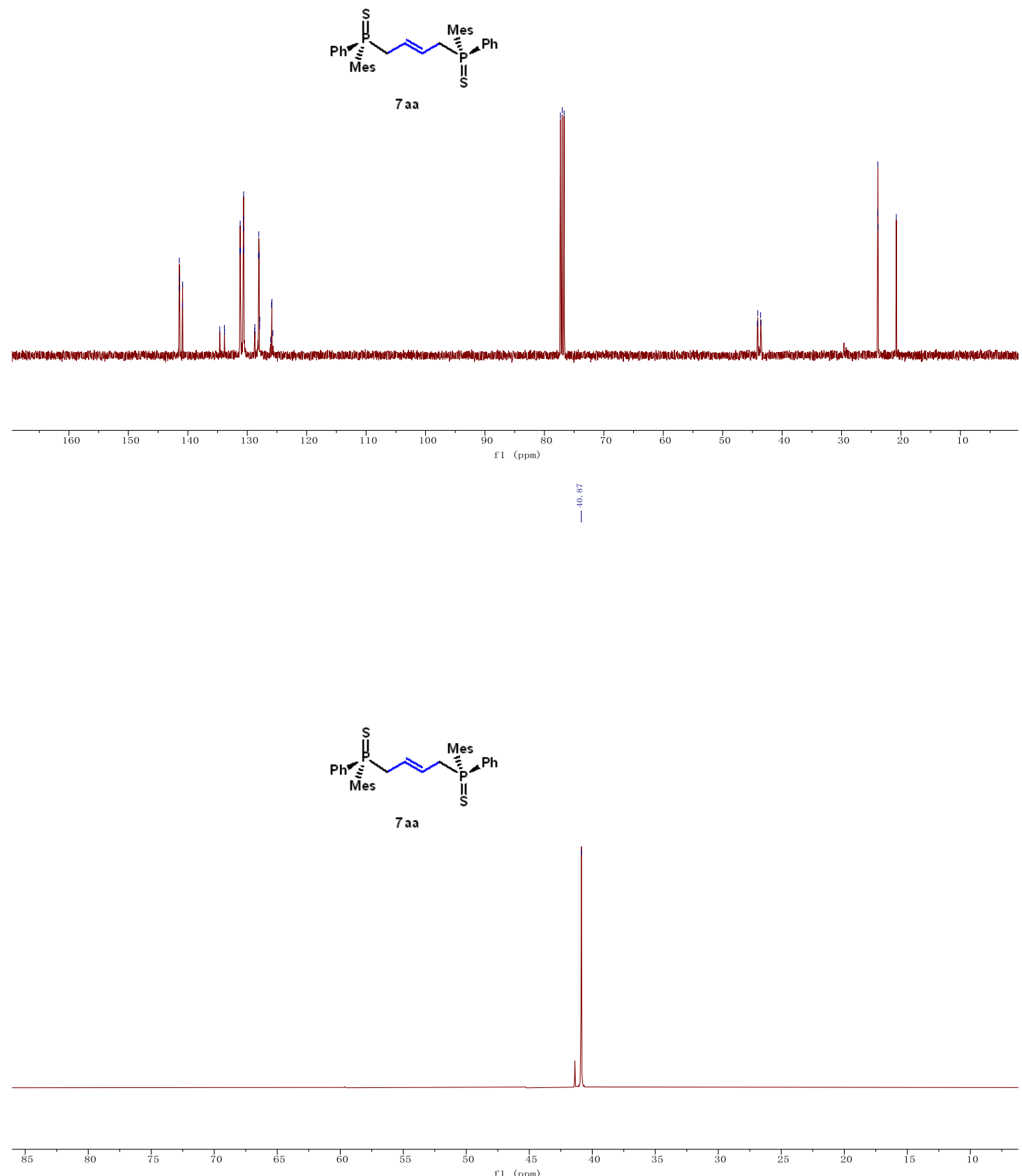

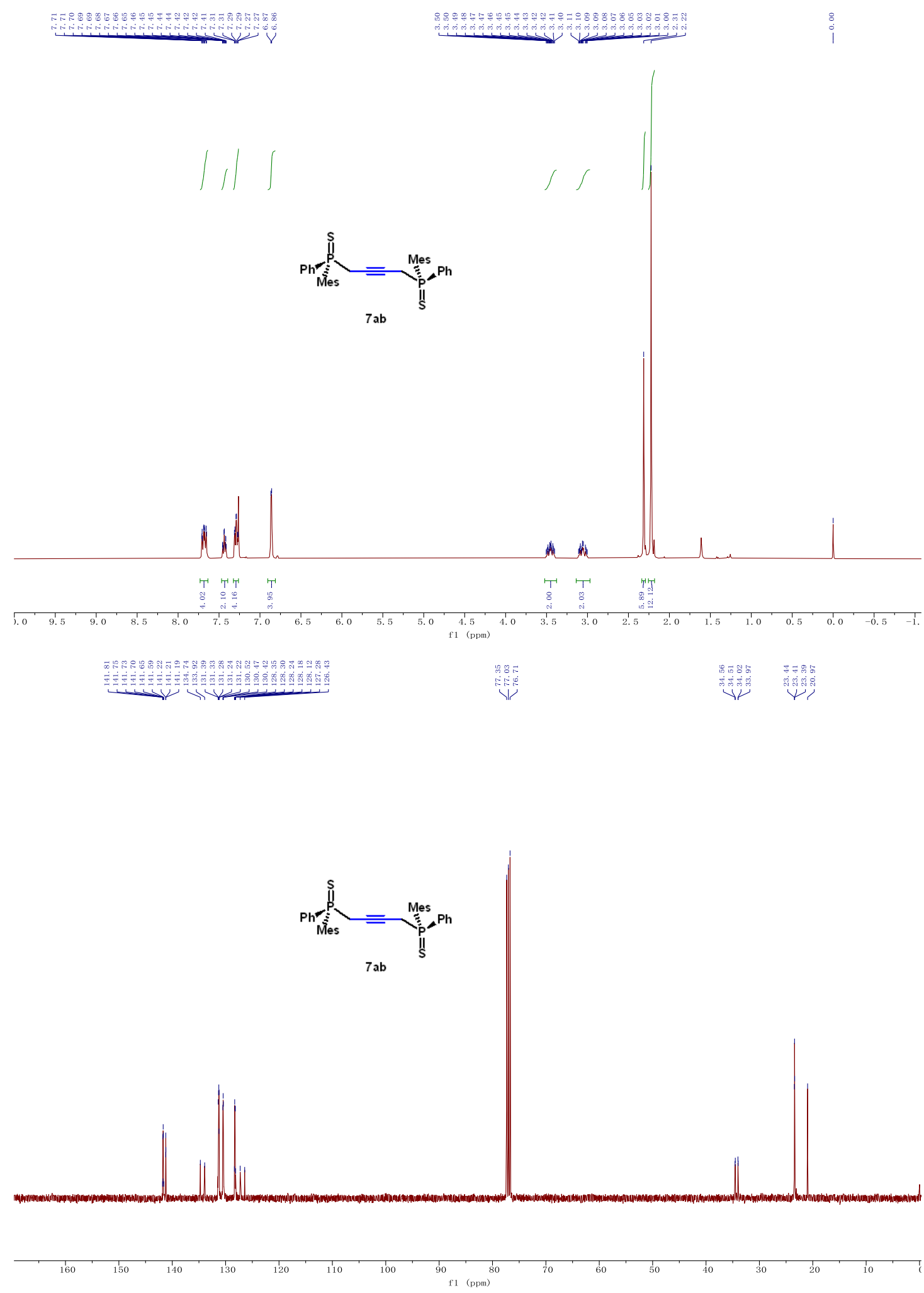

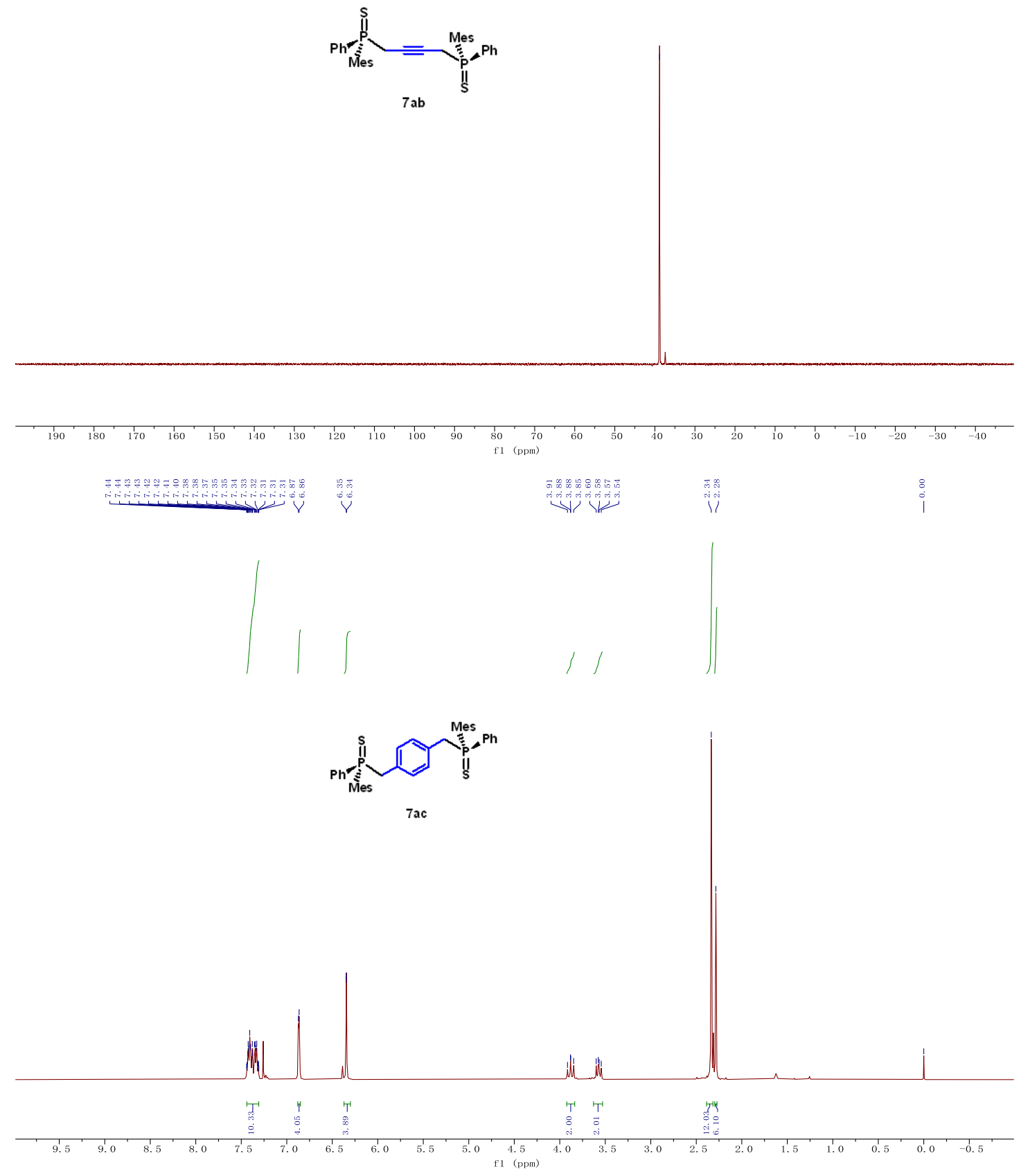

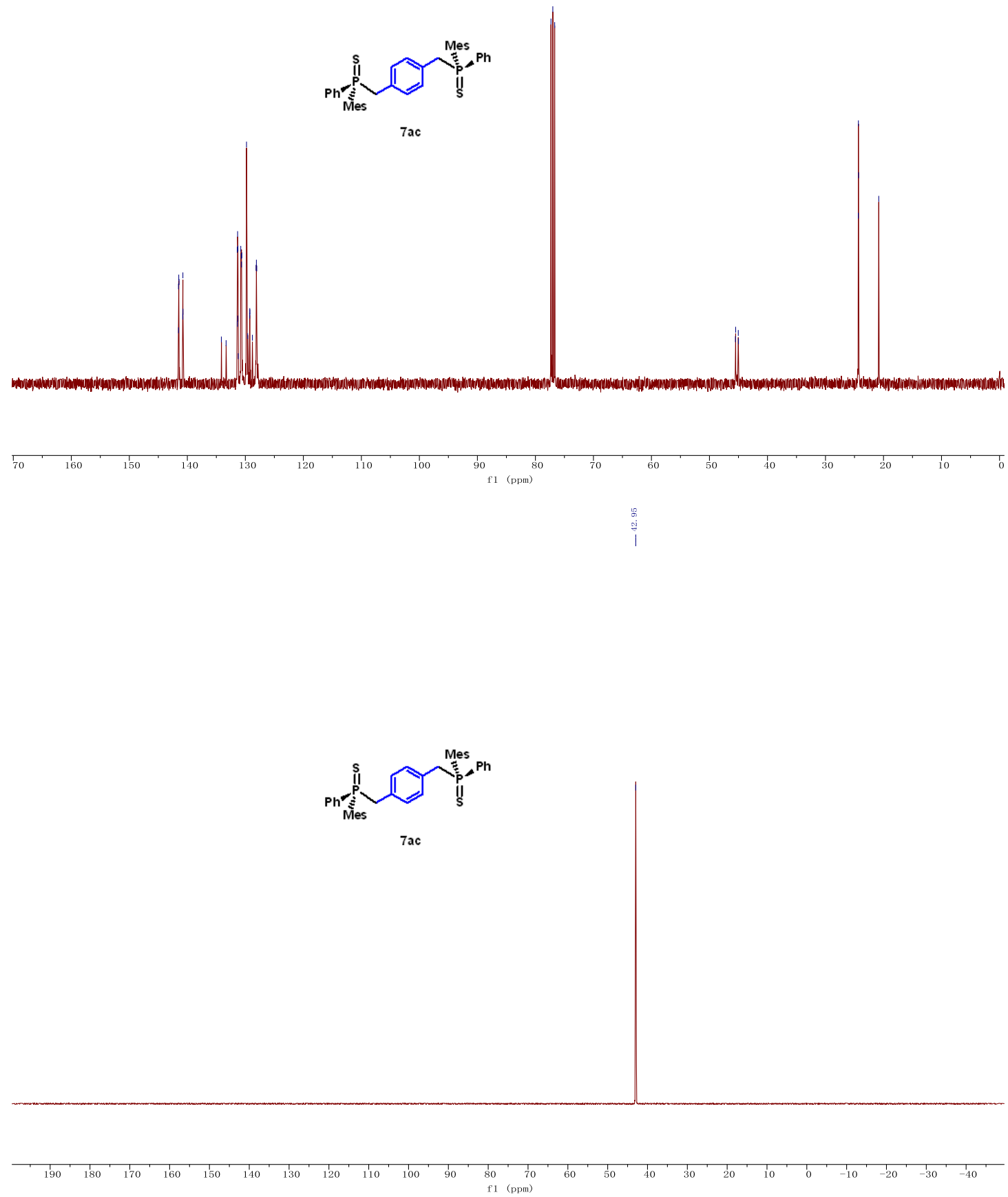

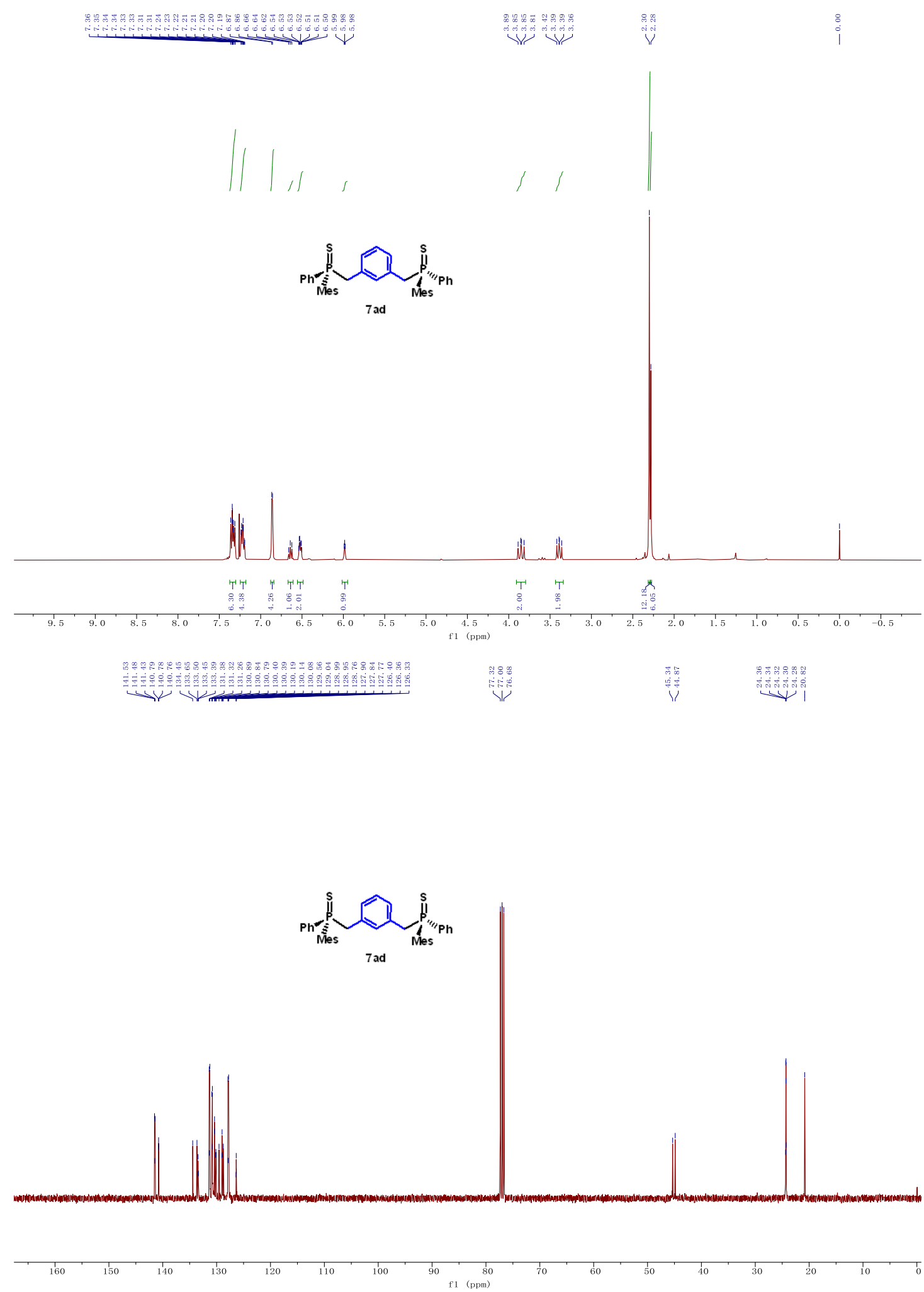

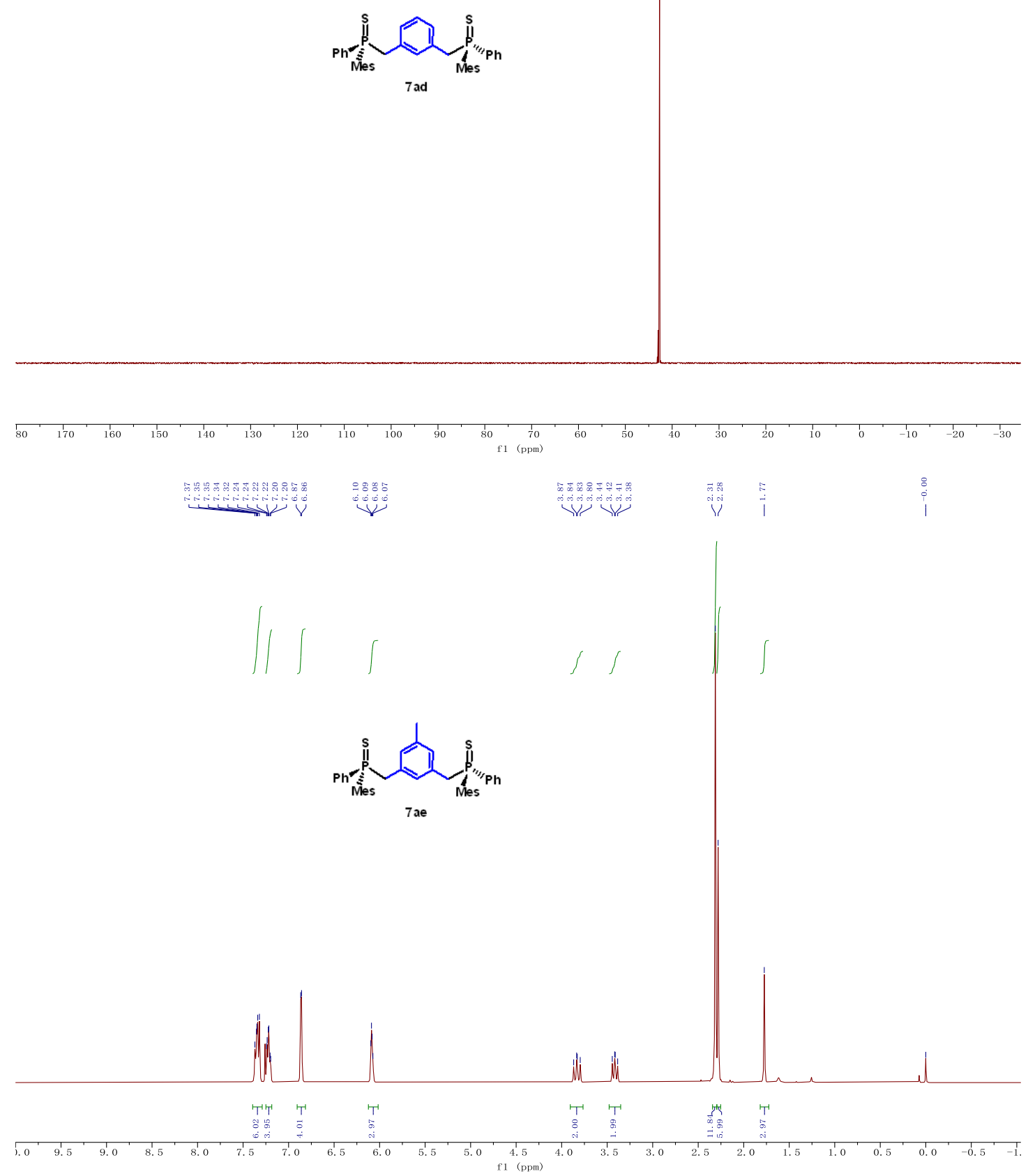

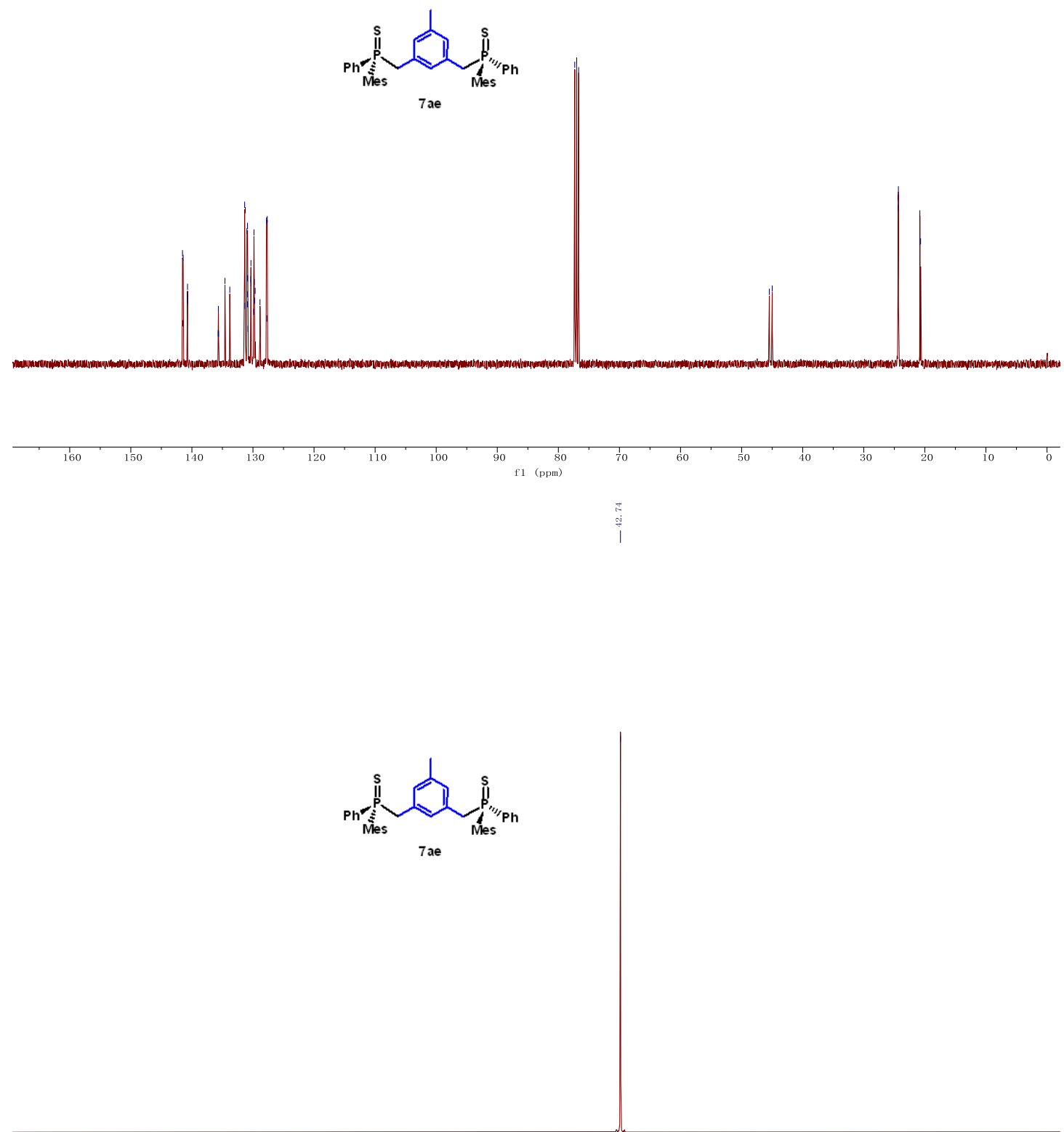

$\frac{1}{80}$ ${ }_{50}^{1}{ }_{\mathrm{f1}}(\mathrm{ppm}) \quad 45$ 


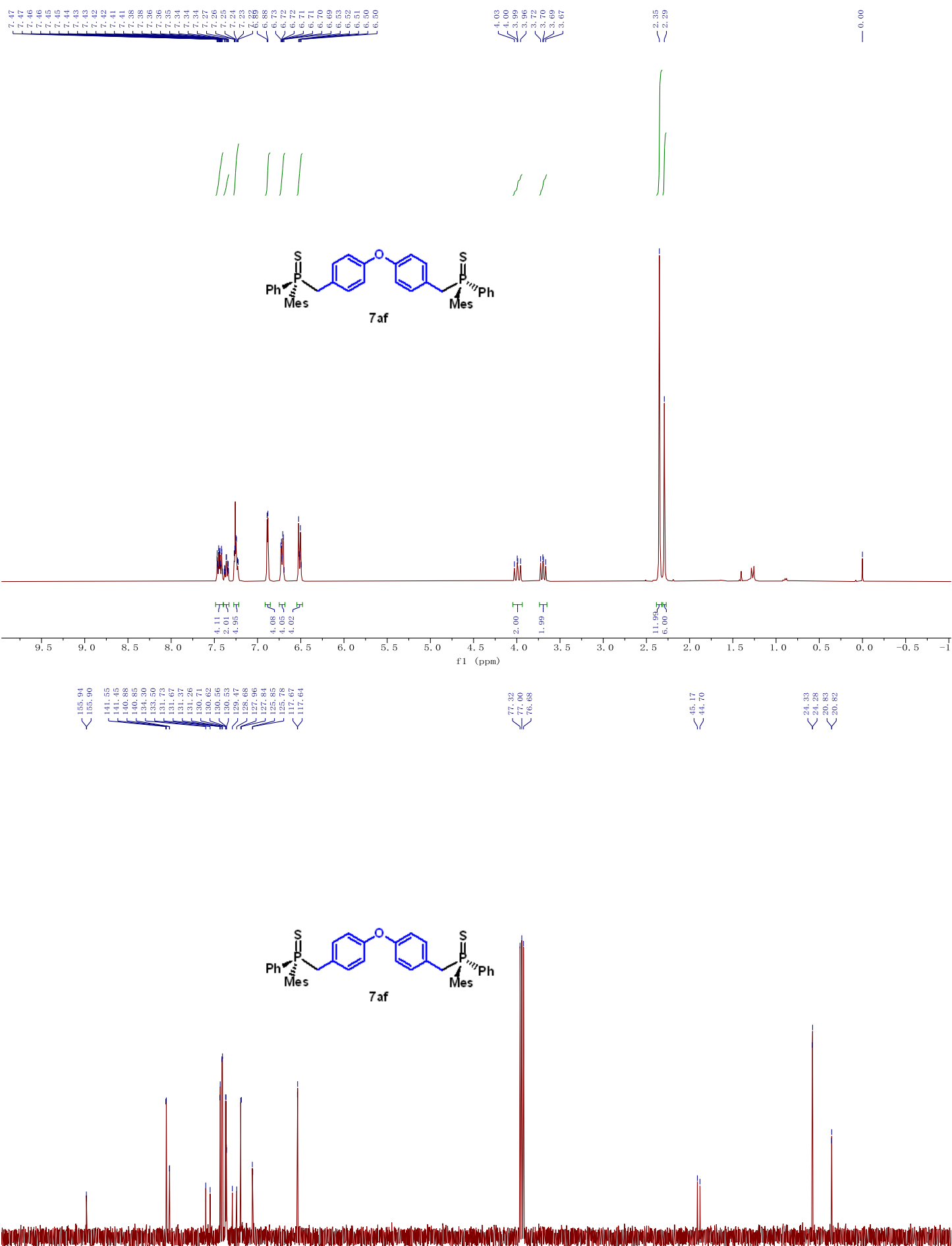

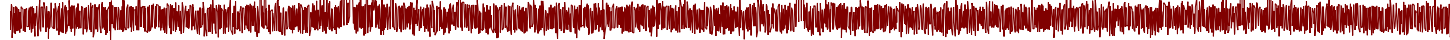

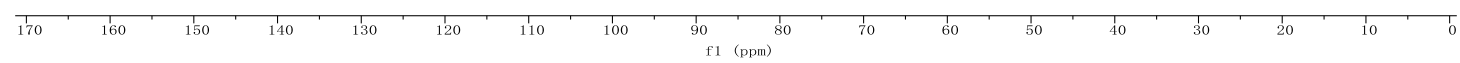



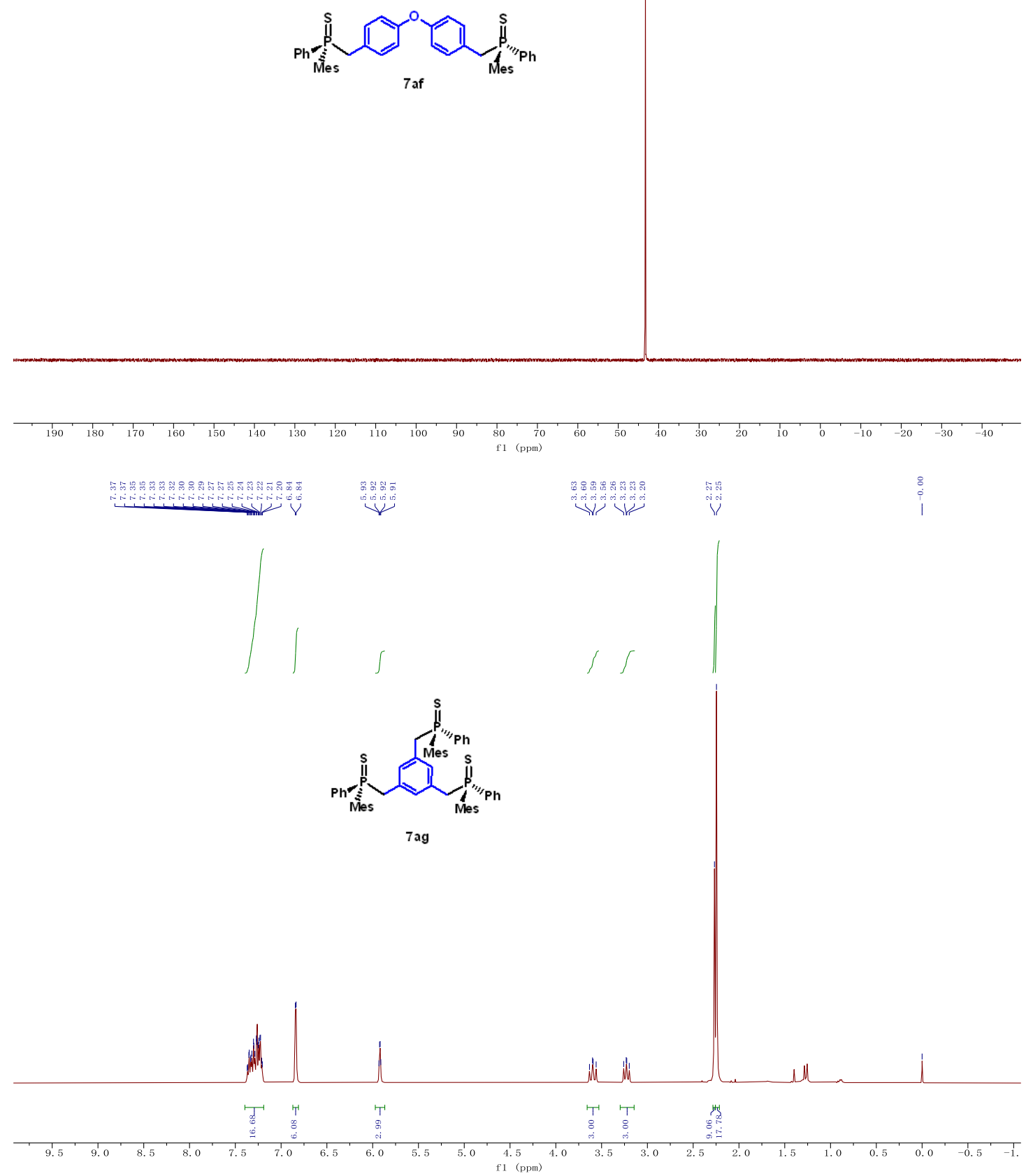


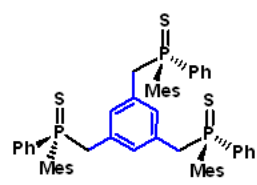

$7 \mathrm{ag}$

(1)
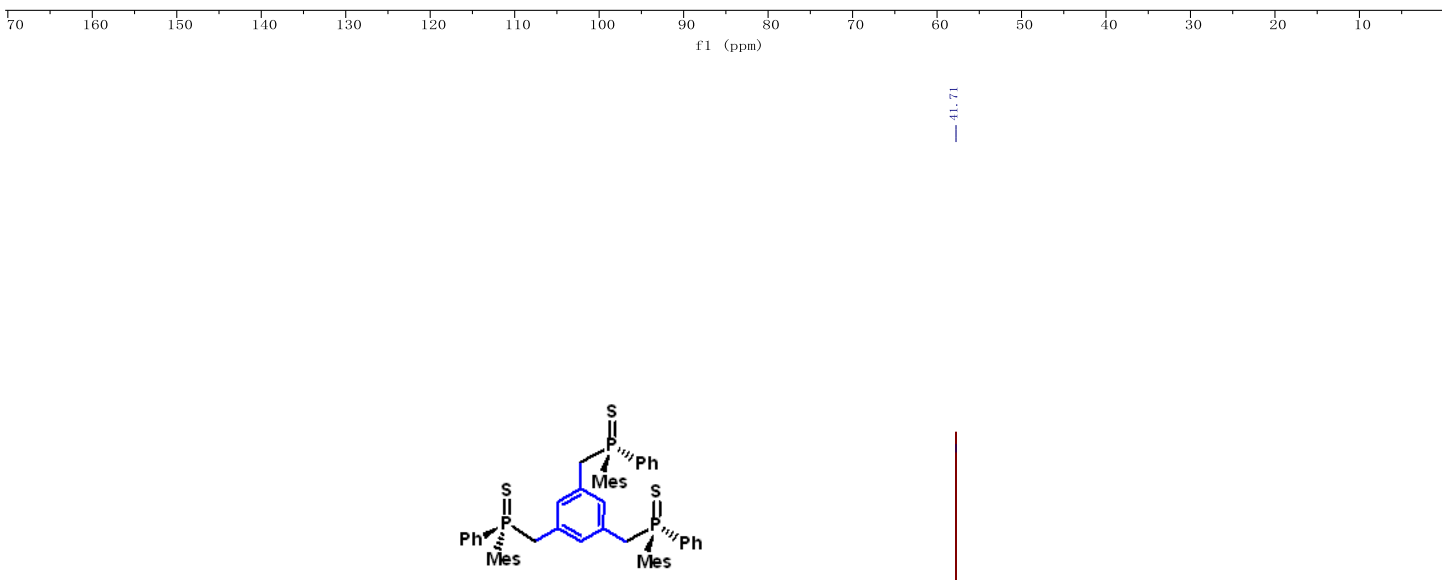

7 ag

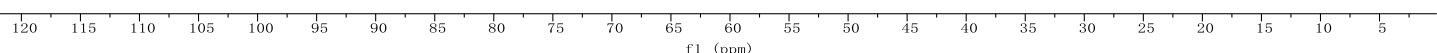




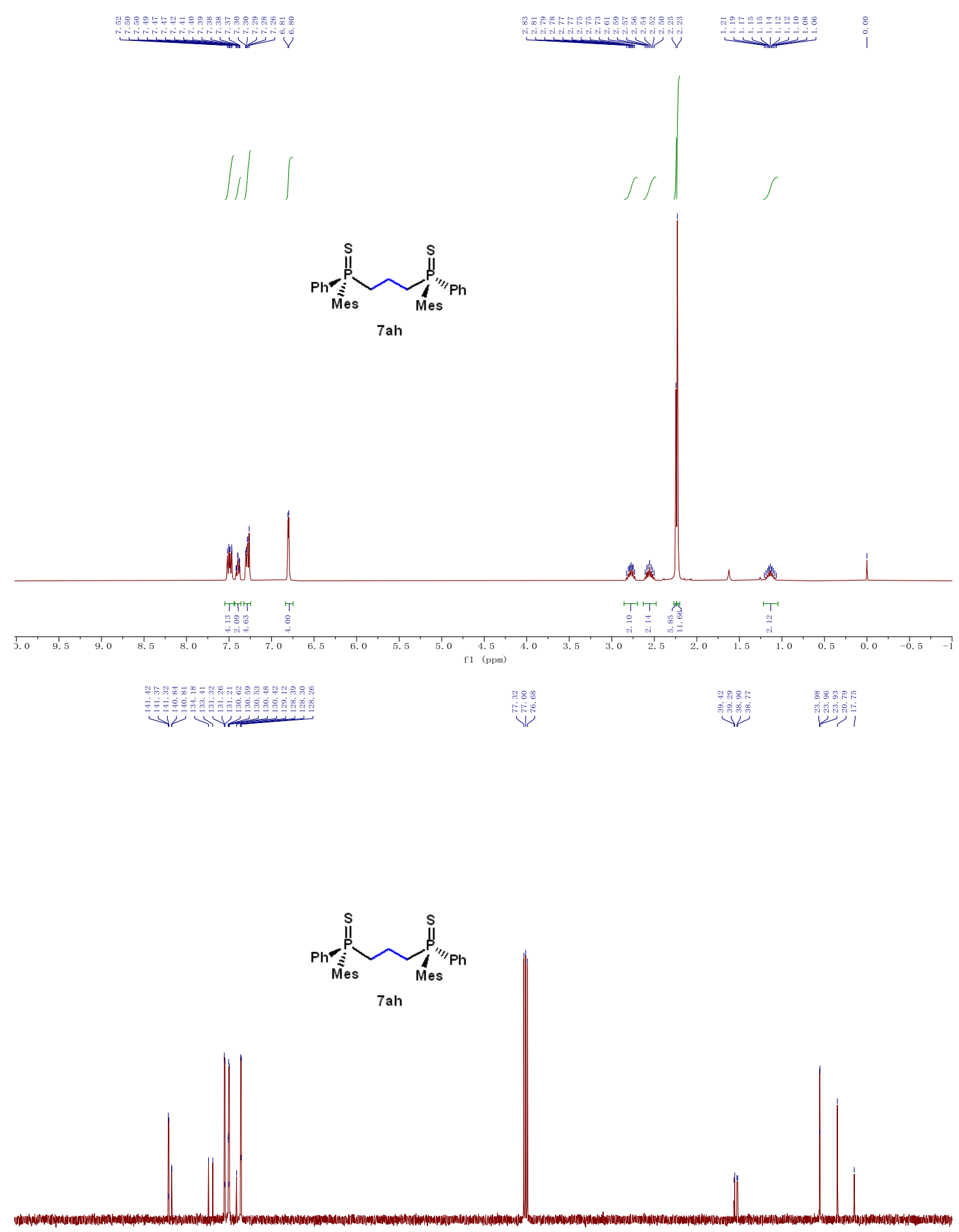

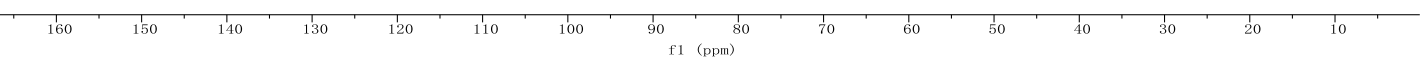



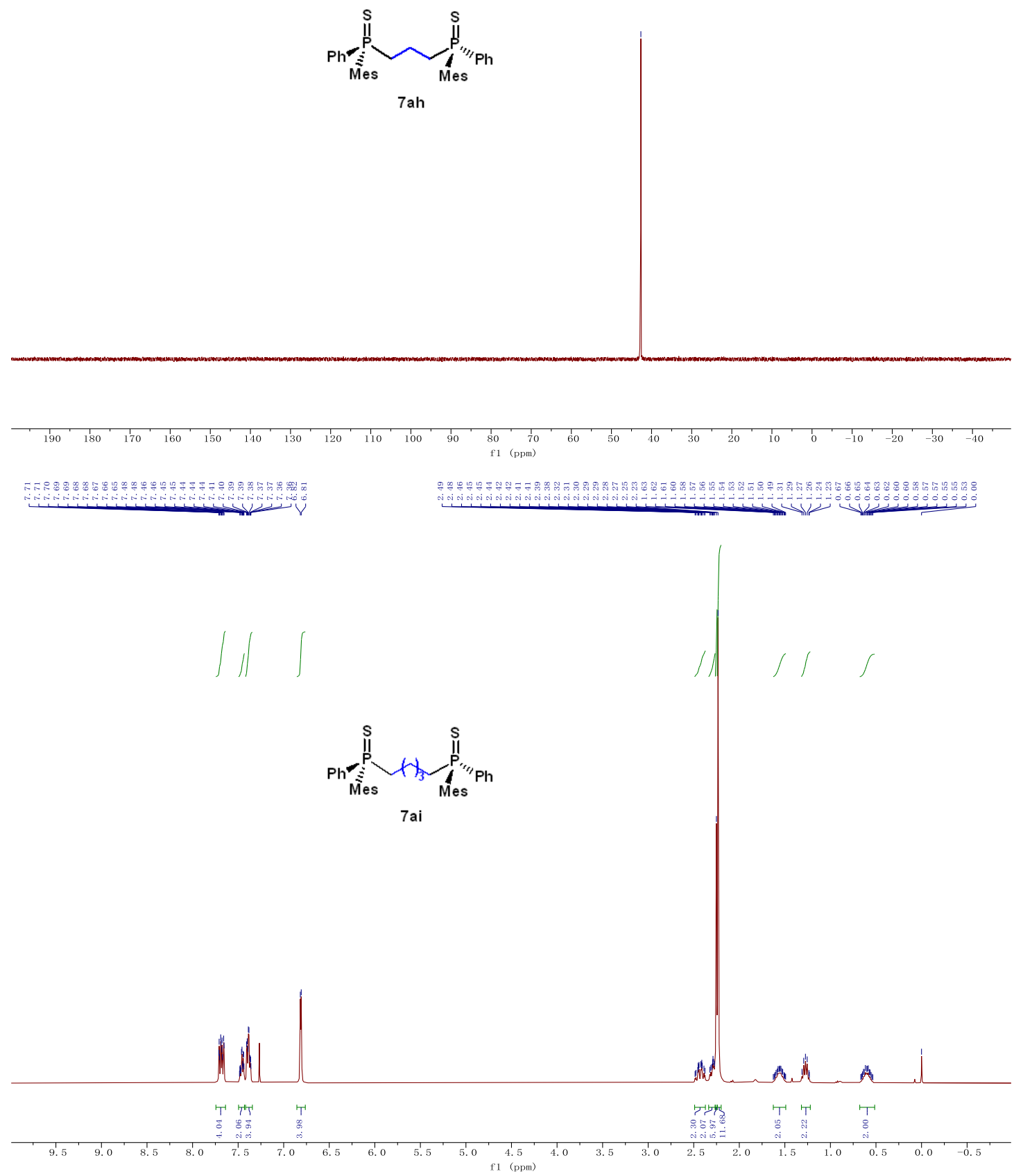

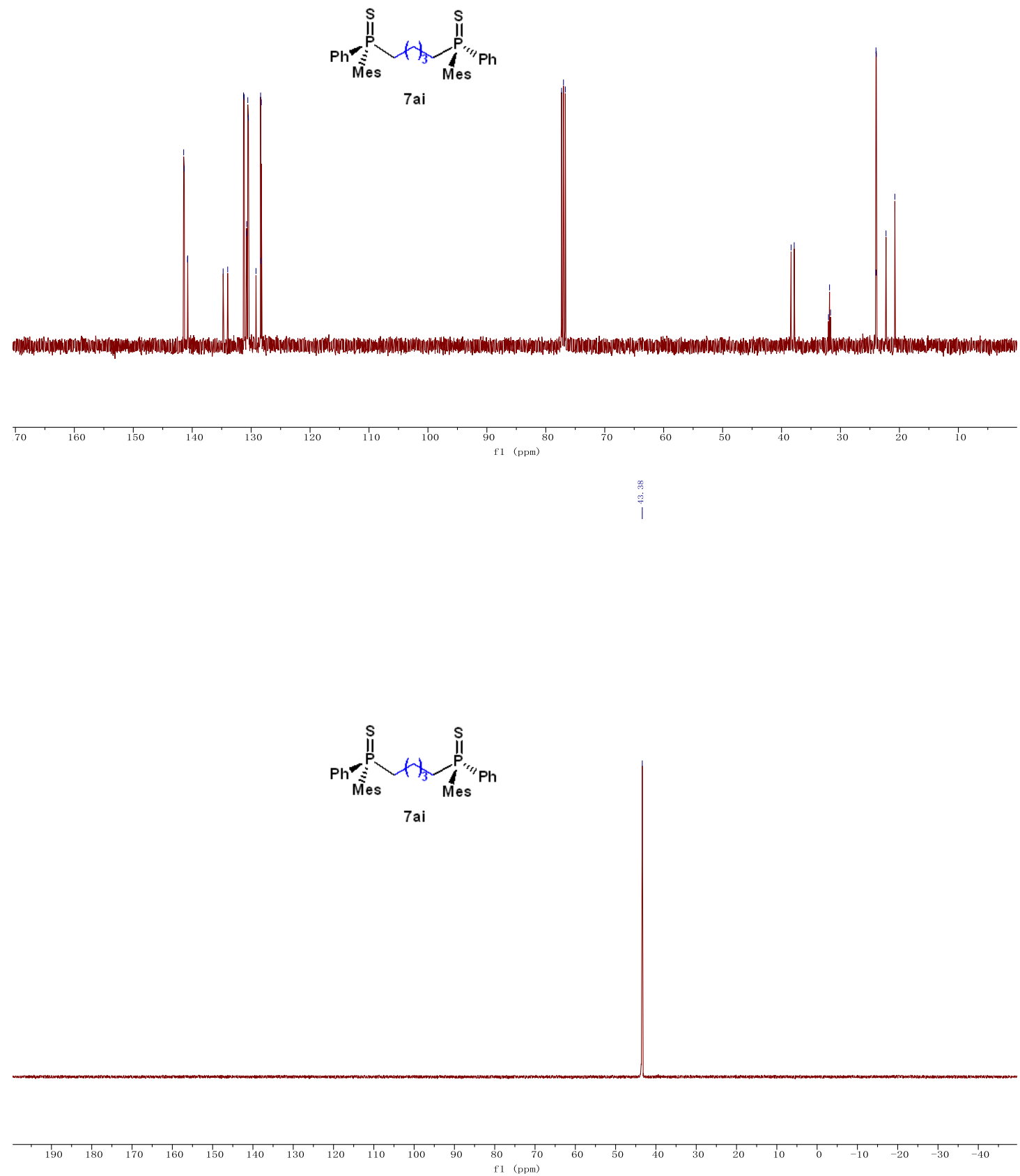

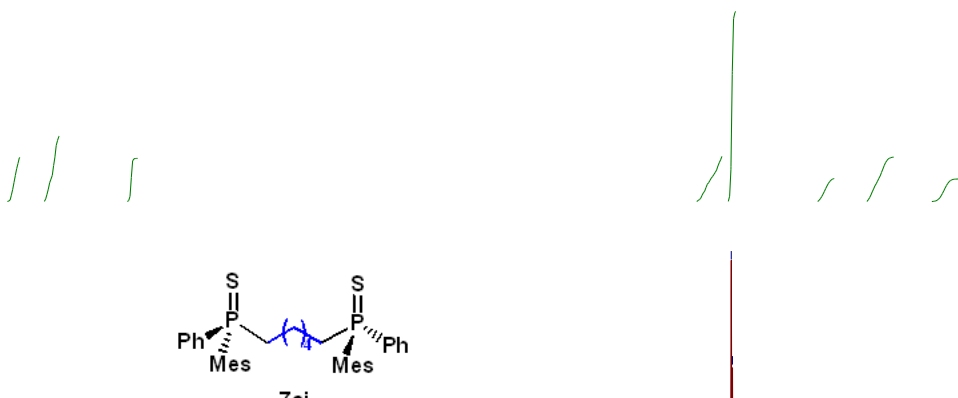

7aj
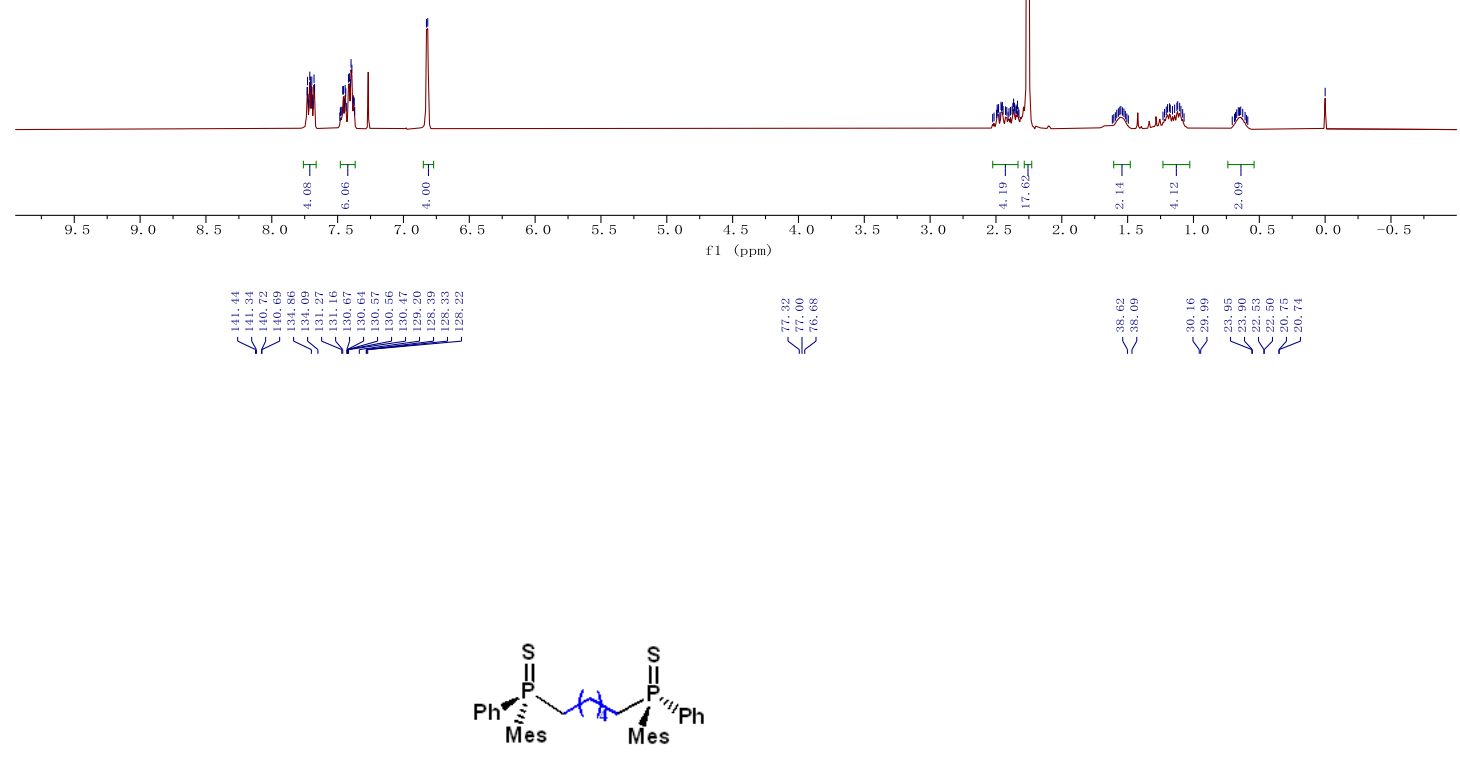

7aj
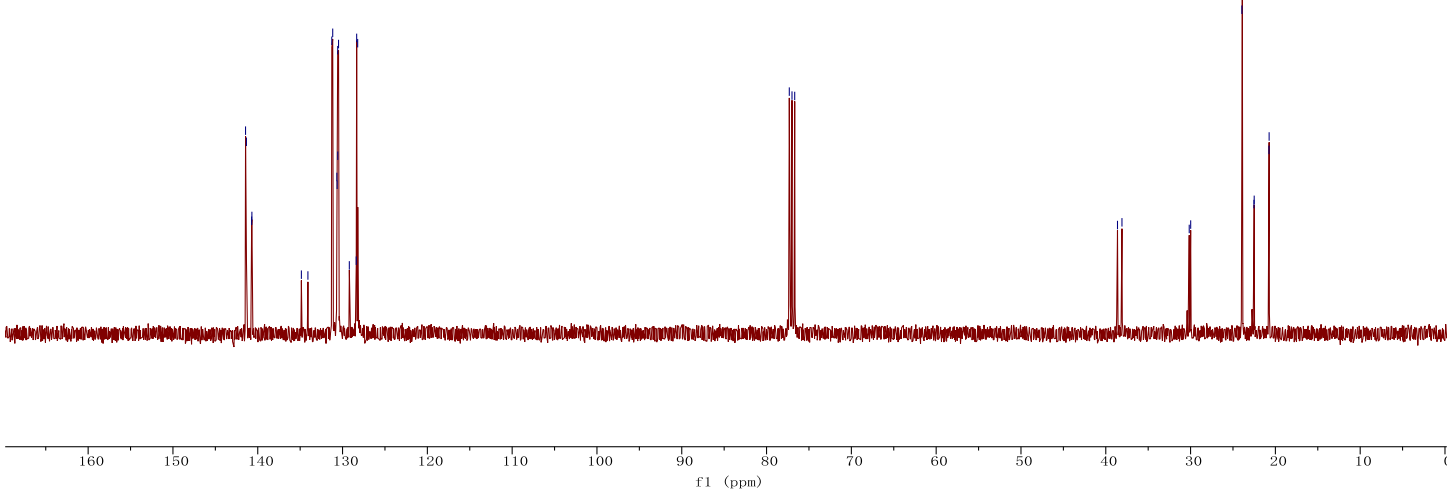


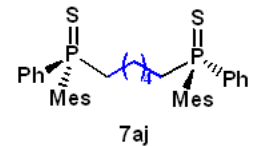

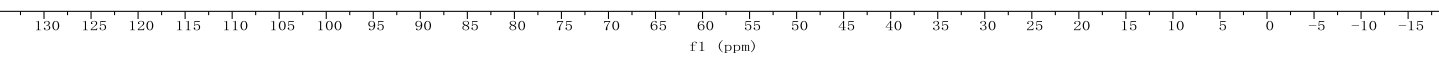

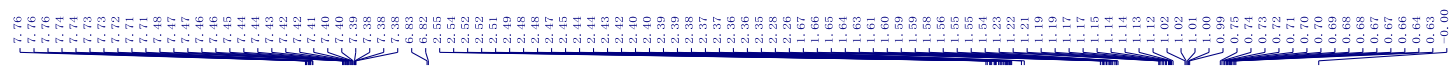

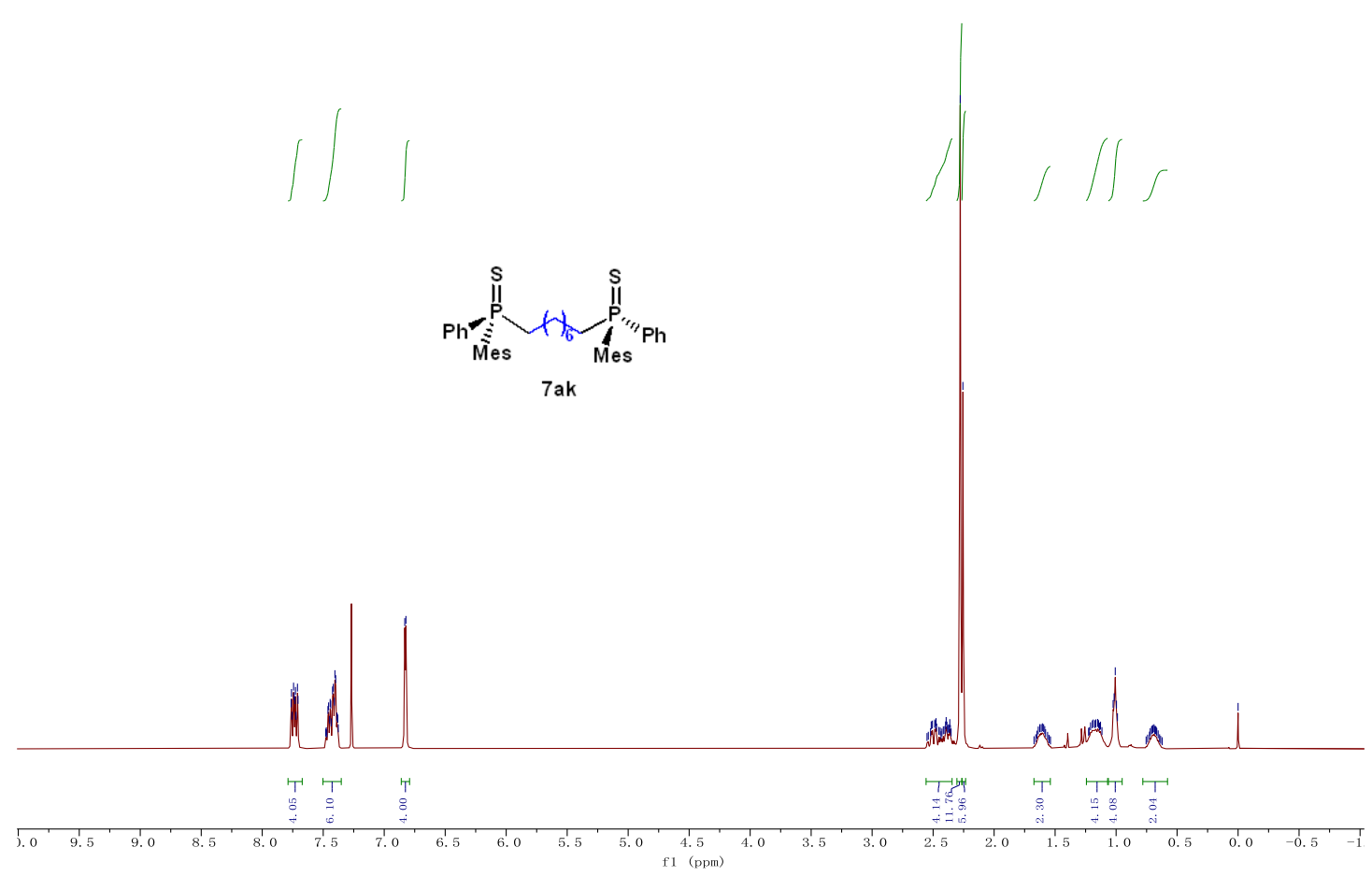




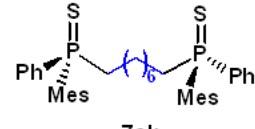
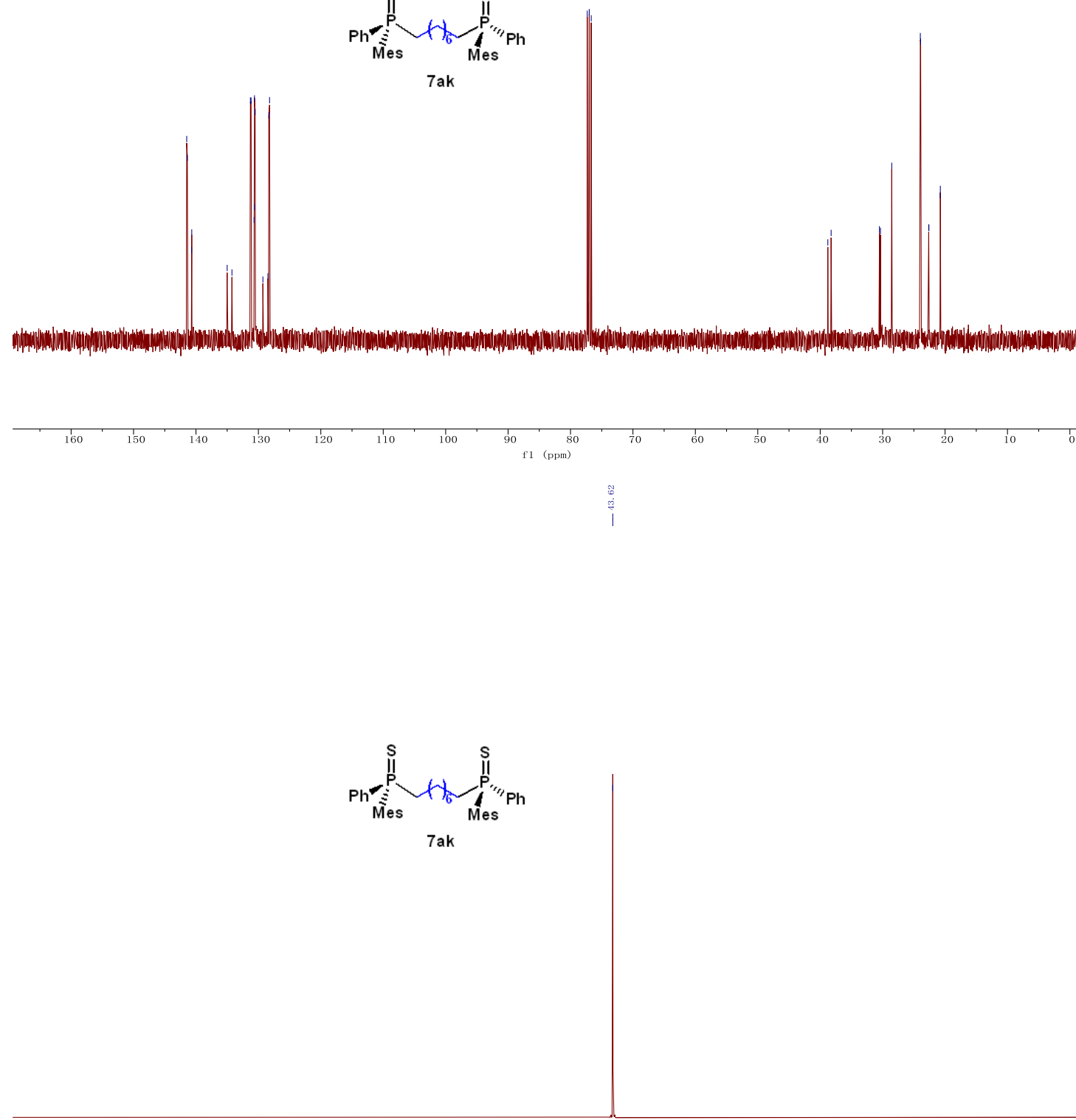

\begin{tabular}{llllllllllllllll}
\hline 120 & 115 & 110 & 105 & 100 & 95 & 90 & $\frac{1}{1}$ & $\frac{1}{1}$ & $\frac{1}{1}$ & $\frac{1}{1}$ & 1 & $\frac{1}{1}$ & $\frac{1}{1}$ \\
\hline
\end{tabular} 

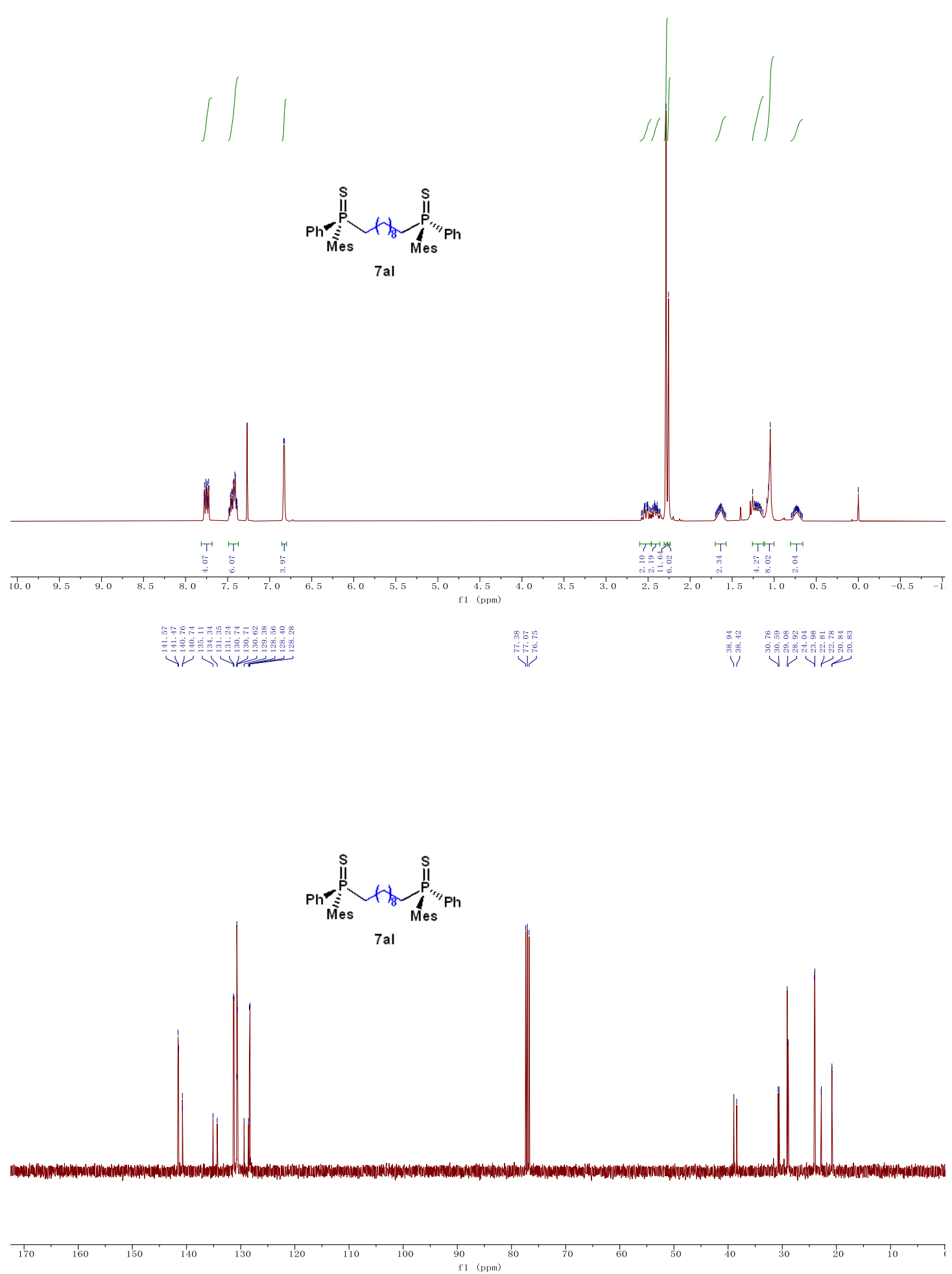
Phes

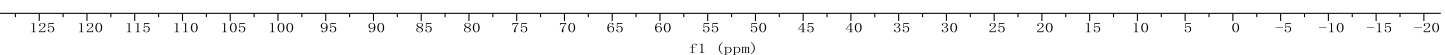

